\title{
RECENT PROGRESS IN
}

\section{HORMONE RESEARCH}

Edited by

ANTHONY R. MEANS

VOLUME 57

Reproductive Hormones \& Human Health

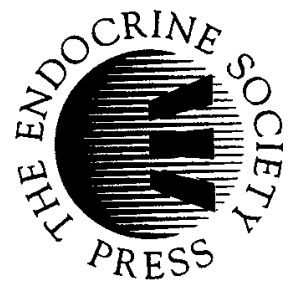

"Recent Progress in Hormone Research" is an annual publication of The Endocrine Society that is published under the editorial auspices of Endocrine Reviews. 
Copyright 2002 by The Endocrine Society

All Rights Reserved

The reproduction or utilization of this work in any form or in any electronic, mechanical, or other means, now known or hereafter invented, including photocopying or recording and in any information storage or retrieval system, is forbidden, except as may be expressly permitted by the 1976 Copyright Act or by permission of the publisher.

ISBN 0-879225-45-X 


\section{CONTENTS}

Senior Author Correspondence Information $\quad$ V

1. Cellular and Molecular Pathways Regulating Mammalian Sex Determination 1 Christopher Tilmann and Blanche Capel

2. Steroidogenic Factor 1: An Essential Mediator of Endocrine Development Keith L. Parker, Douglas A. Rice, Deepak S. Lala, Yayoi Ikeda, Xunrong Luo, Margaret Wong, Marit Bakke, Liping Zhao, Claudia Frigeri, Neil A. Hanley, Nancy Stallings, and Bernard P. Schimmer

3. Molecular Basis of Mammalian Gamete Binding David J. Miller, Xudong Shi, and Heather Burkin

4. Regulation of the Mitotic and Meiotic Cell Cycles in the Male Germ Line Debra J. Wolgemuth, Erika Laurion, and Karen M. Lele

5. Male Germ Cell Gene Expression Edward M. Eddy

6. The Expression of the Follicle-stimulating Hormone Receptor in Spermatogenesis Leslie L. Heckert and Michael D. Griswold

7. Identification of Specific Sites of Hormonal Regulation in Spermatogenesis in Rats, Monkeys, and Man

R.I. McLachlan, L. O’Donnell, S.J. Meachem, P.G. Stanton, D.M. de Kretser, K. Pratis, and D.M. Robertson

8. Molecular Defects of the Androgen Receptor Michael J. McPhaul

9. Novel Signaling Pathways That Control Ovarian Follicular Development, Ovulation, and Luteinization

JoAnne S. Richards, Darryl L. Russell, Scott Ochsner, Minnie Hsieh,

Kari H. Doyle, Allison E. Falender, Yuet K. Lo, and S. Chidananda Sharma

10. Transcription Factors Underlying the Development and Endocrine Functions of the Placenta

James C. Cross, Lynn Anson-Cartwright, and Ian C. Scott

11. Neuroendocrine Modulation and Repercussions of Female Reproductive Aging Phyllis M. Wise, Matthew J. Smith, Dena B. Dubal, Melinda E. Wilson, Shane W. Rau, Adrienne B. Cashion, Martina Böttner, and Katherine L. Rosewell 
12. Hormonal Changes in the Menopause Transition

Henry G. Burger, Emma C. Dudley, David M. Robertson,

and Lorraine Dennerstein

13. Role of Hormonal and Reproductive Factors in the Etiology and Treatment of Uterine Leiomyoma

Cheryl L. Walker

14. Definition of the Molecular and Cellular Mechanisms Underlying the Tissue-selective Agonist/Antagonist Activities of Selective Estrogen Receptor Modulators

Donald P. McDonnell, Caroline E. Connor, Ashini Wijayaratne,

Ching-yi Chang, and John D. Norris

15. Aromatase and Its Inhibitors: Significance for Breast Cancer Therapy

Evan R. Simpson and Mitch Dowsett

16. Reproductive Functions of Progesterone Receptors

Orla M. Conneely, Biserka Mulac-Jericevic, Francesco DeMayo,

John P. Lydon, and Bert W. O’Malley

17. Estrogen Actions Throughout the Brain

Bruce McEwen

18. Sex Steroids and Bone

S.C. Manolagas, S. Kousteni, and R.L. Jilka

19. Anabolic Steroids

Cynthia M. Kuhn

20. Prolactin Modulation of Immune and Inflammatory Responses

Li-yuan Yu-Lee

Author Index

Subject Index 


\section{Senior Author \\ Correspondence Information}

Henry G. Burger

Prince Henry's Institute of Medical Research Level 4, Block E

Monash Medical Centre

246 Clayton Road

Clayton, Victoria 3168 Australia

Phone: 011-61-3-9594-3553

Fax: 011-61-3-9594-3558

E-mail: henry.burger@med.monash.edu.au

Blanche Capel

Department of Cell Biology

Duke University Medical Center

340 Nanaline Duke

Box 3709

Durham, NC 27710

Phone: 919-684-6390

Fax: 919-681-7978

E-mail: b.capel@cellbio.duke.edu

Orla M. Conneely

Department of Molecular and Cellular

Biology

M-511 Debakey Building

Baylor College of Medicine

Houston, TX 77030

Phone: 713-798-6233

E-mail: orlac@bcm.tmc.edu
James Cross

Department of Biochemistry \& Molecular Biology

University of Calgary Faculty of Medicine HSC Room 2279

3330 Hospital Drive, NW

Calgary, Alberta T2N 4N1 Canada

Phone: 403-220-6876

Fax: 403-270-0737

E-mail: jcross@ucalgary.ca

\section{Edward M. Eddy}

Laboratory of Reproductive and

Developmental Toxicology

National Institute of Environmental Health

Sciences

National Institutes of Health

111 T.W. Alexander Drive, Building 101, Room C422a

Research Triangle Park, NC 27709-2233

Phone: 919-541-3015

Fax: 919-541-3800

E-mail: eddy@niehs.nih.gov

Michael D. Griswold

School of Molecular Biosciences

Center for Reproductive Biology

Washington State University

630 Fulmer Hall

Pullman, WA 99164-4660

Phone: 509-335-6281

Fax: 509-335-9688

E-mail: griswold@mail.wsu.edu 
Cynthia M. Kuhn

Department of Pharmacology and Cancer

Biology

Duke University Medical Center

4011 Bryan Research Building

Durham, NC 27710

Phone: 919-684-8828

Fax: 919-681-8609

E-mail: ckuhn@duke.edu

\section{S.C. Manolagas}

Center for Osteoporosis and Metabolic Bone Diseases

Division of Endocrinology and Metabolism University of Arkansas Medical School 4301 W. Markham Street, Slot 587

Little Rock, AR 72205

Phone: 501-686-5130

Fax: 501-296-1002

E-mail: manolagasstavros@uams.edu

Donald P. McDonnell

Department of Pharmacology and Cancer Biology

Duke University Medical Center

Box 3813

Levine Science Research Center, C259

Research Drive

Durham, NC 27710

Phone: 919-684-6035

Fax: 919-681-7139

E-mail: donald.mcdonnell@duke.edu

Bruce S. McEwen

Harold and Margaret Milliken Hatch

Laboratory of Neuroendocrinology

The Rockefeller University

1230 York Avenue

New York, NY 10021

Phone: 212-327-8624

Fax: 212-327-8634

E-mail: mcewen@ rockefeller.edu
Robert I. McLachlan

Male Reproductive Endocrinology Group Prince Henry's Institute of Medical Research Level 4, Block E

Monash Medical Centre

246 Clayton Road

Clayton, Victoria, 3168, Australia

Phone: 011-61-3-9594-4372

Fax: 011-61-3-9594-6125

E-mail: rob.mclachlan@med.monash.edu.au

Michael J McPhaul

Department of Internal Medicine

Division of Endocrinology and Metabolism

University of Texas Southwestern Medical

Center

5323 Harry Hines Boulevard

Dallas, Texas 75390-8857

Phone: 214-648-3494

Fax: 214-648-8917

E-mail:

Michael.mcphaul@utsouthwestern.edu

David J Miller

Department of Animal Sciences

University of Illinois at Urbana-Champaign 328 Animal Sciences Laboratory

1207 West Gregory Drive

Urbana, Illinois 61801

Phone: 217-333-3408

Fax: 217-333-8286

E-mail: d-mille@uiuc.edu

Keith L Parker

Division of Endocrinology

Room J6.106

University of Texas Southwestern Medical

Center

5323 Harry Hines Boulevard

Dallas, TX 75390-8857

Phone: 214-648-5027

Fax: 214-648-5044

E-mail: keith.parker@email.swmed.edu 
JoAnne S Richards

Department of Molecular and Cellular

Biology

Baylor College of Medicine

One Baylor Plaza

Houston, TX 77030

Phone: 713-798-6238

Fax: 713-790-1275

E-mail: joanner@bcm.tmc.edu

Evan R. Simpson

Prince Henry's Institute of Medical Research

Monash Medical Centre

Level 4, Block E

246 Clayton Road

Clayton, Victoria 3168 Australia

Phone: 011-61-3-9594-4397

Fax: 011-61-3-9594-6376

E-mail: evan.simpson@med.monash.edu.au

Cheryl L Walker

Department of Carcinogenesis

University of Texas MD Anderson Cancer

Center

Science Park-Research Division

Park Road 1C

Smithville, TX 78957

Phone: 512-237-9550

Fax: 512-237-2475

E-mail: cwalker@odin.mdacc.tmc.edu
Phyllis M Wise

Division of Biological Sciences

University of California-Davis

One Shields Avenue

Davis, CA 95616-8536

Phone: 530-752-4460

Fax: 530-752-2604

E-mail: pmwise@ucdavis.edu

\section{Debra J Wolgemuth}

Department of Genetics and Development

Columbia University College of Physicians

\& Surgeons

630 West $168^{\text {th }}$ Street

Black Building 1613

New York, NY 10032

Phone: 212-305-7900

Fax: 212-305-6084

E-mail: djw3@columbia.edu

\section{Li-yuan Yu-Lee}

Department of Medicine

Section on Immunology, Allergy and

Rheumatology

Baylor College of Medicine

1 Baylor Plaza

Houston, TX 77030

Phone: 713-798-4770

Fax: 713-798-5780

E-mail: yulee@bcm.tmc.edu 



\title{
Cellular and Molecular Pathways Regulating Mammalian Sex Determination
}

\author{
Christopher Tilmann and Blanche Capel \\ Department of Cell Biology, Duke University Medical Center, Durham, North Carolina 27710
}

\begin{abstract}
In mammals, sex is determined by the presence or absence of a single gene on the $\mathrm{Y}$ chromosome, Sry. Sry, a member of the high mobility group family of transcription factors, is required to initiate male-specific pathways and repress female-specific pathways. Expression of Sry in the gonad, beginning at 10.5 days postcoitum, leads to the differentiation of the somatic supporting cell precursors as Sertoli cells. These cells direct the other cells of the gonad into their respective lineages. Currently, no direct targets of Sry are known. A number of cellular pathways initiated by Sry are required for testis development. These include the proliferation of pre-Sertoli cells and commitment to the Sertoli lineage, migration of cells from the adjacent mesonephros, and formation of a male-specific vasculature. Work is underway to identify genes controlling these processes. These genes will then be linked to Sry.
\end{abstract}

\section{Introduction}

Organisms that display sexually dimorphic phenotypes have evolved a variety of mechanisms for determining sex. In worms and flies, sex is determined by the ratio of X chromosomes to autosomes (Parkhurst and Meneely, 1994; Cline and Meyer, 1996). Reptiles and fish use hormones, temperature, or environmental cues to determine sex (Shapiro, 1990; Pieau et al., 2001). In mammals, sex is determined genetically by the presence or absence of a $\mathrm{Y}$ chromosome (Swain and Lovell-Badge, 1999; Capel, 2000).

In 1947, Alfred Jost showed that removal of the gonads from fetal rabbits resulted in the development of female secondary sex characteristics, whether the animal was $\mathrm{XX}$ or $\mathrm{XY}$. These experiments showed that male secondary sex characteristics depend upon the presence of a testis and led to the hypothesis that female development was the "default" state, since no gonads were required to specify female characteristics. Subsequently, the Y chromosome was found to be consistently associated with male sexual development, regardless of the number of $\mathrm{X}$ chromosomes present. The hypothetical factor on the $\mathrm{Y}$ chromosome required for male differentiation was termed $T d y$ (for testis-determining factor on the Y chromosome). 
In 1990, studies performed in humans on sex-reversed XX males revealed that a $35-\mathrm{kb}$ region of the $\mathrm{Y}$ chromosome was capable of causing sex reversal when transferred to an X chromosome (Gubbay et al., 1990; Sinclair et al., 1990). A single gene common to all mammals was found in this region and named Sry (for sex-determining region of the $\mathrm{Y}$ chromosome). Expression studies in mice revealed that Sry is expressed in the gonad from 10.5 days postcoitum (dpc) to approximately $12.0 \mathrm{dpc}$, coincident with formation of the gonad and initiation of testis development (Koopman et al., 1990; Hacker et al., 1995; Bullejos and Koopman, 2001). Further studies showed that when a 14-kb region containing this gene was introduced into $\mathrm{XX}$ mice as a transgene, testis development and male secondary sexual differentiation occurred, proving that Sry is Tdy (Koopman et al., 1991). However, these mice are sterile because other genes on the $\mathrm{Y}$ chromosome are required for spermatogenesis.

The Sry gene encodes a member of the high mobility group (HMG) family of transcription factors that has been shown to activate transcription by binding and bending DNA (Kamachi et al., 2000). More recent experiments have begun to reveal a role for HMG proteins in transcriptional repression as well (Zorn et al., 1999). Thus, Sry may initiate the male pathway by activating testis-specific genes and/or repressing genes that are specifically involved in ovarian development. Other members of this family include Tcfl (T-cell factor 1), Tcf4, Lefl (lymphoid enhancer binding factor), and Sox (Sry-related box) genes. These genes are involved in a number of developmental processes, ranging from cartilage formation to specification of neural identity (Pevny and Lovell-Badge, 1997).

\section{Origin and Development of the Gonads}

In the mouse, the urogenital ridge develops from intermediate mesoderm, beginning around $9.5 \mathrm{dpc}$. This starts with formation of the pronephros at the anterior end of the body cavity. The mesonephros develops centrally and gives rise to the gonads and adrenal glands. The metanephros, the area in which the ureteric bud branches to form the kidney, develops more posteriorly. At $10.0 \mathrm{dpc}$, the gonads begin to develop on the ventromedial surface of the mesonephroi. The somatic cells of the gonad originate from both the mesonephros and the coelomic epithelium, a single layer of epithelial cells that lines the coelomic cavity (Byskov, 1986; Karl and Capel, 1998). In both XX and XY animals, primordial germ cells (PGCs) first form at $7.5 \mathrm{dpc}$ at the base of the allantois. As the embryo undergoes morphogenesis, PGCs migrate through the gut mesentary and populate the urogenital ridge at $9.5 \mathrm{dpc}$. The PGCs then populate the gonads as they are beginning to form at $10.0 \mathrm{dpc}$ (Ginsburg et al., 1990; Gomperts et al., 1994). Until $11.5 \mathrm{dpc}$, the gonads in XX and XY individuals appear identical and are capable of forming either testes or ovaries. By $12.5 \mathrm{dpc}$, the formation of testis 
cords can be seen in XY gonads, whereas no obvious changes occur in $\mathrm{XX}$ gonads. XY gonads are noticeably larger than XX gonads by this time and acquire a characteristic pattern of vasculature.

\section{Cell Types of XX and XY Gonads}

Available evidence indicates that the cells in $\mathrm{XX}$ and $\mathrm{XY}$ gonads are bipotential and capable of differentiating along either the male or female pathway (Koopman et al., 1990; Palmer and Burgoyne, 1991a; Albrect et al., 2001). Expression of Sry in the XY gonadal primordium causes the somatic supporting cell precursors of the gonad to differentiate as Sertoli cells. In the absence of Sry, the somatic supporting cell precursors follow the ovarian pathway and eventually become follicle cells.

The development of PGCs into either sperm or oocytes depends upon the environment in which they reside (for a review, see McLaren, 1995). Regardless of their chromosomal makeup (XX or XY), PGCs synchronously enter meiosis by $13.5 \mathrm{dpc}$ in the absence of any interference from Sertoli cells. In the ovary, PGCs arrest in the first prophase of meiosis I until later ovulatory cycles (McLaren and Southee, 1997; McLaren, 2000). Many experiments have shown that PGCs are required for the differentiation of follicle cells; in the absence of PGCs, follicles never form (Huang et al., 1993; Bedell et al., 1995). In contrast, the differentiation of Sertoli cells and formation of testis cords are not dependent upon PGCs; in the absence of PGCs, testis cords form normally. The differentiation of Sertoli cells in the gonad leads to the enclosure of PGCs inside testis cords and their arrest in mitosis before $13.5 \mathrm{dpc}$ (Kurohmaru et al., 1992; McLaren and Southee, 1997).

Sertoli cells are believed to act as an organizing center for the testis by directing all other cell types into their respective lineages. Experiments with XX $\leftrightarrow \mathrm{XY}$ mosaic mice revealed that the only cell type with a strong bias for a $\mathrm{Y}$ chromosome is the Sertoli cell (Palmer and Burgoyne, 1991a). In mosaic gonads that became testes, $90 \%$ of Sertoli cells were XY and $10 \%$ were XX. All other somatic cell types displayed a 50/50 XX:XY distribution. From these results, it was concluded that Sry-expressing Sertoli cells are capable of initiating the differentiation of all other cell types within the testis. These experiments also revealed that Sry is not required cell autonomously for Sertoli differentiation, since $10 \%$ of Sertoli cells in these experiments were XX. It is likely that Sry-expressing somatic cells are capable of recruiting other non-Sry-expressing somatic cells into the Sertoli lineage. Sertoli cells - and probably follicle cell precursors - have been shown to arise from the coelomic epithelium before 11.5 dpc. This led us to hypothesize that coelomic epithelial cells would express Sry (Karl and Capel, 1998; Schmahl et al., 2000). However, more recent experiments have localized Sry expression to a population of somatic cells that lies underneath 
the coelomic epithelium at 11.2 to 11.5 dpc (Bullejos and Koopman, 2001; Albrecht et al., 2001). This expression pattern suggests that pre-Sertoli cells turn on Sry and other genes required for Sertoli differentiation after they delaminate from the coelomic epithelium and enter the gonad. It is also possible that an additional population of Sertoli cells that express Sry arise from the mesonephros.

The Leydig cells of the XY gonad are required for production of testosterone, which initiates development of the male reproductive tract and male secondary sex characteristics. All available data suggest that these cells originate from the mesonephros before 11.5 dpc. Steroidogenic factor 1 (Sf1), a gene expressed in steroid-producing cells of the adrenal and Leydig cells of the testis, labels cells in the anterior end of the mesonephros prior to $11.5 \mathrm{dpc}$. These cells contribute to the adrenal gland and the XY gonad and represent a likely source of Leydig precursors (Hatano et al., 1996; Morohashi, 1997). In one electron microscopy study, Leydig cells were identified among a marked population of cells that had originated from the mesonephros (Merchant-Larios and MorenoMendoza, 1998). Finally, experiments in which XY gonads were separated from their mesonephroi at $11.5 \mathrm{dpc}$ and cultured several days revealed that testosterone still was produced by these gonads. This indicated that Leydig precursors already are present in the gonad at $11.5 \mathrm{dpc}$ (Merchant-Larios, 1979). The counterpart of the Leydig cell in the ovary is the theca cell. Precursors of theca cells are not identifiable early in the ovary but their origins are believed to be parallel to that of Leydig cells.

The other known somatic lineages in the $\mathrm{XY}$ gonad include peritubular myoid cells and endothelial cells. Myoid cells, a smooth muscle lineage, have been shown to collaborate with Sertoli cells in setting up the tubule structure of the testis (Skinner et al., 1985). In the adult, they generate a peristaltic action to pump sperm through the tubules. Myoid cells have no analogous lineage in XX gonads. Endothelial cells are present in both XX and XY gonads; however, by $12.5 \mathrm{dpc}$, the vasculature of $\mathrm{XY}$ gonads undergoes major structural changes. The most notable of these vascular reorganizations is the formation of a large coelomic surface vessel.

\section{Genes Required for Testis and/or Ovary Development}

Several genes have been identified that play roles in the early formation of the gonads in both sexes. These include Sf1, Wilm's tumor (Wt1), Liml, Lhx9, and Emx2. Sf1 encodes a member of the nuclear hormone receptor family that is expressed in the somatic cells of $\mathrm{XX}$ and $\mathrm{XY}$ genital ridges as the gonad begins to form at $10.0 \mathrm{dpc}$ (Ikeda et al., 1994). Expression continues in the precursors of Leydig and Sertoli cells in XY gonads until $12.5 \mathrm{dpc}$, then gradually becomes restricted to Leydig cells. In XX gonads, SfI expression can be seen in somatic 
cells until $13.0 \mathrm{dpc}$ (Ikeda et al., 1996). Sf1 -/- mice initiate development of the gonadal primordium; however, by $12.5 \mathrm{dpc}$, the gonads of both XX and XY individuals regress by apoptosis (Luo et al., 1994). The Wt1 gene also is expressed in the somatic cells of the gonadal primordium of both sexes (Rackley et al., 1993). In Wt1 -/- mice, neither kidneys nor gonads form (Kriedberg et al., 1993). Recent in vitro assays using human forms of WT1 and a reporter driven by the $S R Y$ promoter suggest that WT1 is capable of upregulating $S R Y$ expression (Hossain and Saunders, 2001). Lim 1, Lhx9, and Emx2 also are involved in the early formation of the gonad in both sexes. Liml and Lhx9 are homeodomain proteins and Emx2 encodes a homologue of a Drosophila head gap gene. Mice carrying null mutations for any of these genes do not form gonads (Shawlot and Behringer, 1995; Miyamoto et al., 1997; Birk et al., 2000). Studies have suggested that $L h x 9$ may lie upstream of $S f l$, since $S f l$ expression in $L h x 9$ null mice is dramatically reduced (Birk et al., 2000).

A number of genes are expressed specifically in the XY gonad after $11.5 \mathrm{dpc}$ and have been identified as early players in the sex-determination cascade. Sox 9 is expressed in both $\mathrm{XX}$ and $\mathrm{XY}$ gonads beginning at $10.5 \mathrm{dpc}$ but persists only in the nuclei of Sertoli cells of XY gonads after 11.5 dpc (daSilva et al., 1996; Kent et al., 1996). Humans heterozygous for mutations in Sox9 display varying degrees of sex reversal (Cameron et al., 1996). However, mice heterozygous for mutations in Sox9 form normal testes. Mice homozygous for null mutations in Sox9 have been generated but die before $11.5 \mathrm{dpc}$, preventing study of the gonads. Efforts are underway to generate chimaeric mice with Sox $9+/+$ and $-/-$ cells, which may allow analysis of Sox9 function during gonadogenesis (R. Behringer, personal communication). Experiments in which Sox9 is ectopically expressed in XX gonads under the control of the Wt1 promoter have shown that Sox9 can cause sex reversal in the absence of Sry (Vidal et al., in press). Recently, a transgene insertion upstream of the Sox9 promoter, odsex (for ocular degeneration with sex reversal), was shown to result in misexpression of Sox9 and sex reversal of XX gonads (Bishop et al., 2000). Thus, activation of Sox9 seems to account for all the effects of Sry. Integration of the transgene in odsex resulted in the deletion of sequences one to two megabases upstream of the Sox 9 coding region. From these results, it was proposed that the portion of DNA deleted in odsex mice is the binding site for a negative regulator of Sox9. In normal males, the presence of Sry antagonizes this regulator, allowing Sox 9 to be transcribed. However, in normal females, where Sry is not present, Sox9 is silenced in the presence of the negative regulator. In the odsex deletion, removal of the binding site from a distant regulatory region would allow for the ectopic expression of Sox9 in the $\mathrm{XX}$ gonad.

Müllerian inhibiting substance (MIS), a member of the transforming growth factor beta (TGF $\beta$ ) family, is expressed specifically in the Sertoli cells of the XY gonad, beginning between 11.5 and 12.5 dpc (Munsterberg and Lovell-Badge, 
1991). At $11.5 \mathrm{dpc}$, the mesonephros contains two ducts, the Müllerian duct and the mesonephric duct, which are the precursors of the female and male reproductive tracts, respectively (Josso and Picard, 1986; Donahoe et al., 1987). In females, the Müllerian duct develops in the absence of MIS, whereas in males, MIS production leads to the regression of this duct by apoptosis. Misexpression of MIS in the XX gonad leads to regression of the Müllerian duct, while in XY Mis -/- mice, the Müllerian duct fails to regress and both male and female reproductive tracts develop (Behringer et al., 1990,1994). The mesonephric, or Wolffian duct, develops into the male reproductive tract. Testosterone is required for the development of this duct. In the absence of testosterone in females, the mesonephric duct regresses.

Another gene expressed specifically in Sertoli cells beginning at $12.5 \mathrm{dpc}$ is Desert hedgehog (Dhh). XY Dhh -/- mice are sterile (Bitgood et al., 1996) and show defects in Leydig cell formation and Sertoli-myoid cell interactions in the adult testis (Clark et al., 2000). Patchedl (Ptc1), which encodes a receptor for $D h h$, is expressed by numerous cells of the interstitium, including peritubular myoid cells and Leydig cells. Dhh and Ptcl are not known to be expressed by XX gonads at any stage of development.

$F g f 9$ is expressed specifically in $\mathrm{XY}$ gonads at 11.5 and $12.5 \mathrm{dpc}$. Analysis of $\mathrm{Fgf} 9$-/- mice revealed that the majority of XY mice developed as phenotypic females (Colvin et al., 2001). In these XY mice, $80 \%$ of the gonads developed as ovaries and $20 \%$ developed some abnormal testis cords. Analysis of proliferation in these mice after $12.5 \mathrm{dpc}$ showed that proliferation rates for interstitial cells were less than $70 \%$ that of controls. Furthermore, culturing XX gonads in the presence of FGF9 resulted in the recruitment of mesonephric cells into the $\mathrm{XX}$ gonad. These data suggest that $\mathrm{Fg} f 9$ plays an early role in regulating male-specific pathways downstream of Sry.

Another gene that, when deleted in mice, causes male-to-female sex reversal is M33. M33 is a homologue of the Drosophila polycomb group genes (KatohFukui et al., 1998). It is not clear where in the sex-determination hierarchy M33 acts. Since both polycomb and HMG proteins have been shown to alter chromatin structure, there may be interactions between M33 and Sry that regulate domains of gene expression.

DMRT1 was identified by its homology to genes with DNA-binding motifs termed DM domains. Two such genes are mab-3 in C. elegans and doublesex $(d s x)$ in Drosophila, genes that play roles in sex determination and male-specific differentiation in these organisms (Raymond et al., 1998). The DMRT1 gene in humans maps to a cluster of DMRT genes in a region of chromosome 9 associated with defective testicular development. In mice, Dmrtl is expressed in the gonads of both sexes at the early stages of gonad development. By 13.5 to $14.5 \mathrm{dpc}$, it becomes specific to the testis (Raymond et al., 1999). Testes in mice null for Dmrtl are normal at the early stages of testis development. At later stages 
after birth, the seminiferous tubules of Dmrtl -/- XY gonads begin to degenerate. Ovarian development is normal in Dmrtl null mice (Raymond et al., 2000). While these results do not place Dmrtl early in the sex-determination pathway in mammals, it is possible that there is redundancy with other Dmrt family members.

Daxl and Wnt4 are genes expressed specifically in XX gonads after 11.5 dpc. Daxl was cloned from an X chromosomal region in humans responsible for dosage-sensitive sex reversal (DSS) (Bardoni et al., 1994; Zanaria et al., 1994). Studies by Swain et al. (1998) have suggested that Daxl plays a role in ovarian development by inhibiting Sry action. However, removing the Daxl gene from mice had no effect on ovary development but instead led to male sterility and Leydig cell hyperplasia (Yu et al., 1998). Therefore, the function of Daxl during gonadogenesis is not entirely clear. Wnt4 -/- females exhibit ectopic Leydig cell development, whereas XY Wnt4 -/- mice have no obvious defects in testis development (Vanio et al., 1999). These results suggest that Wnt4 plays an inhibitory role in XX gonads by repressing a portion of the male pathway that leads to the differentiation of Leydig cells. In XY gonads, expression of Sry and Sox9 somehow leads to the repression of Wnt4, allowing Leydig cell development to proceed. (See Table I for a list of genes involved in testis and ovary development.)

\section{Genetic Models of Sex Determination}

Analysis of the formation of ovotestes in C57BL/6 (B6) XYpos mice has provided insight into the mechanisms governing the decision to become a testis or an ovary. When the $\mathrm{Y}^{\mathrm{pos}}$ chromosome from Mus musculus poschiavinus is crossed onto a B6 background, $\mathrm{XY}^{\text {pos }}$ mice display a delay in testis cord formation that leads to varying degrees of sex reversal, ranging from the formation of ovotestes (gonads containing both testis and ovarian regions) to ovaries (Eicher et al., 1982; Palmer and Burgoyne, 1991b). To account for the occurrence of ovotestes in $\mathrm{B} 6 \mathrm{XY}^{\mathrm{pos}}$ mice, it has been proposed that male-specific pathways must be initiated by $11.5 \mathrm{dpc}$ in order to repress female-specific pathways; otherwise, ovarian development proceeds. Several studies have attempted to link the delay in testis cord formation to a delay in Sry expression, with inconsistent results (Nagamine et al., 1999; Lee and Taketo, 2000). However, evidence from transgenic animals also supports this idea. Experiments attempting to rescue the defect in $\mathrm{B} 6 \mathrm{XY}^{\mathrm{pos}}$ mice with an Sry transgene are only successful if Sry is expressed at sufficient levels and in the proper temporal pattern (Eicher et al., 1995). The $\mathrm{Y}^{\mathrm{pos}}$ chromosome is capable of inducing normal testis formation on other genetic backgrounds. This has led to the hypothesis that incompatibilities exist between the $\mathrm{Y}^{\mathrm{pos}}$ Sry gene and alleles of other genes that are specific to B6, termed TDAs (testis-determining autosomal genes). Mapping 
TABLE I

Genes Involved in Mammalian Gonad Development

\begin{tabular}{|c|c|c|c|}
\hline Gene & Expression & Function & Reference(s) \\
\hline Sf1 & $\mathrm{M}, \mathrm{F}$ & $\begin{array}{l}\text { Early establishment of gonads, } \\
\text { regulator of genes for steroid } \\
\text { production }\end{array}$ & $\begin{array}{l}\text { Luo et al., 1994; } \\
\text { Ikeda et al., 1994,1996 }\end{array}$ \\
\hline$W t 1$ & $\mathrm{M}, \mathrm{F}$ & $\begin{array}{l}\text { Early establishment of gonads } \\
\text { and kidneys, possible regulator of } \\
\text { Sry expression }\end{array}$ & $\begin{array}{l}\text { Rackley, 1993; Kriedberg et al., } \\
1993\end{array}$ \\
\hline Lim1 & $\mathrm{M}, \mathrm{F}$ & Early establishment of gonads & Shawlot and Behringer, 1995 \\
\hline Emx2 & $\mathrm{M}, \mathrm{F}$ & Early establishment of gonads & Miyamoto et al., 1997 \\
\hline $\operatorname{Lh} x 9$ & $\mathrm{M}, \mathrm{F}$ & Early establishment of gonads & Birk et al., 2000 \\
\hline $\operatorname{Sox} 9$ & M & $\begin{array}{l}\text { Sertoli cell differentiation, } \\
\text { initiation of testis development }\end{array}$ & $\begin{array}{l}\text { Cameron et al., 1996; daSilva } \\
\text { et al., 1996; Kent et al., 1996; } \\
\text { Bishop et al., } 2000\end{array}$ \\
\hline Mis & M & Regression of Müllerian duct & $\begin{array}{l}\text { Josso and Picard, 1986; } \\
\text { Donahoe et al., 1987; Behringer } \\
\text { et al., 1990,1994; Munsterberg } \\
\text { and Lovell-Badge, } 1991\end{array}$ \\
\hline Dhh & M & $\begin{array}{l}\text { Regulator of Sertoli-myoid, } \\
\text { Sertoli-Leydig interactions, } \\
\text { development of male germ cells }\end{array}$ & $\begin{array}{l}\text { Bitgood et al., 1996; } \\
\text { Clark et al., } 2000\end{array}$ \\
\hline$F g f 9$ & M & Proliferation in male gonad & Colvin et al., 2001 \\
\hline M33 & Unknown & Required for testis development & Katoh-Fukui et al., 1998 \\
\hline Dmrt1 & M & $\begin{array}{l}\text { Maintenance of seminiferous } \\
\text { tubules after birth }\end{array}$ & $\begin{array}{l}\text { Raymond et al., } \\
1998,1999,2000\end{array}$ \\
\hline $\operatorname{Daxl}$ & $\mathrm{F}$ & Unclear & $\begin{array}{l}\text { Swain et al., 1998; Yu et al., } \\
1998\end{array}$ \\
\hline Wnt4 & $\mathrm{F}$ & $\begin{array}{l}\text { Repression of Leydig cell } \\
\text { differentiation in female gonads }\end{array}$ & Vanio et al., 1999 \\
\hline
\end{tabular}

studies have identified regions on chromosomes 2, 4, and 5 that are associated with the formation of ovotestes (Eicher et al., 1996).

\section{Cellular Pathways Downstream of SRY}

Despite the discovery of multiple genes involved in the testis pathway, no direct targets of SRY have been identified. In this 24-hour period, SRY estab- 
lishes the expression of genes involved in the rapid patterning of the gonadal cells into testis cords and a male-specific pattern of vasculature. Virtually nothing is known about the genes that SRY activates to govern the cellular changes that occur in the XY gonad between 11.5 and $12.5 \mathrm{dpc}$. An alternative approach is to characterize the cellular pathways initiated by SRY. Knowledge of these pathways and how they operate in testis formation can be used to identify genes required for these processes and to link them to SRY.

Experiments have revealed several cellular pathways downstream of Sry that play a role in testis formation. These include cell proliferation, commitment of coelomic epithelial cells to the Sertoli lineage, mesonephric cell migration, and vascularization. All of these pathways have been shown to be required for normal testis formation and depend upon the expression of Sry in the genital ridge.

\section{A. PROLIFERATION OF SERTOLI PRECURSORS AND COMMITMENT TO THE SERTOLI LINEAGE}

The Sertoli cell plays a central role in testis development by directing all other cell types into their respective lineages. For this reason, it is important to understand the origin of this cell type. Previous studies have suggested two sources of Sertoli cells: the coelomic epithelium and the mesonephros. By labeling cells of the coelomic epithelium with DiI, experiments have shown that, before $11.5 \mathrm{dpc}$, the cells of the coelomic epithelium contribute to the Sertoli lineage (Karl and Capel, 1998). Furthermore, prior to $11.5 \mathrm{dpc}$, the cells of the coelomic epithelium can give rise to daughter cells of two lineages: Sertoli cells and unidentified interstitial cell types. After $11.5 \mathrm{dpc}$, coelomic epithelial cells still contribute to the XY gonad but only become interstitial cells.

In agreement with DiI studies, experiments using bromodeoxyuridine (BrdU) to label dividing cells showed that the coelomic epithelium is highly proliferative prior to $12.0 \mathrm{dpc}$ (Schmahl et al., 2000). These experiments defined two stages of proliferation in the XY gonad. The first stage of proliferation occurred in a population of cells that expresses high levels of SfI and contributes to the Sertoli lineage as well as an interstitial lineage. A second stage of proliferation occurred at the coelomic epithelium after $11.5 \mathrm{dpc}$ in a population of cells that expressed $S f l$ at low levels and contributed to interstitial lineages. To determine if proliferation was dependent upon Sry expression, the proliferation pattern of XX gonads from mice carrying an Sry transgene was examined and found to be identical to that of $\mathrm{XY}$ gonads. Conversely, $\mathrm{XY}$ gonads from B6XY ${ }^{\text {pos }}$ mice, which become ovaries or ovotestes, displayed levels of proliferation similar to XX gonads.

From these data, it was concluded that Sry induces the upregulation of proliferation in cells at the coelomic epithelium. Recent expression studies have shown that Sry is not expressed in coelomic epithelial cells (Bullejos and 
Koopman, 2001; Albrecht et al., submitted). Therefore, Sry-expressing Sertoli precursors beneath the coelomic epithelium are likely to expand their population in a noncell-autonomous manner by recruiting non-Sry-expressing cells at the coelomic epithelium into the Sertoli lineage. Prior to $11.5 \mathrm{dpc}$, cell divisions at the coelomic epithelium result in daughter cells that follow different fates, as revealed in the DiI injection experiments. This might occur through the asymmetric distribution of cellular components during the division process, by lateral signaling between daughter cells, or through interactions with other somatic cells of the gonad once the division is complete. After $11.5 \mathrm{dpc}$, proliferation at the coelomic epithelium results in an expansion of the population of interstitial cells (for a diagram of male-specific proliferation, see Figure 1).

\section{B. MESONEPHRIC CELL MIGRATION}

Early electron microscopy studies on the structure of the gonad revealed similarities in morphology and cell staining between mesonephric and gonadal cells, suggesting that gonadal cells may have origins in the mesonephros. Experiments demonstrated that mesonephric cells contribute to the XY gonad and that culture of XY gonads without their adjoining mesonephroi resulted in a failure of testis cord formation (Buehr et al., 1993; Merchant-Larios et al., 1993). More recent experiments showed that cells migrated into the XY gonad between $11.5 \mathrm{dpc}$ and at least $16.5 \mathrm{dpc}$ and that this event did not occur in XX gonads. Migrating cells are represented in at least three lineages: peritubular myoid cells, endothelial cells, and cells associated with the endothelium (Martineau et al., 1997). To determine whether cell migration directly depends upon Sry expression, this event was examined in XY mice lacking Sry and XX mice carrying Sry as a transgene. These experiments showed that cell migration did not occur in $\mathrm{XY}$ gonads destined to form ovaries. In contrast, XX gonads carrying an Sry transgene, which always formed testes, did recruit cells from the mesonephros (Capel et al., 1999). Furthermore, XY gonads with weak alleles of Sry that result in ovotestes recruited cells only into testicular regions of the gonad (Albrecht $e t$ al., 2000). These experiments showed that cell migration from the adjacent mesonephros is one of the earliest events induced by Sry and strongly suggested a role for cell migration in the formation of testis cords.

Initial attempts to characterize the signal(s) for cell migration demonstrated that culturing an XX gonad with an XY gonad at its coelomic surface resulted in migration of mesonephric cells into the XX tissue (Martineau et al., 1997). Likewise, culture of XX gonads with beads coated with proteins from XY gonads led to the induction of cell migration. This assay was used to further investigate the role of cell migration in the developing gonad. Examination of the structure of XX gonads that had been cultured with an XY gonad at their coelomic surface 
$15-16$ ts

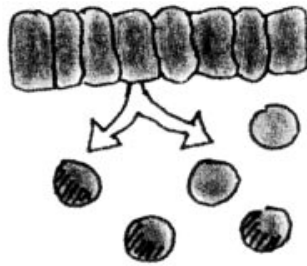

18 ts

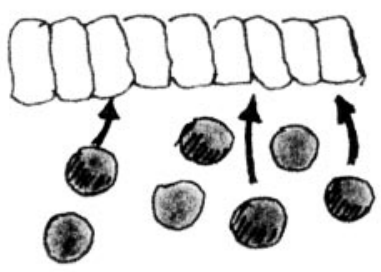

24 ts

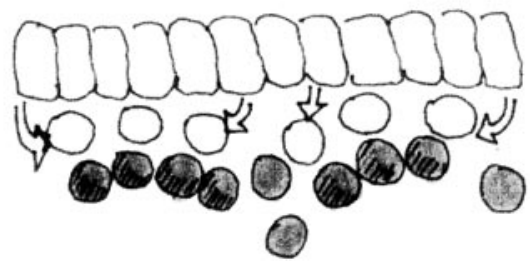

coelomic epithelial cell, high S 1

coelomic epithelial cell, low Sf1

pre-Sertoli cell, high Sf1

Interstitial precursor, high Sf1

Interstitial precursor, low Sf1

FIG. 1. Model for stages of male-specific proliferation in the mammalian gonad. At 15-16 tail somites (ts), corresponding to $11.25 \mathrm{dpc}$, cells of the coelomic epithelium, expressing Sf1 at high levels, divide (outlined arrow) and give rise to cells destined to become Sertoli cells as well as interstitial cells. At 18 ts, corresponding to $11.5 \mathrm{dpc}$, pre-Sertoli cells, expressing high levels of Sf1, signal to cells at the coelomic epithelium (solid arrows), expressing Sf1 at low levels, to continue dividing. At 24 ts, corresponding to $12.0 \mathrm{dpc}$, cells at the coelomic epithelium give rise (outlined arrows) to cells of interstitial lineages.

as a "sandwich" to induce mesonephric cell migration revealed that the XX gonadal cells organized into structures similar to testis cords. Laminin was deposited around cord structures and germ cells were enclosed within these cords. Furthermore, induced XX gonads upregulated the expression of the male-specific gene Sox 9 and downregulated the female-specific gene Daxl. These experiments showed that mesonephric cell migration is capable of inducing Sertoli cell differentiation in the absence of Sry in the XX supporting cell precursors (Tilmann and Capel, 1999). These results suggested that interactions between migrating cells and supporting cell precursors play an important role in 
the establishment of the Sertoli fate (for a diagram of male-specific cell migration, see Figure 2).

\section{TESTIS DEVELOPMENT MUST BE INITIATED WITHIN A NARROW WINDOW}

"Sandwich" experiments also defined a window in which cord formation could be induced in the XX gonad. While $11.5 \mathrm{dpc}$ XX gonads were capable of organizing cords and expressing male-specific genes, $12.5 \mathrm{dpc} \mathrm{XX}$ gonads were not. This timeframe is consistent with the models proposed based on ovotestis formation in $\mathrm{B} 6 \mathrm{XY} \mathrm{Y}^{\mathrm{pos}}$ mice. Interestingly, the window in which cords could be induced to form in "sandwich" experiments coincides with the timing of PGC entry into meiosis. Previous experiments showed that PGCs enter meiosis by $13.5 \mathrm{dpc}$, unless enclosed within testis cords (McLaren and Southee, 1997). Because it is known that PGCs are required for the initiation of follicle formation (Huang et al., 1993; Bedell et al., 1995), it is likely that important interactions are
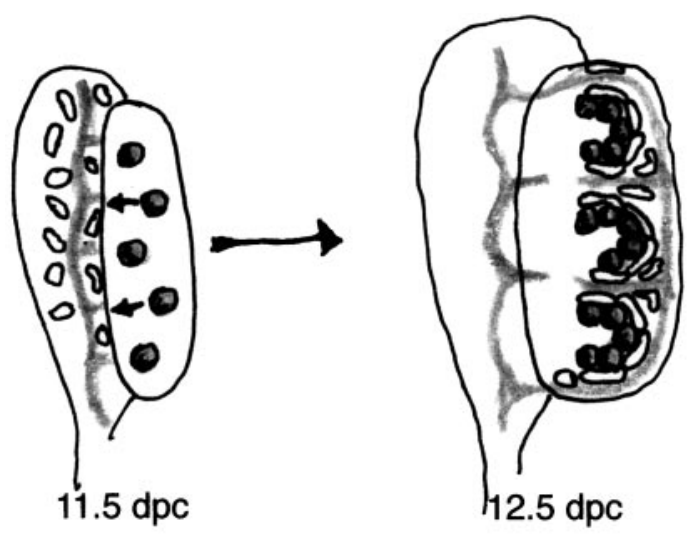

\section{จ pre-Sertoli cell}

\section{0 migrating mesonephric cell}

\section{endothelium}

FIG. 2. Model for male-specific cell migration in the mammalian gonad. At $11.5 \mathrm{dpc}$, pre-Sertoli cells of the XY gonad (solid arrows) signal endothelial cells and other cells in the mesonephros to begin migrating into the gonad. By $12.5 \mathrm{dpc}$, interactions between migrating mesonephric cells and pre-Sertoli cells establish testis cords. Migrating cells are represented in at least three lineages: peritubular myoid cells, endothelial cells, and cells associated with the endothelium. 
established between the somatic and germ cells of the XX gonad by $12.5 \mathrm{dpc}$ that antagonize male-specific pathways. Several characterized cases of XX sex reversal result in the formation of tubules in ovaries older than $12.5 \mathrm{dpc}$ and all are associated with the elimination of meiotic germ cells from the ovary (Vigier et al., 1987; Merchant-Larios and Taketo, 1991; Whitworth et al., 1996). These data support a model in which male-specific pathways must be initiated prior to $12.5 \mathrm{dpc}$ in order to prevent PGCs from entering meiosis, an event that antagonizes testis formation by initiating follicle cell differentiation.

\section{VASCULARIZATION}

One of the most noticeable structures that appears in the XY gonad by 12.5 $\mathrm{dpc}$ is a large vessel just under the coelomic epithelium, termed the coelomic vessel. Early experiments designed to study cell migration into the XY gonad revealed that endothelial and associated cells enter the $\mathrm{XY}$ gonad from the mesonephros beginning at $11.5 \mathrm{dpc}$ (Martineau et al., 1997). Cell migration occurs until at least $16.5 \mathrm{dpc}$, specifically in XY gonads. Many of the migrating endothelial cells become part of the coelomic vessel as well as vessels in the interstitium of the testis. The dimorphic development of the vasculature in XX and $\mathrm{XY}$ gonads led to the hypothesis that construction of a male-specific vasculature plays an early role in the patterning and function of the testis.

To begin to address the role of migrating endothelial cells in testis formation, the mechanisms for vascular development in XX and XY gonads were compared and contrasted (Brennan et al., submitted). At $11.5 \mathrm{dpc}$, the pattern of vessels appears similar between $\mathrm{XX}$ and $\mathrm{XY}$ gonads, as revealed by staining of gonads from Tie-2::LacZ mice, which contain an endothelial-specific LacZ transgene. However, by $12.5 \mathrm{dpc}$, the $\mathrm{XY}$ vasculature undergoes rapid reorganization that does not occur in XX gonads. The most noticeable features of the reorganization are the formation of the coelomic vessel, restriction of endothelial cells to the interstitial space between the testis cords, and a generation of a more highly branched system of vessels. The vasculature of the XX gonad expands during this period but its pattern does not change.

Cell migration from the mesonephros into the XY gonad is certainly one mechanism that accounts for this rapid reorganization and growth of the XY gonad. To determine whether endothelial migration from the mesonephros represented a specific subset of the vasculature (e.g., lymphatics, arteries, veins), the expression patterns of markers specific to these populations were examined in XX and XY gonads. Lymphatics did not populate the XX or XY gonad until $17.5 \mathrm{dpc}$. Examination of the arterial and venous specific markers, Ephrin B2 (arterial) and its receptor EphB4 (venous), revealed that, at $11.5 \mathrm{dpc}$, endothelial cells of XX and XY gonads express both arterial and venous markers in similar patterns, suggesting that these two populations have not been distinctly specified. 
Interestingly, by $12.5 \mathrm{dpc}$, the vasculature of the XY gonad labels primarily with the arterial specific marker, Ephrin B2. In contrast, the vasculature of the XX gonad at $12.5 \mathrm{dpc}$ continues to express both arterial and venous markers, suggesting that it has not been specified into distinct populations.

From this analysis, it was concluded that the Sry is required for the recruitment of endothelial cells from the mesonephros. These cells are required for the formation of the coelomic vessel and the respecification of the XY vasculature into an arterial system, which results in the rerouting of blood flow, primarily through the coelomic vessel. One explanation for this pattern of specification and blood flow in the $\mathrm{XY}$ gonad is that this event is necessary for the rapid export of testosterone from the testis, which occurs by $13.5 \mathrm{dpc}$. Future experiments will address the role of genes involved in the specification of the XY vasculature.

\section{Summary}

Defects in sexual development are among the most common birth defects in humans. Since these defects can be a result of early events in the decision to form testes or ovaries occurring improperly, it is important to identify genes operating at these early steps. Identification of the cellular pathways that operate downstream of Sry has provided insight into the mechanisms of testis formation and will provide a foundation for identification of the genes involved in these pathways. Since SOX9 seems to account for all the effects of SRY, it is likely that activation of male-specific genes involved in proliferation, cell migration, and vascularization will depend upon SOX9. These genes are likely to be secreted signals and surface molecules involved in the proper sorting and specification of cells within the XY gonad. These genes must be downregulated in the XX gonad. This is likely to occur through the expression of molecules such as Wnt4 and Daxl.

Differential expression studies have begun to identify genes expressed in both XX and XY gonads that may play roles in testis or ovary development. One such gene is vanin- 1 , which is expressed specifically in the Sertoli cells of the XY gonad (Bowles et al., 2000). Serendipitous phenotypes in both XX and XY null mice (e.g., $F g f 9, M 33$, Wht4) have helped uncover several genes involved in gonad development as well. Mutagenesis screens are underway to identify new genes that influence testis or ovary development (R. Lovell-Badge, personal communication).

\section{REFERENCES}

Albrecht KH, Capel B, Washburn LL, Eicher EM 2000 Mesonephric cell migration and testis cord development coincide in mouse XY gonads that develop abnormally. Dev Biol 225:26-36 
Albrecht KH, Eicher EM 2001 Evidence that Sry is expressed in pre-Sertoli cells and Sertoli and granulosa cells have a common precursor. Dev Biol 240:92-107

Bardoni B, Zanaria E, Guioli S, Floridia G, Worley K, Tonini G, Ferrante E, Chiumello G, McCabe E, Fraccaro M, Zuffardi O, Camerino G 1994 A dosage sensitive locus at chromosome Xp21 is involved in male to female sex reversal. Nature Genet 7:497-501

Bedell MA, Brannan, CI, Evans EP, Copeland NG, Jenkins NA, Donovan PJ 1995 DNA rearrangements located over $100 \mathrm{~kb} \mathrm{5'}$ of the Steel (Sl)-coding region in Steel-panda and Steel-contrasted mice deregulate $\mathrm{Sl}$ expression and cause female sterility by disrupting ovarian follicle development. Genes Dev 9:455-470

Behringer RR, Cate RL, Froelick GJ, Palmiter RD, Brinster RL 1990 Abnormal sexual development in transgenic mice chronically expressing Müllerian inhibiting substance. Nature 345:167-170

Behringer RR, Finegold MJ, Cate RL 1994 Müllerian inhibiting substance function during mammalian sexual development. Cell 79:415-425

Birk O, Casiano D, Wassif C, Cogliati T, Zhao L, Zhao Y, Grinberg A, Huang S, Kreidberg J, Parker K, Porter F, Westphal H 2000 The LIM homeobox gene $L h x 9$ is essential for mouse gonad formation. Nature 403:909-913

Bishop C, Whitworth D, Qin Y, Agoulnik A, Agoulnik I, Harrison W, Behringer R, Overbeek P 2000 A transgenic insertion upstream of Sox 9 is associated with dominant XX sex reversal in the mouse. Nature Genet 26:490-494

Bitgood MJ, Shen L, McMahon AP 1996 Sertoli cell signaling by Desert hedgehog regulates the male germline. Curr Biol 6:298-304

Bowles J, Bullejos M, Koopman P 2000 A subtractive expression screen suggests a role for Vanin-1 in testis development in mice. Genesis 27:124-135

Buehr M, Gu S, McLaren A 1993 Mesonephric contribution to testis differentiation in the fetal mouse. Development 117:273-281

Bullejos M, Koopman, P 2001 Spatially dynamic expression of Sry in mouse genital ridges. Dev Dynam 221:201-205

Byskov AG 1986 Differentiation of the mammalian embryonic gonad. Phys Rev 66:71-117

Cameron FJ, Hageman, RM, Cooke-Yarborough C, Kwok C, Goodwin LL, Sillence DO, Sinclair AH 1996 A novel germline mutation in SOX9 causes familial campomelic dysplasia and sex reversal. Hum Molec Genet 5:1625-1630

Capel B 2000 The battle of the sexes. Mech Dev 92:89-103

Capel B, Albrecht KH, Washburn LL, Eicher EM 1999 Migration of mesonephric cells into the mammalian gonad depends on Sry. Mech Dev 84:127-131

Clark A, Garland K, Russell L 2000 The Desert hedgehog (Dhh) gene is required in the mouse testis for formation of adult-type Leydig cells and normal development of peritubular cells and seminiferous tubules. Biol Reprod 63:1825-1838

Cline T, Meyer B 1996 Vive la difference: males vs females in flies vs worms. Annu Rev Genet 30:637-702

Colvin JS, Green RP, Schmahl J, Capel B, Ornitz DM 2001 Male-to-female sex reversal in mice lacking fibroblast growth factor 9. Cell 104:875-889

daSilva SM, Hacker A, Harley V, Goodfellow P, Swain A, Lovell-Badge R 1996 Sox9 expression during gonadal development implies a conserved role for the gene in testis differentiation in mammals and birds. Nature Genet 14:62-68

Donahoe PK, Cate RL, MacLaughlin DT, Epstein J, Fuller AF, Takahashi M, Coughlin JP, Ninfa EG, Taylor LA 1987 Müllerian inhibiting substance: gene structure and mechanism of action of a fetal regressor. Recent Progr Horm Res 43:431-467

Eicher EM, Washburn LL, Whitney IJ, Morrow KE 1982 Mus poschiavinus Y chromosome in the C57BL/6J murine genome causes sex reversal. Science 217:535-537 
Eicher EM, Shown EP, Washburn LL 1995 Sex reversal in C57BL/6J-Y ${ }^{\mathrm{POS}}$ mice corrected by a Sry transgene. Phil Trans R Soc B 350:263-269

Eicher E, Washburn L, Schork N, Lee B, Shown E, Xu X, Dredge R, Pringle M, Page D 1996 Sex-determining genes on mouse autosomes identified by linkage analysis of C57BL/6J$\mathrm{Y}^{\mathrm{POS}}$. Nature Genet 14:206-209

Ginsburg M, Snow M, McLaren A 1990 Primordial germ cells in the mouse embryo during gastrulation. Development 110:521-528

Gomperts M, Wylie C, Heasman J 1994 Primordial germ cell migration. Ciba Found Symp 182:121-134

Gubbay J, Collignon J, Koopman P, Capel B, Economou A, Munsterberg A, Vivian N, Goodfellow P, Lovell-Badge R 1990 A gene mapping to the sex-determining region of the mouse $\mathrm{Y}$ chromosome is a member of a novel family of embryonically expressed genes. Nature 346:245-250

Hacker A, Capel B, Goodfellow P, Lovell-Badge R 1995 Expression of Sry, the mouse sex determining gene. Development 121:1603-1614

Hatano O, Takakusu A, Nomura M, Morohashi K 1996 Identical origin of adrenal cortex and gonad revealed by expression profiles of $A d 4 B P / S F 1$. Genes Cells 1:663-671

Hossain A, Saunders GF 2001 The human sex-determining gene SRY is a direct target of WT1. J Biol Chem 276:16817-16823

Huang EJ, Manova K, Packer AI, Sanchez S, Bachvarova RF, Besmer P 1993 The murine steel panda mutation affects kit ligand expression and growth of early ovarian follicles. Dev Biol 157:100-109

Ikeda Y, Shen W-H, Ingraham H, Parker K 1994 Developmental expression of mouse steroidogenic factor 1, an essential regulator of the steroid hydroxylases. Mol Endocrinol 8:654-662

Ikeda Y, Swain A, Weber TJ, Hentges KE, Zanaria E, Lalli E, Tamai KT, Sassone-Corsi P, Lovell-Badge R, Camerino G, Parker KL 1996 Steroidogenic factor 1 and Dax-1 colocalize in multiple cell lineages: potential links in endocrine development. Mol Endocrinol 10:12611272

Josso N, Picard JY 1986 Anti-Müllerian hormone. Physiol Rev 66:1038-1090

Jost A 1947 Recherches sur la differenciation sexuelle de l'embryon de lapin. Archs Anat Microsc Morph Exp 36:271-315

Kamachi Y, Uchikawa M, Kandoh H 2000 Pairing SOX off: with partners in the regulation of embryonic development. Trends Genet 16:182-187

Karl J, Capel B 1998 Sertoli cells of the mouse testis originate from the coelomic epithelium. Dev Biol 203:323-333

Katoh-Fukui Y, Tsuchiya R, Shiroishi T, Nakahara Y, Hashimoto N, Noguchi K, Higashinakagawa T 1998 Male to female sex reversal in M33 mutant mice. Nature 393:688-692

Kent J, Wheatley SC, Andrews JE, Sinclair AH, Koopman P 1996 A male-specific role for SOX9 in vertebrate sex determination. Development 122:2813-2822

Koopman P, Munsterberg A, Capel B, Vivian N, Lovell-Badge R 1990 Expression of a candidate sex-determining gene during mouse testis differentiation. Nature 348:450-452

Koopman P, Gubbay J, Vivian N, Goodfellow P, Lovell-Badge R 1991 Male development of chromosomally female mice transgenic for Sry. Nature 351:117-121

Kriedberg JA, Sariola H, Loring JM, Maeda M, Pelletier J, Housman D, Jaenisch R 1993 Wt-1 is required for early kidney development. Cell 74:679-691

Kurohmaru M, Kanai Y, Hayashi Y 1992 A cytological and cytoskeletal comparison of Sertoli cells without germ cells and those with germ cells using the $W / W^{V}$ mutant mouse. Tiss Cell 24:895-903

Lee CH, Taketo T 2001 Low levels of Sry transcripts cannot be the sole cause of B6-Y ${ }^{\mathrm{TIR}}$ sex reversal. Genesis 30:7-11 
Luo X, Ikeda Y, Parker K 1994 A cell-specific nuclear receptor is essential for adrenal and gonadal development and sexual differentiation. Cell 77:481-490

Martineau J, Nordqvist K, Tilmann C, Lovell-Badge R, Capel B 1997 Male-specific cell migration into the developing gonad. Curr Biol 7:958-968

McLaren A 1995 Germ cells and germ cell sex. Phil Trans Royal Soc Lond 350:229-233

McLaren A 2000 Germ and somatic cell lineages in the developing gonad. Mol Cell Endocrinol 163:3-9

McLaren A, Southee D 1997 Entry of mouse embryonic germ cells into meiosis. Dev Biol 187:107-113

Merchant-Larios H 1979 Origin of the somatic cells in the rat gonad: an autoradiographic approach. Ann Biol Anim Biochem Biophys 19:1219-1229

Merchant-Larios H, Taketo T 1991 Testicular differentiation in mammals under normal and experimental conditions. J Electron Microsc Tech 19:158-171

Merchant-Larios H, Moreno-Mendoza N, Buehr M 1993 The role of the mesonephros in cell differentiation and morphogenesis of the mouse fetal testis. Intl J Dev Biol 37:407-415

Merchant-Larios H, Moreno-Mendoza N 1998 Mesonephric stromal cells differentiate into Leydig cells in the mouse fetal testis. Exper Cell Res 244:230-238

Miyamoto N, Yoshida M, Kuratani S, Matsuo I, Aizawa S 1997 Defects of urogenital development in mice lacking Emx2. Development 124:1653-1664

Morohashi K 1997 The ontogenesis of the steroidogenic tissues. Genes Cells 2:95-106

Münsterberg A, Lovell-Badge R 1991 Expression of the mouse Anti-Müllerian hormone gene suggests a role in both male and female sexual differentiation. Development 113:613-624

Nagamine C, Morohashi K, Carlisle C, Chang D 1999 Sex reversal caused by Mus musculus domesticus $\mathrm{Y}$ chromosome linked to variant expression of the testis determining gene. Dev Biol 216:182-194

Palmer SJ, Burgoyne PS 1991a In situ analysis of fetal, prepubertal and adult XX-XY chimaeric mouse testes: Sertoli cells are predominantly, but not exclusively, XY. Development 112:265-268

Palmer SJ, Burgoyne PS 1991b The Mus musculus domesticus Tdy allele acts later than the Mus musculus musculus Tdy allele: a basis for $\mathrm{XY}$ sex reversal in $\mathrm{C} 57 \mathrm{~B} 1 / 6-\mathrm{XY}^{\mathrm{POS}}$ mice. Development 113:709-714

Parkhurst S, Meneely P 1994 Sex determination and dosage compensation: lessons from flies and worms. Science 264:924-932

Pevny L, Lovell-Badge R 1997 Sox genes find their feet. Curr Opin Genet Dev 7:338-344

Pieau C, Dorizzi M, Richard-Mercier N 2001 Temperature-dependent sex determination and gonadal differentiation in reptiles. EXS 91:117-141

Rackley RR, Flenniken AM, Kuriyan NP, Kessler PM, Stoler MH, Williams BR 1993 Expression of the Wilms' tumor suppressor gene, Wt1, during mouse embryogenesis. Cell Growth Different 4:1023-1031

Raymond CS, Shamu C, Shen MM, Seifert K, Hirsch B, Hodgkin J, Zarkower D 1998 Evidence for evolutionary conservation of sex determining genes. Nature 391:691-695

Raymond CS, Kettlewell JR, Hirsch B, Bardwell VJ, Zarkower D 1999 Expression of Dmrt1 in the genital ridge of mouse and chicken embryos suggests a role in vertebrate sexual development. Dev Biol 215:208-220

Raymond CS, Murphy MW, O'Sullivan MG, Bardwell VJ, Zarkower D 2000 Dmrt1, a gene related to worm and fly sexual regulators, is required for mammalian testis differentiation. Genes Dev 14:2587-2595

Schmahl J, Eicher EM, Washburn LL, Capel B 2000 Sry induces cell proliferation in the mouse gonad. Development 127:65-73 
Shapiro DY 1990 Sex-changing fish as a manipulable system for the study of the determination, differentiation, and stability of sex in vertebrates. J Exp Zool 4:132-136

Shawlot W, Behringer R 1995 Requirement for Lim1 in head-organizer function. Nature 374:425430

Sinclair AH, Berta P, Palmer MS, Hawkins JR, Griffiths BL, Smith MJ, Foster JW, Frischauf AM, Lovell-Badge R, Goodfellow PN 1990 A gene from the human sex-determining region encodes a protein with homology to a conserved DNA-binding motif. Nature 346:240-244

Skinner MK, Tung PS, Fritz IB 1985 Cooperativity between Sertoli cells and testicular peritubular cells in the production and deposition of extracellular matrix components. J Cell Biol 100:1941-1947

Swain A, Lovell-Badge R 1999 Mammalian sex determination: a molecular drama. Genes Dev 13:755-767

Swain A, Narvaez S, Burgoyne P, Camerino G, Lovell-Badge R 1998 DAX1 antagonizes SRY action in mammalian sex determination. Nature 391:761-767

Tilmann C, Capel B 1999 Mesonephric cell migration induces testis cord formation and Sertoli cell differentiation in the mammalian gonad. Development 126:2883-2890

Vanio S, Heikkila M, Kispert A, Chin N, McMahon A 1999 Female development in mammals is regulated by Wnt-4 signaling. Nature 397:405-409

Vidal V, Chaboissier MC, de Rooij DG, Schedl A 2001 Sox9 induces testis development in XX transgenic mice. Nature Genet 28:216-217

Vigier B, Watrin F, Magre S, Tran D, Josso N 1987 Purified bovine AMH induces a characteristic freemartin effect in fetal rat prospective ovaries exposed to it in vitro. Development 100:43-55

Whitworth DJ, Shaw G, Renfree MB 1996 Gonadal sex reversal of the developing marsupial ovary in vivo and in vitro. Development 122:4057-4063

Yu R, Ito T, Saunders S, Camper S, Jameson J 1998 Role of Ahch in gonadal development and gametogenesis. Nature Genet 20:353-357

Zanaria E, Muscatelli F, Bardoni B, Strom T, Guioli S, Guo W, Lalli E, Moser C, Walker A, McCabe E, Meitinger T, Monaco A, Sassone-Corsi P, Camerino G 1994 An unusual member of the nuclear hormone receptor superfamily responsible for X-linked adrenal hypoplasia congenita. Nature 372:635-641

Zorn AM, Barish GD, Williams BO, Lavender P, Klymkowsky MW, Varmus HE 1999 Regulation of Wnt signaling by Sox proteins: XSox17 alpha/beta and XSox3 physically interact with $\beta$-catenin. Mol Cell 4:487-498 


\title{
Steroidogenic Factor 1: an Essential Mediator of Endocrine Development
}

\author{
Keith L. Parker,* Douglas A. Rice,* Deepak S. Lala,* Yayoi Ikeda,* \\ Xunrong Luo, $*$ Margaret Wong, ${ }^{*}$ Marit Bakke, ${ }^{*}$ Liping Zhao, ${ }^{*}$ Claudia \\ Frigeri, ${ }^{\dagger}$ Neil A. Hanley, $*$ Nancy Stallings, ${ }^{*}$ and Bernard P. Schimmer ${ }^{\dagger}$ \\ *Departments of Internal Medicine and Pharmacology, University of Texas Southwestern Medical \\ Center, Dallas, Texas 75390; ${ }^{\dagger}$ Banting and Best Department of Medical Research, \\ University of Toronto, Toronto M5G 1 L6 Canada
}

\begin{abstract}
The orphan nuclear receptor steroidogenic factor 1 (SF-1, also called Ad4BP and officially designated NR5A1) has emerged as an essential regulator of endocrine development and function. Initially identified as a tissue-specific transcriptional regulator of the cytochrome P450 steroid hydroxylases, SF-1 has considerably broader roles, as evidenced from studies in knockout mice lacking SF-1. The SF-1-knockout mice lacked adrenal glands and gonads and therefore died from adrenal insufficiency within the first week after birth. In addition, SF-1 knockout mice exhibited male-to-female sex reversal of their internal and external genitalia, impaired expression of multiple markers of pituitary gonadotropes, and agenesis of the ventromedial hypothalamic nucleus (VMH). These studies delineated essential roles of SF-1 in regulating endocrine differentiation and function at multiple levels, particularly with respect to reproduction. This chapter will review the experiments that established SF-1 as a pivotal, global determinant of endocrine differentiation and function. We next discuss recent insights into the mechanisms controlling the expression and function of SF-1 as well as the current status of research aimed at delineating its roles in specific tissues. Finally, we highlight areas where additional studies are needed to expand our understanding of SF-1 action.
\end{abstract}

\section{Initial Isolation of Steroidogenic Factor 1}

Steroid hormones are essential for fluid and electrolyte balance, intermediary metabolism, sexual differentiation, and reproductive function. Once the pathways of steroid hormone biosynthesis were defined and shown to involve the concerted actions of several cytochrome P450 mixed-function oxidases, attention turned to elucidating the mechanisms that regulate the expression of these enzymes. With the isolation of the bovine 21-hydroxylase cDNA (White et al., 1984b), followed shortly thereafter by the cloning of cDNAs encoding the side-chain cleavage enzyme (Matteson et al., 1984; Morohashi et al., 1984) and 11 $\beta$-hydroxylase (John et al., 1984), these questions could be addressed at a molecular level. David Chaplin, a postdoctoral fellow in J.G. Seidman's laboratory who previ- 
ously had isolated cosmids spanning the $\mathrm{S}$ region of the mouse $\mathrm{H}-2$ complex, cloned a cosmid that contained the mouse 21-hydroxylase gene and $\approx 30 \mathrm{~kb}$ of its $5^{\prime}$-flanking region (White et al., 1984a). The Schimmer laboratory had extensive experience with Y1 mouse adrenocortical tumor cells, which expressed several cytochrome P450 steroid hydroxylases - but not steroid 21-hydroxylase - in a hormonally responsive manner and were readily amenable to both stable and transient transfection (Schimmer, 1985). In a collaborative effort, the Schimmer and Seidman laboratories showed that Y1 cells stably transfected with the mouse 21-hydroxylase cosmid recovered hormonally regulated expression of 21-hydroxylase (Parker et al., 1985). Thereafter, 5'-deletion assays localized sequences essential for cell-selective and hormone-regulated expression of the 21-hydroxylase gene to the proximal $330 \mathrm{bp}$ of $5^{\prime}$-flanking DNA (Parker et al., 1986; Handler et al., 1988).

Using similar approaches, a number of groups analyzed the 5'-flanking regions of genes encoding the cytochrome P450 steroid hydroxylases (for reviews of these studies, see Omura and Morohashi, 1995; Parker and Schimmer, 1995). In particular, studies by two groups identified shared AGGTCA promoter elements in the proximal promoter regions of several of the steroid hydroxylases that interacted with the same DNA-binding protein (Rice et al., 1991; Morohashi et al., 1992). This protein, which initially was found only in steroidogenic cell lines, was designated steroidogenic factor 1 (SF-1) or adrenal 4-binding protein (Ad4BP). The selective expression of SF-1 in steroidogenic cells and its regulation of multiple genes encoding steroid hydroxylases provided the first clues that it was an important determinant of the cell-selective expression of the steroidogenic enzymes.

Based on evidence that SF-1 was a key determinant of the expression of the cytochrome P450 steroid hydroxylases, the Parker and Morohashi laboratories independently cloned cDNAs encoding SF-1. Morohashi and colleagues used an oligonucleotide affinity column to purify the protein from bovine adrenal glands, ultimately allowing them to obtain amino acid sequence and clone a bovine cDNA with an oligonucleotide probe (Honda et al., 1993). In contrast, Douglas Rice, a postdoctoral fellow in the Parker laboratory, reasoned that the AGGTCA DNA recognition motif represented a binding site for an atypical member of the nuclear hormone receptor family. Using a hybridization probe comprising the DNA-binding region of retinoid $\mathrm{X}$ receptor, the Parker laboratory isolated a cDNA clone that was expressed in adrenal gland, testes, and ovaries, but not in a variety of other tissues (Lala et al., 1992).

Subsequent studies established that the mouse and bovine cDNAs encoded orthologs of a protein that transactivated the steroid hydroxylase promoters in steroidogenic and nonsteroidogenic cells. As predicted from the cloning strategy used by the Parker group, the sequences of these cDNAs confirmed that SF-1 
belonged to the nuclear hormone receptor family, with striking homology to the Drosophila nuclear receptor fushi tarazu factor 1 (Ftz-F1) and the mouse nuclear receptor embryonal long terminal repeat-binding protein (Tsukiyama et al., 1992). SF-1 homologs have been identified in a diverse group of species that includes humans, marmosets, cows, sheep, horses, mice, rats, pigs, tamarind wallabies, chickens, turtles, salmon, trout, zebrafish, flies, and worms.

\section{Developmental Profile of SF-1 Expression}

To address the potential roles of SF-1 during mammalian development, Yayoi Ikeda in the Parker laboratory used in situ hybridization to analyze its spatial and temporal profiles of expression in mouse embryos (Ikeda et al., 1994). As anticipated, SF-1 transcripts were detected in the adrenal primordium from very early stages of its development (approximately embryonic day (E) 10.5). As the chromaffin cell precursors migrated into the adrenal primordium at $\approx \mathrm{E} 12.5$ to E13.5, SF-1 expression was restricted to the steroidogenic cells in the cortex. The initiation of SF-1 expression before the onset of steroidogenesis supported its key role in steroid hydroxylase expression and suggested additional roles in adrenal development.

In mice, gonadal development first becomes apparent at $\approx \mathrm{E} 9$, when the intermediate mesoderm condenses into the urogenital ridge, which ultimately contributes cell lineages to the gonads, adrenal cortex, and kidneys. At this time, developing testes and ovaries are indistinguishable histologically and thus are termed indifferent or bipotential. By $\approx \mathrm{E} 12.5$, the fetal testes have organized into the testicular cords, which contain fetal Sertoli cells and primordial germ cells, and the surrounding interstitial region, which contains the Leydig cells. Faint expression of SF-1 was seen in both male and female embryos from the inception of the indifferent stage (E9.0-E9.5), persisting thereafter throughout the indifferent gonad stage. Coincident with formation of the testicular cords at E12.5, SF-1 expression persisted in the testes but diminished in ovaries (Hatano et al., 1994; Ikeda et al., 1994). In addition, SF-1 transcripts were detected in both the interstitial region, where Leydig cells produce steroid hormones, and the testicular cords, where fetal Sertoli cells produce anti-Müllerian hormone. SF-1 transcripts also were detected in the embryonic diencephalon - the precursor to the endocrine hypothalamus - and the anterior pituitary gland (Ikeda et al., 1994). Taken together, these findings suggested roles for SF-1 in gonadal development that extended beyond its effects on the expression of the steroidogenic enzymes and actions to regulate multiple levels of the hypothalamic-pituitary-steroidogenic organ axis. 


\section{The Roles of SF-1 in Vivo}

\section{A. KNOCKOUT MOUSE STUDIES}

To address the role of SF-1 in vivo, three groups (Parker, Morohashi, and Milbrandt) used targeted gene disruption in embryonic stem cells to generate SF-1 knockout mice. In the Parker laboratory, Xunrong Luo generated the SF-1 knockout mice in collaboration with Drs. Beverly Koller and Ann Latour (Luo et al., 1994). Consistent with the model that SF-1 was required for adrenal and gonadal steroidogenesis, SF-1 knockout mice died shortly after birth from adrenocortical insufficiency and exhibited male-to-female sex reversal of the external genitalia (Luo et al., 1994; Sadovsky et al., 1995). By analogy with human subjects with impaired expression of the steroid hydroxylases, the Parker laboratory anticipated that the adrenal glands of SF-1 knockout mice would be hyperplastic due to their inability to make glucocorticoids and the consequent exposure to high levels of corticotropin (ACTH). In a major surprise, shown in Figure 1, the adrenal glands and gonads were completely absent in newborn SF-1 knockout mice (Luo et al., 1994; Sadovsky et al., 1995). Subsequent studies showed that the initial stages of adrenal and gonadal development occurred in the absence of SF-1, followed by their regression at a specific stage of development. Because their gonads regressed before male sexual differentiation normally occurs, the internal and external urogenital tracts of SF-1 knockout mice were female, irrespective of genetic sex.

The gonadotropes of SF-1 knockout mice also had impaired expression of a number of genes that regulate reproduction, including luteinizing hormone $\beta$ (LH- $\beta$ ), follicle-stimulating hormone $\beta$ (FSH- $\beta$ ), the $\alpha$-subunit of glycoprotein hormones $(\alpha \mathrm{GSU})$, and the receptor for gonadotropin-releasing hormone (Ingraham et al., 1994; Shinoda et al., 1995). As shown in Figure 2, these knockout mice also lacked the ventromedial hypothalamic nucleus (VMH), a hypothalamic region linked to feeding and appetite regulation and female reproductive behavior (Ikeda et al., 1995; Shinoda et al., 1995). Finally, although the functional consequences remain to be defined, the SF-1 knockout mice had defects in their splenic parenchyma (Morohashi et al., 1999).

Guided in part by studies in human subjects with clinical disorders due to haploinsufficiency of SF-1 or other genes involved in gonadal development (see below), Ingraham and colleagues examined more carefully the phenotype of heterozygous SF-1 knockout mice. These studies, which revealed decreased adrenal volume associated with impaired corticosterone production in response to stress (Bland et al., 2000), suggested that the level of SF-1 expression may be very important for optimal adrenal development.

Because the original SF-1 knockout mice are globally deficient in SF-1, they cannot be used to delineate the roles of SF-1 at specific sites of expression. For 

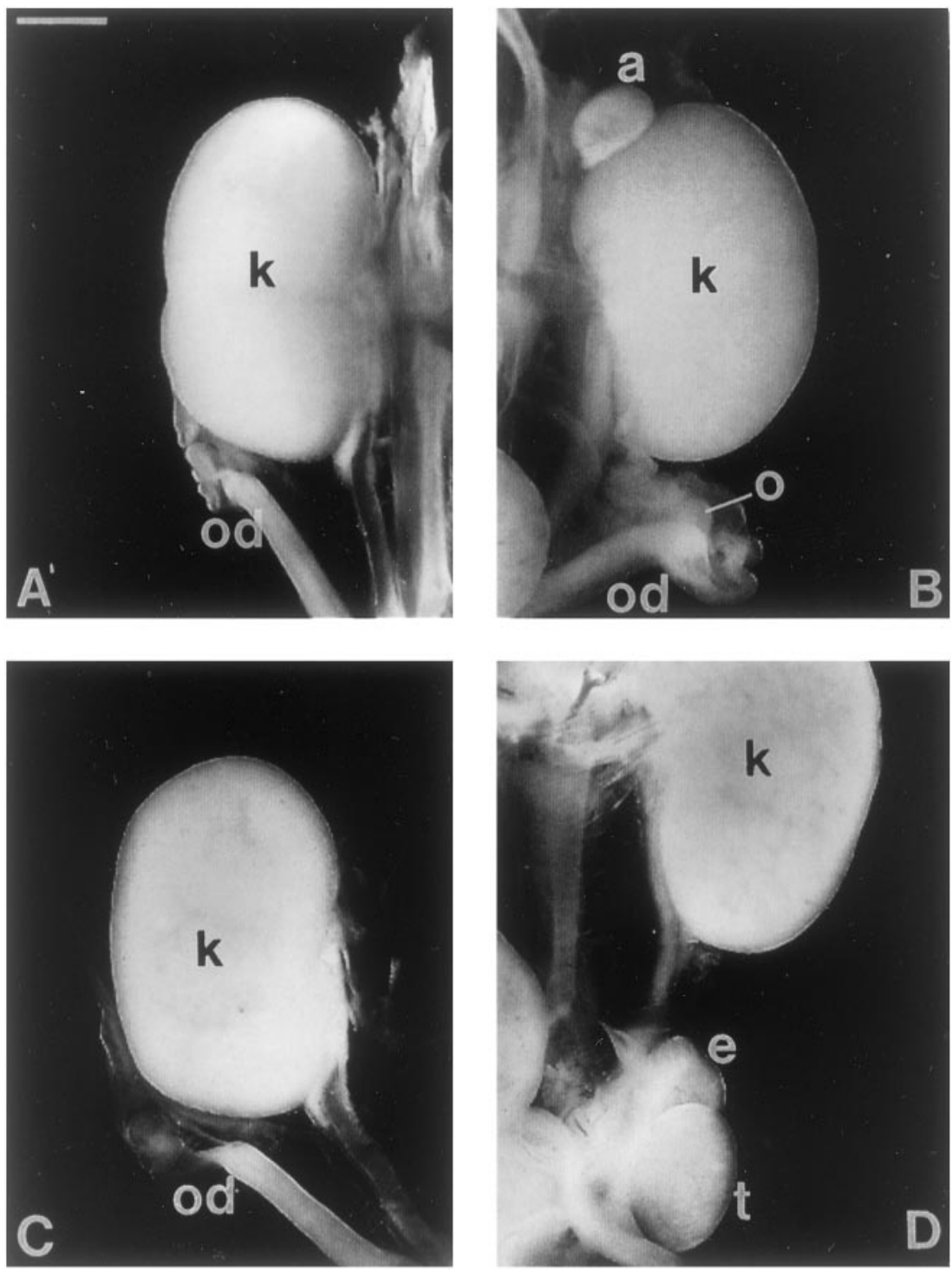

FIG. 1. SF-1 knockout mice lack adrenal glands and gonads and have female internal genitalia. The dissected genitourinary tracts of wild-type female (B) and male (D) and SF-1 knockout female (A) and male (C) mice are shown. Note the absence of adrenal glands and gonads in SF-1-deficient mice and the presence of oviducts in both males and females. a, adrenal gland; k, kidney; o, ovary; t, testis; e, epididymis; od, oviduct. [Reprinted from Luo X, Ikeda Y, Parker KL 1994 A cell specific nuclear receptor is required for adrenal and gonadal development and for male sexual differentiation. Cell 77:481-490, with permission from Elsevier Science.] 

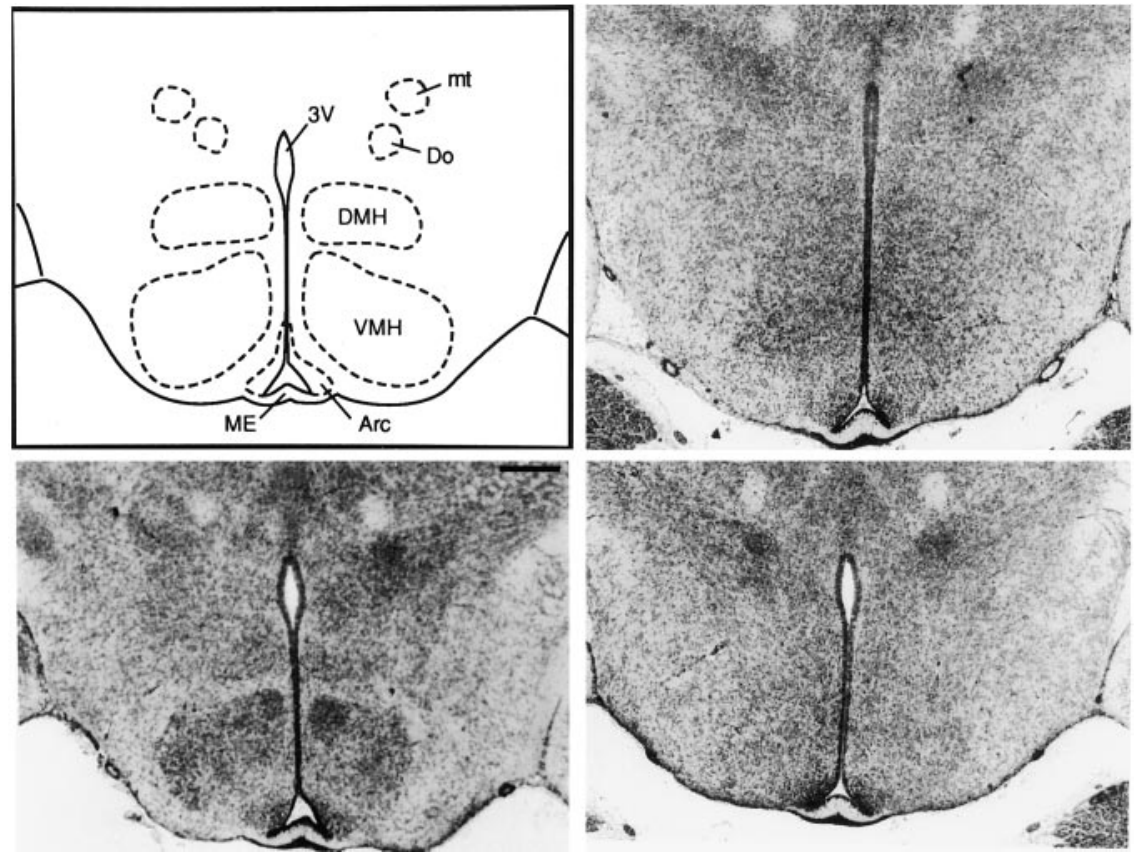

FIG. 2. SF-1 knockout mice lack the ventromedial hypothalamic nucleus (VMH). Serial coronal sections from wild-type (lower left) and -/- male (upper right) and female (lower right) mice were stained and analyzed histologically. Shown at the upper left is a schematic diagram of anatomical regions found within these sections. VMH, ventromedial hypothalamic nucleus; DMH, dorsomedial hypothalamic nucleus; ME, median eminance; Arc, arcuate nucleus; Do, dorsal hypothalamic nucleus; $3 \mathrm{~V}$, 3rd ventricle; $\mathrm{mt}$, mammilothalamic tract. [Modified with permission from Ikeda Y, Luo X, Abbud R, Nilson JH, Parker KL 1995 The nuclear receptor steroidogenic factor 1 is essential for the formation of the ventromedial hypothalamic nucleus. Mol Endocrinol 9:478-486. Copyright The Endocrine Society.]

example, the apparent defect in gonadotrope function could merely reflect the absence of gonadal steroids. Another limitation of the original SF-1 knockout mice is their early postnatal death from adrenocortical insufficiency, which precludes efforts to examine the roles of SF-1 after differentiation has occurred. Finally, the need to administer exogenous corticosteroids to keep SF-1 knockout mice alive complicates considerably our ability to use these mice to assess the role of the VMH in feeding and weight regulation. To obviate such limitations, the Parker laboratory has begun to use the Cre/loxP system to produce tissue-specific knockouts of SF-1. Marit Bakke initiated this process by modifying the SF-1 locus in embryonic stem cells to insert recognition sites for the bacteriophage Cre recombinase (termed loxP sites) around the last exon of SF-1, which encodes an essential domain for 
transcriptional activation and transcription termination sequences. The second essential step in the tissue-specific knockout was to generate a Cre transgene selectively active in a subset of SF-1-expressing cells. Lisa Cushman in the Camper laboratory generated a transgenic mouse line in which Cre expression was directed to the anterior pituitary gland by the 5 '-flanking sequences of the $\alpha$ subunit of glycoprotein hormones (Cushman et al., 2000). Liping Zhao bred the loxP-modified SF-1 line with the Cre transgenic mice, ultimately generating mice with pituitary-specific disruption of SF-1.

As shown in Figure 3, the $\alpha \mathrm{GSU}-\mathrm{Cre} /$ loxP mice selectively lacked SF-1 immunoreactivity in the anterior pituitary (Zhao et al., 2001) but had normal levels at other sites, including the adrenal cortex and VMH. These mice had markedly diminished levels of pituitary gonadotropins and exhibited severe gonadal hypoplasia secondary to impaired gonadotropin stimulation. These pituitary-specific SF-1 knockout mice demonstrated that the local production of SF-1 in mice is essential for normal gonadotrope function, strongly supporting a direct role for SF-1 in gonadotropin gene expression.

\section{B. SF-1 AND HUMAN DISEASE}

The sequence of the human gene encoding SF-1 closely resembled that of the mouse gene (Oba et al., 1996; Wong et al., 1996) and SF-1 expression during human embryological development closely paralleled that in mice (Hanley et al., 1999; de Santa Barbara et al., 2000). Thus, it was plausible that mutations in the human SF-1 gene on chromosome 9q33 (Taketo et al., 1995) might cause endocrine disease. Many groups looked for SF-1 mutations in patients with clinical disorders of adrenocortical development and/or sexual differentiation. To date, only two subjects with diseases associated with SF-1 mutations have been described, suggesting that SF-1 mutations occur only rarely. The first subject with a SF-1 mutation presented with adrenocortical insufficiency and 46,XY gonadal dysgenesis (Achermann et $a l ., 1999)$. In contrast to SF-1 knockout mice, which had diminished gonadotropin levels, this subject had elevated levels of gonadotropins. The second subject also presented with adrenocortical insufficiency, but had a 46,XX karyotype with apparently normal prepubertal ovaries (Biason-Lauber and Schoenle, 2000). Surprisingly, each patient had one apparently normal SF-1 allele and different mutated alleles that resulted in loss-of-function rather than dominant-negative effects. This apparent haploinsufficiency raises the intriguing possibility that gene dosage may be a critical component of SF-1 function in humans. These results further suggest, as seen in other knockout mouse models, that the phenotypes in patients with SF-1 mutations need not correlate precisely with observations in SF-1 knockout mice. 


\section{SF-1}
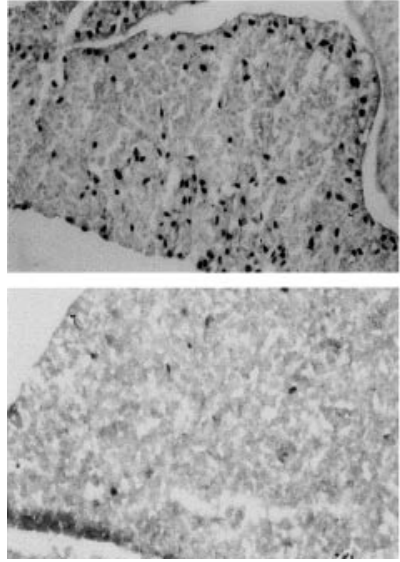
us to inactivate SF-1 specifically in the anterior pituitary gland. The top panels show wild-type sections and the bottom panels show sections from mice with a pituitary-specific knockout of SF-1. Note that immunoreactivities for SF-1 and LH are both virtually abolished and that the testes and ovaries are markedly hypoplastic secondary to deficient gonadotropin stimulation.
Testis
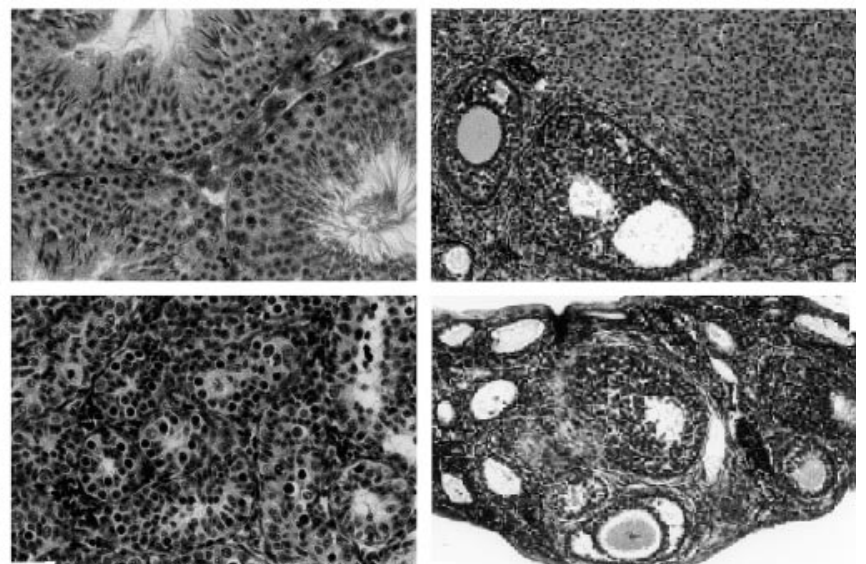


\section{Regulation of SF-1 Expression and Function}

\section{A. REGULATION OF SF-1 EXPRESSION}

Relatively little is known about the mechanisms that regulate the expression of SF-1 within specific cell lineages. To date, there are no published successes with transgenic promoter analyses, so our insights largely are limited to transfection analyses in cell-culture models. In one published report, a conserved E-box motif in the 5'-flanking region of the rat gene encoding SF-1 was shown to regulate promoter activity in transfected mouse Y1 adrenocortical or I-10 Leydig tumor cells (Nomura et al., 1995). Moreover, a protein that interacted with this E-box motif in gel mobility shift assays was expressed at higher levels in embryonic testes than ovaries. The same E-box motif was implicated as an important regulator of SF-1 promoter activity in Sertoli cells (Daggett et al., 2000) and in Y1 adrenocortical cells and $\alpha \mathrm{T} 3$ gonadotropes (Harris and Mellon, 1998). In the latter study, the transcription factor USF-1 was shown to regulate SF-1 expression. In view of the known developmental roles of basic helix-loophelix proteins that bind these E-box motifs, these studies suggest an important role for basic helix-loop-helix proteins in regulating SF-1 expression in several cell types.

Other promoter elements implicated in SF-1 promoter activity include a GC-rich sequence that may represent a binding site for Sp1 and a CCAAT-box motif (Woodson et al., 1997). The precise roles of these elements in different tissues remain to be defined. Moreover, the lack of success in the reported transgenic expression studies suggests that other elements also play important roles in vivo.

One might predict that distinct mechanisms regulate SF-1 expression in the adrenal cortex and gonads, which are believed to arise from the same embryonic lineage (Hatano et al., 1996), versus the anterior pituitary and hypothalamus, which are contiguous structures that interact reciprocally during development (Rosenfeld et al., 1996). In support of this, the pituitary transcripts in some species arise from a transcription initiation site distinct from that used in other sites (Ninomiya et al., 1995; Kimura et al., 2000). In an effort to explore the mechanisms that regulate SF-1 expression in vivo, the Parker laboratory recently used a 50-kb fragment derived from a bacterial artificial chromosome to direct expression of a green fluorescent protein (GFP) reporter gene in transgenic mice. As shown in Figure 4, GFP expression in the urogenital ridge was detected at E9.5, closely paralleling the onset of SF expression during gonadogenesis (Ikeda et al., 1994). Although GFP expression in the adrenal primordium and $\mathrm{VMH}$ also corresponded to the known expression profile of SF-1, GFP was not expressed in the anterior pituitary. These results document that $50 \mathrm{~kb}$ of $5^{\prime}$-flanking region 


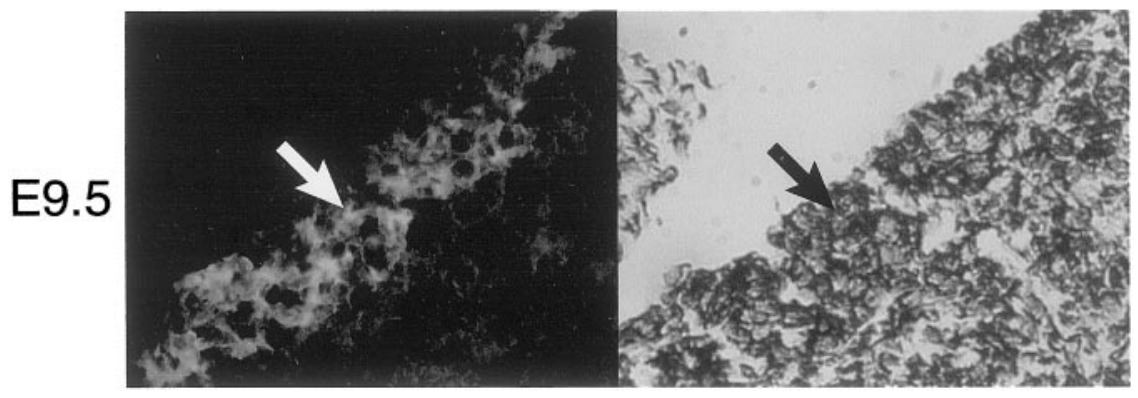

FIG. 4. A green fluorescent protein reporter (GFP) transgene is targeted to the embryonic gonad by SF-1 $5^{\prime}$-flanking sequences. A transgene containing $50 \mathrm{~kb}$ of $5^{\prime}$-flanking sequences from the mouse locus encoding SF-1 was placed upstream of coding sequences for enhanced green fluorescent protein (eGFP). This construct was microinjected into pronuclei to generate a transgenic founder. A section from an E9.5 embryo was analyzed by fluorescence microscopy, revealing GFP expression in the urogenital ridge (arrows).

suffice to direct regulated expression in most sites but also suggest that additional regulatory elements are required for activation of the pituitary-specific promoter.

\section{B. MODULATION OF SF-1 ACTIVITY}

Limited data also are available regarding the regulation of SF-1 activity in cells where it is expressed. As a member of the nuclear hormone receptor family, one obvious possibility is that a ligand regulates SF-1 transcriptional activity in a manner analogous to many former "orphan" members (Kliewer et al., 1999). Using a cotransfection assay, Lala and colleagues noted that hydroxycholesterol derivatives increased SF-1 transcriptional activity by about 10-fold, suggesting that intermediates in the steroidogenic pathway might modulate SF-1 activity (Lala et al., 1997). Others, however, have suggested that the effects of oxysterols are unique to certain nonsteroidogenic cells and may not be relevant to steroidogenic cells (Christenson et al., 1998; Mellon and Bair, 1998). Thus, the precise role of ligands in SF-1 activity remains to be defined.

In the absence of a clear-cut ligand, others have examined the possibility that post-translational modifications alter SF-1 transcriptional activity. In their initial description of the bovine sequence, Morohashi and colleagues noted a potential phosphorylation site for cAMP-dependent protein kinase. Mellon and colleagues subsequently reported that recombinantly expressed SF-1 was phosphorylated in vitro by cAMP-dependent protein kinase (Zhang and Mellon, 1996). Mutation of the serine residue in the consensus motif did not impair SF-1 function in transfection assays (Lopez et al., 2001), suggesting that this site is not a key regulator of SF-1 activity. 
In a separate line of investigations, the Ingraham laboratory used peptide mapping to define a site of SF-1 phosphorylation (Ser203) that mapped within a consensus motif for phosphorylation by mitogen-activated protein (MAP) kinase (Hammer et al., 1999). They further showed that activating the MAP kinase pathway increased SF-1 transcriptional activation, while mutation of Ser203 diminished SF-1 activity. Collectively, these findings raise the possibility that SF-1 function is modulated by posttranslational modification through extracellular signals that act via the MAP kinase pathway.

\section{REGULATION THROUGH PROTEIN-PROTEIN INTERACTIONS}

Analyses of human patients and knockout mouse models have identified a number of other genes that play key roles in the development of SF-1-expressing tissues (for a review, see Parker et al., 1999). It is almost certain that these genes interact with SF-1 - either in hierarchical cascades of gene regulation or via protein-protein interactions - to mediate endocrine development. Indeed, a number of factors have been shown to interact directly with SF-1, including the Wilm's tumor related tumor suppressor gene WT-1 (Nachtigal et al., 1998), GATA-4 (Tremblay and Viger, 1999), Ptx1 (Tremblay et al., 1999), SOX9 (de Santa-Barbara et al., 1999), and EGR1 (Halvorson et al., 1998; Dorn et al., 1999). In contrast, SF-1 expression is markedly decreased in the gonads - but not the adrenal glands - of Lhx 9 knockout mice, suggesting that Lhx9 regulates SF-1 expression in the gonads (Birk et al., 2000). Finally, DAX-1 and SF-1 apparently interact both hierarchically (i.e., SF-1 regulates DAX1 expression) (Yu et al., 1998; Kawabe et al., 1999) and by direct protein-protein interactions wherein DAX1 inhibits SF-1 transcriptional activation (Ito et al., 1997; Crawford et al., 1998).

Besides interactions with other tissue-specific transcription factors, it is apparent that coactivators and co-repressors are critical modulators of nuclear receptor transcriptional activity (for a review, see Xu et al., 1999). Predictably, a number of co-regulators have been reported to interact with SF-1, including CBP/P300 (Monte et al., 1998), GRIPI (Hammer et al., 1999), SRC-1 (Crawford et al., 1997a), MBP1 (Kabe et al., 1999), SMRT (Hammer et al., 1999), and N-CoR (Crawford et al., 1998; Nachtigal et al., 1998). It is possible that differential interactions with these co-regulators, as well as with the tissuespecific transcription factors described above, specify the differential expression of SF-1 target genes in various tissues. An increased understanding of the ways in which these genes interact to regulate the expression of specific target genes undoubtedly will provide important new insights into processes of endocrine development. 


\section{Directions for Future Research}

The studies summarized here have defined essential roles of SF-1 at multiple levels of endocrine differentiation and function, particularly within the reproductive axis. Specifically, SF-1 is the first transcriptional regulator shown to play key roles at all levels of the hypothalamic-pituitary-steroidogenic organ axis. Despite this considerable progress, a number of important questions remain to be answered. We still do not completely understand the factors that govern the expression of SF-1 or that regulate its activity in different tissues. Nor do we understand the specific roles of SF-1 at the different sites where it is expressed. As noted earlier, the pituitary-specific knockout of SF-1 has helped establish the functional importance of SF-1 within mouse gonadotropes. Similar efforts now are underway to inactivate SF-1 specifically in the VMH (using neuron-specific promoters), in Leydig/theca cells (using steroid 17 $\alpha$-hydroxylase or Mullerian inhibiting substance (MIS) receptor promoters), and in Sertoli/granulosa cells (using the MIS or inhibin promoters). These experiments should provide novel insights into the specific functions of SF-1 at these sites.

What is the relationship of SF-1 to other closely related members of the NR5 nuclear receptor family? SF-1 most closely resembles another orphan nuclear receptor, NR5A2, particularly within the DNA-binding domain, suggesting that these two transcription factors may regulate overlapping target genes. Indeed, both SF-1 and NR5A2 can activate promoter activity of the small heterodimerization partner (SHP) nuclear receptor (Lee et al., 1999), which is expressed in the adrenal cortex, liver, and other tissues. Interestingly, NR5A2 transcripts are expressed at high levels in the corpus luteum of the ovary (Boerboom et al., 2000), where it may replace SF-1 as a critical regulator of the cytochrome P450 steroid hydroxylases at certain stages of the ovulatory cycle.

While many laboratories have identified a diverse group of SF-1 target genes, as summarized in Table I, these analyses largely have focused on transient transfection assays using relatively limited stretches of promoter/regulatory DNA. These studies may overemphasize the importance of SF-1 in gene regulation and verification of important roles in vivo ultimately is needed. Among the SF-1 target genes in Table I, evidence supporting such in vivo roles has been provided for MIS (Giuili et al., 1997; Arango et al., 1999) and LH $\beta$ (Keri and Nilson, 1996). Moreover, important questions about specific roles of SF-1 in development versus differentiated function may be too subtle to be addressed in either global or tissue-specific knockouts. Although it was anticipated that SF-1 knockout mice might provide in vivo evidence for the importance of SF-1 in gene expression, particularly for those genes involved in steroidogenesis, the failure of the knockout mice to develop the steroidogenic organs and the VMH precluded such analyses at these sites. Strong evidence for a developmental role of SF-1 came from studies in which forced expression of SF-1 in embryonic stem cells 
TABLE I

Sites of Action and Target Genes for Steroidogenic Factor-1

\begin{tabular}{|c|c|}
\hline Ventromedial hypothalamic nucleus & $\mathrm{N}$-methyl-D-aspartate receptor \\
\hline Gonadotropes & $\begin{array}{l}\alpha \text { subunit of glycoprotein hormones } \\
\text { Luteinizing hormone (LH) } \beta \\
\text { Follicle-stimulating hormone (FSH) } \beta \\
\text { Gonadotropin-releasing hormone receptor }\end{array}$ \\
\hline Adrenal cortex & $\begin{array}{l}\text { Cytochrome } \mathrm{P} 450 \text { steroid hydroxylases } \\
3 \beta \text {-hydroxysteroid dehydrogenase } \\
\text { Steroidogenic acute regulatory protein (StAR) } \\
\text { Corticotropin receptor } \\
\text { Scavenger receptor-B1 } \\
\text { Hydromethylglutaryl-CoA reductase } \\
\text { DAX-1 } \\
\text { Aldose reductase-like protein }\end{array}$ \\
\hline Gonads & \\
\hline Leydig cells & $\begin{array}{l}\text { Cytochrome P450 steroid hydroxylases } \\
\text { StAR } \\
\text { LH receptor } \\
\text { Insulin-like polypeptide } 3 \\
\text { Prolactin receptor } \\
\text { Mullerian inhibiting substance (MIS) receptor }\end{array}$ \\
\hline Sertoli cells & $\begin{array}{l}\text { MIS } \\
\text { Inhibin } \\
\text { FSH receptor } \\
\text { Sex-determining region Y (SRY) } \\
\text { SOX9 (SRY box) }\end{array}$ \\
\hline Theca and granulosa cells & $\begin{array}{l}\text { Cytochrome P450 steroid hydroxylases } \\
\text { StAR } \\
\text { Inhibin } \\
\text { Oxytocin }\end{array}$ \\
\hline
\end{tabular}


induced the expression of the cholesterol side-chain cleavage enzyme (Crawford et al., 1997b). In contrast, analyses of mutant Y1 cells with defects affecting SF-1 function provided evidence for multiple roles in differentiated function. The Schimmer laboratory showed that a SF-1 mutation was associated with decreased expression of the ACTH receptor, $11 \beta$-hydroxylase, cholesterol side-chain cleavage enzyme, and steroidogenic acute regulator protein (StAR) (Frigeri et al., 2000). Interestingly, the SF-1 defect affected the expression of the ACTH receptor and $11 \beta$-hydroxylase to a much greater degree than cholesterol sidechain cleavage enzyme or StAR, suggesting further subtleties among the target genes in their regulation by SF-1.

The ability to disrupt SF-1 expression or function in a temporally specific manner after development of the steroidogenic tissues is completed would help to assess the importance of SF-1 in gene expression in a more physiological context. To this end, transgenic systems in which Cre recombinase is fused to ligand-inducible proteins (e.g., mutated versions of estrogen receptor or progesterone receptor) provide an opportunity to induce Cre pharmacologically by treatment with synthetic agonists. Such temporally regulated knockouts should provide a novel approach to examine the roles of SF-1 in this continuum of differentiated function. A clear delineation of SF-1 specific target genes, an increased understanding of the factors governing SF-1 function and expression, and the temporal staging of SF-1 action undoubtedly will provide important new insights into processes of endocrine differentiation and development.

\section{ACKNOWLEDGMENTS}

We thank the past and present members of the Parker and Schimmer laboratories, who have contributed to many of the studies reviewed here, and our many colleagues who communicated unpublished results. We especially acknowledge Professors Tsuneo Omura and Ken Morohashi, who independently provided many of the critical experiments that defined the multiple roles of SF-1 and who - even as competitors - were unfailingly generous in their sharing of reagents and unpublished data. Our studies were supported by the Howard Hughes Medical Institute and the National Institutes of Health (KLP) and the Medical Research Council of Canada and the Canadian Institutes of Health Research (BPS).

\section{REFERENCES}

Achermann JC, Hindmarsh PC, Ito M, Ito M, Jameson JL 1999 A mutation in the gene encoding steroidogenic factor-1 causes $46, \mathrm{XY}$ sex reversal and adrenal failure in humans. Nature Genet 22:125-126

Arango NA, Lovell-Badge R, Behringer RR 1999 Targeted mutagenesis of the endogenous mouse Mis gene promoter: in vivo definition of genetic pathways of vertebrate sexual development. Cell 99:409-419

Biason-Lauber A, Schoenle EJ 2000 Apparently normal ovarian differentiation in a prepubertal girl with transcriptionally inactive steroidogenic factor 1 (NR5A1/SF-1) and adrenocortical insufficiency. Am J Hum Genet 67:1563-1568 
Birk OS, Casiano DE, Wassif CA, Cogliatti T, Zhao L, Zhao Y, Grinberg A, Huang SP, Kreidberg JA, Parker KL, Porter FD, Westphal H 2000 The LIM homeobox gene Lhx 9 is essential for mouse gonad formation. Nature 403:909-912

Bland ML, Jamieson CA, Akana SF, Bornstein SR, Eisenhofer G, Dallman MF, Ingraham HA 2000 Haploinsufficiency of steroidogenic factor-1 in mice disrupts adrenal development leading to an impaired stress response. Proc Natl Acad Sci USA 97:14488-14493

Boerboom D, Pilon N, Behdjani R, Silversides DW, Sirois J 2000 Expression and regulation of transcripts encoding two members of the NR5A nuclear receptor subfamily of orphan nuclear receptors, steroidogenic factor-1 and NR5A2, in equine ovarian cells during the ovulatory process. Endocrinology 141:4647-4656

Christenson LK, McAllister JM, Martin KO, Javitt NB, Osborne TF, Strauss JF III 1998 Oxysterol regulation of steroidogenic acute regulatory protein gene expression. Structural specificity and transcriptional and posttranscriptional actions. J Biol Chem 273:30729-30735

Crawford P, Polish J, Ganpule G, Sadovsky Y 1997a The activation function-2 hexamer of steroidogenic factor-1 is required but not sufficient for potentiation by SRC-1. Mol Endocrinol 11:1626-1635

Crawford PA, Sadovsky Y, Milbrandt J 1997b Nuclear receptor steroidogenic factor 1 directs embryonic stem cells toward the steroidogenic lineage. Mol Cell Biol 17:3997-4006

Crawford PA, Dorn C, Sadovsky Y, Milbrandt J 1998 Nuclear receptor DAX-1 recruits nuclear receptor corepressor N-CoR to steroidogenic factor 1. Mol Cell Biol 18: 2949-5629

Cushman L, Burrows H, Seasholtz A, Lewandoski M, Muzyczka J, Martin G, Camper S 2000 Cre-mediated recombination in the pituitary gland. Genesis 28:167-174

Daggett MA, Rice DA, Heckert LL 2000 Expression of steroidogenic factor 1 in the testis requires an E box and CCAAT box in its promoter proximal region. Biol Reprod 62:670-679

de Santa Barbara P, Bonneaud N, Boizet B, Desclozeaux M, Moniot B, Sudbeck P, Scherer G, Poulat F, Berta P 1999 Direct interaction of SRY-related protein SOX9 and steroidogenic factor 1 regulates transcription of the human anti-Mullerian hormone gene. Mol Cell Bio 18:6653-6665

de Santa Barbara P, Moniot B, Poulat F, Berta P 2000 Expression and subcellular localization of SF-1, SOX9, WT1, and AMH proteins during early human testicular development. Devel Dyn 217:293-298

Dorn C, Ou Q, Svaren J, Crawford PA, Sadovsky Y 1999 Activation of luteinizing hormone beta gene by gonadotropin-releasing hormone requires the synergy of early growth response- 1 and steroidogenic factor-1. J Biol Chem 274:13870-13876

Frigeri C, Tsao J, Czerwinski W, Schimmer BP 2000 Impaired steroidogenic factor 1 (NR5A1) activity in mutant Y1 mouse adrenocortical tumor cells. Mol Endocrinol 14:535-544

Giuili G, Shen WH, Ingraham HA 1997 The nuclear receptor SF-1 mediates sexually dimorphic expression of Mullerian inhibiting substance, in vivo. Development 124:1799-1807

Halvorson L, Ito M, Jameson JL, Chin W 1998 Steroidogenic factor-1 and early growth response protein 1 act through two composite DNA binding sites to regulate luteinizing hormone beta-subunit. J Biol Chem 273:14712-14720

Hammer GD, Krylova I, Zhang Y, Darimont BD, Simpson K, Weigel NL, Ingraham HA 1999 Phosphorylation of the nuclear receptor SF-1 modulates cofactor recruitment: integration of hormone signaling in reproduction and stress. Molec Cell 3:521-526

Handler JD, Schimmer BP, Flynn TR, Szyf M, Seidman JG, Parker KL 1988 An enhancer element and a functional cAMP-dependent protein kinase are required for expression of adrenocortical 21-hydroxylase. J Biol Chem 263:13068-13073

Hanley NA, Ball SG, Clement-Jones M, Hagan DM, Strachan T, Lindsay S, Robson S, Ostrer H, Parker KL, Wilson DI 1999 Expression of steroidogenic factor 1 and Wilms' tumour 1 during early human gonadal development and sex determination. Mech Dev 87:175-180 
Harris AN, Mellon PL 1998 The basic helix-loop-helix, leucine zipper transcription factor, USF (upstream stimulatory factor), is a key regulator of SF-1 (steroidogenic factor-1) gene expression in pituitary gonadotrope and steroidogenic cells. Mol Endocrinol 12:714-726

Hatano O, Takayama K, Imai T, Waterman MR, Takakusu A, Omura T, Morohashi, K-I 1994 Sex-dependent expression of a transcription factor, Ad4BP, regulating steroidogenic P-450 genes in the gonads during prenatal and postnatal rat development. Development 120:27872797

Hatano O, Takakusu A, Nomura M, Morohashi K-I 1996 Identical origin of adrenal cortex and gonads as revealed by expression profiles of Ad4BP/SF-1. Genes Cells 1:663-671

Honda S-I, Morohashi K-I, Nomura M, Takeya H, Kitajima M, Omura T 1993 Ad4BP regulating steroidogenic $\mathrm{P}-450$ gene is a member of the steroid receptor superfamily. J Biol Chem 268:7494-7502

Ikeda Y, Shen W-H, Ingraham HA, Parker KL 1994 Developmental expression of mouse steroidogenic factor 1 , an essential regulator of the steroid hydroxylases. Mol Endocrinol $8: 654-662$

Ikeda Y, Luo X, Abbud R, Nilson JH, Parker KL 1995 The nuclear receptor steroidogenic factor 1 is essential for the formation of the ventromedial hypothalamic nucleus. Mol Endocrinol 9:478-486

Ingraham HA, Lala DS, Ikeda Y, Luo X, Shen W-H, Nachtigal MW, Abbud R, Nilson JH, Parker KL 1994 The nuclear receptor SF-1 acts at multiple levels of the reproductive axis. Genes Dev 8:2302-2312

Ito M, Yu R, Jameson JL 1997 DAX-1 inhibits SF-1-mediated transactivation via a carboxyterminal domain that is deleted in adrenal hypoplasia congenita. Mol Cell Biol 17:1476-1483

John ME, John MC, Ashley P, McDonald RJ, Simpson ER, Waterman MR 1984 Identification and characterization of cDNA clones specific for cholesterol side chain cleavage cytochrome P450. Proc Natl Acad Sci USA 81:5628-5632

Kabe Y, Goto M, Shima D, Imai T, Wada T, Morohashi K-I, Shirakawa M, Hirose S, Handa H 1999 The role of human MBF1 as a transcriptional coactivator. J Biol Chem 274:3419634202

Kawabe K, Shikayama T, Tsuboi H, Oka S, Oba K, Yanase T, Nawata H, Morohashi K-I 1999 Dax-1 as one of the target genes of Ad4BP/SF-1. Mol Endocrinol 13:1267-1284

Keri RA, Nilson JH 1996 A steroidogenic factor-1 binding site is required for activity of the luteinizing hormone beta subunit promoter in gonadotropes of transgenic mice. J Biol Chem 271:10782-10785

Kimura R, Yoshii H, Nomura M, Kotomura N, Mukai T, Ishihara S, Ohba K, Yanase T, Gotoh O, Nawata H, Morohashi K-I 2000 Identification of novel first exons in Ad4BP/SF-1 (NR5A1) gene and their tissue- and species-specific usage. Biochem Biophy Res Comm 278:63-71

Kliewer SA, Lehmann JM, Willson TM 1999 Orphan nuclear receptors: shifting endocrinology into reverse. Science 284:757-760

Lala DS, Rice DA, Parker KL 1992 Steroidogenic factor 1, a key regulator of steroidogenic enzyme expression, is the mouse homolog of fushi tarazu-factor 1. Mol Endocrinol 6:1249-1258

Lala DS, Syka PM, Lazarchik SB, Mangelsdorf DJ, Parker KL, Heyman RA 1997 Activation of the orphan nuclear receptor steroidogenic factor 1 by oxysterols. Proc Natl Acad Sci USA 94:4895-4900

Lee YK, Parker KL, Choi HS, Moore DD 1999 Activation of the promoter of the orphan receptor SHP by orphan receptors that bind DNA as monomers. J Biol Chem 274:20869-20873

Lopez D, Nackley AC, Shea-Eaton W, Xue J, Schimmer BP, McLean MP 2001 Effects of mutating different steroidogenic factor-1 protein regions on gene regulation. Endocrine $14: 353-362$ 
Luo X, Ikeda Y, Parker KL 1994 A cell specific nuclear receptor is required for adrenal and gonadal development and for male sexual differentiation. Cell 77:481-490

Matteson KJ, Chung BC, Miller WL 1984 Molecular cloning of DNA complementary to bovine adrenal P450scc mRNA. Biochem Biophys Res Commun 120:264-270

Mellon SH, Bair SR 1998 25-Hydroxycholesterol is not a ligand for the orphan nuclear receptor steroidogenic factor-1 (SF-1). Endocrinology 139:3026-3029

Monte D, DeWitte F, Hum DW 1998 Regulation of the human P450scc gene by steroidogenic factor 1 is mediated by CBP/p300. J Biol Chem 273:4585-4891

Morohashi K, Fujii-Kuriyama Y, Okada Y, Sogawa K, Hirose T, Inayama S, Omura T 1984 Molecular cloning and nucleotide sequence of cDNA for mRNA of mitochondrial cytochrome P-450(SCC) of bovine adrenal cortex. Proc Natl Acad Sci USA 81:4647-4651

Morohashi K-I, Honda S-I, Inomata Y, Handa H, Omura T 1992 A common trans-acting factor, Ad4-binding protein, to the promoters of steroidogenic P-450s. J Biol Chem 267:1791317919

Morohashi K, Lida H, Nomura M, Hatano O, Honda S, Tsukiyama T, Niwa O, Hara T, Takakusu A, Shibata Y, Omura T 1994 Functional differences between Ad4BP and ELP and their distributions in steroidogenic tissues. Mol Endocrinol 8:643-653

Morohashi K, Tsuboi-Asai H, Matsushita S, Suda, M, Nakashima M, Sasano H, Hataba Y, Li CL, Fukata J, Irie J, Watanabe T, Nagura H, Li E 1999 Structural and functional abnormalities in the spleen of an mFtz-F1 gene-disrupted mouse. Blood 93:1586-1594

Nachtigal MW, Hirokawa Y, Enjeart-VanHouten DL, Flanagan JN, Hammer GD, Ingraham HA 1998 Wilms' tumor 1 and Dax-1 modulate the orphan nuclear receptor SF-1 in sex-specific gene expression. Cell 93:445-454

Ninomiya Y, Okada M, Kotomura N, Suzuki K, Tsukiyama T, Niwa O 1995 Genomic organization and isoforms of the mouse ELP gene. J Biochem (Tokyo) 118:380-389

Nomura, M, Bartsch S, Nawata H, Omura T, Morohashi K-I 1995 An E box element is required for expression of the ad4bp gene, a mammalian homologue of ftz-f1 gene, which is essential for adrenal and gonadal development. J Biol Chem 270:7453-7461

Oba K, Yanase T, Nomura M, Morohashi K, Takayanagi R, Nawata H 1996 Structural characterization of human Ad4BP (SF-1) gene. Biochem Biophys Res Commun 226:261-267

Omura T, Morohashi K 1995 Gene regulation of steroidogenesis. J Steroid Biochem Mol Biol $53: 19-25$

Parker KL, Schimmer BP 1995 Transcriptional regulation of the genes encoding the cytochrome P-450 steroid hydroxylases. Vit Horm 51:339-370

Parker KL, Chaplin DD, Wong M, Seidman JG, Smith JA, Schimmer BP 1985 Expression of murine 21-hydroxylase in mouse adrenal glands and in transfected Y-1 adrenocortical tumor cells. Proc Natl Acad Sci USA 82:7860-7864

Parker KL, Schimmer BP, Chaplin DD, Seidman JG 1986 Characterization of a regulatory region of the steroid 21-hydroxylase gene. J Biol Chem 261:15353-15355

Parker KL, Schimmer BP, Schedl A 1999 Gene interactions in gonadal development. Ann Rev Physiol 61:417-433

Rice DA, Mouw AR, Bogerd A, Parker KL 1991 A shared promoter element regulates the expression of three steroidogenic enzymes. Mol Endocrinol 5:1552-1561

Rosenfeld MG, Bach I, Erkman L, Li P, Lin C, Lin S, McEvilly R, Ryan A, Rhodes S, Schonnemann M, Scully K 1996 Transcriptional control of cell phenotypes in the neuroendocrine system. Rec Prog Horm Res 51:217-238

Sadovsky Y, Crawford P, Woodson K, Polish J, Clements M, Tourtellotte L, Simburger K, Milbrandt J 1995 Mice deficient in the orphan receptor steroidogenic factor 1 lack adrenal glands and gonads but express P450 side-chain-cleavage enzyme in the placenta and have normal embryonic serum levels of corticosteroids. Proc Natl Acad Sci USA 92:10939-10943 
Schimmer BP 1985 Isolation of ACTH-resistant Y1 adrenal tumor cells. Meth Enzymol 109:350356

Shinoda K, Lei H, Yoshii H, Nomura M, Nagano M, Shiba H, Sasaki H, Osawa Y, Ninomiya Y, Niwa O, Morohashi K-I, Li E 1995 Developmental defects of the ventromedial hypothalamic nucleus and pituitary gonadotroph in the Ftz-F1-disrupted mice. Devel Dynam 204:22-29

Taketo M, Parker KL, Howard TA, Seldin MF 1995 Homologs of Drosophila fushi tarazu factor 1 map to mouse chromosome 2 and human chromosome 9q33. Genomics 25:565-567

Tremblay JJ, Viger RS 1999 Transcription factor GATA-4 enhances Mullerian inhibiting substance gene transcription through a direct interaction with the nuclear receptor SF-1. Mol Endocrinol 13:1388-1401

Tremblay JJ, Marcil A, Gauthier Y, Drouin J 1999 Ptx1 regulates SF-1 activity by an interaction that mimics the role of the ligand-binding domain. EMBO J 18:3431-3441

Tsukiyama T, Ueda H, Hirose S, Niwa O 1992 Embryonal long terminal repeat binding protein is a murine homolog of FTZ-F1, a member of the steroid receptor superfamily. Mol Cell Biol 12:1286-1291

White PC, Chaplin DD, Weis JH, Dupont B., New MI, Seidman JS 1984a Two steroid 21-hydroxylase genes are located in the murine S region. Nature 312:465-467

White PC, New MI, Dupont B 1984b Cloning and expression of cDNA encoding a bovine adrenal cytochrome P-450 specific for steroid 21-hydroxylation. Proc Natl Acad Sci USA 81:19861990

Wong M, Ramayya MS, Chrousos GP, Driggers PH, Parker KL 1996 Cloning and sequence analysis of the human gene encoding steroidogenic factor 1. J Molec Endocrinol 17:139-147

Woodson KG, Crawford PA, Sadovsky Y, Milbrandt J 1997 Characterization of the promoter of SF-1, an orphan nuclear receptor required for adrenal and gonadal development. Mol Endocrinol 11:117-126

Xu L, Glass CK, Rosenfeld MG 1999 Coactivator and corepressor complexes in nuclear receptor function. Curr Opin Genet Dev 9:140-147

Yu RN, Ito M, Jameson JL 1998 The murine Dax-1 promoter is stimulated by SF-1 (steroidogenic factor-1) and inhibited by COUP-TF (chicken ovalbumin upstream promoter-transcription factor) via a composite nuclear receptor regulatory element. Mol Endocrinol 12:1010-1022

Zhang P, Mellon SH 1996 The orphan nuclear receptor steroidogenic factor-1 regulates the cyclic adenosine $3^{\prime}, 5^{\prime}$-monophosphate-mediated transcriptional activation of rat cytochrome P450c17 (17 alpha-hydroxylase/c17-20 lyase). Mol Endocrinol 10:147-158

Zhao L, Bakke M, Cushman LJ, Burrows HL, Parlow AF, Camper SA, Parker KL 2001 Steroidogenic factor 1 is essential for pituitary gonadotrope function. Development 128:147154 


\title{
Molecular Basis of Mammalian Gamete Binding
}

\author{
David J. Miller, Xudong Shi, And Heather Burkin
}

Department of Animal Sciences, University of Illinois at Urbana-Champaign, Urbana, Illinois 61801

\begin{abstract}
Despite the importance of fertilization for controlling human reproduction, regulating animal production, and promoting preservation of endangered species, the molecular basis underlying gamete binding and fertilization has been perplexing. More progress has been made in the mouse than in other mammals and, recently, targeted deletion of specific genes in the mouse has yielded intriguing results. This review will emphasize research performed by our laboratory and others done primarily with mouse gametes but will include some interesting observations from other mammals. Studies of murine fertilization indicate that oligosaccharides on the egg coat glycoprotein ZP3 bind sperm. The precise oligosaccharides that bind sperm are the subject of considerable debate. ZP3 also induces exocytosis of the sperm acrosome, allowing sperm to penetrate through the egg coat (zona pellucida). A number of candidate ZP3 receptors have been proposed and studies of $\beta 1$,4galactosyltransferase-I (GalT-I) are reviewed here in the most detail. Sperm from mice with a targeted deletion of GalT-I still are able to bind the zona pellucida but are unable to acrosome react and penetrate through the zona. Therefore, the unique role of GalT-I appears to be in signal transduction. GalT-I forms a complex with heterotrimeric $\mathrm{G}$ proteins and activates signaling, leading to exocytosis in sperm and in heterologous cells expressing GalT-I. Other signaling steps triggered by GalT-I are under active investigation; this receptor forms a complex with a protein kinase anchoring protein. After exocytosis of the acrosome, sperm penetrate the zona pellucida and fuse with the oocyte plasma membrane using ADAM family members on sperm and integrins on oocytes. These proteins, along with the tetraspanins on oocytes, may form a complex web at gamete fusion. Targeted deletion of specific genes in this putative complex has provided important information about their redundancy. After the oocyte is fertilized, the binding site for GalT-I is lost from ZP3, preventing additional sperm from binding to the zona pellucida. New technical advances and creative ideas offer the opportunity to make important advances and to solve the conundrum of fertilization.
\end{abstract}

\section{Introduction}

Fertilization is one of the most fascinating processes in biology. This interaction between highly specialized cells provides a unique example of many cellular processes, including specific cell adhesion, cell signaling, regulated exocytosis, cell migration, cell fusion, and regulation of the cell cycle (Figure 1). For example, gamete recognition proteins that mediate species-restricted spermoocyte interactions provide a model of highly specific cell recognition events. At fertilization, sperm must bind to the oocyte extracellular matrix (zona pellucida), 

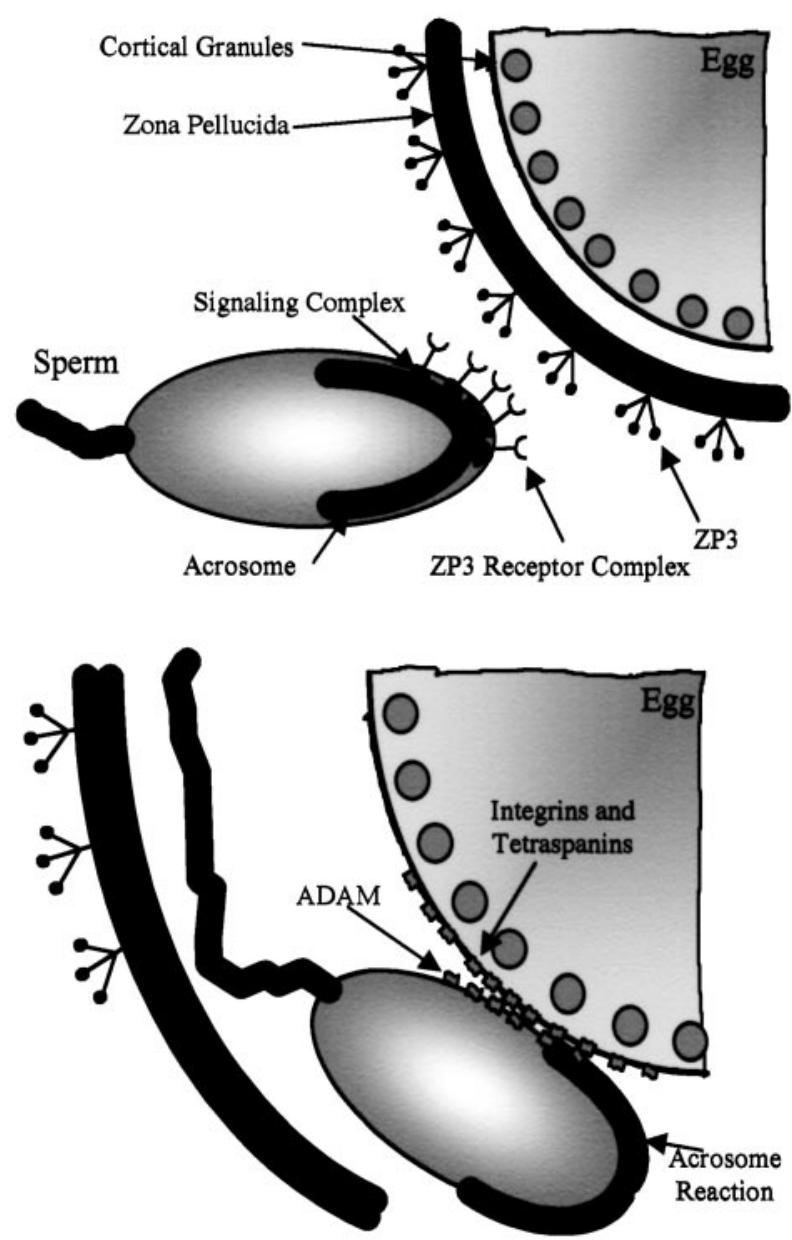

FIG. 1. Schematic working model of fertilization. Acrosome-intact sperm complete capacitation and bind to the zona pellucida. ZP3 binds mouse sperm through a receptor or receptor complex that includes GalT-I (upper panel, see text). GalT-I binding activates heterotrimeric G proteins and perhaps other signaling proteins in a complex and triggers the exocytosis of the sperm acrosome. Once the fertilizing sperm completes the acrosome reaction, it migrates through the zona pellucida and binds and fuses with the oocyte plasma membrane, using ADAM family members on sperm that interact with integrins in a complex with tetraspanins on the oocyte plasma membrane (lower panel). Fusion activates the oocyte, releasing cortical granules whose secretion modifies the zona pellucida so that additional sperm do not bind the zona.

activate the release of a large, specialized secretory vesicle (the acrosome), and penetrate through the tough zona pellucida (Yanagimachi, 1994). Oocytes from some species retain a layer of somatic cells at fertilization called cumulus cells. 
These cells and their matrix are an added barrier that sperm must traverse. After moving through the cumulus matrix and the zona pellucida, the fertilizing sperm must bind to and fuse with the oocyte plasma membrane, resulting in oocyte activation. During activation, the oocyte, previously suspended in metaphase II, completes meiosis and triggers mechanisms to stop additional sperm from penetrating through the zona pellucida and causing polyspermy (Yanagimachi, 1994). This complex series of cell interactions allows the formation of a new diploid cell (zygote) that can develop into the wide variety of tissues found in adult animals.

A better understanding of fertilization is vital to improving or controlling fertility. Once the molecular basis underlying fertilization is elucidated, one can develop specific tests to diagnose the causes of reduced fertility and therapies to treat the specific cause. One could also develop new alternatives for contraception, to regulate the population of humans and pests. Despite the importance of fertilization, a clear understanding of the molecular details has been elusive. This review will present our work and that of others aimed at understanding this process. Excellent reviews emphasizing other aspects of fertilization have been published recently (Rankin and Dean, 2000; Nixon et al., 2001; Wassarman et al., 2001).

\section{Sperm-Zona Pellucida Binding}

The initial step in gamete interaction in oocytes that lack cumulus cells is sperm binding to the oocyte's zona pellucida. Although sperm-zona binding is not completely species specific, it appears to be largely species restricted (Schmell and Gulyas, 1980; Moller et al., 1990; Rankin and Dean, 2000). The zona pellucida is a porous but tough and rigid extracellular matrix. Sperm bind to this formidable barrier and must exocytose the acrosome in order to pass through the matrix (Yanagimachi, 1994). Considerable evidence produced during the last 20 years suggests that sperm binding to the zona pellucida is a carbohydrate-mediated process (Nixon et al., 2001; Wassarman and Litscher, 2001; Wassarman et al., 2001). Lectin-like proteins on sperm bind to oligosaccharides on the zona pellucida proteins. This paradigm is probably similar to the binding of sperm to invertebrate oocytes as well as lymphocyte homing, binding of pathogens to their cellular hosts, and binding of pollen to plant stigma (Wassarman and Litscher, 2001). The plethora of possible combinations of monosaccharides that can be synthesized may provide the observed species restrictions in sperm-zona binding.

Because each zona pellucida component is embedded in a matrix, investigators initially had to solubilize and purify each zona protein to study its function. The individual components of the mouse zona pellucida were studied following heat or acid solubilization and separation by size. The original 
approach to identify the function of zona proteins was to add each protein to sperm and determine which would bind sperm and competitively block sperm binding to oocytes (Bleil and Wassarman, 1980). Under these conditions, only one of the three zona proteins, ZP3, blocked binding of sperm to oocytes (Bleil and Wassarman, 1980). Similarly, in direct binding assays, ZP3 bound to acrosome-intact sperm but lower binding was observed to acrosome-reacted sperm (Bleil and Wassarman, 1986; Mortillo and Wassarman, 1991). ZP3 is the smallest of the proteins, with an apparent molecular weight of $83 \mathrm{kDa}$ (Bleil and Wassarman, 1980). The other two proteins, ZP1 and ZP2, have molecular weights of about 200 and $120 \mathrm{kDa}$, respectively. All three zona proteins are glycoproteins and appear as broad bands by SDS-PAGE (sodium dodecyl sulfate-polyacrylamide gel electrophoresis) due to heterogeneous glycosylation (Rankin and Dean, 2000; Wassarman and Litscher, 2001). Oligosaccharides are found on all three glycoproteins on both asparagine (N-linked) and serine/ threonine (O-linked) residues (Shimizu et al., 1983; Florman and Wassarman, 1985). Most evidence indicates that O-linked oligosaccharides of ZP3 bind mouse sperm (Florman and Wassarman, 1985), although there is recent evidence that N-linked oligosaccharides also may have binding activity in other species (Nakano and Yonezawa, 2001). Based on site-directed mutagenesis, the O-linked oligosaccharides that bind mouse sperm appear to be linked to ZP3 at serine-332 and serine-334, found in the carboxy terminal half of ZP3 (Chen et al., 1998). This region appears to be the most polymorphic among species (discussed in greater detail below) (Wassarman and Litscher, 2001; Wassarman et al., 2001).

\section{A. IDENTITY OF SPERM-BINDING OLIGOSACCHARIDES IN THE ZONA PELLUCIDA}

The precise oligosaccharides on ZP3 that bind sperm have not been identified. There are conflicting data regarding the nonreducing terminal monosaccharide that is necessary to bind sperm. Removing or blocking $\mathrm{N}$-acetylglucosamine residues from ZP3 blocks sperm binding (Shur and Hall, 1982a; Lopez et al., 1985; Miller et al., 1992). Similarly, removal of mannose, $\alpha$-galactose, or fucose residues from ZP3 blocks sperm binding (Bleil and Wassarman, 1988; Cornwall et al., 1991). Synthetic oligosaccharides containing $\alpha$-galactose, $\beta$-galactose, or mannose residues as terminal monosaccharides inhibit sperm binding competitively (Cornwall et al., 1991; Litscher et al., 1995; Johnston et al., 1998). Recent analysis of the composition of the entire mouse zona pellucida suggested structures for over 20 high mannose and complex N-linked structures, many of which contained these residues. In contrast, these investigators recovered less total O-linked oligosaccharide, the oligosaccharides that are believed to bind mouse sperm. Structures for only 10 to 13 O-linked oligosaccharides could be proposed (Easton et al., 2000). A problem with analyzing the total composition 
of the zona pellucida or purified ZP3 is that oligosaccharides in the zona pellucida are not distributed homogeneously. For example, terminal $\alpha$-galactosyl residues are distributed primarily in the inner half of the zona pellucida, whereas other terminal monosaccharides such as $\mathrm{N}$-acetylglucosamine residues are distributed homogeneously throughout the zona pellucida (Aviles et al., 2000). This heterogeneity is also a problem when interpreting the effect of adding soluble ZP3 or glycosidase-treated ZP3 because some of the ZP3 would be from the inner portion of the zona and not be exposed to sperm at initial binding. The necessity of $\alpha$-galactosyl residues is also in doubt because oocytes from mice bearing null mutations in $\alpha 1,3$-galactosyltransferase have normal fertility (Thall et al., 1995). The precise structure of the oligosaccharides that bind sperm remains unresolved. Additional studies of intact oocytes from mice engineered to have specific mutations or deletions in glycosyltransferases will probably provide the most compelling information about which oligosaccharides are crucial for sperm binding.

While it is generally agreed that, in mice, oligosaccharides of ZP3 are responsible for binding sperm, in other animals, the situation is less clear. In domestic pigs and cattle, there is evidence that ZP1, perhaps in a complex with ZP3, binds sperm (Kudo et al., 1998; Yurewicz, et al., 1998; Yonezawa et al., 2001). The sperm-binding activity of ZP1 (sometimes called ZPB or ZP3 $\alpha$ ) is contrary to the lack of sperm-binding activity of ZP1 in mice. A nagging problem with the porcine and bovine zona and the zona pellucida proteins from some other species is that they have not been purified without partial deglycosylation. All studies performed with individual porcine zona proteins have used endo- $\beta$ galactosidase-treated proteins. Although endo- $\beta$-galactosidase-treated porcine ZP1 retains some sperm-binding activity, partial deglycosylation of other zona proteins may influence their sperm receptor activity. A second consideration is that removal of some oligosaccharides from ZP1 by endo- $\beta$-galactosidase may expose cryptic sperm-binding oligosaccharides. Conclusions about the biological activity of purified porcine zona proteins have this caveat. Another difference between the porcine and mouse zona is evidence that the sperm-binding oligosaccharides may be linked to the porcine zona protein through both asparagine and serine/threonine linkages (Yurewicz et al., 1992; Yonezawa et al., 1997; Nakano and Yonezawa, 2001). Further study is necessary to determine whether the glycosylation differences may contribute to species specificity during spermoocyte binding. The possibility that each species may process ZP3 differently is supported by evidence from mice that express human ZP3 but have a null mutation in mouse ZP3. Oocytes from these mice form a normal-appearing zona but do not bind human sperm; instead, they bind mouse sperm (Rankin et al., 1998). One interpretation is that mouse oocytes process ZP3 in a unique way, allowing human ZP3 to have the biological activity of mouse ZP3. 
The evidence that porcine and bovine ZP1 homologues bind sperm raises the issue that mouse ZP1 or ZP2 may bind sperm. One would not expect that species would use different zona proteins to bind sperm but, instead, species might use unique variants of the same gamete receptors. In the initial experiments testing their activity, denatured soluble ZP1, ZP2, and ZP3 were individually tested for their ability to bind sperm and competitively inhibit sperm binding to oocytes. Only ZP3 was active (Bleil and Wassarman, 1980). Soluble ZP2 bound to acrosome-reacted sperm (Bleil et al., 1988) but neither ZP1 or ZP2 had an effect on acrosome-intact sperm. A proviso is that denaturation may have negated any biological activity of ZP1 and ZP2. Because zona proteins are deposited into an insoluble matrix, they are denatured to solubilize and purify them. An alternative is to synthesize recombinant zona proteins individually. Importantly, the recombinant zona proteins must be post-translationally processed by the cell as they would be by the oocyte, the cell that synthesizes zona proteins in most mammals (Rankin and Dean, 2000; Wassarman and Litscher, 2001). In the absence of careful oligosaccharide analysis, one must question results with recombinant zona proteins. Recombinant ZP3 produced by embryonal carcinoma cells and Chinese hamster ovary $(\mathrm{CHO})$ cells appears to be processed by these cells similarly but not exactly the way oocytes process ZP3. Like oocyte-produced $\mathrm{ZP} 3$, recombinant mouse ZP3 produced by embryonal carcinoma and $\mathrm{CHO}$ cells binds mouse sperm (Kinloch et al., 1991; van Duin et al., 1994; Brewis et al., 1996). But, unlike oocyte-produced mouse ZP3, which binds hamster sperm (Moller et al., 1990), recombinant mouse ZP3 expressed in somatic cells does not bind hamster sperm (Kinloch et al., 1991; Litscher and Wassarman, 1996). The glycosylation of other recombinant zona proteins has not been analyzed and their biological activity in purified native form has not been tested. But oocytes from mice with null mutations in ZP1 still are able to bind sperm (Rankin et al., 1999). Clearly, ZP1 is not necessary for mouse sperm binding.

\section{B. RECEPTORS FOR ZONA PELLUCIDA PROTEINS}

A number of zona receptors on sperm have been studied but how they may function individually or collectively is not yet clear. Identifying zona receptors has proved to be more difficult than identifying the zona ligands, probably because of the greater complexity of the sperm surface, as compared to the zona pellucida. There may be multiple receptors to account for the multiple affinities of ZP3 to sperm (Thaler and Cardullo, 1996). We will discuss zona receptors based upon how they were isolated and whether they appear to be primary receptors (found on plasma membrane of acrosome-intact sperm) or secondary receptors (on acrosome-reacted sperm), although data on the location of all the candidates are not available. 
Several receptor candidates have been isolated, based on their affinity for the zona pellucida. From mouse sperm, sp56 was isolated based on its affinity for ZP3 and $\alpha$-galactose residues (Cheng et al., 1994; Bookbinder et al., 1995) while zonadhesin, proacrosin, sp38, P47, and a group of proteins called spermadhesins (all studied most on porcine sperm) were isolated based on their affinity for whole zona pellucida (Hardy and Garbers, 1995; Ensslin et al., 1998; Jansen et $a l ., 2001)$. The specific zona ligand for the latter group is unknown. Further study revealed that sp56 was found within the acrosome (Foster et al., 1997; Kim et al., 2001a). In this location, its function may be to tether sperm onto the zona pellucida as they are undergoing the acrosome reaction. Proacrosin, P-selectin, and $\mathrm{sp} 38$ are found within the acrosome and exposed during the acrosome reaction, where they can serve as secondary zona receptors (Mori et al., 1995; Geng et al., 1997; Jones et al., 1988). Although mice lacking either P-selectin or proacrosin are fertile, careful analysis of proacrosin null sperm shows that these sperm penetrate the zona pellucida more slowly (Mayadas et al., 1993; Adham et al., 1997). This is probably because the activated form of proacrosin is important for dispersal of the acrosomal matrix during the acrosome reaction (Yamagata et al., 1999). The spermadhesins and P47 are peripheral membrane proteins (Ensslin et al., 1998; Jansen et al., 2001). Many of the spermadhesins are produced by seminal vesicles (Jansen et al., 2001). Because sperm from the cauda epididymis that have not been exposed to secretions from the seminal vesicles have normal fertility, spermadhesins that are seminal vesicle products do not appear to be necessary for fertilization. Whether zonadhesin is intracellular or on the plasma membrane has not been reported (Hickox et al., 2001).

Another approach to identify zona receptors has been to develop antibodies that block sperm-zona binding. A monoclonal antibody to sperm PH-20 blocks fertilization, implicating PH-20 in zona binding (Myles and Primakoff, 1997). PH-20 is an interesting molecule that has both hyaluronidase and zona binding activity (Hunnicutt et al., 1996). It appears to have a dual role. PH-20 on the postacrosomal region of sperm and perhaps $\mathrm{PH}-20$ released by acrosomereacting sperm disperses the cumulus cells, allowing sperm passage (Hunnicutt et al., 1996). After the acrosome reaction, PH-20 binding is necessary for sperm to bind the zona pellucida (Hunnicutt et al., 1996). Therefore, PH-20 appears to be a secondary zona receptor, although the zona ligand is unknown. Sperm Sp17 is an acrosomal protein first isolated using antibodies. It has affinity for the zona pellucida and other sulfated glycoproteins (Yamasaki et al., 1995; Wen et al., 2001).

P95 is a mouse sperm protein identified by SDS-PAGE, transfer to nitrocellulose, and ligand blotting, using ZP3 as a ligand (Leyton and Saling, 1989a). Whether it is acrosomal or on the plasma membrane is not known. A sequence of the putative human homologue has been reported but the sequence may, in 
fact, be the sequence of c-mer (Burks et al., 1995; Bork, 1996; Tsai and Silver, 1996).

\section{C. $\beta 1,4$ GALACTOSYLTRANSFERASE AS A MULTIFUNCTIONAL PROTEIN}

The zona receptor candidate that has been investigated most extensively is an enzyme with two forms that have roles at two distinct regions of the cell. $\beta 1,4 \mathrm{Galactosyltransferase}$ (GalT) was named for its ability to add galactose to glycoproteins and glycolipids with terminal $\mathrm{N}$-acetylglucosamine residues. Like other glycosyltransferases, GalT-I is a type II membrane glycoprotein whose catalytic domain is in the internal compartment of the Golgi (Joziasse, 1992). In addition to its Golgi location, some GalT was discovered as a component of the plasma membrane of specific cells (Shur, 1991). More recently, other glycosyltransferases have been found on the plasma membrane, so GalT appears not to be unique (Borsig et al., 1996; Close and Colley, 1998; Mandel et al., 1999). On the plasma membrane, the catalytic domain is externally oriented. Because the typical galactose donor, uridine diphosphate (UDP)-galactose, is not present in the extracellular fluid, GalT may not be able to carry out a synthetic function. Rather, it appears to act as a lectin and bind to galactose acceptors, specific glycoproteins that have appropriately presented $\mathrm{N}$-acetylglucosamine residues at nonreducing termini (Shur, 1993).

Recently, several other gene products were discovered with GalT activity. At least six additional genes encode proteins capable of adding galactose to $\mathrm{N}$-acetylglucosamine; some of these enzymes prefer specific substrates (e.g., glycolipid rather than glycoprotein) (Amado et al., 1999). Of the seven GalTs identified, to date, only the first GalT sequenced, referred to as GalT-I, has been shown to have both plasma membrane and Golgi distributions. A variety of experiments have demonstrated that GalT-I is found on the surface of some cells. Antibodies to recombinant GalT-I or GalT-I peptides bind to the plasma membrane of live sperm and some somatic cells, as assessed by confocal microscopy (Youakim et al., 1994a; Larson and Miller, 1997; Tengowski et al., 2001). GalT-I can be detected as a biotinylated protein when sperm plasma membrane proteins are biotinylated by nonpermeable biotin analogs (Larson and Miller, 1997). Enzyme assays of intact cells demonstrate that GalT-I is on the surface and the enzyme is detected on enriched plasma membrane fractions (FayrerHosken et al., 1991; Shur, 1991).

The GalT-I gene is unusual because, in the four mammals with sequence information, it has two translation start sites that encode two slightly different proteins (Shaper et al., 1988; Russo et al., 1990; Mengel-Gaw et al., 1991; E.A. Landers and D.J. Miller, unpublished results). One start site of the mouse gene is 39 bases upstream of the second site. When translated, this results in a long 
form of the protein that is identical to the short form, except that it has 13 additional amino acids, yielding 24 amino acids at its amino terminal cytoplasmic domain rather than 11 . Both forms share a noncleavable signal-anchor/transmembrane domain, a stem region, and long extracellular/lumenal catalytic domain. The long form is found on the plasma membrane as well as in the Golgi. This was demonstrated most convincingly using antibodies generated to the sequence specific to the long form (Youakim et al., 1994a). Only the long form is able to activate signal transduction in response to binding of a ligand (discussed below). Cells are able to specifically regulate transcription of each form. The long form appears to be constitutively synthesized in many cells. The short form can be specifically upregulated by the mammary gland during lactation, where it forms the lactose synthase complex (Shaper et al., 1998). The functions in cell adhesion, fertilization, lactation, and glycosylation demonstrate the broad importance of GalT-I. This multifunctional protein was first named for its enzyme activity in the Golgi but, in view of the breadth of actions GalT-I carries out, the name implies too narrow a function.

How GalT-I is targeted to two different locations on the cell is an intriguing question. Indeed, how glycosyltransferases are retained in the Golgi is an active area of research. There are two prominent models proposed to explain glycosyltransferase retention (Colley, 1997). One hypothesis, known as the bilayer thickness model, is that the length of the glycosyltransferase transmembrane domain mediates retention in the Golgi membrane. The shorter transmembrane domains of Golgi proteins may prevent them from entering the cholesterol-rich membranes of transport vesicles that would otherwise carry the enzymes to the plasma membrane. A second hypothesis is referred to as kin recognition. In this model, glycosyltransferases form oligomers or perhaps large hetero-oligomers that do not enter secretory vesicles. Neither model completely explains the observations in the literature. The stem region or cytoplasmic tail also may influence Golgi retention of some glycosyltransferases (Milland et al., 2001; Sasai et al., 2001). In any case, the Golgi retention mechanism must be altered or overridden for GalT-I to move to the plasma membrane.

\section{D. $\beta 1,4$ GALACTOSYLTRANSFERASE AS AN ADHESION RECEPTOR}

As a plasma membrane molecule, GalT-I can act as a receptor for specific glycoproteins, including ZP3. All known ligands for GalT-I have terminal $\mathrm{N}$-acetylglucosamine residues. Therefore, it appears that, as expected, GalT-I recognizes glycoproteins that could be galactose acceptors in the Golgi. On the other hand, terminal $\mathrm{N}$-acetylglucosamine is not sufficient for a glycoprotein to be a sperm GalT-I ligand. For example, both ZP1 and ZP2 have N-acetylglucosamine at nonreducing termini but they are not ligands for sperm GalT-I 
(Miller et al., 1992). The additional ligand requirements for sperm membranebound GalT-I are unknown and could include oligosaccharide or protein determinants. What regulates the specificity of GalT-I is not certain but it is possible that being embedded in the plasma membrane influences substrate specificity. Detergent solubilization of GalT-I from somatic cells allows the enzyme to bind to a much wider variety of ligands (Begovac et al., 1991). The soluble forms of some glycosyltransferases that are cleaved from the membrane-bound forms glycosylate substrates less efficiently (Zhu et al., 1998). In addition, the binding specificity of GalT-I could be influenced by other proteins in a putative receptor complex in the sperm plasma membrane. The question of binding specificity is an important one to understanding GalT-I function.

During spermatogenesis, GalT-I is synthesized by spermatogonia, primary and secondary spermatocytes, and round spermatids (Pratt and Shur, 1993; Charron et al., 1999). Interestingly, a unique transcript is synthesized in spermatocytes and round spermatids that, when translated, yields only the long form of the protein (Charron et al., 1999). As the round spermatids change their shape to that of mature sperm, GalT-I moves to the developing sperm head and becomes localized to the plasma membrane in the acrosomal region of sperm (Scully et al., 1987; Pratt and Shur, 1993). GalT-I from sperm behaves as a typical integral membrane protein with a molecular weight of $60 \mathrm{kDa}$ (Shur and Neely, 1988). In the epididymis, glycoconjugates in the epididymal fluid bind to GalT-I, blocking the interaction of GalT-I with other ligands such as the zona pellucida. These glycoconjugates are lost from sperm during capacitation, the final sperm maturation process that occurs in the female tract, enabling GalT-I to bind other ligands (Shur and Hall, 1982b). During fertilization, GalT-I on the mouse sperm surface binds to ZP3 but not other zona pellucida glycoproteins (Miller et al., 1992). This fulfills an important criterion for bona fide ZP3 receptors.

The biological importance of the GalT-I and ZP3 adhesion was tested in several in vitro assays. When $\mathrm{N}$-acetylglucosamine residues were either blocked or removed from soluble ZP3, it lost its ability to bind sperm (Miller et al., 1992). This suggested that the interaction between GalT-I and ZP3 was necessary for gamete binding. In similar experiments using the intact zona pellucida, blocking or removing $\mathrm{N}$-acetylglucosamine residues reduced binding of sperm (Shur and Hall, 1982a; Lopez et al., 1985). Blocking GalT-I with antibodies, $\mathrm{F}_{\mathrm{ab}}$ fragments, or $\alpha$-lactalbumin (a protein that modifies the substrate specificity of GalT-I) reduces sperm binding to the zona pellucida (Shur and Hall, 1982a; Lopez et al., 1985). Purified GalT-I from sperm blocks sperm-zona binding when added as a competitor. All these results demonstrate the important function of sperm GalT-I in zona binding.

The oligosaccharides on ZP3 that bind GalT-I have the same characteristics as oligosaccharides shown in independent experiments to bind sperm. The 
binding site for GalT-I on ZP3 was removed by mild alkaline hydrolysis but not peptide-N-glycosidase F, suggesting it was O linked (Miller et al., 1992). The chromatographic behavior of the GalT-I binding site and the active ZP3 O-linked oligosaccharides was similar (Miller et al., 1992). Further characterization of the oligosaccharides that bind sperm and GalT-I has been problematic due to the difficulty in obtaining sufficient zona pellucida glycoproteins.

\section{E. $\beta 1,4$ GALACTOSYLTRANSFERASE ACTIVATES SIGNAL TRANSDUCTION AND THE ACROSOME REACTION}

In addition to its role in binding, ZP3 is also the zona protein that triggers the acrosome reaction in sperm (Figure 1). The acrosome is a membrane-bound organelle located in the anterior portion of the head between the nucleus and the plasma membrane. During the acrosome reaction, the outer membrane of the acrosome fuses with the overlying plasma membrane and the contents of the acrosome are dispersed. This relatively slow exocytosis includes a gradual dissolution of the acrosomal matrix stored within the acrosome (Kim et al., 2001b). The acrosome reaction is required for sperm to penetrate through the zona pellucida. Isolated ZP3, purified in a soluble form from the zona pellucida or expressed in heterologous cells, induces the acrosome reaction when added to sperm (Bleil and Wassarman, 1983; Kinloch et al., 1991; Wassarman et al., 2001).

Although oligosaccharides on ZP3 bind sperm, the oligosaccharides or small glycopeptides from ZP3 are unable to induce the acrosome reaction (Florman et al., 1984; Leyton and Saling, 1989b). However, addition of antibodies to the ZP3 glycopeptides induces the acrosome reaction, supporting the model that ZP3 requires a multivalent interaction with receptors on sperm to induce signal transduction (Leyton and Saling, 1989b). Consistent with this model, each ZP3 molecule can bind two or three GalT-I molecules (Miller et al., 1992). This appears similar to the behavior of somatotropin and its receptor (Cunningham et al., 1991).

At some point during the acrosome reaction, sperm lose their affinity for ZP3 and develop an affinity for ZP2 (Bleil et al., 1988). This so-called secondary binding may maintain sperm on the zona pellucida and aid in penetration of sperm through the zona pellucida. Whereas ZP2 and ZP3 have functional roles in both zona formation and sperm binding, ZP1's major function is only structural. ZP1 appears to crosslink the other zona proteins to provide form to the matrix. In the absence of ZP1, ZP2 and ZP3 form a loose, fragile zona pellucida (Rankin et al., 1999).

The first indication that GalT-I may act as a signaling receptor was the result of experiments with GalT-I antibodies that apparently can mimic ZP3 (Macek et al., 1991). Intact immunoglobulin $\mathrm{G}$ ( $\mathrm{IgG})$ induces the acrosome reaction in 
capacitated mouse sperm but $\mathrm{F}_{\mathrm{ab}}$ fragments do not, even though they still inhibit sperm-zona binding. However, if the $\mathrm{F}_{\mathrm{ab}}$ are cross-linked with a secondary IgG, they trigger the acrosome reaction on sperm. Although this is intriguing, an alternative explanation is that $\operatorname{IgG}$ can affect sperm viability and sperm often release their acrosomes as a consequence of death. This is unlikely because control IgG did not induce the acrosome reaction. If GalT-I aggregation is sufficient to induce the acrosome reaction, a further prediction is that other multivalent GalT-I ligands would act in the same manner. This prediction was borne out in two ways. Bovine serum albumin (BSA) derivatized with $\mathrm{N}$ acetylglucosamine induces the acrosome reaction in mouse sperm but unconjugated $\mathrm{N}$-acetylglucosamine does not (Loeser and Tulsiani, 1999). The specificity of the response is in question because these authors also found that $\mathrm{N}$-acetylgalactosamine or mannose coupled to BSA induces the acrosome reaction in mouse sperm. Using polyacrylamide as a matrix, $\mathrm{N}$-acetylglucosamine on polyacrylamide triggers acrosomal exocytosis. Free $\mathrm{N}$-acetylglucosamine, polyacrylamide, or polyacrylamide derivatized with galactose have no effect (Nixon et al., 2001).

Although multivalent binding appears necessary for signal transduction through GalT-I, the state of GalT-I in the plasma membrane is not certain. In the Golgi membrane, GalT-I appears to have the ability to form multimers but its state in the plasma membrane is uncertain (Yamaguchi and Fukuda, 1995). At least two models are possible. On the plasma membrane, GalT-I may exist as a monomer and cross-linking by ZP3 or GalT-I IgG would induce signal transduction. Alternately, GalT-I in the membrane may exist as a multimer and multivalent binding induces a response in the GalT-I complex that activates signal transduction.

The signaling response elicited in sperm by ZP3 includes some components found in somatic cells. For example, during capacitation, there is an increase in tyrosine phosphorylation of sperm proteins (Visconti and Kopf, 1998). Presumably, this is caused by activation of either receptor or nonreceptor tyrosine kinases, although the identity of the activated kinases is unclear. The role of G proteins in the acrosome reaction has been studied more extensively. Induction of the acrosome reaction by ZP3 can be blocked by pertussis toxin, demonstrating that a subclass of heterotrimeric $\mathrm{G}$ proteins is necessary for the acrosome reaction (Ward and Kopf, 1993). The pertussis toxin substrates $\mathrm{G}_{\mathrm{i} 1}$ and $\mathrm{G}_{\mathrm{i} 2}$ are activated by ZP3 binding to sperm (Ward et al., 1994). G protein activation may lead to transient intracellular alkalinization, enabling a robust influx of calcium in response to zona pellucida binding (Florman et al., 1998).

If some GalT-I antibodies mimic ZP3 by acting as GalT-I agonists, one would expect they would activate signal transduction steps normally activated by ZP3. Pharmacological agents that block the ZP3-induced acrosome reaction might also block the GalT-I antibody-induced acrosome reaction. In concordance 
with this prediction, pertussis toxin blocks the GalT-I antibody-induced acrosome reaction (Gong et al., 1995). GalT-I antibodies also activate sperm G proteins, assessed by an increase in high-affinity guanosine triphosphate (GTP) binding (Gong et al., 1995). Activated G proteins hydrolyze GTP as they are inactivated. Measurement of GTP hydrolysis often is used to confirm G protein activation. Membrane preparations from sperm also increased GTP hydrolysis in response to GalT-I antibodies, supporting the hypothesis that GalT-I activates heterotrimeric $\mathrm{G}$ proteins (Figure 2).

The results suggesting that GalT-I activates G proteins were unexpected because most $G$ protein-coupled receptors span the plasma membrane seven times and GalT-I has only a single transmembrane sequence. To confirm that GalT-I interacts with G proteins, we expressed GalT-I in heterologous cells. We selected Xenopus laevis oocytes to express GalT-I because they synthesize a wide variety of heterotrimeric G proteins (Olate et al., 1990) and G protein activation leads to exocytosis (Kline et al., 1991). As expected, expression of GalT-I allowed oocytes to bind ZP3 but not ZP1 or ZP2 (Figure 3) (Shi et al., 2001). The calculated dissociation constant, although variable between ZP3 preparations, was $9 \mathrm{nM}$, intermediate between the expected affinity for low- and high-affinity ZP3 receptors (Thaler and Cardullo, 1996).

In sperm, ZP3 and antibodies to GalT-I activate G proteins and trigger the exocytosis of the acrosome. Likewise, oocytes exocytose their cortical granules when traditional G protein-coupled receptors are expressed in oocytes and the ligand is added (Kline et al., 1991). When we expressed GalT-I on Xenopus

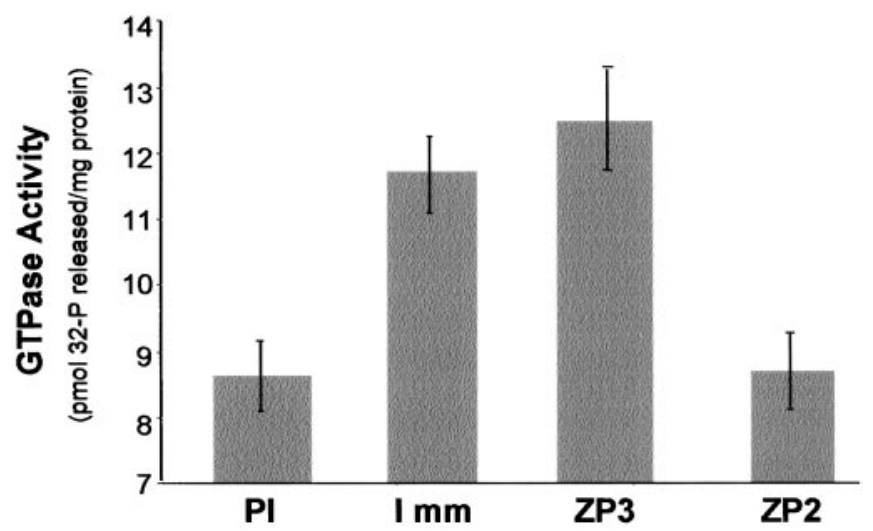

FIG. 2. Membrane preparations from sperm increase GTP hydrolysis in response to GalT-I antibodies. GTPase assays were performed using methods similar to those described elsewhere (Ward et al., 1992). GalTase antibodies or solubilized zona proteins were added to sperm membranes in reaction buffer. The samples were incubated at $30^{\circ} \mathrm{C}$ for 15 minutes. The assay was stopped and released ${ }^{32} \mathrm{P}$ was counted. Background counts were obtained from samples without membranes. 


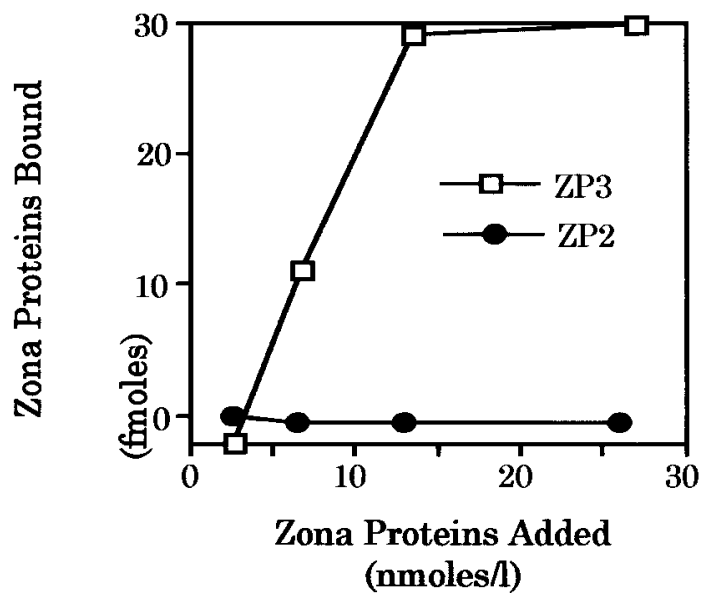

FIG. 3. ZP3, but not ZP2, bound to Xenopus oocytes expressing GalT-I. Increasing concentrations of each zona glycoprotein were added to Xenopus oocytes expressing GalT-I and water-injected control eggs. With increasing zona protein concentration, ZP3 binding increased to saturation but ZP2 binding did not differ between RNA-injected and water-injected eggs. In this ZP3 preparation, the $K_{D}$ was approximately $9 \mathrm{nM}$ but affinity was variable between ZP3 preparations. [Adapted with permission from Shi X, Amindari S, Paruchuru K, Skalla D, Burkin H, Shur BD, Miller DJ 2001 Cell surface $\beta$-1,4-galactosyltransferase-I activates $\mathrm{G}$ protein-dependent exocytotic signaling. Development 128:645-654. Copyright Company of Biologists, Ltd.]

oocytes and added GalT-I ligands, such as antibodies or ZP3, the oocytes released their cortical granules (Figures 4 and 5) (Shi et al., 2001). Indeed, the oocytes showed other indications of activation, including the elevation of the vitelline envelope and the cortical contraction. Pertussis toxin blocked oocyte activation, demonstrating that the $G$ proteins activated by GalT-I on sperm and oocytes were related. GalT-I activation by agonistic antibodies or ZP3 also activated G proteins, as indicated by increased GTP binding and GTP hydrolysis (Figures 6 and 7). Expressing GalT-I and perhaps other candidate zona receptors on Xenopus oocytes may be a fruitful way to identify the motifs of the receptors involved in signal transduction.

We have used the oocyte expression system to begin studies of the GalT-I motifs that are necessary for signal transduction and exocytosis. Although most $\mathrm{G}$ protein-coupled receptors have the traditional seven transmembrane domains, there are at least three examples of receptors that appear to couple to $\mathrm{G}$ proteins that have a single transmembrane domain (Okamoto et al., 1990, Liang and Garrison, 1991; Yang et al., 1991; Nishimoto et al., 1993; Sun et al., 1997). They are the insulin-like growth factor-II/mannose-6-phosphate receptor (IGF-II/ M6PR), amyloid protein precursor, and epidermal growth factor receptor, although the results with IGF-II/M6PR are controversial (Korner et al., 1995). 

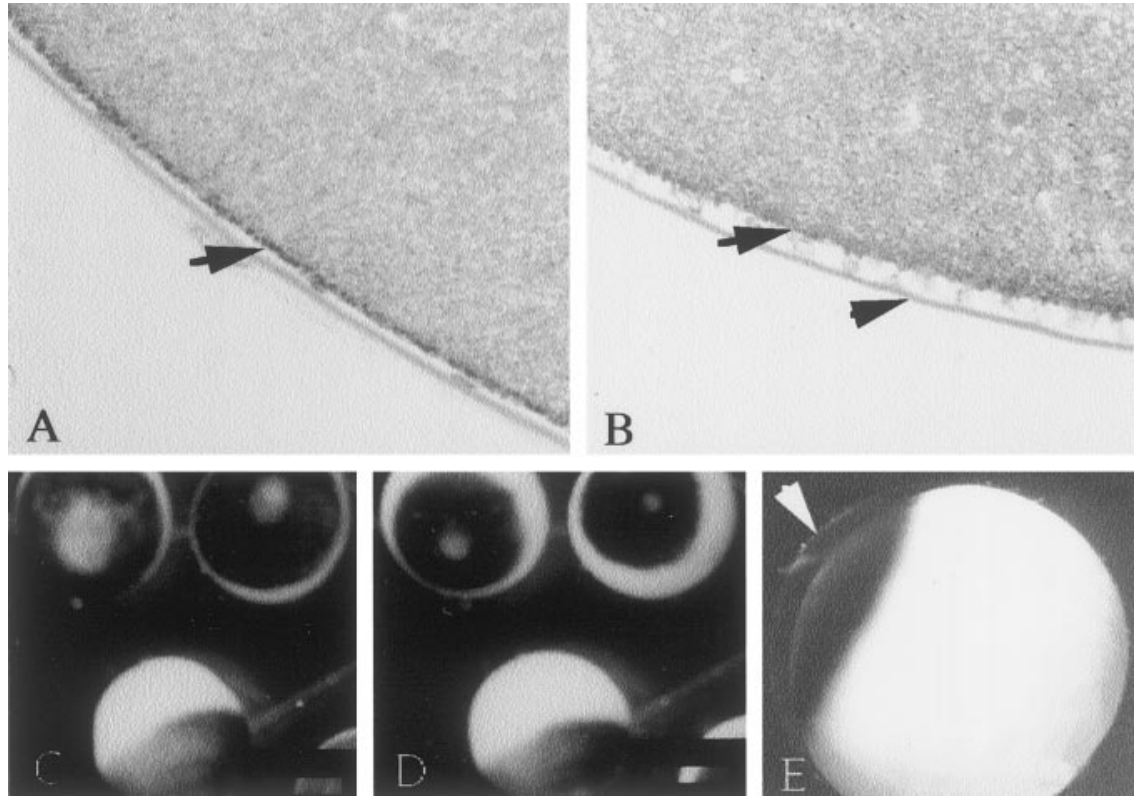

FIG. 4. Changes observed as Xenopus eggs expressing GalT-I are activated. Eggs were sectioned and cortical granules stained with periodic acid-Schiffs reagent (PAS). (A) Control egg injected with water and treated with GalT-I antibodies. Cortical granules are evident just under the plasma membrane, labeled with an arrow. (B) GalT-I-expressing egg treated with GalT-I antibody showing release of cortical granules. The arrow shows the exocytosis of cortical granules. The vitelline envelope has separated from the plasma membrane, as shown by the arrowhead. (C) Eggs expressing GalT-I prior to addition of GalT-I antibody. (D) The same eggs as in (C) 10 minutes after addition of GalT-I antibody showed contraction of the pigmented zone of the animal pole. (E) GalT-Iexpressing egg treated with GalT-I antibody illustrating the elevation of the vitelline envelope, shown by the arrow. [Adapted with permission from Shi X, Amindari S, Paruchuru K, Skalla D, Burkin H, Shur BD, Miller DJ 2001 Cell surface $\beta$-1,4-galactosyltransferase-I activates G protein-dependent exocytotic signaling. Development 128:645-654. Copyright Company of Biologists, Ltd.]

Studies of the first two examples and mastoparan, a peptide that activates $\mathrm{G}_{\mathrm{i}}$ and $\mathrm{G}_{\mathrm{o}}$ proteins, have led to a proposed structural motif (Okamoto et al., 1990; Nishimoto et al., 1993). The minimal motif is from 14-23 amino acids long and contains two basic amino acids near the $\mathrm{N}$-terminus and the sequence BBXB or $\mathrm{BBXXB}$ near the $\mathrm{C}$-terminus, where $\mathrm{B}$ is a basic residue and $\mathrm{X}$ is any residue. More recent studies have identified similar clusters of basic domains in traditional seven transmembrane-spanning receptors that are necessary for $\mathrm{G}$ protein activation (Lee et al., 1996; Xie et al., 1997; Frandberg et al., 1998; Wade et al., 1999; Wang, 1999). The cytoplasmic domain of long GalT-I is 24 amino acids in length and has clusters of basic residues at each end of the cytoplasmic 
A

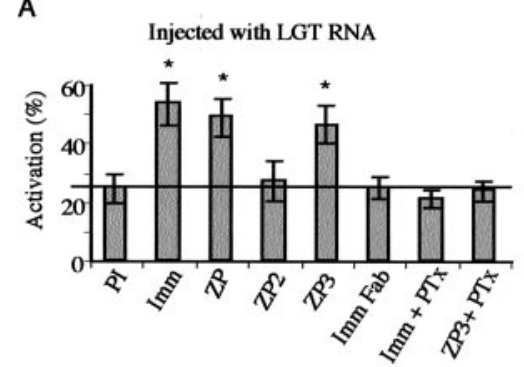

C

Injected with SGT RNA

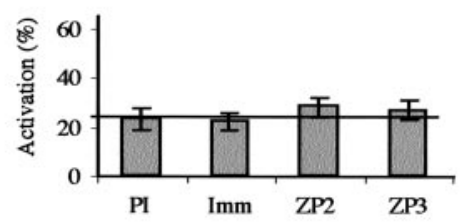

B

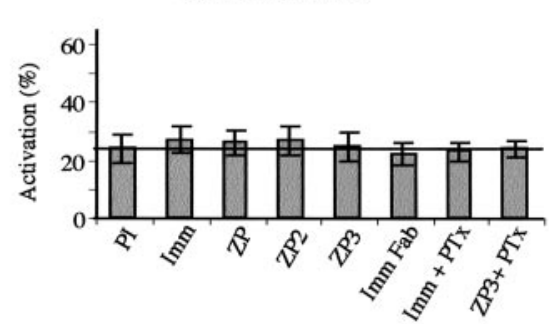

D

Injected with $\triangle$ LGT RNA

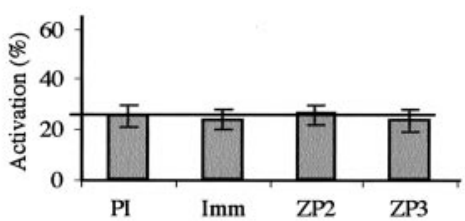

FIG. 5. GalT-I agonists activated Xenopus eggs expressing long GalT-I. (A) Addition of total zona pellucida (ZP) glycoproteins, ZP3, or GalT-I antibodies (Imm) to eggs expressing GalT-I triggered cortical granule exocytosis, cortical contraction, and vitelline envelope elevation. Ligands were added to GalT-I-expressing eggs and signs of activation were observed for 20 minutes. GalT-Iexpressing eggs treated with preimmune antibodies $(\mathrm{PI})$, monovalent $\mathrm{F}_{\mathrm{ab}}$ fragments $\left(\mathrm{Imm} \mathrm{F}_{\mathrm{ab}}\right.$ ) or ZP2 had background activation rates. Pertussis toxin (PTx) pretreatment of eggs prevented activation. (B) Eggs injected with water had low activation rates. (C) Eggs expressing the short GalT-I protein (SGT) or (D) expressing long GalT-I with a mutation in the putative G protein activation domain ( $\Delta$ LGT) did not activate in response to agonists. An asterisk above the bars indicates a significant difference from the groups without an asterisk $(P<0.01)$. [Adapted with permission from Shi $\mathrm{X}$, Amindari $\mathrm{S}$, Paruchuru K, Skalla D, Burkin H, Shur BD, Miller DJ 2001 Cell surface $\beta$-1,4-galactosyltransferase-I activates $G$ protein-dependent exocytotic signaling. Development 128:645-654. Copyright Company of Biologists, Ltd.]

sequence. The cytoplasmic domain of short GalT-I is only 11 amino acids and lacks the N-terminal cluster of basic residues. Short GalT-I was unable to activate $\mathrm{G}$ proteins and exocytosis when expressed in oocytes (Figures 5 and 6). Therefore, we mutated the two arginine residues near the N-terminus found only in the long form and expressed this mutant in oocytes. The mutant did not activate $\mathrm{G}$ proteins and did not activate oocytes or trigger the release of cortical granules (Figures 5 and 6; Shi et al., 2001). This suggests that GalT-I may interact with $\mathrm{G}$ proteins using motifs related to those found in seven transmembrane receptors that interact with $\mathrm{G}$ proteins. 
A

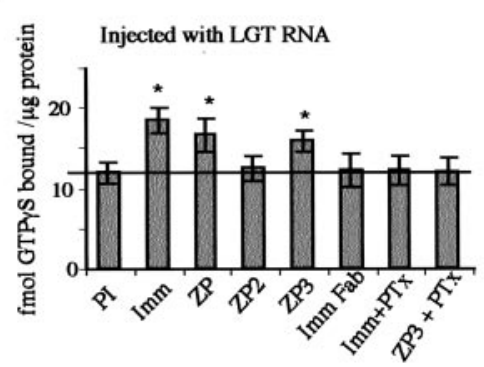

C

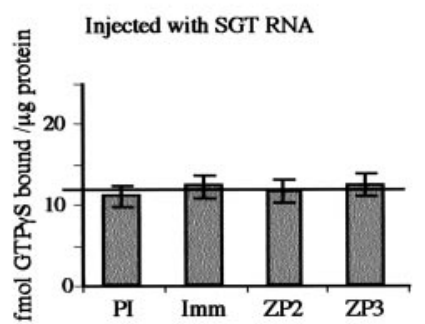

B

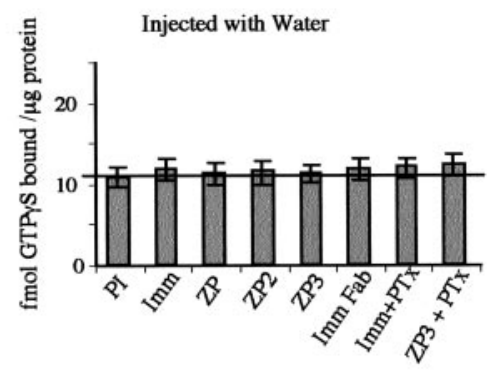

D

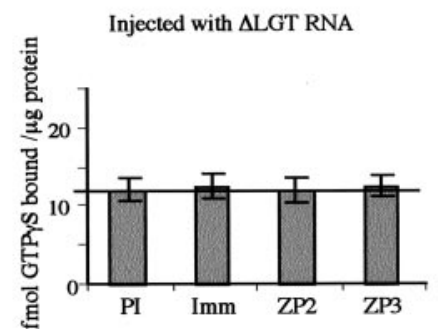

FIG. 6. GalT-I agonists increase GTP $\gamma\left[{ }^{35} \mathrm{~S}\right]$ binding to membranes from Xenopus oocytes expressing long GalT-I. (A) Addition of total zona pellucida (ZP) glycoproteins, ZP3, or GalT-I antibodies (Imm) to eggs expressing GalT-I increased GTP $\gamma\left[{ }^{35} \mathrm{~S}\right]$ binding. Addition of preimmune antibodies (PI), monovalent $\mathrm{F}_{\mathrm{ab}}$ fragments $\left(\operatorname{Imm} \mathrm{F}_{\mathrm{ab}}\right.$ ) or ZP2 activated only background levels of GTP $\gamma\left[{ }^{35} \mathrm{~S}\right]$ binding. Pertussis toxin (PTx) pretreatment of eggs prevented any change in GTP $\gamma\left[{ }^{35} \mathrm{~S}\right]$ binding in response to GalT-I agonists. (B) Membranes from water-injected control eggs showed no change in GTP $\gamma\left[{ }^{35} \mathrm{~S}\right]$ binding. (C) Eggs expressing the short GalT-I protein or (D) eggs expressing long GalT-I with a mutation in the putative G protein-binding domain did not respond to GalT-I agonists. An asterisk above the bars indicates a significant difference from the groups without an asterisk $(P<0.05)$. [Adapted with permission from Shi X, Amindari S, Paruchuru K, Skalla D, Burkin H, Shur BD, Miller DJ 2001 Cell surface $\beta$-1,4-galactosyltransferase-I activates G proteindependent exocytotic signaling. Development 128:645-654. Copyright Company of Biologists, Ltd.]

If GalT-I signaling proceeds through $G$ protein activation, there may be a direct interaction between GalT-I and a specific G protein complex. Alternately, GalT-I could bind to a type of adapter protein that could, in subsequent steps, activate $\mathrm{G}$ proteins. Therefore, it was important to determine whether there was a protein-protein connection between GalT-I and G proteins. The sequence corresponding to the long form of the GalT-I cytoplasmic domain was coupled to Sepharose and used to precipitate proteins that associated with the long form of GalT-I. Both $\mathrm{G}_{\mathrm{i} \alpha}$ and $\mathrm{G}_{\beta \gamma}$ were precipitated with GalT-I (Gong et al., 1995). 


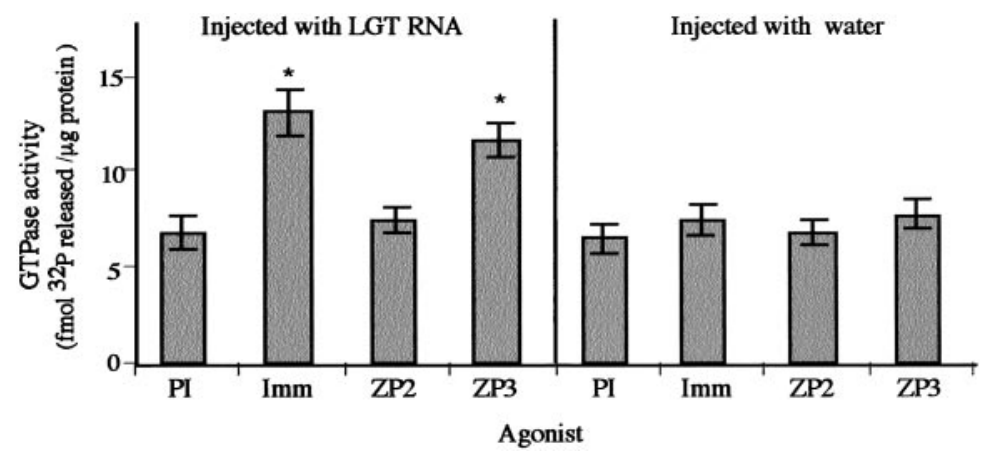

FIG. 7. Addition of ZP3 or GalT-I antibodies (Imm) to eggs expressing GalT-I increased GTP hydrolysis. Preimmune antibodies (PI) or ZP2 stimulated only background levels of GTPase activity (left panel). Control eggs injected with water did not respond to GalT-I agonists (right panel). An asterisk above the bars indicates a significant difference from the groups without an asterisk $(P<$ 0.05). [Adapted with permission from Shi X, Amindari S, Paruchuru K, Skalla D, Burkin H, Shur BD, Miller DJ 2001 Cell surface $\beta$-1,4-galactosyltransferase-I activates $\mathrm{G}$ protein-dependent exocytotic signaling. Development 128:645-654. Copyright Company of Biologists, Ltd.]

This demonstrated that $\mathrm{G}$ proteins could form complexes with the GalT-I cytoplasmic domain but, unfortunately, this technique does not identify the specific protein-protein interactions. A valuable approach to identify protein partners that interact directly is the yeast two-hybrid system. Among several proteins identified as GalT-I binding partners using the yeast two-hybrid system is a protein called SSeCKS (Wassler et al., 2001). SSeCKS is expressed in the testis and found on the periacrosomal area of mature sperm, the same region as GalT-I (Erlichman et al., 1999; Nixon et al., 2001). Immunoprecipitation studies confirmed that GalT-I interacts with SSeCKS (Wassler et al., 2001). SSeCKS is particularly interesting because it can serve as an anchoring protein for both protein kinase $\mathrm{A}$ and $\mathrm{C}$ and may thereby regulate signal transduction (Lin et al., 1996; Erlichman et al., 1999).

\section{Signal Transduction Steps During the Acrosome Reaction}

The sequence of events that triggers the acrosome reaction is not certain but some pieces are starting to fit together into a model. The target of the activated $\mathrm{G}$ proteins is not clear. Members of the phospholipase $\mathrm{C}$ family are frequent targets of $\mathrm{G}$ proteins. It is intriguing that targeted deletion of one phospholipase $\mathrm{C} \delta$ isoform (PLC $\delta 4)$ reduces male fertility and fertilization rates (Fukami et al., 2001). The effect on fertility was apparently related to the inability of sperm to release the acrosome in response to binding the zona pellucida. Whether PLC $\delta 4$ is activated by $\mathrm{G}$ proteins or some other mechanism is unknown. Other phos- 
pholipase C isoforms are present in sperm (Walensky and Snyder, 1995). How any PLC isoform is activated in sperm is unclear because of the gaps in our knowledge of signaling through ZP3 receptors. Inositol 1,4,5-trisphosphate (IP3) produced by PLC can bind to and activate IP3 receptors on the acrosome (Walensky and Snyder, 1995). Release of calcium from the acrosome depletes the internal stores of calcium, activating store-operated calcium channels and allowing calcium influx. The store-operated channels include the protein Trp2 and perhaps other Trp proteins (Jungnickel et al., 2001). Additionally, ZP3 binding triggers membrane depolarization and a transient influx of calcium through what behave as low-voltage-activated T-type calcium channels that are regulated by capacitation (Arnoult et al., 1999). The increase in intracellular calcium is necessary for docking and fusion of the outer acrosomal and plasma membranes during acrosomal exocytosis. Membrane fusion appears to include SNAREs, Rab3A, and other molecules involved with membrane fusion in somatic cells (Schulz et al., 1997; Iida et al., 1999; Ramalho-Santos et al., 2000).

\section{Genetic Modulation of GalT-I Activity in Mouse Sperm}

Studies of transgenic mice with elevated or absent GalT-I enzyme activity have provided considerable insight into the function of GalT-I. As expected, sperm from mice that overexpress GalT-I bind greater amounts of purified soluble ZP3 (Youakim et al., 1994b). Unexpected was the observation that sperm with high GalT-I activity were less able to bind to oocytes (Youakim et al., 1994b). This appears to reflect two different phenomena. First, sperm with increased GalT-I either bind more glycosides from epididymal fluid or bind them with higher affinity. These glycosides often are referred to as decapacitation factors because they block sperm capacitation and fertilization (Yanagimachi, 1994). After removing these glycosides, more sperm are able to bind the zona pellucida. The second causative phenomenon is that sperm with increased GalT-I undergo precocious acrosome reactions. Sperm overexpressing GalT-I undergo greater zona-induced $\mathrm{G}$ protein activation, more rapid acrosome reactions, but bind more tenuously to the zona pellucida (Youakim et al., 1994b). It appears that acrosome-reacted sperm have lower affinity for the zona pellucida because free-swimming, acrosome-reacted mouse sperm do not initiate binding to the zona (Florman and Storey, 1982). Therefore, the most likely explanation of the lower affinity binding to the zona pellucida of GalT-I transgenic sperm is that they have undergone the acrosome reaction very quickly and are removed when the oocytes are washed to remove sperm bound by low affinity.

Targeted disruption of GalT-I from mouse sperm yielded intriguing results. Most animals with disruption of both long and short forms of GalT-I die perinatally but the males that survive produce sperm with negligible levels of GalT activity (Lu and Shur, 1997). Sperm from GalT-I null mice bind a reduced 
amount of purified soluble ZP3 and their ability to undergo the zona-induced acrosome reaction is severely compromised. The defect appears relatively specific, as sperm still can undergo the acrosome reaction in response to calcium ionophore, which bypasses the ZP3-binding step. As a result of the defect in the acrosome reaction, GalT-I null sperm show flawed penetration of the zona pellucida (Lu and Shur, 1997). Surprisingly, sperm showed no decrease in binding to the intact zona pellucida. The reason binding to the intact zona is not affected is perhaps that ZP3 in the zona is modified by oviduct fluid. Purified ZP3 usually is collected from oocytes that have not been exposed to oviduct fluid. In view of the zona penetration defect in vitro, also unforeseen was that there was no obvious defect in male fertility in vivo in GalT-I null sperm (Asano et al., 1997; Lu and Shur, 1997; Lu et al., 1997). Perhaps some compensatory mechanisms exist in vivo that overcome the defect. Alternately, similar to the results with acrosin-null mice, the defect in GalT-I null sperm may be more subtle in vivo. Carefully designed assays may detect a delay or a competitive disadvantage when GalT-I null sperm are compared to wild-type sperm.

Elimination of both long and short forms of GalT-I may have global effects on glycoprotein processing in the Golgi and thereby affect sperm fertility. To minimize alterations in glycosylation, the long form of GalT-I was selectively disrupted. Sperm lacking the long isoform still have GalT activity, reflecting enzyme synthesized from the short transcript, and protein glycosylation appears normal (Lu and Shur, 1997). But these sperm are unresponsive to ZP3. Thus, the fertilization defects in long GalT-I null sperm are not due to disruption of protein glycosylation. Furthermore, the short form of GalT-I is not able to activate signal transduction; the longer cytoplasmic domain is required for normal signal transduction. This is consistent with results from expressing both forms in Xenopus oocytes. Only the long form is able to activate $\mathrm{G}$ proteins and trigger exocytotic signaling when expressed on Xenopus oocytes (Shi et al., 2001).

Considering that sperm from GalT-I null mice are still able to bind to oocytes and that GalT-I null males are fertile, other zona receptors must be capable of serving as adhesion molecules during sperm-zona binding. Other receptors may impart species specificity to gamete recognition. Sea urchins and abalone provide some insight to the problem of species specificity. In abalone, a sperm protein called lysin creates a hole in the egg coat, known as the vitelline envelope (Swanson and Vacquier, 1998). Regions within lysin show divergence between species and some of these divergent segments impart species specificity (Lyon and Vacquier, 1999). Similarly, the sea urchin sperm protein bindin is polymorphic at distinct regions within the molecule. Even females within a species indicate a preference for sperm carrying specific bindin genotypes (Palumbi, 1999). In female mammals, there are corresponding divergent regions in $\mathrm{ZP} 2$ and $\mathrm{ZP} 3$ that are under positive selection. Some divergent regions are found in the region in which the sperm-binding oligosaccharides are attached 
(Swanson et al., 2001). No specific segment of GalT-I has been identified that is hypervariable between species. Furthermore, in addition to GalT-I, porcine sperm have other redundant zona receptors, as described earlier (Rebeiz and Miller, 1999). Therefore, a reasonable model is that the role of GalT-I is to cooperate with other zona receptors that have divergent segments that could contribute towards the species-restricted nature of gamete binding. Based on the knockout results, the more necessary role of GalT-I appears to be signaling to trigger the acrosome reaction. The identity of receptors that cooperate with GalT-I in a putative complex is unknown.

\section{Zona Penetration}

During the acrosome reaction, sperm remain bound to the zona pellucida, perhaps through the interaction of acrosomal proteins that bind ZP3 such as sp56 (Kim et al., 2001a,b). After the acrosome has completely dispersed, sperm lose affinity for ZP3 and gain affinity for ZP2 (Bleil and Wassarman, 1986; Mortillo and Wassarman, 1991). The interaction with ZP2 apparently retains sperm on the zona pellucida so that the sperm can begin penetration through the zona. Penetration may involve enzymatic hydrolysis of the zona pellucida but also requires the forward physical force of sperm motility (Bedford, 1998). Although the acrosomal protease acrosin is not required, other acrosomal proteases may be important for zona penetration. But the sea abalone egg coat is dissolved without hydrolysis by the lysin from sperm (Swanson and Vacquier, 1997). Whether zona pellucida penetration absolutely requires lysis of zona proteins is uncertain.

\section{Sperm Binding and Fusion with the Oocyte Plasma Membrane}

After zona penetration, sperm attach to the oocyte plasma membrane and the two cells fuse together. A number of studies suggest that ADAM family members bind to integrin receptors on oocytes. The ADAM family members most heavily implicated are two found in the fertilin dimer (fertilin $\alpha$ or ADAM- 1 and fertilin $\beta$ or ADAM-2) and cyritestin (ADAM-3). Synthetic peptides corresponding to specific fertilin $\beta$ and cyritestin sequences inhibit sperm binding and fusion to the oocyte plasma membrane (Myles et al., 1994; Evans et al., 1995; Yuan et al., 1997). Recombinant proteins containing portions of ADAM members and corresponding antibodies also inhibit sperm binding and fusion (Almeida et al., 1995; Evans et al., 1997; Bigler et al., 2000; Takahashi et al., 2001). The role of fertilin $\alpha$ may be unique. Fertilin $\alpha$ has a hydrophobic peptide sequence that resembles fusion peptides found in viral proteins and it has been proposed to mediate sperm fusion with the oocyte membrane (Martin et al., 1998; Wolfe et al., 1999). 
Results using mice with specific deletions of fertilin $\beta$ or cyritestin yielded surprising results. Fertilin $\beta$-null mice are infertile. Their sperm fail to adhere to the egg plasma membrane (13\% of wild-type level) and have reduced ability to fuse with oocytes (45-50\% of wild-type level), although some fusion occurs (Cho et al., 1998). What is surprising is that sperm from fertilin $\beta$-null mice also have defects in sperm migration from the uterus into the oviduct and in binding to the zona pellucida. Cyritestin -/- sperm have reduced zona and egg membrane binding but these defects did not affect fusion with the oocyte membrane and fertilization (Nishimura et al., 2001). Both knockouts cause a reduction in abundance of other proteins on sperm, including fertilin $\alpha$ (Nishimura et al., 2001). The effects of deleting these genes on sperm migration through the reproductive tract and on zona binding could be caused by defects in transport of proteins to the sperm plasma membrane or on their function at the plasma membrane.

The protein partner on oocytes to which fertilin $\beta$ binds was believed to be $\alpha 6 \beta 1$ integrin (Almeida et al., 1995). Antibodies to $\alpha 6$ blocked binding of sperm, although this appeared to depend on the conditions used (Almeida et al., 1995; Evans et al., 1997). More recently, it was discovered that oocytes lacking $\alpha 6$ are still able to bind and fuse with sperm (Miller et al., 2000). Perhaps other integrins that are reported to bind ADAM family members are found on oocytes and could fulfill that function. In contrast, a protein on oocytes called CD9, a member of the tetraspanin family of proteins (four transmembrane domains), is necessary for sperm fusion. CD9 associates with $\beta 1$ integrins and antibodies to CD9 inhibit sperm-oocyte binding and fusion (Chen et al., 1999). Oocytes from mice with targeted deletion of CD9 bind sperm normally but are defective in sperm fusion (Kaji et al., 2000; Le Naour et al., 2000; Miyado et al., 2000). $\beta 1$ integrin associates with other tetraspanins, including CD81 and CD98. Antibodies to both these tetraspanins inhibit fertilization (Takahashi et al., 2001). It has been proposed that a web of ADAMs and multiple $\beta 1$ integrin-associated proteins are formed to mediate sperm-oocyte membrane interactions (Takahashi et al., 2001). In fact, the tetraspanins, integrins, and ADAM family members include a large number of proteins and several are present on gametes (Evans, 2001). Dissecting their individual functions will be challenging.

\section{Egg Activation and the Block to Polyspermic Zona Binding}

After sperm bind and fuse with the oocyte plasma membrane, the oocyte is "reawakened," a process referred to as activation. One of the earliest hallmarks of this activation is a release of calcium from intracellular stores (Stricker, 1999; Carroll, 2001). There is considerable debate about what triggers activation but there is accumulating evidence in mammals that a sperm component released at fusion can activate a series of calcium transients and trigger development (Perry 
et al., 2000; Wu et al., 2001). As eggs are activated, the cortical granules are released and their secretions act on the zona pellucida. Following cortical granule release, the zona pellucida loses its ability to bind sperm and bound sperm cease penetration through the zona. ZP3 purified from fertilized eggs is inactive; it does not competitively inhibit sperm binding to unfertilized oocytes, although no change in behavior on SDS-PAGE is detectable (Bleil and Wassarman, 1980). In contrast, ZP2 is cleaved by an unidentified protease released at egg activation (Moller and Wassarman, 1989).

We found that ZP3 from fertilized eggs loses its ability to bind sperm GalT-I. This is due to secretion of hexosaminidase from the cortical granules. Hexosaminidase B is found abundantly in cortical granules of mouse and Xenopus oocytes (Miller et al., 1993; Greve et al., 1985). As oocytes are activated, it removes specific $\mathrm{N}$-acetylglucosamine residues from ZP3 so that ZP3 does not bind additional sperm (Miller et al., 1993). If this hexosaminidase is blocked with specific inhibitors of $\mathrm{N}$-acetylglucosaminidase or antibodies during egg activation, multiple sperm bind to activated eggs (Miller et al., 1993).

\section{Fertilization in Other Mammals}

\section{A. FUNCTION OF ZONA PELLUCIDA RECEPTORS ON PORCINE AND BOVINE SPERM}

Although the mouse has been the most studied mammal, there is considerable information about the molecular biology of fertilization in the pig. One reason it has been a popular animal is that large numbers of porcine gametes can be acquired. Studies of the porcine zona pellucida have been hampered by the inability to purify zona proteins without denaturing and deglycosylating the glycoproteins and perhaps affecting their biological activity. After partial deglycosylation, a mixture of porcine ZP1 and ZP3 appear most effective in binding sperm. Perhaps a heterodimer of these two zona glycoproteins is necessary for binding porcine sperm (Yurewicz et al., 1998).

Several candidate zona receptors on porcine sperm have been identified and discussed earlier in this review. GalT-I has been identified on a number of mammalian sperm, including that from rabbits, horses, cattle, rats, guinea pigs, and domestic pigs (Larson and Miller, 1997). Its location has been studied on cattle and porcine sperm, where it is found on the plasma membrane overlying the acrosomal region of sperm (Fayrer-Hosken et al., 1991; Larson and Miller, 1997; Tengowski et al., 2001). Antibodies to GalT-I inhibit sperm-zona binding and fertilization of bovine oocytes (Tengowski et al., 2001). Studies of porcine fertilization have yielded intriguing results. To determine if GalT-I bound the porcine zona pellucida, porcine sperm were incubated with soluble whole zona proteins and the sugar donor, uridine-diphospho- $\left[{ }^{3} \mathrm{H}\right]$-galactose. In this experi- 
ment, the zona proteins bound to GalT-I would be labeled by $\left[{ }^{3} \mathrm{H}\right]$-galactose. The labeled galactose was attached to porcine zona proteins with a broad range in molecular weights from $50-75 \mathrm{kDa}$, the region in which ZP1 and ZP3 migrate (Rebeiz and Miller, 1999). Therefore, either one or both are ligands for porcine sperm GalT-I. But, surprisingly, reagents that blocked GalT-I or its zona ligand did not inhibit porcine sperm-zona binding (Rebeiz and Miller, 1999). These results suggested that GalT-I is a redundant zona receptor in the domestic pig.

Since GalT-I appeared to be a redundant zona receptor in porcine fertilization, it was important to ascertain its specific role as part of a zona receptor complex. It was important to determine if GalT-I, by itself, was sufficient to bind sperm to the zona pellucida. To answer this question, we removed the GalT-I binding sites from soluble porcine zona proteins using hexosaminidase. These enzyme-treated zona proteins could bind to other receptors on sperm but not to GalT-I. When the enzyme-treated zona proteins were added to sperm, leaving only GalT-I exposed, these sperm were unable to bind to oocytes (Rebeiz and Miller, 1999). Therefore, GalT-I on porcine sperm is not sufficient to bind sperm to the zona pellucida (Figure 8). GalT-I can bind soluble zona proteins but perhaps the affinity for zona proteins is inadequate for GalT-I to hold a highly motile sperm on the zona pellucida.

\section{B. LOCALIZATION OF ZONA RECEPTORS ON PORCINE SPERM}

When assessing the functional role of candidate zona receptors, one important criterion is that the protein be localized to the region of sperm that binds the zona pellucida. ZP3 binds to acrosome-intact but not acrosome-reacted mouse sperm. The precise region of mouse sperm that binds ZP3 was examined using gold-labeled ZP3 (Mortillo and Wassarman, 1991). Gold particles were bound throughout the head of acrosome-intact sperm. Gold-labeled ZP2 bound to the postacrosomal region of acrosome-intact sperm and to the inner acrosomal membrane of acrosome-reacted sperm. The region of sperm from other mammals that bound zona proteins has been controversial.

To resolve this issue on porcine sperm, we directly labeled soluble total zona proteins with Alexa, an intense fluorescent probe, and incubated them with sperm (Burkin and Miller, 2000). Labeled zona proteins retained normal biological activity, assessed by inhibition of sperm binding to oocytes and by induction of the acrosome reaction (Burkin and Miller, 2000). Dead sperm were identified by labeling with propidium iodide. Zona proteins bound primarily to the apical edge of the plasma membrane covering the acrosome of sperm (Figure 9A\&B). Labeled control proteins did not bind sperm and binding of zona proteins was eliminated by an excess of unlabeled zona proteins (Figure 9C-F). After induction of the acrosome reaction, zona proteins bound to a larger portion of the sperm head, to the anterior half of sperm (Figure 10A). The time course of the 

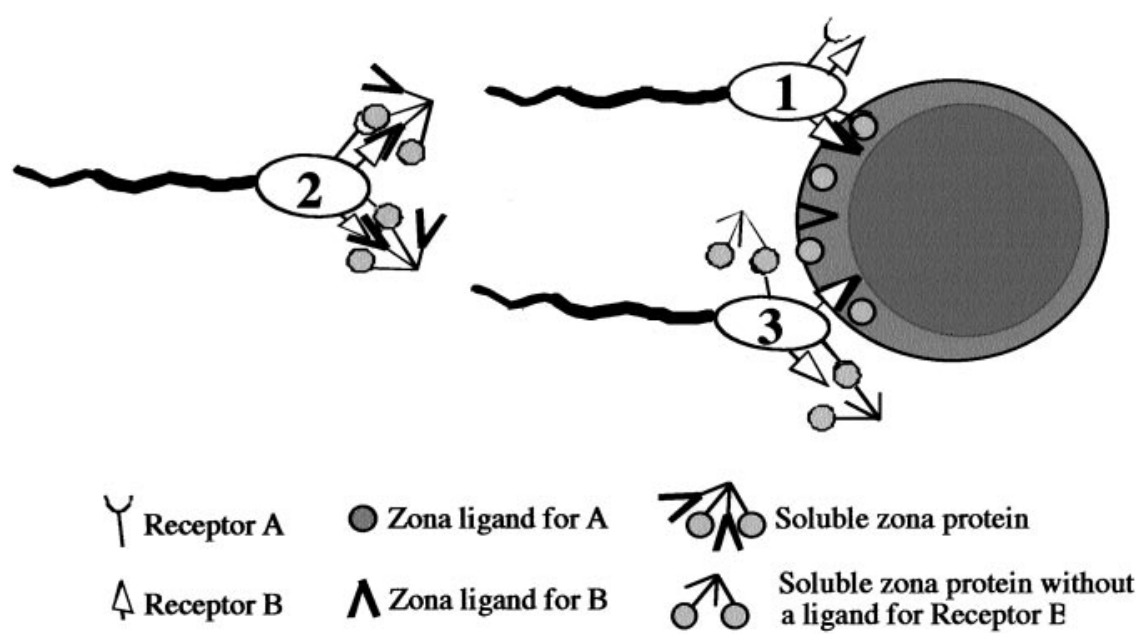

FIG. 8. Schematic drawing of experiment to determine the importance of redundant receptors. When performing competitive binding assays, one can determine if a single receptor in a redundant system is adequate to allow gamete binding. Suppose there are two receptors on the surface of the porcine spermatozoa named A and B (B represents GalT-I in our experiments). Normally, A and B are both able to bind their individual ligands on the porcine zona pellucida, allowing normal sperm-zona attachment (Sperm \#1). If soluble zona is added, both A and B will bind their respective ligands on the soluble zona pellucida and sperm egg binding will be decreased (Sperm \#2). If an enzyme able to cleave the ligand for receptor B is used to digest soluble zona, the result is soluble zona deficient in the ligand for $\mathrm{B}$. These soluble zona will inhibit the action of A but, because they cannot compete for the binding of receptor B, B is free to bind its ligand on the intact oocyte zona pellucida (Sperm \#3). Using this approach, it can be determined if B alone is sufficient to mediate sperm-zona binding.

acrosome reaction matched the change in zona binding pattern (Figure 10B). It also appeared that more total zona protein was bound to sperm following the acrosome reaction. Based on these results, we expect to find primary zona receptor candidates on the apical ridge of acrosome-intact sperm. Secondary zona receptor candidates on acrosome-reacted sperm are expected to be found over the entire acrosomal region.

Although zona receptors were detected on porcine ejaculated sperm, the maturational stage at which sperm acquired the ability to bind zona proteins was controversial. We collected sperm from the caput, corpus, and cauda epididymis and incubated these sperm with labeled zona proteins (Figure 11). Whereas 6\% of the caput sperm bound zona proteins, $75 \%$ and $93 \%$ of the corpus and cauda sperm, respectively, bound zona proteins in the same localization pattern as ejaculated sperm (Burkin and Miller, 2000). Therefore, it appears that either zona receptors already on sperm are transformed into an active state or that sperm acquire zona-binding proteins as they pass through the epididymis. 


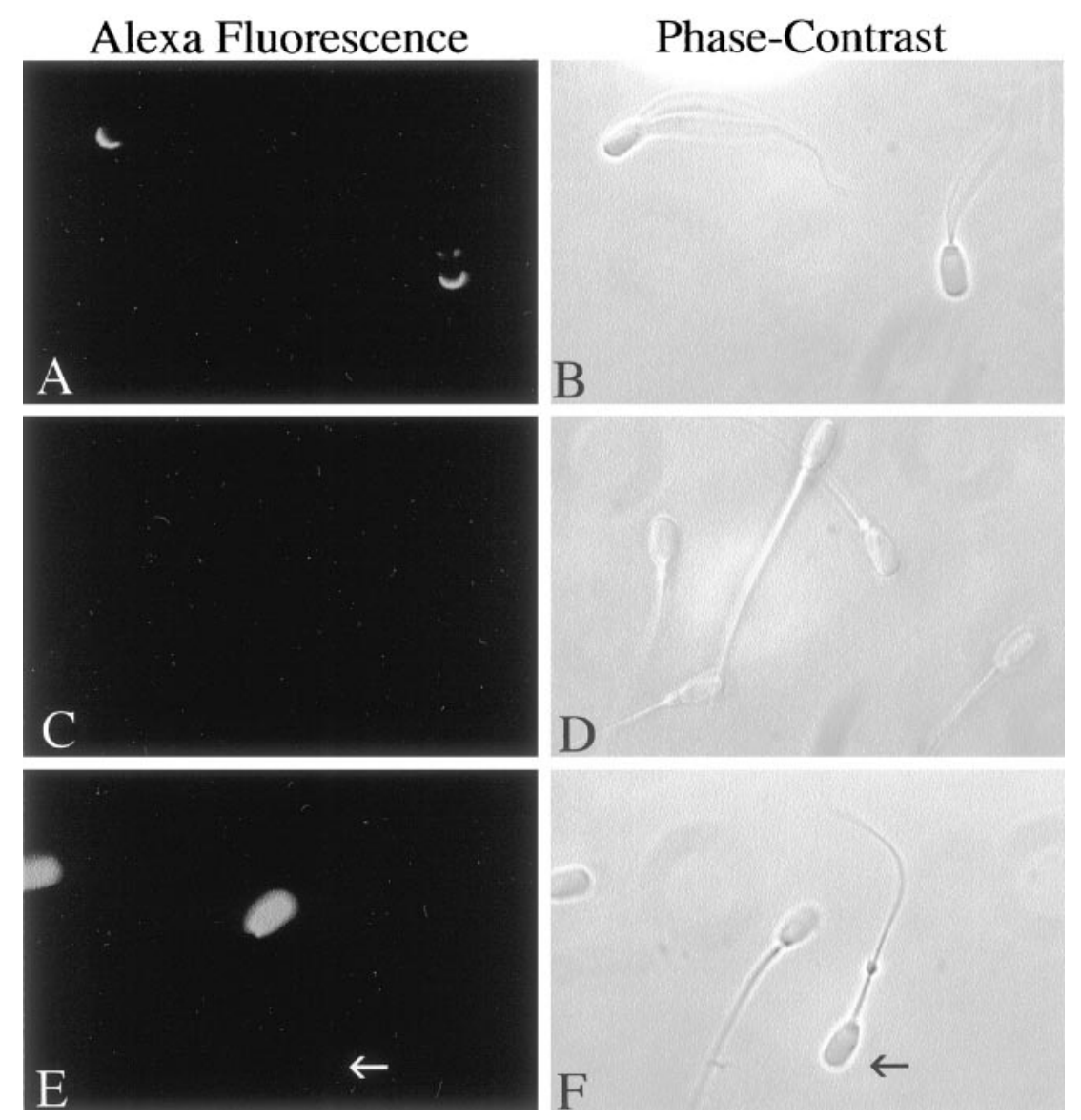

FIG. 9. Localization of solubilized zona pellucida proteins on live sperm. Alexa-labeled zona proteins and propidium iodide were added to live boar sperm. Alexa-zona proteins bound live, acrosomeintact sperm over the anterior head region concentrated over the acrosomal ridge (A). Addition of a 100 -fold excess of unlabeled zona proteins displaced the signal (C). The Alexa-labeled control glycoprotein, transferrin, did not bind to live sperm indicated by the arrow in (E). Dead sperm stained with propidium iodide over the entire head are visible, since fluorescence images were captured using a filter set that allowed detection of both red and green fluorochromes simultaneously (A,C,E). Corresponding phase-contrast images are shown (B,D,F). [Adapted with permission from Burkin HR, Miller DJ 2000 Zona pellucida protein binding ability of porcine sperm during epididymal maturation and the acrosome reaction. Dev Biol 222:99-109. Copyright Academic Press.]

\section{Conclusions}

Despite 20 years of investigation, the molecular basis underlying fertilization remains an enigma. Although the role of ZP3 in mouse fertilization is well 


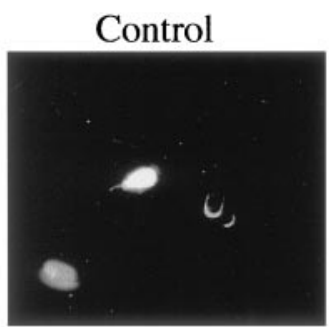

\section{Ionophore}
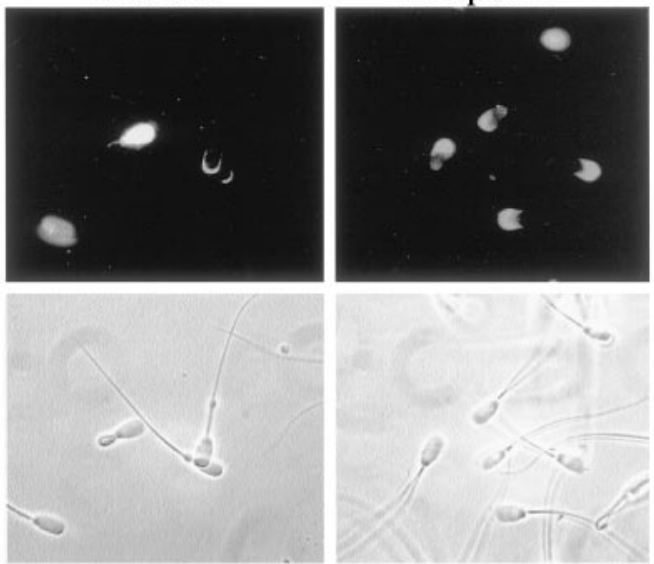

B

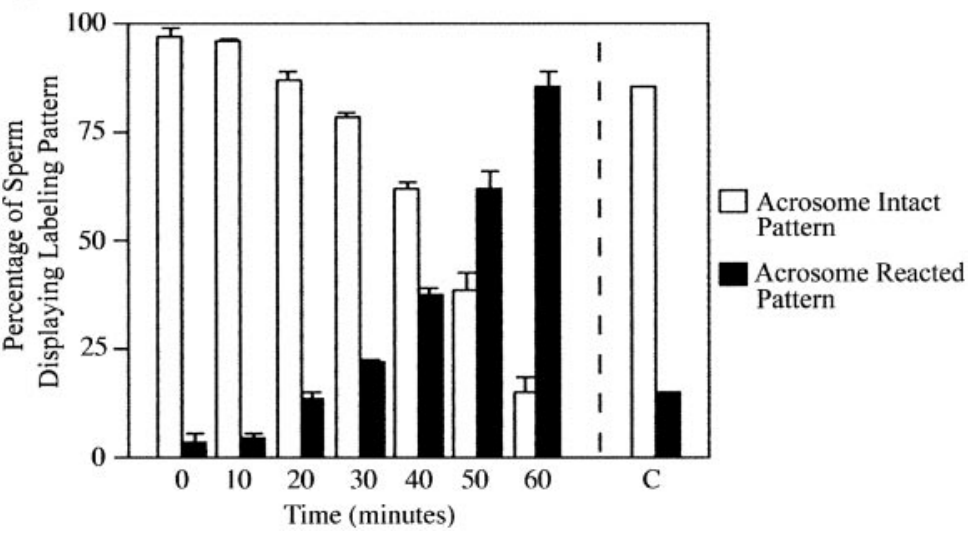

FIG. 10. Binding of zona pellucida proteins is increased in acrosome-reacted sperm. Live, capacitated boar sperm were incubated with the calcium ionophore A23187 to induce the acrosome reaction, followed by the addition of Alexa-zona proteins and propidium iodide. The upper panels in (A) show fluorescence images of Alexa-zona and propidium iodide, the lower panels show corresponding phase-contrast images. Acrosome-reacted sperm showed an increased area of zona protein binding, extending from the acrosomal ridge to the equatorial region of live sperm, whereas controls without ionophore did not. Intense sperm head staining in the left panel and light staining at the base of the head in the right panel are due to propidium iodide. For time course experiments (B), samples were removed and at least 200 sperm counted at 10-minute intervals after the addition of ionophore. The percentage of live sperm displaying zona binding in a thin band over the acrosomal ridge decreased with time (white bars), while the percentage displaying strong acrosomal fluorescence increased (black bars). Controls to which ionophore was not added ("C") were counted after 60 minutes. [Adapted with permission from Burkin HR, Miller DJ 2000 Zona pellucida protein binding ability of porcine sperm during epididymal maturation and the acrosome reaction. Dev Biol 222:99-109. Copyright Academic Press.] 

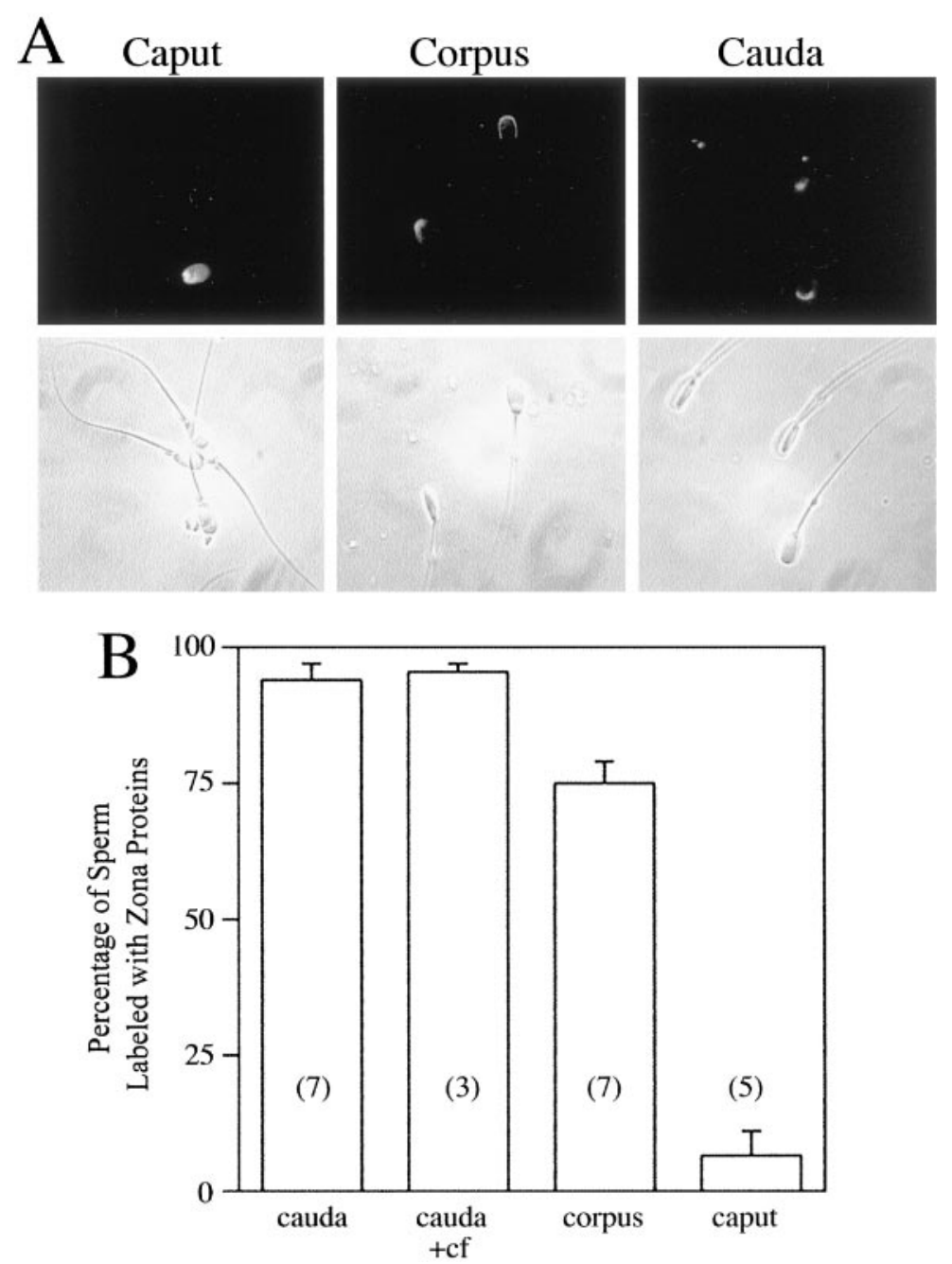

FIG. 11. Boar sperm acquire the ability to bind zona pellucida proteins during epididymal maturation. Sperm from the caput, corpus, and cauda regions of the boar epididymis were capacitated and incubated with Alexa-zona proteins and propidium iodide. (A) Most caput sperm do not bind zona proteins. Those that do display a very faint signal. The intensely labeled sperm is labeled with propidium iodide. In contrast, most corpus and cauda sperm exhibit intense zona protein binding. (B) Quantitation of live, acrosome-intact sperm displaying zona fluorescence. Approximately $93 \%$ of cauda sperm and $75 \%$ of corpus sperm bound zona proteins. In contrast, only about $6.4 \%$ of caput sperm bind zona proteins after extensive washing. Ninety-five percent of cauda sperm incubated for 4 hours in caput fluid $(+\mathrm{cf})$ retained the ability to bind zona proteins. [Adapted with permission from Burkin HR, Miller DJ 2000 Zona pellucida protein binding ability of porcine sperm during epididymal maturation and the acrosome reaction. Dev Biol 222:99-109. Copyright Academic Press.] 
established, there is debate about its role in other species. ZP3 is glycosylated variably between species and it seems apparent that how ZP3 is glycosylated will impact the species specificity of sperm binding. There are a number of candidate $\mathrm{ZP} 3$ receptors whose function is still unclear. GalT-I is the ZP3 receptor that has been studied in most detail. Recent evidence suggests its role is more necessary in signal transduction leading to the acrosome reaction than in initial ZP3 binding. After zona penetration, members of the ADAM, integrin, and tetraspanin family may form a complex, allowing sperm to fuse with the oocyte plasma membrane and activate development. Further studies of GalT-I knockout mice as well as knockouts of ADAM, integrin, and tetraspanin members should provide valuable clues about the molecular biology of gamete interaction. The enhanced ability to identify important genes and then to delete them from the animal genome offers opportunities to assemble the puzzle and solve the complicated process of mammalian fertilization.

\section{ACKNOWLEDGMENTS}

Research in the author's laboratory was supported by the National Institutes of Health, the National Science Foundation, the U.S. Department of Agriculture, the University of Illinois Agricultural Experiment Station, and the Illinois Council for Food and Agricultural Research. The authors thank the members of the Miller laboratory for careful review of the manuscript and for creative and critical review of the original research.

\section{REFERENCES}

Adham IM, Nayernia K, Engel W 1997 Spermatozoa lacking acrosin protein show delayed fertilization. Mol Reprod Dev 46:370-376

Almeida EA, Huovila AP, Sutherland AE, Stephens LE, Calarco PG, Shaw LM, Mercurio AM, Sonnenberg A, Primakoff P, Myles DG 1995 Mouse egg integrin $\alpha 6 \beta 1$ functions as a sperm receptor. Cell 81:1095-1104

Amado M, Almeida R, Schwientek T, Clausen H 1999 Identification and characterization of large galactosyltransferase gene families: galactosyltransferases for all functions. Biochim Biophys Acta 1473:35-53

Arnoult C, Kazam IG, Visconti PE, Kopf GS, Villaz M, Florman HM 1999 Control of the low voltage-activated calcium channel of mouse sperm by egg ZP3 and by membrane hyperpolarization during capacitation. Proc Natl Acad Sci USA 96:6757-6762

Asano M, Furukawa K, Kido M, Matsumoto S, Umesaki Y, Kochibe N, Iwakura Y 1997 Growth retardation and early death of $\beta 1,4$-galactosyltransferase knockout mice with augmented proliferation and abnormal differentiation of epithelial cells. EMBO J 16:1850-1857

Aviles M, Okinaga T, Shur BD, Ballesta J 2000 Differential expression of glycoside residues in the mammalian zona pellucida. Mol Reprod Dev 57:296-308

Bedford JM 1998 Mammalian fertilization misread? Sperm penetration of the eutherian zona pellucida is unlikely to be a lytic event. Biol Reprod 59:1275-1287

Begovac PC, Hall DE, Shur BD 1991 Laminin fragment E8 mediates PC12 cell neurite outgrowth by binding to cell surface $\beta 1$,4galactosyltransferase. J Cell Biol 113:637-644 
Bigler D, Takahashi Y, Chen MS, Almeida EA, Osbourne L, White JM 2000 Sequence-specific interaction between the disintegrin domain of mouse ADAM 2 (fertilin $\beta$ ) and murine eggs. Role of the $\alpha(6)$ integrin subunit. J Biol Chem 275:11576-11584

Bleil JD, Wassarman PM 1980 Mammalian sperm-egg interaction: identification of a glycoprotein in mouse egg zonae pellucidae possessing receptor activity for sperm. Cell 20:873-882

Bleil JD, Wassarman PM 1983 Sperm-egg interactions in the mouse: sequence of events and induction of the acrosome reaction by a zona pellucida glycoprotein. Dev Biol 95:317-324

Bleil JD, Wassarman PM 1986 Autoradiographic visualization of the mouse egg's sperm receptor bound to sperm. J Cell Biol 102:1363-1371

Bleil JD, Wassarman PM 1988 Galactose at the nonreducing terminus of O-linked oligosaccharides of mouse egg zona pellucida glycoprotein ZP3 is essential for the glycoprotein's sperm receptor activity. Proc Natl Acad Sci USA 85:6778-6782

Bleil JD, Greve JM, Wassarman PM 1988 Identification of a secondary sperm receptor in the mouse egg zona pellucida: role in maintenance of binding of acrosome-reacted sperm to eggs. Dev Biol 128:376-385

Bookbinder LH, Cheng A, Bleil JD 1995 Tissue- and species-specific expression of sp56, a mouse sperm fertilization protein. Science 269:86-89

Bork P 1996 Sperm-egg binding protein or proto-oncogene? Science 271:1431-1432

Borsig L, Kleene R, Dinter A, Berger EG 1996 Immunodetection of alpha 1-3 fucosyltransferase (FucT-V). Eur J Cell Biol 70:42-53

Brewis IA, Clayton R, Barratt CL, Hornby DP, Moore HD 1996 Recombinant human zona pellucida glycoprotein 3 induces calcium influx and acrosome reaction in human spermatozoa. Mol Hum Reprod 2:583-589

Burkin HR, Miller DJ 2000 Zona pellucida protein binding ability of porcine sperm during epididymal maturation and the acrosome reaction. Dev Biol 222:99-109

Burks DJ, Carballada R, Moore HDM, Saling PM 1995 Interaction of a tyrosine kinase from human sperm with the zona pellucida at fertilization. Science 269:83-86

Carroll J 2001 The initiation and regulation of $\mathrm{Ca}^{2+}$ signalling at fertilization in mammals. Semin Cell Dev Biol 12:37-43

Charron M, Shaper NL, Rajput B, Shaper JH 1999 A novel 14-base-pair regulatory element is essential for in vivo expression of murine beta4-galactosyltransferase-I in late pachytene spermatocytes and round spermatids. Mol Cell Biol 19:5823-5832

Chen J, Litscher ES, Wassarman PM 1998 Inactivation of the mouse sperm receptor, mZP3, by site-directed mutagenesis of individual serine residues located at the combining site for sperm. Proc Natl Acad Sci USA 95:6193-6197

Chen MS, Tung KS, Coonrod SA, Takahashi Y, Bigler D, Chang A, Yamashita Y, Kincade PW, Herr JC, White JM 1999 Role of the integrin-associated protein CD9 in binding between sperm ADAM 2 and the egg integrin alpha6beta1: implications for murine fertilization. Proc Natl Acad Sci USA 96:11830-11835

Cheng A, Le T, Palacios M, Bookbinder LH, Wassarman PM, Suzuki F, Bleil JD 1994 Sperm-egg recognition in the mouse: characterization of sp56, a sperm protein having specific affinity for ZP3. J Cell Biol 125:867-878

Cho C, Bunch DO, Faure JE, Goulding EH, Eddy EM, Primakoff P, Myles DG 1998 Fertilization defects in sperm from mice lacking fertilin $\beta$. Science 281:1857-1859

Close BE, Colley KJ 1998 In vivo autopolysialylation and localization of the polysialyltransferases PST and STX. J Biol Chem 273:34586-34593

Colley KJ 1997 Golgi localization of glycosyltransferases: more questions than answers. Glycobiology 7:1-13

Cornwall GA, Tulsiani DR, Orgebin-Crist MC 1991 Inhibition of the mouse sperm surface $\alpha$-D-mannosidase inhibits sperm-egg binding in vitro. Biol Reprod 44:913-921 
Cunningham BC, Ultsch M, de Vos AM, Mulkerrin MG, Clauser KR, Wells JA 1991 Dimerization of the extracellular domain of the human growth hormone receptor by a single hormone molecule. Science 254:821-825

Easton RL, Patankar MS, Lattanzio FA, Leaven TH, Morris HR, Clark GF, Dell A 2000 Structural analysis of murine zona pellucida glycans. Evidence for the expression of core 2-type O-glycans and the $\mathrm{Sd}^{\mathrm{a}}$ antigen. J Biol Chem 275:7731-7742

Ensslin M, Vogel T, Calvete JJ, Thole HH, Schmidtke J, Matsuda T, Topfer-Petersen E 1998 Molecular cloning and characterization of $\mathrm{P} 47$, a novel boar sperm- associated zona pellucidabinding protein homologous to a family of mammalian secretory proteins. Biol Reprod 58:1057-1064

Erlichman J, Gutierrez-Juarez R, Zucker S, Mei X, Orr GA 1999 Developmental expression of the protein kinase $\mathrm{C}$ substrate/binding protein (clone 72/SSeCKS) in rat testis identification as a scaffolding protein containing an A-kinase-anchoring domain which is expressed during late-stage spermatogenesis. Eur J Biochem 263:797-805

Evans JP 2001 Fertilin beta and other ADAMs as integrin ligands: insights into cell adhesion and fertilization. Bioessays 23:628-639

Evans JP, Schultz RM, Kopf GS 1995 Mouse sperm-egg plasma membrane interactions: analysis of roles of egg integrins and the mouse sperm homologue of PH-30 (fertilin) beta. J Cell Sci 108:3267-3278

Evans JP, Kopf GS, Schultz RM 1997 Characterization of the binding of recombinant mouse sperm fertilin $\beta$ subunit to mouse eggs: evidence for adhesive activity via an egg $\beta 1$ integrinmediated interaction. Dev Biol 187:79-93

Fayrer-Hosken RA, Caudle AB, Shur BD 1991 Galactosyltransferase activity is restricted to the plasma membranes of equine and bovine sperm. Mol Reprod Dev 28:74-78

Florman HM, Storey BT 1982 Mouse gamete interactions: the zona pellucida is the site of the acrosome reaction leading to fertilization in vitro. Dev Biol 91:121-130

Florman HM, Wassarman PM 1985 O-linked oligosaccharides of mouse egg ZP3 account for its sperm receptor activity. Cell 41:313-324

Florman HM, Bechtol KB, Wassarman PM 1984 Enzymatic dissection of the functions of the mouse egg's receptor function for sperm. Dev Biol 106:243-255

Florman HM, Arnoult C, Kazam I, Li C, O'Toole CMB 1998 A perspective on the control of mammalian fertilization by egg-activated ion channels in sperm: a tale of two channels. Biol Reprod 59:12-16

Foster J, Friday B, Maulit M, Blobel C, Winfrey V, Olson G, Kim K, Gerton G 1997 AM67, a secretory component of the guinea pig sperm acrosomal matrix, is related to mouse sperm protein sp56 and the complement component 4-binding proteins. J Biol Chem 272: $12714-12722$

Frandberg P-A, Doufexis M, Kapas S, Chhajlani V 1998 Amino acid residues in third intracellular loop of melanocortin 1 receptor are involved in G-protein coupling. Biochem Mol Biol Int 46:913-922

Fukami K, Nakao K, Inoue T, Kataoka Y, Kurokawa M, Fissore RA, Nakamura K, Katsuki M, Mikoshiba K, Yoshida N, Takenawa T 2001 Requirement of phospholipase C $\delta 4$ for the zona pellucida-induced acrosome reaction. Science 292:920-923

Geng JG, Raub TJ, Baker CA, Sawada GA, Ma L, Elhammer AP 1997 Expression of a P-selectin ligand in zona pellucida of porcine oocytes and P-selectin on acrosomal membrane of porcine sperm cells. Potential implications for their involvement in sperm-egg interactions. J Cell Biol 137:743-754

Gong XH, Dubois DH, Miller DJ, Shur BD 1995 Activation of a G protein complex by aggregation of $\beta-1,4$ galactosyltransferase on the surface of sperm. Science 269:1718-1721 
Greve LC, Prody GA, Hedrick JL 1985 N-Acetyl- $\beta$-D-glucosaminidase activity in the cortical granules of Xenopus laevis eggs. Gamete Res 12:305-312

Hardy DM, Garbers DL 1995 A sperm membrane protein that binds in a species-specific manner to the egg extracellular matrix is homologous to von Willebrand factor. J Biol Chem 270:26025-26028

Hickox JR, Bi M, Hardy DM 2001 Heterogeneous processing and zona pellucida-binding activity of pig zonadhesin. J Biol Chem 276:41502-41509

Hunnicutt GR, Primakoff P, Myles DG 1996 Sperm surface protein PH-20 is bifunctional: one activity is a hyaluronidase and a second, distinct activity is required in secondary sperm-zona binding. Biol Reprod 55:80-86

Iida H, Yoshinaga Y, Tanaka S, Toshimori K, Mori T 1999 Identification of Rab3A GTPase as an acrosome-associated small GTP-binding protein in rat sperm. Dev Biol 211:144-155

Jansen S, Ekhlasi-Hundrieser M, Topfer-Petersen E 2001 Sperm adhesion molecules: structure and function. Cells Tiss Organs 168:82-92

Johnston DS, Wright WW, Shaper JH, Hokke CH, Van den Eijnden DH, Joziasse DH 1998 Murine sperm-zona binding, a fucosyl residue is required for a high affinity sperm-binding ligand. J Biol Chem 273:1888-1895

Jones R, Brown CR, Lancaster RT 1988 Carbohydrate-binding properties of boar sperm proacrosin and assessment of its role in sperm-egg recognition and adhesion during fertilization. Development 102:781-792

Joziasse DH 1992 Mammalian glycosyltransferases: genomic organization and protein structure. Glycobiology 2:271-277

Jungnickel MK, Marrero H, Birnbaumer L, Lemos JR, Florman HM 2001 Trp2 regulates entry of $\mathrm{Ca}^{2+}$ into mouse sperm triggered by egg ZP3. Nature Cell Biol 3:499-502

Kaji K, Oda S, Shikano T, Ohnuki T, Uematsu Y, Sakagami J, Tada N, Miyazaki S, Kudo A 2000 The gamete fusion process is defective in eggs of Cd9-deficient mice. Nature Genet 24:279-282

Kim KS, Cha MC, Gerton GL 2001a Mouse sperm protein sp56 is a component of the acrosomal matrix. Biol Reprod 64:36-43

Kim KS, Foster JA, Gerton GL 2001b Differential release of guinea pig sperm acrosomal components during exocytosis. Biol Reprod 64:148-156

Kinloch RA, Mortillo S, Stewart CL, Wassarman PM 1991 Embryonal carcinoma cells transfected with ZP3 genes differentially glycosylate similar polypeptides and secrete active mouse sperm receptor. J Cell Biol 115:655-664

Kline D, Kopf GS, Muncy LF, Jaffe LA 1991 Evidence for the involvement of a pertussis toxin-insensitive $\mathrm{G}$ protein in egg activation of the frog, Xenopus laevis. Dev Biol 143: $218-229$

Korner C, Nurnberg B, Uhde M, Braulke T 1995 Mannose 6-phosphate/insulin-like growth factor II receptor fails to interact with G-proteins. J Biol Chem 270:287-295

Kudo K, Yonezawa N, Katsumata T, Aoki H, Nakano M 1998 Localization of carbohydrate chains of pig sperm ligand in the glycoprotein ZPB of egg zona pellucida. Eur J Biochem 252:492-499

Larson JL, Miller DJ 1997 Sperm from a variety of mammalian species express $\beta 1$,4-galactosyltransferase on their surface. Biol Reprod 57:442-453

Le Naour F, Rubinstein E, Jasmin C, Prenant M, Boucheix C 2000 Severely reduced female fertility in CD9-deficient mice. Science 287:319-321

Lee NH, Geoghagen NSM, Cheng E, Cline RT, Fraser CM 1996 Alanine scanning mutagenesis of conserved arginine/lysine-arginine/lysine-X-X-arginine/lysine $\mathrm{G}$ protein-activating motifs on $\mathrm{m} 1$ muscarinic acetylcholine receptors. Mol Pharmacol 50:140-148 
Leyton L, Saling P 1989a 95 kd sperm proteins bind ZP3 and serve as tyrosine kinase substrates in response to zona binding. Cell 57:1123-1130

Leyton L, Saling P 1989b Evidence that aggregation of mouse sperm receptors by ZP3 triggers the acrosome reaction. J Cell Biol 108:2163-2168

Liang M, Garrison JC 1991 The epidermal growth factor receptor is coupled to a pertussis toxin-sensitive guanine nucleotide regulatory protein in rat hepatocytes. J Biol Chem 266: 13342-13349

Lin X, Tombler E, Nelson PJ, Ross M, Gelman IH 1996 A novel src- and ras-suppressed protein kinase C substrate associated with cytoskeletal architecture. J Biol Chem 271:28430-28438

Litscher ES, Wassarman PM 1996 Recombinant hamster sperm receptors that exhibit speciesspecific binding to sperm. Zygote 4:229-236

Litscher ES, Juntunen K, Seppo A, Penttila L, Niemela R, Renkonen O, Wassarman PM 1995 Oligosaccharide constructs with defined structures that inhibit binding of mouse sperm to unfertilized eggs in vitro. Biochemistry 34:4662-4669

Loeser CR, Tulsiani DR 1999 The role of carbohydrates in the induction of the acrosome reaction in mouse spermatozoa. Biol Reprod 60:94-101

Lopez LC, Bayna EM, Litoff D, Shaper NL, Shaper JH, Shur BD 1985 Receptor function of mouse sperm surface galactosyltransferase during fertilization. J Cell Biol 101:1501-1510

Lu Q, Shur BD 1997 Sperm from $\beta$ 1,4-galactosyltransferase-null mice are refractory to ZP3-induced acrosome reaction and penetrate the zona pellucida poorly. Development 124:4121-4131

Lu Q, Hasty P, Shur BD 1997 Targeted mutation in $\beta 1$,4-galactosyltransferase leads to pituitary insufficiency and neonatal lethality. Dev Biol 181:257-267

Lyon JD, Vacquier VD 1999 Interspecies chimeric sperm lysins identify regions mediating species-specific recognition of the abalone egg vitelline envelope. Dev Biol 214:151-159

Macek MB, Lopez LC, Shur BD 1991 Aggregation of $\beta$-1,4-galactosyltransferase on mouse sperm induces the acrosome reaction. Dev Biol 147:440-444

Mandel U, Hassan H, Therkildsen MH, Rygaard J, Jakobsen MH, Juhl BR, Dabelsteen E, Clausen H 1999 Expression of polypeptide GalNAc-transferases in stratified epithelia and squamous cell carcinomas: immunohistological evaluation using monoclonal antibodies to three members of the GalNAc-transferase family. Glycobiology 9:43-52

Martin I, Epand RM, Ruysschaert JM 1998 Structural properties of the putative fusion peptide of fertilin, a protein active in sperm-egg fusion, upon interaction with the lipid bilayer. Biochemistry 37:17030-17039

Mayadas TN, Johnson RC, Rayburn H, Hynes RO, Wagner DD 1993 Leukocyte rolling and extravasation are severely compromised in P selectin-deficient mice. Cell 74:541-554

Mengel-Gaw L, McCoy-Maman M, Tiemeier D 1991 Genomic structure and expression of human $\beta$-1,4-galactosyltransferase. Biochem Biophys Res Commun 176:1269-1276

Milland J, Taylor SG, Dodson HC, McKenzie IF, Sandrin MS 2001 The cytoplasmic tail of $\alpha 1,2$-fucosyltransferase contains a sequence for golgi localization. J Biol Chem 276: 12012-12018

Miller BJ, Georges-Labouesse E, Primakoff P, Myles DG 2000 Normal fertilization occurs with eggs lacking the integrin $\alpha 6 \beta 1$ and is CD9-dependent. J Cell Biol 149:1289-1296

Miller DJ, Macek MB, Shur BD 1992 Complementarity between sperm surface $\beta$-1,4-galactosyltransferase and egg-coat ZP3 mediates sperm-egg binding. Nature 357:589-593

Miller DJ, Gong XH, Decker G, Shur BD 1993 Egg cortical granule $N$-acetylglucosaminidase is required for the mouse zona block to polyspermy. J Cell Biol 123:1431-1440

Miyado K, Yamada G, Yamada S, Hasuwa H, Nakamura Y, Ryu F, Suzuki K, Kosai K, Inoue K, Ogura A, Okabe M, Mekada E 2000 Requirement of CD9 on the egg plasma membrane for fertilization. Science 287:321-324 
Moller CC, Bleil JD, Kinloch RA, Wassarman PM 1990 Structural and functional relationships between mouse and hamster zona pellucida glycoproteins. Dev Biol 137:276-286

Moller CC, Wassarman PM 1989 Characterization of a proteinase that cleaves zona pellucida glycoprotein ZP2 following activation of mouse eggs. Dev Biol 132:103-112

Mori E, Kashiwabara S, Baba T, Inagaki Y, Mori T 1995 Amino acid sequences of porcine Sp38 and proacrosin required for binding to the zona pellucida. Dev Biol 168:575-583

Mortillo S, Wassarman PM 1991 Differential binding of gold-labeled zona pellucida glycoproteins mZP2 and mZP3 to mouse sperm membrane components. Development 113:141-149

Myles DG, Primakoff P 1997 Why did the sperm cross the cumulus? To get to the oocyte. Functions of the sperm surface proteins $\mathrm{PH}-20$ and fertilin in arriving at, and fusing with, the egg. Biol Reprod 56:320-327

Myles DG, Kimmel LH, Blobel CP, White JM, Primakoff P 1994 Identification of a binding site in the disintegrin domain of fertilin required for sperm-egg fusion. Proc Natl Acad Sci USA 91:4195-4198

Nakano M, Yonezawa N 2001 Localization of sperm ligand carbohydrate chains in pig zona pellucida glycoproteins. Cells Tiss Organs 168:65-75

Nishimoto I, Okamoto T, Matsuura Y, Takahashi S, Okamoto T, Murayama Y, Ogata E 1993 Alzheimer amyloid protein precursor complexes with brain GTP-binding protein $\mathrm{G}_{\mathrm{o}}$. Nature 362:75-79

Nishimura H, Cho C, Branciforte DR, Myles DG, Primakoff P 2001 Analysis of loss of adhesive function in sperm lacking cyritestin or fertilin beta. Dev Biol 233:204-213

Nixon B, Lu Q, Wassler MJ, Foote CI, Ensslin MA, Shur BD 2001 Galactosyltransferase function during mammalian fertilization. Cells Tiss Organs 168:46-57

Okamoto T, Katada T, Murayama Y, Ui M, Ogata E, Nishimoto I 1990 A simple structure encodes G-protein activating function of the IGF-II mannose 6-phosphate receptor. Cell 62:709-717

Olate J, Martinez S, Purcell P, Jorquera H, Codina J, Birnbaumer L, Allende J 1990 Molecular cloning and sequence determination of four different cDNA species coding for alpha-subunits of $\mathrm{G}$ proteins from Xenopus laevis oocytes. FEBS Lett 268:27-31

Palumbi SR 1999 All males are not created equal: fertility differences depend on gamete recognition polymorphisms in sea urchins. Proc Natl Acad Sci USA 96:12632-12637

Perry AC, Wakayama T, Cooke IM, Yanagimachi R 2000 Mammalian oocyte activation by the synergistic action of discrete sperm head components: induction of calcium transients and involvement of proteolysis. Dev Biol 217:386-393

Pratt SA, Shur BD $1993 \beta$-1,4-Galactosyltransferase expression during spermatogenesis: stagespecific regulation by $t$ alleles and uniform distribution in + -spermatids and $t$-spermatids. Dev Biol 156:80-93

Ramalho-Santos J, Moreno RD, Sutovsky P, Chan AW, Hewitson L, Wessel GM, Simerly CR, Schatten G 2000 SNAREs in mammalian sperm: possible implications for fertilization. Dev Biol 223:54-69

Rankin T, Dean J 2000 The zona pellucida: using molecular genetics to study the mammalian egg coat. Rev Reprod 5:114-121

Rankin TL, Tong ZB, Castle PE, Lee E, Gore-Langton R, Nelson LM, Dean J 1998 Human ZP3 restores fertility in Zp3 null mice without affecting order-specific sperm binding. Development 125:2415-2424

Rankin T, Talbot P, Lee E, Dean J 1999 Abnormal zonae pellucidae in mice lacking ZP1 result in early embryonic loss. Development 126:3847-3855

Rebeiz M, Miller DJ 1999 Porcine sperm surface $\beta 1$,4galactosyltransferase binds to the zona pellucida but is not necessary or sufficient to mediate sperm-zona pellucida binding. Mol Reprod Dev 54:379-387 
Russo RN, Shaper NL, Shaper JH 1990 Bovine $\beta 1$,4-galactosyltransferase: two sets of mRNA transcripts encode two forms of the protein with different amino-terminal domains. J Biol Chem 265:3324-3331

Sasai K, Ikeda Y, Tsuda T, Ihara H, Korekane H, Shiota K, Taniguchi N 2001 The critical role of the stem region as a functional domain responsible for the oligomerization and Golgi localization of $\mathrm{N}$-acetylglucosaminyltransferase $\mathrm{V}$. The involvement of a domain homophilic interaction. J Biol Chem 276:759-765

Schmell ED, Gulyas BJ 1980 Mammalian sperm-egg recognition and binding in vitro. I. Specificity of sperm interactions with live and fixed eggs in homologous and heterologous insemination of hamster, mouse, and guinea pig oocytes. Biol Reprod 23:1075-1085

Schulz JR, Wessel GM, Vacquier VD 1997 The exocytosis regulatory proteins syntaxin and VAMP are shed from sea urchin sperm during the acrosome reaction. Dev Biol 191:80-87

Scully NF, Shaper JH, Shur BD 1987 Spatial and temporal expression of cell surface galactosyltransferase during mouse spermatogenesis and epididymal maturation. Dev Biol 124:111-124

Shaper NL, Hollis GF, Douglas JG, Kirsch IR, Shaper JH 1988 Characterization of the full length cDNA for murine $\beta 1,4$-galactosyltransferase. Novel features at the $5^{\prime}$-end predict two translational start sites at two in-frame AUGs. J Biol Chem 263:10420-10428

Shaper NL, Charron M, Lo NW, Shaper JH 1998 $\beta 1,4$-Galactosyltransferase and lactose biosynthesis: recruitment of a housekeeping gene from the nonmammalian vertebrate gene pool for a mammary gland specific function. J Mammary Gland Biol Neoplasia 3:315-324

Shi X, Amindari S, Paruchuru K, Skalla D, Burkin H, Shur BD, Miller DJ 2001 Cell surface $\beta$-1,4-galactosyltransferase-I activates G protein-dependent exocytotic signaling. Development 128:645-654

Shimizu S, Tsuji M, Dean J 1983 In vitro biosynthesis of three sulfated glycoproteins in murine zonae pellucidae by oocytes grown in follicle culture. J Biol Chem 258:5858-5863

Shur BD 1991 Cell surface $\beta 1$,4galactosyltransferase: twenty years later. Glycobiology 1:563-575

Shur BD 1993 Glycosyltransferasess as cell adhesion molecules. Curr Opin Cell Biol 5:854-863

Shur BD, Hall NG 1982a A role for mouse sperm surface galactosyltransferase in sperm binding to the egg zona pellucida. J Cell Biol 95:574-579

Shur BD, Hall NG 1982b Sperm surface galactosyltransferase activities during in vitro capacitation. J Cell Biol 95:567-573

Shur BD, Neely CA 1988 Plasma membrane association, purification, and partial characterization of mouse sperm $\beta 1$,4-galactosyltransferase. J Biol Chem 263:17706-17714

Stricker SA 1999 Comparative biology of calcium signaling during fertilization and egg activation in animals. Dev Biol 211:157-176

Sun H, Chen Z, Poppleton H, Scholich K, Mulenix J, Weipz GJ, Fulgham DL, Bertics PJ, Patel TB 1997 The juxtamembrane, cytosolic region of the epidermal growth factor receptor is involved in association with $\alpha$-subunit of $\mathrm{G}_{\mathrm{s}}$. J Biol Chem 272:5413-5420

Swanson WJ, Vacquier VD 1997 The abalone egg vitelline envelope receptor for sperm lysin is a giant multivalent molecule. Proc Natl Acad Sci USA 94:6724-6729

Swanson WJ, Vacquier VD 1998 Concerted evolution in an egg receptor for a rapidly evolving abalone sperm protein. Science 281:710-712

Swanson WJ, Yang Z, Wolfner MF, Aquadro CF 2001 Positive Darwinian selection drives the evolution of several female reproductive proteins in mammals. Proc Natl Acad Sci USA 98:2509-2514

Takahashi Y, Bigler D, Ito Y, White JM 2001 Sequence-specific interaction between the disintegrin domain of mouse ADAM 3 and murine eggs: role of $\beta 1$ integrin-associated proteins CD9, CD81, and CD98. Mol Biol Cell 12:809-820 
Tengowski MW, Wassler MJ, Shur BD, Schatten G 2001 Subcellular localization of $\beta 1,4-$ galactosyltransferase on bull sperm and its function during sperm-egg interactions. Mol Reprod Dev 58:236-244

Thaler CD, Cardullo RA 1996 The initial molecular interaction between mouse sperm and the zona pellucida is a complex binding event. J Biol Chem 271:23289-23297

Thall AD, Maly P, Lowe JB 1995 Oocyte Gal $\alpha 11,3 \mathrm{Gal}$ epitopes implicated in sperm adhesion to the zona pellucida glycoprotein ZP3 are not required for fertilization in the mouse. J Biol Chem 270:21437-21440

Tsai J-Y, Silver L 1996 Sperm-egg binding protein or proto-oncogene. Science 271:1431-1432

van Duin M, Polman JE, De Breet IT, van Ginneken K, Bunschoten H, Grootenhuis A, Brindle J, Aitken RJ 1994 Recombinant human zona pellucida protein ZP3 produced by chinese hamster ovary cells induces the human sperm acrosome reaction and promotes sperm-egg fusion. Biol Reprod 51:607-617

Visconti PE, Kopf GS 1998 Regulation of protein phosphorylation during sperm capacitation. Biol Reprod 59:1-6

Wade SM, Lim WK, Lan K-L, Chung DA, Nanamori M, Neubig RR 1999 G $_{\mathrm{i}}$ activator region of $\alpha_{2 \mathrm{~A}}$-adrenergic receptors: distinct basic residues mediate $\mathrm{G}_{\mathrm{i}}$ versus $\mathrm{G}_{\mathrm{s}}$ activation. Mol Pharmacol 56:1005-1013

Walensky LD, Snyder SH 1995 Inositol 1,4,5-trisphosphate receptors selectively localized to the acrosomes of mammalian sperm. J Cell Biol 130:857-869

Wang H-L 1999 A conserved arginine in the distal third intracellular loop of the micro-opioid receptor is required for $\mathrm{G}$ protein activation. J Neurochem 72:1307-1314

Ward CR, Kopf GS 1993 Molecular events mediating sperm activation. Dev Biol 158:9-34

Ward CR, Storey BT, Kopf GS 1992 Activation of $\mathrm{G}_{\mathrm{i}}$ protein in mouse sperm membranes by solubilized proteins of the zona pellucida, the egg's extracellular matrix. J Biol Chem 267:14061-14070

Ward C, Storey B, Kopf GS 1994 Selective activation of $\mathrm{G}_{\mathrm{i} 1}$ and $\mathrm{G}_{\mathrm{i} 2}$ in mouse sperm by the zona pellucida, the egg's extracellular matrix. J Biol Chem 269:13254-13258

Wassarman PM, Litscher ES 2001 Towards the molecular basis of sperm and egg interaction during mammalian fertilization. Cells Tiss Organs 168:36-45

Wassarman PM, Jovine L, Litscher ES 2001 A profile of fertilization in mammals. Nature Cell Biol 3:E59-E64

Wassler MJ, Foote CI, Gelman IH, Shur BD 2001 Functional interaction between the SSeCKS scaffolding protein and the cytoplasmic domain of $\beta 1$,4-galactosyltransferase. J Cell Sci 114:2291-2300

Wen Y, Richardson RT, Widgren EE, O'Rand MG 2001 Characterization of Sp17: a ubiquitous three domain protein that binds heparin. Biochem J 357:25-31

Wolfe CA, Cladera J, Ladha S, Senior S, Jones R, O'Shea P 1999 Membrane interactions of the putative fusion peptide $(\mathrm{MF} \alpha \mathrm{P})$ from fertilin- $\alpha$, the mouse sperm protein complex involved in fertilization. Mol Membr Biol 16:257-263

Wu H, Smyth J, Luzzi V, Fukami K, Takenawa T, Black SL, Allbritton NL, Fissore RA 2001 Sperm factor induces intracellular free calcium oscillations by stimulating the phosphoinositide pathway. Biol Reprod 64:1338-1349

Xie W, Jiang H, Wu Y, Wu D 1997 Two basic amino acids in the second inner loop of the interleukin-8 receptor are essential for G $\alpha 16$ coupling. J Biol Chem 272:24948-24951

Yamagata K, Murayama K, Okabe M, Toshimori K, Nakanishi T, Kashiwabara S, Baba T 1999 Acrosin accelerates the dispersal of sperm acrosomal proteins during acrosome reaction. J Biol Chem 273:10470-10474 
Yamaguchi N, Fukuda MN 1995 Golgi retention mechanism of $\beta$-1,4-galactosyltransferase. Membrane-spanning domain-dependent homodimerization and association with $\alpha$ - and $\beta$ tubulins. J Biol Chem 270:12170-12176

Yamasaki N, Richardson RT, O'Rand MG 1995 Expression of the rabbit sperm protein Sp17 in COS cells and interaction of recombinant Sp17 with the rabbit zona pellucida. Mol Reprod Dev 40:48-55

Yanagimachi R 1994 Mammalian fertilization. In: Knobil E, Neill JD, eds. Physiology of Reproduction, 2nd edit. New York: Raven Press; A189-A317

Yang L, Baffy G, Rhee SG, Manning D, Hansen CA, Williamson JR 1991 Pertussis toxinsensitive $G_{i}$ protein involvement in epidermal growth factor-induced activation of phospholipase C- $\gamma$ in rat hepatocytes. J Biol Chem 266:22451-22458

Yonezawa N, Mitsui S, Kudo K, Nakano M 1997 Identification of an N-glycosylated region of pig zona pellucida glycoprotein ZPB that is involved in sperm binding. Eur J Biochem 248:86-92

Yonezawa N, Fukui N, Kuno M, Shinoda M, Goko S, Mitsui S, Nakano M 2001 Molecular cloning of bovine zona pellucida glycoproteins ZPA and ZPB and analysis for sperm-binding component of the zona. Eur J Biochem 268:3587-3594

Youakim A, Dubois D, Shur B 1994a Localization of the long form of $\beta$-1,4-galactosyltransferase to the plasma membrane and Golgi complex of 3T3 and F9 cells by immunofluorescence confocal microscopy. Proc Natl Acad Sci USA 91:10913-10917

Youakim A, Hathaway HJ, Miller DJ, Gong X, Shur BD 1994b Overexpressing sperm surface $\beta 1,4$-galactosyltransferase in transgenic mice affects multiple aspects of sperm-egg interactions. J Cell Biol 126:1573-1583

Yuan R, Primakoff P, Myles DG 1997 A role for the disintegrin domain of cyritestin, a sperm surface protein belonging to the ADAM family, in mouse sperm-egg plasma membrane adhesion and fusion. J Cell Biol 137:105-112

Yurewicz EC, Pack BA, Sacco AG 1992 Porcine oocyte zona pellucida Mr 55,000 glycoproteins: identification of O-glycosylated domains. Mol Reprod Dev 33:182-188

Yurewicz EC, Sacco AG, Gupta SK, Xu N, Gage DA 1998 Hetero-oligomerization-dependent binding of pig oocyte zona pellucida glycoproteins ZPB and ZPC to boar sperm membrane vesicles. J Biol Chem 273:7488-7494

Zhu G, Allende ML, Jaskiewicz E, Qian R, Darling DS, Worth CA, Colley KJ, Young WW Jr 1998 Two soluble glycosyltransferases glycosylate less efficiently in vivo than their membrane bound counterparts. Glycobiology 8:831-840 



\title{
Regulation of the Mitotic and Meiotic Cell Cycles in the Male Germ Line
}

\author{
Debra J. Wolgemuth, ${ }^{*}{ }^{\dagger}+$ III Erika Laurion, ${ }^{\dagger}$ and Karen M. Lele ${ }^{\dagger}$ \\ *Department of Genetics and Development; ${ }^{\dagger}$ The Institute of Human Nutrition; \\ *Department of Obstetrics and Gynecology; "The Center for Reproductive Sciences; \\ and ${ }^{\|}$The Herbert Irving Comprehensive Cancer Center, Columbia University College of \\ Physicians and Surgeons, New York, New York 10032
}

\begin{abstract}
Mammalian gametogenesis provides a unique system in which to study cell-cycle regulation. Furthermore, understanding the genetic program controlling the mitotic and meiotic divisions of the germ line will provide insight into understanding infertility and new directions for contraception. Male and female germ cells have stages of cell-cycle regulation in common, including a mitotic proliferative stage, entry into meiosis, completion of a reductive division, and entry into a quiescent state awaiting signals at fertilization. However, the timing of these events - and, indeed, even the stage of development at which these events occurs - differs in the two sexes. The genes involved in controlling these specialized mitotic and meiotic cycles of mammalian germ cell differentiation are only now being identified. They include a complex array of kinases, phosphatases, regulatory proteins (e.g., cyclins), and an equally complex array of substrates, including components of the nuclear and cytoplasmic structures involved in cell division. This chapter provides an overview of our current understanding of cell-cycle regulation in mammalian mitotic cells and the importance of restriction points. A summary of observations regarding the expression of various cell-cycle regulatory genes in mouse gametes is provided, along with comments on interesting differences between mitotic and meiotic cells. Finally, the role of the novel A-type cyclin, cyclin A1, during male meiosis is discussed in depth.
\end{abstract}

\section{Introduction}

Understanding the genetic program controlling the mitotic and meiotic divisions of the germ line represents a unique opportunity for providing insight into cell-cycle control in vivo during development and differentiation. Meiosis itself is, of course, restricted to germ line cells and as such represents potential cell-cycle control points that simply do not exist in somatic cells. Furthermore, higher organisms are characterized by sexually dimorphic gametes. Male and female germ cells have stages of cell-cycle regulation in common, including a mitotic proliferative stage, entry into meiosis, completion of a reductive division, and entry into a quiescent state prior to fertilization. However, the timing of these 
events - and, indeed, even the stage of development at which these events occurs - differs in the two sexes (reviewed in Wolgemuth et al., 1995) (Figure 1). For example, female germ cells enter meiosis during fetal development, whereas this is a postnatal event in the male. Once the male germ cell has entered meiosis, the process continues without interruption until the haploid spermatozoon is produced. In contrast, the oocyte is arrested in the diplotene stage of meiotic prophase I, where it can remain for months or years. Following a growth period, the oocyte resumes meiosis, only to arrest at a second point, metaphase II. Fertilization then triggers the completion of meiosis and extrusion of the second polar body. Yet another curiosity of mammalian germ cells is the fact that the two haploid pronuclei commence DNA synthesis independently after fertilization, prior to a fusion event. These and other critical control points in the specialized cell cycles of mammalian germ cells are depicted in Figure 1.

The genetic hierarchy controlling mitosis and meiosis in gamete formation is only recently being elucidated, even in less-complicated systems such as the yeasts. A key player in both mitosis and meiosis is maturation promoting factor

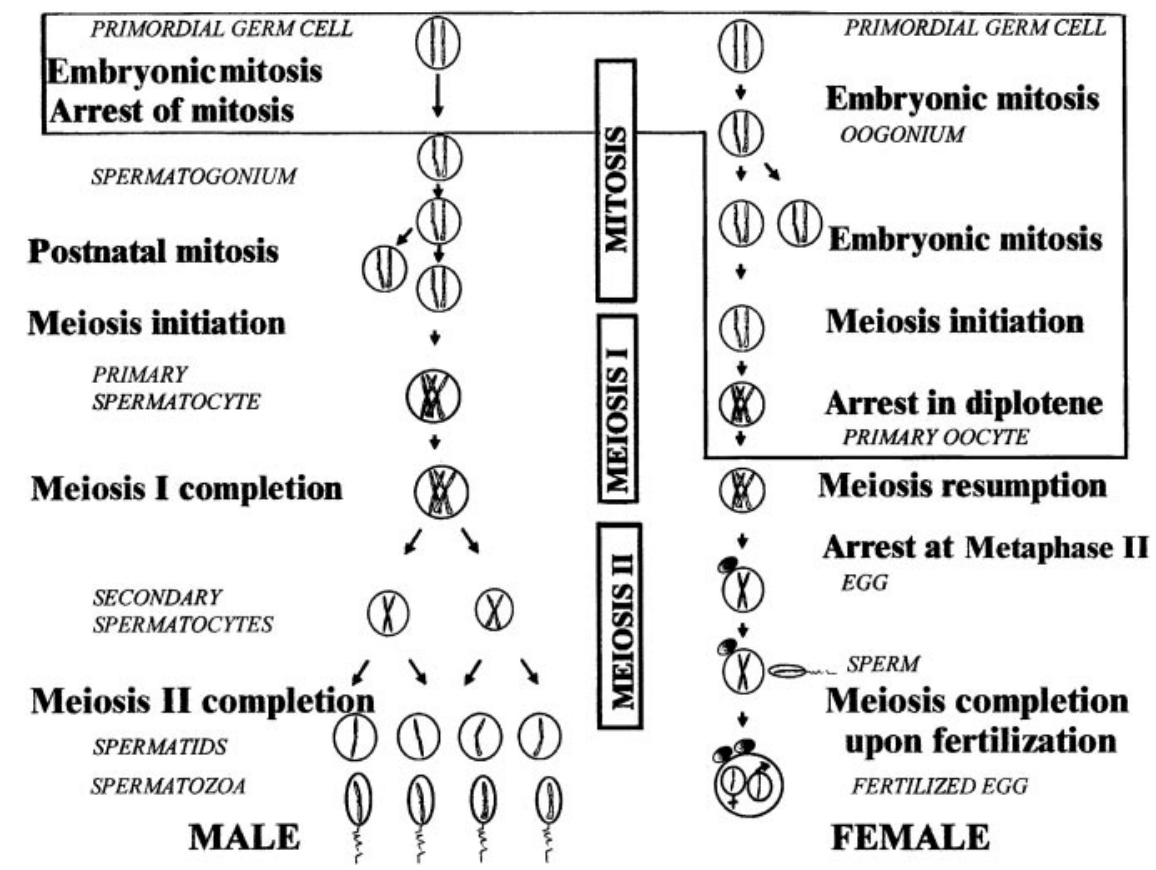

FIG. 1. Flowchart of differences in the cell-cycle progression during mammalian spermatogenesis and oogenesis (Wolgemuth et al., 1995). [Modified from Alberts B, Bray D, Lewis J, Raff M, Roberts K, Watson J 1983 Molecular Biology of the Cell. New York: Garland Publishing, Inc.] 
or M-phase promoting factor (MPF), originally described as an activity found in unfertilized frog eggs capable of inducing germinal vesicle breakdown and resumption of meiosis when injected into immature oocytes (Masui and Markert, 1971). MPF is composed of a regulatory subunit, cyclin $B$, and p34cdc2 (Cdk1), the catalytic serine/threonine protein kinase. These proteins have been detected in all higher eukaryotes examined. Given the high level of conservation of these key molecules across a diverse range of organisms, it is likely that they will also be important for both mitosis and meiosis in mammals, as they are in the yeasts, in which they have been best characterized. However, it is clear that there are control points and "checkpoints" in the cell cycles of higher eukaryotes that do not exist in simpler organisms and that may differ, depending on the developmental and tissue origin of the cells. For example, the oocytes of mammals exhibit unique cell-cycle control points: the signals to enter meiosis are different from those found in yeast, there is an arrest in diplotene of meiosis, and the cell pauses again in metaphase II, awaiting fertilization to complete meiosis (Figure 1). The spermatocyte shares some of these control points but, strikingly, does not normally arrest in either diplotene or at metaphase of meiosis II. It therefore is likely that there will be genes uniquely involved in these regulatory checkpoints that will be distinct from the yeast genes thus far studied.

In this review, we briefly discuss key aspects of cell-cycle control in mammalian cells, most of which have been derived from studies in cultured cells. We then survey what is known about the expression of known mitotic cell-cycle regulators in the testis and ovary, based on previous studies primarily from our lab. We then focus on the A-type cyclins, studies that have occupied much of our attention in the last 5 years and have provided insight into meiosis-specific and sex-specific differences in cell-cycle regulation. Finally, we highlight some of the many unanswered questions regarding the function of the A-type cyclins. These questions are likely to be relevant to other components of the cell-cycle machinery and will provide insight into infertility and possibly identify new targets for contraception.

\section{Overview of the Cell Cycle and Components of the Mammalian Cell-cycle Machinery}

The mammalian mitotic cell cycle involves a sequence of transitions and can be divided into four discrete phases (Figure 2). These stages include S-phase, in which DNA synthesis occurs, and M-phase, in which the cells complete mitosis. Between these stages are two gap (G) phases, G2 (S/M) and G1 (M/S). The progression through these stages is controlled by a series of sequential enzymatic reactions, the activity of which is highly ordered and tightly controlled. Restriction points at the G1/S and G2/M boundaries ensure that the requisite steps have taken place properly (discussed below). The primary enzymatic machinery 


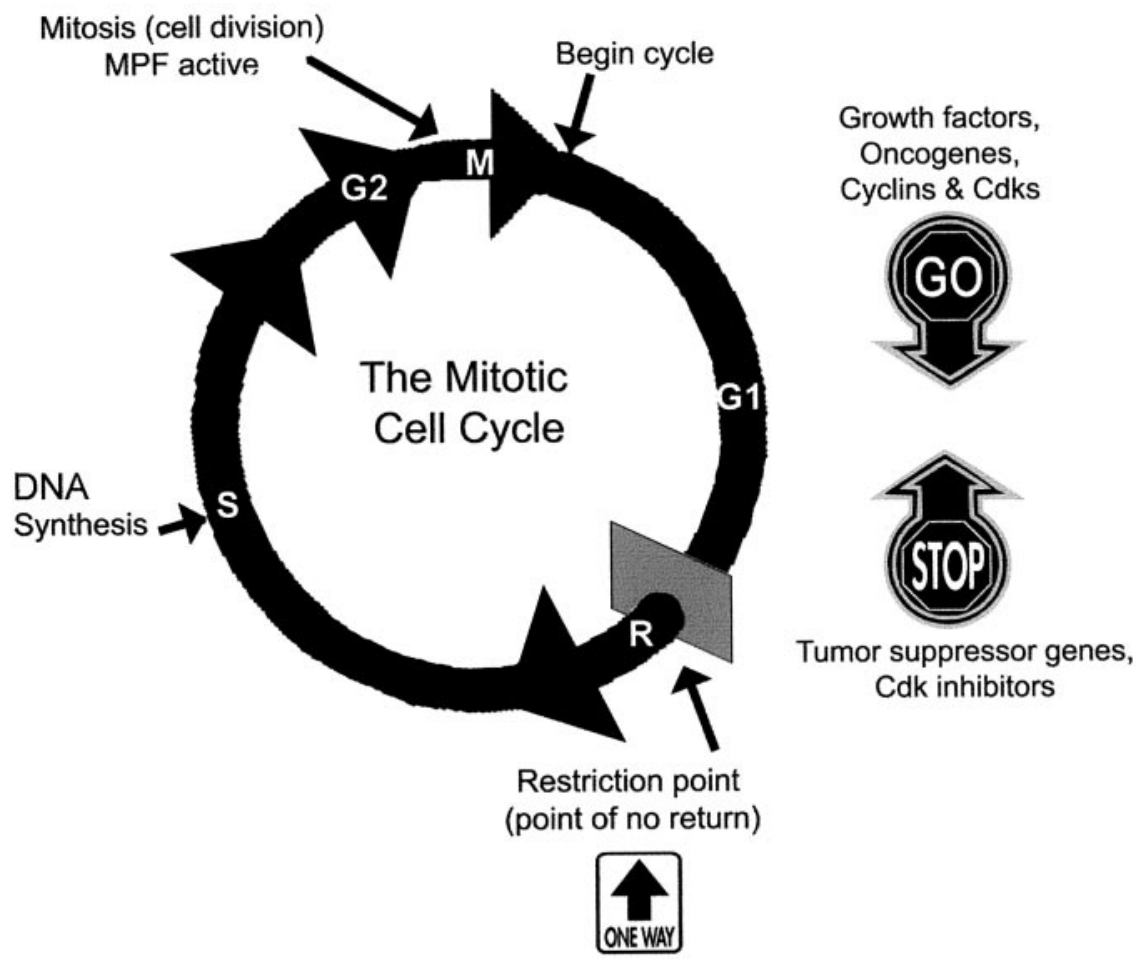

FIG. 2. Cartoon of key players in regulating the mitotic cell cycle.

orchestrating this progression consists of a regulatory subunit (cyclin) and a catalytic subunit, the cyclin-dependent kinase (Cdk).

Cyclins have been identified in a variety of organisms ranging from yeast to man, based on amino acid homology and by functional complementation of yeast cell-cycle mutants. The cyclins have been divided into at least eight classes in higher vertebrates, cyclins $\mathrm{A}$ to $\mathrm{H}$, based on their amino acid similarity and the timing of their appearance during the cell cycle (reviewed in Sherr, 1993; Pines, 1995b; Roberts, 1999). The complexity of the mammalian system is underscored by the existence not only of multiple classes of cyclins but also of multiple members of the A-, B-, and D-type cyclin families. Although their role in mammalian germ cell development is only beginning to be investigated, observations from our lab and others have shown that various cyclins exhibit distinct patterns of expression in the male and female germ cell lineages as well as in the somatic compartments of the gonads (Chapman and Wolgemuth, 1992,1993; Chesnel and Eppig, 1995; Ravnik et al., 1995; Ravnik and Wolgemuth, 1996,1999; Sweeney et al., 1996; Taieb et al., 1997; D. Liu et al., 1998). These 
studies have revealed patterns of cell-type and cell-cycle-stage specificity of in vivo expression of several of the cyclins that had not been detected previously in cultured cell systems (see Section V and Figure 5).

The Cdks are members of a serine/threonine kinase family that share the common properties of a conserved catalytic domain and phosphorylation sites that can modulate their subsequent activity. At least nine distinct Cdks have been identified in mammalian cells and been shown to exhibit differences in their preference for a cyclin partner (Pines, 1995a). The choice of cyclin partner may affect the enzymatic activity of the complex and possibly the substrates as well (Roberts, 1999). There are also Cdk-family members for which no cyclin partners have yet been identified. While they share structural homology to the bona fide Cdks, primarily through their shared PSTAIRE motif (or variations thereof), whether they also participate in cell-cycle regulation remains to be determined.

\section{Modes of Regulation of Activity of Cyclin/Cdk Complexes}

Progression through cell-cycle transitions is due to the sequential appearance of cyclins and activation of their Cdk partner. Several modes of regulating the presence of the cyclins are used, as outlined briefly in the following paragraphs.

\section{A. TRANSCRIPTION}

Cyclin abundance is regulated mainly at the level of transcription and proteolysis. The modes of transcriptional regulation vary among and within the different classes of cyclins. For example, transcription of D-type cyclins is growth factor dependent. When mitogens are withdrawn from cultured cells that have not completed a cell cycle, D-type cyclin mRNA and protein are degraded rapidly and the cells arrest in G1 (Sherr, 1993). Expression of genes for other cyclins and the Cdk1, which function later in the cell cycle, is mediated by elements that resemble, in part, E2F binding sites. The promoter of cyclin E contains a bipartite element named the cyclin E repressor module (CERM). The CERM element binds a protein complex that includes E2F-4 and a pocket protein, which then inhibits transcription (Le Cam et al., 1999). In vivo footprinting revealed that the CERM repressor is occupied only when the gene is not transcribed (in early G1). The onset of expression of the genes for cyclin A2, Cdk1, and cyclin B2 occurs later in the cell cycle than the gene for cyclin E and is mediated by a different bipartite repressor element termed the cell-cycledependent element/cell-cycle genes homology region (CDE/CHR) (Zwicker et al., 1995a; Lange-zu Dohna et al., 2000). The CDE/CHR is occupied when the genes are not transcribed in G1 and a mutation in either the CDE or CHR abolishes repression of corresponding promoter/reporter constructs early in the cell cycle (Zwicker et al., 1995a; Lange-zu Dohna et al., 2000). 


\section{B. TRANSLATION}

Relatively little is known about translational control of cyclin mRNAs, especially in mammalian cells. In budding yeast, initiation of a new cell cycle requires attainment of a critical cell mass and is responsive to the rate of protein synthesis. $\operatorname{Cln} 3$ translation in early G1 is regulated by the cap-binding protein eIF4E, which controls the rate of G1 protein synthesis and is inhibited by rapamycin (Pyronnet et al., 2001). Given the importance of translational regulation of a wide variety of genes during gametogenesis, especially in postmeiotic cells, it would be surprising if mammalian cyclin mRNAs were not regulated, at least in part, at the translational level. In this light, it was interesting to note that only low levels of mouse cyclin B1 were present in postmeiotic spermatids, as compared to meiotically dividing spermatocytes, even though cyclin B1 mRNA was readily detected (Chapman and Wolgemuth, 1994). Furthermore, recent studies on the translation regulatory factor CPEB revealed that it is involved in synapsis and progression through pachytene of meiosis (Mendez and Richter, 2001).

\section{TURNOVER}

The timely degradation of cyclins is equally important to exit a transition and appears to involve a ubiquitin-mediated proteolysis (reviewed in Hershko and Ciechanover, 1998). In this process, a ubiquitin-activating enzyme (E1) activates and transfers ubiquitin to a ubiquitin-conjugating enzyme (E2). E2, along with a substrate recognition protein (E3), assembles a ubiquitin chain on its substrate. Multi-ubiquitinated substrates are rapidly degraded by the 26 s proteasome. There are two types of E3 complexes: the SCF complex and the anaphase-promoting complex (APC). SCF-mediated proteolysis is best studied in yeast. Its substrates include proteins expressed early in the cell cycle, such as the G1 cyclins. Substrates targeted by SCF must first be phosphorylated, suggesting that their stability is controlled by activity of a protein kinase. In mammals, proteolysis of cyclin D1 and cyclin E is controlled by phosphorylation-dependent ubiquitination (Elledge and Harper, 1998). The mitotic cyclins are proteolyzed after APC recognition, which is essential for exit from mitosis. Cyclin A2 is degraded after breakdown of the nuclear envelope (den Elzen and Pines, 2001) and proteolysis of cyclin B is required for exit from M-phase (Gallant and Nigg, 1992).

\section{PHOSPHORYLATION AND DEPHOSPHORYLATION}

The activation state of Cdk complexes is regulated not only by cyclin association but also by $\mathrm{Cdk}$ phosphorylation, which can inhibit or enhance its kinase activity and therefore regulate the timing of Cdk activity (Figure 3). For example, MPF is not active until the G2/M transition, although Cdk1 is present 


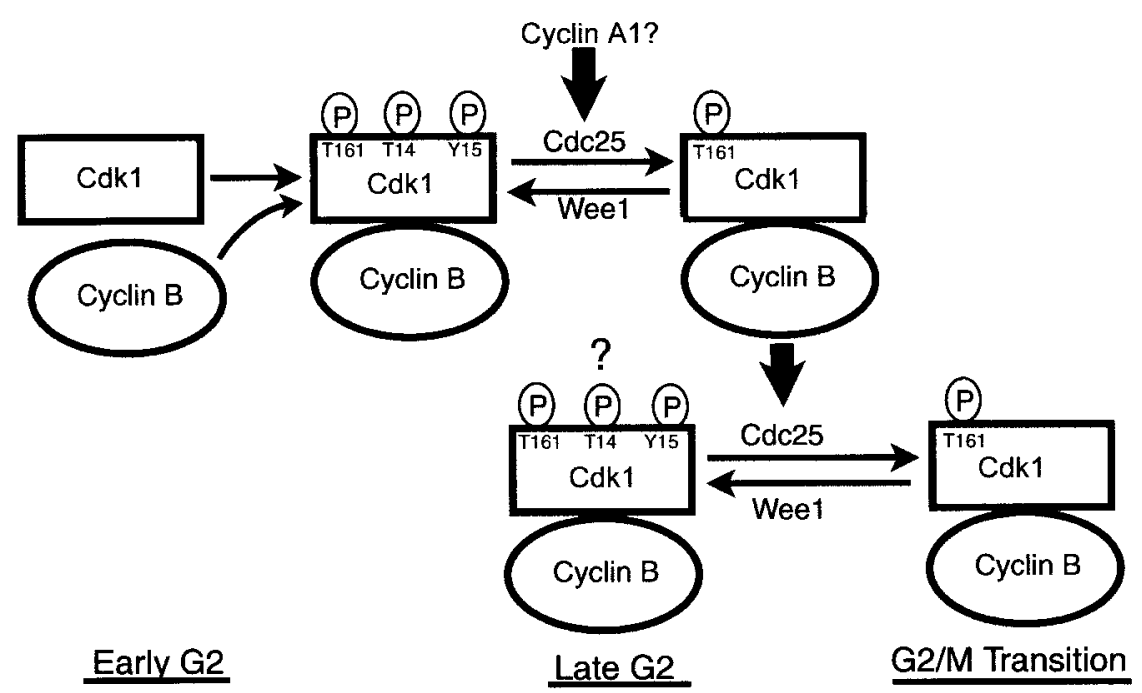

FIG. 3. Cartoon of factors involved in activating and inhibiting MPF.

throughout the cell cycle. In S and G2 phases, Cdk1 complexes with B-type cyclins and becomes phosphorylated on three residues, two of which inhibit activity. At the onset of $\mathrm{M}$ phase, inhibitory phosphorylation is removed by the activity of a protein phosphatase of the Cdc 25 family. In mice, the three Cdc25 genes (Cdc25A, Cdc25B, Cdc25C) are expressed in overlapping but distinct patterns (Kakizuka et al., 1992; Nargi and Woodford-Thomas, 1994; Wu and Wolgemuth, 1995). Cdc25C is activated by phosphorylation by Cdk1/cyclin B1, suggesting that a positive feedback loop exists between $\mathrm{Cdk} 1$ and $\mathrm{Cdc} 25$ (Hoffmann et al., 1993).

\section{E. INHIBITORS}

In mammals and other higher eukaryotes, there are two classes of relatively small (i.e., $16-57 \mathrm{kDa}$ ), tightly binding proteins that function as Cdk inhibitors (CKIs) (reviewed in Sherr and Roberts, 1995,1999). They are grouped according to their structure and their Cdk targets and include the CIP/KIP family consisting of p21CIP1/WAF1, p27KIP1, and p57KIP2 - and the INK family consisting of p16INK4a, p15INK4b, p18INK4c, and p19INK4d. The CKIs appear to exhibit their inhibitory function by binding in a stoichiometric manner, usually to cyclin-Cdk complexes but sometimes to noncomplexed Cdks. The INK family contains ankyrin repeats and binds only to Cdk4 and Cdk6 but not to other Cdks or to the D-type cyclins. The Cip/Kip families are broader with regard to their inhibiting activity, affecting the activity of the cyclin D-, E-, and 
A-type cyclin-dependent kinases. This class contains motifs in their aminoterminal regions that enable them to interact with both cyclin and Cdk proteins. Recent studies suggest that interaction with a Cip/Kip family member may actually positively regulate cyclin D-Cdk complexes but inhibit cyclin A- or E-Cdk complexes (reviewed in Sherr and Roberts, 1999).

\section{F. SUBCELLULAR LOCALIZATION}

A final level of control involves the distribution of the cyclin/Cdk proteins within cells, particularly whether they are in the nuclear or cytoplasmic compartments. For example, cyclin A2 apparently is constitutively nuclear in cultured cells (Pines and Hunter, 1991). However, cyclin B1 accumulates almost exclusively in the cytoplasm and undergoes a striking translocation to the nucleus as the cells enter prophase. This nuclear accumulation of Cdk1-cyclin B appears to be required for mitosis to proceed. Several recent studies have implicated the exclusion of $\mathrm{Cdk} 1 /$ cyclin B in the function of checkpoints that prevent entry into mitosis in the presence of DNA damage (Toyoshima et al., 1998). Nuclear transport factors have been identified that appear to be involved in ferrying some of the cyclins and Cdks in and out of the nucleus. Such factors might have been predicted to exist, since no classic nuclear localization sequences have yet been identified in the primary sequences of any of the vertebrate cyclins or Cdks. As noted, they exhibit striking differences in their subcellular localization (reviewed in Yang and Kornbluth, 1999). In fact, deletion studies have identified sequences in the B-type cyclins that may function as both cytoplasmic-retention sequences and/or nuclear-export sequences (reviewed in Yang and Kornbluth, 1999).

The state of phosphorylation also may be important for the nuclear localization of at least some of the cyclin/Cdk complexes. For example, during meiotic maturation in frog oocytes, phosphorylation of four conserved serine residues in cyclin B1 correlates with the nuclear accumulation and activity of MPF (Li et al., 1995). A model for the role of cyclin B1 localization to the nucleus has been proposed in which phosphorylation of cyclin B1 inhibits its interaction with proteins that appear to favor nuclear export, thus promoting the accumulation of cyclin B1 in the nucleus. While the role of changes in the subcellular distribution of cyclin B1 during meiotic maturation in spermatocytes remains to be demonstrated, we note that cyclin B1 is predominantly cytoplasmic in meiotic prophase cells and shifts to the nucleus just before the entry into the first meiotic division (Liu et al., 2000).

\section{Restriction Points During Mitosis and Meiosis}

Signaling pathways operate at cell-cycle transitions to monitor genomic integrity and the successful completion of upstream events, which ensures 
orderly progress through the cell cycle. In the mitotic cell cycle, response to damaged or improperly replicated or aligned DNA activates pathways that arrest cells at G1-S, G2-M, and at the metaphase-anaphase transition (Figure 2). Recent findings suggest there are two types of cell-cycle checkpoint responses that delay the G1-S transition, both of which target Cdk2 activity. An initial, transient response leads to rapid inhibition of Cdk2 activity due to degradation of Cdc25A (Mailand et al., 2000). A second, sustained response is carried out by p53dependent production of $\mathrm{p} 21$, which binds to and blocks activity of Cdk2/cyclin E complexes (Stewart and Pietenpol, 2001). Arrest at the G2-M phase transition targets MPF by preventing the removal of Cdk1/cyclin B inhibitory phosphorylation. Inhibition of $\mathrm{Cdc} 25 \mathrm{C}$ phosphatase activity is mediated by damage-induced activation of the protein kinase Chk1, which appears to be a primary mechanism of MPF inhibition, as studied in fission yeast (Lopez-Girona et al., 2001) and in human cells (Blasina et al., 1999). Signaling pathways also activate p53, which activates the gene for p21 (Bunz et al., 1998). Interestingly, DNA damage also may repress transcription of genes for cell-cycle regulators. That is, radiationinduced G2 arrest is accompanied by a p53-dependent downregulation of the gene for Cdk1 via the CDE/CHR element (Badie et al., 2000) and the promoters for cyclin B1, cyclin B2, and $\mathrm{Cdc} 25 \mathrm{C}$, which is mediated by NF-Y-binding CAAT boxes (Manni et al., 2001).

The spindle checkpoint is activated at the metaphase-anaphase transition and prevents entry into anaphase until all chromosomes are properly aligned by monitoring microtubule attachment on kinetochores and their tension (Rieder et al., 1994). Components of the spindle checkpoint inhibit activity of APC, which, in the absence of checkpoint activation, degrades proteins, allowing sister chromatid separation and mitosis exit (Clarke and Gimenez-Abian, 2000).

Some evidence exists for the presence of checkpoints in meiotic cell cycles, primarily from studies in yeast. Cells that divide meiotically can arrest in prophase of metaphase I. In yeast, arrest occurs at the pachytene stage of meiotic prophase in response to incomplete chromosome synapsis or recombination (reviewed in Roeder and Bailis, 2000). There may be an analogous checkpoint in mammalian meiotic cells, since failure to enter into meiosis I has been observed when defects occur in prophase (Figure 4; Table I). Male mice lacking genes that function in recombination, DNA repair, or cell-cycle regulation exhibit defects in chromosomal synapsis or recombination during prophase that, unlike observations in yeast, result in apoptosis (Table I). However, it should be noted that the cells arrest at different stages of prophase, suggesting multiple rather than a single checkpoint. In response to low levels of radiation, a G2 delay is observed in spermatocytes that is not observed in spermatocytes that are p53 deficient, suggesting a role for p53 in the process (Schwartz et al., 1999). A spindle checkpoint may be active during meiosis in Drosophila spermatocytes. Entry into 


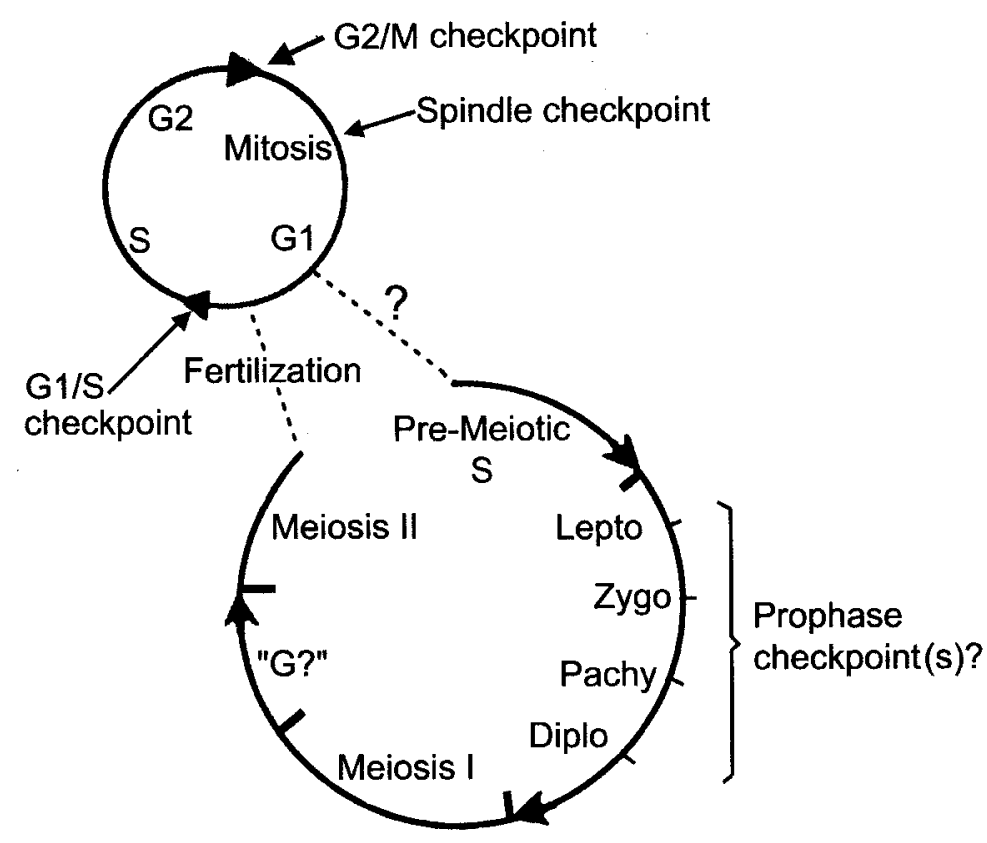

FIG. 4. Possible restriction points during mitosis and meiosis.

anaphase is delayed in spermatocytes that are either colchicine treated or contain misaligned chromosomes (Rebollo and Gonzalez, 2000).

\section{Which of the Cell-cycle Components Are Expressed in the Testis?}

\section{A. OVERVIEW OF THE EXPERIMENTS}

We initiated our studies on identifying the cell-cycle genes involved in mammalian gametogenesis with a very simple hypothesis having two major points: 1) meiosis is a highly conserved process across organisms, thus the regulatory components may also be conserved; and 2) control points exists in mammalian meiosis that simply do not exist in lower forms, hence, novel cell-cycle regulators may have evolved to fulfill these specialized functions. As will be discussed in the following sections, both parts of this hypothesis have been shown to be true.

\section{B. SUMMARY OF CELL-CYCLE GENES EXPRESSED IN THE GERM LINE}

A variety of experimental approaches have been used to identify cellcycle genes expressed during gametogenesis, from mRNA expression and 
TABLE I

Genetic Models in Male Mice Exhibiting Meiotic Prophase Arrest and Apoptosis

\begin{tabular}{|c|c|c|c|}
\hline Mutation & Arrest point & Synapsis phenotype & Reference \\
\hline A-myb & Early pachytene & Not reported & Toscani et al., 1997 \\
\hline Atm & Zygotene/early pachytene & Frequent asynapsis & Xu et al., 1996 \\
\hline Dmc1 & Zygotene/early pachytene & Asynapsis & Yoshida et al., 1998 \\
\hline Msh4 & Zygotene & $\begin{array}{l}\text { Frequent asynapsis; } \\
\text { Nonhomologous } \\
\text { pairing }\end{array}$ & Kneitz et al., 2000 \\
\hline Msh5 & Zygotene/early pachytene & $\begin{array}{l}\text { Frequent asynapsis; } \\
\text { Nonhomologous } \\
\text { pairing }\end{array}$ & Edelmann et al., 1999 \\
\hline Scp3 & Zygotene & Asynapsis & Yuan et al., 2000 \\
\hline Spo11 & Zygotene & Asynapsis & $\begin{array}{l}\text { Baudat et al., 2000; } \\
\text { Romanienko and } \\
\text { Camerini-Otero, } 2000\end{array}$ \\
\hline XSxr(a)0 & Late prophase/meiosis I & $\begin{array}{l}\text { Asynapsis of the sex } \\
\text { chromosome }\end{array}$ & Odorisio et al., 1998 \\
\hline Hsp70.2 & Late pachytene & Failure to desynapse & Dix et al., 1997 \\
\hline Mlh1 & Late pachytene & Failure to desynapse & Edelmann et al., 1996 \\
\hline Cyclin A1 & Late pachytene/diplotene & Incomplete desynapsis & D. Liu et al., 1998 \\
\hline
\end{tabular}

protein analysis using several methods and known probes and reagents to screens to identify new genes. A summary of the overall expression patterns of various categories of cell-cycle regulators that have been investigated in our lab is presented in Figure 5. It should be recognized that the data were obtained from studies in the rodent model system, where precise identification of cell types is easier than in other mammals (e.g., humans). However, in several studies, we have been able to extend the expression analysis to humans. For example, cyclin A1 is expressed in human late pachytene to diplotene spermatocytes, very much as it is in the mouse (C. Liao, S.Q. Li, S. Mulrad, X.Y. Wang, and D.J. Wolgemuth, submitted).

The genes examined generally fall into four classes. First, genes that are expressed in both germline and somatic cells of gonads and are rather ubiquitously expressed in other cells. Second, genes that are much more abundant in the germ line and third, those that are apparently specific to the germ line. Finally, 

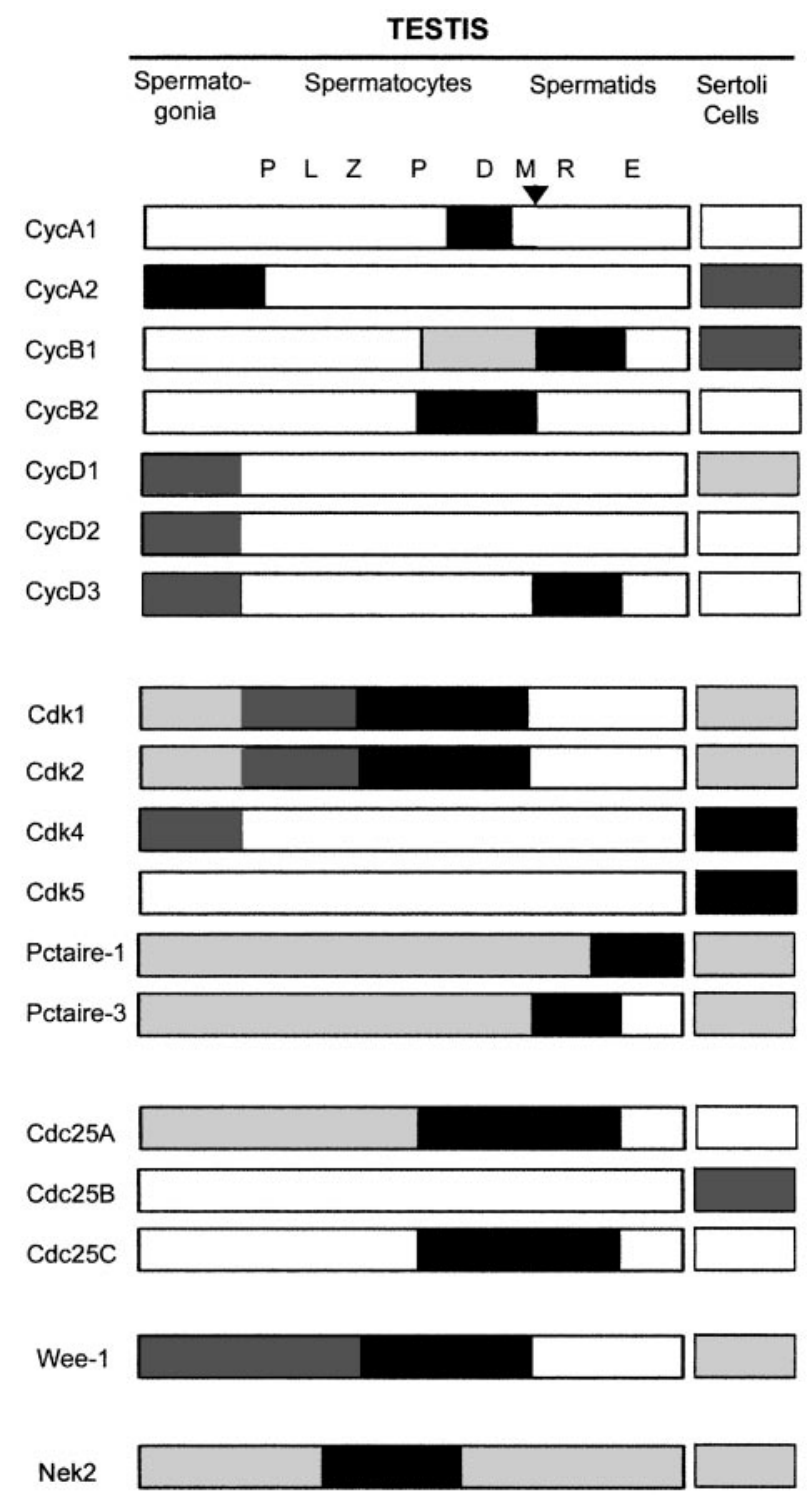

FIG. 5. Summary of the expression of selected cell-cycle-regulating genes in the mouse testis and ovary. This figure represents a composite of the results of studies from our laboratory on the levels of expression of the genes denoted on the left. The references containing the data supporting this compilation may be found in a review by Wolgemuth et al. (1995) and in references cited in this review. The relative levels of expression are depicted by shading of the bars: solid black, most robust expression of a particular gene; white bar, no detection of expression. 
genes that are specific to one or the other germ line (i.e., exhibiting sexual dimorphism in their patterns of expression).

\section{HIGHLIGHTS AND INTERESTING FEATURES OF THESE EXPRESSION PATTERNS THAT MAY BE IMPORTANT FOR SPERMATOGENESIS}

One of the earliest observations of unique differences between meiotic and somatic cells came from the observations of expression of the B-type cyclins (Chapman and Wolgemuth, 1992). The two mammalian B-type cyclins had been shown to be coexpressed in virtually all cultured cells analyzed. Surprisingly, their expression at the mRNA level was shown to be uncoupled in prophase spermatocytes. Cyclin B2 transcripts were detected earlier in prophase, at about the zygotene to early pachytene stage, and were followed by the appearance of cyclin B1 later in prophase. Further, cyclin B1 transcripts were observed in early round spermatids, which are postmeiotic. Whether these transcripts have any functional significance is not known, since subsequent studies using highly specific antibodies against the two B-type cyclins did not reveal significant accumulation of B1 protein in spermatids (Brandeis et al., 1998; D. Liu et al., 1998). The cyclin B1 null mutation results in embryonic lethality (Brandeis et al., 1998), thus obviating a direct test of its function in early spermatids in the adult. In contrast, cyclin B2 does not appear to be essential in that cyclin B2 knockout animals are viable and fertile, although a bit smaller and yielding slightly reduced litter sizes (Brandeis et al., 1998).

Another curiosity is the expression of cell-cycle genes, which are intimately tied to proliferation in mitotic cells, in testicular cells that are not dividing. In addition to the detection of cyclin B1 in spermatids, several of the Cdks have been observed to be expressed in nondividing cells in the testis. For example, both Cdk4 (Rhee and Wolgemuth, 1997) and Cdk5 (Q. Zhang, K. Rhee, and D.J. Wolgemuth, unpublished observations; Session et al., 2001) have been shown to be expressed in Sertoli cells in the adult mouse and human testis - cells that are no longer dividing and are highly differentiated.

Although the cyclins and Cdks are the major focus of this review, other pathways, possibly acting in parallel with cyclin/Cdk complexes, could play roles in mitosis and possibly in meiosis. For example, we and others have investigated the role of the putative homologues of the Aspergillus never in mitosis (NimA) gene (Osmani et al., 1991; Rhee and Wolgemuth, 1997; reviewed in Fry and Nigg, 1995). Although there appears to be as many as seven mammalian homologues of NimA (designated Nek genes), based on structural similarities, none has been shown to complement NimA in vivo (Schultz and Nigg, 1993; Kandli et al., 2000;). The pattern of expression of Nek1 and Nek2, in particular, during mammalian gametogenesis strongly 
suggests roles in meiosis (Letwin et al., 1992; Rhee and Wolgemuth, 1997; Tanaka et al., 1997; Arama et al., 1998).

\section{Focus on the A-type Cyclins}

\section{A. IDENTIFICATION OF TWO A-TYPE CYCLINS IN HIGHER ORGANISMS}

Of the mammalian cyclins identified to date, cyclin A has remained somewhat enigmatic, in part due to the fact that no homologues exist in the yeasts. We have shown that there are two distinct cyclin A genes in mammals, one of which, cyclin A1, is most highly expressed in the testis where it is restricted to the germ line (Ravnik and Wolgemuth, 1996; Sweeney et al., 1996). In contrast, cyclin A2 was expressed in all tissues analyzed. Two A-type cyclin genes have been documented in human and frog (Howe et al., 1995; Yang et al., 1997). Mouse cyclin A1 and A2 share 44\% identity at the amino acid level; however, they share a much higher level of identity (84\%) within the two highly conserved cyclin boxes (Ravnik and Wolgemuth, 1996; Sweeney et al., 1996).

\section{B. EXPRESSION OF THE A-TYPE CYCLINS IN MAMMALIAN GAMETOGENESIS}

In the adult mouse, Northern blot hybridization analysis revealed that expression of cyclin A1 was restricted to the testis, specifically, to meiotic germ cells. In situ hybridization analysis and immunohistochemistry were used to localize their expression to specific testicular cells. The levels of cyclin A1 mRNA (Sweeney et al., 1996; D. Liu et al., 1998) and protein (D. Liu et al., 1998; Ravnik and Wolgemuth, 1999) rise dramatically in late pachytene spermatocytes and become undetectable soon after completion of the first meiotic division. Thus, its expression is cell-cycle regulated. At both the RNA and protein levels, the predominant sites of cyclin A2 expression in the adult testis were the mitotic germ line stem cells, the spermatogonia, and in preleptotene spermatocytes, cells in which premeiotic DNA synthesis occurs (Ravnik and Wolgemuth, 1996,1999). Cyclin A2 expression also is detected in Leydig cells (Ge and Hardy, 1997). The concurrent localization of mRNA and protein for both of the A-type cyclins further suggested that both are regulated primarily at the level of transcription in the testis. The observed cellular specificity of cyclin A2 expression is consistent with its function during mitosis in the stem-cell stage of this lineage, while the restricted preleptotene stage localization suggests function in $\mathrm{G} 1 / \mathrm{S}$ or $\mathrm{S}$ but not in the meiotic divisions per se. No cyclin A1 mRNA was detected in ovaries or oocytes by Northern or in situ hybridization analysis (C. Liao, S.E. Ravnik, Q. Zhang, S. Mulrad, and D.J. Wolgemuth, in preparation). 
However, both somatic and germ cells of the adult ovary express cyclin A2 mRNA and protein. The different expression patterns suggest distinct functions for cyclin A1 and cyclin A2 in the somatic and germinal lineages, which also differ between the male and female.

Cyclin A2 is expressed ubiquitously in cultured cells and in a broad variety of tissues in the adult mouse and during embryogenesis (Ravnik and Wolgemuth, 1996; Sweeney et al., 1996). Perhaps not surprisingly then, targeted mutagenesis of encoding murine cyclin A2 resulted in early embryonic lethality, apparently around the peri-implantation stage (Murphy et al., 1997). This embryonic lethality has obviated understanding the role of cyclin A2 in other aspects of mammalian development, including the germ line. In contrast, the strikingly restricted expression of cyclin A1 led us to hypothesize that its primary site of function is in the male germ line, specifically, at the first meiotic division.

\section{REGULATION OF EXPRESSION OF THE A-TYPE CYCLINS}

Regulation of cyclin A2 has been extensively studied in cultured cells and shown to be first expressed during the mitotic cell cycle at the G1/S transition (reviewed in Pines and Hunter, 1991). The regulatory elements required for efficient cell-cycle-regulated transcription are contained within a small fragment of the proximal promoter ( 89 base pairs upstream and 11 base pairs downstream from its transcriptional start site) (Schulze et al., 1995). Periodic occupation of the CDE/CHR element, located in this fragment, by a repressor controls the timing of its expression (Zwicker et al., 1995b). Activities have been isolated from nuclear extracts that bind to the CDE/CHR (Liu et al., 1997) or to the CHR (Philips et al., 1999) in gel-shift experiments. E2F can bind the CDE of CDE/CHR (Zerfass-Thome et al., 1997). The CDE/CHR binding activity, termed CDE/CHR binding factor-1 (CDF-1), could not be supershifted by antibodies directed against E2F or DP-1 proteins. Mutating the first nucleotide of the CDE can abrogate E2F binding, while having little effect on the binding of CDF-1 in gel-shift experiments (N. Liu et al., 1998). The corresponding mutant reporter construct showed unchanged repression in G0, suggesting that E2F does not mediate repression of the gene for cyclin A2. This led to the hypothesis that E2F may compete with and displace CDF-1 in late G1/S phase, leading to transcriptional activation of the gene (N. Liu et al., 1998). Transcriptional activation upon E2F binding also can explain the observed upregulation of the cyclin A2 promoter following ectopic expression of E2F-1 (Schulze et al., 1995). The CHR-binding activity, CHR-binding factor (CHF), was isolated from nuclear extract from quiescent cells (Philips et al., 1999). CHF, when microinjected into cells, was able to downregulate the cyclin A2 promoter/reporter construct (Philips et al., 1999). 
It is speculated that the repressor occupying the CDE/CHR may interfere with the activity of factors bound upstream. In vivo footprinting revealed upstream elements that were protected constitutively during the cell cycle (Desdouets et al., 1995; Zwicker et al., 1995a). These elements bind Sp1, NF-Y, and CBP/cycA (CCAAT binding protein for cyclin A gene) and members of the ATF/CREB/CREM family (Desdouets et al., 1995; Zwicker et al., 1995a; Kramer et al., 1997). CBP/cycA is implicated in adhesion-dependent cyclin A2 transcription (Kramer et al., 1997), while members of ATF/CREB/CREM mediate regulation of the cyclin A2 promoter by cAMP (Desdouets et al., 1995). There may be cell specificity of binding of ATF/CREB/CREM family factors to the cAMP response element in the cyclin A2 promoter, as shown in gel-shift assays using extracts from various types of cells (Blanchard, 2000).

Almost nothing is known about the regulation of the cyclin A1 gene, especially in vivo. A 1.3-kb fragment of the human cyclin A1 promoter appears to reproduce the highly restricted tissue expression pattern in three of four transgenic mouse lines carrying human cyclin A1 reporter gene constructs (Muller et al., 2000a). Potential regulatory elements and factors that control expression have been studied in the context of a smaller promoter fragment that is comprised of 190 base pairs of upstream promoter sequence in cultured cells. This fragment can induce expression of a reporter gene mediated by GC boxes that can bind Sp1 in HeLa cells (Muller et al., 1999) and by coexpression with B-myb and cyclin A1 in CV-1 cells (Muller et al., 2000b). Although it was initially suggested that the tissue-specific expression of mouse cyclin A1 correlates with methylation of its CpG-rich proximal promoter (Muller et al., 2000a), subsequent studies revealed that this is not likely (Muller-Tidow et al., 2001). Cyclin A1 frequently is overexpressed in human acute promyelocytic leukemia, a subtype of acute myeloid leukemia that is characterized by the presence of the PML-RAR $\alpha$ fusion protein (Yang et al., 1999). A recent study has suggested that PML-RAR $\alpha$ ectopically expressed in human myeloid leukemia cell lines led to induction of co-transfected human cyclin A1 promoter/reporter constructs with either 190 base pairs of promoter or with $1.3 \mathrm{~kb}$ (Muller et al., 2000b). Whether such constructs will be activated in myeloid cells in vivo remains to be determined, as does the question of whether these flanking regions are active in spermatocytes.

\section{PREFERENCE OF CDK PARTNER FOR THE A-TYPE CYCLINS IN TESTICULAR CELLS}

To extend our studies on the function of the A-type cyclins during meiosis in the male at the biochemical level, we examined the developmental and cellular distribution of their candidate cyclin-dependent kinase partners, Cdk1 and Cdk2, in the spermatogenic lineage (Ravnik and Wolgemuth, 1999). As noted previ- 
ously, immunohistochemical localization revealed that cyclin A1 is present only in male germ cells just prior to or during the first, but not the second, meiotic division. By contrast, cyclin A2 was expressed in spermatogonia and was most abundant in preleptotene spermatocytes, cells about to enter the meiotic pathway. Immunohistochemical detection of Cdk1 was most apparent in early pachytene spermatocytes, while staining intensity diminished in diplotene and meiotically dividing spermatocytes, the cells in which cyclin A1 expression was strongest. Cdk2 was highly expressed in all spermatocytes. Notably, in cells undergoing the meiotic reduction divisions, Cdk2 appeared to localize specifically to the chromatin. This was not the case for spermatogonia undergoing mitotic divisions. We also showed that, in the testis, cyclin A1 binds both Cdk1 and Cdk2 (Sweeney et al., 1996; D. Liu et al., 1998,2000). In contrast, cyclin A2 strongly preferred to bind to Cdk2 in testicular lysates. These results suggest that the A-type cyclins have differences in their partner preference and may exhibit unique target substrate specificity.

\section{E. TARGETED MUTAGENESIS OF CYCLIN A1}

To begin to address possible redundancy of the two A-type cyclin genes, we generated cyclin A1-deficient mice by targeted mutagenesis of the cyclin A1 gene (D. Liu et al., 1998). The remarkable restriction of cyclin A1 suggested a very specific function only at the G2/M transition of the first meiotic division. Cyclin A1-deficient males were sterile due to a block of spermatogenesis before the first meiotic division, whereas females were normal. Meiosis arrest in males lacking cyclin A1 was associated with increased germ-cell apoptosis and desynapsis abnormalities (D. Liu et al., 1998,2000). There was a striking reduction in the activation of MPF kinase at the end of meiotic prophase, although both Cdk1 and cyclin B proteins were present. Cyclin A1 is therefore essential for spermatocyte passage into the first meiotic division in male mice, a function that cannot be complemented by the concurrently expressed B-type cyclins.

We then explored the possibility that a cyclin A1-dependent process dictates the activation of MPF (D. Liu et al., 1998). There are several pieces of evidence in the literature that supported a role for A-type cyclins in the activation of MPF. Xenopus cyclin A1 is synthesized before cyclin B and accumulates as an active kinase complex (Minshull et al., 1990). Cyclin A1 bound to Cdk1 in Xenopus egg extracts is immediately active, independent of Cdc25 activity (Clarke et al., 1992). The addition of cyclin A1 into such cell-free extracts potentiated MPF activity (Devault et al., 1992). Further, blocking Cdk2 activity, either by 21 or by immunodepletion, prevented activation of MPF and mitosis in cultured cells (Guadagno and Newport, 1996).

We asked whether the expression of the B-type cyclins that comprise MPF was retained in cyclin A1-deficient spermatocytes (Liu et al., 2000). Both cyclin 
B1 and B2 proteins were readily detected, although their associated kinases were kept at inactive states. We then turned to an in vitro, short-term culture system developed by Handel and colleagues (Wiltshire et al., 1995) in which treatment of pachytene spermatocytes with the protein phosphatase type 1 and type $2 \mathrm{~A}$ inhibitor okadaic acid can induce premature chromosome condensation and entry into the first meiotic division. We asked if treatment of the arrested diplotene spermatocytes in cyclin A1-deficient testes could drive the cells into meiosis I and activate MPF activity. Indeed, okadaic acid treatment restored MPF activity and induced entry into $\mathrm{M}$ phase and the formation of overtly normally condensed chromosome bivalents (Figure 4), concomitant with hyperphosphorylation of Cdc25 proteins (Liu et al., 2000).

Conversely, inhibition of tyrosine phosphatases, including Cdc25s, by vanadate suppressed the okadaic acid-induced metaphase induction. The highest levels of $\mathrm{Cdc} 25 \mathrm{~A}$ and $\mathrm{Cdc} 25 \mathrm{C}$ expression and their subcellular localization during meiotic prophase coincide with that of cyclin A1. When overexpressed in HeLa cells, cyclin A1 coimmunoprecipitates with Cdc25A. Furthermore, the protein kinase complexes consisting of cyclin A1 and either $\mathrm{Cdk} 1$ or $\mathrm{Cdk} 2$ phosphorylate both Cdc25A and Cdc25C in vitro (Liu et al., 2000). These results suggest that, in normal meiotic male germ cells, cyclin A1 participates in the regulation of other protein kinases or phosphatases critical for the G2-M transition. In particular, it may be directly involved in the initial amplification of MPF through the activating phosphorylation on Cdc25 phosphatases (Figure 3).

\section{Unanswered Questions}

Our investigations on the role of the A-type cyclins in the development, differentiation, and function of the germ line have provided some clues as to their in vivo function; however, much remains to be elucidated. Outlined below are several of the questions we would like to address.

\section{A. IS CYCLIN A2 REQUIRED IN MITOSIS IN SPERMATOGONIA AND FOR PRELEPTOTENE DNA SYNTHESIS?}

The striking differences in the patterns of expression between cyclin A1 and cyclin A2 in spermatogenic cells suggested that they would have distinct functions (Ravnik and Wolgemuth, 1999). Our targeted mutagenesis of the cyclin A1 gene revealed the clear requirement for this gene, specifically in meiosis in the male but not in the female germ line. The role of cyclin A2 is more difficult to assess because, as shown very elegantly by Murphy et al. (1997), loss of cyclin A2 results in early embryonic lethality. Embryos lacking cyclin A2 can develop from the two-cell to blastocyst stage but then die (Winston et al., 2000). Therefore, to study its function during spermatogenesis in the adult animal, a 
cell-specific targeted loss of function strategy will be needed. One such approach would involve the generation of transgenic mice expressing the Cre recombinase uniquely in spermatogonia and/or preleptotene spermatocytes, under the direction of a promoter specific to these cells. These animals would then be crossed to mice carrying the cyclin $\mathrm{A} 2$ gene into which loxP sites have been introduced (floxed cyclin A2). Excision of portions of the cyclin A2 gene between the loxP sites would result in a mutant cyclin A2 gene but only in spermatogonia or preleptotene spermatocytes. The critical missing link in this approach is the lack of well-characterized, spermatogonia-specific promoters.

\section{B. IS CYCLIN A2 ESSENTIAL FOR OOGENESIS?}

Ongoing studies in our lab and the work of others have shown that cyclin A2 is expressed in the mouse oocyte (Winston et al., 2000; C. Liao, S.E. Ravnik, Q. Zhang, S. Mulrad, and D.J. Wolgemuth, in preparation). As noted previously, the early embryonic lethality of the cyclin A2 null mutation (Murphy et al., 1997; Winston et al., 2000) obviates a direct test of its function in the germ line in either male or female. The Cre-lox strategies discussed earlier - using, in this case, oocyte-specific promoters to drive Cre recombinase - might be fruitful to address the role of cyclin A2 in oocyte mitosis and especially meiosis. Alternatively, the recent, very exciting application of double-stranded RNAs (RNAi) to interfere with gene expression in oocytes might permit a direct test of the cyclin A2 protein in the resumption of meiosis (Svoboda et al., 2000). In this approach, double-stranded RNAs for the c-mos proto-oncogene were shown to inhibit the translation of c-mos mRNA in a time- and concentration-dependent manner. Importantly, this inhibition resulted in parthenogenetic activation of the oocytes, similar to the phenotype observed in c-mos knockout mouse oocytes (Colledge et al., 1994; Hashimoto et al., 1994).

\section{ARE THE A-TYPE CYCLINS FUNCTIONALLY REDUNDANT?}

One hypothesis regarding the possible functional redundancy of the two mammalian A-type cyclin proteins is that they have essentially identical functions as part of cyclin $\mathrm{A} / \mathrm{Cdk}$ complexes and that differences in their function are reflected only by where they are expressed. A prediction of this hypothesis is that the two A-type cyclins would have identical biochemical properties as part of a Cdk1 or Cdk2 complex and be functionally interchangeable in vivo. An alternative hypothesis is that the two mammalian A-type cyclins have distinct functions as well as distinct regulation of expression. Yet a third possibility is that they may have both common and distinct functions, including unique functions of cyclin A1 in meiotic progression.

One approach for assessing these possibilities would ask whether functional differences between cyclin A1- and A2-containing kinase complexes can be 
detected with regard to their activity on specific substrates. One might also examine the interaction of cyclin A1 with proteins known to interact with cyclin A2 in vivo, including upstream inhibitors and activators and/or downstream targets. A systematic screen of known targets of cyclinA2/Cdk complexes for differences in the specificity of kinase activity of cyclin A1-containing versus cyclin A2-containing complexes might be quite informative. In such a scenario, particular attention would be paid to substrates whose expression and function, if known, might suggest a role during spermatogenesis.

A second strategy might utilize a molecular genetic approach to address the question of functional redundancy between the cyclin A1 and A2 proteins. As noted before, we have generated a targeted mutation in the cyclin A1 gene that resulted in an arrest in spermatogenesis specifically at the late pachytenediplotene stage of meiosis. The first meiotic division is prevented and the cells undergo a wave of apoptosis. One might now ask if cyclin A2 can rescue the meiotic arrest caused by the lack of cyclin A1 expression in the knockout mice. The use of either a transgenic approach using regulatory sequences that drive the expression of cyclin A1 to the appropriate cell types in vivo (K.M. Lele and D.J. Wolgemuth, unpublished observations) to drive coding sequences for cyclin A2 or alternatively, a knock-in approach (Hanks et al., 1995) could be envisioned. The technically more-complicated "knock-in" strategy would involve placing cyclin A2 coding sequences within the context of the cyclin A1 gene and then substituting into the genome by targeted mutagenesis.

\section{WHAT ARE THE SUBSTRATES FOR CYCLIN A1/CDK COMPLEXES IN GERM CELLS?}

As mentioned before, cyclin A2 functions during both G1-S and G2-M phases in cultured cells (Girard et al., 1991; Pagano et al., 1992; Pines and Hunter, 1994). The activity of the cyclin A2/Cdk2 kinase complex has been shown to be regulated by either inhibitory or activating proteins. Cyclin A2/Cdk2 shares some common properties with other G1/S or G2/M-phase cyclin/kinase complexes as well as possessing several features that are unique. Unlike cyclin B1, cyclin A2 can bind and activate both Cdk1 and Cdk2 proteins (Elledge et al., 1992; Kobayashi et al., 1992; Rosenblatt et al., 1992). While both cyclin A2/Cdk and cyclin $\mathrm{B} / \mathrm{Cdk} 1$ phosphorylate histone $\mathrm{H} 1$, only cyclin $\mathrm{A} 2 / \mathrm{Cdk}$ complexes were shown to associate with and phosphorylate the Rb-related protein p107 in vitro (Peeper et al., 1993). Differences in the consensus phosphorylation site for cyclin A2 versus cyclin E/Cdk2 complexes have been determined (Higashi et al., 1995). Cyclin A2/Cdk2 was more efficient than cyclin $\mathrm{E} / \mathrm{Cdk} 2$ in phosphorylating the transcription factor E2f-1 (Kitagawa et al., 1995). Cyclin A or E/Cdk2 and cyclin D/Cdk4 can all phosphorylate pRB; however, the phosphorylation sites are different (Kitagawa et al., 1996). Much less is known about cyclin A1, 
in particular as to how it may differ from cyclin A2 with respect to its interacting proteins and substrates. Our studies on the role of okadaic acid in overcoming the block in the first meiotic division in the absence of cyclin A1 led us to explore the role of the Cdc25 phosphatases as potential downstream targets of cyclin $\mathrm{A} 1 / \mathrm{Cdk}$. We previously had shown that $\mathrm{Cdc} 25 \mathrm{C}$ is expressed in late meiotic prophase cells in the mouse testis (Wu and Wolgemuth, 1995) and, recently, that Cdc25A is also expressed in spermatocytes at this stage (as is cyclin A1) (Liu et al., 2000). We have gone on to demonstrate that cyclin A1 can co-precipitate Cdc25A in co-transfection experiments and that testicular cyclin A1/Cdk2 kinase complexes can phosphorylate recombinant $\mathrm{Cdc} 25 \mathrm{~A}$ and $\mathrm{Cdc} 25 \mathrm{C}$ in vitro (Liu et al., 2000). Whether the Cdc25s are critical substrates in vivo in pachytene spermatocytes remains to be determined.

\section{E. WHAT ARE THE MECHANISMS BY WHICH THE LACK OF CYCLIN A1 TRIGGERS APOPTOSIS?}

A striking aspect of the loss of cyclin A1 function during male meiosis is the abrupt entry of the late-pachytene/diplotene spermatocytes into cell death or apoptosis, as assessed by TUNEL (terminal-deoxy UTP nick end labeling) assay (D. Liu et al., 1998). In fact, we have noted that apoptosis is characteristic of loss of function of several genes that have been shown by targeted mutagenesis to be required for the progression of meiotic prophase. Table I lists several of these genes and the stage at which the cells arrest. There are several pathways by which cells can undergo cell death, including pathways mediated by p53, members of the Bcl2/Bax family, Fas/Fas ligand, and the TNF-R superfamily (reviewed in Raff, 1998, and Strasser et al., 2000). A role for p53-mediated pathway in the apoptosis observed in Atm-deficient spermatocytes is suggested by the elevated levels of p53, Bax, and p21 observed in Atm-deficient testes and the decrease in apoptosis seen in mice lacking both Atm and 553 function (Barlow et al., 1997). There was not a complete loss of apoptosis, however, which raises the possibility of multiple pathways. The pathways of apoptosis for the other mutant strains listed in Table I remain to be elucidated.

\section{ACKNOWLEDGMENTS}

This work was supported in part by grants from the National Institutes of Health, HD34915 (DJW) and T32 DK07647 (KML). We thank former members of the Wolgemuth lab whose experimental and intellectual contributions helped to form the foundation on which our current and future studies on cell-cycle control are based. In particular, we wish to acknowledge Drs. Debbie Chapman, Kunsoo Rhee, Shuang Wu, Qi Zhang, Valerie Besset, Stuart Ravnik, Dong Liu, and Ching Liao. 


\section{REFERENCES}

Alberts B, Bray D, Lewis J, Raff M, Roberts K, Watson J 1983 Molecular Biology of the Cell. New York: Garland Publishing, Inc.

Arama E, Yanai A, Kilfin G, Bernstein A, Motro B 1998 Murine NIMA-related kinases are expressed in patterns suggesting distinct functions in gametogenesis and a role in the nervous system. Oncogene 16:1813-1823

Badie C, Itzhaki JE, Sullivan MJ, Carpenter AJ, Porter AC 2000 Repression of CDK1 and other genes with $\mathrm{CDE}$ and CHR promoter elements during DNA damage-induced $\mathrm{G}(2) / \mathrm{M}$ arrest in human cells. Mol Cell Biol 20:2358-2366

Barlow C, Liyanage M, Moens PB, Deng CX, Ried T, Wynshaw-Boris A 1997 Partial rescue of the prophase I defects of Atm-deficient mice by p53 and p21 null alleles. Nature Genet 17:462-466

Baudat F, Manova K, Yuen JP, Jasin M, Keeney S 2000 Chromosome synapsis defects and sexually dimorphic meiotic progression in mice lacking spo11. Mol Cell 6:989-998

Blanchard J 2000 Cyclin A2 transcriptional regulation: modulation of cell cycle control at the G1/S transition by peripheral cues. Biochem Pharmacol 60:1179-1184

Blasina A, Price BD, Turenne GA, McGowan CH 1999 Caffeine inhibits the checkpoint kinase ATM. Curr Biol 9:1135-1138

Brandeis M, Rosewell I, Carrington M, Crompton T, Jacobs MA, Kirk J, Gannon J, Hunt T 1998 Cyclin B2-null mice develop normally and are fertile whereas cyclin B1-null mice die in utero. Proc Natl Acad Sci USA 95:4344-4349

Bunz F, Dutriaux A, Lengauer C, Waldman T, Zhou S, Brown JP, Sedivy JM, Kinzler KW, Vogelstein B 1998 Requirement for p53 and p21 to sustain G2 arrest after DNA damage. Science 282:1497-1501

Chapman DL, Wolgemuth DJ 1992 Identification of a mouse B-type cyclin which exhibits developmentally regulated expression in the germ line. Mol Reprod Dev 33:259-269

Chapman DL, Wolgemuth DJ 1993 Isolation of the murine cyclin B2 cDNA and characterization of the lineage and temporal specificity of expression of the B1 and B2 cyclins during oogenesis, spermatogenesis and early embryogenesis. Development 118:229-240

Chapman DL, Wolgemuth DJ 1994 Expression of proliferating cell nuclear antigen in the mouse germ line and surrounding somatic cells suggests both proliferation-dependent and -independent modes of function. Intl J Dev Biol 38:491-497

Chesnel F, Eppig JJ 1995 Synthesis and accumulation of p34cdc2 and cyclin B in mouse oocytes during acquisition of competence to resume meiosis. Mol Reprod Dev 40:503-508

Clarke DJ, Gimenez-Abian JF 2000 Checkpoints controlling mitosis. Bioessays 22:351-363

Clarke PR, Leiss D, Pagano M, Karsenti E 1992 Cyclin A- and cyclin B-dependent protein kinases are regulated by different mechanisms in Xenopus egg extracts. EMBO J 11:1751-1761

Colledge WH, Carlton MB, Udy GB, Evans MJ 1994 Disruption of c-mos causes parthenogenetic development of unfertilized mouse eggs [see comments]. Nature 370:65-68

den Elzen N, Pines J 2001 Cyclin a is destroyed in prometaphase and can delay chromosome alignment and anaphase. J Cell Biol 153:121-136

Desdouets C, Matesic G, Molina CA, Foulkes NS, Sassone-Corsi P, Brechot C, Sobczak-Thepot J 1995 Cell cycle regulation of cyclin A gene expression by the cyclic AMP-responsive transcription factors CREB and CREM. Mol Cell Biol 15:3301-3309

Devault A, Fesquet D, Cavadore JC, Garrigues AM, Labbe JC, Lorca T, Picard A, Philippe M, Doree M 1992 Cyclin A potentiates maturation-promoting factor activation in the early Xenopus embryo via inhibition of the tyrosine kinase that phosphorylates cdc2. J Cell Biol 118:1109-1120 
Dix DJ, Allen JW, Collins BW, Poorman-Allen P, Mori C, Blizard DR, Brown PR, Goulding EH, Strong BD, Eddy EM 1997 HSP70-2 is required for desynapsis of synaptonemal complexes during meiotic prophase in juvenile and adult mouse spermatocytes. Development 124:4595-4603

Edelmann W, Cohen PE, Kane M, Lau K, Morrow B, Bennett S, Umar A, Kunkel T, Cattoretti G, Chaganti R, Pollard JW, Kolodner RD, Kucherlapati R 1996 Meiotic pachytene arrest in MLH1-deficient mice. Cell 85:1125-1134

Edelmann W, Yang K, Kuraguchi M, Heyer J, Lia M, Kneitz B, Fan K, Brown AM, Lipkin M, Kucherlapati R 1999 Tumorigenesis in Mlh1 and Mlh1/Apc1638N mutant mice. Cancer Res 59:1301-1307

Elledge SJ, Harper JW 1998 The role of protein stability in the cell cycle and cancer. Biochim Biophys Acta 1377:M61-M70

Elledge SJ, Richman R, Hall FL, Williams RT, Lodgson N, Harper JW 1992 CDK2 encodes a $33-\mathrm{kDa}$ cyclin A-associated protein kinase and is expressed before CDC2 in the cell cycle. Proc Natl Acad Sci USA 89:2907-2911

Fry AM, Nigg EA 1995 Cell cycle. The NIMA kinase joins forces with Cdc2. Curr Biol 5:1122-1125

Gallant P, Nigg EA 1992 Cyclin B2 undergoes cell cycle-dependent nuclear translocation and, when expressed as a non-destructible mutant, causes mitotic arrest in HeLa cells. J Cell Biol 117:213-224

Ge RS, Hardy MP 1997 Decreased cyclin A2 and increased cyclin G1 levels coincide with loss of proliferative capacity in rat Leydig cells during pubertal development. Endocrinology 138: 3719-3726

Girard F, Strausfeld U, Fernandez A, Lamb NJ 1991 Cyclin A is required for the onset of DNA replication in mammalian fibroblasts. Cell 67:1169-1179

Guadagno TM, Newport JW 1996 Cdk2 kinase is required for entry into mitosis as a positive regulator of Cdc2-cyclin B kinase activity. Cell 84:73-82

Hanks M, Wurst W, Anson-Cartwright L, Auerbach AB, Joyner AL 1995 Rescue of the En-1 mutant phenotype by replacement of En-1 with En-2 [see comments]. Science 269:679-682

Hashimoto N, Watanabe N, Furuta Y, Tamemoto H, Sagata N, Yokoyama M, Okazaki K, Nagayoshi M, Takeda N, Ikawa Y 1994 Parthenogenetic activation of oocytes in c-mosdeficient mice. Nature 370:68-71

Hershko A, Ciechanover A 1998 The ubiquitin system. Annu Rev Biochem 67:425-479

Higashi H, Suzuki-Takahashi I, Taya Y, Segawa K, Nishimura S, Kitagawa M 1995 Differences in substrate specificity between Cdk2-cyclin A and Cdk2-cyclin E in vitro. Biochem Biophys Res Commun 216:520-525

Hoffmann I, Clarke PR, Marcote MJ, Karsenti E, Draetta G 1993 Phosphorylation and activation of human cdc25-C by cdc2-cyclin B and its involvement in the self-amplification of MPF at mitosis. EMBO J 12:53-63

Howe JA, Howell M, Hunt T, Newport JW 1995 Identification of a developmental timer regulating the stability of embryonic cyclin A and a new somatic A-type cyclin at gastrulation. Genes Dev 9:1164-1176

Kakizuka A, Sebastian B, Borgmeyer U, Hermans-Borgmeyer I, Bolado J, Hunter T, Hoekstra MF, Evans RM 1992 A mouse cdc25 homolog is differentially and developmentally expressed. Genes Dev 6:578-590

Kandli M, Feige E, Chen A, Kilfin G, Motro B 2000 Isolation and characterization of two evolutionarily conserved murine kinases (Nek6 and nek7) related to the fungal mitotic regulator, NIMA. Genomics 68:187-196

Kitagawa M, Higashi H, Suzuki-Takahashi I, Segawa K, Hanks SK, Taya Y, Nishimura S, Okuyama A 1995 Phosphorylation of E2F-1 by cyclin A-cdk2. Oncogene 10:229-236 
Kitagawa M, Higashi H, Jung HK, Suzuki-Takahashi I, Ikeda M, Tamai K, Kato J, Segawa K, Yoshida E, Nishimura S, Taya Y 1996 The consensus motif for phosphorylation by cyclin D1-Cdk4 is different from that for phosphorylation by cyclin A/E-Cdk2. EMBO J 15:70607069

Kneitz B, Cohen PE, Avdievich E, Zhu L, Kane MF, Hou H Jr, Kolodner RD, Kucherlapati R, Pollard JW, Edelmann W 2000 MutS homolog 4 localization to meiotic chromosomes is required for chromosome pairing during meiosis in male and female mice. Genes Dev 14:1085-1097

Kobayashi H, Stewart E, Poon R, Adamczewski JP, Gannon J, Hunt T 1992 Identification of the domains in cyclin A required for binding to, and activation of, p34cdc2 and $\mathrm{p} 32 \mathrm{cdk} 2$ protein kinase subunits. Mol Biol Cell 3:1279-1294

Kramer A, Carstens CP, Wasserman WW, Fahl WE 1997 CBP/cycA, a CCAAT-binding protein necessary for adhesion-dependent cyclin A transcription, consists of NF-Y and a novel $\mathrm{Mr}$ 115,000 subunit. Cancer Res 57:5117-5121

Lange-zu Dohna C, Brandeis M, Berr F, Mossner J, Engeland K 2000 A CDE/CHR tandem element regulates cell cycle-dependent repression of cyclin B2 transcription. FEBS Lett 484:77-81

Le Cam L, Polanowska J, Fabbrizio E, Olivier M, Philips A, Ng Eaton E, Classon M, Geng Y, Sardet C 1999 Timing of cyclin E gene expression depends on the regulated association of a bipartite repressor element with a novel E2F complex. EMBO J 18:1878-1890

Letwin K, Mizzen L, Motro B, Ben-David Y, Bernstein A, Pawson T 1992 A mammalian dual specificity protein kinase, Nek1, is related to the NIMA cell cycle regulator and highly expressed in meiotic germ cells. EMBO J 11:3521-3531

Li J, Meyer AN, Donoghue DJ 1995 Requirement for phosphorylation of cyclin B1 for Xenopus oocyte maturation. Mol Biol Cell 6:1111-1124

Liu D, Matzuk MM, Sung WK, Guo Q, Wang P, Wolgemuth DJ 1998 Cyclin A1 is required for meiosis in the male mouse. Nature Genet 20:377-380

Liu D, Liao C, Wolgemuth DJ 2000 A role for cyclin A1 in the activation of MPF and G2-M transition during meiosis of male germ cells in mice. Dev Biol 224:388-400

Liu N, Lucibello FC, Korner K, Wolfraim LA, Zwicker J, Muller R 1997 CDF-1, a novel E2F-unrelated factor, interacts with cell cycle-regulated repressor elements in multiple promoters. Nucleic Acids Res 25:4915-4920

Liu N, Lucibello FC, Engeland K, Muller R 1998 A new model of cell cycle-regulated transcription: repression of the cyclin A promoter by CDF-1 and anti-repression by E2F. Oncogene 16:2957-2963

Lopez-Girona A, Kanoh J, Russell P 2001 Nuclear exclusion of Cdc25 is not required for the DNA damage checkpoint in fission yeast. Curr Biol 11:50-54

Mailand N, Falck J, Lukas C, Syljuasen RG, Welcker M, Bartek J, Lukas J 2000 Rapid destruction of human Cdc25A in response to DNA damage. Science 288:1425-1429

Manni I, Mazzaro G, Gurtner A, Mantovani R, Haugwitz U, Krause K, Engeland K, Sacchi A, Soddu S, Piaggio G 2001 NF-Y mediates the transcriptional inhibition of the cyclin B1, cyclin B2, and cdc25C promoters upon induced G2 arrest. J Biol Chem 276:5570-5576

Masui Y, Markert CL 1971 Cytoplasmic control of nuclear behavior during meiotic maturation of frog oocytes. J Exp Zool 177:129-145

Mendez R, Richter JD 2001 Translational control by CPEB: a means to the end. Nature Rev Mol Cell Biol 2:521-529

Minshull J, Golsteyn R, Hill CS, Hunt T 1990 The A- and B-type cyclin associated cdc2 kinases in Xenopus turn on and off at different times in the cell cycle. EMBO J 9:2865-2875

Muller C, Yang R, Beck-von-Peccoz L, Idos G, Verbeek W, Koeffler HP 1999 Cloning of the cyclin A1 genomic structure and characterization of the promoter region. GC boxes are 
essential for cell cycle-regulated transcription of the cyclin A1 gene. J Biol Chem 274:1122011228

Muller C, Readhead C, Diederichs S, Idos G, Yang R, Tidow N, Serve H, Berdel WE, Koeffler HP 2000a Methylation of the cyclin A1 promoter correlates with gene silencing in somatic cell lines, while tissue-specific expression of cyclin A1 is methylation independent. Mol Cell Biol 20:3316-3329

Muller C, Yang R, Park DJ, Serve H, Berdel WE, Koeffler HP 2000b The aberrant fusion proteins PML-RAR alpha and PLZF-RAR alpha contribute to the overexpression of cyclin A1 in acute promyelocytic leukemia. Blood 96:3894-3899

Muller-Tidow C, Bornemann C, Diederichs S, Westermann A, Klumpen S, Zuo P, Wang W, Berdel WE, Serve H 2001 Analyses of the genomic methylation status of the human cyclin A1 promoter by a novel real-time PCR-based methodology. FEBS Lett 490:75-78

Murphy M, Stinnakre MG, Senamaud-Beaufort C, Winston NJ, Sweeney C, Kubelka M, Carrington M, Brechot C, Sobczak-Thepot J 1997 Delayed early embryonic lethality following disruption of the murine cyclin A2 gene [published erratum appears in Nature Genet 1999 23(4):481]. Nature Genet 15:83-86

Nargi JL, Woodford-Thomas TA 1994 Cloning and characterization of a cdc25 phosphatase from mouse lymphocytes. Immunogenetics 39:99-108

Odorisio T, Rodriguez TA, Evans EP, Clarke AR, Burgoyne PS 1998 The meiotic checkpoint monitoring synapsis eliminates spermatocytes via p53-independent apoptosis. Nature Genet 18:257-261

Osmani AH, McGuire SL, Osmani SA 1991 Parallel activation of the NIMA and p34cdc2 cell cycle-regulated protein kinases is required to initiate mitosis in A. nidulans. Cell 67:283-291

Pagano M, Pepperkok R, Verde F, Ansorge W, Draetta G 1992 Cyclin A is required at two points in the human cell cycle. EMBO J 11:961-971

Peeper DS, Parker LL, Ewen ME, Toebes M, Hall FL, Xu M, Zantema A, van der Eb AJ, Piwnica-Worms H 1993 A- and B-type cyclins differentially modulate substrate specificity of cyclin-cdk complexes. EMBO J 12:1947-1954

Philips A, Chambeyron S, Lamb N, Vie A, Blanchard JM 1999 CHF: a novel factor binding to cyclin A CHR corepressor element. Oncogene 18:6222-6232

Pines J 1995a Cyclins and cyclin-dependent kinases: a biochemical view. Biochem J 308:697-711

Pines J 1995b Cyclins, CDKs and cancer. Semin Cancer Biol 6:63-72

Pines J, Hunter T 1991 Human cyclins A and B1 are differentially located in the cell and undergo cell cycle-dependent nuclear transport. J Cell Biol 115:11-17

Pines J, Hunter T 1994 The differential localization of human cyclins A and B is due to a cytoplasmic retention signal in cyclin B. EMBO J 13:3772-3781

Pyronnet S, Dostie J, Sonenberg N 2001 Suppression of cap-dependent translation in mitosis. Genes Dev 15:2083-2093

Raff M 1998 Cell suicide for beginners. Nature 396:119-122

Ravnik SE, Wolgemuth DJ 1996 The developmentally restricted pattern of expression in the male germ line of a murine cyclin A, cyclin A2, suggests roles in both mitotic and meiotic cell cycles. Dev Biol 173:69-78

Ravnik SE, Wolgemuth DJ 1999 Regulation of meiosis during mammalian spermatogenesis: the A-type cyclins and their associated cyclin-dependent kinases are differentially expressed in the germ-cell lineage. Dev Biol 207:408-418

Ravnik SE, Rhee K, Wolgemuth DJ 1995 Distinct patterns of expression of the D-type cyclins during testicular development in the mouse. Dev Genet 16:171-178

Rebollo E, Gonzalez C 2000 Visualizing the spindle checkpoint in Drosophila spermatocytes. EMBO Rep 1:65-70 
Rhee K, Wolgemuth DJ 1997 The NIMA-related kinase 2, Nek2, is expressed in specific stages of the meiotic cell cycle and associates with meiotic chromosomes. Development 124:21672177

Rieder CL, Schultz A, Cole R, Sluder G 1994 Anaphase onset in vertebrate somatic cells is controlled by a checkpoint that monitors sister kinetochore attachment to the spindle. J Cell Biol 127:1301-1310

Roberts JM 1999 Evolving ideas about cyclins. Cell 98:129-132

Roeder GS, Bailis JM 2000 The pachytene checkpoint. Trends Genet 16:395-403

Romanienko PJ, Camerini-Otero RD 2000 The mouse spo11 gene is required for meiotic chromosome synapsis. Mol Cell 6:975-987

Rosenblatt J, Gu Y, Morgan DO 1992 Human cyclin-dependent kinase 2 is activated during the S and G2 phases of the cell cycle and associates with cyclin A. Proc Natl Acad Sci USA 89:2824-2828

Schultz SJ, Nigg EA 1993 Identification of 21 novel human protein kinases, including 3 members of a family related to the cell cycle regulator nimA of Aspergillus nidulans. Cell Growth Differ 4:821-830

Schulze A, Zerfass K, Spitkovsky D, Middendorp S, Berges J, Helin K, Jansen-Durr P, Henglein B 1995 Cell cycle regulation of the cyclin A gene promoter is mediated by a variant E2F site. Proc Natl Acad Sci USA 92:11264-11268

Schwartz D, Goldfinger N, Kam Z, Rotter V 1999 p53 controls low DNA damage-dependent premeiotic checkpoint and facilitates DNA repair during spermatogenesis. Cell Growth Differ 10:665-675

Session DR, Fautsch MP, Avula R, Jones WR, Nehra A, Wieben ED 2001 Cyclin-dependent kinase 5 is expressed in both Sertoli cells and metaphase spermatocytes. Fertil Steril 75:669-673

Sherr CJ 1993 Mammalian G1 cyclins. Cell 73:1059-1065

Sherr CJ, Roberts JM 1995 Inhibitors of mammalian G1 cyclin-dependent kinases. Genes Dev 9:1149-1163

Sherr CJ, Roberts JM 1999 CDK inhibitors: positive and negative regulators of G1-phase progression. Genes Dev 13:1501-1512

Stewart ZA, Pietenpol JA 2001 p53 Signaling and cell cycle checkpoints. Chem Res Toxicol $14: 243-263$

Strasser A, O'Connor L, Dixit VM 2000 Apoptosis signaling. Annu Rev Biochem 69:217-245

Svoboda P, Stein P, Hayashi H, Schultz RM 2000 Selective reduction of dormant maternal mRNAs in mouse oocytes by RNA interference. Development 127:4147-4156

Sweeney C, Murphy M, Kubelka M, Ravnik SE, Hawkins CF, Wolgemuth DJ, Carrington M 1996 A distinct cyclin A is expressed in germ cells in the mouse. Development 122:53-64

Taieb R, Thibier C, Jessus C 1997 On cyclins, oocytes, and eggs. Mol Reprod Dev 48:397-411

Tanaka K, Parvinen M, Nigg EA 1997 The in vivo expression pattern of mouse Nek2, a NIMA-related kinase, indicates a role in both mitosis and meiosis. Exp Cell Res 237:264-274

Toscani A, Mettus RV, Coupland R, Simpkins H, Litvin J, Orth J, Hatton KS, Reddy EP 1997 Arrest of spermatogenesis and defective breast development in mice lacking A-myb. Nature 386:713-717

Toyoshima F, Moriguchi T, Wada A, Fukuda M, Nishida E 1998 Nuclear export of cyclin B1 and its possible role in the DNA damage-induced G2 checkpoint. EMBO J 17:2728-2735

Wiltshire T, Park C, Caldwell KA, Handel MA 1995 Induced premature G2/M-phase transition in pachytene spermatocytes includes events unique to meiosis. Dev Biol 169:557-567

Winston N, Bourgain-Guglielmetti F, Ciemerych MA, Kubiak JZ, Senamaud-Beaufort C, Carrington M, Brechot C, Sobczak-Thepot J 2000 Early development of mouse embryos 
null mutant for the cyclin A2 gene occurs in the absence of maternally derived cyclin A2 gene products. Dev Biol 223:139-153

Wolgemuth DJ, Rhee K, Wu S, Ravnik SE 1995 Genetic control of mitosis, meiosis and cellular differentiation during mammalian spermatogenesis. Reprod Fertil Dev 7:669-683

Wu S, Wolgemuth DJ 1995 The distinct and developmentally regulated patterns of expression of members of the mouse Cdc25 gene family suggest differential functions during gametogenesis. Dev Biol 170:195-206

Xu Y, Ashley T, Brainerd EE, Bronson RT, Meyn MS, Baltimore D 1996 Targeted disruption of ATM leads to growth retardation, chromosomal fragmentation during meiosis, immune defects, and thymic lymphoma. Genes Dev 10:2411-2422

Yang J, Kornbluth S 1999 All aboard the cyclin train: subcellular trafficking of cyclins and their CDK partners. Trends Cell Biol 9:207-210

Yang R, Morosetti R, Koeffler HP 1997 Characterization of a second human cyclin A that is highly expressed in testis and in several leukemic cell lines. Cancer Res 57:913-920

Yang R, Nakamaki T, Lubbert M, Said J, Sakashita A, Freyaldenhoven BS, Spira S, Huynh V, Muller C, Koeffler HP 1999 Cyclin A1 expression in leukemia and normal hematopoietic cells. Blood 93:2067-2074

Yoshida K, Kondoh G, Matsuda Y, Habu T, Nishimune Y, Morita T 1998 The mouse RecA-like gene Dmc1 is required for homologous chromosome synapsis during meiosis. Mol Cell 1:707-718

Yuan L, Liu JG, Zhao J, Brundell E, Daneholt B, Hoog C 2000 The murine SCP3 gene is required for synaptonemal complex assembly, chromosome synapsis, and male fertility. Mol Cell 5:73-83

Zerfass-Thome K, Schulze A, Zwerschke W, Vogt B, Helin K, Bartek J, Henglein B, JansenDurr P 1997 p27KIP1 blocks cyclin E-dependent transactivation of cyclin A gene expression. Mol Cell Biol 17:407-415

Zwicker J, Gross C, Lucibello FC, Truss M, Ehlert F, Engeland K, Muller R 1995a Cell cycle regulation of cdc $25 \mathrm{C}$ transcription is mediated by the periodic repression of the glutaminerich activators NF-Y and Sp1. Nucleic Acids Res 23:3822-3830

Zwicker J, Lucibello FC, Wolfraim LA, Gross C, Truss M, Engeland K, Muller R 1995b Cell cycle regulation of the cyclin $\mathrm{A}, \mathrm{cdc} 25 \mathrm{C}$ and cdc2 genes is based on a common mechanism of transcriptional repression. EMBO J 14:4514-4522 



\title{
Male Germ Cell Gene Expression
}

\author{
EDWARD M. EDDY \\ Laboratory of Reproductive and Developmental Toxicology, National Institute of Environmental \\ Health Sciences, National Institutes of Health, Research Triangle Park, North Carolina 27709
}

\begin{abstract}
Formation of the male gamete occurs in sequential mitotic, meiotic, and postmeiotic phases. Many germ cell-specific transcripts are produced during this process. Their expression is developmentally regulated and stage specific. Some of these transcripts are product of genes that are male germ cell-specific homologs of genes expressed in somatic cells, while some are expressed from unique genes unlike any others in the genome. Others are alternate transcripts derived from the same gene as transcripts in somatic cells but differing from them in size and/or overall sequence. They are generated during gene expression by using promoters and transcription factors that activate transcription at different start sites upstream or downstream of the usual site, by incorporation of alternate exons, by germ cell-specific splicing events, and by using alternate initiation sites for polyadenylation. Male germ cell development consists of an assortment of unique processes, including meiosis, genetic recombination, haploid gene expression, formation of the acrosome and flagellum, and remodeling and condensation of the chromatin. These processes are intricate, highly ordered, and require novel gene products and a precise and well-coordinated program of gene expression to occur.

The regulation of gene expression in male germ cells occurs at three levels: intrinsic, interactive, and extrinsic. A highly conserved genetic program "intrinsic" to germ cells determines the sequence of events that underlies germ cell development. This has been underscored by recent studies showing that meiosis involves many genes that have been conserved during evolution from yeast to man. During meiosis and other processes unique to germ cells, the intrinsic program determines which genes are utilized and when they are expressed. In the postmeiotic phase, it coordinates the expression of genes whose products are responsible for constructing the sperm. The process of spermatogenesis occurs in overlapping waves, with cohorts of germ cells developing in synchrony. The intrinsic program operating within a particular germ cell requires information from and provides information to neighboring cells to achieve this coordination. Sertoli cells are crucial for this "interactive" process as well as for providing essential support for germ cell proliferation and progression through the phases of development. The interactive level of regulation is dependent on "extrinsic" influences, primarily testosterone and follicle-stimulating hormone (FSH). Studies during the last 4 years have established that FSH is not essential for germ cell development but instead serves an important supportive role for this process. While testosterone is essential for maintenance of spermatogenesis, it acts on Sertoli cells and peritubular cells and has indirect effects on germ cells. The extrinsic and interactive processes are extremely important for establishing and maintaining an optimum environment within which gametogenesis occurs. Nevertheless, an intrinsic evolutionarily conserved genetic program regulates male germ cell gene expression and development.
\end{abstract}




\section{Introduction}

Spermatogenesis is a paradigm of development that continues throughout adult life in most mammals. This process occurs in seminiferous tubules containing an epithelium populated by a mixture of germ cells and Sertoli cells, surrounded by a thin wall of peritubular cells (Figure 1). Sertoli cells serve a crucial nurturing role for germ cells and are believed to help coordinate important events of spermatogenesis (Griswold, 1998). Sertoli cells also divide the seminiferous epithelium into two compartments: a basal compartment where cells are exposed to the surrounding milieu and a luminal compartment where cells are sequestered behind a "blood-testis" barrier formed by junctional complexes between Sertoli cells. Although the focus here is on the mouse, most of what is discussed applies to mammals in general.

Germ cell development occurs in successive mitotic, meiotic, and postmeiotic phases (Figure 2), with the germ cells moving from the periphery to the lumen of the seminiferous tubule during this process. The mitotic phase occurs in the basal compartment, while the meiotic and postmeiotic phases occur in the luminal compartment. This takes about 35 days in the mouse, with the mitotic phase lasting approximately 11 days, the meiotic phase lasting approximately 10

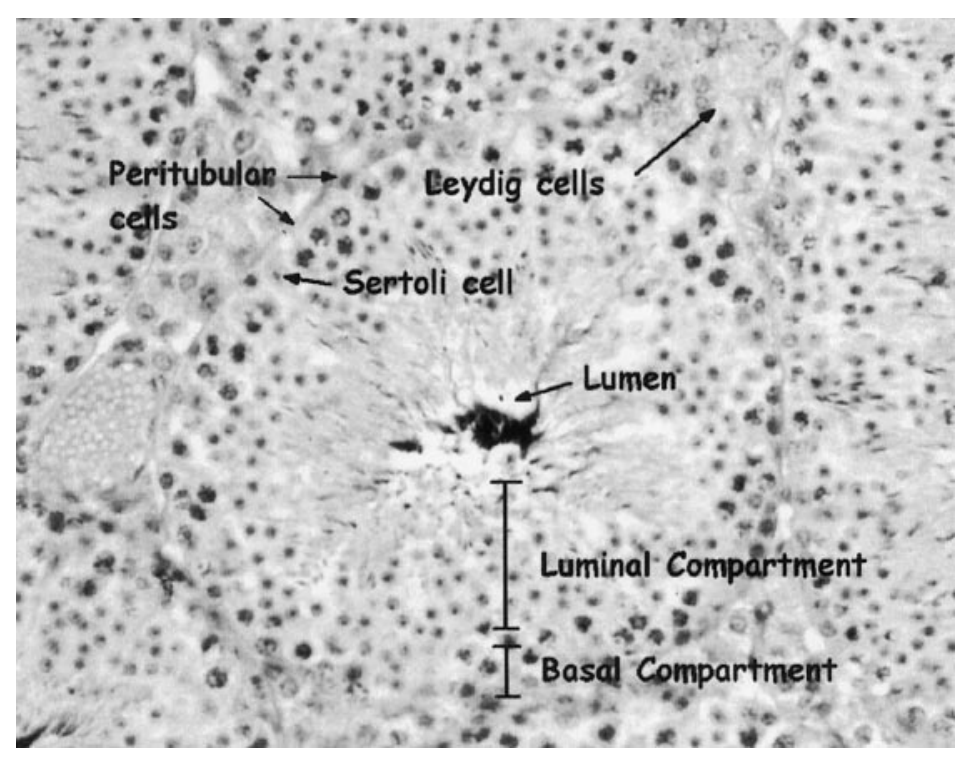

FIG. 1. A cross-section of a seminiferous tubule in the mouse testis. A thin layer of peritubular cells surrounds the tubule and Leydig cells are located in the areas between tubules. Sertoli cells divide the seminiferous tubule into the basal compartment containing germ cells in the mitotic phase and a luminal compartment containing germ cells in meiotic and postmeiotic phases. 


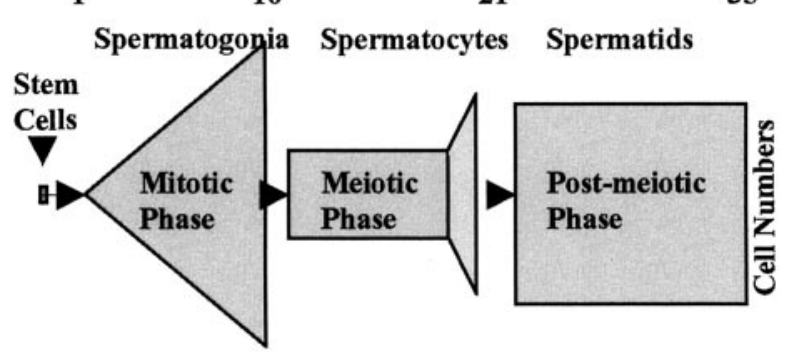

\section{Apoptosis Meiotic Divisions}

FIG. 2. The three phases of germ cell development. Stem cells give rise to spermatogonia that undergo several mitotic divisions over the next 10 days. More than half of the spermatogonia undergoes apoptosis during the latter part of the mitotic phase. During much of the meiotic phase, the spermatocytes are in the $\mathrm{G} 2$ phase of the cell cycle. After the two meiotic divisions at the end of this phase, germ cells enter the postmeiotic phase. Transcription occurs throughout germ cell development until the midpoint of the postmeiotic phase. During the latter half of this phase, proteins are synthesized from transcripts that have been stored since the early part of the postmeiotic phase.

days, and the postmeiotic phase lasting approximately 14 days (Clermont and Trott, 1969). The process begins every 8.7-8.9 days. The duration of these phases varies slightly between mouse strains. It includes processes unique to germ cells, including meiosis, genetic recombination, haploid gene expression, formation of the acrosome and flagellum, and remodeling and condensation of chromatin, all of which must occur at the correct time to produce the male gamete.

The highly ordered process of spermatogenesis, in turn, requires a precise and well-coordinated program that regulates the constantly changing patterns of gene expression. Although many questions remain to be answered, the regulation of this process can be thought of as occurring in three concentric levels: intrinsic, interactive, and extrinsic (Figure 3). Intrinsic regulation is managed by an evolutionarily conserved genetic program that underlies the development of germ cells of diverse species, ranging from nematodes to flies to mammals. This program is responsible for the sequence of events of differentiation and morphogenesis that occur throughout the progression of spermatogenesis. For meiosis and other processes specific to germ cells in particular, the intrinsic program determines which genes are utilized and when they are expressed.

The development of male germ cells occurs in overlapping waves in mammals and other vertebrates, with cohorts of mitotic, meiotic, and postmeiotic germ cells developing in synchrony. The intrinsic program operating within a given cell requires information from, and provides information to, neighboring cells to achieve this coordination. This interactive regulation of germ cell gene 


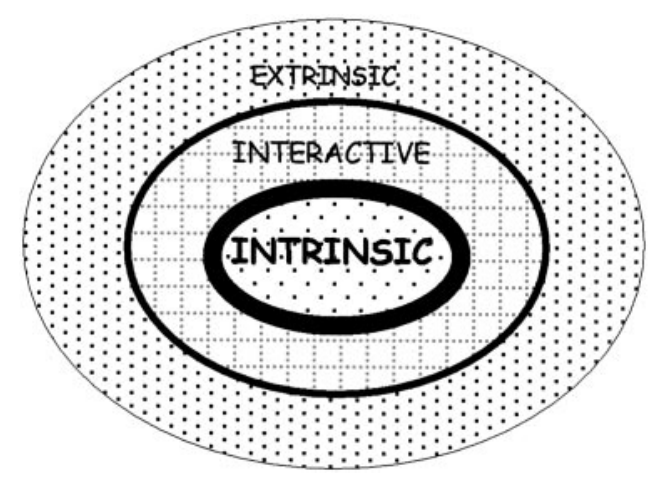

FIG. 3. The three levels of regulation of gene expression in germ cells. An intrinsic genetic program is responsible for regulating gene expression in male germ cells. Germ cells develop in overlapping waves, with cohorts of germ cells developing in synchrony. This requires coordination of the intrinsic programs for the cohorts of germ cells. The Sertoli cells serve a crucial role in this process. The interactive level of influence over germ cell development, in turn, is responsive to extrinsic cues that influence gene expression in Sertoli and Leydig cells, thereby regulating the milieu within which germ cells develop and indirectly influencing gene expression in germ cells. The extrinsic and interactive programs are essential for supporting germ cell development but gene expression is regulated by the germ cells themselves.

expression is far from being well understood but in vitro and in vivo studies indicate that Sertoli cells are key players in the information-exchange process. Each Sertoli cell is in contact simultaneously with three or four layers of germ cells that are in all three phases of spermatogenesis and is well situated to integrate and modulate the various signals to, from, and between germ cells.

Interactive regulation is, in turn, subject to extrinsic regulation by the endocrine system, primarily through testosterone and FSH. These hormones directly influence gene expression in somatic cells of the testis, thereby regulating the milieu within which interactive regulation occurs and indirectly influencing gene expression in germ cells.

\section{Intrinsic Regulation of Germ Cell Gene Expression}

The ultimate regulation of gene expression in male germ cells, as in all other cells, occurs first at the level of transcription, next at the level of translation, and thereafter at the post-translational level. The fundamental apparatus for these processes and the mechanisms that control them vary little between cell types but the specific features of the individual proteins involved and how their actions are modulated greatly influence the progression and outcome of the cell's development. There are three important features of the intrinsic genetic program underlying the process of male germ cell development. First, the processes of 
meiosis and spermatogenesis have been conserved throughout evolution. Second, many unique genes and variant transcripts are expressed during male germ cell development. Third, the expression of such genes is developmentally regulated and stage specific.

\section{A. GENETIC CONSERVATION}

Many of the general features of germ cell development are well conserved between phyla (Roosen-Runge, 1977). It is becoming increasingly clear that the same is true for the genes involved. The genome of the budding yeast, Saccharomyces cerevisiae, has been sequenced and the temporal profile of gene expression during sporulation, when meiosis occurs, has been determined (Chu et al., 1998). A subset of 164 genes induced in the early, middle, and later periods of meiosis was identified and a computer-based search was used to identify homologs, genes that have evolved from a common ancestor, in C. elegans, Drosophila, and mammals (Hwang et al., 2001). Over $70 \%$ of the yeast genes were found to have homologs in C. elegans, Drosophila, mice and/or humans, providing strong evidence that a substantial amount of the genetic program of meiosis is well conserved between phyla. More than half of these genes are represented by expressed sequence tags (ESTs) from mouse cDNA libraries that span the period from before until after meiosis in the female germ cell line (Hwang et al., 2001).

Expression of many of the same genes presumably occurs during meiosis in the male. However, systematic and comprehensive sequencing of cDNA libraries covering all phases of spermatogenesis needs to be done before this can be verified. There are many differences between oogenesis and spermatogenesis, with different genes being expressed during these processes. This was demonstrated in recent studies that evaluated the expression of testis-specific genes for C. elegans and Drosophila (Andrews et al., 2000; Reinke et al., 2000). On microarrays that contained nearly two thirds of the predicted genes for $C$. elegans, $11.8 \%$ were germline-enriched genes, of which $46 \%$ were expressed specifically in the male gonad, $18 \%$ in the female gonad, and 36\% in both (Reinke et al., 2000). Comparisons of ESTs generated by sequencing cDNA libraries of Drosophila found that nearly half of testis ESTs did not match with any nongonadal Drosophila EST sequences and about two thirds of those differed from ovary ESTs (Andrews et al., 2000). These studies indicate that several hundred male gonad-specific genes are expressed in C. elegans and Drosophila. It remains to be determined how many of these genes have homologs in mouse and/or human but the studies by Hwang et al. (2001) suggest that a majority of the genes are conserved.

The principal role of the intrinsic program in germ cell development was shown quite dramatically when rat spermatogonial stem cells were transplanted 
into the seminiferous tubules of the mouse testis. The rat stem cell gave rise to normal-appearing rat sperm (Clouthier et al., 1996) that developed at the same rate as in the rat testis, which is slower than in the mouse (Franca et al., 1998). These studies show that germ cells carry all of the information and instructions needed for their own development.

\section{B. GERM CELL-SPECIFIC TRANSCRIPTS}

It was estimated that up to 20,000 different transcripts are present in a given cell population (Zhang et al., 1997). Most of these are products of ubiquitously expressed "housekeeping genes" that are responsible for the basic structures and functions common to all cells, while fewer are responsible for the specific features and activities of the particular cell type. As of this writing, the NCBI Mouse UniGene database (http://www.ncbi.nlm.nih.gov/UniGene/Mm.Home. $\mathrm{html}$ ) contains 18,673 sequence clusters that include cDNAs from testis libraries, with 2701 sequence clusters identified as containing cDNAs specifically from spermatogonia, spermatocyte, or spermatid libraries. However, these numbers are probably inflated. Most of the ESTs are only 300 to 600 bp in length, very few have been confirmed by other methods to be expressed in testis or male germ cells, and it is likely that many are ubiquitously expressed genes. In addition, an unknown fraction consists of nonoverlapping ESTs from different regions of full-length cDNA sequences. On the other hand, there are undoubtedly many cDNAs in germ cell libraries that have not yet been sequenced and the short cDNA sequences that are determined probably do not identify variant transcripts.

There are three principal ways that male germ cell-specific transcripts and proteins are produced. The first is by expression of genes only in spermatogenic cells that are homologs of genes expressed in somatic cells, or in somatic cells and germ cells. The second is by expression of unique genes, those without significant similarity to any other gene in the genome. The third is by expression of variant transcripts. These are transcribed from genes also expressed in somatic cells but are often smaller or larger than their somatic cell counterparts and are the result of using one or more alternate transcript start sites, transcript splice sites, exons, or polyadenylation signals.

\section{Male Germ Cell-specific Gene Homologs}

Genes expressed only in male germ cells frequently are homologs of genes expressed in somatic cells (reviewed in Eddy and O'Brien, 1998). In some cases, a gene that is expressed in somatic cells is inactivated and a germ cell homolog is activated. An example of this is glyceraldehyde 3-phosphate dehydrogenase $(G a p d)$, a highly conserved gene for an essential enzyme in the glycolytic pathway. Gapd usually is considered to be a housekeeping gene and its mRNA is commonly used as a loading control on northern blots. However, this gene is 
inactivated during the meiotic phase and the spermatogenic cell-specific Gapds gene is activated early in the postmeiotic phase of male germ cell development (Mori et al., 1992; Welch et al., 1992). The amino acid sequence of GAPDS is over $70 \%$ identical to that of GAPD and contains the appropriate amino acids in the locations necessary to form the binding pocket for the $\mathrm{NAD}^{+}$cofactor and the thioester substrate-binding site. Although the exon-intron structure of mouse Gapds is similar to that of Gapd (Welch et al., 1995), GAPDS has a large proline-rich domain at the N-terminus that is not present in GAPD.

It seems inefficient for male germ cells to use a new gene instead of an existing one that encodes a nearly identical protein. However, there are often significant advantages for male germ cells to have their "own" genes. One is that a germ cell homolog may compensate for a gene that is inactivated in male germ cells. An example of this is the phosphoglycerate kinase-1 ( $P g k l)$ gene that encodes another essential enzyme in the glycolytic pathway. Pgkl is located on the $\mathrm{X}$ chromosome, which is inactivated during the meiotic phase of male germ cell development. However, the germ cell-specific $P g k 2$ gene is located on an autosome and is activated soon thereafter. The $P g k 2$ gene lacks introns and was hypothesized to have evolved as a functional retroposon of the X-linked $P g k 1$ gene (Boer et al., 1987; McCarrey and Thomas, 1987). Expression of the Pgk2 gene is restricted to germ cells in most eutherian mammals but is expressed ubiquitously or in a subset of somatic tissues and in germ cells of different marsupials. It was hypothesized that tissue-specific enhancer activity and tissuespecific demethylation led to the restriction of $P g k 2$ expression to germ cells, thereby maintaining glycolysis when $P g k l$ expression ceases due to $\mathrm{X}$ chromosome inactivation (McCarrey, 1994).

Another advantage of germ cell-specific genes is that the encoded protein may fulfill the same role as that of the somatic cell's product but also have other structural or functional properties that serve unique roles in male germ cells. For example, GAPDS is anchored to the fibrous sheath by the proline-rich $\mathrm{N}$-terminal domain that is absent in GAPD (Bunch et al., 1998). Furthermore, most of the other enzymes in the glycolytic pathway in postmeiotic germ cells and sperm also have unique structural or functional properties (reviewed in Eddy et al., 1994). Several are known to be concentrated in the principal piece (Bunch et al., 1998). Glycolytic enzymes in sperm cofractionate with sperm tail components (Mohri et al., 1965) and are associated as a complex (Storey and Kayne, 1978). The unique structural and functional features of the other glycolytic enzymes may serve a role similar to the proline-rich domain of GAPDS for assembling this complex. Molecular modeling studies indicate that important differences occur in specific amino acids surrounding the $\mathrm{NAD}^{+}$cofactor-binding pocket and glyceraldehyde 3-phosphate (G3P) substrate-binding pocket of GAPD and GAPDS (unpublished observations). Earlier investigators were unaware that sperm contain GAPDS rather than GAPD but observed that sperm were signif- 
icantly more vulnerable than other cells to damage by environmental chemicals structurally similar to G3P (Jones, 1978; Stevenson and Jones, 1985). However, glycolysis is ineffective in spermatids. Addition of glucose to spermatids in vitro leads to an increase in the concentration of intermediates upstream of GAPD in the glycolytic pathway, while those downstream of GAPD decrease, leading to a substantial drop in adenosine triphosphate (ATP) levels (Nakamura et al., 1982; Jutte et al., 1983). This is strikingly different from the situation in sperm, where glucose is required for fertilization to succeed (Hoppe, 1976; Fraser and Quinn, 1981; Cooper, 1984). Differences in molecular structure between GAPDS and GAPD may provide the ability for GAPDS to serve as the switch from inactive glycolysis in postmeiotic germ cells to active glycolysis in sperm (Eddy et al., 1994).

Activation of germ cell-specific gene expression may also occur without the associated inactivation of a homolog. An example of this occurs for two members of the 70-kDa heat-shock protein (HSP70) family. The HSP70 proteins are chaperones that assist other proteins to fold as they emerge from the ribosome, escort them through the cytoplasm, help them assemble into complexes, and facilitate the refolding of proteins partially denatured by heat or other stresses. The genes for most HSP70 proteins are expressed in all tissues, either constitutively or following induction by heat shock and other stresses. However, a major $70-\mathrm{kDa}$ protein is present in germ cells that migrates to the same position on two-dimensional (2-D) gels as the HSP70 protein induced by heat shock in 3T3 cells (Allen et al., 1988a,b). The protein is synthesized in spermatocytes (O'Brien, 1987) and was recognized on immunoblots with a pan-HSP70 antibody. The cDNA was cloned and the sequence was found to correspond to a region in a genomic clone (Hsp70-2) isolated previously (Zakeri et al., 1988). Northern and in situ hybridization analysis found that transcription began in the early part of the meiotic phase and immunoblot and immunohistochemical analysis determined that the protein was expressed shortly thereafter (Rosario et al., 1992).

Mice with a targeted mutation in the $H s p 70-2$ gene were produced to determine if the HSP70-2 protein has a unique role in germ cells. Male mice homozygous for the mutation are infertile (Dix et al., 1996a), demonstrating that HSP70-2 serves a different role than the other HSP70 proteins present in germ cells. Germ cells in the mitotic and meiotic phases were present in the testis but development was arrested in late pachytene spermatocytes, at the G2/M phase transition of the meiotic cell cycle. This transition occurs in all cells and requires Cdc2 kinase activity, acquired when a cyclin A or B regulatory subunit binds to the $\mathrm{Cdc} 2$ catalytic subunit and triggers changes in $\mathrm{Cdc} 2$ phosphorylation. This suggested that HSP70-2 is a molecular chaperone required for Cdc2 activation (Zhu et al., 1997). HSP70-2 was found to associate with Cdc2, but not with cyclin $\mathrm{B} 1$ or the cyclin B1/Cdc2 heterodimer, in germ cells of wild-type mice. 
While cyclin B1 and Cdc2 were present in germ cells of male mice homozygous for the Hsp70-2 mutation, they failed to assemble into heterodimers, lacked Cdc2 kinase activity, and did not undergo the changes in phosphorylation seen in wild-type mice. This suggested that HSP70-2 is required for Cdc2 to dimerize with cyclin B1 and become an active kinase in male germ cells. This was verified by showing that addition of recombinant HSP70-2 protein to a homogenate of germ cells from homozygous mutant mice restored the ability of $\mathrm{Cdc} 2$ to form heterodimers with cyclin B1 and to become an active kinase (Zhu et al., 1997). It was suggested that HSP70-2 enables Cdc2 to acquire the necessary conformation to form a heterodimer with cyclin $\mathrm{B} 1$, thereby providing the protein kinase activity necessary for the G2/M transition to occur. However, Cdc2 serves the same role at the G2/M transition during mitosis in somatic cells and of meiosis in female germ cells, in the absence of HSP70-2. Although it is not yet understood why HSP70-2 is required only in male germ cells to achieve the G2/M transition, it apparently serves a chaperone role that is specifically required in male germ cells and is not provided by homologs.

Transcription of $H s c 70 t$ also is activated in postmeiotic male germ cells without changes in expression of other HSP70 family genes. A monoclonal antibody against a protein spot extracted from $2 \mathrm{D}$ gels of isolated male germ cells recognized a $70-\mathrm{kDa}$ protein present only in spermatids. The protein had immunoblotting and peptide map characteristics similar to HSC70, a constitutively expressed member of the HSP70 family (Maekawa et al., 1989). The monoclonal antibody was used to screen a mouse testis cDNA library and clones were isolated with high sequence homology to an HSP70 family gene expressed only during the postmeiotic phase of spermatogenesis (Matsumoto and Fujimoto, 1990). Northern analysis demonstrated that Hsc70t transcripts are present throughout much of the postmeiotic phase, while immunostaining and immunblot analysis indicated that HSC70T protein is not synthesized until late in the postmeiotic phase (Tsunekawa et al., 1999).

It was hypothesized that HSC70T has a role similar to that of HSC70-2, serving as a chaperone for a protein or proteins of male germ cells essential during the late postmeiotic phase. The gene targeting approach was used to test this hypothesis. However, male mice homozygous for a mutation in $\mathrm{Hsc} 70 \mathrm{t}$ had normal fertility and histological studies indicated that germ cell development during the late postmeiotic phase was not altered (unpublished observations). Nevertheless, when sperm from homozygous mice were placed in vitro, they became immotile within an hour, while sperm from wild-type mice remained motile for several hours. ATP levels are low in these sperm and their ability to produce ATP appears to be compromised (E.M. Eddy, unpublished observations). This suggests that HSC70t possesses unique chaperone capabilities that are required by postmeiotic germ cells for the assembly and function of protein complexes involved in energy production. 
A variation on the theme of activating a gene without inactivating its homolog is seen for A-kinase anchoring proteins (AKAPs). They tether cAMPdependent protein kinase A (PKA), serving to localize its signal transduction activity within a particular region of the cell. The AKAPs differ substantially in sequence but all contain PKA-binding domains. Two AKAPs unique to male germ cells are located in the fibrous sheath, a cytoskeletal component of the sperm flagellum that contains at least six major proteins and more minor proteins in the mouse (Eddy et al., 1991). AKAP4 is the major structural protein of the fibrous sheath and the Akap4 gene is expressed only during the postmeiotic phase (Carrera et al., 1994; Fulcher et al., 1995). The Akap4 gene is located on the X chromosome, making it a single-copy gene in the male (Moss et al., 1997). The AKAP4 protein appears to serve as a scaffold for proteins that are tightly associated with the fibrous sheath or localized to the principal piece, including GAPDS. The Akap3 gene was identified recently and found to be expressed only during the postmeiotic phase (Mandal et al., 1999; Vijayaraghavan et al., 1999). The presence of two AKAPs in the fibrous sheath suggests that PKA activation and function lead to phosphorylation of proteins in this region that regulates flagellar function.

\section{Unique Genes}

Some genes expressed only in male germ cells do not have homologs in other cells. Most of these genes are expressed during the postmeiotic phase, when the specialized structural components of spermatozoa are produced. During this period, dramatic remodeling of the nucleus and condensation of the chromatin occurs, the acrosome forms, and the flagellar axoneme and accessory fiber proteins are synthesized and assembled.

The round spermatid nucleus is remodeled into a falciform-shaped nucleus in the mouse during the last part of the postmeiotic phase. This occurs by removal of the histones, the major nuclear proteins in all other cell types, and their replacement with transition proteins and those, in turn, with protamines. The transition proteins and protamines are products of unique genes expressed only in male germ cells. The protamines are small, basic proteins that package the sperm DNA into a volume about one twentieth of that of a nucleus of somatic cells (reviewed by Balhorn et al., 1999). Most mammals have only one protamine gene but mouse, human, and a few other mammals have two. Gene targeting was used to determine if both protamines are necessary or if protamine 2 (Prm2) is redundant to protamine 1 ( $\mathrm{Prml})$ in the mouse. One copy of either the Prml or Prm2 gene was disrupted in embryonic stem (ES) cells that were then injected into blastocysts to produce chimeras carrying the mutated gene. Although many male chimeras were produced containing a copy of the mutated Prm1 or Prm2 gene, no offspring heterozygous for the mutated gene were sired (Cho et al., 
2001). One possible explanation was that the chimeras did not produce sperm containing the mutated gene. The ES cells were derived from 129Sv strain mice and the blastocysts were from C57BL6 mice, which allowed the use of a polymerase chain reaction (PCR) assay based on microsatellite polymorphisms to genotype the sperm produced by chimeras. It was found that chimeras were producing 129-genotype sperm, of which half had a disrupted Prm1 or Prm2 gene. Subsequent studies determined that a decrease in either of the protamines disrupted sperm nuclear compaction, processing of protamine 2 from precursor to mature form, and normal sperm function. These changes occurred in all 129-genotype sperm, whether they had a copy of the intact gene or a copy of the mutated gene.

Although postmeiotic germ cells are haploid, spermatids remain interconnected by intercellular bridges and form a syncytium that allows sharing of mRNA and protein. It was concluded that transcript and/or protein sharing, coupled with lack of production of Prm1 or Prm2 in half of the spermatids, led to a reduction in amount of the protein in all of the spermatids within a syncytial cluster. This reduction compromised sperm development and function and caused the failure to transmit the intact or mutated Prm1 or Prm2 genes to offspring. These studies show that protamine 2 is not a functionally redundant protein and that both protamine 1 and protamine 2 are essential for the production of normally functioning sperm in the mouse (Cho et al., 2001).

Unique structures are present in germ cells during the meiotic phase, the major one being the synaptonemal complex. This structure, present in oocytes as well as spermatocytes, zippers together the homologous chromosomes to form the structural substrate upon which the processes of crossing over and genetic recombination occur. Its composition and function remain to be fully characterized but unique genes encode the SCP1 and COR1 proteins that are major structural components of the synaptonemal complex (Meuwissen et al., 1992; Dobson et al., 1994). The sex body or XY body is another unique structure present during meiosis in the male. It is located within the nucleus of spermatocytes and is the domain within which the $\mathrm{X}$ and $\mathrm{Y}$ chromosomes pair. Several antibodies have been used to identify proteins unique to the $\mathrm{XY}$ body (Kralewski and Benavente, 1997; Escalier and Garchon, 2000) but the identity of the genes has not been reported. Additional genes are expressed during meiosis that encode proteins involved in recombination and DNA repair but most are also expressed in somatic cells (reviewed in Eddy and O'Brien, 1998). However, the major phenotype for knockouts of several of these genes occurs in germ cells (reviewed in Eddy, 1999). The Dmcl gene is a homolog of yeast S. cerevisiae "disrupted in meiosis" gene and is expressed only during meiosis in male and female mice (Habu et al., 1996). A knockout of the Dmcl gene results in infertility due to failure of synapsis between homologous chromosomes during meiosis (Pittman et al., 1998; Yoshida et al., 1998). 


\section{Alternate Transcripts}

Male germ cell-specific proteins also may be synthesized from alternate transcripts that are derived from the same gene as transcripts in somatic cells but differ from them in size and/or overall sequence. They are generated during gene expression by using promoters and transcription factors that activate transcription at different start sites upstream or downstream of the usual site, by incorporation of alternate exons, by germ cell-specific splicing events, and by using alternate initiation sites for polyadenylation. Alternate transcripts in germ cells often are found serendipitously on northern blots as transcripts that are a different size in testis than in other cells.

Examples of alternate transcripts produced by several of these processes are seen for type 1 hexokinase. Hexokinase metabolizes glucose and is the first enzyme in the glycolytic pathway. Three germ cell-specific transcripts (Hkl-sa, $H k 1-s b, H k 1-s c)$ were identified. Genomic sequencing determined that the germ cell-specific $H k 1-s$ mRNAs in male mice are transcribed from the same gene as somatic cell $H k 1$ mRNA. The spermatogenic cell-specific domain replaced the $\mathrm{N}$-terminal mitochondrial porin-binding domain present in HK1 in somatic cells (Mori et al., 1993). The three cDNAs also differed from each other in the $5^{\prime}$ untranslated region. Northern analysis indicated that $H k 1$-sa transcripts are first present at low levels during meiosis, while the more abundant $H k 1$-s $b$ transcripts are detected only in postmeiotic germ cells. In addition, $H k l-s b$ contains a novel 23-residue sequence within the coding region that is not present in the Hkl-sa or $H k I$-sc sequences. Sequence analysis also showed that the common spermatogenic cell-specific sequence of the three $H k l-s$ transcripts was derived from two exons upstream of the exon encoding the porin-binding domain. Furthermore, the $5^{\prime}$ untranslated regions are found within other exons farther upstream (Mori et al., 1993). The HK1-S protein is present mainly within the principal piece region of the flagellum (Mori et al., 1998; Travis et al., 1998), the region where GAPDS is localized. Production of the three alternate transcripts for HK1-S involves utilization of germ cell-specific promoters, germ cell-specific transcription start sites, germ cell-specific exons, and alternate splice events (Mori et al., 1998).

Alternate transcripts also result from use of alternate polyadenylation sites in the 3' untranslated region. The cation-dependent mannose 6-phosphate receptor (CD-MPR) mediates transport of acid hydrolyases to lysosomes. Three transcripts for this receptor are present in spermatogenic cells. There is a 2.4-kb transcript comparable to that found in somatic cells, a $1.2-\mathrm{kb}$ transcript in pachytene spermatocytes, and a 1.4-kb transcript in round spermatids (O'Brien et al., 1994). The shorter transcripts in germ cells contain the full-length coding region present in the 2.4-kb transcript. By using probes for different regions of the 3'untranslated sequence on northern blots, it was found that the 1.4-kb and $1.2-\mathrm{kb}$ transcripts are produced by utilization of an alternate polyadenylation site. 
The difference in length of these two transcripts is due to a longer poly(A) tail on the 1.4-kb transcript (O'Brien et al., 1994).

Alternate transcripts are present in other cell types but are particularly abundant in male germ cells (reviewed in Eddy et al., 1993; Hecht, 1993; Eddy and O'Brien, 1998). There are at least four reasons that alternate transcripts may be beneficial to male germ cells. First, addition of new domains can expand the function of the protein and allow it to perform germ cell-specific functions. An example described previously is the proline-rich $\mathrm{N}$-terminal domain that tethers GAPDS to the fibrous sheath (Bunch et al., 1998). Second, removal of one domain and replacement with another can change the distribution of the protein within the cell. This occurs for HK-1 where the porin-binding domain that usually targets the enzyme to mitochondria is lost and a germ cell-specific domain is added (Mori et al., 1998), which presumably is responsible for HK1-S being located mainly in the principal piece. Third, a long poly(A) tail often is found on transcripts in postmeiotic germ cells for which there is a delay of several days between transcription and translation. These transcripts undergo shortening of the poly(A) tail coincident with becoming associated with polysomes and translationally active (Kleene et al., 1984). However, there are substantial differences in this process between various germ cell transcripts (Kleene, 1996) and it is unclear whether transcript shortening is a cause or an effect of translational activation of stored mRNAs. Fourth, alternate transcripts can arise by utilization of a promoter within an intron, resulting in a functionally different protein in male germ cells than in somatic cells. Examples of this are the truncated versions of angiotensin-converting enzyme (ACE) (Howard et al., 1990) and calcium ${ }^{+2} /$ calmodulin-dependent kinase IV (CaMKIV) (Means et al., 1991) that are found only in male germ cells and have enzymatic properties different from the longer forms.

\section{Developmentally Regulated Gene Expression}

The expression of many genes in male germ cells is developmentally regulated during the meiotic and postmeiotic phases. This probably is true for all germ cell-specific homologs, genes expressed only in male germ cells, and germ cell-specific transcripts. However, many other genes that are developmentally regulated in male germ cells are expressed in somatic cells as well (see reviews by Willison and Ashworth, 1987; Eddy et al., 1991,1993; Wolgemuth and Watrin, 1991; Hecht, 1993; Eddy and O'Brien, 1998).

Developmentally regulated gene expression in male germ cells may have evolved by more widely expressed genes becoming restricted in expression to male germ cells (McCarrey, 1994) or as the result of gene duplications or swapping of exons between precursor genes. In either case, these genes would need to incorporate promoters that drive their expression in male germ cells and 
not in other cells. Such promoters may have evolved from regulatory elements for meiosis-specific genes conserved from ancestral unicellular organisms. In addition, genes expressed in somatic cells also have undergone modifications to become developmentally regulated in germ cells. Two possible ways this may have occurred were either by incorporation of promoter elements regulating developmental expression in germ cells or by utilizing unique transcription factor combinations in germ cells that act through existing promoter elements. It is notable that gene-targeting studies involving genes expressed in somatic cells and germ cells often have found that the sole or major phenotype produced is in germ cells (reviewed in Eddy, 1999). A gene does not have to be expressed only in germ cells to be essential for germ cell development.

The regulation of transcription has not been studied in depth for any germ cell-specific genes, mainly because permanent germ cell lines are not available for use in conventional promoter-reporter assays. In lieu of this, transgenic mice have been used for promoter mapping for some germ cell-specific genes. Although this approach is cumbersome, the regions containing key regulatory elements for germ cell-specific and stage-specific gene expression have been delimited for several genes (Dix et al., 1996b; Iannello et al., 1997; Li et al., 1998; Reddi et al., 1999).

A diverse group of transcription factors are expressed in male germ cells. Some have been implicated in the transcriptional regulation of genes in specific stages of spermatogenesis (see Famia et al., 1999; Daniel and Habener, 2000; Liu et al., 2000; Han et al., 2001; and references therein). However, experimental evidence is available for only a few demonstrating a specific role in germ cells. One example is the Erg4 transcription factor. The only effect of a targeted mutation in the Erg4 gene was disruption of male germ cell development during the meiotic phase (Tourtellotte et al., 1999). Also, a knockout of the gene for SPRM-1, a POU-homeodomain gene expressed only in male germ cells during the postmeiotic phase, produced subnormal fertility (Pearse et al., 1997). In addition, a germ cell-specific subunit of the general transcription factor TFIIA was found to be upregulated in pachytene spermatocytes, along with several RNA polymerase II and III factors (Han et al., 2001). This coordinated expression of general transcription factor genes was hypothesized to regulate a particular subset of genes in germ cells (Han et al., 2001). An interesting parallel is that the general transcription factor TFIID is required for high level transcription of a set of stage-specific and tissue-specific target genes during male germ cell development in Drosophila (Hiller et al., 2001).

It has been recognized for nearly 20 years that activation of expression of some genes occurs specifically during the meiotic or postmeiotic phases. More recent studies indicate that developmentally regulated gene expression also occurs during the mitotic phase. The use of library subtraction methods to identify cDNAs present in a mouse primitive type A spermatogonia library led to 
the identification of 19 novel genes expressed only in the testis (Wang et al., 2001). Seven of these appeared to be involved in transcriptional or posttranscriptional regulation of gene expression. A relatively high fraction of these genes mapped to the $\mathrm{X}$ chromosome. It was suggested that the $\mathrm{X}$ chromosome has a pre-eminent role in male germ cell development that was acquired as the $\mathrm{X}$ chromosome evolved from an autosome (Wang et al., 2001).

\section{Interactive Regulation of Germ Cell Gene Expression}

Sertoli cells are essential for germ cells to proliferate and to progress through the phases of development. The general functions of Sertoli cells in these processes are well known, such as dividing the seminiferous epithelium into basal and luminal compartments and providing a suitable milieu within which germ cell development occurs. In addition, Sertoli cells are more amenable to in vitro study than germ cells. This has allowed the identification of a variety of proteins that are synthesized and exported by Sertoli cells, at least some of which are involved in the interaction with germ cells (Griswold, 1995,1998). However, what role most of these proteins serve in regulating germ cell development remains to be determined.

The relationship between germ cells and Sertoli cells is bi-directional. The patterns of gene expression in Sertoli cells change as neighboring germ cells progress through the stages of spermatogenesis (Zabludoff et al., 2001). This suggests that one aspect of germ cell-Sertoli cell interactions involves diverse signals coming from the associated cohort of germ cells to act on Sertoli cells and cause changes in gene expression, which alters feedback signals that modulate the intrinsic regulatory program driving germ cell development.

\section{A. SERTOLI CELL EFFECTS ON GERM CELLS}

Sertoli cells secrete more than 100 different proteins detectable by 2-D gel electrophoresis of conditioned media from primary Sertoli cell cultures (see reviews by Griswold, 1993,1998; Jégou, 1993; Dupaix et al., 1996), and seminiferous tubule fluid has a 10 -fold higher potassium concentration than blood (Hinton and Setchell, 1993). Sertoli cell products for which the identity is known include transport or binding proteins, proteases, energy metabolites, components of the extracellular matrix and junctional complexes, and a variety of factors that may influence germ cell division, differentiation, and metabolism (Jégou, 1993). The factors include several cytokines, a variety of growth factors, signaling molecules, bioactive peptides, and hormones (Griswold, 1993; Skinner, 1993). Although assumed to be involved in signaling from Sertoli to germ cell, experimental evidence demonstrating effects on gene expression in germ cells has been shown for only a few. Gene knockout studies have provided evidence 
that two factors produced by Sertoli cells - desert hedgehog (Dhh) (Bitgood et al., 1996) and glial cell line-derived neurotrophic factor (GDNF) (Meng et al., 2000) - are involved in signaling.

One of the receptors known to participate in Sertoli-to-germ-cell signaling is the bi-functional insulin-like growth factor-II/cation-independent mannose 6-phosphate (IGF-II/M6P) receptor. This receptor is present on the surface of spermatogenic cells and Sertoli cells secrete ligands for the receptor, including IGF-II and 10 or more M6P-bearing glycoprotein ligands that remain to be characterized (O'Brien et al., 1993; Tsuruta and O'Brien, 1995). Furthermore, proliferin, leukemia inhibitory factor (LIF), and the precursor forms of transforming growth factor beta (TGF $\beta$ ) are M6P-glycoprotein-containing growth factors found in the testis and may act through the IGFII/M6P receptor on germ cells. Addition of either IGF-II or of M6P-glycoproteins affinity-purified from Sertoli cell-conditioned medium to isolated spermatogenic cells produces dosedependent increases in mRNA for the immediate-early gene $c$-fos and in rRNA (Tsuruta and O'Brien, 1995; Tsuruta et al., 2000). This effect is seen in spermatogonia (Tsuruta et al., 2000), spermatocytes, and round spermatids (Tsuruta and O'Brien, 1995), indicating that interactions can occur between M6P-containing ligands and germ cells in both the basal and luminal compartments of the seminiferous epithelium, with the possibility that different ligands are secreted into each compartment.

Another example of Sertoli-to-germ-cell signaling is provided by stem cell factor (SCF) ligand and c-kit receptor interaction. The c-kit trans-membrane tyrosine kinase receptor is present on spermatogonia (Manova et al., 1990; Yoshinaga et al., 1991). Sertoli cells produce SCF (Rossi et al., 1991) and a mutation in the Kit gene or in Kitl, the gene for SCF, disrupts male germ cell development (reviewed in Besmer et al., 1993). SCF exists in two forms, membrane-associated and soluble, and Sertoli cells predominantly produce the membrane-associated form. This suggests that Sertoli cells communicate directly with spermatogonia through the association between SCF ligand and c-kit receptor.

\section{B. GERM CELL EFFECTS ON SERTOLI CELLS}

Addition of germ cells to Sertoli cell primary cultures can stimulate or inhibit different Sertoli cell functions (Jégou, 1993; Dupaix et al., 1996). Some of the germ cell factors that affect Sertoli cells are known to be proteins but have not been identified. In addition, meiotic and postmeiotic germ cells produce the growth factors $\beta \mathrm{NGF}$ (nerve growth factor), bFGF (fibroblast growth factor), IGF-I, and TGF $\beta$; and the cytokines interleukin-1 (IL-1), interferon (INF), and tumor necrosis factor alpha (TNF $\alpha$ ) (reviewed in Skinner, 1993; Dupaix et al., 1996; Gnessi et al., 1997) that are likely to signal Sertoli cells. A gene trap 
strategy to identify genes involved in signaling from germ cells to Sertoli cells found that Fral mRNA was induced in Sertoli cells by addition of either round spermatids or NGF to Sertoli cells. The NGF effect was mediated by the TrkA receptor and the ERK1-ERK2 kinase pathway (Vidal et al., 2001). In addition, mRNA differential display studies identified four genes that were upregulated and three genes that were downregulated in primary Sertoli cells when cocultured for 24 hours with germ cells. The same studies found that three genes were downregulated in germ cells in the cocultures, demonstrating the reciprocal nature of Sertoli and germ cell interactions (Syed and Hecht, 1997).

\section{GERM CELL EFFECTS ON GERM CELLS}

The effects of one population of germ cells on another is probably the least understood and certainly the least studied of the interactive regulatory processes of germ cell development. However, the results of loss-of-function and gain-offunction studies provide indirect evidence of communication between different populations of germ cells.

The bone morphogenetic protein 8B (BMP8B) is a member of the TGF $\beta$ growth factor superfamily and shows bimodal expression in developing germ cells. It is expressed in spermatogonia at 1 week after birth but not in those cells after BMP8A expression begins in round spermatids (Zhao et al., 1996). A knockout of the $B m p 8 b$ gene caused two separate effects on gene cells: a failure or reduction in proliferation that coincides with the early BMP8B expression and delayed differentiation, causing a reduction in mitotic and meiotic germ cells. BMP8B may act through two receptors that bind BMPs, ActRIIA, expressed mainly in spermatogonia and early meiotic cells, and ActRIIB, expressed mainly in pachytene spermatocytes and round spermatids. It was hypothesized that BMP8B produced by round spermatids acts on primary spermatocytes to regulate their survival and differentiation (Zhao et al., 1998).

The Bcl-2 family of proteins can have either positive or negative effects on the regulation of apoptosis (Korsmeyer, 1995). BAX forms a heterodimer with Bcl-2 and counters the apoptosis-repressing role of Bcl-2, thereby promoting apoptosis. BAX is expressed at high levels in spermatogonia (Rodriguez et al., 1997). Bax knockout mice are infertile, have atrophic testes, and lack sperm in the epididymis (Knudson et al., 1995). The substantial increase in apoptosis that occurs near the end of the mitotic phase is suppressed in Bax knockout mice. The increased number of spermatogonia has a detrimental effect on germ cells in other phases of spermatogenesis. Furthermore, transgenic mice that express high levels of the apoptosis-inhibiting proteins Bcl-2 or BclxL in spermatogonia also have increased numbers of spermatogonia and disrupted spermatogenesis (Furuchi et al., 1996; Rodriguez et al., 1997). In another study using the genetrapping procedure, a mutation was produced in the gene for Bclw, an apoptosis- 
inhibiting protein expressed during the postmeiotic phase (Ross et al., 1998). This resulted in a block in germ cell development in late spermatids in juvenile mice, followed by an increase in apoptosis in spermatocytes, which led to the loss of spermatids and degeneration of the seminiferous epithelium. In each of these transgenic or knockout mice, spermatogenesis was most affected in a phase of germ cell development different from the phase when expression of a Bcl-2 family protein was altered. A common feature of these studies is that the ratios between germ cells in mitotic, meiotic, and postmeiotic phases of germ cell development were altered. This suggests that an imbalance in reciprocal signals between germ cells that coordinate their development leads to disruption of spermatogenesis.

\section{BIOACTIVE PEPTIDES}

The genes for many bioactive peptides, originally considered to be neuroendocrine regulators, as well as the genes for many of their receptors are expressed in the testis. The peptides are produced in Leydig cells, Sertoli cells, peritubular myoid cells, and germ cells, and often in more than one cell type. Germ cells have been reported to have RNA or protein for the proenkephalin (pENK) pituitary adenylate cyclase-activating peptide (PACAP), pro-opiomelanocortin (POMC), and gonadotropin-releasing hormone $(\mathrm{GnRH})$ peptides and gastrinrelated peptide (GRP) receptor (reviewed by Gnessi et al., 1997). A larger number of peptides and receptors are present in the other cell types, perhaps suggesting a major role of the peptides and receptors in coordinating the activities of Leydig cells, Sertoli cells, and peritubular cells, and a lesser role in germ cell interactions. However, germ cells contain RNA or protein for bFGF, IGF-I, $\beta$-NGF and TGF growth factors, and the receptors for epidermal growth factor (EGF)/TGF $\alpha$, bFGF, stem cell factor (SCF), and IGF-I. This might indicate that growth factors have a greater role than bioactive peptides in regulating germ cells. The presence of growth hormone releasing hormone (GHRH), growth hormone (GH), IGF-I, and IGF binding proteins in the testis suggests an intra-testicular GH axis (DiMeglio et al., 1998). It is quite likely that these and probably other peptides and receptors are part of the intratesticular network that coordinates the biological activities of the various cell types but how significant a role they play remains to be determined.

\section{Extrinsic Regulation of Germ Cell Gene Regulation}

The dogma has been that FSH and testosterone regulate spermatogenesis, sometimes resulting in the view that germ cell development is a black box, with hormones going in one end and sperm coming out the other end. However, it has become increasingly apparent that FSH and testosterone do not regulate germ 
cell development but instead serve an important supportive role for this process. FSH is not essential for spermatogenesis, as shown by the findings that male mice with a knockout of the gene for FSH- $\beta$ are fertile (Kumar et al., 1997) and that men with an inactivating mutation in the FSH receptor had varying degrees of spermatogenic failure but were not infertile (Tapanainen et al., 1997). In addition, testis weight and epididymal sperm numbers were reduced in FSH receptor knockout mice (Krishnamurthy et al., 2000) but males were fertile. It has become apparent in the last 4 years that germ cell development is considerably less dependent on FSH than was previously thought.

The other major player in the extrinsic regulation of germ cell development, testosterone, is essential for this process. LH regulates the production of testosterone by Leydig cells and testosterone acts through androgen receptors (ARs) in Sertoli cells and peritubular cells to influence the Sertoli cell functions that support germ cell development (reviewed by Sar et al., 1993). In the absence of LH, spermatogenesis can be maintained by administering testosterone but not quite at the same level as in the normal state (reviewed by Sharpe, 1994). There are several studies indicating that androgen receptors are present in germ cells and several indicating that they are not. However, it has been shown recently that if there are ARs in germ cells, they are not essential for germ cell development. Using the germ cell transplantation technique, germ cells from the testis of Tfm mice lacking functional androgen receptors but carrying a $L a c Z$ transgene were transplanted into the testes of mice with functional ARs. Colonies of donor-derived spermatogenic cells were seen in the seminiferous tubules of recipient mice, indicating that mouse germ cells do not require functional ARs to carry out spermatogenesis (Johnston et al., 2001).

Not all extrinsic regulators of germ cell development act through Sertoli cells. Vitamin A is required for spermatogenesis. Vitamin A-deficient rats have only spermatogonia and Sertoli cells in their seminiferous tubules. However, treatment with retinol restores spermatogenesis in these animals (reviewed by Kim and Akmal, 1996). The retinoid acid receptor (RAR) $\alpha$ is present in spermatogenic cells (Wang and Kim, 1993) and the retinoid X receptor (RXR) $\beta$ is present in Sertoli cells (Kastner et al., 1996). These are members of the steroid receptor superfamily and gene knockout studies have demonstrated that each is essential for spermatogenesis (Lufkin et al., 1993; Kastner et al., 1996), strongly suggesting that vitamin A may have direct effects on germ cell development and function.

\section{Conclusions}

There is strong evidence that regulation of gene expression in male germ cells is largely an independent process. Male germ cells carry their own marching orders that have been refined and handed down over the millennia. Given the appropriate environment, spermatogonial stem cells can do their job of producing 
spermatogonia and/or other stem cells. The spermatogonia proliferate, then squeeze through a bottleneck where many die but some become spermatocytes. These cells carry out the complex process of meiosis, an ancient series of events that is good for the species but a dangerous prospect for the cell because of the intentional DNA damage that occurs during recombination. The next hurdle is for these cells to divide. Cell division is not an unusual process but germ cells make it complicated by dividing twice without making new copies of their chromosomes.

The postmeiotic cells are responsible for synthesizing and assembling a host of novel proteins that make up the sperm. Hidden away in the genome is a curious group of genes that are quiescent through most of the lifecycle of the organism. Only in spermatids do these genes become activated to carry out the construction of a hydrodynamically specialized cell containing a payload of tightly packaged DNA, an elaborate propulsion system, and special enzymes that help deliver the DNA to the egg. Most, if not all, of these processes are the responsibility of a program that operates without outside direction. Of course, germ cells need considerable help from other cells for all of this to happen and they are dependent on a remarkably complex support system. There is a hierarchy of local to distant systems responsible for ensuring that germ cells have the appropriate environment for producing the highly specialized cells necessary for continuation of the next and future generations of the species.

Sertoli cells in particular provide essential and highly effective support that is responsive and highly interactive, but not directive, for gene expression in male germ cells. The major functions of the Sertoli cells are heavily influenced by and dependent on other cells, particularly their neighbors, the peritubular myoid cells and Leydig cells. The functions of Sertoli cells are also modulated directly and indirectly by $\mathrm{FSH}, \mathrm{LH}$, and other hormones, whose influences range from crucial to beneficial at different times during the development and function of the seminiferous tubules. Although these extrinsic signals are of immeasurable importance for establishing and maintaining the environment within which male germ cells develop, it needs to remembered that they support, rather than regulate, male germ cell development.

\section{REFERENCES}

Allen RL, O'Brien DA, Jones CC, Rockett DL, Eddy EM 1988a Expression of heat shock proteins by isolated mouse spermatogenic cells. Mol Cell Biol 8:3260-3266

Allen RL, O'Brien DA, Eddy EM 1988b A novel hsp70-like protein (P70) is present in mouse spermatogenic cells. Mol Cell Biol 8:828-832

Andrews J, Bouffard G, Cheadle C, Lu J, Becker K, Oliver B 2000 Gene discovery using computation and microarray analysis of transcription in the Drosophila melanogaster testis. Genome Res 10:2030-2043 
Balhorn R, Cosman M, Thornton K, Krishnan V, Corzett M, Bench G, Kramer C, Lee J IV, Hud N, Allen M, Priety M, Meyer-IIse W, Brown J, Kirz J, Zhang X, Bradbury E, Maki G, Braun R, Breen W 1999 Protamine mediated condensation of DNA in mammalian sperm. In: Gagnon C, ed. The Male Gamete: From Basic Science to Clinical Applications. Vienna, IL: Cache River Press; 55-70

Besmer P, Manova K, Duttlinger R, Huang EJ, Packer A, Hyssler C, Bachvarova RF 1993 The kit-ligand (steel factor) and its receptor $c$-kit/W: pleiotropic roles in gametogenesis and melanogenesis. Development (suppl):125-137

Bitgood MJ, Shen L, McMahon AP 1996 Sertoli cell signaling by Desert hedgehog regulates the male germline. Current Biol 5:298-304

Boer PH, Adra CN, Lau Y-F, McBurney MW 1987 The testis-specific phosphoglycerate kinase gene pgk-2 is a recruited retroposon. Mol Cell Biol 7:3107-3112

Bunch DO, Welch JE, Magyar PL, Eddy EM, O'Brien DA 1998 Glyceraldehyde 3-phosphate dehydrogenase-S protein distribution during mouse spermatogenesis. Biol Reprod 58:834841

Carrera A, Gerton GL, Moss SB 1994 The major fibrous sheath polypeptide of mouse sperm: structural and functional similarities to the A-kinase anchoring proteins. Dev Biol 165:272284

Cho C, Willis W, Goulding EH, Jung-Ha H, Choi Y-C, Hecht NB, Eddy EM 2001 Haploinsufficiency of protamine-1 or -2 causes infertility in mice. Nature Genet 28:82-86

Chu S, Dervan PA, Botstein DBP, Herskowitz I 1998 The transcriptional program of sporulation in budding yeast. Science 282:699-705

Clermont Y, Trott M 1969 Duration of the cycle of the seminiferous epithelium in the mouse and hamster determined by means of 3H-thymidine and radioautography. Fertil Steril 20:805-817

Clouthier DE, Averbock MA, Maika SD, Hammer RE, Brinster RL 1996 Rat spermatogenesis in mouse testis. Nature 381:418-421

Cooper TG 1984 The onset and maintenance of hyperactivated motility of spermatozoa in the mouse. Gamete Res 9:55-74

Daniel PB, Habener JF 2000 Pituitary adenylate cyclase-activating polypeptide gene expression regulated by a testis-specific promoter in germ cells during spermatogenesis. Endocrinology 141:1218-1227

DiMeglio LA, Steinmetz R, Pescovitz OH 1998 Variations on a theme: testis-derived neuropeptide hormones. Eur J Endocrinol 139:361-362

Dix DJ, Allen JW, Collins BW, Mori C, Nakamura N, Poorman-Allen P, Goulding EH, Eddy EM 1996a Targeted gene disruption of Hsp70-2 results in failed meiosis, germ cell apoptosis, and male infertility. Proc Natl Acad Sci USA 93:3264-3268

Dix DJ, Rosario-Herrle M, Gotoh H, Mori C, Goulding EH, Barrett CV, Eddy EM 1996b Developmentally regulated expression of $H s p 70-2$ and a $H s p 70-2 / l a c Z$ transgene during spermatogenesis. Dev Biol 174:310-321

Dobson MJ, Pearlman RE, Karaiskakis A, Spyropoulos B, Moens PB 1994 Synaptonemal complex proteins: occurrence, epitope mapping and chromosome disjunction. J Cell Sci 107:2749-2760

Dupaix A, Pineau C, Piquet-Pellorce C, Jégou B 1996 Paracrine and autocrine regulations of spermatogenesis. In: Hamamah S, Mieusset R, eds. Research in Male Gametes: Production and Quality. Rennes, France: INSERM; 47-63

Eddy EM 1999 The effects of gene knockouts on spermatogenesis. In: Gagnon C, ed. The Male Gamete: From Basic Science to Clinical Applications. Vienna, IL: Cache River Press; 23-36

Eddy EM, O'Brien DA 1994 The spermatozoon. In: Knobil E, Neill JD, eds. The Physiology of Reproduction. New York: Raven Press; 29-77 
Eddy EM, O’Brien DA 1998 Gene expression during mammalian meiosis. In: Handel MA, ed. Meiosis and Gametogenesis. San Diego: Academic Press; 141-200

Eddy EM, O'Brien DA, Fenderson BA, Welch JE 1991 Intermediate filament-like proteins in the fibrous sheath of the mouse sperm flagellum. Ann NY Acad Sci 637:224-239

Eddy EM, Welch JE, O'Brien DA 1993 Gene expression during spermatogenesis. In: De Kretser DM, ed. Molecular Biology of the Male Reproductive System. Orlando, FL: Academic Press; 181-232

Eddy EM, Welch JE, Mori C, Fulcher KD, O'Brien DA 1994 Role and regulation of spermatogenic cell-specific gene expression: enzymes of glycolysis. In: Bartke A, ed. Function of Somatic Cells in the Testis. New York: Springer-Verlag; 362-372

Escalier D, Garchon H-J 2000 XMR is associated with the asynapsed segments of sex chromosomes in the XY body of mouse primary spermatocytes. Chromosoma 109:259-265

Famia G, De Casare D, Sassone-Corsi P 1999 CBP-independent activation of CREM and CREB by the LIM-only protein ACT. Nature 398:165-169

Franca LR, Ogawa T, Averbock MR, Brinster RL, Russell LD 1998 Germ cell genotype controls cell cycle during cell cycle during spermatogenesis in the rat. Biol Reprod 59:1371-1377

Fraser LR, Quinn PJ 1981 A glycolytic product is obligatory for initiation of the sperm acrosome reaction and whiplash motility required for fertilization in the mouse. J Reprod Fertil 61:25-35

Fulcher KD, Mori C, Welch JE, O'Brien DA, Klapper DG, Eddy EM 1995 Characterization of Fsc1 cDNA for a mouse sperm fibrous sheath component. Biol Reprod 52:41-49

Furuchi T, Masuko K, Nishimune Y, Obinata M, Matsui Y 1996 Inhibition of testicular germ cell apoptosis and differentiation in mice misexpression Bcl-2 in spermatogonia. Development 122:1703-1709

Gnessi L, Fabbri A, Spera G 1997 Gonadal peptides as mediators of development and functional control of the testis: an integrated system with hormones and local environment. Endocr Rev 18:541-609

Griswold MD 1993 Protein secretion by Sertoli cells: general considerations. In: Russell LD, Griswold MD, eds. The Sertoli Cell. Clearwater, FL: Cache River Press; 195-200

Griswold MD 1995 Interactions between germ cells and Sertoli cells in the testis. Biol Reprod 52:211-216

Griswold MD 1998 The central role of Sertoli cells in spermatogenesis. Semin Cell Dev Biol 9:411-416

Habu T, Taki T, West A, Nishimune Y, Morita T 1996 The mouse and human homologs of DMC1, the yeast meiosis-specific homologous recombination gene, have a common unique form of exon-skipped transcripts in meiosis. Nucleic Acids Res 24:470-477

Han S, Zhou L, Upadhyaya A, Lee S, Parker K, DeJong J 2001 TFII $\alpha / \beta$-like factor is encoded by a germ cell-specific gene whose expression is up-regulated with other general transcription factors during spermatogenesis in the mouse. Biol Reprod 64:507-517

Hecht NB 1993 Gene expression during male germ cell development. In: Desjardins C, Ewing LL, eds. Cell and Molecular Biology of the Testis. Oxford: Oxford University Press; 464-503

Hiller M, Lin T, Wood C, Fuller M 2001 Developmental regulation of transcription by a tissue-specific TAF homolog. Genes Dev 15:1021-1030

Hinton B, Setchell B 1993 Fluid secretion and movement. In: Russell LD, Griswold MD, eds. The Sertoli Cell. Clearwater, FL: Cache River Press; 249-267

Hoppe PC 1976 Glucose requirement for mouse sperm capacitation in vitro. Biol Reprod 15:39-45

Howard TE, Shai S-Y, Langford KG, Martin BM, Bernstein KE 1990 Transcription of testicular angiotensin-converting enzyme (ACE) is initiated within the 12th intron of the somatic ACE gene. Mol Cell Biol 10:4294-4302 
Hwang SY, Oh B, Knowles BB, Solter D, Lee JS 2001 Expression of genes involved in mammalian meiosis during the transition from egg to embryo. Mol Reprod Dev 59:144-158

Iannello R, Young J, Sumarsono S, Tymms M, Dahl H-H, Gould J, Hedger M, Kola I 1997 Regulation of Pdha-2 expression is mediated by proximal promoter sequences and $\mathrm{CpG}$ methylation. Mol Cell Biol 17:612-619

Jegou B 1993 The Sertoli-germ cell communication network in mammals. Intl Rev Cytol 147:25-96

Johnston DS, Russell LD, Friel PF, Griswold MD 2001 Murine germ cells do not require functional androgen receptors to complete spermatogenesis following spermatogonial stem cell transplantation. Endocrinology 142:2405-2408

Jones AR 1978 The antifertility actions of $\alpha$-chlorohydrin in the male. Life Sci 23:1625-1646

Jutte NHPM, Jansen R, Grootegoed JA, Rommerts FFG, van der Molen HJ 1983 FSH stimulation of the production of pyruvate and lactate by rat Sertoli cells may be involved in hormonal regulation of spermatogenesis. J Reprod Fertil 68:219-226

Kastner P, Mark M, Leid M, Gansmuller A, Chin W, Grondona JM, Decimo D, Krezel W, Dierich A, Chambon P 1996 Abnormal spermatogenesis in RXR $\beta$ mutant mice. Genes Dev 10:80-92

Kim KH, Akmal KM 1996 Role of vitamin A in male germ cell development. In: Desjardins C, ed. Cellular and Molecular Regulation of Testicular Cells. New York: Springer; 83-98

Kleene KC 1996 Patterns of translational regulation in the mammalian testis. Mol Reprod Devel 43:268-281

Kleene KC, Distel RJ, Hecht NB 1984 Translational regulation and deadenylation of a protamine mRNA during spermiogenesis in the mouse. Dev Biol 105:71-79

Knudson CM, Tung KSK, Tourtellotte WG, Brown GA, Korsmeyer SJ 1995 Bax-deficient mice with lymphoid hyperplasia and male germ cell death. Science 270:96-99

Korsmeyer SJ 1995 Regulators of cell death. Trends Genet 11:101-105

Kralewski M, Benavente R 1997 XY body formation during rat spermatogenesis: an immunocytochemical study using antibodies against XY body-associated proteins. Chromosoma 106: 304-307

Krishnamurthy H, Danilovich N, Morales CR, Sairam MR 2000 Qualitative and quantitative decline in spermatogenesis of the follicle-stimulating hormone receptor knockout (FORKO) mouse. Biol Reprod 62:1146-1159

Kumar TR, Wang Y, Lu N, Matzuk MM 1997 Follicle stimulating hormone is required for ovarian follicle maturation but not male fertility. Nature Genet 15:201-204

Li S, Zhou W, Doglio L, Goldberg E 1998 Transgenic mice demonstrate a testis-specific promoter for lactate dehydrogenase, LDHC. J Biol Chem 273:31191-31194

Liu F, Kondova I, Kilpatrick D 2000 Detection of PACH1, a nuclear factor implicated in the transcriptional regulation of meiotic and early haploid stages of spermatogenesis. Mol Reprod Dev 57:224-231

Lufkin T, Lohnes D, Mark M, Dierich M, Gorry P, Gaub M-P, LeMeur M, Chambon P 1993 High postnatal lethality and testis degeneration in retinoic acid receptor $\alpha$ mutant mice. Proc Natl Acad Sci USA 90:7225-7229

Maekawa M, O'Brien DA, Allen RL, Eddy EM 1989 Heat-shock cognate protein (hsc71) and related proteins in mouse spermatogenic cells. Biol Reprod 40:843-852

Mandal A, Naaby-Hansen S, Wolkowicz M, Klotz K, Shetty J, Retief J, Coonrod S, Kinter M, Sherman N, Cesar F, Flickinger C, Herr J 1999 FSP95, a testis-specific 95-kilodalton fibrous sheath antigen that undergoes tyrosine phosphorylation in capacitated human spermatozoa. Biol Reprod 61:1184-1197

Manova K, Nocka K, Besmer P, Bachvarova RF 1990 Gonadal expression of c-kit encoded at the $W$ locus of the mouse. Development 110:1057-1069 
Matsumoto M, Fujimoto H 1990 Cloning of a hsp70-related gene expressed in mouse spermatids. Biochem Biophys Res Comm 166:43-49

McCarrey JR 1994 Evolution of tissue-specific gene expression in mammals. BioScience 44:20-27

McCarrey JR, Thomas K 1987 Human testis-specific PGK gene lacks introns and possesses characteristics of a processed gene. Nature 326:501-505

Means AR, Cruzalegui F, LeMagueresse B, Needleman DS, Slaughter GR, Ono T 1991 A novel $\mathrm{Ca}^{2+} /$ calmodulin-dependent protein kinase and a male germ cell-specific calmodulin-binding protein are derived from the same gene. Mol Cell Biol 11:3960-3971

Meng X, Lindahl M, Hyvonen ME, Parvinen M, De Rooij DG, Hess MW, Raatikainen-Ahokas A, Sainio K, Rauvala H, Lakso M, Pichel JG, Weshphal H, Saarma M, Sariola H 2000 Regulation of cell fate decision of undifferentiated spermatogonia by GDNF. Science 287:1489-1493

Meuwissen RLJ, Offenberg HH, Dietrich AJJ, Riesewijk A, Van Iersel M, Heyting C 1992 A coiled-coil related protein specific for synapsed regions of meiotic prophase chromosomes. EMBO J 11:5091-5100

Mohri H, Mohri T, Ernster L 1965 Isolation and enzymatic properties of the midpiece of bull spermatozoa. Exp Cell Res 38:217-246

Mori C, Welch JE, Sakai Y, Eddy EM 1992 In situ localization of spermatogenic cell-specific glyceraldehyde 3-phosphate dehydrogenase (Gapd-s) messenger ribonucleic acid in mice. Biol Reprod 46:859-868

Mori C, Welch JE, Fulcher KD, O'Brien DA, Eddy EM 1993 Unique hexokinase messenger ribonucleic acids lacking the porin-binding domain are developmentally expressed in mouse spermatogenic cells. Biol Reprod 49:191-203

Mori C, Nakamura N, Welch JE, Gotoh H, Goulding EH, Fajioka M, Eddy EM 1998 Mouse spermatogenic-cell specific type 1 hexokinase $(m H k l-s)$ transcripts are expressed by alternative splicing from the $m H k 1$ gene and the HK1-S protein is localized mainly in the sperm tail. Mol Reprod Dev 49:374-385

Moss SB, VanScoy H, Gerton GL 1997 Mapping of a haploid transcribed and translated spermspecific gene to the mouse X chromosome. Mamm Genome 8:37-38

Nakamura M, Fujiwara A, Yasamasu I, Okinaga S, Arai K 1982 Regulation of glucose metabolism by adenine nucleotides in round spermatids from rat testes. $\mathrm{J}$ Biol Chem 257:13945-13950

O'Brien DA 1987 Stage-specific protein synthesis by isolated spermatogenic cells throughout meiosis and early spermiogenesis in the mouse. Biol Reprod 37:147-157

O'Brien DA, Gabel CA, Eddy EM 1993 Mouse Sertoli cells secrete mannose 6-phosphate containing glycoproteins that are endocytosed by spermatogenic cells. Biol Reprod 49:10551065

O'Brien DA, Welch JE, Fulcher KD, Eddy EM 1994 Expression of mannose 6-phosphate receptor messenger ribonucleic acids in mouse spermatogenic and Sertoli cells. Biol Reprod 50:429435

Pearse RVI, Drolet EW, Kalla KA, Hooshmand F, Bermingham JRJ, Rosenfeld MG 1997 Reduced fertility in mice deficient for the POU protein sperm-1. Proc Natl Acad Sci USA 94:7555-7560

Pittman DL, Cobb J, Schimenti KJ, Wilson LA, Cooper DM, Brugnull E, Handel MA, Schimenti JC 1998 Meiotic prophase arrest with failure of chromosome synapsis in mice deficient for Dmc1, a germline-specific RecA homolog. Mol Cell 1:697-705

Reddi PP, Flickinger CJ, Herr JC 1999 Round spermatid-specific transcription of the mouse SP-10 gene is mediated by a 294-base pair proximal promoter. Biol Reprod 61:1256-1266 
Reinke V, Smith H, Nance J, Wang J, Van Doren C, Begley R, Jones S, Davis E, Scherer S, Ward S, Kim S 2000 A global profile of germline gene expression in C. elegans. Mol Cell 6:605-616

Rodriguez I, Ody C, Araki K, Garcia I, Vassalli P 1997 An early and massive wave of germinal cell apoptosis is required for the development of functional spermatogenesis. EMBO J 9:2262-2270

Roosen-Runge EC 1977 The Process of Spermatogenesis. London: Cambridge University Press

Rosario MO, Perkins SL, O'Brien DA, Allen RL, Eddy EM 1992 Identification of the gene for the developmentally expressed $70 \mathrm{kDa}$ heat-shock protein (P70) of mouse spermatogenic cells. Dev Biol 150:1-11

Ross AJ, Waymire KG, Moss JE, Parlow AF, Skinner MK, Russell LD, MacGregor GR 1998 Testicular degeneration in Bclw-deficient mice. Nature Genet 18:251-256

Rossi P, Albanesi C, Grimaldi P, Geremia R 1991 Expression of the mRNA for the ligand of c-kit in mouse Sertoli cells. Biochem Biophys Res Commun 176:910-914

Sar M, Hall SH, Wilson EM, French FS 1993 Androgen regulation of Sertoli cells. In: Russell LD, Griswold MD, eds. The Sertoli Cell. Clearwater, FL: Cache River Press; 509-516

Sharpe RM 1994 Regulation of spermatogenesis. In: Knobil E, Neill J, eds. The Physiology of Reproduction. New York: Raven Press; 1363-1434

Skinner MK 1993 Secretion of growth factors and other regulatory factors. In: Russell LD, Griswold MD, eds. The Sertoli Cell. Clearwater, FL; Cache River Press; 237-247

Stevenson D, Jones AR 1985 Production of (S)-3-chloroactaldehyde from (S)-a-chlorohydrin by boar spermatozoa and the inhibition of glyceraldehyde-3-phosphate dehydrogenase in vitro. J Reprod Fertil 74:157-165

Storey BT, Kayne FJ 1978 Energy metabolism of spermatozoa. VII. Interactions between lactate, pyruvate and malate as oxidative substrates for rabbit sperm mitochondria. Biol Reprod 18:527-536

Syed V, Hecht NB 1997 Up-regulation and down-regulation of genes expressed in cocultures of rat Sertoli cells and germ cells. Mol Reprod Dev 47:380-389

Tapanainen JS, Aittomaki K, Mui J, Vaskivuo T, Huhtaniemi IT 1997 Men homozygous for an inactivating mutation of the follicle-stimulating hormone (FSH) receptor gene present variable suppression of spermatogenesis and fertility. Nature Genet 15:205-206

Tourtellotte W, Nagarajan J, Auyeung A, Mueller C, Milbrandt J 1999 Infertility associated with incomplete spermatogenic arrest and oligozoospermia in Efr4-deficient mice. Development 126:5061-5071

Travis AJ, Foster JA, Rosenbaum NA, Visconti PE, Gerton GL, Moss SB 1998 Targeting of a germ cell-specific type 1 hexokinase lacking a porin-binding domain to the mitochondria as well as to the head and fibrous sheath of murine spermatozoa. Mol Biol Cell 9:263-276

Tsunekawa N, Matsumoto M, Tone S, Nishida T, Fumimoto H 1999 The Hsp70 homolog gene, $H s c 70 t$, is expressed under translational control during mouse spermiogenesis. Mol Reprod Dev 52:383-391

Tsuruta JK, O'Brien DA 1995 Sertoli cell-spermatogenic cell interaction: the insulin-like growth factor-II/cation-independent mannose 6-phosphate receptor mediates changes in spermatogenic cell gene expression in mice. Biol Reprod 53:1454-1464

Tsuruta JK, Eddy EM, O'Brien DA 2000 Insulin-like growth factor-II/cation-independent mannose 6-phosphate receptor mediates paracrine interactions during spermatogonial development. Biol Reprod 63:1006-1013

Vidal F, Lopez P, Lopez-Fernandez LA, Ranc F, Scimeca JC, Cuzin F, Rassoulzadegan M 2001 Gene trap analysis of germ cell signaling to Sertoli cells: NGF-TrkA mediated induction of Fra1 and Fos by post-meiotic germ cells. J Cell Sci 114:435-443 
Vijayaraghavan S, Liberty G, Mohan J, Winfrey V, Olson G, Carr D 1999 Isolation and molecular characterization of AKAP110, a novel, sperm-specific protein kinase A-anchoring protein. Mol Endocrinol 13:705-717

Wang P, McCarrey JR, Yang F, Page DC 2001 An abundance of X-linked genes expressed in spermatogonia. Nature Genet 27:422-426

Wang Z, Kim KH 1993 Vitamin A-deficient testis germ cells are arrested at the end of S phase of the cell cycle: a molecular study of the origin of synchronous spermatogenesis in regenerated seminiferous tubules. Biol Reprod 48:1157-1165

Welch JE, Schatte EC, O'Brien DA, Eddy EM 1992 Expression of a glyceraldehyde 3-phosphate dehydrogenase gene specific to mouse spermatogenic cells. Biol Reprod 46:869-878

Welch JE, Brown PR, O'Brien DA, Eddy EM 1995 Genomic organization of a mouse glyceraldehyde 3-phosphate dehydrogenase gene (Gapd-s) expressed in post-meiotic spermatogenic cells. Dev Genet 16:179-189

Willison K, Ashworth A 1987 Mammalian spermatogenic gene expression. Trends Genet 3:351-355

Wolgemuth DJ, Watrin F 1991 List of cloned mouse genes with unique expression patterns during spermatogenesis. Mamm Genome 1:283-288

Yoshida K, Kondoh G, Matsuda Y, Habu T, Nishimune Y, Morita T 1998 The mouse RecA-like gene $\mathrm{Dmcl}$ is required for homologous chromosome synapsis during meiosis. Mol Cell 1:707-718

Yoshinaga K, Nishikawa S, Ogawa M, Hayashi S-I, Kunisada T, Fujimoto T, Nishikawa S-I 1991 Role of c-kit in mouse spermatogenesis: identification of spermatogonia as a specific site of c-kit expression and function. Development 113:689-699

Zabludoff S, Charron M, DeCerbo J, Simukova N, Wright W 2001 Male germ cells regulation transcription of the cathepsin 1 gene by rat Sertoli cells. Endocrinology 142:2318-2327

Zakeri ZF, Wolgemuth DJ, Hunt CR 1988 Identification and sequence analysis of a new member of the mouse HSP70 gene family and characterization of its unique cellular and developmental pattern of expression in the male germ line. Mol Cell Biol 8:2925-2932

Zhang L, Zhou W, Velculescu VE, Kern SE, Hruban RH, Hamilton SR, Vogelstein B, Kinzler KW 1997 Gene expression profiles in normal and cancer cells. Science 276:1268-1272

Zhao G-Q, Deng K, Labosky PA, Liaw L, Hogan BLM 1996 The gene encoding bone morphogenetic protein $8 \mathrm{~B}$ is required for the initiation and maintenance of spermatogenesis in the mouse. Genes Dev 10:1657-1669

Zhao G-Q, Liaw L, Hogan BLM 1998 Bone morphogenetic protein 8A plays a role in the maintenance of spermatogenesis and the integrity of the epididymis. Development 125:11031112

Zhu D, Dix DJ, Eddy EM 1997 HSP70-2 is required for CDC2 kinase activity in meiosis I of mouse spermatocytes. Development 124:3007-3014 


\title{
The Expression of the Follicle-stimulating Hormone Receptor in Spermatogenesis
}

\author{
Leslie L. Heckert* and Michael D. Griswold ${ }^{\dagger}$ \\ *Department of Molecular and Integrative Physiology, University of Kansas Medical Center, \\ Kansas City, Kansas 66160; 'School of Molecular Biosciences, Center for Reproductive Biology, \\ Washington State University, Pullman, Washington 99164-4660
}

\begin{abstract}
Results from experiments using mouse models suggest that the role of follicle-stimulating hormone (FSH) in spermatogenesis is the regulation of Sertoli cell proliferation and, ultimately, the size and spermatogenic capacity of the testis. The regulation of the expression of the FSH receptor (FSHR) gene is very cell specific and plays an initial role in the ultimate response of the Sertoli cells to FSH. The extreme cell specificity and the importance of the FSH response to spermatogenesis have led to an extensive characterization of the promoter of the FSHR gene. Several widely expressed transcription factors - including USF 1 and 2, GATA-1, and SF-1 and potential elements such as an E2F site and an Inr region - have been shown to contribute to the maximal transcription of the transfected FSHR gene. However, these experiments have failed to provide clues as to the cell-specific expression of the FSHR gene. In both cell transfections and in transgenic mice, the promoter can direct expression of transgenes promiscuously. The rodent FSHR promoter contains conserved $\mathrm{CpG}$ dinucleotides that were shown to be methylated in nonexpressing cells and tissue but unmethylated in Sertoli cells. The methylated $\mathrm{CpG}$ sites could interfere with the binding of general transcription factors and/or lead to a repressive chromatin structure in the nonexpressing cells. While yet-undiscovered cell-specific factors may play a role in the expression of the FSHR gene, repression and activation of local chromatin structure are likely to be involved.
\end{abstract}

\section{Biology of the Follicle-stimulating Hormone (FSH) Receptor in Spermatogenesis}

The primary hormonal controls on spermatogenesis involve the action of FSH and testosterone on Sertoli cells. Many of the studies examining the actions of FSH have been done on cultured Sertoli cells from 10- to 30-day-old rats. The Sertoli cells from rats of this age are easily placed in culture and respond to FSH with increased levels of cyclic AMP (cAMP), increased protein synthesis, and increased estradiol production (Fritz et al., 1976). As the age of the rat increases to 40 days or more, the response of Sertoli cells both in culture and in vivo changes. There is a large increase in the phosphodiesterase activity in the cells and the accumulation of cAMP and the subsequent stimulation of specific protein 
synthesis are curtailed (Means et al., 1976; Griswold, 1993). Responses to FSH in Sertoli cells from the adult rat are usually only measurable in the presence of a phophodiesterase inhibitor. In other words, at least in some rodents, the biochemical activities of FSH in the prepubertal animal appear to be assumed by testosterone in the adult. These findings have led to considerable controversy concerning the role of FSH in male reproduction (Zirkin et al., 1994).

In the past several years, some key experiments have clarified the overall biological role of FSH in rodent testes. First, Handelsman and colleagues administered testosterone alone to the gonadotropin-releasing hormone ( $\mathrm{GnRH})$ deficient mouse and showed that this treatment was sufficient for testicular maturation and fertility (Singh et al., 1995). GnRH-deficient mutant mice treated with testosterone implants had normal spermatogenesis but reduced testis size and germ cell numbers. Second, in a parallel study the same group showed that FSH treatment, in addition to testosterone treatment, resulted in GnRH-deficient mice with quantitatively normal spermatogenesis and testes of normal size (Singh and Handelsman, 1996). The exogenous FSH treatment increased testis size by $43 \%$. They concluded from these studies that FSH treatment during the first 2 weeks of life increased Sertoli cell numbers and total sperm production by the mouse testis. Third, it has been demonstrated that prepubertal treatment of normal rats with additional FSH produced larger-than-normal testes and higher total germ cell numbers (Meachem et al., 1996). Finally, several gene knockout experiments have contributed to this issue. A FSH $\beta$ gene knockout has resulted in a line of mice where the males are fertile but have smaller-than-normal testes and reduced germ cell numbers (Kumar et al., 1997). The FSH null mutants had testes that were about half normal size and, at 6-7 weeks of age, the number of epididymal sperm was reduced by $75 \%$. Two groups have produced FSH receptor null mice (FORKO) and have shown that while the males were fertile, the size of the testis and the total number of sperm produced were decreased significantly (Dierich et al., 1998; Abel et al., 2000). A FSH receptor null mutation $(566 \mathrm{C} \rightarrow \mathrm{T})$ has been reported in five male humans (Tapanainen et al., 1997). The men showed variable degrees of spermatogenic failure but were fertile. None of the five had normal sperm parameters and testicular size was reduced but two of the men had fathered two children each.

The use of the mice with the null mutations in the GnRH and FSH genes and the discovery of fertile men with inactivating mutations in the FSH receptor gene have shown clearly that FSH is not required for fertility in mice or men. However, these experiments also support the role for FSH in testis size and, ultimately, in spermatogenic capability. In addition, there is some evidence that, in the FORKO mice, the quality of the sperm produced is decreased (Krishnamurthy et al., 2000). The proliferation of Sertoli cells is maximal in 20- and 21-day-old rat fetuses and declines steadily until the second week after birth, when further cell division is rare (Orth, 1984). The role of FSH in the prenatal 
and newborn rat as a Sertoli cell mitogen is critical in the ultimate spermatogenic capability of the testis. When cytosine arabinoside, an inhibitor of DNA synthesis, was injected into testes of newborn rats, there was an overall $54 \%$ decrease in the size of the Sertoli cell population in the subsequent adults but the ratio of round spermatids to Sertoli cells was similar to controls (Orth et al., 1988). Thus, the Sertoli cell population was limiting to overall sperm production and underscored the importance of FSH in the establishment of the number of Sertoli cells and, therefore, the spermatogenic capacity of the adult (Orth et al., 1988).

\section{The FSH Receptor (FSHR) Gene}

The structure and organization of the FSHR gene was elucidated in both rats and humans, revealing that the receptor is encoded within 10 exons spanning at least $84 \mathrm{~kb}$ of DNA (Figure 1) (Heckert et al., 1992; Gromoll et al., 1996). The first nine exons encode the large extracellular amino-terminal domain of the receptor, while exon 10 encodes the transmembrane and intracellular portions of the protein. Exons 2 through 8 are both similar in length (68-77 bp) and amino acid sequence and, when aligned with each other, a similar repeated motif known as a leucine-rich repeat emerges from the sequences (Sprengel et al., 1990; Heckert et al., 1992). This motif, which is characterized by a pattern of commonly positioned aliphatic amino acids (leucine, isoleucine, valine, and phenylalanine), also was present in exon 9 but the sequence diverged from the patterns observed in exons 2-8. Typically, leucine-rich repeats are involved in cell-cell-specific adhesion and protein-protein interactions. With the FSH receptor, these repeated units are known to be involved in hormone binding and selectivity (Davy et al., 1977; Braun et al., 1991; Kobe and Deisendorfer, 1994). Interestingly, this repeated motif is present in other receptors within the larger superfamily of G protein-coupled receptors, of which FSHR is a member. This includes the other two glycoprotein hormone receptors, luteinizing hormone receptor (LHR) and thyroid stimulating hormone receptor (TSHR), and several more recently described receptors. Together, they make up the growing family of leucine-rich-repeat-containing, G protein-coupled receptors (LGRs) (Loosfelt et al., 1989; McFarland et al., 1989; Parmentiar, 1989; Akamizu et al., 1990; Hsu et al., 1998,2000).

Phylogenetic analysis of members within the LGR family identified three subgroups in which one subgroup is made up of the glycoprotein hormone receptors and the LRG receptors identified in sea anemone, Caenorhabditis elegans, and Drosophila (Hsu et al., 2000). When the gene structures are compared between family members, some striking similarities are unveiled, indicating that a receptor gene identified in cnidarians (e.g., sea anemones, corals, Hydra), the lowest animal group having a nervous system, and the mammalian glycoprotein receptor genes are evolutionarily linked (Figure 1) (Vibede et al., 


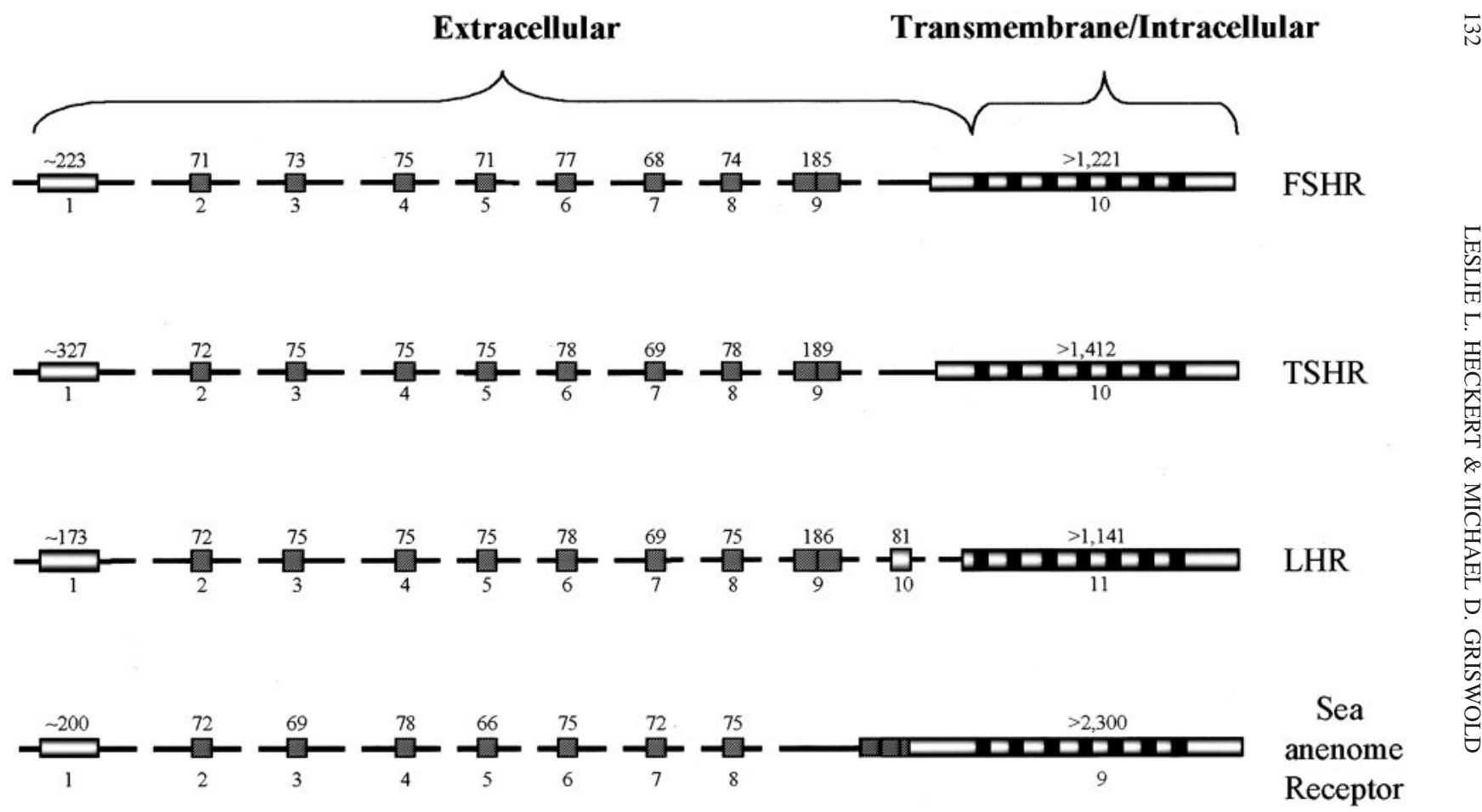

FIG. 1. Comparison of the gene structures for the glycoprotein hormone receptors and sea anenome receptor. Exons are depicted as boxes with their number given below and their size in bp given above. Hatched boxes represent leucine-rich repeats and the black boxes, the seven membrane-spanning domains. For the glycoprotein hormone receptor genes, the structures were derived from the rat FSHR (Heckert et al., 1992), human TSHR (Gross et al., 1991), and rat LHR (Koo et al., 1991; Tsai-Morris et al., 1991). The gene structure for the receptor from sea anemone was derived from studies by Vibede et al. (1998) (Accession nos. AF084384-AF084390). 
1998). While small differences in the number of exons exist among these receptors, the general structure follows that described for FSHR, whereby the leucine-rich motifs within the amino-terminal domain are encoded by multiple small exons and the seven membrane-spanning, C-terminal intracellular domain is encoded by the last and largest exon (Gross et al., 1991; Koo et al., 1991; Tsai-Morris et al., 1991; Vibede et al., 1998). Of note, the phasing of the exons within each of the genes is identical (i.e., they are all in phase 2). Thus, it appears that the FSHR, TSHR, and LHR genes share a common ancestor that arose prior to cnidarian evolution (Vibede et al., 1998).

\section{Transcriptional Regulation of FSHR}

\section{A. TRANSCRIPTION FACTORS AND TRANSFECTIONS}

The expression of FSHR is remarkably cell specific. It is found in testicular Sertoli cells and ovarian granulosa cells, where receptor levels help determine the physiological response to FSH by communicating the pituitary-derived FSH signal to these cells in the gonads. The mechanisms that regulate FSHR gene transcription are of interest because they not only provide insight into endocrine regulation but also into cell-specific gene expression. Most of the data concerning the expression of the FSHR gene were generated by evaluation of its promoter in transient transfection assays and transgenic mice. While progress has been made regarding the mechanisms that control basal transcription and regulation by the hormone FSH, the mechanisms that drive cell-specific expression remain elusive.

Studies on the rat gene have provided the vast majority of information on FSHR transcription and thus, for simplicity, we will review findings on the rat promoter and add important detail and support from the other species where appropriate. Alignment of the DNA sequences of the FSHR promoter regions from rat, mouse, human, and ovine revealed a high degree of sequence conservation spanning from the translational start codon to approximately $1050 \mathrm{bp}$ upstream (Figure 2) (Huhtaniemi et al., 1992; Gromoll et al., 1994; Sairam and Subbarayan, 1997; Heckert et al., 1998). Thereafter, the sequence similarity drops significantly. In the rat gene, transcriptional start sites were mapped to positions -80 and -98 relative to the translational start codon (Heckert et al., 1998) (Figure 2). These sites corresponded well to those identified for the human gene but differed significantly from those originally reported for the mouse and ovine genes, which were located at positions $-534 \mathrm{bp}$ and $-163 \mathrm{bp}$, respectively (Figure 2) (Huhtaniemi et al., 1992; Gromoll et al., 1994; Sairam and Subbarayan, 1997). Examination of the sequences within the proximal promoters revealed that the FSHR gene is a member of a class of genes whose promoters lack a canonical TATA motif. Different classes of TATA-less promoters have been described and include the housekeeping genes, which are a groups of genes 
†ouse (-534)

rat -513 A-TATCAGATGGGACACAGGAAAACAGCAACACCAAATACT----AAAGGTAAATTTTGT mouse -543 A-TAACAGATAGGTCACAGGAAAACAGCAATGCAAAATACT----AAAGGTAAATTTTGT human -453 TCTCTCAGGTGGGCTGGTTGAAGAAATTGATGCTGAAGACT----GAAGGTCCCAG---ovine -511 TGPATCAAGCAGCCTGGAGGAMGACATTGACACC-AAGACTGGAAACAGGTCCCTG----

rat -458 TCCCTTCACATCAOTCGT-------TAGAGATGTGTCATGT----GGATGTG-CTTG-mouse -488 CCCCTTCATGTCAGTAGTACA-----TTAGAGATGTGTCATAT----GGATGTA-CTTGCC human -401 -CCCTTCAC-TTATVAGTACCCCTCTTAGTGATGTGTCATATTGTGGGTTOTGTCTTTTT ovine -456 -ACCTTCAC-TGAGGAACTC---TCTTPATGATOTTTCACACTGCAGATTGCATCTGTTT

rat -413 GGGAGAGGGTCAA-----TTATGTCACTGAGG----AG---AAGACAGTAGTGACCAGTAG mouse -437 GGGA-AGGGACGAACCCTGATACACA human -343 TGGAGAAAGCAA----TCATGTCACTCTGTT--GAG---AaGAGAATGGTGAACAGCAA OVine -401 TGGAGAAAOTCAA----GCGTGTCACTCTETTTTGAGAGAAAAAAAATAGTGACCCACAG rat -364 GGACCCCTGTGCAGCAAAAT-AATGTGAATCTGCTGCTAT---------AGACTGATATC mouse -388 G-ACCTCCATGCAGTAAAAT-AATGTGAATCTGCTGATATCAGTCCATTAGACTGATATC

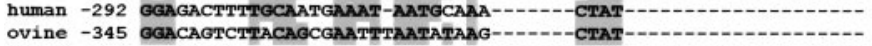

rat -314 TTCAAATATGCACCAAGTTTCTCTTYTCTGTCATTTTGGGGGTCAAGGAATAAAAAATAT mouse -330 TCTAAATATGCACCAAGTTTCTACTTGTTGTCATTTTGG-GGTCAAGGAATAGAAAATAT human -260 TCCAGACATGCCTAATGGTTCTATTTGCTGTGTGCCTTA-GGTCAGGGTGTAAGAAACCC ovine -312 TCTAGACATGCATCAAGTTTCAATTTGCAAAC---------CCAACC---AAAAAAGGT

AP-1

rat -254 AGGTCTTGAAGGATAAAGCAGAAGATtATTGACACACATTAGTCACAT---:------mouse -271 AGGTCTTGAAGGATAAGACAGGTGCTTATTGACAAATATTAATCACATTTCAATCATGTA human -201 AA-TCTTGAAGGAAAACAGAGTAGCTTAT---
ovine -265 A---AAGG--ACAGCGTATCTTCCACGCCCTCTACCTCTCCCACCCCACCCCCA $\longleftrightarrow$ human $(-184)$

rat -205 TTAATATATATAATCACTATTGACACATATTAATTrTACT---TGCCTGGAAGC̈GAC--mouse -211 TTAATACATATAGTTACTACGGACACATATTAATTTTACT---TGCCTGGAAGCGAC--human -173 -.--_ovine -214 cCAA----AGTCACTGCTGTCACTCAGAAATTCTGCTATTTGTCTGGAAGTGACCGA
ovine $(-163)$

E box

rat -151 -TAA-----AAAAAA---AGGCATCTCTTGGTGGGTCAC̈GTGACTTTGCCC̈GTTCTCCA mouse -157 -AAAAGAAAAAAAAA---AAGCATCCTTTAGTGGGTCACGTGACTTTGCT-GTCCTCCA

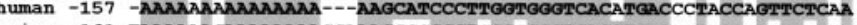
ovine -161 TAAAAAAGAAAAAAAAGGAAGCGGCCCTGGGCGGTCACGTGACCCTACCAGCTCCCAA

$$
\text { Inr GATA }
$$

E.2F rat -101 AGCA-GATCTCP-CTPATCCEGACAGTGTGTGGAGGAGCCTGGGGATCTGTGGAAGTTY mouse -102 AGCA-GATCTCT-CTTATCCGGACAGTGTGTGGAGGAGCCTGGGGAATCCGTGGAGGTTP human -101 GTCA-GATCTCTTCTCATAAGGGCACTETGTGGAG---CTTCTGAGATCTGTGGAGGTTT ovine -101 CGCAAGACCTCTTCTCAAAAGGGCTCAGTGTGGAG---CCTCTGAAATCTGGGCAGGATT $\longrightarrow$ rat $(-98) \underset{\text { human }(-99)}{\longrightarrow} \longrightarrow$ humat $(-89)$

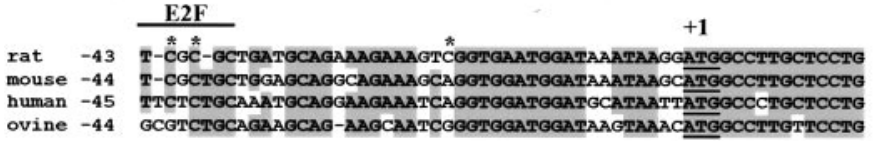

FIG. 2. Sequence comparison of the rat, mouse, human, and ovine FSHR promoter regions. Aligned sequences of the FSHR promoter regions from rat, mouse, human, and ovine, including the translational start site $(+1)$ and approximately $500 \mathrm{bp}$ upstream. Base positions are indicated in the left margin and are relative to the translational start codon (ATG underlined), where adenosine is represented as +1 . Arrows indicate transcriptional start sites identified in each species. DNA elements revealed as important for FSHR regulation are marked with a line over top of the sequence (E2F, Inr, GATA, E box, and AP1 sites). Asterisks mark cytosines within the rat FSHR promoter that were differentially methylated in nonexpressing and expressing cell types. 
that are constitutively active and ubiquitously expressed, and genes that are more restricted in their developmental and tissue-specific expression patterns. The FSHR gene more closely resembles the latter. Like the FSHR, genes that lack a TATA motif often initiate transcription from multiple sites and employ an initiator element (Inr) that assists in the positioning of RNA polymerase II (Smale and Baltimore, 1989; Weis and Reinberg, 1992).

Characterization of the FSHR promoter by transient transfection analysis has provided important insight into basal promoter function but revealed little with regards to the mechanisms responsible for cell-specific expression (Linder et al., 1994; Goetz et al., 1996; Heckert et al., 1998). Promoter evaluation in various cell types, both FSHR expressing and nonexpressing, showed that the promoter activity did not correlate with the cell's ability to express the endogenous FSHR gene. Thus, in primary cultures of Sertoli cells, where the endogenous receptor is actively expressed, promoter activity was only modestly higher than in some nonexpressing cell types but lower than in others (i.e., the MA-10 Leydig cell line). Thus, transient transfection analysis has failed to provide any clear-cut evidence that the $5^{\prime}$ flanking region of the rat gene imparts cell-specific expression. In part, this appears to be upheld in transgenic mice (see below).

Deletion and block-replacement mutagenesis have identified several important response elements within the FSHR promoter region (Linder et al., 1994; Goetz et al., 1996; Heckert et al., 1998). Promoters of various lengths have been included in the studies on FSHR transcription, with the largest fragment spanning out $5000 \mathrm{bp} 5^{\prime}$ to the start of translation (Linder et al., 1994; Heckert et al., 1998). Promoter deletion studies showed that most, if not all, of the positive regulatory elements participating in basal promoter function reside within the first $200 \mathrm{bp}$ of the promoter and suggested that repressor elements also contribute to control of FSHR transcription. Accordingly, the FSHR promoter could be deleted to approximately $200 \mathrm{bp}$ upstream of the translational start site without adversely impacting promoter activity when assayed in either primary cultures of Sertoli cells or the mouse Sertoli cell line, MSC-1 (Linder et al., 1994; Goetz et al., 1996; Heckert et al., 1998). In fact, promoter activity increased with some deletions, suggesting that transcriptional repressors bind elements located between -5000 and -2700 and between -2700 and -200 bp. Similar transcriptional increases were observed when the human and ovine promoters were truncated to within a few hundred bp of the translational start site (Gromoll et al., 1994). No further characterization of the potential repressor elements has been reported.

Elements that have been identified in the regulation of FSHR include an E box, AP-1 site, Inr, E2F site, GATA site, and two steroidogenic factor-1 (SF-1)-like binding sites (Figures 2 and 3) (Goetz et al., 1996; Heckert et al., 1998; Kim and Griswold, 2001; Levallet et al., 2001). Extensive block replacement analysis performed on the first $200 \mathrm{bp}$ of the promoter revealed the E box, 


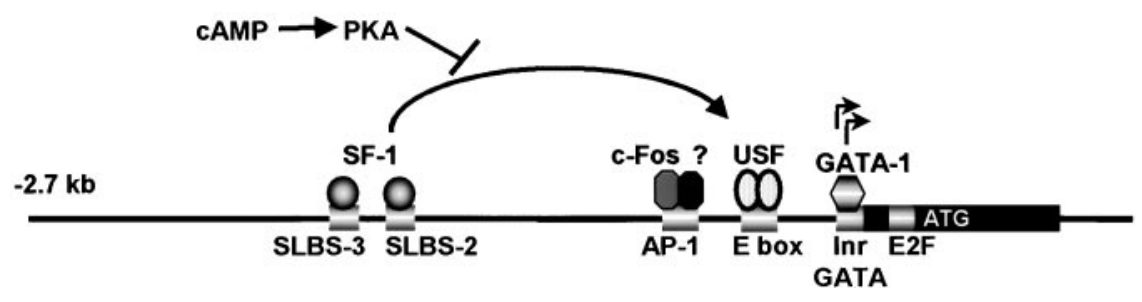

FIG. 3. Model for regulation of the FSHR promoter. Exon 1 of the FSHR gene is indicated as a black rectangle; important regulatory elements are shown by shaded white rectangles. Straight arrows indicate the transcriptional start sites. Regulatory elements, indicated below the depicted promoter, are the Inr, GATA, and E2F sites, located within exon 1, and the E box, AP-1 site, and two SF-1-like binding sites, SLBS-3 and SLBS-2, located within the promoter region. GATA-1 binds the GATA site, the upstream factor (USF) proteins (USF1 and USF2) bind and activate transcription through the E box, while SF1 induces FSHR transcription through the SLBS-2 and SLBS-3 elements. Following binding to SLBS-2 and SLBS-3, SF-1 is postulated to interact either directly or indirectly with the USF proteins, resulting in transcriptional stimulation. Activation of protein kinase A (PKA) by cAMP interferes with the ability of SF1 to transactivate the FSHR promoter. In the absence of the upstream SF-1 binding sites, cAMP stimulation results in the induction of cfos-containing complexes that bind the AP-1 site and stimulate FSHR promoter activity.

Inr, and E2F sites (Goetz et al., 1996; Heckert et al., 1998). A mutation through the $\mathrm{E}$ box had the single greatest impact on rat FSHR promoter activity, while more modest effects were observed with mutations in the E2F and Inr sites. In the rat gene, the E box $\left(5^{\prime}\right.$-CACGTG- $\left.3^{\prime}\right)$ is located at position -120 and shows a high degree of sequence conservation with the other species. Except for the human gene, which has a single G-to-A change at the first guanosine of the core sequence (5'-CACGTG-3' to $5^{\prime}$-CACATG-3'), each of the core bases is conserved across species (Figure 2). A requirement for the $\mathrm{E}$ box also was shown in studies with the ovine promoter (Xing and Sairam, 2001). Mutagenesis of both the core and flanking sequence of the E box revealed that a change in the core's central CG dinucleotide (CACGTG) to either GC or GA diminished promoter activity $60-80 \%$ but was tolerated to a greater extent than a change in the core's flanking bases (5'-GGTCACGTGACTT-3' to 5'-GtaCACGTGtaTT-3') (Heckert et al., 1998). These studies also showed that the sequence difference within the human E box had little impact on promoter function (Figure 2). Furthermore, in vivo genomic footprinting revealed that the $\mathrm{E}$ box within the endogenous FSHR gene is bound by proteins but only in cells that expressed the receptor (Heckert et al., 2000).

DNA/protein binding analysis identified the ubiquitously expressed transcription factors USF1 and USF2 as the predominant proteins binding the E box in Sertoli cells and showed that the bases required for binding to the element mirrored those needed for promoter activity (Figure 3) (Goetz et al., 1996; Heckert et al., 1998). Direct functional support for the USF proteins in transcrip- 
tional regulation of FSHR was obtained when co-transfection of wild-type or mutant forms of the USF proteins with FSHR reporter constructs confirmed that the USF proteins activated the FSHR promoter by binding the proximal E box (Heckert et al., 2000). Furthermore, the first 40 amino acids of the USF proteins, as well as regions encoded by exon 5 and the USF-specific region (USR), were necessary for FSHR transcription. The observations that ubiquitously expressed USF proteins regulate transcription through the $\mathrm{E}$ box and that the $\mathrm{E}$ box of the endogenous FSHR gene is occupied by proteins only in cells that express the receptor suggest that the chromatin structure in expressing cells somehow allows access of the USF proteins to the $\mathrm{E}$ box, while, in nonexpressing cells, the structure is different and nonpermissive for USF binding.

Like the E box, the E2F, GATA, and Inr sites were implicated in the regulation of endogenous FSHR gene activity by in vivo genomic footprint analysis (Kim and Griswold, 2001). In addition, these elements have been examined on the FSHR promoter by mutagenesis and binding analysis but not as extensively as the E box. In addition to the footprint analysis, the E2F site (5'-TTTTCGCGC-3'), located from -38 to -46 , was implicated by a blockreplacement mutation that encompassed the entire site as well as by more refined mutations that specifically changed bases within the conserved element (Heckert et al., 1998). Examination of the sequence of this element revealed that it is significantly less conserved across species than the E box (Figure 2). The largest impact of this element was observed with a mutation within the second GC pair (underlined above), which decreased promoter activity to the same degree $(\approx 50 \%)$ as the full block-replacement mutant (Heckert et al., 1998). Interestingly, the position of this dinucleotide is conserved in each of the four species (Figure 2). While specific protein complexes were shown to bind the E2F site, they do not appear to contain the E2F1 protein, as antibodies against this factor failed to produce a supershifted complex (Griswold and Kim, 2001; Kim and Griswold, 2001).

A role for the Inr was determined from analysis of 3' deletion mutants and specific mutations within the element (Goetz et al., 1996; Heckert et al., 1998). This region of the promoter encompasses both transcriptional start sites, spanning from -103 to -80 . Specific protein complexes were shown to bind this region of the promoter and were competed by the well-characterized Inr from the deoxynucleotidyltransferase gene (Goetz et al., 1996). Interestingly, mutation of a GATA site located within this region significantly diminished FSHR promoter activity, suggesting that a member of the GATA family of transcription factors is important for FSHR promoter function (Kim and Griswold, 2001). In nuclear extracts from Sertoli cells, the GATA-1 transcription factor was found to bind this element, further supporting this suggestion. While GATA-1 binds within the Inr region of the promoter, several other proteins that interact with this element 
remain unidentified and may also contribute to FSHR transcription (Kim and Griswold, 2001).

Recently, studies on both the rat and mouse FSHR promoters demonstrated that the orphan nuclear receptor SF-1 regulates transcription of the FSHR gene. SF-1 is a key regulator of endocrine function and sex determination. Its expression is limited primarily to cells of the gonads, adrenal, pituitary, and ventral medial hypothalamus, where it is thought to contribute to cell-specific properties of genes expressed within these tissues (Hatano et al., 1994; Ikeda et al., 1994; Ingraham et al., 1994; Shen et al., 1994; Morohashi et al., 1995; Roselli et al., 1997). SF-1 is known to regulate transcription of several key steroidogenic enzymes as well as genes involved in the production of the gonadotropin hormones, including the luteinizing hormone (LH) $\beta$ subunit, the GnRH receptor, and the inhibin $\alpha$ subunit (Rice et al., 1991; Morohashi et al., 1992; Barnhart and Mellon, 1994; Asa et al., 1996; Keri and Nilson, 1996; Caron et al., 1997; Duval et al., 1997; Halvorson et al., 1998,1999; Dorn et al., 1999; Ngan et al., 1999; Tremblay and Drouin, 1999; Wolfe, 1999; Wolfe and Call, 1999; Ito et al., 2000). Identification of FSHR as a target gene for SF-1 regulation revealed yet another level in which SF-1 acts to control endocrine homeostasis.

Transfection studies with both the mouse and rat FSHR promoters showed that transcriptional activity increased substantially when co-transfected with an expression vector for SF-1 (Heckert, 2001; Levallet et al., 2001). In the mouse promoter, two SF-1-like binding sites (SLBS-2 and SLBS-3) (Figure 3) were required for response to the orphan receptor. In the rat promoter, SF-1 response was mapped to several regions upstream of -743 and within the first 200 bases of the promoter. Within the latter region, the $\mathrm{E}$ box was shown to be critical for response to SF-1, not only for the proximal promoter but also for response generated through the upstream site(s). In the absence of SF-1 binding to the $\mathrm{E}$ box, a role for the USF proteins in integrating the SF-1 response was implicated and subsequently substantiated through co-transfection studies with mutant USF proteins (Heckert, 2001). Notably, the requirement for USF in response to SF-1 was unique to the FSHR promoter, as USF levels in the cell did not influence SF-1 activation of the equine $\mathrm{LH} \beta$ promoter. Unlike the mouse promoter, upstream SF-1 binding sites were not identified in the rat gene but analysis of a series of truncated promoters suggested that multiple response elements were required in the upstream region.

Although the mechanisms by which SF-1 and USF cooperate to regulate FSHR are not fully understood, the finding that these transcription factors act in concert to activate transcription provides important insight into FSHR gene regulation, the transactivation function of SF-1, and the expanding physiological roles of SF-1 in the hypothalamic-pituitary-gonadal axis. Within this axis, SF-1 appears to regulate both FSH production and response. Thus, SF-1 can directly influence FSH production in the pituitary through its regulation of the $\alpha$ subunit 
of the FSH regulatory protein inhibin. SF-1 can also influence FSH response in the testis through its regulation of FSHR. Interestingly, activation of the inhibin $\alpha$ subunit by SF-1 has been shown to dramatically increase in the presence of activated protein kinase A (PKA), the major downstream effector of FSH action in the gonads (Ito et al., 2000). Thus, increasing levels of FSH in the presence of SF-1 should dramatically stimulate inhibin levels produced by the gonads, which would then feed back on the pituitary to specifically decrease FSH production. Remarkably, studies with the rat promoter revealed that activated PKA blocked SF-1-stimulated transcription of the FSHR promoter (Heckert, 2001). Thus, with SF-1 poised to regulate these two genes, FSH stimulation can lead to opposite transcriptional effects, resulting in a rise in $\alpha$ inhibin and a fall in FSHR, a result that would dramatically decrease FSH signaling in the gonads.

\section{B. REGULATION BY CAMP}

In males, FSHR mRNA levels are significantly reduced in response to FSH or agents that stimulate the cAMP pathway (Themmen et al., 1991; Maguire et al., 1997; Griswold et al., 2001). While earlier studies have suggested that post-transcriptional mechanisms are responsible, two more recent experiments implicate the involvement of transcriptional events (Maguire et al., 1997; Griswold et al., 2001). These latter studies showed that the transcriptional inhibitor actinomycin D blocked receptor mRNA downregulation and that FSHR hnRNA decreased in the presence of FSH (Maguire et al., 1997; Griswold et al., 2001). However, an FSHR promoter construct containing sequence from -383 bp to -1 bp was not negatively regulated by FSH treatment (Griswold et al., 2001). In fact, promoter activity increased approximately three-fold in the presence of FSH, an event that required an AP-1 site located at position - 213 in the promoter (Figures 2 and 3). The AP-1 element also was shown to specifically bind FSH-induced protein complexes that contained the transcription factor c-fos. While these studies did not resolve the mechanism by which FSHR transcription is diminished by FSH, they did appear to rule out a role for the transcriptional repressor inducible cAMP early repressor (ICER), which is also known to bind the AP-1 site (Monaco et al., 1995; Griswold et al., 2001). As mentioned above, a mechanism for the inhibitory effects of the cAMP pathway on FSHR transcription was suggested recently by the observation that the SF-1 activation of the FSHR promoter was blocked when this pathway was stimulated (Heckert, 2001). Thus, under conditions where SF-1 is activating the gene, stimulation of the cells with FSH would result in decreased transcription.

\section{TRANSGENIC MICE}

Studies in transgenic mice have been used to help delineate the region of the FSHR gene needed for cell-specific expression. In two separate studies, $5000 \mathrm{bp}$ 
of the rat FSHR promoter were used to drive expression of a reporter gene, either $\beta$-galactosidase or Cre recombinase, in transgenic mice (Linder et al., 1994; Heckert et al., 2000). In the first study, $\beta$-galactosidase expression was measured in various tissues by Northern blot analysis to help assess cell-specific expression (Linder et al., 1994). In two independent transgenic lines, evaluation of liver, thyroid, kidney, ovary, and epididymal RNA samples revealed that only testis and ovary expressed the transgene. While the cell types expressing the transgene were not determined, the results indicated that tissue-specific expression is directed by the first $5000 \mathrm{bp}$ of FSHR 5' flanking sequence, indirectly suggesting that cell specificity is directed by this portion of the gene as well.

In the second study, Cre recombinase expression was evaluated by reverse transcriptase polymerase chain reaction (RT-PCR) in RNA samples collected from testis, ovary, heart, lung, liver, kidney, brain, bladder, stomach, spleen, and eyes (Heckert et al., 2000). A second transgene containing only $198 \mathrm{bp}$ of 5' flanking sequence also was examined in this study. Of eight transgenic lines examined, the $5000 \mathrm{bp}$ Cre construct expressed in both testis and brain. Three of these lines also had marked expression in other tissues. Further examination revealed that testis expression of the transgene did not follow the same temporal pattern as that of the endogenous FSHR gene in that it was not expressed at postnatal day 10 . Interestingly, the smaller -198 Cre transgene appeared to better restrict expression, as three transgenic lines only expressed Cre recombinase in the testis. However, like the 5000 bp Cre construct, no expression was observed at postnatal day 10, despite expression of the endogenous FSHR gene. Closer inspection of two of the -198 bp Cre lines revealed that testis expression in mice either 27 or 50 days of age was mostly, if not entirely, due to inappropriate expression in germ cells. Thus, the inaccurate temporal expression of the transgenes and their expression in germ cells led to the conclusion that the promoter sequences did not contain enough information to properly restrict or express the receptor to Sertoli cells of the testis. It is important to note, however, that examination of the cell types expressing the transgene was limited to studies on the smaller construct, leaving open the possibility that Cre expressed from the $5000 \mathrm{bp}$ promoter occurred within the Sertoli cells but later than that observed for the endogenous gene. If true, this would indicate that different mechanisms control early (embryonic/early postnatal) and late (postpubertal) expression of the FSHR gene.

Studies on the luteinizing hormone receptor (LHR), a gene evolutionarily linked to FSHR, provided support for this theory, as they implicated different transcriptional mechanisms for early and late LHR expression (Hamalainen et al., 1999). Transgenic mice carrying $2 \mathrm{~kb}$ of the murine LHR promoter exhibited remarkable similarities to the FSHR transgenic mice. Thus, three out of five LHR transgenic lines exhibited expression within the testis but failed to express in the ovary, while all lines showed ectopic expression in the brain. Also of note was 
the finding that testis expression of the LHR transgene was not observed between weeks 1 and 3 after birth but appeared at 5 weeks of age in both the germ cells and Leydig cells. Because the transgene did not follow the temporal expression of the endogenous gene, separate transcriptional mechanisms for early and late expression were implicated. Further evaluation of the FSHR transgenes is necessary to determine if this is true for FSHR too. Thus, while it is currently unclear if elements sufficient for adult Sertoli cell expression of FSHR reside in the 5000-bp promoter, we can conclude from these studies that the evaluated promoters lack sufficient information to direct Sertoli cell expression in early postnatal testis as well as the information needed to prevent expression outside of this cell type.

\section{Role of Methylation and Chromatin Structure in FSHR Expression}

As described above, the region of the core promoter between -383 and +1 bp relative to the translational start site contains two Inr-mediated transcriptional start sites, an E box, and several other sites involved in transcriptional regulation of this gene. No cell-specific DNA-protein complexes have been found in the FSHR promoter that could mitigate the extreme cell specificity of transcription (Heckert et al., 1992,1998; Linder et al., 1994; Goetz et al., 1996). Reporter gene assays driven by the rat FSHR promoter have shown that this region could promote the promiscuous transcription of the gene in several cell lines in which the expression of the endogenous FSHR gene was originally repressed (Linder et al., 1994).

In mammals, DNA methylation patterns appear to play a critical role in terms of gene regulation in differentiating and differentiated cells (el-Deiry et al., 1991; Tate and Bird, 1993; Eden and Cedar, 1994). There is considerable evidence that a variety of cell-specific genes have distinctive DNA methylation patterns in expressing and nonexpressing cells, and undergo demethylation at the stage of gene activation (el-Deiry et al., 1991; Eden and Cedar, 1994; Beard et al., 1995; Nickel et al., 1995). The core promoter of the rat FSHR gene does not contain $\mathrm{CpG}$ islands but does contain a few specific $\mathrm{CpG}$ dinucleotides that are potential methylation sites (Heckert et al., 1992). Using bisulfite-based DNA sequencing, seven specific CpG sites within the FSHR core promoter were shown to be methylated in cells that did not express the gene and unmethylated in Sertoli cells (Figure 2) (McGuinness et al., 1994; Griswold and Kim, 2001).

In addition, the mouse Sertoli cell line (MSC-1) derived from a testicular tumor has an inactive FSHR promoter (McGuinness et al., 1994) and the inactive state of transcription in MSC-1 cells correlated with the cytosine methylation of the core promoter of the FSHR gene (Griswold and Kim, 2001). Treatment of MSC-1 cells with 5-azacytidine (5-azaCdR) resulted in the demethylation of the 
mouse FSHR promoter and reactivated the transcription of the FSHR gene. The drug 5-azaCdR is known to block DNA methylation in newly replicated DNA molecules and has been used to activate silent genes in cells whose expression of that gene is controlled by DNA methylation (Ottaviano et al., 1994; Bender et al., 1998; Bovenzi et al., 1999).

DNA methylation in the proximal promoter could potentially lead to regulation of the expression of the FSHR gene by directly interfering with or changing protein-DNA interactions at those sites. This changed interaction could lead to changes in transcription factor-DNA interactions or altered chromatin structure. Within the 320-bp region of the rat FSHR core promoter, four of the seven potential 5-methylcytosine residues are located within known protein-DNA binding sites (Figure 2). The methyl group(s) within cis-acting regulatory elements could interrupt the binding of trans-acting factors to their target sites on the promoter. For example, DNA methylation of the E box element (CACGTG, -124/-119) in the FSHR promoter region inhibited the binding of nuclear extracts from Sertoli cells by 3.5 -fold (Griswold and Kim, 2001). It also is clear that cytosine methylation can exert inhibitory effects through altering chromatin structure and not by inhibition of the transcription machinery directly (Tate and Bird, 1993; Richardson and Yung, 1999). A repressive chromatin structure can result from the recruitment of histone deacetylases (HDACs) by methylated cytosine binding protein (MeCP2) in nucleosomal DNA (Jones et al., 1998; Nan et al., 1998). It is possible that the FSHR promoter is an example of a gene where $\mathrm{CpG}$ methylation both inhibits transcription factor binding directly and promotes the formation of a repressive chromatin structure.

The chromatin structure surrounding the proximal promoter in the rat FSHR receptor has been examined in nucleosome reconstitution experiments (M.D. Griswold, unpublished data). The proximal promoter was reconstituted into nucleosomes using $319 \mathrm{bp}$ and chicken erythrocyte histones. The resulting nucleosomal locations were then mapped by hydroxyl radical footprinting. Examination of the sequence in the core promoter reveals the presence of possible strong nucleosome positioning sequences just $5^{\prime}$ of the $\mathrm{E}$ box. The hydroxyl radical footprinting showed that one nucleosome positioned very tightly over the region of the promoter from approximately -200 to $-400 \mathrm{bp}$. The second nucleosome positioned very weakly over the region from -100 to +100 and the $E$ box appeared to be localized to the linker region. The seven $\mathrm{CpG}$ methylation sites were positioned mainly within the second nucleosome but results of reconstitution experiments with promoter DNA where the $\mathrm{CpG}$ sites were methylated were unchanged from the first experiment. These results lead to a model (Figure 4) whereby the tightly positioned nucleosome helps to define the promoter site. The repressed state of the promoter could be achieved when the methylated DNA binds 


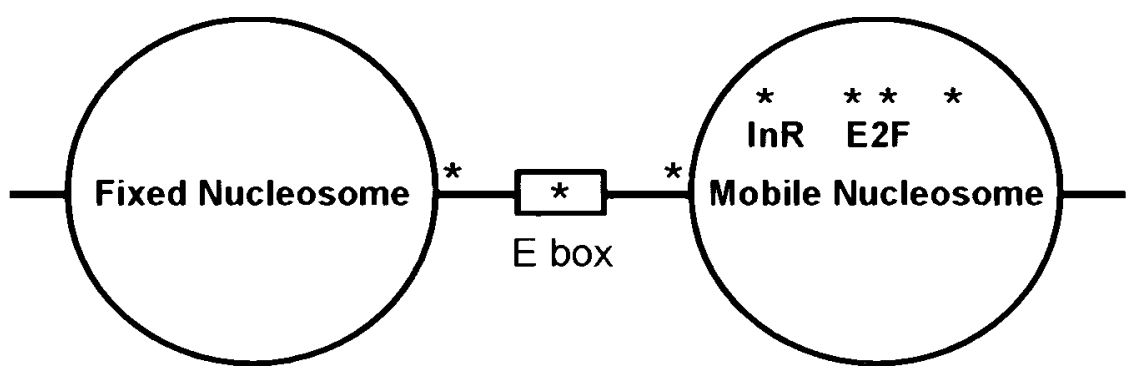

FIG. 4. Positioning of nucleosomes over the proximal FSHR promoter. Nucleosomes were reconstituted using 319 base pairs of the proximal FSHR promoter and chicken erythrocyte histones (Reeves and Wolffe, 1996). The positions of the reconstituted nucleosomes on the FSHR promoter were then mapped using hydroxyl radical footprinting (Tullius et al., 1987). The results from this analysis are shown schematically. One nucleosome positioned very tightly over the region of the promoter from approximately -200 to $-400 \mathrm{bp}$, while the second nucleosome positioned very weakly over the region from -100 to +100 . The $\mathrm{E}$ box appeared to be localized to the linker region and the location of the seven CpG methylation sites $\left(^{*}\right)$ are shown. The model suggests that the upstream nucleosome positions the start site, while the nucleosome over the promoter is much more mobile and can be displaced by demethylation and recruitment of the transcriptional machinery. The methylation or demethylation of the $\mathrm{CpG}$ sites could ultimately determine the ability of cells to express FSHR.

methyl binding proteins and ultimately histone deacetylases and other transcription inactivators. This binding activity could serve to fix the second nucleosome located over the promoter region in place. In addition, methylation of the E box in the linker DNA and perhaps methylation of other sites such as the E2F site could directly prevent binding of a transcription factor. Activation occurs when the DNA is demethylated. Transcription factors could then bind to the DNA and the second nucleosome could be displaced.

Altogether, the data on methylation and chromatin structure support the notion that the transcription of the rat/mouse FSHR gene is regulated by methylation/demethylation of $\mathrm{CpG}$ dinucleotides in the proximal promoter. Although CpG dinucleotides are not abundant in the rat/mouse FSHR promoter, DNA methylation at very specific sites within the promoter correlated with gene inactivation. The maintenance of tissue specificity in gene expression probably requires multiple mechanisms. Our studies support the idea that DNA methylation plays a major role in this process in the regulation of the rat/mouse FSHR gene. It is noteworthy that the proximal $231 \mathrm{bp}$ of the promoter for the human FSHR gene, while $80 \%$ homologous to the promoter for the rat/mouse gene in this region, lacks all of the seven specific $\mathrm{CpG}$ sites and must be regulated by some other mechanism (Gromoll et al., 1996). 


\section{REFERENCES}

Abel MH, Wootton AN, Wilkins V, Huhtaniemi I, Knight PG, Charlton HM 2000 The effect of a null mutation in the follicle-stimulating hormone receptor gene on mouse reproduction. Endocrinology 141(5):1795-1803

Akamizu T, Kosugi S, Kohn LD 1990 Thyrotropin receptor processing and interaction with thyrotropin. Biochem Biophys Res Comm 169(3):947-952

Asa SL, Bamberger AM, Cao B, Wong M, Parker KL, Ezzat S 1996 The transcription activator steroidogenic factor-1 is preferentially expressed in the human pituitary gonadotroph. J Clin Endocrinol Metab 81(6):2165-2170

Barnhart KM, Mellon PL 1994 The orphan nuclear receptor, steroidogenic factor-1, regulates the glycoprotein hormone alpha-subunit gene in pituitary gonadotropes. Mol Endocrinol 8(7): $878-885$

Beard C, Li E, Jaenisch R 1995 Loss of methylation activates Xist in somatic but not in embryonic cells. Genes Dev 9(19):2325-2334

Bender CM, Pao MM, Jones PA 1998 Inhibition of DNA methylation by 5-aza-2'-deoxycytidine suppresses the growth of human tumor cell lines. Cancer Res 58(1):95-101

Bovenzi V, Le NL, Cote S, Sinnett D, Momparler LF, Momparler RL 1999 DNA methylation of retinoic acid receptor beta in breast cancer and possible therapeutic role of 5-aza-2'deoxycytidine. Anticancer Drugs 10(5):471-476

Braun T, Schofield PR, Sprengel R 1991 Amino-terminal leucine rich repeats in gonadotropin receptors determine hormone selectivity. EMBO J 10:1885-1890

Caron KM, Clark BJ, Ikeda Y, Parker KL 1997 Steroidogenic factor 1 acts at all levels of the reproductive axis. Steroids 62(1):53-56

Davy M, Torjesen PA, Aakavaag A 1977 Demonstration of an FSH receptor in a functioning granulosa cell tumour. The effect of gonadotrophin treatment on its viability following transplantation to nude mice. Acta Endocrinol (Copenh) 85(3):615-623

Dierich A, Sairam MR, Monaco L, Fimia GM, Gansmuller A, LeMeur M, Sassone-Corsi P 1998 Impairing follicle-stimulating hormone (FSH) signaling in vivo: targeted disruption of the FSH receptor leads to aberrant gametogenesis and hormonal imbalance. Proc Natl Acad Sci USA 95(23):13612-13617

Dorn C, Ou Q, Svaren J, Crawford PA, Sadovsky Y 1999 Activation of luteinizing hormone beta gene by gonadotropin-releasing hormone requires the synergy of early growth response-1 and steroidogenic factor-1. J Biol Chem 274(20):13870-13876

Duval DL, Nelson SE, Clay CM 1997 A binding site for steroidogenic factor-1 is part of a complex enhancer that mediates expression of the murine gonadotropin-releasing hormone receptor gene. Biol Reprod 56(1):160-168

Eden S, Cedar H 1994 Role of DNA methylation in the regulation of transcription. Curr Opin Genet Dev 4(2):255-259

el-Deiry WS, Nelkin BD, Celano P, Yen RW, Falco JP, Hamilton SR, Baylin SB 1991 High expression of the DNA methyltransferase gene characterizes human neoplastic cells and progression stages of colon cancer. Proc Natl Acad Sci USA 88(8):3470-3474

Fritz KB, Rommerts FG, Louis BG, Dorrington JH 1976 Regulation by FSH and dibutyryl cyclic AMP of the formation of androgen-binding protein in Sertoli cell-enriched cultures. J Reprod Fertil 46:17-24

Goetz TL, Lloyd TL, Griswold MD 1996 Role of E box and initiator region in the expression of the rat follicle-stimulating hormone receptor. J Biol Chem 271(52):33317-33324

Griswold MD 1993 Action of FSH on mammalian Sertoli cells. In: Griswold MD, Russell LD, eds. The Sertoli Cell. Clearwater, FL: Cache River Press; 493-508 
Griswold MD, Kim JS 2001 Site-specific methylation of the promoter alters deoxyribonucleic acidprotein interactions and prevents follicle-stimulating hormone receptor gene transcription. Biol Reprod 64(2):602-610

Griswold MD, Kim J, Tribley WA 2001 Mechanisms involved in the homologous down-regulation of transcription of the follicle-stimulating hormone receptor gene in Sertoli cells. Mol Cell Endocrinol 173(1-2):95-107

Gromoll J, Ried T, Holtgreve-Grez H, Nieschlag E, Gudermann T 1994 Localization of the human FSH receptor to chromosome 2 p21 using a genomic probe comprising exon 10. J Mol Endocrinol 12(3):265-271

Gromoll J, Pekel E, Nieschlag E 1996 The structure and organization of the human folliclestimulating hormone receptor (FSHR) gene. Genomics 35(2):308-311

Gross B, Misrahi M, Sar S, Milgrom E 1991 Composite structure of the human thyrotropin receptor gene. Biochem Biophys Res Comm 177:679-687

Halvorson LM, Ito M, Jameson JL, Chin WW 1998 Steroidogenic factor-1 and early growth response protein 1 act through two composite DNA binding sites to regulate luteinizing hormone beta- subunit gene expression. J Biol Chem 273(24):14712-14720

Halvorson LM, Kaiser UB, Chin WW 1999 The protein kinase C system acts through the early growth response protein 1 to increase LHbeta gene expression in synergy with steroidogenic factor-1. Mol Endocrinol 13(1):106-116

Hamalainen T, Poutanen M, Huhtaniemi I 1999 Age- and sex-specific promoter function of a 2-kilobase 5 '-flanking sequence of the murine luteinizing hormone receptor gene in transgenic mice. Endocrinology 140(11):5322-5329

Hatano O, Takayama K, Imai T, Waterman MR, Takakusu A, Omura T, Morohashi K 1994 Sex-dependent expression of a transcription factor, Ad4BP, regulating steroidogenic P-450 genes in the gonads during prenatal and postnatal rat development. Development 120(10): 2787-2797

Heckert LL 2001 Activation of the rat follicle-stimulating hormone receptor promoter by steroidogenic factor 1 is blocked by protein kinase a and requires upstream stimulatory factor binding to a proximal e box element. Mol Endocrinol 15(5):704-715

Heckert LL, Daley IJ, Griswold MD 1992 Structural organization of the follicle-stimulating hormone receptor gene. Mol Endocrinol 6(1):70-80

Heckert LL, Daggett MA, Chen J 1998 Multiple promoter elements contribute to activity of the follicle-stimulating hormone receptor (FSHR) gene in testicular Sertoli cells. Mol Endocrinol 12(10): 1499-1512

Heckert LL, Sawadogo M, Daggett MA, Chen JK 2000 The USF proteins regulate transcription of the follicle-stimulating hormone receptor but are insufficient for cell-specific expression. Mol Endocrinol 14(11):1836-1848

Hsu SY, Liang SG, Hsueh AJ 1998 Characterization of two LGR genes homologous to gonadotropin and thyrotropin receptors with extracellular leucine-rich repeats and a $\mathrm{G}$ proteincoupled, seven-transmembrane region. Mol Endocrinol 12(12):1830-1845

Hsu SY, Kudo M, Chen T, Nakabayashi K, Bhalla A, van der Spek PJ, van Duin M, Hsueh AJ 2000 The three subfamilies of leucine-rich repeat-containing $\mathrm{G}$ protein-coupled receptors (LGR): identification of LGR6 and LGR7 and the signaling mechanism for LGR7. Mol Endocrinol 14(8):1257-1271

Huhtaniemi IT, Eskola V, Pakarinen P, Matikainen T, Sprengel R 1992 The murine luteinizing hormone and follicle-stimulating hormone receptor genes: transcription initiation sites, putative promoter sequences and promoter activity. Mol Cell Endocrinol 88:55-66

Ikeda Y, Shen WH, Ingraham HA, Parker KL 1994 Developmental expression of mouse steroidogenic factor-1, an essential regulator of the steroid hydroxylases. Mol Endocrinol $8(5): 654-662$ 
Ingraham HA, Lala DS, Ikeda Y, Luo X, Shen WH, Nachtigal MW, Abbud R, Nilson JH, Parker KL 1994 The nuclear receptor steroidogenic factor 1 acts at multiple levels of the reproductive axis. Genes Dev 8(19):2302-2312

Ito M, Park Y, Weck J, Mayo KE, Jameson JL 2000 Synergistic activation of the inhibin alpha-promoter by steroidogenic factor-1 and cyclic adenosine $3{ }^{\prime}, 5^{\prime}$-monophosphate. Mol Endocrinol 14(1):66-81

Jones PL, Veenstra GJ, Wade PA, Vermaak D, Kass SU, Landsberger N, Strouboulis J, Wolffe AP 1998 Methylated DNA and MeCP2 recruit histone deacetylase to repress transcription. Nature Genet 19(2):187-191

Keri RA, Nilson JH 1996 A steroidogenic factor-1 binding site is required for activity of the luteinizing hormone beta subunit promoter in gonadotropes of transgenic mice. J Biol Chem 271(18):10782-10785

Kim JS, Griswold MD 2001 E2F and GATA-1 are required for the Sertoli cell-specific promoter activity of the follicle-stimulating hormone receptor (FSHR) gene. J Androl 22(4):629-639

Kobe B, Deisendorfer J 1994 The leucine-rich repeat: a versatile binding motif. Trends Biochem Sci 19:415-421

Koo YB, Ji I, Slaughter RG, Ji TH 1991 Structure of the leuteinizing hormone receptor gene and multiple exons of the coding sequence. Endocrinology 128:2297-2650

Krishnamurthy H, Danilovich N, Morales CR, Sairam MR 2000 Qualitative and quantitative decline in spermatogenesis of the follicle-stimulating hormone receptor knockout (FORKO) mouse. Biol Reprod 62(5):1146-1159

Kumar TR, Wang Y, Lu N, Matzuk M 1997 Follicle stimulating hormone is required for ovarian follicle maturation but not male fertility. Nature Genet 15:201-204

Levallet J, Koskimies P, Rahman N, Huhtaniemi I 2001 The promoter of murine folliclestimulating hormone receptor: functional characterization and regulation by transcription factor steroidogenic factor 1. Mol Endocrinol 15(1):80-92

Linder CC, Heckert LL, Goetz TL, Griswold MD 1994 Follicle-stimulating hormone receptor gene promoter activity. Endocr J 2:957-966

Loosfelt H, Misrahi M, Atger M, Salesse R, Thi MTVH-L, Jolivet A, Guiochon-Mantel A, Sar S, Jallal B, Garnier J, Milgrom E 1989 Cloning and sequencing of porcine LH-hCG receptor cDNA: variants lacking transmembrane domain. Science 245:525-528

Maguire SM, Tribley WA, Griswold MD 1997 Follicle-stimulating hormone (FSH) regulates the expression of FSH receptor messenger ribonucleic acid in cultured Sertoli cells and in hypophysectomized rat testis. Biol Reprod 56(5):1106-1111

McFarland KC, Sprengel R, Phillips HS, Köhler M, Rosemblit N, Nikolics K, Segaloff DL, Seeburg PH 1989 Lutropin-choriogonadotropin receptor: an unusual member of the G protein-coupled receptor family. Science 245:494-499

McGuinness MP, Linder CC, Morales CR, Heckert LL, Pikus J, Griswold MD 1994 Relationship of a mouse Sertoli cell line (MSC-1) to normal Sertoli cells. Biol Reprod 51(1):116-124

Meachem SJ, McLachlan R, de Kretser D, Robertson DM, Wreford NG 1996 Neonatal exposure of rats to recombinant follicle stimulating hormone increases adult Sertoli cell and spermatogenic cell numbers. Biol Reprod 54:36-44

Means AR, Fakunding JL, Huckins C, Tindall DJ, Vitale R 1976 Follicle-stimulating hormone, the Sertoli cell, and spermatogenesis. Recent Progr Horm Res 32(477):477-527

Monaco L, Foulkes NS, Sassone-Corsi P 1995 Pituitary follicle-stimulating hormone (FSH) induces CREM gene expression in Sertoli cells: involvement in long-term desensitization of the FSH receptor. Proc Natl Acad Sci USA 92(23):10673-10677

Morohashi K, Honda S, Inomata Y, Handa H, Omura T 1992 A common trans-acting factor, Ad4-binding protein, to the promoters of steroidogenic P-450s. J Biol Chem 267(25):1791317919 
Morohashi K, Hatano O, Nomura M, Takayama K, Hara M, Yoshii H, Takakusu A, Omura T 1995 Function and distribution of a steroidogenic cell-specific transcription factor, Ad4BP. J Steroid Biochem Mol Biol 53(1-6):81-88

Nan X, Ng HH, Johnson CA, Laherty CD, Turner BM, Eisenman RN, Bird A 1998 Transcriptional repression by the methyl-CpG-binding protein MeCP2 involves a histone deacetylase complex. Nature 393(6683):386-389

Ngan ES, Cheng PK, Leung PC, Chow BK 1999 Steroidogenic factor-1 interacts with a gonadotrope-specific element within the first exon of the human gonadotropin-releasing hormone receptor gene to mediate gonadotrope-specific expression. Endocrinology 140(6): 2452-2462

Nickel J, Short ML, Schmitz A, Eggert M, Renkawitz R 1995 Methylation of the mouse M-lysozyme downstream enhancer inhibits heterotetrameric GABP binding. Nucleic Acids Res 23(23):4785-4792

Orth J 1984 The role of follicle-stimulating hormone in controlling Sertoli cells proliferation in testes of fetal rats. Endocrinology 115:1248-1255

Orth JM, Gunsalus GL, Lamperti AA 1988 Evidence from Sertoli cell-depleted rats indicates that spermatid number in adults depends on numbers of Sertoli cells produced during perinatal development. Endocrinology 122(3):787-794

Ottaviano YL, Issa JP, Parl FF, Smith HS, Baylin SB, Davidson NE 1994 Methylation of the estrogen receptor gene $\mathrm{CpG}$ island marks loss of estrogen receptor expression in human breast cancer cells. Cancer Res 54(10):2552-2555

Parmentiar M 1989 Molecular cloning of the thyrotropin receptor. Science 246:1620-1622

Reeves R, Wolffe A 1996 Substrate structure influences binding of the non-histone protein HMI-Y to free and nucleosomal DNA. Biochemistry 35:5063-5074

Rice DA, Mouw AR, Bogerd AM, Parker KL 1991 A shared promoter element regulates the expression of three steroidogenic enzymes. Mol Endocrinol 5(10):1552-1561

Richardson B, Yung R 1999 Role of DNA methylation in the regulation of cell function. J Lab Clin Med 134(4):333-340

Roselli CE, Jorgensen EZ, Doyle MW, Ronnekleiv OK 1997 Expression of the orphan receptor steroidogenic factor-1 mRNA in the rat medial basal hypothalamus. Brain Res Mol Brain Res 44(1):66-72

Sairam MR, Subbarayan VS 1997 Characterization of the $5^{\prime}$ flanking region and potential control elements of the ovine follitropin receptor gene. Mol Reprod Dev 48(4):480-487

Shen WH, Moore CC, Ikeda Y, Parker KL, Ingraham HA 1994 Nuclear receptor steroidogenic factor 1 regulates the mullerian inhibiting substance gene: a link to the sex determination cascade. Cell 77(5):651-661

Singh J, Handelsman DJ 1996 Neonatal administration of FSH increases Sertoli cell numbers and spermatogenesis in gonadotropin-deficient $(h p g)$ mice. J Endocrinol 151:37-48

Singh J, O'Neill C, Handelsman DJ 1995 Induction of spermatogenesis by androgens in gonadotropin-deficient ( $h p g$ ) mice. Endocrinology 136(12):5311-5321

Smale S, Baltimore D 1989 The "initiator" as a transcription control element. Cell 57:103-113

Sprengel R, Braun T, Nikolics K, Segaloff D, Seeburg P 1990 The testicular receptor for follicle stimulating hormone: structure and functional expression of cloned cDNA. Mol Endocrinol 4:525-530

Tapanainen JS, Aittomaki K, Min J, Vaskivuo T, Huhtaniemi IT 1997 Men homozygous for an inactivating mutation of the follicle-stimulating hormone (FSH) receptor gene present variable suppression of spermatogenesis and fertility. Nature Genet 15(2):205-206

Tate PH, Bird AP 1993 Effects of DNA methylation on DNA-binding proteins and gene expression. Curr Opin Genet Dev 3(2):226-231 
Themmen A, Blok L, Post M, Baarends W, Hoogerbrugge J, Parmentier M, Vassart G, Grootegood A 1991 Follitropin receptor down regulation involves a cAMP-dependent post-transcriptional decrease of receptor mRNA expression. Mol Cell Endocrinol 78:R7-R13

Tremblay JJ, Drouin J 1999 Egr-1 is a downstream effector of GnRH and synergizes by direct interaction with Ptx1 and SF-1 to enhance luteinizing hormone beta gene transcription. Mol Cell Biol 19(4):2567-2576

Tsai-Morris C, Buczko E, Wang W, Xie X, Dufau M 1991 Structural organization of the rat leutinizing hormone receptor gene. J Biol Chem 266:11,355-11,359

Tullius TD, Dombroski BA, Churchill MEA, Kam L 1987 Hydroxyl radical footprinting: a high resolution method for mapping protein-DNA contacts. Meth Enzymol 155:537-558

Vibede N, Hauser F, Williamson M, Grimmelikhuijzen CJ 1998 Genomic organization of a receptor from sea anemones, structurally and evolutionarily related to glycoprotein hormone receptors from mammals. Biochem Biophys Res Commun 252(2):497-501

Weis L, Reinberg D 1992 Transcription by RNA polymerase II: initiator-directed formation of transcription-competent complexes. FASEB J 6(14):3300-3309

Wolfe MW 1999 The equine luteinizing hormone beta-subunit promoter contains two functional steroidogenic factor-1 response elements. Mol Endocrinol 13(9):1497-1510

Wolfe MW, Call GB 1999 Early growth response protein 1 binds to the luteinizing hormone-beta promoter and mediates gonadotropin-releasing hormone-stimulated gene expression. Mol Endocrinol 13(5):752-763

Xing W, Sairam MR 2001 Characterization of regulatory elements of ovine follicle-stimulating hormone (FSH) receptor gene: the role of E-box in the regulation of ovine FSHreceptor expression. Biol Reprod 64(2):579-589

Zirkin BR, Awoniyi C, Griswold MD, Russell LD, Sharpe R 1994 Is FSH required for adult spermatogenesis? J Androl 15(4):273-276 


\title{
Identification of Specific Sites of Hormonal Regulation in Spermatogenesis in Rats, Monkeys, and Man
}

\author{
R.I. McLachlan,* L. O’Donnell,* S.J. Meachem,* P.G. Stanton,* \\ D.M. de Kretser, ${ }^{\dagger}$ K. Pratis, ${ }^{*}$ and D.M. Robertson* \\ *Prince Henry's Institute of Medical Research, Monash Medical Centre, Clayton, Victoria, 3168, \\ Australia; ${ }^{\dagger}$ Monash Institute of Reproduction and Development, Monash University, \\ Monash Medical Centre, Clayton, Victoria, 3168, Australia
}

\begin{abstract}
A detailed understanding of the hormonal regulation of spermatogenesis is required for the informed assessment and management of male fertility and, conversely, for the development of safe and reversible male hormonal contraception. An approach to the study of these issues is outlined based on the use of well-defined in vivo models of gonadotropin/androgen deprivation and replacement, the quantitative assessment of germ cell number using stereological techniques, and the directed study of specific steps in spermatogenesis shown to be hormone dependent. Drawing together data from rat, monkey, and human models, we identify differences between species and formulate an overview of the hormonal regulation of spermatogenesis. There is good evidence for both separate and synergistic roles for both testosterone and follicle-stimulating hormone (FSH) in achieving quantitatively normal spermatogenesis. Based on relatively selective withdrawal and replacement studies, FSH has key roles in the progression of type A to B spermatogonia and, in synergy with testosterone, in regulating germ cell viability. Testosterone is an absolute requirement for spermatogenesis. In rats, it has been shown to promote the adhesion of round spermatids to Sertoli cells, without which they are sloughed from the epithelium and spermatid elongation fails. The release of mature elongated spermatids from the testis (spermiation) is also under FSH/testosterone control in rats. Data from monkeys and men treated with steroidal contraceptives indicate that impairment of spermiation is a key to achieving azoospermia. The contribution of $5 \alpha$-reduced androgens in the testis to the regulation of spermatogenesis is also relevant, as $5 \alpha$-reduced androgens are maintained during gonadotropin suppression and may act to maintain low levels of germ cell development. These concepts are also discussed in the context of male hormonal contraceptive development.
\end{abstract}

\section{Introduction}

Despite impressive technical advances in human reproductive medicine (e.g., assisted reproductive technologies, new drugs, and recombinant hormones), there remain many important questions about male fertility regulation. In the area of spermatogenesis, such deficiencies exist in the areas of hormonal male contraception, complete spermatogenic failure, damage from toxic/environmen- 
tal agents, and in understanding hypothalamo-pituitary-testicular relationships in infertile or aging men.

We have focused on the hormonal regulation of spermatogenesis with a view to testing, whenever possible, observations made in lower mammals (primarily rodents) for their relevance to man. In this review, we will provide an overview of the hormonal regulation of spermatogenesis in all these species, drawing together common features and highlighting important differences, particularly in regard to monkeys and man.

\section{A. OVERVIEW OF THE HYPOTHALAMIC-PITUITARY-TESTIS AXIS}

The production of spermatozoa (fertility) and the secretion of testosterone (virility) by the testis are both dependent on stimulation by the pituitary gonadotropins, follicle-stimulating hormone (FSH) and luteinising hormone (LH), which are secreted in response to hypothalamic gonadotropin-releasing hormone $(\mathrm{GnRH})$. Testosterone $(\mathrm{T})$, which is essential for the initiation and maintenance of spermatogenesis, is secreted by the adult Leydig cell under LH stimulation. Testosterone acts via androgen receptors (ARs) on Sertoli, Leydig, and peritubular cells. The fact that $\mathrm{T}$ exerts its effects on somatic cells rather than germ cells was highlighted by recent germ cell transplantation studies (Johnston et al., 2001) in which spermatogonia from AR-deficient animals developed into spermatozoa in wild-type recipients. FSH acts via specific G protein-coupled surface receptors located exclusively on Sertoli cells. FSH has a key role in the development of the immature testis, particularly by controlling Sertoli cell proliferation (Orth, 1993). Following many conflicting data in animal and human models, there is now general agreement that some degree of complete spermatogenesis can be initiated and maintained in the apparent absence of FSH. However, quantitatively normal spermatogenesis in adulthood is dependent on FSH, certainly in man and monkeys. FSH secretion is regulated by negative feedback from the testicular hormone, inhibin B, and through testosterone, either alone or by its aromatisation to estradiol (Hayes et al., 2001).

\section{B. OVERVIEW OF SPERMATOGENESIS AND APPROACH TO ITS STUDY}

Many in vitro and in vivo model systems have been used to study regulation of spermatogenesis by FSH and T, each with varying strengths and weaknesses. Often, reports using these models extrapolate the principle findings to other species, with limited justification. In addition, conclusions may be affected by whether the model system is one of congenital deficiency of hormone secretion or action, as opposed to one involving spermatogenic restoration or maintenance in adulthood. The degree of gonadotropin deficiency may be difficult to establish due to the limited sensitivity of gonadotropin assays used in many test species. 
Finally, the descriptions of the changes in germ cell populations may be only qualitative, while properly validated quantitative methods (stereological methods) are preferred. Before undertaking a review of the effects and sites of hormone action on spermatogenesis, we will briefly highlight some aspects of the underlying physiology and the approaches to its study.

Spermatogenesis involves four basic processes: spermatogonial development (stem cell and subsequent cell mitotic divisions), meiosis (DNA synthesis and two meiotic divisions to yield haploid spermatids), spermiogenesis (spermatid development involving differentiation of head and tail structures), and spermiation (the process of release of mature sperm into the tubule lumen). These occur along similar lines in all mammals and are well described at the morphological level (Leblond and Clermont, 1952; Clermont, 1972; de Kretser and Kerr, 1988; Russell et al., 1990). In rodents and some primate species (e.g., Macaca fascicularis), germ cell development occurs in orderly and recognizable cell associations (or stages) along the seminiferous tubule, such that a single stage can be seen within a tubule cross section (Russell et al., 1990). However, in humans and some other primates (e.g., marmosets), the stages are arranged in an intertwining helical pattern such that a single tubule cross section may have up to six identified stages represented (Schulze and Rehder, 1984). Such an arrangement makes the systematic stage-based counting of germ cell populations, as well as the stage-specific detection of proteins or mRNA species of interest, difficult, although possible.

\section{THE STEREOLOGICAL APPROACH TO THE STUDY OF SPERMATOGENESIS}

Relative to primates, a greater range of experimental paradigms are available in rodents for the study of the relative contributions of FSH and LH/T. The ease of obtaining testicular tissue in lower mammals for quantitative analyses has made it easier to ascribe specific FSH or T effects to particular stages or germ cell types. To provide quantitative data on germ cell number, we have focused on the use of unbiased stereological approaches, particularly the thick section optical disector model, in combination with a systematic random sampling scheme (for a review, see Wreford, 1995). This procedure involves the visualisation of complete cell profiles in thick $(25 \mu \mathrm{m})$ sections and has the particular advantage of allowing assessment of irregularly shaped cellular forms, such as differentiating spermatids and spermatozoa, which was not possible with earlier geometric-based methods. In our studies, we have expressed the data as germ cell number per testis. In primates and man, where biopsies are normally used, data are expressed on a per Sertoli cell basis, as the number of Sertoli cells does not alter in response to hormonal manipulation in either primates (Zhengwei et al., 1998c) or humans (Zhengwei et al., 1998b). The expression of data on a 
per-tubule cross-sectional basis is not appropriate as a general rule, as a reduction in tubule volume is seen following hormonal withdrawal (Zhengwei et al., 1998c).

Inherent in the interpretation of germ cell data derived by stereological techniques is the knowledge that the duration of spermatogenesis under the various treatments proposed is known and that it remains unchanged. Animal models based on bromodeoxyuridine or tritiated thymidine labeling have been used to determine the duration of the spermatogenic cycle in monkey and humans. The duration of one cycle of the seminiferous epithelium is $\approx 10$ days in monkeys (de Rooij et al., 1986; Aslam et al., 1999) and 16 days in the human (Heller and Clermont, 1963). Rodent, primate, and human data suggest that the duration of spermatogenesis cannot be altered by modulation of the gonadotropin environment (e.g., hypophysectomy) (Clermont and Harvey, 1965) or by GnRHantagonist treatment of rats or cynomolgus monkeys (Aslam et al., 1999). Our studies in testosterone-treated cynomolgus monkeys suggest that stage frequency (which approximates stage duration) is not affected by the resulting gonadotropin suppression (O'Donnell et al., 2001a). Limited studies in man also suggest that the length of the spermatogenic cycle is not affected by hormone treatment (Heller and Clermont, 1964).

\section{Models Used to Explore Rat Spermatogenesis}

\section{A. MODELS OF TESTOSTERONE DEFICIENCY}

The concentration of testosterone in the rat testis is normally 50 -fold higher than that in serum. It exerts a biphasic effect on spermatogenesis by both inhibiting and promoting the process in vivo, depending on the dose administered (Walsh and Swerdloff, 1973; Sun et al., 1989; Zirkin et al., 1989). Administration of a low dose of T, often as 2.5- to 3-cm T-filled Silastic implants, in combination with a low dose of estradiol (E) (0.1- to 0.4-cm implant, TE implants) causes slightly supraphysiological circulating $\mathrm{T}$ levels that suppress LH, but not FSH secretion (Awoniyi et al., 1989b,1990; McLachlan et al., 1994a; O'Donnell et al., 1994). Accordingly this "TE model" is one of isolated LH/T suppression, a response that is peculiar to rats, and a fact that underlies the very different spermatogenic response to similar treatments in primates (see below). Restoration of sperm production occurs in a dose-responsive manner by the administration of higher doses of T (Awoniyi et al., 1989b,1990; McLachlan et al., 1994a; O'Donnell et al., 1994).

The administration of TE implants to adult rats for 6-12 weeks causes the suppression of testicular T levels to approximately 3\% of normal (O'Donnell et al., 1994,1999) and testicular elongated spermatid production ceases (McLachlan et al., 1994a; O'Donnell et al., 1994). Quantitation of testicular germ cell 
populations during TE suppression and high-dose $\mathrm{T}$ restoration has allowed an understanding of the sites of $\mathrm{T}$ action in germ cell development in the presence of FSH (McLachlan et al., 1994a; Meachem et al., 1998). Using the optical disector stereological approach, studies in TE-treated rats show that spermatogonia and early spermatocytes are suppressed to $\approx 80 \%$ of control, with less suppression of pachytene spermatocytes in stages I-VIII $(\approx 60 \%$ of control $)$ and pachytene spermatocytes in later stages ( $\approx 33 \%$ of control) (Figure 1$)$ (Meachem et al., 1997,1998). Early round spermatids in stages I-VII are suppressed to $\approx 20 \%$ of normal, yet round spermatids in stage VIII are more markedly suppressed to $5 \%$ of normal and elongated spermatids are undetectable (Meachem et al., 1998). Spermatogenesis can be restored to near-normal levels with the replacement of higher-dose T implants (McLachlan et al., 1994a).

FSH is also important in maintaining germ cell development in the TE model. During the restoration of spermatogenesis using higher-dose $\mathrm{T}$ implants, the co-administration of an FSH antibody (see section II-B) to suppress the action of FSH results in significant reductions in various germ cells, most notably, spermatocytes and early spermatids (Meachem et al., 1998). As will be discussed below, there is evidence for complementary and synergistic effects of FSH and $\mathrm{T}$ on germ cell development.

These stereological studies have identified a progressive decline in germ cell number throughout spermatogenesis with $\mathrm{T}$ withdrawal. It has become apparent that sperm release (spermiation) is also affected by this treatment; instead of detaching from the epithelium, mature spermatids are retained by the Sertoli cell, then phagocytosed and thus fail to spermiate. The appearance of mature spermatids retained within the seminiferous epithelium after hormone suppression is well known (Russell and Clermont, 1977; Russell, 1991). We recently used TE suppression and stereological techniques to quantify the extent to which sperm fail to be released and showed that $16 \%, 45 \%, 70 \%$, and $97 \%$ of sperm failed to be released after 1, 2, 3, and 4 weeks of TE treatment, respectively (Saito et al., 2000). After 6 weeks of treatment, however, earlier germ cell populations decreased to a point where no elongated spermatids are produced and thus disruptions to spermiation are not as evident in chronic suppression models (Figure 1). Therefore spermiation failure is an early feature of gonadotropin suppression in the rat (Saito et al., 2000), although is a feature of both acute and chronic gonadotropin suppression in primates (Section III).

\section{B. ACTIVE IMMUNISATION AGAINST GNRH OR GNRH ANTAGONIST TREATMENT IN RATS}

The loss of GnRH action following either of these modalities results in severe combined FSH and LH deficiency, with serum FSH levels falling to below the limit of assay detection and intratesticular $\mathrm{T}$ levels to $\approx 1-2 \%$ of control, 


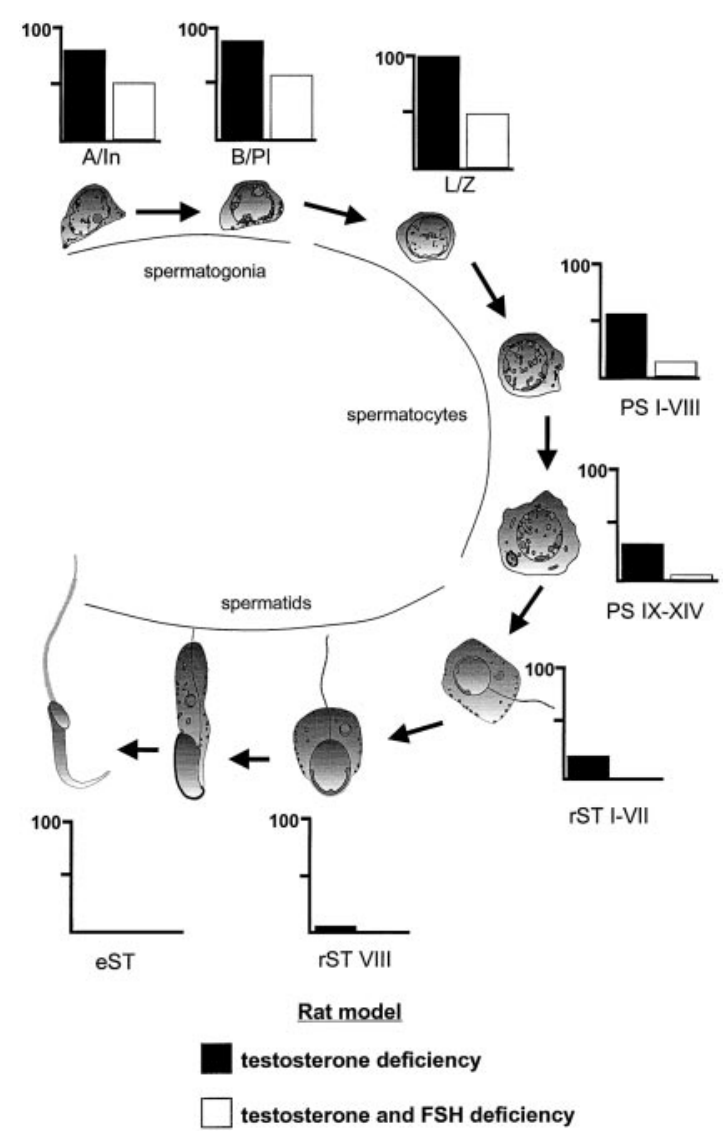

FIG. 1. Comparison of germ cell populations in rats after long-term testosterone (T) suppression or long-term suppression of both T and FSH. Germ cell development from immature spermatogonia through to elongated spermatids is shown, together with stereological data on each germ cell population. Germ cell numbers per testis were determined by the optical disector technique and are expressed as a percentage of an untreated control group. Data were adapted from a previous study (Meachem et al., 1998). The model of $\mathrm{T}$ deficiency utilised adult rats $(\mathrm{n}=6)$ given $3-\mathrm{cm} \mathrm{T}$ and 0.4-cm E implants for 9 weeks to cause the suppression of LH to undetectable levels (O'Donnell et al., 1994) and testicular T to <3\% of controls (O'Donnell et al., 1994; Meachem et al., 1998). Serum FSH levels in this model are either slightly or not significantly altered (O'Donnell et al., 1994,1996a; Meachem et al., 1998). Thus, this is a model primarily of LH/T deficiency. The model of T and FSH deficiency utilised adult rats $(n=6)$ administered a GnRH immunogen for 3 months to suppress LH and FSH to undetectable levels (McLachlan et al., 1994b) and testicular T to $<4 \%$ of controls (Meachem et al., 1998). Abbreviations: A, type A spermatogonia; In, intermediate spermatogonia; B, type B spermatogonia; Pl, preleptotene spermatocytes; L, leptotene spermatocytes; Z, zygotene spermatocytes; PS I-VIII, pachytene spermatocytes in stages I-VIII; PS IX-XIV, pachytene spermatocytes in stages IX-XIV; rST I-VII, round spermatids in stages I-VII; rST VIII, round spermatids in stage VIII; eST, elongated spermatids. 
resulting in severe spermatogenic impairment (Sinha Hikim and Swerdloff, 1993; McLachlan et al., 1994b; Kangasniemi et al., 1995). While this may appear a good model for the study of selective FSH and LH/T replacement, in rats, the restoration of serum $\mathrm{T}$ by exogenous $\mathrm{LH}$ or $\mathrm{T}$ treatment also normalises serum FSH (McLachlan et al., 1994b) by a direct action on pituitary FSH $\beta$ secretion (Wierman and Wang, 1990). Thus, to study the effects of LH/T on spermatogenesis, simultaneous neutralisation of serum FSH must be achieved, such as by passive FSH immunisation (Meachem et al., 1998). We have shown that passive immunisation of adult rats with an FSH antiserum for 7 days by subcutaneous (sc) daily injection at a dose of $2 \mathrm{mg} / \mathrm{kg}$ rat results in immunoabsorption and neutralisation of at least $90 \%$ of circulating FSH, with no changes in serum or testicular $\mathrm{T}$ levels. However, it cannot be ruled out that lower levels of biologically active FSH are still circulating. Since rats rapidly develop neutralising antibodies to the antisera using this approach, only short-term effects of FSH withdrawal can be studied (i.e., $\approx 8$ days).

Spermatogenic failure after GnRH immunisation is characterised by the abolition of round spermatids beyond stage VII, with the numbers of earlier germ cells being severely reduced (Awoniyi et al., 1989a; Sinha Hikim and Swerdloff, 1993; McLachlan et al., 1994b). Three months of GnRH immunisation results in spermatogonial number being reduced to $\approx 50 \%$ of normal (Figure 1). Interestingly, spermatogonial number does not fall further, suggesting that only part of the spermatogonial population is regulated by gonadotropins, although it is possible that residual FSH and testicular T may provide some support. Major losses are also seen during spermatocyte development with early spermatocytes (leptotene-zygotene), pachytene spermatocytes in stages I-VIII, and pachytene spermatocytes in stages IX-XIV, with reductions to $45 \%, 13 \%$, and $4 \%$ of control, respectively (McLachlan et al., 1995; Meachem et al., 1998) (Figure 1). Round spermatids are markedly reduced to $<1 \%$ of control and elongated spermatids are not seen. When compared to the TE model (LH/T deficiency), there is a more-marked loss of spermatogonia and spermatocytes, probably due to the effects of FSH withdrawal on spermatogonial proliferation/survival and germ cell apoptosis in the GnRH-immunised model (Figure 1).

In GnRH-immunised animals, the spermatogenic process can be restored to normal (Awoniyi et al., 1989a) or near normal by T treatment (24-cm Silastic implants) (McLachlan et al., 1994b), as determined by elongated sperm content of the testis. We have prevented the T-induced restoration of serum FSH levels in GnRH-immunised rats by co-treatment with an FSH antiserum, thereby enabling the study of the effects of $\mathrm{T}$ alone on the restoration of spermatogenesis (Meachem et al., 1998). Treatment with FSH antiserum blocked the ability of T to restore spermatogenic cell populations, suggesting that FSH is required for the initial phase of spermatogenic restoration in adult rats following chronic gonadotropin suppression (Meachem et al., 1998). 
We have investigated the restorative effects of FSH on germ cell populations by the administration of recombinant human FSH (rhFSH) after gonadotropin suppression. It is clear from such studies that FSH plays a major role in spermatogonial development, as FSH promptly restores spermatogonial number to normal levels after 7 days, while a partial restoration of spermatocyte and spermatid number was observed (Meachem et al., 1998). When rhFSH was administered for up to 14 days, very few round spermatids underwent elongation and mature, elongated (step 15-19) spermatids were almost never seen (McLachlan et al., 1995), supporting the need for $\mathrm{T}$ in spermatid elongation. Further studies are required to determine the long-term effects of FSH on spermatogenic restoration but would require the availability of recombinant rat FSH to counter the bioneutralisation of administered heterologous FSH.

In summary, chronic $\mathrm{T} \pm \mathrm{FSH}$ suppression results in disordered spermatogonial development and disordered progression through meiosis and spermatid development, with evidence for FSH-specific effects on spermatogonia and of T-specific effects on spermiogenesis in stages VII-VIII.

\section{MODELS EXPLORING THE ACUTE WITHDRAWAL OF HORMONES}

Acute suppression models, such as hypophysectomy and GnRH antagonist treatment, have also been useful in clarifying the effects of FSH and T on germ cell development and have been used to demonstrate the role of these hormones in the maintenance of germ cell viability. Acute gonadotropin suppression results in germ cell death, particularly in stages VII and VIII (Russell and Clermont, 1977; Sinha Hikim and Swerdloff, 1993) via the apoptotic pathway (Sinha Hikim et al., 1995; Sinha Hikim and Swerdloff, 1999). Apoptosis is particularly evident in preleptotene and pachytene spermatocytes in stages VII-VIII, which would account for the marked losses during spermatocyte development after chronic suppression of either $\mathrm{T}$ alone or of FSH and T in rats (Figure 1).

It seems clear from various studies that apoptosis/viability of germ cells can be regulated by FSH and/or T. Germ cell death/apoptosis can be prevented by either T or FSH, with both hormones having a synergistic effect, suggesting that germ cell death in the seminiferous epithelium is regulated by $\mathrm{T}$ and FSH via similar pathways (Russell et al., 1987; Tapanainen et al., 1993; El Shennawy et al., 1998). While acute FSH and T suppression certainly causes an increase in the appearance of degenerating/apoptotic germ cells (Russell and Clermont, 1977), this may or may not lead to significant changes to the viable cell population. For example, 1 week of gonadotropin suppression induced by a GnRH antagonist treatment caused significant decreases in germ cell numbers in stage VII (Sinha Hikim and Swerdloff, 1993), yet 1 week of gonadotropin suppression induced by TE treatment in combination with an FSH antibody did not produce a significant fall in germ cells in this stage (Saito et al., 2000). 
Acute models of gonadotropin suppression have revealed that synergistic effects of FSH and T are evident when one considers spermiation. We have shown that suppression of either FSH or T for 1 week caused $10-15 \%$ of spermatids to be retained. Yet, when both hormones were withdrawn, a moremarked failure (50\%) of spermiation was seen (Saito et al., 2000), suggesting that spermiation is regulated by FSH and T, with both hormones having a synergistic effect. Such studies support earlier observations on the ability of LH and FSH to prevent the retention of mature spermatids (Russell and Clermont, 1977).

An important model to investigate the acute suppression of spermatogenesis is the acute and selective disruption of FSH action in rats in vivo by immunoneutralisation of the FSH protein, which has enabled us to pinpoint sites in the spermatogenic process that are sensitive to FSH withdrawal (Meachem et al., 1999). We observed a time-dependent decline in early germ cell populations in normal rats after FSH antibody ( $\mathrm{Ab}$ ) treatment, demonstrating that FSH plays a major role in spermatogonial development, particularly in the maturation of type A3/A4 spermatogonial subtypes. Loss of spermatocytes and spermatids after 8.5 days of FSH withdrawal demonstrated that FSH also supports germ cell maturation in midstages of spermatogenesis (Meachem et al., 1999), probably by supporting germ cell viability. As mentioned above, $15 \%$ of spermatids fail to spermiate following 1 week of FSH Ab treatment (Saito et al., 2000). Thus, these short-term studies highlight the importance of FSH for quantitatively normal spermatogenesis. The precise mechanisms by which FSH acts on each phase of germ cell development are yet to be elucidated.

\section{OTHER EXPERIMENTAL PARADIGMS}

While we have approached the issue of the regulation of spermatogenesis using hormonal manipulation in adult rats, other approaches using congenital or transplantation techniques have been described in the past 10 years that also provide novel insights and are briefly described below for completeness. As with all such models of congenital deficiency, they provide valuable insight into the roles of the gonadotropins during sexual development and in the initiation of spermatogenesis. Their utility in studying the control of spermatogenesis in the adult, however, is reduced due to the likelihood of defects in reproductive development during the fetal and postnatal period, which could confound observations in adult animals.

Knockout mouse models have been produced for the key components of the hypothalamo-pituitary-testicular axis, including the GnRH gene (hpg), FSH and LH receptors, FSH and LH $\beta$ subunits, estrogen receptors, and the aromatase enzyme. The reproductive phenotypes of such transgenic animals have been the subject of recent reviews and thus will not be considered here (Huhtaniemi and Bartke, 2001; O’Donnell et al., 2001b). Germ cell transplantation models are 
based on the transplantation of spermatogonial stem cells from wild-type or factor-deficient animals into infertile hetero- or homozygous recipients. This innovative approach to the study of spermatogonia has been recently reviewed (McLean et al., 2001; Meachem et al., 2001).

\section{Models Used to Explore Human and Monkey Spermatogenesis}

Exogenous $\mathrm{T}$ markedly suppresses both FSH and $\mathrm{LH}$ in primates and profoundly impairs spermatogenesis, thereby providing a steroidal basis for male hormonal contraception (see below). The addition of progestin also appears to accelerate and perhaps augment the degree of gonadotropin withdrawal (Handelsman et al., 1996; Meriggiola and Bremner, 1997). Using ultra-sensitive assays, serum LH is found to be $<0.3 \%$ of control but FSH remains detectable at 1-2\% (Robertson et al., 2001). This residual FSH secretion appears to be constitutive (i.e., GnRH independent). Exogenous $\mathrm{T}$ does not result in a restoration of serum FSH as in rats; thus, both exogenous LH (in the form of human chorionic gonadotropin (hCG)) and/or FSH can be administered to study their effects on spermatogenesis (Matsumoto and Bremner, 1989). An interesting difference exists between man and monkeys in regard to testicular $\mathrm{T}$ levels in response to such treatment, as $\mathrm{T}$ levels fall to $\approx 2 \%$ of normal in human (McLachlan et al., in press) but to only $\approx 25 \%$ of normal (and many-fold those in serum) in monkeys (Weinbauer et al., 1988; Zhengwei et al., 1998c). This implies a substantial degree of LH-independent androgen secretion in monkeys.

A feature of many T-based contraceptive formulations in man has been the variable induction of azoospermia, ranging from 70 to $95 \%$, depending on the regimen and ethnic group under study (World Health Organization, 1990,1996; Meriggiola et al., 1996; Martin et al., 2000). While sperm counts less than 3 million/ml may provide adequate contraception (World Health Organization, 1996), there is a general consensus that the reliable induction of azoospermia is important to ensure contraceptive efficacy and the widespread acceptance of male hormonal contraception. An understanding of the biological basis for the variable response is essential to this goal.

In order to pursue these issues, we have undertaken a series of studies in man and monkeys aimed at understanding which sites in spermatogenesis are affected by gonadotropin withdrawal and whether various aspects of proposed contraceptive treatments could be modified to augment the degree of suppression. Broadly, these studies have exploited paradigms similar to those being considered for human clinical application. Overall, there are striking similarities between the data from man and monkeys and several directions for further research have emerged. 


\section{A. PRIMATE STUDIES}

The nonhuman primate is an excellent model of human spermatogenesis sharing very similar hormonal dependencies and structural patterns. Studies are very demanding and expensive but allow experimental paradigms not possible in man (e.g., sequential testis biopsies). We have explored their use in studies aimed at understanding the basis of hormonal contraception, namely, $\mathrm{T}$ treatment, either alone or in combination with progestins.

\section{Testosterone Treatment}

We administered $\mathrm{T}$ to adult macaque monkeys using subcutaneous implants for 20 weeks, which provide moderately supraphysiological serum levels in order to suppress gonadotropins. Then, we determined germ cell populations using the optical disector stereological method (O'Donnell et al., 2001a). In all animals, the only acute decrease in germ cell numbers observed was a fall in A pale spermatogonia to $45 \%$ of baseline within 2 weeks. The subsequent depletion of later germ cells was manifest by a decline in type B spermatogonia (32-38\% baseline) and spermatocyte/spermatid numbers (20-30\% baseline) after 14 and 20 weeks. While there was evidence of some minor losses of spermatocytes and spermatids, the reduction in later germ cell types was primarily attributed to a decrease in the conversion of type A pale $\rightarrow$ B spermatogonia. Type B spermatogonia were more markedly suppressed in those animals becoming azoospermic, compared to those who did not. Therefore, the conversion of type A pale $\rightarrow$ type B spermatogonia may be a key point in determining the degree of contraceptive efficacy.

A second observation was the abnormal retention of mature elongated spermatids in some monkeys after long-term $\mathrm{T}$ administration (O'Donnell et al., 2001a). The number of retained spermatids was negatively correlated with sperm count in the ejaculate, suggesting that failure of spermiation contributes to the extent of sperm count suppression during chronic $\mathrm{T}$ treatment in monkeys. Thus, it is clear that both the inhibition of A pale and B spermatogonial development and inhibition of spermiation are the major defects caused by long-term $\mathrm{T}$ administration to monkeys. These observations align closely with those seen in humans (see below) but show some differences compared to rodents (Figure 2).

A number of endocrine parameters were investigated as potential markers that might differentiate animals that did or did not achieve azoospermia. Serum bioactive FSH was found to be the only endocrine marker of this effect that was significantly lower in azoospermic animals (A. Narula, Y.-Q. Gu, L. O'Donnell, P. Stanton, D. Robertson, R. McLachlan, W. Bremner, submitted), which correlated with the lower numbers of $\mathrm{B}$ spermatogonia in these animals (O'Donnell et al., 2001a). These observations support a key role for FSH in spermatogonial development and emphasize the need for FSH suppression in 


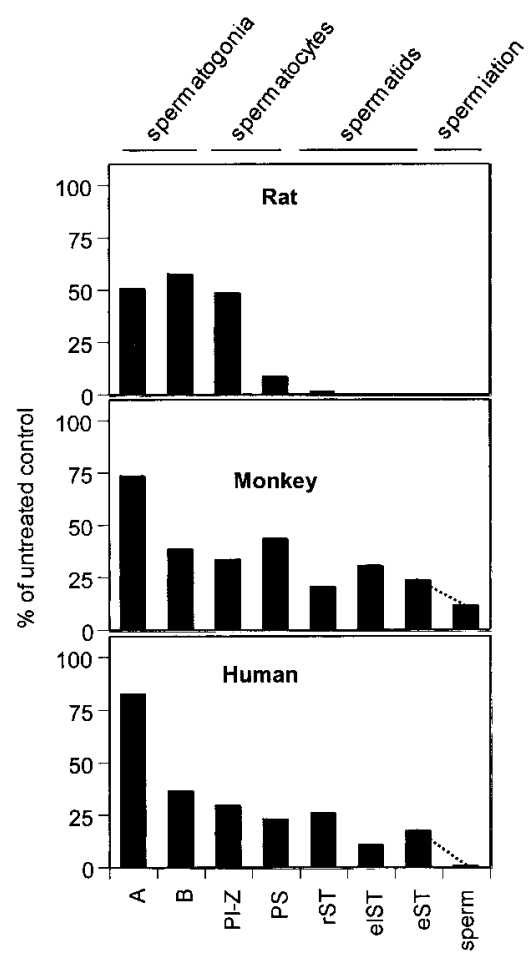

FIG. 2. Comparison of the progression of germ cells through spermatogenesis in rats (upper panel), monkeys (middle panel), and men (lower panel) after 12-14 weeks of combined gonadotropin (LH and FSH) suppression. Germ cell numbers were determined in whole testes in rats and in biopsy material obtained from open testicular biopsies in monkeys and men, using the optical disector stereological approach. Data were expressed on a per-testis or per-Sertoli cell basis. Data in rats are modified from Meachem et al., 1998, in which adult Sprague Dawley rats $(\mathrm{n}=6)$ were immunized against GnRH for 12 weeks, the germ cell populations determined, and expressed as a percentage of a control group of placebo-treated rats $(\mathrm{n}=6)$. Data in monkeys are modified from O'Donnell et al., 2001a, in which adult Macaca fascicularis $(\mathrm{n}=9)$ were administered testosterone implants for 14 weeks. Germ cell populations were determined and expressed as a percentage of germ cells in pretreatment biopsies. Data in humans are modified from our own data on men $(n=5)$ receiving weekly injections of $200 \mathrm{mg} \mathrm{T}$ enanthate for 12 weeks. The germ cell data are expressed as a percentage of an untreated control group $(\mathrm{n}=5$ ) (McLachlan et al., in press). Abbreviations: A, type A spermatogonia; B, type B spermatogonia; PL-Z, preleptotene-zygotene spermatocytes; PS, pachytene spermatocytes; rST, round spermatids; elST, elongating spermatids; eST, elongated spermatids. The dashed line between elongated spermatids (eST) and sperm represents the difference between the number of spermatids in the testis prior to release and the number in the ejaculate, in order to provide insights into the possibility of spermiation failure. These data obtained for monkeys and men after 14 and 12 weeks of gonadotropin suppression respectively, are comparable to the extent of spermatogenic suppression in monkeys and men after 20-24 weeks of gonadotropin suppression (Zhengwei et al., 1998b; O'Donnell et al., 2001a), and thus appear to represent steady-state suppression. 
contraceptive regimens. A similar conclusion that FSH is an important factor in spermatogenic inhibition in T-treated monkeys was recently drawn by Weinbauer and colleagues (2001). Interestingly, in our study, serum inhibin B levels (a potential marker of the Sertoli cell-spermatogenic relationship) declined to about $40 \%$ of baseline but did not differ between azoospermic and non-azoospermic monkeys (A. Narula, Y.-Q. Gu, L. O'Donnell, P. Stanton, D. Robertson, R. McLachlan, W. Bremner, submitted). Testicular androgens also did not differ between groups. However, it was noted that, despite a marked decrease in testicular $\mathrm{T}$ concentrations after exogenous $\mathrm{T}$ administration, the levels of $5 \alpha$-reduced androgenic metabolites were maintained at control levels, suggesting an upregulation of the $5 \alpha$-reductase enzyme in the LH-deprived primate testis (see below).

\section{GnRH Antagonist Treatment}

Our studies of monkeys given a GnRH antagonist for 21 days revealed similar spermatogenic sites for gonadotropin suppression, namely, the conversion of type A pale $\rightarrow$ B spermatogonia and elongated spermatid retention (Zhengwei et al., 1998c). This suggests that GnRH antagonist and moderately supraphysiological $\mathrm{T}$ treatment have similar effects on primate spermatogenesis.

\section{B. HUMAN STUDIES}

\section{Testosterone Treatment}

Testosterone treatment only leads to azoospermia in $\approx 70 \%$ of normal men and to variable degrees of oligospermia in the remainder. However, the basis of this variable response is unclear (World Health Organization, 1990,1996; Handelsman et al., 1995). In order to ascertain the changes in germ cell populations during $\mathrm{T}$ treatment, we undertook a stereological assessment of spermatogenesis in men receiving the same contraceptive regimen as used in the WHO multicentre trial (World Health Organization, 1990). Ten normal, fertile men, already planning to undergo vasectomy, received $\mathrm{T}$ enanthate $(200 \mathrm{mg}$ intramuscularly (im) weekly) for $\approx 20$ weeks prior to testicular biopsy (Zhengwei et al., 1998b). Type B spermatogonia fell markedly to $10 \%$ of the untreated control group and later germ cell types to 11-18\% of controls. Despite the presence of elongated spermatids ( $0.6-20 \%$ of control) in the testis, four men became azoospermic. Two T-treated subjects with similar early germ cell complements and elongated spermatids numbers had sperm counts of $<0.1$ and $21 \mathrm{million} / \mathrm{ml}$. The latter man demonstrated marked variability in germ cell numbers between adjacent tubules. Overall, we concluded that the principal spermatogenic lesion in T-treated men is the marked inhibition of type $\mathrm{A} \rightarrow \mathrm{B}$ spermatogonial maturation, although other sites were also affected, particularly the release of elongated spermatids. It was also striking that a similar degree of gonadotropin withdrawal was associated 
with widely variable spermatogenic patterns, both between and within individuals, the latter being evident in different histological patterns between adjacent tubules, despite exposure to presumably an identical endocrine milieu (Zhengwei et al., 1998b).

\section{Testosterone + Progestin: Effects on Spermatogenesis and Reproductive Endocrinology}

We have also examined the proposition that the co-administration of a progestin with $\mathrm{T}$ enhances the suppression of spermatogenesis. We hypothesised that the greater speed and/or extent of suppression of germ cell number would be correlated with an enhanced suppression of serum gonadotropins and/or testicular androgens levels. Normal, fertile men received either T enanthate $200 \mathrm{mg}$ im weekly alone or in combination with the depot progestin, medroxyprogesterone acetate (DMPA) (McLachlan et al., in press) for 2, 6, or 12 weeks prior to stereological assessment of testis biopsy material. The inclusion of DMPA led to a more rapid fall in serum FSH/LH levels, achieving nadir levels in about half the time. Yet, the mean time to sperm count below 1 million/ $\mathrm{ml}$ (around 25 days) and the maximum extent of FSH/LH suppression (mean serum FSH 1.2-1.6\%, and mean LH $0.2-0.3 \%$ of baseline) did not differ. In both groups, intratesticular $\mathrm{T}$ levels declined similarly to $\approx 2 \%$ of control levels but, as in monkeys, the testicular $5 \alpha$-reduced androgens dihydrotestosterone (DHT) and $5 \alpha$-androstane$3 \alpha 17 \beta$-diol (Adiol) did not fall significantly. The only difference in germ cell numbers was seen at 2 weeks, when type B spermatogonia and early spermatocytes were significantly lower in the T enanthate + DMPA group, presumably reflecting the lower gonadotropin levels at this time. In the longer term, a marked inhibition of A pale $\rightarrow$ B spermatogonial maturation was seen, along with a striking inhibition of spermiation, but no difference was seen in germ cell suppression with or without DMPA.

In summary, it is clear that spermatogonial inhibition is a consistent feature of both acute and chronic gonadotropin withdrawal in these contraceptive models. However, spermiation inhibition is also striking within the first month of treatment and appears to be a major determinant of sperm output. Despite marked reductions in spermatogonia and subsequent germ cells, appreciable germ cell development (10-30\% of normal) continues even after long-term gonadotropin suppression (Figure 2), in comparison to a similar milieu in rats. What factors account for this? Androgen action may support some degree of germ cell development by virtue of the persistence of testicular DHT and Adiol levels. The fact that $\mathrm{T}$ administration with or without added progestin is associated with measurable levels of FSH (Robertson et al., 2001) may suggest some FSHmediated maintenance of spermatogenesis. Yet, to date, a relationship has not 
been seen between the achievement of either azoospermia or oligospermia and residual serum FSH levels in contraceptive trials (Handelsman et al., 1995).

\section{Current Studies Characterising the Mechanisms of Regulation of Hormone-sensitive Sites in Spermatogenesis}

The above studies on the role of FSH and/or $\mathrm{T}$ in the suppression and restoration of adult rat spermatogenesis indicate that both hormones have independent and synergistic effects on germ cell development. Our further studies in primates and humans have demonstrated the relevance of several of these processes - notably, spermatogonial development, sperm release, and testicular androgen biosynthesis - to the contraceptive-treated man. The comparison between rat, monkey, and human spermatogenesis after long-term gonadotropin suppression reveals the similarities between monkeys and humans and the similarities and differences between rats and primates (Figure 2). The identification of hormone-dependent steps has led to further research into the molecular mechanisms by which these processes are regulated. The next section discusses the focus of our more recent work on specific processes in spermatogenesis.

\section{A. REGULATION OF SPERMATOGONIAL DEVELOPMENT}

Spermatogonial stem cells provide a mitotically active lineage committed to both differentiation and renewal of the stem cell population. Both stem cells and differentiating spermatogonia are difficult to study due to their small populations and the lack of morphological and biochemical/molecular markers for identifying their various developmental phases. To date, their basal position and nuclear morphology, together with their stage associations, are the main features used to distinguish each spermatogonial subclass. Three models of spermatogonial renewal have been proposed in rodents (Meistrich and van Beek, 1993), with four subclasses of rat type A spermatogonia (denoted A1-4) as well as intermediate and type B spermatogonia (Clermont, 1972). In the monkey and human, there are two morphologically distinct type A spermatogonial subtypes, A dark (Ad) and A pale (Ap), as well as type B spermatogonia (Clermont, 1972) (Figure 3). Type Ap are proposed to divide to give rise to type $\mathrm{B}$ as well as to renew their own population (Clermont, 1969; van Alphen et al., 1988a,b; Schlatt and Weinbauer, 1994). Type Ad are considered to be the nonproliferative reserve spermatogonial population (Clermont, 1969; van Alphen et al., 1988a,b; Schlatt and Weinbauer, 1994) that may be able to undergo transition to Ap following testicular insult, thereby allowing repopulation of the testis (van Alphen et al., 1988a,b). Ap have been suggested to be the true stem cell of the testis because Ap (not Ad) are seen in humans after radiation (Schulze, 1979), after long-term estrogen therapy, and 


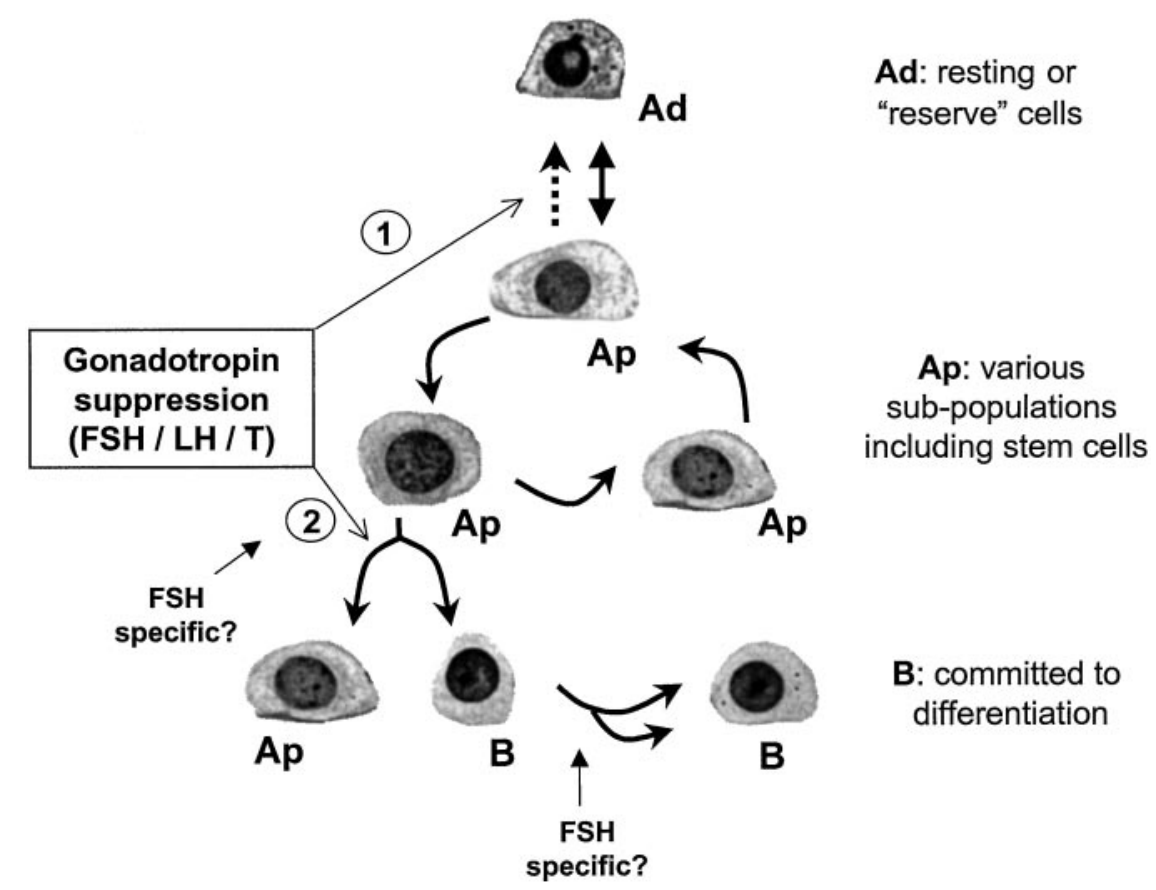

FIG. 3. Hypothesis of the effects of gonadotropin suppression on spermatogonial subtypes in primates. There are three subtypes of spermatogonia, type A pale (Ap) and A dark (Ad) spermatogonia and type B spermatogonia. Ap spermatogonia divide in the later stages of the spermatogenic cycle to produce type B spermatogonia as well as to renew their own population (indicated by the branched arrow). It is likely that different subtypes exist within the Ap category. Since a proportion of Ap spermatogonia are considered to be the true "stem cells" of the testis, there must be subpopulations of stem cell Ap spermatogonia as well as Ap spermatogonia that will give rise to B spermatogonia. Ad spermatogonia rarely divide and are considered to be "resting" or "reserve" stem cells. Type B spermatogonia are produced from the final mitosis of Ap spermatogonia and thus are considered as committed to differentiation. Type B spermatogonia then undergo a series of mitotic divisions (indicated by the branched arrow) before entering meiosis. It should be noted that the exact number of divisions is not indicated. Gonadotropin suppression, in which FSH, LH, and testicular T are suppressed, produces changes in these spermatogonial subtypes. However, in these suppression models, it is not possible to dissect the specific effects of each hormone (see section IV-A). Our data in monkeys and men suggest that gonadotropin suppression causes the "transdifferentiation" of Ap to Ad spermatogonia (dashed arrow), since increases in Ad and decreases in Ap are evident. Gonadotropin suppression also causes an inhibition of the final mitosis of Ap to B spermatogonia, which then leads to decreases in B spermatogonial numbers. This disruption of the final mitosis of Ap spermatogonia may also explain the fact that Ap spermatogonial number decrease with increasing time of gonadotropin suppression. There is evidence for FSH-specific effects (indicated by the arrow) on both the division of Ap into B spermatogonia and within the type B spermatogonial population (see section IV-A). This diagram shows primate (Macaca fascicularis) spermatogonia but is applicable to human spermatogonia. We believe it is likely that some species differences exist in the relative sensitivities of each spermatogonial type to gonadotropin/FSH suppression (see section IV-A). 
in the postpubertal cryptorchid testes (Schulze, 1981). Various studies have suggested that Ap can undergo transition without division into Ad (Fouquet and Dadoune, 1986; van Alphen et al., 1988a,b).

Following FSH/LH withdrawal, type Ap spermatogonia are the first cells to decrease in monkeys, followed by a subsequent decrease in B spermatogonia (O'Donnell et al., 2001a). In man, type B spermatogonia are the first cells to decrease, followed by decreases in type Ap (McLachlan et al., in press). The basis for this species difference is unclear but may relate to different sensitivities of Ap and B spermatogonia to gonadotropin suppression. The fall in the number of type B spermatogonia could be due to an inhibition of Ap spermatogonial mitosis, such as was demonstrated in GnRH antagonist-treated monkeys (Schlatt and Weinbauer, 1994), or by a direct effect on B spermatogonial mitosis (Figure 3).

Studies in cynomolgus monkeys showed that short-term (i.e., 2 weeks) $\mathrm{T}$ administration caused a decrease in type Ap spermatogonia, an increase in Ad spermatogonia, while type B spermatogonia were unchanged (O'Donnell et al., 2001a). This suggests gonadotropin withdrawal results in type Ap spermatogonia ceasing to proliferate into B spermatogonia but instead differentiating into $\mathrm{Ad}$ spermatogonia (Figure 3), as has been suggested by others (Fouquet and Dadoune, 1986). Studies in rhesus monkeys have shown decreases in Ad spermatogonia and increases in B spermatogonia in response to FSH treatment in juvenile monkeys (Ramaswamy et al., 2000b) or in response to the FSH rise induced by unilateral castration (Ramaswamy et al., 2000a). These data support our contention that Ap spermatogonia can produce B spermatogonia upon gonadotropic (presumably FSH) stimulation but can be shunted to "resting" Ad spermatogonia in the absence of such stimulus (Figure 3). Our studies on T-induced gonadotropin suppression in monkeys and man do not allow us to dissect out the relative effects of FSH versus LH/T suppression on spermatogonial subtypes in these species. However, other studies have administered FSH to monkeys to show that FSH alone can increase B spermatogonia (van Alphen et al., 1988c; Marshall et al., 1995; Ramaswamy et al., 2000b), suggesting that some or all of the effects of gonadotropin suppression on spermatogonia is due to the loss of FSH (Figure 3). Certainly, our data in rats would suggest that spermatogonia are regulated primarily by FSH (McLachlan et al., 1995; Meachem et al., 1998,2001).

In fact, there has been little support for the notion that sex steroids or Leydig cell factors stimulate spermatogonial development. In the rat, we found no evidence that $\mathrm{T}$ supports spermatogonial development after long-term gonadotropin depletion (Meachem et al., 1997,1998). Conversely, we have suggested that high serum $\mathrm{T}$ levels produced by exogenous $\mathrm{T}$ administration inhibits the restoration of spermatogonial number (Meachem et al., 1997,1998). Consistent with this, others have provided evidence that high testicular $\mathrm{T}$ levels are 
detrimental to spermatogonial development (Meistrich and Kangasniemi, 1997) and that suppression of testicular $\mathrm{T}$ levels is required to promote spermatogonial development in the irradiated rat and in the juvenile spermatogonial depletion (jsd) mutant mouse (Matsumiya et al., 1999).

\section{B. THE REGULATION OF SPERMIOGENESIS}

Several studies have demonstrated that $\mathrm{T}$ is critical for spermiogenesis (Awoniyi et al., 1989b; Sun et al., 1989; McLachlan et al., 1994a; O'Donnell et al., 1994). As described in Section IIA and Figure 4, suppression of intratesticular T levels impairs the conversion of step $7 \rightarrow 8$ round spermatids due to the premature detachment of step 8 round spermatids from the epithelium. These cells are subsequently found in the epididymis, where they degenerate (O'Donnell et al., 1996a). Based on these data, we (McLachlan et al., 1996; O'Donnell et al., 1996a) and others (Cameron et al., 1993) have hypothesised that androgens regulate adhesion between Sertoli cells and step 8 round spermatids, either via effects on the cell adhesion molecules (CAMs) located between the two cell types, or on the intracellular junctional apparatus located in the Sertoli cell.

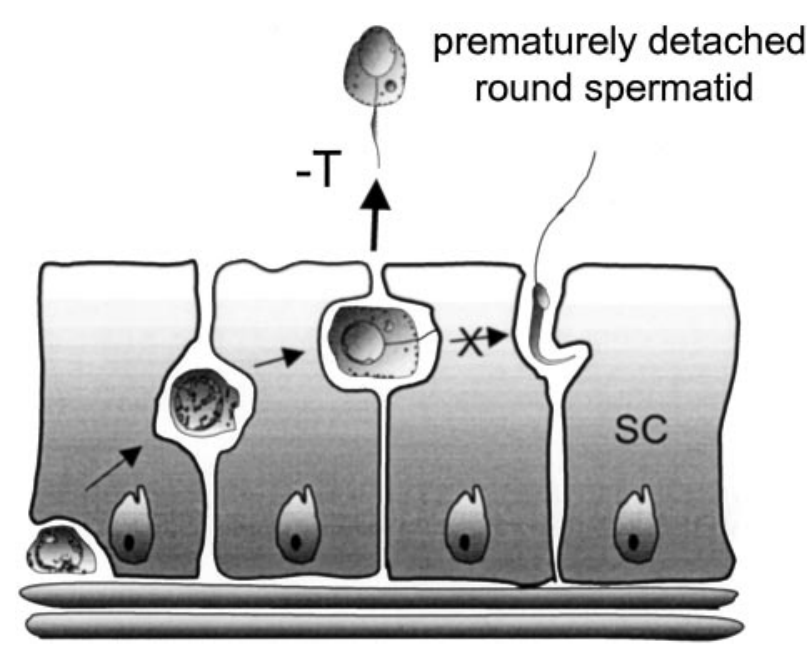

FIG. 4. Diagram of the effects of testicular testosterone (T) suppression by TE treatment on the association between step 8 round spermatids and the seminiferous epithelium. Testicular T suppression causes step 8 round spermatids to lose their attachment from the Sertoli cell (SC) within 3 weeks of testicular $\mathrm{T}$ suppression. The round spermatids proceed to the epididymis, where they degenerate (O’Donnell et al., 1996a). Thus, round spermatids prematurely detach and are unable to complete their elongation into the mature spermatid form, as indicated by the cross. This detachment can be reversed by 4 days of high-dose T replacement (O'Donnell et al., 1994). 
The contribution of FSH to round spermatid adhesion in this rodent model is expected to be permissive rather than regulatory, as FSH levels remain near normal in the above rodent model. However, it is well known that the intracellular organisation of various Sertoli cell cytoskeletal proteins is FSH dependent (Muffly et al., 1994). In vitro evidence has shown that FSH as well as T is required for adhesion between rat Sertoli cells and purified round spermatids (Cameron and Muffly, 1991; Perryman et al., 1996).

Coincident with the appearance of step 8 round spermatids in the normal epithelium is the formation of an adjacent specialised Sertoli cell junctional apparatus called the ectoplasmic specialisation (ES) (see Vogl et al., 2000, for a recent review), which remains during the elongation process and is removed just prior to spermiation. The ES comprises the Sertoli cell plasma membrane, a layer of hexagonally packed, noncontractile actin filaments and an underlying endoplasmic reticulum (Russell et al., 1988; Vogl et al., 2000). The ES is a hormone-sensitive structure, as it is disorganised in hypophysectomised adult rats (Muffly et al., 1993) and can be restored by treatment with FSH (Muffly et al., 1994). We postulated that the detachment of step 8 round spermatids when testicular T levels are low may have been due to the absence of the ES but have recently found that the actin-containing intracellular domain associated with the ES remains qualitatively normal under these circumstances (O'Donnell et al., 2000). This evidence suggests that the androgen-dependent lesion leading to detachment of step 8 round spermatids may lie in the intercellular CAM domain.

Despite extensive morphological data describing the ES, little is known about the identity(ies) or regulation of CAMs at this junction. Linkages between Sertoli cells and the spermatid acrosome have been observed by electron microscopy (Russell et al., 1988), which presumably contribute to a strong adhesive domain as mechanical disruption of the seminiferous epithelium results in spermatids with attached fragments of Sertoli cell cytoplasm containing ES (Romrell and Ross, 1979). One candidate CAM is $\alpha 6 \beta 1$-integrin, which has been immunolocalised to the junction between Sertoli cells and both round and elongating spermatids (Palombi et al., 1992; Salanova et al., 1995; Mulholland et al., 2001) and may be involved in a signaling complex with integrin-linked kinase (Mulholland et al., 2001). Other data support an involvement for CAMs from the cadherin (Byers et al., 1994; Wine and Chapin, 1999) and protocadherin (Johnson et al., 2000) families. We have demonstrated that N-cadherin production by Sertoli cells in vitro is dose dependent for $\mathrm{T}$ in the presence of FSH (Perryman et al., 1996). As an N-cadherin-specific antibody will also block androgen-stimulated adhesion between Sertoli cells and isolated round spermatids in vitro (Perryman et al., 1996), N-cadherin may be one of the CAMs that subserves this process. Proof of the importance of the androgenic regulation of CAM function in round spermatid adhesion in vivo is lacking. However, we have recently demonstrated that the mRNAs for $\beta 1$-integrin, $\mathrm{N}$-cadherin, and $\beta$-cate- 
nin are all significantly upregulated by $\mathrm{T}$ replacement in the rat model (P.G. Stanton, N.F. Cahir, L. O'Donnell, D.M. Robertson, unpublished data), highlighting their potential significance.

In contrast to the T-deficient rat, there is no evidence to support a major midspermiogenic lesion in monkeys or man following suppression of gonadotropins (Zhengwei et al., 1998b,c; O'Donnell et al., 2001a) (Figure 2). Although some round spermatids are seen in ejaculates of men following gonadotropin withdrawal, their number is low and does not correlate with the rapid fall in sperm count (Zhengwei et al., 1998a). However, it is important to note that $\mathrm{T}$ treatment suppresses both LH and FSH in man, a situation that is analogous to the GnRH-immunised rat, where spermiogenesis is fully suppressed prior to the production of step 8 spermatids (see Figures 1 and 2). Against this background, an appreciable degree of step 8 round spermatid detachment would not be apparent.

\section{THE COMBINED ROLE OF FSH AND TESTOSTERONE IN THE REGULATION OF SPERMIATION}

Although normal spermiation is clearly important for determining the sperm output from the testis, relatively little is known of the molecular control of this process. Immunocytochemical localisation studies have revealed the presence of several cell adhesion molecules and their associated proteins encompassing the spermatid head prior to release, such as $\mathrm{N}$-cadherin and catenin (Wine and Chapin, 1999), $\beta 1$-integrin (Palombi et al., 1992; Salanova et al., 1995; Mulholland et al., 2001) and its associated kinase integrin-linked kinase (ILK) (Mulholland et al., 2001). Other cytoskeletal and signaling molecules are present in the Sertoli cell at this stage (Wine and Chapin, 1999), which may be important in the control of adhesion between the spermatid and the Sertoli cell as well as for the subsequent disengagement of the spermatid during spermiation. Several lines of data suggest that sperm release is mediated by the Sertoli cell and that FSH and T activate similar pathways. These include 1) spermiation in rats in vivo appears to be regulated synergistically by FSH and T (Saito et al., 2000); 2) only Sertoli cells contain the receptors for these hormones; and 3) mature elongated spermatids are transcriptionally inactive.

Given that little is known of the molecular processes controlling normal spermiation, the regulation of spermiation failure is equally unclear. Marked spermiation failure occurs within a matter of days after hormone suppression, suggesting that the loss of FSH and T action on Sertoli cells either results in the loss of a "spermiation signal" and/or the initiation of processes required for spermatid retention. The fact that spermatids and Sertoli cells seem to interact via CAMs, and that most of the morphological events leading up to spermatid disengagement appear relatively normal on light microscopy during spermiation failure (A. Beardsley, 
L. O'Donnell, unpublished data), leads us to speculate that it is the actual disengagement (i.e., loss of adhesion) process that is impaired during spermiation failure, as has been speculated by others (Wine and Chapin, 1999).

Thus, it seems that the Sertoli cell fails to release the spermatid and phagocytosis then follows. Our efforts are now focused on characterising the changes in the expression, localisation, and phosphorylation status of putative spermiation-associated molecules, including CAMs, related downstream signaling molecules and kinases, both in normal rats and in those in which spermiation failure has been induced.

Further studies on the hormonal regulation of spermiation in humans are necessary to understand the relative sensitivities of this process to FSH and T suppression and whether various contraceptive regimes have differential effects on spermiation. The hypothesis that more profound suppression of gonadotropins would be more likely to lead to spermiation failure is supported by studies using combined $\mathrm{T}$ plus progestin contraceptive regimes in which the rapid suppression of sperm counts (<6 weeks) was seen (Meriggiola et al., 1996). It remains to be seen whether spermiation failure contributes to the heterogeneity in the suppression of sperm counts: Do men who remain oligospermic do so because spermiation failure does not occur? Further consideration ought be given to contraceptive formulations that target spermiation in humans, as they may provide more rapid and effective suppression of sperm count.

\section{THE ROLE OF $5 \alpha$-REDUCED ANDROGENS IN REGULATING SPERMATOGENESIS}

Our previous studies (O'Donnell et al., 1999) showed that blockade of androgen action in the testis by the administration of the AR antagonist, flutamide, increased the production of testicular $5 \alpha$-reduced metabolites, such that a significant increase in the concentration of testicular DHT and Adiol was observed, compared to vehicle-treated animals. This increase in $5 \alpha$-reduced metabolites occurred in the absence of changes in testicular $\mathrm{T}$ or serum $\mathrm{LH}$, suggesting that T may negatively regulate the $5 \alpha$-reductase $(5 \alpha \mathrm{R})$ enzyme. This was particularly interesting in view of the fact that exogenous $\mathrm{T}$ treatment to monkeys and men causes a marked decrease in testicular $\mathrm{T}$ levels, yet a maintenance of testicular $5 \alpha$-reduced androgens (see section III). These studies prompted further examination of testicular $5 \alpha$-reductase expression and regulation.

Two $5 \alpha \mathrm{R}$ genes - termed type $1(5 \alpha \mathrm{R}-1)$ and type $2(5 \alpha \mathrm{R}-2)$ - have been identified in humans and rats (for a review, see Russell and Wilson, 1994). $5 \alpha \mathrm{R}-1$ has a micromolar affinity for steroid substrates and a broad neutral $\mathrm{pH}$ range of activity, whereas $5 \alpha \mathrm{R}-2$ has a nanomolar affinity for steroid substrates and optimum activity at pH 5.0 (Normington and Russell, 1992). The cellular site and 
type of $5 \alpha \mathrm{R}$ isoform expressed in the rat testis is unclear, with conflicting evidence to show that either $5 \alpha \mathrm{R}-1$ or $5 \alpha \mathrm{R}-2$ is the predominant enzyme expressed in the testis (Normington and Russell, 1992; Viger and Robaire, 1995). Our studies show that both isoenzymes are present in the testis but that $5 \alpha \mathrm{R}-1$ is the predominant form (Pratis et al., 2000).

To examine the role of the $5 \alpha \mathrm{R}$ in the regulation of rat spermatogenesis, we studied the restoration of spermiogenesis following $\mathrm{T}$ treatment in the presence or absence of a $5 \alpha \mathrm{R}$ inhibitor. When submaximal levels of $\mathrm{T}$ were administered ( $\leq 10$-cm implants), the co-treatment with a $5 \alpha \mathrm{R}$ inhibitor impaired the restoration of step 8 round spermatids (O'Donnell et al., 1996b) and, subsequently, the production of mature spermatids (O'Donnell et al., 1999).

In humans, there is evidence to suggest that the inability of T-based contraceptive regimens to consistently reach azoospermia is due to differences in $5 \alpha \mathrm{R}$ activity between subjects. Anderson and colleagues (1996) showed that, in response to $\mathrm{T}$ enanthate, plasma DHT levels increased in men, compared to pretreatment. Interestingly, plasma DHT levels in men achieving oligospermia were significantly greater than those men who achieved azoospermia, despite no differences being seen in plasma T levels, presumably reflecting their higher $5 \alpha \mathrm{R}$ activity. These findings provide evidence to suggest that ongoing low levels of sperm production in oligospermic men may be due to upregulation of $5 \alpha \mathrm{R}$ activity in the reproductive tract.

To summarise, data in rats (O'Donnell et al., 1999; and K. Pratis, L. O'Donnell, G. Ooi, P. Stanton, R. McLachlan, D. Robertson, unpublished data) and humans (Anderson et al., 1996,1997) suggest that $5 \alpha$-reduction of $\mathrm{T}$ to DHT in a setting of reduced testicular T concentrations (i.e., during contraceptive administration), via up-regulation of the $5 \alpha \mathrm{R}$ enzyme, may provide an increased androgen stimulus to promote low levels of sperm production in the presence of reduced concentrations of gonadotropins. The preferred pathway of androgen action may depend on the concentration of intratesticular $\mathrm{T}$ (Figure 5). In the normal testis, we suggest that $\mathrm{T}$ would be the preferred ligand for the androgen receptor; however, during suppression of intratesticular $\mathrm{T}$, the more potent androgen DHT would be the preferred ligand.

Two recent human clinical trials have employed a $5 \alpha \mathrm{R}$ inhibitor during T-based hormonal contraception in order to see whether better contraceptive suppression is achieved. The $5 \alpha \mathrm{R}-2$ specific inhibitor, finasteride, was coadministered with T pellets (McLachlan et al., 2000) or T pellets in combination with desogestrel (Kinniburgh et al., 2001). Both studies showed that the addition of finasteride did not enhance spermatogenic suppression. Although these studies do not support the proposition that the inclusion of $5 \alpha \mathrm{R}$ inhibitors assists in obtaining consistent azoospermia, it must be noted that inhibition of both $5 \alpha \mathrm{R}-1$ and $5 \alpha \mathrm{R}-2$ (by combined single or dual inhibitors) may provide a better alternative to finasteride. 
(a) Normal testis

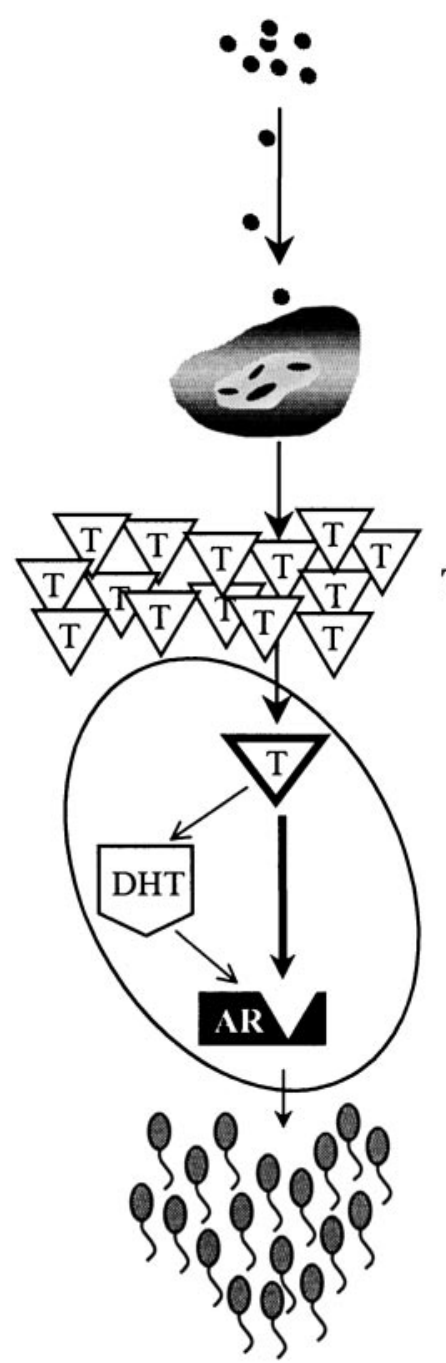

(b) T-suppressed testis

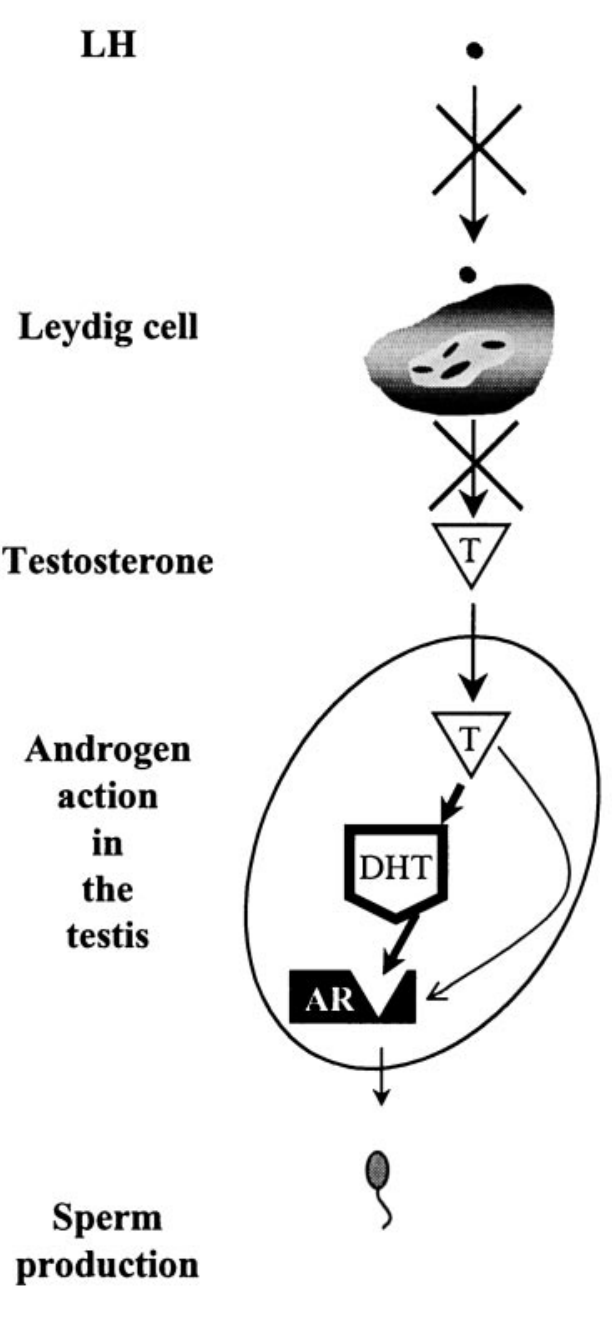

FIG. 5. Androgen action in normal and testosterone-suppressed testes. (A) In the normal testis, LH stimulation of Leydig cell steroidogenesis provides the testis with high local concentrations of testosterone, to act directly on the AR to maintain sperm production. (B) In the testosteronesuppressed testis, the low concentration of testosterone is unable to maintain spermatogenesis. However, conversion of testosterone to the more potent androgenic DHT, via the $5 \alpha$ R enzyme, allows low-level sperm production, despite gonadotropin and testosterone suppression. 


\section{E. RELEVANCE TO RESEARCH INTO MALE FERTILITY REGULATION}

Our present view of potential sites for hormonal manipulation of spermatogenesis centre on two steps in the process: the inhibition of spermatogonial replication and maturation, and spermiation failure. It appears that FSH withdrawal is critical for the former, while both FSH and T are critical for the latter. It is apparent, however, that the inhibitory processes involved in both cases are poorly understood, leading to variability in degree and time to full suppression.

Wider testing of hormonal contraception with regimens including $\mathrm{T}$ plus progestin is underway worldwide and is likely to provide effective contraception in $>95 \%$ of men. After the "proof-of-concept" work undertaken over recent years by public sector agencies, notably WHO and Contraceptive Research and Development Program, funded by the U.S. Agency for International Development, there is now pharmaceutical interest in product development. However, the fundamental goal of achieving uniform induction of azoospermia has not been achieved. Furthermore, differences between men in the speed of induction and recovery of spermatogenesis remain unexplained.

Our future studies will be directed to clarifying the underlying factors responsible for this variability, with the following hormonal and spermatogenic issues being considered worthy of study:

1. The need for the suppression of serum gonadotropins at a maximum rate and as profoundly as possible. Better suppression of gonadotropins is likely to be important for a more rapid onset of suppression of sperm counts (due to the induction of spermiation failure) and a more uniform induction of azoospermia. It is clear that sperm count suppression is poorer when gonadotropins are only partially suppressed (Matsumoto, 1990; Handelsman et al., 1996; McLachlan et al., 2000). However, the hypothesis that failure to fully suppress serum gonadotropins (i.e., to $<5 \%$ of baseline) correlates with the failure to achieve azoospermia has so far not been borne out (Handelsman et al., 1995). Alternative agents that more profoundly suppress gonadotropin levels, such as specific inhibitors and receptor antagonists, may be useful in this respect.

2. As discussed above, there is a need to consider larger trials of $5 \alpha \mathrm{R}$ inhibitors in conjunction with contraceptive formulations. Combined type 1 and $25 \alpha \mathrm{R}$ inhibition would ensure more profound suppression of testicular androgen action, which may be important for the maintenance of spermatogenesis and is likely to be beneficial for the prevention of adverse androgen-dependent effects, particularly on prostate and skin.

3. Finally, a better understanding of the molecular mechanisms of spermiation may allow the design of strategies to specifically block sperm release, thereby providing for a more rapid onset of effectiveness, which would be desirable in clinical practice. Further understanding of spermatogonial prolifer- 
ation and differentiation is needed to allow the development of strategies to more profoundly inhibit the entry of spermatogonia into the spermatogenic process. Such therapies are necessary for the more uniform induction of azoospermia, as indicated in monkeys.

\section{Conclusions}

We have used a range of rat, monkey, and human models to show that FSH and androgens act both separately and synergistically to support a range of key events in spermatogenesis, from spermatogonial stem cell division through to final sperm release. All of these events have been demonstrated using objective and quantitative techniques of germ cell enumeration. We continue to examine the basic mechanisms of these specific hormonal-dependent events using biochemical and molecular approaches. Through identification of these processes, new developments in male hormonal contraception can be expected. The majority of idiopathic male infertility is most likely due to genetic factors, with little or no hormonal basis (de Kretser et al., 2000). But it must be stressed that a better understanding of the physiology of normal spermatogenesis will provide valuable leads in areas such as infertility due to the failure of stem cell proliferation or of germ cells to mature beyond a particular developmental point. Knowledge of the regulation of normal spermatogenesis will facilitate the interpretation of genotype-phenotype relationships in male infertility and permit the definition of new diagnostic categories and perhaps treatments in this difficult field.

\section{ACKNOWLEDGMENTS}

This review was supported by a Program Grant (\#983212) from the National Health and Medical Research Council of Australia (NH\&MRC) and the Wellcome Trust Fellowship Scheme (SM \#058479).

\section{REFERENCES}

Anderson RA, Wallace AM, Wu FC 1996 Comparison between testosterone enanthate-induced azoospermia and oligozoospermia in a male contraceptive study. III. Higher 5 alpha-reductase activity in oligozoospermic men administered supraphysiological doses of testosterone. J Clin Endocrinol Metab 81:902-908

Anderson RA, Kelly RW, Wu FC 1997 Comparison between testosterone enanthate-induced azoospermia and oligozoospermia in a male contraceptive study. V. Localization of higher 5 alpha-reductase activity to the reproductive tract in oligozoospermic men administered supraphysiological doses of testosterone. J Androl 18:366-371

Aslam H, Rosiepen G, Krishnamurthy H, Arslan M, Clemen G, Nieschlag E, Weinbauer GF 1999 The cycle duration of the seminiferous epithelium remains unaltered during GnRH antagonist-induced testicular involution in rats and monkeys. J Endocrinol 161:281-288 
Awoniyi CA, Santulli R, Chandrashekar V, Schanbacher BD, Zirkin BR 1989a Quantitative restoration of advanced spermatogenic cells in adult male rats made azoospermic by active immunization against luteinizing hormone or gonadotropin-releasing hormone. Endocrinology 125:1303-1309.

Awoniyi CA, Santulli R, Sprando RL, Ewing LL, Zirkin BR 1989b Restoration of advanced spermatogenic cells in the experimentally regressed rat testis: quantitative relationship to testosterone concentration within the testis. Endocrinology 124:1217-1223

Awoniyi CA, Sprando RL, Santulli R, Chandrashekar V, Ewing LL, Zirkin BR 1990 Restoration of spermatogenesis by exogenously administered testosterone in rats made azoospermic by hypophysectomy or withdrawal of luteinizing hormone alone. Endocrinology 127:177-184

Byers SW, Sujarit S, Jegou B, Butz S, Hoschutzky H, Herrenknecht K, MacCalman C, Blaschuk OW 1994 Cadherins and cadherin-associated molecules in the developing and maturing rat testis. Endocrinology 134:630-639

Cameron DF, Muffly KE 1991 Hormonal regulation of spermatid binding. J Cell Sci 100:623-633

Cameron DF, Muffly KE, Nazian SJ 1993 Reduced testosterone during puberty results in a midspermiogenic lesion. Proc Soc Exp Biol Med 202:457-464.

Clermont Y 1969 Two classes of spermatogonial stem cells in the monkey (Cercopithecus aethiops). Am J Anat 126:57-71

Clermont Y 1972 Kinetics of spermatogenesis in mammals: seminiferous epithelium cycle and spermatogonial renewal. Physiol Rev 52:198-236

Clermont Y, Harvey SC 1965 Duration of the cycle of the seminiferous epithelium of normal, hypophysectomized and hypophysectomized-hormone treated albino rats. Endocrinology 76:80-89

de Kretser DM, Kerr JB 1988 The cytology of the testis. In: Knobil E, Neill JD, eds. The Physiology of Reproduction, vol. 1. New York: Raven Press Ltd.; 837-932

de Kretser DM, O'Bryan MK, Cram D, McLachlan RI 2000 Expanding our understanding of spermatogenesis: the future genetic tests for infertility. Intl J Androl 23:30-33

de Rooij DG, van Alphen MM, van de Kant HJ 1986 Duration of the cycle of the seminiferous epithelium and its stages in the rhesus monkey (Macaca mulatta). Biol Reprod 35:587-591

El Shennawy A, Gates RJ, Russell LD 1998 Hormonal regulation of spermatogenesis in the hypophysectomized rat: cell viability after hormonal replacement in adults after intermediate periods of hypophysectomy. J Androl 19:320-334; discussion 341-322

Fouquet JP, Dadoune JP 1986 Renewal of spermatogonia in the monkey (Macaca fascicularis). Biol Reprod 35:199-207

Handelsman DJ, Farley TM, Peregoudov A, Waites GM 1995 Factors in nonuniform induction of azoospermia by testosterone enanthate in normal men. World Health Organization Task Force on Methods for the Regulation of Male Fertility. Fertil Steril 63:125-133

Handelsman DJ, Conway AJ, Howe CJ, Turner L, Mackey MA 1996 Establishing the minimum effective dose and additive effects of depot progestin in suppression of human spermatogenesis by a testosterone depot. J Clin Endocrinol Metab 81:4113-4121

Hayes FJ, DeCruz S, Seminara SB, Boepple PA, Crowley WF Jr 2001 Differential regulation of gonadotropin secretion by testosterone in the human male: absence of a negative feedback effect of testosterone on follicle-stimulating hormone secretion. J Clin Endocrinol Metab 86:53-58

Heller CG, Clermont Y 1963 Spermatogenesis in man: an estimation of its duration. Science 140:184-186

Heller CG, Clermont Y 1964 Kinetics of the germinal epithelium in man. Recent Prog Horm Res 20:545-575

Huhtaniemi I, Bartke A 2001 Perspective: male reproduction. Endocrinology 142:2178-2183 
Johnson KJ, Patel SR, Boekelheide K 2000 Multiple cadherin superfamily members with unique expression profiles are produced in rat testis. Endocrinology 141:675-683

Johnston DS, Russell LD, Friel PJ, Griswold MD 2001 Murine germ cells do not require functional androgen receptors to complete spermatogenesis following spermatogonial stem cell transplantation. Endocrinology 142:2405-2408

Kangasniemi M, Wilson G, Parchuri N, Huhtaniemi I, Meistrich ML 1995 Rapid protection of rat spermatogenic stem cells against procarbazine by treatment with a gonadotropin-releasing hormone antagonist (Nal-Glu) and an antiandrogen (flutamide). Endocrinology 136:28812888

Kinniburgh D, Anderson RA, Baird DT 2001 Suppression of spermatogenesis with desogestrel and testosterone pellets is not enhanced by addition of finasteride. J Androl 22:88-95

Leblond CP, Clermont Y 1952 Definition of the stages of the cycle of the seminiferous epithelium in the rat. Ann NY Acad Sci 55:548-573

Marshall GR, Zorub DS, Plant TM 1995 Follicle-stimulating hormone amplifies the population of differentiated spermatogonia in the hypophysectomized testosterone-replaced adult rhesus monkey (Macaca mulatta). Endocrinology 136:3504-3511

Martin CW, Riley SC, Everington D, Groome NP, Riemersma RA, Baird DT, Anderson RA 2000 Dose-finding study of oral desogestrel with testosterone pellets for suppression of the pituitary-testicular axis in normal men. Hum Reprod 15:1515-1524

Matsumiya K, Meistrich ML, Shetty G, Dohmae K, Tohda A, Okuyama A, Nishimune Y 1999 Stimulation of spermatogonial differentiation in juvenile spermatogonial depletion (jsd) mutant mice by gonadotropin-releasing hormone antagonist treatment. Endocrinology 140: 4912-4915

Matsumoto AM 1990 Effects of chronic testosterone administration in normal men: safety and efficacy of high dosage testosterone and parallel dose-dependent suppression of luteinizing hormone, follicle-stimulating hormone, and sperm production. J Clin Endocrinol Metab 70:282-287.

Matsumoto AM, Bremner WJ 1989 Endocrine control of human spermatogenesis. J Steroid Biochem 33:789-790

McLachlan RI, Wreford NG, Meachem SJ, De Kretser DM, Robertson DM 1994a Effects of testosterone on spermatogenic cell populations in the adult rat. Biol Reprod 51:945-955

McLachlan RI, Wreford NG, Tsonis C, De Kretser DM, Robertson DM 1994b Testosterone effects on spermatogenesis in the gonadotropin-releasing hormone-immunized rat. Biol Reprod 50:271-280

McLachlan RI, Wreford NG, de Kretser DM, Robertson DM 1995 The effects of recombinant follicle-stimulating hormone on the restoration of spermatogenesis in the gonadotropinreleasing hormone-immunized adult rat. Endocrinology 136:4035-4043

McLachlan RI, Wreford NG, O'Donnell L, de Kretser DM, Robertson DM 1996 The endocrine regulation of spermatogenesis: independent roles for testosterone and FSH. J Endocrinol 148:1-9

McLachlan RI, McDonald J, Rushford D, Robertson DM, Garrett C, Baker HW 2000 Efficacy and acceptability of testosterone implants, alone or in combination with a 5alpha-reductase inhibitor, for male hormonal contraception. Contraception 62:73-78

McLachlan RI, O'Donnell L, Stanton PG, Balourdos G, Frydenberg M, de Kretser DM, Robertson DM 2002 Effects of testosterone plus medroxyprogesterone acetate on semen quality, reproductive hormones, and germ cell populations in normal young men. J Clin Endocrinol Metab, in press

McLean DJ, Johnston DS, Russell LD, Griswold MD 2001 Germ cell transplantation and the study of testicular function. Trends Endocrinol Metab 12:16-21. 
Meachem S, von Schonfeldt V, Schlatt S 2001 Spermatogonia: stem cells with a great perspective. Reproduction 121:825-834.

Meachem SJ, Wreford NG, Robertson DM, McLachlan RI 1997 Androgen action on the restoration of spermatogenesis in adult rats: effects of human chorionic gonadotrophin, testosterone and flutamide administration on germ cell number. Intl J Androl 20:70-79

Meachem SJ, Wreford NG, Stanton PG, Robertson DM, McLachlan RI 1998 Follicle-stimulating hormone is required for the initial phase of spermatogenic restoration in adult rats following gonadotropin suppression. J Androl 19:725-735

Meachem SJ, McLachlan RI, Stanton PG, Robertson DM, Wreford NG 1999 FSH immunoneutralization acutely impairs spermatogonial development in normal adult rats. J Androl 20:756-762; discussion 755

Meistrich ML, Kangasniemi M 1997 Hormone treatment after irradiation stimulates recovery of rat spermatogenesis from surviving spermatogonia. J Androl 18:80-87

Meistrich ML, van Beek ME 1993 Spermatogonial stem cells. In: Desjardins C, Ewing LL, eds. Cell and Molecular Biology of the Testis. New York: Oxford University Press; 266-295

Meriggiola MC, Bremner WJ, Paulsen CA, Valdiserri A, Incorvaia L, Motta R, Pavani A, Capelli M, Flamigni C 1996 A combined regimen of cyproterone acetate and testosterone enanthate as a potentially highly effective male contraceptive. J Clin Endocrinol Metab 81:3018-3023

Meriggiola MC, Bremner WJ 1997 Progestin-androgen combination regimens for male contraception. J Androl 18:240-244

Muffly KE, Nazian SJ, Cameron DF 1993 Junction-related Sertoli cell cytoskeleton in testosteronetreated hypophysectomized rats. Biol Reprod 49:1122-1132

Muffly KE, Nazian SJ, Cameron DF 1994 Effects of follicle-stimulating hormone on the junction-related Sertoli cell cytoskeleton and daily sperm production in testosterone-treated hypophysectomized rats. Biol Reprod 51:158-166

Mulholland DJ, Dedhar S, Vogl AW 2001 Rat seminiferous epithelium contains a unique junction (ectoplasmic specialization) with signaling properties both of cell/cell and cell/matrix junctions. Biol Reprod 64:396-407

Normington K, Russell DW 1992 Tissue distribution and kinetic characteristics of rat steroid 5 alpha-reductase isozymes. Evidence for distinct physiological functions. J Biol Chem 267: $19548-19554$

O'Donnell L, McLachlan RI, Wreford NG, Robertson DM 1994 Testosterone promotes the conversion of round spermatids between stages VII and VIII of the rat spermatogenic cycle. Endocrinology 135:2608-2614

O'Donnell L, McLachlan RI, Wreford NG, de Kretser DM, Robertson DM 1996a Testosterone withdrawal promotes stage-specific detachment of round spermatids from the rat seminiferous epithelium. Biol Reprod 55:895-901

O'Donnell L, Stanton PG, Wreford NG, Robertson DM, McLachlan RI 1996b Inhibition of 5 alpha-reductase activity impairs the testosterone-dependent restoration of spermiogenesis in adult rats. Endocrinology 137:2703-2710

O’Donnell L, Pratis K, Stanton PG, Robertson DM, McLachlan RI 1999 Testosterone-dependent restoration of spermatogenesis in adult rats is impaired by a 5alpha-reductase inhibitor. $\mathrm{J}$ Androl 20:109-117

O'Donnell L, Stanton PG, Bartles JR, Robertson DM 2000 Sertoli cell ectoplasmic specializations in the seminiferous epithelium of the testosterone-suppressed adult rat. Biol Reprod 63:99108

O'Donnell L, Narula A, Balourdos G, Gu YQ, Wreford NG, Robertson DM, Bremner WJ, McLachlan RI 2001a Impairment of spermatogonial development and spermiation after 
testosterone-induced gonadotropin suppression in adult monkeys (Macaca fascicularis). J Clin Endocrinol Metab 86:1814-1822

O'Donnell L, Robertson KM, Jones ME, Simpson ER 2001b Estrogen and spermatogenesis. Endocr Rev 22:289-318

Orth JM 1993 Cell biology of testicular development in the fetus and neonate. In: Desjardins C, Ewing LL, eds. Cell and Molecular Biology of the Testis. New York: Oxford University Press; 3-43

Palombi F, Salanova M, Tarone G, Farini D, Stefanini M 1992 Distribution of beta 1 integrin subunit in rat seminiferous epithelium. Biol Reprod 47:1173-1182

Perryman KJ, Stanton PG, Loveland KL, McLachlan RI, Robertson DM 1996 Hormonal dependency of neural cadherin in the binding of round spermatids to Sertoli cells in vitro. Endocrinology 137:3877-3883

Pratis K, O'Donnell L, Ooi GT, McLachlan RI, Robertson DM 2000 Enzyme assay for 5alpha-reductase type 2 activity in the presence of 5alpha-reductase type 1 activity in rat testis. J Steroid Biochem Mol Biol 75:75-82

Ramaswamy S, Marshall GR, McNeilly AS, Plant TM 2000a Dynamics of the follicle-stimulating hormone (FSH)-inhibin B feedback loop and its role in regulating spermatogenesis in the adult male rhesus monkey (Macaca mulatta) as revealed by unilateral orchidectomy. Endocrinology 141:18-27

Ramaswamy S, Plant TM, Marshall GR 2000b Pulsatile stimulation with recombinant single chain human luteinizing hormone elicits precocious Sertoli cell proliferation in the juvenile male rhesus monkey (Macaca mulatta). Biol Reprod 63:82-88

Robertson DM, Pruysers E, Stephenson T, Pettersson K, Morton S, McLachlan RI 2001 Sensitive LH and FSH assays for monitoring low serum levels in men undergoing steroidal contraception. Clin Endocrinol 55:331-339

Romrell LJ, Ross MH 1979 Characterization of Sertoli cell-germ cell junctional specializations in dissociated testicular cells. Anat Rec 193:23-41.

Russell DW, Wilson JD 1994 Steroid 5 alpha-reductase: two genes/two enzymes. Annu Rev Biochem 63:25-61

Russell LD 1991 The perils of sperm release - "let my children go." Intl J Androl 14:307-311

Russell LD, Clermont Y 1977 Degeneration of germ cells in normal, hypophysectomized and hormone treated hypophysectomized rats. Anat Rec 187:347-366

Russell LD, Alger LE, Nequin LG 1987 Hormonal control of pubertal spermatogenesis. Endocrinology 120:1615-1632

Russell LD, Goh JC, Rashed RM, Vogl AW 1988 The consequences of actin disruption at Sertoli ectoplasmic specialization sites facing spermatids after in vivo exposure of rat testis to cytochalasin D. Biol Reprod 39:105-118

Russell LD, Ettlin RA, Sinha Hikim AP, Clegg ED 1990 Histological and Histopathological Evaluation of the Testis. Clearwater, FL: Cache River Press

Saito K, O'Donnell L, McLachlan RI, Robertson DM 2000 Spermiation failure is a major contributor to early spermatogenic suppression caused by hormone withdrawal in adult rats. Endocrinology 141:2779-2785

Salanova M, Stefanini M, De Curtis I, Palombi F 1995 Integrin receptor alpha 6 beta 1 is localized at specific sites of cell-to-cell contact in rat seminiferous epithelium. Biol Reprod 52:79-87

Schlatt S, Weinbauer GF 1994 Immunohistochemical localization of proliferating cell nuclear antigen as a tool to study cell proliferation in rodent and primate testes. Intl $\mathrm{J}$ Androl $17: 214-222$

Schulze C 1979 Morphological characteristics of the spermatogonial stem cells in man. Cell Tiss Res 198:191-199 
Schulze C 1981 Survival of human spermatogonial stem cells in various clinical conditions. Fortschritte Der Andrologie 7:58-68

Schulze W, Rehder U 1984 Organization and morphogenesis of the human seminiferous epithelium. Cell Tiss Res 237:395-407

Sinha Hikim AP, Swerdloff RS 1993 Temporal and stage-specific changes in spermatogenesis of rat after gonadotropin deprivation by a potent gonadotropin-releasing hormone antagonist treatment. Endocrinology 133:2161-2170.

Sinha Hikim AP, Wang C, Leung A, Swerdloff RS 1995 Involvement of apoptosis in the induction of germ cell degeneration in adult rats after gonadotropin-releasing hormone antagonist treatment. Endocrinology 136:2770-2775

Sinha Hikim AP, Swerdloff RS 1999 Hormonal and genetic control of germ cell apoptosis in the testis. Rev Reprod 4:38-47

Sun YT, Irby DC, Robertson DM, de Kretser DM 1989 The effects of exogenously administered testosterone on spermatogenesis in intact and hypophysectomized rats. Endocrinology 125: $1000-1010$

Tapanainen JS, Tilly JL, Vihko KK, Hsueh AJ 1993 Hormonal control of apoptotic cell death in the testis: gonadotropins and androgens as testicular cell survival factors. Mol Endocrinol 7:643-650

van Alphen MM, van de Kant HJ, de Rooij DG 1988a Depletion of the spermatogonia from the seminiferous epithelium of the rhesus monkey after X irradiation. Radiat Res 113:473-486

van Alphen MM, van de Kant HJ, de Rooij DG 1988b Repopulation of the seminiferous epithelium of the rhesus monkey after X irradiation. Radiat Res 113:487-500

van Alphen MM, van de Kant HJ, de Rooij DG 1988c Follicle-stimulating hormone stimulates spermatogenesis in the adult monkey. Endocrinology 123:1449-1455

Viger RS, Robaire B 1995 Steady state steroid 5 alpha-reductase messenger ribonucleic acid levels and immunocytochemical localization of the type 1 protein in the rat testis during postnatal development. Endocrinology 136:5409-5415

Vogl AW, Pfeiffer DC, Mulholland D, Kimel G, Guttman J 2000 Unique and multifunctional adhesion junctions in the testis: ectoplasmic specializations. Arch Histol Cytol 63:1-15

Walsh PC, Swerdloff RS 1973 Biphasic effect of testosterone on spermatogenesis in the rat. Invest Urol 11:190-193

Weinbauer GF, Gockeler E, Nieschlag E 1988 Testosterone prevents complete suppression of spermatogenesis in the gonadotropin-releasing hormone antagonist-treated nonhuman primate (Macaca fascicularis). J Clin Endocrinol Metab 67:284-290

Weinbauer GF, Schlatt S, Walter V, Nieschlag E 2001 Testosterone-induced inhibition of spermatogenesis is more closely related to suppression of FSH than to testicular androgen levels in the cynomolgus monkey model (Macaca fascicularis). J Endocrinol 168:25-38

Wierman ME, Wang C 1990 Androgen selectively stimulates follicle-stimulating hormone-beta mRNA levels after gonadotropin-releasing hormone antagonist administration. Biol Reprod 42:563-571

Wine RN, Chapin RE 1999 Adhesion and signaling proteins spatiotemporally associated with spermiation in the rat. J Androl 20:198-213

World Health Organization Task Force on Methods for the Regulation of Male Fertility 1990 Contraceptive efficacy of testosterone-induced azoospermia in normal men. Lancet 336:955959

World Health Organization Task Force on Methods for the Regulation of Male Fertility 1996 Contraceptive efficacy of testosterone-induced azoospermia and oligozoospermia in normal men. Fertil Steril 65:821-829

Wreford NG 1995 Theory and practice of stereological techniques applied to the estimation of cell number and nuclear volume in the testis. Microsc Res Tech 32:423-436 
Zhengwei Y, Wreford NG, Bremner WJ, Matsumoto AM, Anawalt BA, McLachlan RI 1998a Immature spermatids are not prevalent in semen from men who are receiving androgen-based contraceptive regimens. Fertil Steril 69:89-95

Zhengwei Y, Wreford NG, Royce P, de Kretser DM, McLachlan RI 1998b Stereological evaluation of human spermatogenesis after suppression by testosterone treatment: heterogeneous pattern of spermatogenic impairment. J Clin Endocrinol Metab 83:1284-1291

Zhengwei Y, Wreford NG, Schlatt S, Weinbauer GF, Nieschlag E, McLachlan RI 1998c Acute and specific impairment of spermatogonial development by GnRH antagonist-induced gonadotrophin withdrawal in the adult macaque (Macaca fascicularis). J Reprod Fertil 112:139147

Zirkin BR, Santulli R, Awoniyi CA, Ewing LL 1989 Maintenance of advanced spermatogenic cells in the adult rat testis: quantitative relationship to testosterone concentration within the testis. Endocrinology 124:3043-3049 



\title{
Molecular Defects of the Androgen Receptor
}

\author{
Michael J. McPhaul \\ Department of Internal Medicine, Division of Endocrinology and Metabolism, The University of \\ Texas Southwestern Medical School, Dallas, Texas 75390-8857
}

\begin{abstract}
Mutations in the androgen receptor (AR) gene cause a range of phenotypic abnormalities of male sexual development. At one end of the spectrum are individuals with complete androgen insensitivity (complete testicular feminization) who exhibit normal breast development and female external genitalia. At the other extreme are individuals with male phenotypes that are characterized by either subtle undervirilization or infertility. Studies in a number of different laboratories have identified mutations of the AR gene in subjects with androgen resistance syndromes. Defects that interrupt the AR open-reading frame have been traced to a number of distinct types of genetic alterations, have been identified in widely separated segments of the AR gene, and are invariably associated with the phenotype of complete androgen insensitivity. By contrast, mutations that cause single amino acid substitutions within the AR are localized to the DNA- or ligand-binding domains of the receptor protein and have been associated with the full range of androgen-resistant phenotypes. Regardless of the nature of the mutation, functional studies and assays of AR abundance suggest that the phenotypic abnormalities that result from mutation of the AR are the result of the impairment of receptor function, decreases in receptor concentration, or both.
\end{abstract}

\section{Background}

The description of androgen-resistant states built upon the recognition that endocrine disorders would likely result from abnormalities in the capacity of target tissues to respond appropriately to selected hormones (Albright, 1942). In 1953, Morris provided a detailed modern description of the syndrome that he termed testicular feminization, which he identified as a "recognizable syndrome found in patients who are essentially normal-appearing women, but who have undescended testes in place of ovaries" (Morris, 1953). Subsequent investigators presented evidence suggesting the insensitivity of end organs to the actions of androgens (Wilkins, 1957). Further investigators amassed information suggesting that distinct phenotypes might be the result of similar, but less severe, abnormalities (Reifenstein 1947, Rosewater et al., 1965; Morris and Mahesh, 1968) Careful endocrine studies, coupled with family studies, suggested that these syndromes were likely the result of variable defects of the same or similar genes (Wilson et al., 1974). 
Defects of the androgen receptor (AR) are common, compared to mutations of other members of the nuclear receptor family. In addition to the frequency of patients with clinical androgen resistance, the AR is unusual in the range of abnormal phenotypes that have been identified and traced to AR defects. Several features of the AR are believed to contribute to the frequency and diversity of the disorders that have been identified. First, the AR gene is located on the human X-chromosome (Migeon et al., 1981). For this reason, in normal 46,XY males, only a single copy of the AR gene is present. Consequently, any defect of function of the single AR gene present in normal genetic males and which is responsible for mediating the responses to androgen will result in an abnormality of male phenotypic development. Second, such defects of virilization are frequently evident at birth and thus are likely to precipitate investigations to determine their cause. Finally, even complete defects of the AR are compatible with life and individuals affected by such disorders are available for ascertainment.

\section{Classification on Clinical and Biochemical Grounds}

The phenotypic abnormalities that accompany androgen-resistant states can is be viewed as a continuum that relates directly to the extent to which the androgen-mediated processes of male sexual development have been disturbed. In patients in whom AR function is completely absent, none of the androgendependent internal or external male structures develop. By contrast, a range of intermediate phenotypes results in cases where AR function is partially preserved (Griffin, 1992; Quigley et al., 1995; Griffin et al., 2001). As was noted, these syndromes have been identified clinically using a variety of terms, including the Reifenstein syndrome, partial androgen insensitivity, and incomplete testicular feminization. Some authors have defined more-detailed systems with which to grade the degree of virilization in patients with partial forms of androgen insensitivity (Quigley et al., 1995).

In parallel with the recognition of the clinical syndromes, the study and diagnosis of AR defects was greatly aided by the development of methods to measure the intracellular protein that binds androgens with high affinity. These methods - applied to whole-cell and broken-cell assays - permitted the initial characterization of the receptor that mediated the actions of androgens and the definition of methods to measure and characterize the level and physical properties of this protein in tissues and in cells (Pinsky et al., 1981; Griffin and Durant, 1982; Brown et al., 1992). When applied to fibroblasts established from patients with these disorders, these techniques led to the recognition of a number of quantitative and qualitative abnormalities of androgen binding in patients with androgen resistance (Figure 1). 


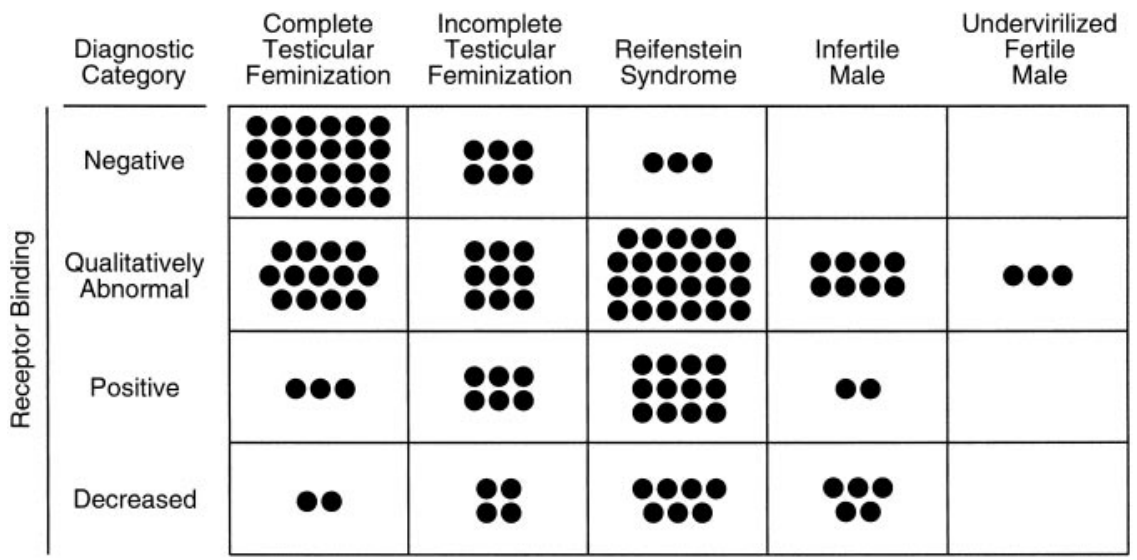

FIG. 1. Diagnostic and phenotypic categories of androgen resistance. Monolayer-binding assays were performed on genital skin fibroblasts established from individual subjects with varying degrees of androgen resistance. The samples were studied to assess the level and character of the androgen receptor (AR) expressed. "Qualitative binding abnormalities" denotes samples in which the level of AR detected was within the normal range but in which qualitative defects of androgen binding were detected (i.e., reduced affinity, accelerated ligand dissociation, thermal instability). While a preponderance of complete androgen resistance is associated with the absence of ligand binding (androgen binding negative), no direct relationship is evident between the type of ligand binding defect and the observed phenotype. [Reprinted with permission from Griffin JE 1992 Androgen resistance - the clinical and molecular spectrum. N Engl J Med 326:611-618. Copyright The Massachusetts Medical Society.]

Several features became apparent from such studies. First, the binding abnormalities fell into three broad categories: absent ligand binding, decreased levels of qualitatively normal ligand binding, and qualitative ligand-binding defects. In approximately $20 \%$ of patients in one series (Griffin et al., 2001), no quantitative or qualitative defect of the AR could be discerned. Second, such studies made it clear that although some types of defect were more frequent in some categories (e.g., a predominance of samples in which ligand binding was undetectable), no direct relationship existed between the clinical phenotype and the abnormality of ligand binding that was observed (Figure 1).

\section{Cloning and Structure of the Androgen Receptor}

Although progress in the characterization of many steroid receptors progressed rapidly in the 1970s and 1980s, the AR proved to be a difficult protein to purify. For this reason, specific antibodies were not available and information regarding the nature of the AR abnormalities causing the different states of $\mathrm{AR}$ 
resistance did not advance until the cloning of cDNAs encoding the AR (Chang et al., 1988; Lubahn et al., 1988; Faber et al., 1989; Tilley et al., 1989).

These studies revealed that the AR is a prototypic member of a large gene family: the nuclear receptor family (Mangelsdorf et al., 1995; Zoppi et al., in press). This family includes the classic steroid receptors as well as a large number of related proteins such as the receptors for thyroid hormone, vitamin D, and retinoic acid.

Members of this gene family possess a similar organization. Each protein contains a central DNA-binding domain and a carboxyl terminal ligand-binding domain (LBD). In addition, each predicted protein sequence contains an aminoterminal segment that is of variable length. This amino terminus is required for full transcriptional activity and is believed to represent the sites of important intramolecular and intermolecular contacts. In the case of the AR, the cDNAs isolated for the human AR predicted a protein approximately 917 amino acids long (Figure 2). In keeping with its large size, the human AR contains a large amino terminus that comprises nearly half of the molecule. Of interest, this amino-terminal segment contains three direct repeats of amino acid residues: one each containing glutamine, proline, and glycine residues. Expansion of the size

AR-A

AR-B 1

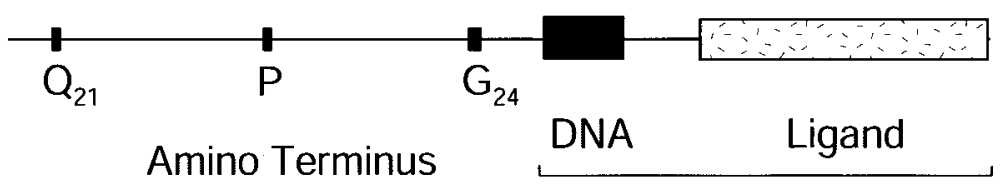

Binding Domains

FIG. 2. The predicted amino acid sequence of the human AR. The organization of the human AR is similar to that of the other nuclear receptors. The receptor contains a central DNA-binding domain (DBD) (residues 553-623) that mediates the high-affinity binding of the AR to target DNA sequences and a carboxyl terminal ligand-binding domain (LBD) (residues 692-919) that mediates the high-affinity binding of hormone. The amino terminus of the receptor comprises approximately half of the molecule (residues 1-550) and is required for full transcriptional activity (Gao et al., 1996). The predicted amino acid sequence of this segment of the human AR is unusual in that it contains three segments that are composed of direct repeats of single amino acid residues (homopolymeric repeats). These repeat elements, each located within the amino terminus of the protein, are not unique to the AR and have been identified in other transcription factors, including other members of the steroid receptor family. The amino acid coordinates employed here and throughout the text are those of Lubahn (Lubahn et al., 1988). 
of this glutamine homopolymeric segment has been associated with the pathogenesis of the spinal and bulbar muscular atrophy (Kennedy's disease) (La Spada et al., 1991).

\section{Mutations of the Androgen Receptor Gene and Androgen Resistance Syndromes}

As noted earlier, the AR was originally identified and characterized using assays to measure levels of tritiated androgen binding in intact cells and in cell homogenates. As the techniques to measure AR function and to identify mutations within the AR gene have become widely available, it has become possible to view the androgen-resistant states from perspectives that are more directly related to the effects that such mutations exert on receptor expression levels, structure, and function.

\section{A. INTERRUPTION OF THE ANDROGEN RECEPTOR OPEN-READING FRAME}

A number of different types of genetic defects can cause interruptions of the primary sequence of the human AR, including large- or small-scale deletions, insertions, and mutations that result in alterations of AR mRNA splicing. Although each of these mechanisms is the result of different genetic events, in each instance, the resulting mutant AR protein differs from the normal human $\mathrm{AR}$, either by premature truncation of the receptor protein or by the addition or removal of amino acids from the receptor sequence. As noted above, the critical hormone-binding and DNA-binding domains of the AR are localized to the carboxyl-terminus of receptor protein. For this reason, mutations that result in premature termination of the receptor have a dramatic effect on receptor function when introduced at virtually any position within the primary amino acid sequence, as these critical segments will be lacking. By contrast, alterations that serve to insert or remove single or multiple amino acids (small insertions or deletions) cause androgen resistance only when occurring within the DNA- or hormone-binding segments of the receptor protein (Figure 3).

\section{B. MUTATIONS IN THE DNA-BINDING DOMAIN OF THE ANDROGEN RECEPTOR}

In up to $20 \%$ of patients with clinical androgen resistance supported by endocrine studies or family histories consistent with a defect of the AR, abnormalities were not identified in monolayer assays of ligand binding by the AR (Griffin et al., 2001). AR gene structure from a number of such pedigrees has been analyzed. In most instances, amino acid substitutions have been detected within the DNA-binding domain of the AR. In studies by Zoppi (Zoppi et al., 


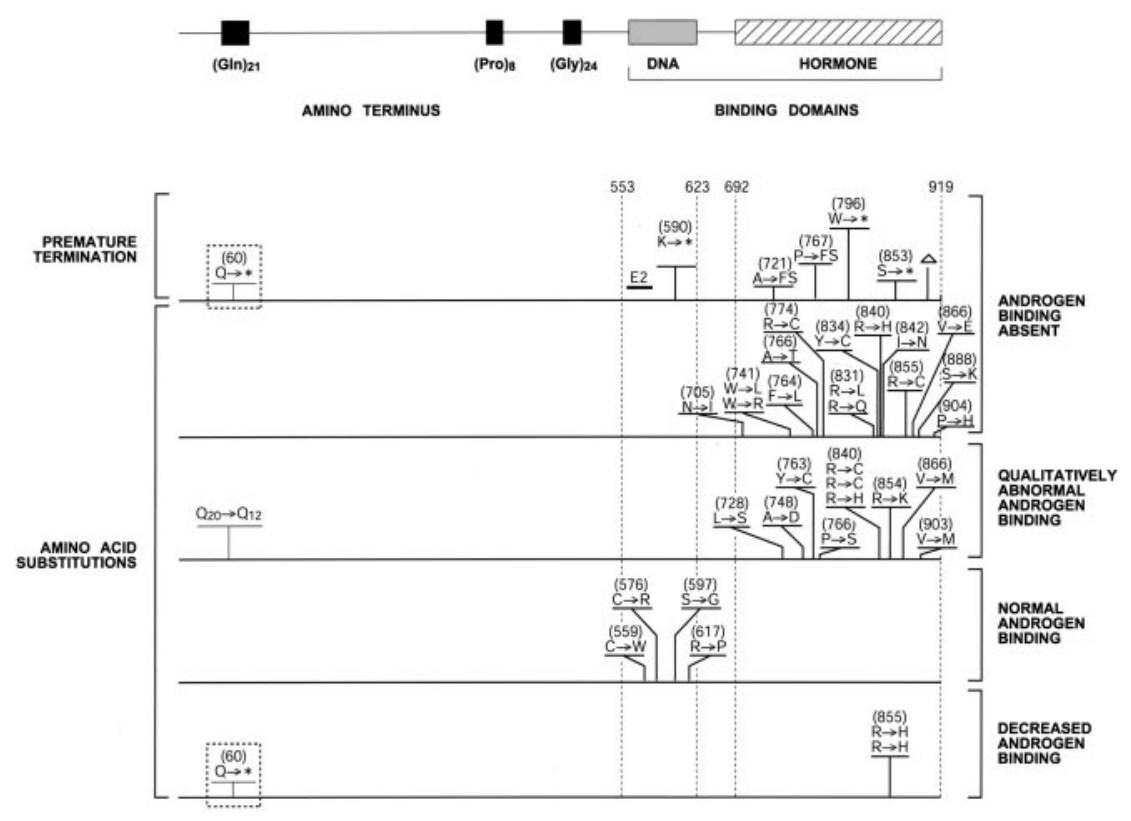

FIG. 3. Genetic mutations of the AR genes cause different abnormalities of androgen binding. The AR gene mutations identified in patients with androgen resistance exhibiting distinct abnormalities of androgen binding in genital skin fibroblast monolayer-binding assays are presented. Gene defects that interrupt the primary amino acid sequence of the AR are associated with the absence of androgen binding, whether caused by the insertion of premature termination codons, deletions, insertions, or alterations of AR mRNA splicing. Amino acid substitutions in specific segments of the AR LBD also can lead to the expression of undetectable androgen binding in genital skin fibroblast cultures, although, in most instances, the levels of immunoreactive AR are near normal (Avila et al., in press). Qualitative abnormalities of androgen binding are caused by amino acid substitutions in the LBD of the receptor protein. It is evident that the positions of the amino acid replacements causing absent androgen binding and qualitative defects are similar. It appears that the differences between these two categories reflect the degree to which the structure of the AR LBD is disrupted. Amino acid substitutions within the DBD of the AR are frequently identified in samples exhibiting normal levels of qualitatively normal androgen binding. Decreased levels of qualitatively normal androgen binding are traced to mutations that diminish the level of intact AR that is synthesized. The coordinates utilized in this figure and throughout the text are those of Lubahn (Lubahn et al., 1988). [Modified with permission from McPhaul MJ, Marcelli M, Zoppi S, Griffin JE, Wilson JD 1993 The spectrum of mutations in the androgen receptor gene that causes androgen resistance. J Clin Endocrinol Metab 76:17-23. Copyright The Endocrine Society.]

1992), amino acid substitutions were localized to critical residues within the DNA-binding domain of the AR in four unrelated subjects with complete or nearly complete forms of androgen resistance. Experiments examining the physical attributes of these mutant receptors in heterologous cells demonstrated that these mutant receptors bound ligand with normal or near-normal affinity, as 
was observed in fibroblast samples from the same patients. Although these studies confirmed the normal ligand-binding properties of the mutant receptors, transfection studies demonstrated that each was markedly impaired in terms of function. In vitro experiments to measure the capacity of the mutant ARs to bind to DNA showed that each was unable to bind normally to target DNA sequences. Similar results have been obtained in studies conducted in a number of different laboratories to analyze similar groups of patients with complete or partial forms of androgen resistance (Lumbroso et al., 1993; Mowszowicz et al., 1993; Sultan et al., 1993; Beitel et al., 1994b; De Bellis et al., 1994). These findings suggest that the AR defects in this patient group comprise a relatively homogeneous group from a mechanistic standpoint. Mutations of this type alter the structure of the DNA-binding domain of the receptor and interfere with the capacity of the receptor to recognize specific target DNA sequences.

\section{MUTATIONS IN THE LIGAND-BINDING DOMAIN OF THE ANDROGEN RECEPTOR}

Single amino acid substitutions in the LBD of the AR represent the most-frequent type of mutation that is identified in patients with androgen resistance. These individual amino acid replacements account for approximately $60 \%$ of the mutations identified in patients with clinical defects of androgen action and are most often the result of single nucleotide substitution mutations. Substitution mutations of the AR LBD have been identified in patients with the entire range of androgen-resistant phenotypes. The mutations can be divided into two categories, based on the effects on the binding of ligand in patient fibroblast samples: absence of detectable ligand binding and qualitative abnormalities of ligand binding.

\section{UNDETECTABLE LEVELS OF ANDROGEN BINDING IN FIBROBLAST MONOLAYER-BINDING ASSAYS}

Although identical when analyzed at the level of monolayer-binding assays (undetectable levels of androgen binding), amino acid substitution mutations in the LBD of the AR in this category are of two types. The first is less frequent and represents the replacement of amino acid residues in critical segments of the LBD. Amino acid substitutions of this type presumably induce major alterations of the structure of the LBD, to the extent that it is no longer capable of interacting with ligand. The mutant receptor described by Marcelli et al. (1994) is one well-characterized mutation of this type. As a result of this amino acid substitution (W741R), a hydrophobic residue within the amino terminal end of helix 5 is replaced that would be predicted to make important contacts with the $\mathrm{C}$ ring of testosterone (Mathias et al., 2000; McPhaul, 2000). In addition to the steric effects that such changes might be expected to exert, the introduction of such a 
charged residue into this hydrophobic region of the LBD is likely to have dramatic effects on the tertiary structure of the ligand-binding pocket. In functional analyses, mutant ARs of this type cannot bind hormone and cannot activate model reporter genes, even when expressed in heterologous cells (Marcelli et al., 1994).

Mutant ARs that are completely incapable of binding hormone when assayed following expression in heterologous cells are uncommon. More frequently, when cDNAs encoding such mutant ARs are created and expressed in heterologous cells, they are found to be capable of binding hormone. For example, one strain (strain 105) was categorized originally as ligand-binding negative, based on monolayer ligand-binding assay results. Analysis of the AR gene in this subject revealed a single amino acid substitution (R774C). When cDNAs encoding this mutant receptor were expressed in heterologous cells, however, ligand binding was easily measurable, although altered kinetics and stability were evident (Marcelli et al., 1991). Additional mutant receptors have been described that display similar properties: 1) relatively normal or near-normal levels of immunoreactive receptor, 2) markedly diminished levels of ligand binding, and 3) measurable levels of ligand binding when expressed in heterologous cells. These discrepancies likely reflect differences in the level of expression and the sensitivity of the assays that are employed to analyze the samples.

\section{E. QUALITATIVE LIGAND-BINDING ABNORMALITIES}

Analysis using monolayer-binding assays of the AR present in fibroblast samples from individuals with androgen resistance believed due to AR defects demonstrated normal levels of androgen binding. When fibroblasts from some of these patients were analyzed carefully, discernible differences could be identified when qualitative tests of ligand binding were applied. Such tests included measurements of the affinity of ligand binding, the rapidity with which ligand dissociates from the receptor (accelerated ligand dissociation), and increased susceptibility to thermal denaturation.

With very few exceptions, the naturally occurring mutations causing qualitative abnormalities of ligand binding are single amino acid substitutions localized to the hormone-binding domain of the receptor protein. Furthermore, the distribution of the amino acid substitutions identified in ARs exhibiting qualitative abnormalities of ligand binding is similar to that of amino acid replacements identified in strains in which no androgen binding is detected (McPhaul et al., 1992). This observation suggests that the type of binding abnormality that is observed is related directly to the degree that the structure of the ligand-binding domain is disrupted. Findings from the study of mutant receptors from pedigrees in which the same amino acid has been mutated to 
different residues have supported this concept. This concept is most evident in the work of Prior, in which replacement of arginine 774 by cysteine residue leads to androgen resistance and undetectable ligand binding in patient fibroblasts (Prior et al., 1992). Substitution of the same residue by histidine, a more conservative amino acid replacement, leads to normal levels of androgen binding in fibroblasts that displays a marked thermal lability in in vitro assays. Additional pedigrees have been described in which different amino acid substitution mutations at a single residue have differing effects on the binding of ligand by the receptor, depending of the nature of the amino acid substitution (Ris-Stalpers et al., 1991; Kazemi-Esfarjani et al., 1993; Beitel et al., 1994a).

The analysis of mutant ARs in assays is straightforward when the receptor protein possesses little or no functional activity. The assessment of qualitatively abnormal ARs is considerably more difficult, as mutant receptors of this class are capable of binding ligand with varying affinity and stability. In this context, the cellular environment and the specific ligands will exert important effects on the results of assays performed to analyze AR function. The work of Marcelli clearly demonstrated this effect (Marcelli et al., 1994). These investigators examined the properties of a range of mutant ARs that carried different amino acid substitution mutations in the hormonebinding domain. The responsiveness of these ARs was examined using several agonist ligands, including 5 alpha dihydrotestosterone (DHT), testosterone $(\mathrm{T})$, and mibolerone. The experiments were performed using transfection assays in a cell strain in which the metabolism of testosterone and 5 alpha dihydrotestosterone is rapid, similar to the degradation of androgen that occurs in most mammalian androgen target tissues. Results of these experiments varied, depending on the type of ligand employed. When physiologic androgens (e.g., testosterone, dihydrotestosterone) were used to stimulate the mutant receptors, greater deficiencies of function were observed for most of the mutant receptors. In each instance, the nonmetabolizable androgen, mibolerone, was observed to be more potent than either of the two androgens that were subject to metabolic inactivation. The relative ineffectiveness of $T$ and DHT in these experiments could be overcome by repeated additions of these hormones during the course of the hormone stimulation. These results suggested that the stability of the hormone-receptor complexes plays a critical role in the function of the AR and that AR mutations that destabilize the hormone-receptor complex will have a major effect on receptor activity. These results also demonstrate that the environment in which AR function is assessed can have an important modifying influence on the levels of function that are observed, particularly in response to physiologic, metabolizable androgens. These results also indicate that mutant receptors that display qualitative abnormalities of ligand binding can be manipulated pharmacologically. Consistent with this concept, biological responses have been observed 
to the administration of supraphysiologic levels of androgen (Grino et al., 1989; Tincello et al., 1997).

\section{F. ANDROGEN RECEPTOR MUTATIONS CAUSING DECREASED LEVELS OF LIGAND BINDING}

It appears that the types of mutations identified in this category will be quite heterogeneous and reflect alterations that affect how much AR protein is synthesized.

The first mutation of this class was discovered during the analysis of the AR genes of affected subjects in a pedigree with complete androgen insensitivity. While monolayer-binding assays of fibroblasts established from affected individuals demonstrated measurable, but reduced, levels of a qualitatively normal AR protein, immunoblots were unable to detect immunoreactive AR using an antibody directed at the amino terminus of the AR. Sequence analysis of the AR gene from affected individuals in this pedigree revealed a single-nucleotide substitution that resulted in the insertion of a premature termination codon in place of amino acid residue 60 (Zoppi et al., 1993). Subsequent analyses using antibodies directed at epitopes within the amino terminus revealed that a smaller form of the AR was synthesized in fibroblasts from affected individuals in this pedigree, accounting for the residual ligand binding that was detected in monolayer-binding assays. More recent studies have established that this shortened form of the AR (termed AR-A, on the basis of its similarity to the A-form of the progesterone receptor) is synthesized by internal initiation at methionine 189 and is expressed at low levels in normal fibroblasts and tissues (Wilson and McPhaul, 1994,1996). Transfection experiments demonstrated that this AR-A isoform displays subtle differences in function on selected response elements (Gao and McPhaul, 1998).

The studies of Choong et al. (1996) suggest a somewhat-different mechanism mutation leading to a phenotype of partial androgen insensitivity syndrome (AIS). Analysis of the AR gene in affected subjects identified a single-nucleotide substitution that altered the second amino acid residue (lysine residue in place of the normal aspartate residue) of the AR open-reading frame. Although it was not possible to examine the effects of this mutation in cultured fibroblasts from affected individuals in this pedigree, experiments employing the expression of cDNAs encoding the mutant AR in heterologous cells suggested that the AIS phenotype was caused primarily by the reduced levels of AR expressed due to reduced efficiency of translational initiation.

Additional studies have suggested that alterations in the synthesis of normal AR mRNA can lead to reduced levels of functional AR protein. In the studies reported by Sammarco (Sammarco et al., 2000), partial androgen insensitivity was associated with reduced levels of apparently normal AR. Analysis of the AR 
gene in this individual identified an alteration at position +5 of the donor splice site at the junction between exon 6 and intron 6. Analysis of the AR mRNA expressed in cells from the affected individual demonstrated that intron 6 was retained in a large proportion of the AR mRNA. Immunoblot analysis revealed only a small quantity of intact AR. A similar mechanism was proposed to account for the androgen resistance observed in a patient with the Reifenstein phenotype (Ris-Stalpers et al., 1994).

\section{G. CLINICAL PHENOTYPE AND LOSS-OF-FUNCTION MUTATIONS OF THE ANDROGEN RECEPTOR}

The number of AR gene mutations that have been analyzed permits general conclusions regarding the nature of the relationship between clinical phenotype and AR mutation. First, mutations that interrupt the AR open-reading frame whether caused by premature termination, aberrant splicing, or deletion of partial or complete exon segments - are associated with a phenotype of complete androgen resistance. This relationship is the result of the locations of the DNA- and hormone-binding domains at the carboxyl terminus of the AR protein. As a consequence, alterations that truncate the receptor protein at any point during its synthesis will remove portions of one or both of these important functional domains.

In contrast to mutations that interrupt the AR open-reading frame, amino acid substitutions within the AR sequence are capable of causing all androgenresistant phenotypes. The degree of clinical resistance observed does not appear to correlate with the nature or location of specific mutation. Instead, the phenotype appears to reflect the level of residual AR function that is expressed in the androgen target tissues. In different instances, this diminished level of function may be caused by alterations in the functional capacity of the receptor protein, its level of expression, or combinations of both factors.

\section{ACKNOWLEDGMENTS}

The original work described herein was supported by National Institutes of Health grant 32820 and by grant I-1090 from the Robert A. Welch Foundation.

\section{REFERENCES}

Albright F, Burnett CH, Smith PH, Parson W 1942 Pseudo-hypoparathyroidism - an example of the "Seabright Bantam Syndrome." Endocrinology 30:922-932

Avila DM, Wilson CM, Nandi N, Griffin JE, Wilson JD, McPhaul MJ 2002 Immunoreactive androgen receptor (AR) in genital skin fibroblasts from subjects with androgen resistance and undetectable levels of AR in ligand binding assays. J Clin Endocrinol Metabol, in press

Beitel LK, Kazemi-Esfarjani P, Kaufman M, Lumbroso R, DiGeorge AM, Killinger DW, Trifiro MA, Pinsky L 1994a Substitution of arginine-839 by cysteine or histidine in the 
androgen receptor causes different receptor phenotypes in cultured cells and coordinate degrees of clinical androgen resistance. J Clin Invest 94:546-554

Beitel LK, Prior L, Vasiliou DM, Gottlieb B, Kaufman M, Lumbroso R, Alvarado C, McGillivray B, Trifiro M, Pinsky L 1994b Complete androgen insensitivity due to mutations in the probable alpha-helical segments of the DNA-binding domain in the human androgen receptor. Hum Mol Genet 3:21-27

Brown TR, Maes M, Rothwell SW, Migeon CJ 1982 Human complete androgen insensitivity with normal dihydrotestosterone receptor binding capacity in cultured genital skin fibroblasts: evidence for a qualitative abnormality of the receptor. J Clin Endocrinol Metabol 55:61-69

Chang CS, Kokontis J, Liao ST 1988 Structural analysis of complementary DNA and amino acid sequences of human and rat androgen receptor. Proc Natl Acad Sci USA 85:7211-7215

Choong CS, Quigley CA, French FS, Wilson EM 1996 A novel missense mutation in the amino-terminal domain of the human androgen receptor gene in a family with partial androgen insensitivity syndrome causes reduced efficiency of protein translation. J Clin Invest 98:1423-1431

De Bellis A, Quigley CA, Marschke KB, el-Awady MK, Lane MV, Smith EP, Sar M, Wilson EM, French FS 1994 Characterization of mutant androgen receptors causing partial androgen insensitivity syndrome. J Clin Endocrinol Metab 78:513-522

Faber PW, Kuiper GG, van Rooij HC, van der Korput JA, Brinkmann AO, Trapman J 1989 The N-terminal domain of the human androgen receptor is encoded by one, large exon. Mol Cell Endocrinol 61:257-262

Gao TS, McPhaul MJ 1998 Functional activities of the A- and B-forms of the human androgen receptor in response to androgen receptor agonists and antagonists. Mol Endocrinol 12:654663

Gao TS, Marcelli M, McPhaul MJ 1996 Transcriptional activation and transient expression of the human androgen receptor J Steroid Biochem Mol Biol 59:9-20

Griffin JE 1992 Androgen resistance - the clinical and molecular spectrum. N Engl J Med 326:611-618

Griffin JE, Durrant JL 1982 Qualitative receptor defects in families with androgen resistance: failure of stabilization of the fibroblast cytosol androgen receptor. J Clin Endocrinol Metab 55:465-474

Griffin JE, McPhaul MJ, Russell DW, Wilson JD 2001 The androgen resistance syndromes: steroid 5-reductase 2 deficiency, testicular feminization, and related disorders. In: Scriver CR, Beaudet AL, Sly WS, Valle D, eds. The Metabolic and Molecular Bases of Inherited Disease, 8th edit. New York: McGraw-Hill; 4117-4146

Grino PB, Isidro-Gutierrez RF, Griffin JE 1989 Androgen resistance associated with a qualitative abnormality of the androgen receptor and responsive to high dose androgen therapy. J Clin Endocrinol Metab 68:578-584

Kazemi-Esfarjani P, Beitel LK, Trifiro M, Kaufman M, Rennie P, Sheppard P, Matusik R, Pinsky L 1993 Substitution of valine-865 by methionine or leucine in the human androgen receptor causes complete or partial androgen insensitivity, respectively with distinct androgen receptor phenotypes. Mol Endocrinol 7:37-46

La Spada AR, Wilson EM, Lubahn DB, Harding AE, Fischbeck KH 1991 Androgen receptor gene mutations in X-linked spinal and bulbar muscular atrophy. Nature 352:77-79

Lubahn DB, Joseph DR, Sar M, Tan J, Higgs HN, Larson RE, French FS, Wilson EM 1988 The human androgen receptor: complementary deoxyribonucleic acid cloning, sequence analysis and gene expression in prostate. Mol Endocrinol 2:1265-1275

Lumbroso S, Lobaccaro JM, Belon C, Belon C, Martin D, Chaussain JL, Sultan C 1993 A new mutation within the deoxyribonucleic acid-binding domain of the androgen receptor gene in a family with complete androgen insensitivity syndrome. Fertil Steril 60:814-819 
Mangelsdorf DJ, Thummel C, Beato M, Herrlich P, Schutz G, Umesono K, Blumberg B, Kastner P, Mark M, Chambon P 1995 The nuclear receptor superfamily: the second decade. Cell 83:835-839

Marcelli M, Tilley WD, Zoppi S, Griffin JE, Wilson JD, McPhaul M 1991 Androgen resistance associated with a mutation of the androgen receptor at amino acid 772 (Arg $\rightarrow$ Cys) results from a combination of decreased messenger ribonucleic acid levels and impairment of receptor function. J Clin Endocrinol Metab 73:318-325

Marcelli M, Zoppi S, Wilson CM, Griffin JE, McPhaul MJ 1994 Amino acid substitutions in the hormone-binding domain of the human androgen receptor alter the stability of the hormone receptor complex. J Clin Invest 94:1642-1650

Matias PM, Donner P, Coelho R, Thomaz M, Peixoto C, Macedo S, Otto N, Joschko S, Scholz P, Wegg A, Basler S, Schafer M, Egner U, Carrondo M 2000 Structural evidence for ligand specificity in the binding domain of human androgen receptor. Implications for pathogenic gene mutations. J Biol Chem 275:26164-26171

McPhaul MJ 2000 The androgen receptor in syndromes of androgen insensitivity and in prostate cancer. In: Shupnik, MA, ed. Gene Engineering and Molecular Models in Endocrinology. Totowa, NJ: Humana Press; 339-372

McPhaul MJ, Marcelli M, Zoppi S, Wilson CM, Griffin JE, Wilson JD 1992 Mutations in the ligand-binding domain of the androgen receptor gene cluster in two regions of the gene. J Clin Invest 90:2097-2101

McPhaul MJ, Marcelli M, Zoppi S, Griffin JE, Wilson JD 1993 The spectrum of mutations in the androgen receptor gene that causes androgen resistance. J Clin Endocrinol Metab 76:17-23

Migeon BR, Brown TR, Axelman J, Migeon CJ 1981 Studies of the locus for androgen receptor: localization on the human X chromosome and evidence for homology with the Tfm locus in the mouse. Proc Natl Acad Sci USA 78:6339-6343

Morris JM 1953 The syndrome of testicular feminization in male pseudohermaphrodites. Am J Obstet Gynecol 65:1192-1211

Morris JM, Mahesh VB 1968 Further observations on the syndrome, "testicular feminization." Am J Obstet Gynecol 87:731-748

Mowszowicz I, Lee HJ, Chen HT, Mestayer C, Portois MC, Cabrol S, Mauvais-Jarvis P, Chang C 1993 A point mutation in the second zinc finger of the DNA-binding domain of the androgen receptor gene causes complete androgen insensitivity in two siblings with receptorpositive androgen resistance. Mol Endocrinol 7:861-869

Pinsky L, Kaufman M, Summitt RL 1981 Congenital androgen insensitivity due to a qualitatively abnormal androgen receptor. Am J Med Genet 10:91-99

Prior L, Bordet S, Trifiro MA, Mhatre A, Kaufman M, Pinsky L, Wrogeman K, Belsham DD, Pereira F, Greenberg C 1992 Replacement of arginine 773 by cysteine or histidine in the human androgen receptor causes complete androgen insensitivity with different receptor phenotypes. Am J Hum Genet 51:143-155

Quigley CA, De Bellis A, Marschke KB, el-Awady MK, Wilson EM, French FS 1995 Androgen receptor defects: historical, clinical, and molecular perspectives. Endocr Rev 16:271-321

Reifenstein EC 1947 Hereditary familial hypogonadism. Proc Am Fed Clin Res 3:86

Ris-Stalpers C, Trifiro MA, Kuiper GG, Jenster G, Romalo G, Sai T, van Rooij HC, Kaufman M, Rosenfield RL, Liao S 1991 Substitution of aspartic acid-686 by histidine or asparagine in the human androgen receptor leads to a functionally inactive protein with altered hormone-binding characteristics. Mol Endocrinol 5:1562-1569

Ris-Stalpers C, Verleun-Mooijman MC, de Blaeij TJ, Degenhart HJ, Trapman J, Brinkmann AO 1994 Differential splicing of human androgen receptor pre-mRNA in X-linked Reifenstein syndrome, because of a deletion involving a putative branch site. Am J Hum Genet $54: 609-617$ 
Rosewater S, Gwinup G, Hamwi GJ 1965 Familial gynecomastia. Ann Intern Med 63:377-385

Sammarco I, Grimaldi P, Rossi P, Cappa M, Moretti C, Frajese G, Geremia R 2000 Novel point mutation in the splice donor site of exon-intron junction 6 of the androgen receptor gene in a patient with partial androgen insensitivity syndrome. J Clin Endocrinol Metab 85:32563261

Sultan C, Lumbroso S, Poujol N, Belon C, Boudon C, Lobaccaro JM 1993 Mutations of androgen receptor gene in androgen insensitivity syndromes. J Steroid Biochem Mol Biol 46:519-530

Tilley WD, Marcelli M, Wilson JD, McPhaul MJ 1989 Characterization and expression of a cDNA encoding the human androgen receptor. Proc Natl Acad Sci USA 86:327-331

Tincello DG, Saunders PT, Hodgins MB, Simpson NB, Edwards CR, Hargreaves TB, Wu FC 1997 Correlation of clinical, endocrine and molecular abnormalities with in vivo responses to high-dose testosterone in patients with partial androgen insensitivity syndrome. Clin Endocrinol 46:497-506

Wilkins L 1957 Abnormal sex differentiation: hermaphroditism and gonadal dysgenesis. In: The Diagnosis and Treatment of Endocrine Disorders in Childhood and Adolescence. Springfield, IL: Charles C. Thomas; 258

Wilson CM, McPhaul MJ 1994 A and B forms of the androgen receptor are present in human genital skin fibroblasts. Proc Natl Acad Sci USA 91:1234-1238

Wilson CM, McPhaul MJ 1996 A and B forms of the androgen receptor are expressed in a variety of human tissues. Mol Cell Endocrinol 120:51-57

Wilson JD Harrod MJ Goldstein JL Hemsell DL MacDonald PC 1974 Familial incomplete male pseudohermaphroditism, type 1. Evidence for androgen resistance and variable clinical manifestations in a family with the Reifenstein syndrome. N Engl J Med 290:1097-1103

Zoppi S, Marcelli JP, Griffin JE, Deslypere JP, Wilson JD, McPhaul M 1992 Amino acid substitutions in the DNA-binding domain of the human androgen receptor are a frequent cause of receptor-binding positive androgen resistance. Mol Endocrinol 6:409-415

Zoppi S, Wilson CM, Harbison MD, Griffin JE, Wilson JD, McPhaul MJ, Marcelli M 1993 Complete testicular feminization caused by an amino-terminal truncation of the androgen receptor with downstream initiation. J Clin Invest 91:1105-1112

Zoppi S, Young M, McPhaul MJ 2002 Regulation of gene expression by the nuclear receptor family. In: Mason JI, ed. The Genetics of Steroid Biosynthesis. London: Taylor \& Francis Publishing Group; in press 


\title{
Novel Signaling Pathways That Control Ovarian Follicular Development, Ovulation, and Luteinization
}

\author{
JoAnne S. Richards, Darryl L. Russell, Scott Ochsner, Minnie Hsieh, \\ Kari H. Doyle, Allison E. Falender, Yuet K. Lo, and \\ S. Chidananda Sharma \\ Department of Molecular and Cellular Biology, Baylor College of Medicine, \\ Houston, Texas 77030
}

\begin{abstract}
The interactions of peptide and steroid hormone signaling cascades in the ovary are critical for follicular growth, ovulation, and luteinization. Although the pituitary gonadotropins follicle-stimulating hormone (FSH) and luteinizing hormone ( $\mathrm{LH})$ play key regulatory roles, their actions are also dependent on other peptide signaling pathways, including those stimulated by insulin-like growth factor-1 (IGF-1), transforming growth factor-beta (TGF- $\beta$ ) family members (e.g., inhibin, activin, growth differentiation factor-9, bone morphogenic proteins), fibroblast growth factor, and Wnts (via Frizzled receptors). Each of these factors is expressed and acts in a cell-specific manner at defined stages of follicular growth. IGF-1, estrogen, and FSH comprise one major regulatory system. The Wnt/Frizzled pathways define other aspects relating to ovarian embryogenesis and possibly ovulation and luteinization. Likewise, the steroid receptors as well as orphan nuclear receptors and their ligands impact ovarian cell function. The importance of these multiple signaling cascades has been documented by targeted deletion of specific genes. For example, mice null for the LH-induced genes progesterone receptor (PR) and cyclo-oxygenase-2 (COX-2) fail to ovulate. Whereas PR appears to regulate the induction of novel proteases, $\mathrm{COX}-2$ appears to regulate cumulus expansion. This review summarizes some new aspects of peptide and steroid hormone signaling in the rodent ovary.
\end{abstract}

\section{Overview}

Ovarian follicular growth is controlled by the production of intraovarian growth regulatory factors such as insulin-like growth factor-1 (IGF-1) (Adashi et al., 1985; Baker et al., 1996; Zhou et al., 1997; Burks et al., 2000; Guidice, 2000), steroids (Richards, 1994), members of the transforming growth factorbeta (TGF- $\beta$ ) family (Elvin et al., 1999; Lewis et al., 2000; Chapman and Woodruff, 2001; Otsuka et al., 2001), and the Wnt/Frizzled family (Vainio et al., 1999; Hsieh et al., in press). These factors act by autocrine, paracrine, and intracrine mechanisms. In addition, follicular growth is controlled by endocrine factors such as the pituitary gonadotropins, follicle-stimulating hormone (FSH) and luteinizing hormone (LH) (Richards, 1994). Recent mutant mouse models as 
well as studies identifying new components of IGF-1, FSH, and estradiol (E) signal transduction pathways provide novel insights into how these hormones might interact to control follicular growth and are the focus of this review.

\section{Interactions of the IGF-1, Estradiol, and FSH Signaling Cascades}

The functional links between the FSH and IGF-1 signal pathways are supported by the observations that IGF-1, IGF-1 receptor (Igf-1r), and FSH receptor co-localize to granulosa cells of small growing follicles and preovulatory follicles (Zhou et al., 1995,1997). In mice null for the FSH receptor or the FSH $\beta$ subunit, follicular growth is impaired beyond the preantral stage and is associated with altered expression of specific genes (Kumar et al., 1997; Burns et al., 2001) (Figure 1). Whereas IGF-1 expression is not altered by hypophysectomy or lack of FSH $\beta$ subunit (Zhou et al., 1997), levels of Igf-1r are reduced and can be restored by treating rats with pregnant mare serum gonadotropin (PMSG), which stimulates follicular growth (Zhou et al., 1997). Mice null for either IGF-1 (Igf-1) or insulin-receptor substrate-2 (Irs-2) exhibit severe growth retardation, including follicular growth in the ovary (Baker et al., 1996; Zhou et al., 1997; Burks et al., 2000). In contrast, although mice null for growth hormone exhibit reduced growth rates and have low serum levels of IGF-1, ovarian function is normal, likely a consequence of GH-independent production of IGF-1 by granulosa cells within the ovary (Zhou et al., 1997; Zaczek et al., 2001). Finally, IGF-1 enhances FSH action in granulosa cells by mechanisms that are not entirely clear (Orly, 2000) but must involve specific interactions of these two signaling pathways (Richards, 2001a; Richards et al., in press (b)) (Figure 1).

IGF-1 impacts multiple signaling cascades, one of which includes the phosphoinositide-3 kinase (PI3-K) cascade (LeRoith et al., 1995; Vanhaesebroeck and Alessi, 2000). PI3-K generates phosphoinositides that activate phosphoinositide-dependent kinases (PDK)1 and -2. PDK1 and -2, in turn, phosphorylate and activate protein kinase $\mathrm{B}$ (PKB), which then phosphorylates and inactivates targets such as glycogen synthase kinase (GSK)-3 $\beta$, BAD, and caspase 9 as well as members of the Forkhead (FKHR) winged-helix transcription factor family (Vanhaesebroeck and Alessi, 2000). The IGF-1 signaling cascade has been highly conserved, as revealed by functional and structural homologies of genes present in mammals (IGF-1R, PI3-K, phosphate and tensin homologue deleted on chromosome 10 (PTEN), PDK1, PKB, FKHR) and $C$. elegans (Daf-2, Age-1, Daf 18, PDK1, Akt, Daf 16) (Guarente and Kenyon, 2000) (Figure 1). In C. elegans, this pathway impacts cell survival, energy homeostasis, and longevity and is intimately linked to the neural and reproductive systems. Lack of the IGF-1 receptor (Daf-2) in C. elegans leads to prolonged cell survival that is dependent on Daf16 and Daf12, homologues of FKHR and 

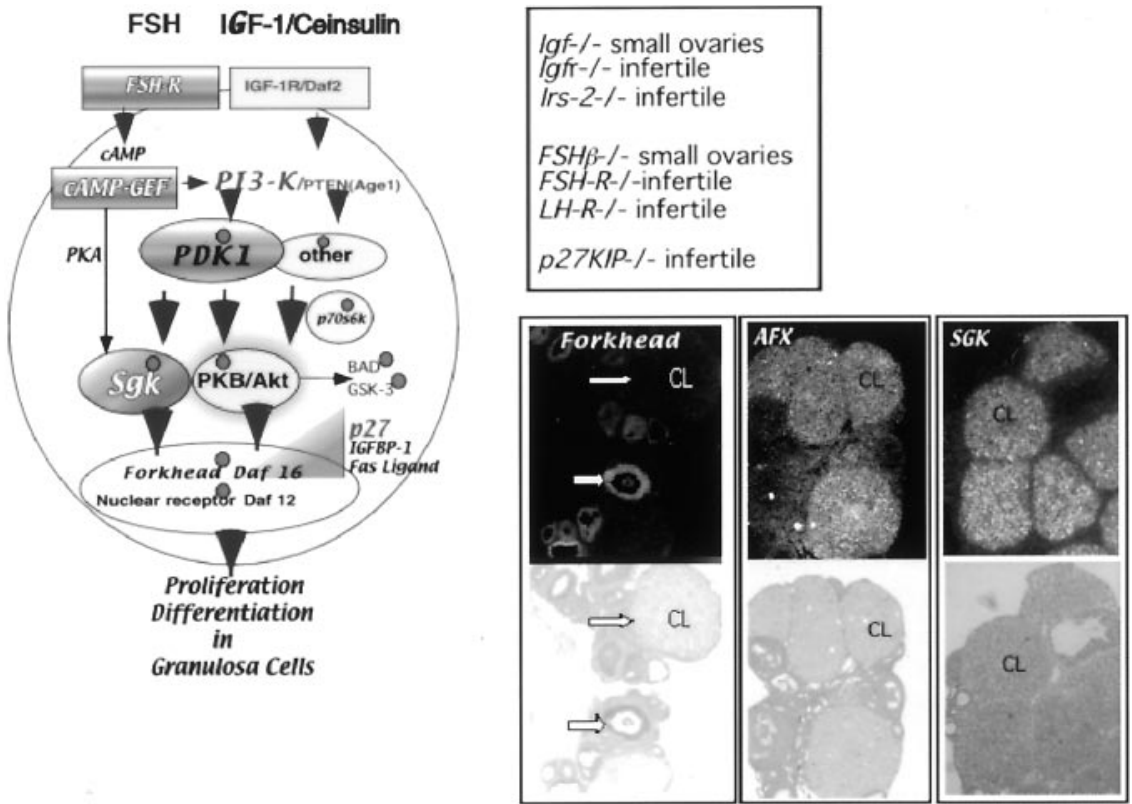

FIG. 1. Follicle-stimulating hormone (FSH) and insulin-like growth factor-1 (IGF-1) signaling pathways operate in granulosa cells. FSH/LH and IGF-1 impact the proliferation and differentiation of granulosa cells, as indicated by specific knockout mouse models and the effects of these hormones in hypophysectomized rats, as reviewed in the text. Note that the IGF-1 pathway is highly conserved from C. elegans to mammals. Common downstream targets of FSH and IGF-1 in granulosa cells include components of the PI3-kinase cascade that lead to the phosphorylation of protein kinase B (PKB) and serum and glutocorticoid-induced kinase (Sgk) as well as the transcription factor, Forkhead (FKHR), which is selectively expressed at high levels in granulosa cells of growing follicles. FSH but not IGF-1 induces Sgk, which reaches its highest levels of expression in luteal cells. These terminally differentiated cells preferentially express AFX (Foxo-4) and FKHRL1 (Foxo-3) but not FKHR - as well as a putative target of AFX, p27KIP. [Adapted with permission from Richards JS, Sharma SC, Falender AE, Lo YH 2002 Expression of FKHR, FKHRL1 and AFX genes in the rodent ovary: evidence for regulation by IGF-1, estrogen and the gonadotropins. Mol Endocrinol, in press; Gonzalez-Robayna IJ, Falender AE, Ochsner S, Firestone GL, Richards JJ 2000 FSH stimulates phosphorylation and activation of protein kinase B (PKB/Akt) and serum and glutocorticoid-induced kinase (Sgk): evidence for A-kinase independent signaling in granulosa cells. Mol Endocrinol 14:1283-1300. Copyright The Endocrine Society.]

a nuclear hormone receptor, respectively (Guarente and Kenyon, 2000; Lin et al., 2001) (Figure 1).

The mammalian homologue of the nuclear receptor Daf12 is not yet known. However, three members of the Forkhead family have been identified in the mouse: FKHR (Foxo-1), FKHRL1 (Foxo-3), and AFX (Foxo-4) (Kaestner et al., 2000; Brunet et al., 2001). A current model of IGF-1 action indicates that 
phosphorylation of Forkhead by PKB (or related kinases) restricts nuclear localization of these factors (Nakae et al., 2000; Brownawell et al., 2001). This impedes transcriptional activation of specific Forkhead target genes, such as Fas ligand (FasL), an inducer of apoptosis (Brunet et al., 1999), p27KIP, an inhibitor of cell cycle progression (Medema et al., 2000) and insulin-like growth factor binding protein-1 (IGFBP-1), a presumed inhibitor of IGF-1 (Kops et al., 1999; Guidice, 2000). Recently, we have shown that FKHR, FKHRL1, and AFX are expressed in the rodent (mouse and rat) ovary in a cell-specific manner at defined stages of follicular growth and luteinization (Richards et al., in press (b)). Expression of FKHR mRNA is restricted to granulosa cells of growing follicles and is not detected in luteal cells. In contrast, FKHRL1 and AFX are highest in corpora lutea, where the PKB-related kinase, serum and glucocorticoid-induced kinase (Sgk), is also expressed in abundance (Figure 1). In addition, we have shown that FKHR expression is regulated by E, IGF-1, and the gonadotropins (Richards et al., in press (b)). E markedly increases FKHR mRNA and protein in granulosa cells of preantral follicles of the hypophysectomized $(\mathrm{H})$ rat. Thus, expression of FKHR mRNA, protein, and phosphorylation are not strictly associated with follicles that are undergoing apoptosis or appear destined for atresia. Rather, FKHR is highest in granulosa cells of follicles in the hypophysectomized and estradiol-treated (HE) rats that exhibit increased proliferation (Rao et al., 1978), increased expression of cyclin D2 (Robker and Richards, 1998a,b) and increased staining for proliferator cell nuclear antigen (PCNA) (Robker and Richards, 1998a,b). FKHR is also elevated in preovulatory follicles of PMSG-treated mice, adult mice, and pregnant mice at day 22 of gestation (Figure 1). The high levels of FKHR in granulosa cells suggest a key role in promoting follicle growth. Moreover, E not only induces FKHR mRNA but also upregulates other notable components of the IGF-1 signaling system, including IGF-1R $\beta$ subunit and the glucose transporter, Glut-1 (Richards et al., in press (b)). The coordinated up-regulation of FKHR with IGF-1R $\beta$ and Glut-1 indicates further that $\mathrm{E}$ enhances granulosa cell function in the $\mathrm{H}$ rat model by regulating three different targets that control cellular energy flow, glucose metabolism, and cell survival. Because IGF-1 helps maintain high expression of estrogen receptor beta $(\mathrm{ER} \beta)$ mRNA, at least in cultured granulosa cells, E and IGF-1 comprise an autocrine regulatory system in granulosa cells that promotes cell survival and proliferation (Richards et al., in press (b)) (Figure 2).

In contrast to $\mathrm{E}$ and basal levels of FSH, ovulatory levels of LH cause dramatic decreases in the expression of FKHR that, in preovulatory follicles, is irreversible as luteinization proceeds (Richards et al., in press (b)). Whether or not this is critical for alterations in granulosa cell function is unknown. However, it is intriguing to note that granulosa cells become resistant to apoptotic insult when they are stimulated with FSH/LH to undergo luteinization (Porter et al., 2000). At this stage of differentiation, factors that impact proliferation (E, IGF-1, 

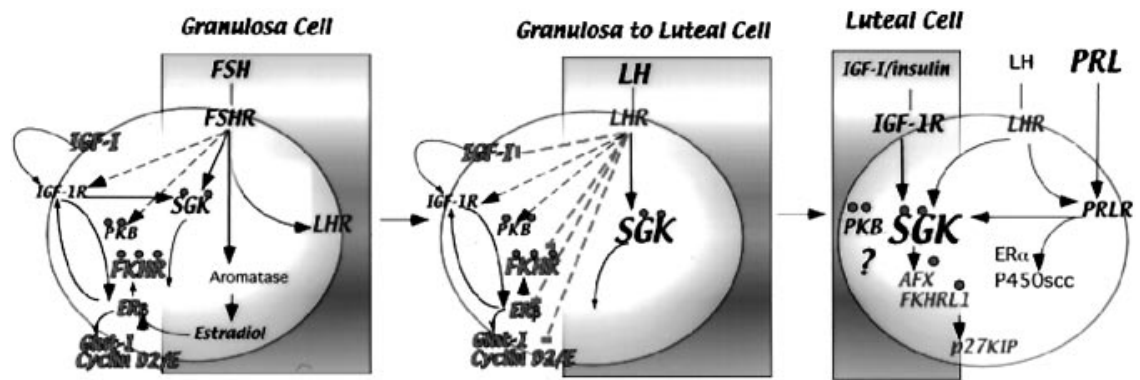

FIG. 2. Schematic of changes in the IGF-1 and FSH pathways in granulosa/luteal cells. Note that IGF-1, FKHR, ER $\beta$, Glut-1, and cyclinD2/E are coexpressed in proliferating granulosa cells. Estradiol (E) upregulates IGF-1R $\beta$, FKHR, and Glut-1, key components of the IGF-1 signaling pathway. IGF-1 upregulates ER $\beta$ and maintains FKHR. FSH at basal concentrations can maintain FKHR directly or indirectly via E. FSH and IGF-1 both stimulate the phosphorylation and activation of PKB and Sgk and their target, FKHR, whereas FSH alone induces Sgk (Alliston et al., 1997,2000; Burns et al., 2001). LH acts to downregulate expression of IGF-1, FKHR, ER $\beta$, and cyclin D2. As granulosa cells luteinize, they gain increased levels of Sgk, AFX (Foxo-4), and FKHRL1 (Foxo-3), which may impact the expression of p27KIP. [Reprinted with permission from Richards JS, Sharma SC, Falender AE, Lo YH 2002b Expression of FKHR, FKHRL1 and AFX genes in the rodent ovary: evidence for regulation by IGF-1, estrogen and the gonadotropins. Mol Endocrinol, in press. Copyright The Endocrine Society.]

cyclin D2) and apoptosis (FKHR, FasL) are lost, whereas factors that are expressed in luteal cells and presumed to impact luteinization (FKHRL1, AFX, Sgk, IGFBP-1, p27KIP, p21CIP, Jun D, Fra2) are acquired (Figure 2) Robker and Richards, 1998a; (Guidice, 2000; Sharma and Richards, 2000; Richards et al., in press (b)). Therefore, it is possible that FKHR, FKHL1, and AFX have different functions that are dependent on the cell type, stage of cell differentiation, or specific associated proteins. In this regard, one study reports that AFX upregulates p27KIP (Medema et al., 2000). In another study, Tanaka et al. (2001) show that expression of FKHRL1 does not activate p21CIP or p27KIP promoter activity or regulate FasL expression. Therefore, the precise relation of Forkhead proteins to the regulation of these genes remains uncertain. Recently, FKHR has been shown to interact with and selectively modify the functional activity of other transcription factors, specifically members of the nuclear steroid receptor superfamily (Schuur et al., 2001; Zhao et al., 2001). FKHRL1 and AFX may interact with the same or different transcription factors. Thus, the function of FKHR proteins likely depends not only their specific transcriptional activities but also on the hormonal milieu, the cell context, and the levels of proapoptotic and antiapoptotic factors (Hsueh et al., 1994; Pru and Tilly, 2001) (Figure 2).

The gonadotropins, as well as IGF-1, impact the phosphorylation of Forkhead proteins and thus can control their functional activity (Richards, 2001a). Specifically, we have shown previously that FSH as well as IGF-1 can impact the 
PI3-K pathway (Gonzalez-Robayna et al., 1999,2000). FSH, like IGF-1, stimulates phosphorylation of PKB Ser-473 that is blocked by the PI3-kinase inhibitor LY294002 but not by the protein kinase A (PKA) inhibitor, H89. Rather H89 enhances FSH and IGF-1 phosphorylation of PKB (Gonzalez-Robayna et al., 2000). FSH also induces the expression of the PKB-related kinase Sgk (Alliston et al., 1997,2000; Gonzalez-Robayna et al., 1999,2000; Burns et al., 2001). Importantly, Sgk reaches its highest levels of expression in corpora lutea in association with the increased levels of FKHRL1 and AFX (Figure 1). Based on these functions of FSH, it is not surprising that FSH stimulates the phosphorylation of FKHR at Thr-24 and Ser-256 in granulosa cells in a kinetic manner that mimics the action of IGF-1 in these same cells (Richards et al., in press (b)). Although the precise mechanism(s) by which FSH stimulates PKA-independent activation of PI3-K remains to be clearly documented, the cAMP-regulated guanine nucleotide exchange factors (cAMP-GEFs) (de Rooij et al., 1998,2000; Kawasaki et al., 1998) provide a potential new link between FSH stimulation of adenylyl cyclase and activation of PI3-K via ras-related small guanine nucleotide triphosphatases (GTPases). Whether PKB, Sgk, or other kinases specifically mediate the phosphorylation of FKHR in granulosa cells in vivo also needs to be verified (Figures 1 and 2).

\section{Regulated Expression of Wnts and Frizzleds in the Ovary}

Other factors that have been shown to impact ovarian cell function and follicular organization are members of the Wnt and Frizzled family of signaling molecules (Figure 3). Wnts are secreted, extracellular signaling molecules that act locally to control diverse developmental processes such as cell fate specification, cell proliferation, and cell differentiation (Cadigan and Nusse, 1997; Miller et al., 1999). Wnts transduce their signals by binding to $G$ protein-coupled receptors of the Frizzled family to activate distinct signaling cascades (Slusarski et al., 1997; Lin and Perrimon, 1999; Liu et al., 2001). In the canonical pathway Wnts/Frizzleds act to hyperphosphorylate dishevelled (Dvl) (Mao et al., 2001), a cytoplasmic scaffolding protein and glycogen synthase kinase 3-beta (GSK-3 $\beta$ ) leading to the release of $\beta$-catenin. Soluble $\beta$-catenin heterodimerizes with members of the T-cell factor/Lymphoid enhancer factor (Tcf/Lef) family of transcription factors to regulate expression of selected target genes such as c-myc (He et al., 1998). Wnt/Frizzled activation of the $\beta$-catenin pathway is further modulated by co-receptors (proteoglycans and/or arrow/LRP-5/LRP-6) (Lin and Perrimon, 1999; Tsuda et al., 1999; Alexander et al., 2000; Pinson et al., 2000; Tamai et al., 2000; Wehrli et al., 2000; Mao et al., 2001) and by antagonists such as secreted frizzled-related proteins (sFRPs) (Rattner et al., 1997). Some Wnts activate Frizzled receptors that signal via intracellular calcium, protein kinase $\mathrm{C}$ (PKC), and/or calmodulin-dependent kinases (CAMK) (Kuhl et al., 2000) 


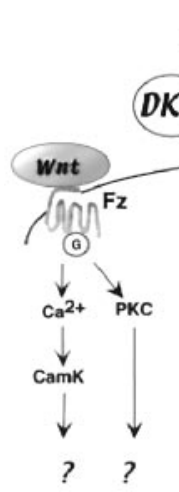

$L R P-5 / 6$
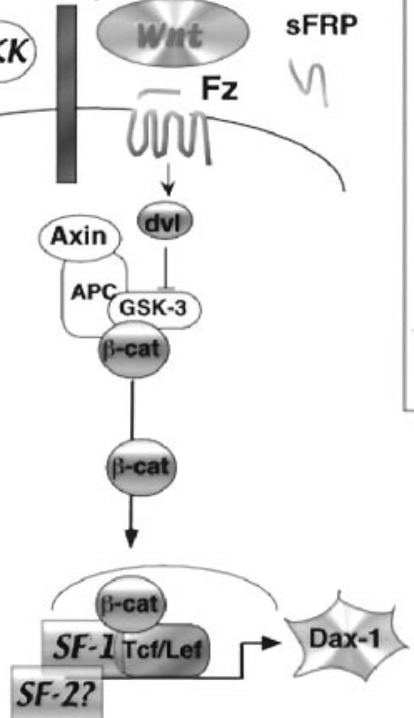

SF-2

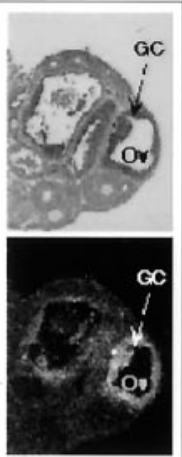

Frizzled-1

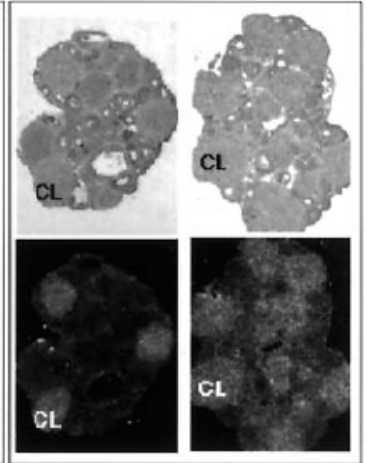

Wnt-4 Frizzled-4
Dax-1
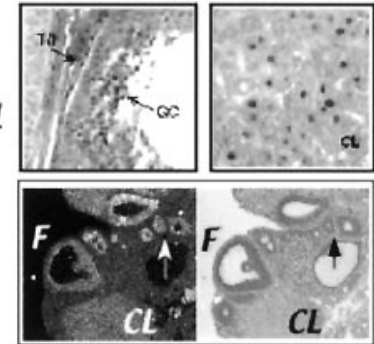

FIG. 3. Wnts and Frizzled receptors are expressed at specific stages of follicular growth and luteinization. Wnts are secreted ligands that activate Frizzled (Fz) receptors. The activation of these G protein-coupled receptors (GPCR) is associated with Wnt activation of the low-density lipoprotein (LDL)-related receptors (LRP5/6). Each of these can be inhibited by other secreted proteins - namely, soluble Frizzeled-related proteins (sFRPs) and Dickkoff (Dkk), respectively. Wnts activate the Axin and dishevelled (Dvl), glycogen synthase kinase (GSK)-3, $\beta$-catenin pathway (via LRP5/6 and Frizzled receptors, respectively), leading to transcriptional activation of T-cell factor/lymphoid enhancer factor (Tcf/Lef )-regulated genes such as c-myc and Dax-1. Wnts also can activate Frizzled receptors that stimulate calcium-mediated pathways. In the ovary, Frizzled-1 is induced by the LH surge in granulosa cells of ovulating follicles. Which Wnt might activate this receptor is not yet known. Wnt 4 is expressed in small primary follicles as well as in corpora lutea and may activate Frizzled-4 that is also expressed in luteal cells. Although Dax-1 expression is increased by overexpression of Wnt-4, its pattern of expression in the adult ovary is not strictly related to Wnt-4. Note that steroidogenic factor (SF)-1 (NR5A1) and possibly SF-2 (NR5A2) act coordinately with Wnt-4 and Tcf/Lef to enhance transcription of DAX. SF-2 is highly expressed in granulosa cells of proliferating follicles as well as corpora lutea but is noticeably absent in theca-interstitial cells of the mouse. These data confirm those published for the mare (Boerboom et al., 2000). [Adapted with permission from Hsieh M, Johnson MA, Greenberg NM, Richards JS 2002 Regulated expression of Wnts and Frizzleds at specific stages of follicular development in the rodent ovary. Endocrinology, in press. Copyright The Endocrine Society.] 
(Figure 3). In this regard, it is of interest that granulosa cells express CAMK IV and mice null for CAMK IV have impaired fertility with abnormal follicular and luteal development (Wu et al., 2000).

The Wnt/Frizzled cellular signaling pathways impact the development of reproductive organs. For example, Wnt-4 is essential for side branching in the mammary gland, a process that likely involves regulation of bone morphogenetic protein (BMP) or fibroblast growth factor (FGF) signaling molecules (ColemanKrnacik and Rosen, 1994; Weber-Hall et al., 1994; Phippard et al., 1996; Brisken et al., 2000; Wakefield et al., 2001). In mammary tissue, Wnt-4 is co-localized with progesterone receptor (PR) and is a target of PR action (Brisken et al., 2000). In the kidney, Wnt-4 is a mesenchymal signal essential for epithelial cell differentiation (Kispert et al., 1998). Furthermore, in this tissue, Wnt-4 can be replaced by Wnt-1, Wnt-3a, Wnt-7a, or Wnt-7b, indicating redundant or overlapping pathways with specific Frizzled receptors (Kispert et al., 1998). In pituitary gland development, Wnt-5a and BMP-4 play critical roles in cell fate, whereas Wnt-4 is important for expansion of ventral pituitary cell phenotypes. Wnt-7a-deficient mice are infertile due to abnormal development of the oviduct and uterus (Parr and McMahon, 1998). Mutations of Wnt-2 and Frizzled-5 were shown to impact placental angiogenesis (Monkley et al., 1996; Ishikawa et al., 2001).

Wnt-4 is also essential for the embryonic development of the ovary. Female mice null for Wnt-4 have sex-reversed ovaries that, at birth, are depleted of oocytes and contain supporting cells expressing genes characteristic of testis development such as Mullerian inhibiting substance (MIS) (Vainio et al., 1999). Since mice null for Wnt-4 die at birth, we have analyzed by reverse transcriptionpolymerase chain reaction (RT-PCR) and in situ hybridization the expression of Wnt- 4 in the adult ovary. Our results show that Wnt-4 is expressed in granulosa cells of small primary follicles containing one or two layers of cells and in granulosa cells of preovulatory follicles (Figure 3) (Hsieh et al., in press). Wnt-4 expression is increased in granulosa cells by the LH surge and reaches its highest level in corpora lutea (Figure 3). Unlike the mammary gland, Wnt-4 is not a target of PR in the ovary (Hsieh et al., in press), perhaps because PRA rather than PRB plays a primary role in the follicle, whereas PRB plays a greater role in mammary tissue (Mulac-Jericevic et al., 2000; Conneely et al., 2001). Wnt-4 may control different aspects of granulosa cell and luteal cell function, depending on which Frizzled receptors are present. Although it is not yet clear which Frizzled receptor is present in primary follicles, our results show that Frizzled-1 is induced transiently by the LH surge (Hsieh et al., in press). It is localized to granulosa cells of ovulating follicles between 4-12 hours after exposure to the LH surge, just prior to ovulation. Thus, Frizzled-1 may control the expression of genes that impact the ovulation process. In contrast, Frizzled-4 is preferentially expressed at elevated levels in corpora lutea (Hsieh et al., in press). Thus, 
Frizzled-4 may be a receptor for Wnt-4 in this tissue (Figure 3). Mice null for Frizzled-4 also exhibit reproductive (ovarian?) defects but the exact nature of these are not yet known (J. Nathans, personal communication).

What are the functions of Wnt- 4 in the adult ovary? In the embryonic gonad, the expression pattern of Wnt-4 is similar to that of DAX-1 (Swain et al., 1996; Vainio et al., 1999). More recently, overexpression of Wnt-4 in gonadal cells upregulated the expression of DAX-1 (Jordan et al., 2001), indicating that Wnt-4 may regulate DAX-1 in the ovary as well. This observation is supported by the ability of $\beta$-catenin to enhance SF-1-stimulated transactivation of the DAX-1 promoter via Tcf/Lef promoter elements (Morohashi et al., 2001). As a corepressor of the orphan nuclear receptor SF-1, ovarian DAX-1 may antagonize the transcriptional activation of genes that are regulated by SF-1 such as aromatase (CYP 19) (Fitzpatrick and Richards, 1994; Carlone and Richards, 1997), P450scc (CYP21) (Clemens et al., 1994; Richards, 1994), 17 $\alpha$-hydroxylase (CYP17) (Zang and Mellon, 1996), FSH receptor (Heckert, 2000; Levallet et al., 2001), MIS (Shen et al., 1994; Watanabe et al., 2000), and inhibin- $\alpha$ (Ito et al., 2000). Since the expression of these genes is low in small growing follicles of immature mice and rats (Richards, 1994,2001b), it is tempting to speculate that a Wnt-4/Frizzled pathway is acting in these follicles to control Dax-1 and hence the activity of SF-1.

However, the role of Wnt-4 in the ovary appears to be complex. DAX-1 remains expressed (albeit at a lower level) in mice null for Wnt-4 (Vainio et al., 1999; Jordan et al., 2001), likely due to the potent control of DAX-1 expression by SF-1. Furthermore, only some but not all SF-1 regulated genes are elevated in the Wnt-4 null mice (Vainio et al., 1999). Mice null for DAX-1 appear to have normal ovarian function (Yu et al., 1998). Recent studies have identified and also shown that the equine ovary expresses not only SF-1 (NR-5A1) but also SF-2 (NR-5A2) (Boerboom et al., 2000). Whereas SF-1 is high in theca cells, SF-2 is highest in granulosa cells and corpora lutea. We have recently confirmed these data in the rat and mouse by RT-PCR and in situ hybridization analyses (Figure 3 ). Thus, although the expression of Wnt-4 in small follicles may suppress steroidogenesis at this stage of development, a critical role for DAX-1 and SF-1 or SF-2 is not entirely clear. Even more striking, Wnt-4 as well as Frizzled-4 are elevated in luteal cells that are highly steroidogenic, contain nuclear Dax-1 protein, and express SF-2 as well as SF-1 (Hsieh et al., in press) (Figure 3). Therefore, it is possible the Wnt-4/Frizzled pathway(s) operating in small follicles is (are) different than the Wnt-4/Frizzled-4 pathway that appears, but has not yet been proven, to be dominant in luteal cells. In addition, the specific downstream effectors of Wnt/Frizzled signaling may change as follicles terminally differentiate to luteal cells, thereby controlling distinct patterns of gene expression. Recent studies have shown that luteal cells exhibit elevated expression of specific kinases, including Sgk (Gonzalez-Robayna et al., 1999; Alliston 
et al., 2000) and a MAP kinase pathway (Maizels et al., 2001). Whether these kinases are targets (or mediators?) of Wnt/Frizzled signaling is also not known. In summary, the localization and regulation of Wnt-4, Frizzled-4, Frizzled-1 and others (Hsieh et al., in press) in the adult rodent ovary, combined with the evidence for critical roles for Wnt-4 (Vainio et al., 1999) and FGF-9 (Colvin et al., 2001) in ovary and testis development, respectively, indicate that Wnt/ Frizzled signaling is important for the growth and development of ovarian follicles. The identification of these ovarian-derived regulatory molecules provides a new intraovarian regulatory network that needs to be more clearly defined.

\section{Genes Involved in Ovulation}

To prepare for ovulation, the ovary undergoes a series of closely regulated events. Small follicles must mature to the preovulatory stage, during which time the oocyte, granulosa cells, and theca cells acquire specific functional characteristics. The oocyte becomes competent to undergo meiosis, granulosa cells acquire the ability to produce $\mathrm{E}$ and respond to $\mathrm{LH}$ via the $\mathrm{LH}$ receptor, and theca cells begin to synthesize increasing amounts of androgens that serve as substrates for the aromatase enzyme in the granulosa cells (for a review, see Eppig, 1991; Richards, 1994). Remarkably, many events are spatially restricted to specific microenvironments within the follicle or surrounding interstitial compartments to allow successful expulsion of the cumulus-oocyte complex from the ruptured follicle (Hess et al., 1999; Hizaki et al., 1999; Sato et al., 2001; Zhou et al., 2001).

\section{A. GENES CONTROLLING CUMULUS EXPANSION}

The cumulus cells surrounding the oocyte and the matrix that the cumulus cells produce prior to ovulation comprise a special functional unit. The pioneering studies of many investigators have shown that the matrix upon which the cumulus cells move is formed by at least three major components (Figure 4). These include hyaluronic acid (HA) (Hess et al., 1999; Salustri et al., 1999) and at least two HA-binding proteins, namely, tumor-necrosis factor-stimulated gene (TSG)-6 (Fulop et al., 1997; Yoshioka et al., 2000) and the serum-derived inter- $\alpha$-inhibitor ( $\mathrm{I} \alpha \mathrm{I}$ ), also known as inter- $\alpha$-trypsin inhibitor (ITI) or serumderived hyaluronic acid binding protein (SHAP) (Hess et al., 1999; Sato et al., 2001; Zhou et al., 2001). HA is a high molecular weight (several million daltons), linear, unbranched glycosaminoglycan. In the ovary, HA is produced by the cumulus cells and granulosa cells adjacent to the antrum (Ochsner et al., 2001). Expansion occurs only when $I \alpha I$ enters the follicle or when serum is added to cumulus-oocyte complexes (COC) to stabilize the matrix by covalent 


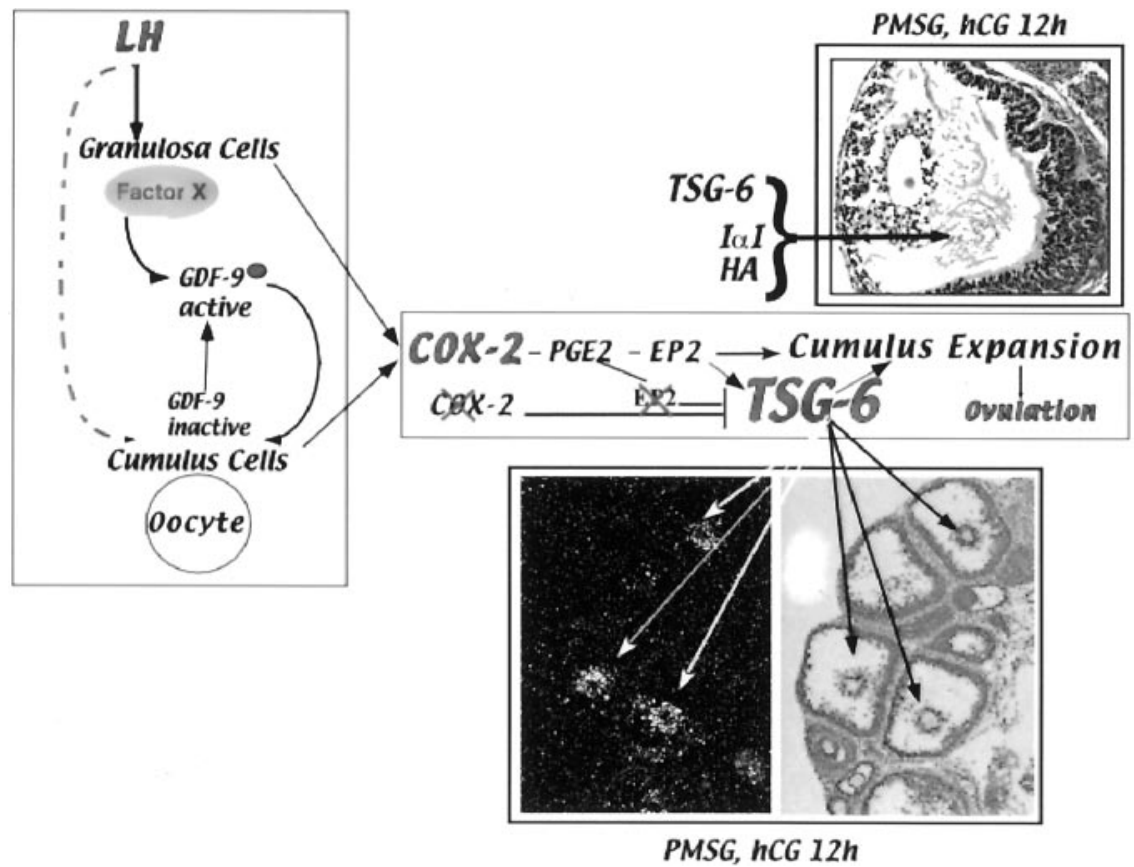

FIG. 4. LH induction of COX-2 is essential for cumulus expansion and ovulation. COX-2 is obligatory for the synthesis of prostaglandins, namely, PGE2 that binds the EP2 receptor. The induction of COX-2 by LH may be direct or indirect via activation of oocyte-derived factor, growth differentiation factor (GDF)-9, as discussed in the text. Mice null for COX-2 and EP2 fail to ovulate and exhibit impaired cumulus cell expansion, a process that requires the synthesis and cross-linking of key matrix components. These are hyaluronic acid (HA) and the HA binding proteins, inter- $\alpha$ inhibitor (I $\alpha \mathrm{I})$ and tumor suppressor gene (TSG)-6. I $\alpha \mathrm{I}$ is provided by serum and enters the follicles upon dissolution of the basement membrane in response to LH. The heavy chain of $\mathrm{I} \alpha \mathrm{I}$ is then covalently linked to HA by a converting enzyme present on granulosa cells. TSG- 6 is induced by LH in cumulus cells of ovulating follicles. The induction of TSG-6 is dependent on the induction of COX-2 and the expression of the EP2 receptor. Of note, TSG-6 expression is absent in cumulus cells of COX-2 and EP2 knockout mice (Ochsner et al., 2001).

coupling to the heavy chain (HC) of I $\alpha \mathrm{I}$ (Hess et al., 1999; Salustri et al., 1999). The cumulus-derived matrix also contains other factors such as the proteoglycans brevican and versican (MacArthur et al., 2000).

Cumulus expansion is induced as a consequence of the LH surge and is dependent on the induction of specific genes (Figure 4). These include cyclooxygenase-2 (COX-2) the rate-limiting enzyme in the synthesis of prostaglandins such as prostaglandin E2 (PGE2) (Sirois et al., 1992; Joyce et al., 2001; Ochsner et al., 2001); HA synthase-2 (HAS-2), which catalyzes the production of HA (Weigel et al., 1997); and TSG-6, which is an HA binding protein (Lee et al., 
1992; Yoshioka et al., 2000). Ovulation is impaired in mice null for COX-2 (Dinchuk et al., 1995; Morham et al., 1995), EP2, the PGE2 receptor (Hizaki et al., 1999; Tilley et al., 1999) as well as $\mathrm{I} \alpha \mathrm{I}$ (Sato et al., 2001; Zhou et al., 2001). In each mutant mouse, the COCs within preovulatory follicles fail to undergo cumulus expansion in response to LH (Davis et al., 1999). Ovulation and cumulus expansion can be restored in the COX-2 mice by exogenous administration of PGE2 or IL-1 $\beta$ (Davis et al., 2001), indicating that prostaglandins and other signaling pathways are obligatory for both events. These observations provide clear evidence that one critical site for PGE2 action in the ovulating follicle is the COC. One target of PGE2 action may be TSG-6, since expression of TSG-6 is selectively reduced in the cumulus cells (but not granulosa cells) of COX-2 and EP2 null mice (Ochsner et al., 2001). Note that TSG-6 remains selectively expressed in cumulus cells but not granulosa cells of ovulating follicles 12 hours after exposure to the LH-like molecule, human chorionic gonadotropin (hCG). These results indicate that PGE2 regulates expression of TSG-6 within the cumulus microenvironment and that TSG-6 may play some critical role in expansion of the matrix. Both TSG-6 and HA are expressed several hours prior to any visible physical expansion of the matrix (i.e., dispersion of the cumulus cells away from the oocyte). This suggests that the presence of these molecules is not sufficient for matrix formation or the movement of cumulus cells away from the oocyte (Figure 4).

The other critical component is $\mathrm{I} \alpha \mathrm{I}$. Ovulation can be restored in the I $\alpha$ I-deficient (bikunin null) mice by adding serum (Sato et al., 2001; Zhou et al., 2001). $\mathrm{I} \alpha \mathrm{I}$ is normally excluded from follicular fluid because of its size and the avascular nature of the granulosa cell layer. It enters upon dissolution of the basal lamina during ovulation (Hess et al., 1999). I $\alpha \mathrm{I}$ is composed of several subunits: the light chain (LC) known as bikunin, which is covalently associated with the heavy chains (HC) via a chondroitin-sulfate moiety. In the presence of $\mathrm{HA}, \mathrm{I} \alpha \mathrm{I}$ undergoes a substitution reaction in which the HC (SHAP) is covalently bound to HA releasing the bikunin (Sato et al., 2001). The high degree of covalent linkage between the heavy chains and HA in the COC is unprecedented (Chen et al., 1996) and suggests that the enzyme activity controlling this process is elevated within the follicle. Indeed, studies by Larsen and colleagues have shown high activity of the HC-HA conversion process in mural granulosa cells (Chen $e t$ al., 1996). Although the enzymatic activity that catalyzes the covalent linkage to HA is essential, the biochemical identity of this converting enzyme is not yet known. Nor is it known if the enzyme is hormonally regulated in the mural granulosa cells. Such a condition would also be an important factor in controlling matrix formation.

Collectively, these observations indicate that $\mathrm{HA}$ and $\mathrm{I} \alpha \mathrm{I}$, as well as COX-2/PGE2/EP2-induced gene products (TSG-6 and others?) are critical for 
COC formation or cumulus cell differentiation; lack of any one of these factors precludes expansion.

The molecular mechanisms by which LH induces expression of HAS-2, COX-2, and TSG-6 genes may be either direct via LH receptors present on cumulus cells (although this possibility is controversial) or indirect via the activation of other signaling events in the follicle (Figure 4). The latter recently has gained credence, since the previously unknown soluble oocytederived factor that is essential for cumulus expansion (Eppig, 1991; Salustri et al., 1999) has been provisionally identified as growth differentiation factor-9, GDF-9 (Elvin et al., 1999). In cultures of rodent granulosa cells, GDF-9 can induce the expression of both HA and COX-2 (Elvin et al., 1999). However, in vivo GDF-9 is expressed in oocytes beginning at the small primary follicle stage and continues in the oocyte after ovulation. If GDF-9 is the stimulatory factor, this raises the dilemma of why the expression of HA and COX-2 is restricted to ovulating follicles (i.e., those stimulated by the LH surge). One possible scenario that would link the obligatory requirement of LH action with that of the soluble ooctye-derived factor (i.e., GDF-9) is that this factor may need to be modified before it becomes activated. GDF-9, like other members of the TGF- $\beta$ family, is synthesized as a pro-peptide and therefore it is likely present at the surface of the oocyte-cumulus cell junctions, possibly attached to proteoglycans (Park et al., 2000) as a latent factor. Induction by LH of a specific protease (factor X) (Figure 4) may be necessary to activate and/or release GDF-9, thereby allowing it to interact with cellular receptors and induce HAS-2 and COX-2 in cumulus cells. Full resolution of the pathways by which LH induces COX-2 in granulosa cells versus cumulus cells will necessitate stage-specific knockouts of GDF-9. Knockouts of TSG-6 also are needed to convincingly show a role for this protein in cumulus expansion in vivo.

\section{B. GENES EXPRESSED IN THECA CELLS}

The specific ovulatory role of the theca cells is not well defined. Whereas matrix metalloproteinase (MMP)2 is expressed exclusively in the theca cells of preantral, preovulatory, and ovulating follicles (Lui et al., 1998), the other MMPs and their inhibitors (TIMPs) exhibit more complex expression patterns for which a function is not yet clear. Based on the regulated expression of several aldo-keto reductase enzymes in the theca cells, it is possible that they act as a protective shield to ensure that toxic levels (Richards et al., in press (a)) of compounds do not reach the granulosa cells or the oocyte at an inopportune time. These changes also may serve to protect the theca cells themselves and the ovary in general from exposure to toxic compounds. 


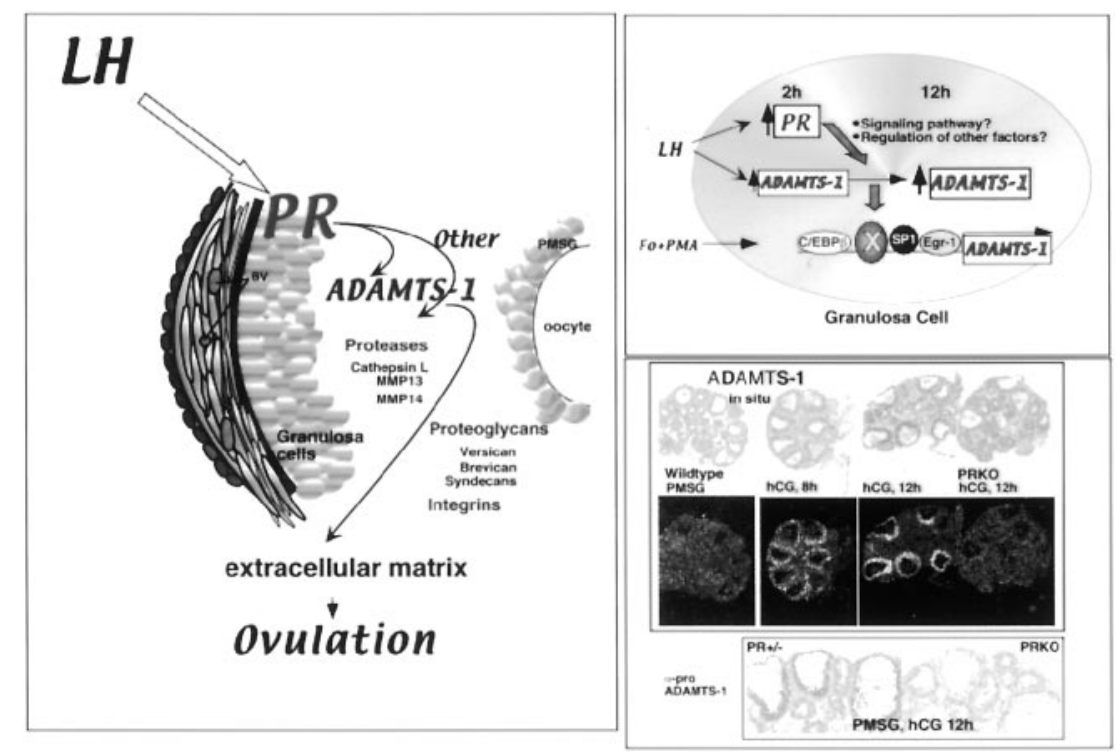

FIG. 5. LH induction of progesterone receptor (PR) and ADAMTS-1 is essential for ovulation. Expression of ADAMTS-1 is low in pregnant mare serum gonadotropin (PMSG)-treated mice but increases markedly by $8-12$ hours after human chorionic gonadotropin (hCG), as shown by in situ hybridization and immunohistochemistry using an antibody to the prodomain of the protein ( $\alpha$-pro ADAMTS-1). Expression of ADAMTS-1 is impaired in the progesterone receptor knockout (PRKO) mice (Robker et al., 2000). The molecular mechanisms by which PR induces ADAMTS-1 appear to involve an indirect pathway, since consensus PR response elements (PRREs) are not found in the proximal ADAMTS-1 promoter. The functional role(s) of ADAMTS-1 remains to be identified, since this protease has multifunctional domains. In other tissues, it is a potent antiangiogenic factor, can degrade aggrecan and brevican, and may impact the integrin system in a manner similar to thrombospondin. Thus, PR and ADAMTS-1 have the potential to regulate many steps in the ovulation process.

\section{LUTEINIZING HORMONE-REGULATED GENES IN GRANULOSA CELLS}

The LH receptor is essential for ovulation and luteinization (Lei et al., 2001). The LH-induced transcription factors in granulosa cells include early growth regulatory factor-1 (Egr-1) (Espey et al., 2000a), CAAT enhancer binding protein beta (C/EBP $\beta$ ) (Sirois and Richards, 1993), and progesterone receptor (PR) (Park and Mayo, 1991; Natraj and Richards, 1993) (Figures 2 and 5). Like COX-2, each of these components of the ovulatory process is induced rapidly but is expressed only transiently, with peak levels of message and protein observed approximately 4 hours after the LH surge. Each of these mediators appears to be involved in the functional activity of granulosa cells of ovulating follicles, as revealed by knockout studies (for a review, see Richards et al., 1998,2000,in 
press (a)). Other transcription factors such as the activator protein-1 (AP1) family members (e.g., Fra2 and JunD) are induced rapidly by the LH surge but then remain elevated in the nondividing, terminally differentiated granulosa cells during the postovulatory luteal phase (Sharma and Richards, 2000).

\section{Progesterone Receptor $(P R)$}

PR is a member of the nuclear receptor superfamily and regulates the numerous functions in reproductive tissues, including the uterus, mammary gland, and ovary. In the ovary, LH rapidly and selectively induces PR in mural granulosa cells of preovulatory follicles (Park and Mayo, 1991; Natraj and Richards, 1993). The molecular mechanisms by which LH acts to induce PR are not yet entirely clear but, in contrast to other tissues, strong evidence for a role of ER is lacking. E alone does not induce $\mathrm{PR}$ in intact cells in vivo or in vitro (Natraj and Richards, 1993). Nor does E induce activity of PR-promoter luciferase constructs transfected into granulosa cells (Clemens et al., 1998; S.C. Sharma and J.S. Richards, in preparation). In addition, PR mRNA and protein can be induced by hCG in granulosa cells of PMSG-treated ER $\beta$ KO mice (J.S. Richards, unpublished observation). Furthermore, ovulation can be restored in the ER $\alpha \mathrm{KO}$ mice by controlling pituitary secretion of LH (Couse and Korach, 1999; Couse et al., 1999). Two regions of the PR promoter previously thought to be involved in mediating LH induction of this gene - namely, the GC-rich region of the distal promoter and the estrogen response element (ERE) 3 site of the proximal promoter (Clemens et al., 1998) - also do not seem to be required in the context of the intact promoter (S.C. Sharma and J.S. Richards, in preparation). Specifically, deletion or mutation of the GC-rich region in the context of the intact murine promoter does not alter functional activity of a luciferase reporter construct when transfected into primary cultures of granulosa cells. Likewise, deletion or mutation of the ERE3 site in the context of the intact promoter does not alter activity. Additional deletional studies and mutational studies have indicated that, in granulosa cells, the critical region of the PR promoter resides downstream relative to the putative transcriptional initiation site. In fact, the putative transcriptional start site can be deleted without affecting PR promoter activity. This critical downstream region contains one of many numerous putative cap sites scattered in the promoter, as indicated by computer-generated sequence homology searches of the PR promoter. In addition, numerous RNA transcripts are expressed in granulosa cells (Natraj and Richards, 1993). Thus, transactivation of the PR promoter in these cells appears to utilize a specific region or can use many different cap sites. Therefore, although nuclear factor $\mathrm{Y}$ (NF-Y), GATA, and Sp-1 binding sites have been characterized by electrophoretic mobility shift assays (EMSAs), none of these binding sites is obligatory for LH activation of PR-promoter-luciferase reporter constructs in granulosa 
cells (S.C. Sharma and J.S. Richards, in preparation). Thus, the key transcription factor(s) remain to be identified.

Mice null for PR fail to ovulate, even when stimulated by exogenous hormones. These findings support other studies that implicated progesterone as a key player in the ovulatory process (Lydon et al., 1995; Rose et al., 1999; Pall et al., 2000). More specifically, mice null for PRA but not PRB exhibit impaired ovulation, indicating the subtype specificity of PR action in the ovulation process (Mulac-Jericevic et al., 2000; Conneely et al., 2001). Despite the failure of ovulation to occur in PRKO/PRAKO mice, the expression of COX-2, cumulus expansion, and luteinization proceed normally (Robker et al., 2000). Recently, two targets of PR action were identified. These are ADAMTS-1 (for a disintegrin and metalloproteinase with thrombospondin-like repeats) that is known as METH-1 in human (Vazquez et al., 1999; Espey et al., 2000b), and cathepsin L (Robker et al., 2000). Expression of ADAMTS-1 mRNA and protein is markedly reduced in granulosa cells of PR null mice (Figure 5).

\section{ADAMTS-1}

ADAMTS-1 is selectively induced by LH in granulosa cells and cumulus cells, with the peak level of mRNA and protein being produced $8-12$ hours after exposure of ovaries to an ovulatory dose of hCG (a gonadotropin that is functionally analogous to LH) (Espey et al., 2000b; Robker et al., 2000). The peak in ADAMTS-1 transcription occurs after the peak of PR expression but before ovulation, which is usually observed at $14-16$ hours after exposure to ovulatory hormones in mice and rats. Quite significantly, there are clear data to show that the induction of ADAMTS- 1 is drastically reduced in rats when the preovulatory synthesis of progesterone is inhibited with epostane (Espey et al., 2000b) or in mice that are null for PR (Robker et al., 2000). Thus, the temporal pattern of these events indicates that ADAMTS-1 has a critical downstream role in mediating the PR-regulated ovarian activity that culminates in the rupture of a follicle. Whether or not PR acts directly or indirectly to control the expression of these two distinct proteases remains to be determined. To date, no consensus PR response element (PRRE) has been identified in the 1.6-kb ADAMTS-1 promoter and evidence for a direct effect of PR has not been observed in culture. Therefore, we propose at the moment that PR controls expression of an intermediary step that may be a specific signaling pathway that impacts transcription factor activity at the ADAMTS-1 promoter (Figure 5).

ADAMTS-1 is a multifunctional protein and, as such, could exert more than one function in the ovary. Which, if any, specifically impacts ovulation needs to be defined. Three major sites of action are likely (Figure 5). ADAMTS-1 is a potent active protease that cleaves, among other substrates, the bait region of $\alpha 2$-macroglobulin (Kuno et al., 1999). As an active secreted protease, it is 
likely to initiate one or more proteolytic cascades that account for the observed phenotype of the mice null for PR. As a protease, ADAMTS-1, like ADAMTS-4 (also expressed in the ovary), may also control the amount and the cellular location of various proteoglycans. Brevican and versican are present in follicular fluid, perlican is present in the thecal compartment, and the cell surface proteoglycans such as syndecan or glypican may be on either granulosa cells or theca cells (Ishiguro et al., 1999; MacArthur et al., 2000). Both ADAMTS-1 and ADAMTS-4 have been shown to degrade aggrecan and brevican (Kuno et al., 2000; Nakamura et al., 2000; Tortorella et al., 2000). The action of ADAMTS-1 on proteoglycans present in ovarian follicles is highly likely. By altering the local concentrations of proteoglycans, ADAMTS- 1 could also regulate the activity of specific growth factors, such as GDF-9, FGF-2 and FGF-7, EGF, TGF- $\alpha$, or Wnts, whose activity is known to be blocked by proteoglycans (Park et al., 2000). Thus, a lack of ADAMTS-1 might prevent the activation of one or more potent bioactive factors in the follicular fluid by preventing their release from the proteoglycans.

The function for ADAMTS- 1 in the follicle may be mediated by its ability to interact with specific cellular signaling molecules through disintegrin or thrombospondin motifs at the carboxy terminus of the protein (Kuno et al., 1999). Like some other ADAM proteins, ADAMTS-1 may be a signaling protein that regulates some aspect of granulosa cell function via interactions with specific cell surface G protein-coupled receptors (GPCRs), integrins, and tetraspan proteins (Bigler et al., 2000; Le Naour et al., 2000). ADAMTS-1, like thrombospondin 1 (TS-1), is also a potent antiangiogenic factor that may interact with integrins (Vazquez et al., 1999). Although TS-1 and -2 are expressed in ovarian follicles (Bagavandoss et al., 1998), the specific roles of thrombspondins and ADAMTS- 1 have not been clearly delineated. Based on our limited understanding of ADAMTS-1 in mammalian cells, it is difficult to predict which of its multiple functions might be critical for impacting the process of ovulation. Mutations of ADAMTS-1, ADAMTS-4, and ADAMTS-9 genes are clearly needed to resolve this important area of ovarian cell function and ovulation (Figure 5).

\section{Cathepsin $L$}

Cathepsin L is another LH- and PR-regulated gene in the ovary that was identified by cDNA array technology (Robker et al., 2000). Cathepsin L is a member of the papain family of enzymes. It is commonly a lysosomal protease but it is also secreted from certain endocrine cells such as Sertoli cells of the testis and placental trophoblasts and from certain tumors (Ishidoh and Kominami, 1998). In the cat uterus, cathepsin $\mathrm{L}$ is also regulated by progesterone (Jaffe et al., 1989). The function of cathepsin L in the ovary appears to be complex. This 
enzyme is expressed in granulosa cells of follicles at several different stages of development in response to both FSH and LH. In addition, its expression in ovulatory follicles is impaired in PR null mice (Robker et al., 2000). A functional link between PR-regulated expression of ADAMTS-1 and cathepsin L is not immediately obvious but this issue will be clarified as more information is gained about the specific roles of these proteases in the ovulation process. Cathepsin L, like cathepsin G, may activate protease-activated receptors (PARs) (Sambrano et al., 2000).

\section{PACAP and the Type-1 PACAP Receptor (PAC1)}

Pituitary adenylate cyclase-activating peptide (PACAP) and PAC1 are also LH-inducible genes that have been shown to be responsive to regulation by progesterone and PR-antagonists in vivo and in vitro (Gras et al., 1999; Ko et al., 1999; Ko and Park-Sarge, 2000; Park et al., 2000). PACAP has been shown to stimulate progesterone production as well as meiotic maturation in follicleenclosed, cumulus-enclosed oocytes (Gras et al., 1999; Ko and Park-Sarge, 2000). Thus, PACAP seemed to be a potential and attractive candidate that might act downstream of PR to regulate transcriptional activation of ADAMTS- 1 . However, expression of PACAP mRNA in ovaries of mice null for PR is identical to that observed in ovaries of wild-type mice, indicating that PR is not essential for LH-induced expression of PACAP (K.H. Doyle and J.S. Richards, unpublished observations). Therefore, other signaling cascades may be regulated by PR that impact the expression of ADAMTS-1 and cathepsin L.

\section{Summary}

The number of factors now known to control the embryonic development of the gonad as well as the regulated progression of follicular growth, ovulation, and luteinization is steadily increasing. Knockout studies have shown that members of the TGF $\beta$ family, the FGF family, and the Wnt/Frizzled pathway are among the newer players to emerge on the scene. In addition, new actions for some of the older players have been identified, such as the ability of FSH to impact the PI3-kinase cascade in a manner independent of PKA. The multiplicity of LH actions in specific microenvironments during ovulation has now documented how complex and finely tuned this process really is. Although many proteases are expressed in the ovary and are hormonally regulated, the novel protease ADAMTS-1 and cathepsin L, rather than the MMPs, have gained particular recognition. New members and new functions for steroid receptors are being unraveled. The roles of ER subtypes $\operatorname{ER} \alpha$ and $\operatorname{ER} \beta$ in the ovary as well as PR are critical for ovarian function. The identification of SF-2 is intriguing and demands that the specific roles of SF-1 and SF-2 be reanalyzed at later stages of 
follicular growth. The future will clearly bring more excitement and resolution to the dynamics of ovarian follicular development, ovulation, and luteinization.

\section{ACKNOWLEDGMENTS}

Supported in part by NIH-HD-16272, HD-16229, and a Specialized Cooperative Centers Program in Reproductive Research (SCCPRR)-HD-07495.

\section{REFERENCES}

Adashi EY, Resnick CE, D'Ercole AJ, Svoboda ME, Van Wyk JJ 1985 Insulin-like growth factors as intraovarian regulators of granulosa cell growth and function. Endocr Rev 6:400420

Alexander CM, Reichman F, Hinkes MT, Lincecum J, Becker KA, Cumberledge S, Bernfield M 2000 Syndecan-1 is required for Wnt-1-induced mammary tumorigenesis in mice. Nature Genet 25:329-332

Alliston TN, Maiyar AC, Buse P, Firestone GL, Richards JS 1997 Follicle stimulating hormoneregulated expression of serum/glucocorticoid-inducible kinase in rat ovarian granulosa cells: a functional role for the $\mathrm{Sp1}$ family in promoter activity. Mol Endocrinol 11:1934-1949

Alliston TN, Gonzalez-Robayna IJ, Buse P, Fireston GL, Richards JS 2000 Expression and localization of serum/glucocorticoid-induced kinase in the rat ovary: relation to follicular growth and differentiation. Endocrinology 141:385-395

Bagavandoss P, Sage EH, Vernon RB 1998 Secreted protein, acidic and rich in cysteine (SPARC) and thrombospondin in the developing follicle and corpus luteum of the rat. J Histochem Cytochem 46:1043-1049

Baker J, Hardy MP, Zhou J, Bondy C, Lupu F, Bellve AR, Efstratiadis A 1996 Effects of an Igf1 gene null mutation on mouse reproduction. Mol Endocrinol 10:903-918

Bigler D, Takahashi Y, Chen MS, Almeda EAC, Osbourne L, White JM 2000 Sequence specific interaction between disintegrin domain of mouse ADAM 2 (fertilin $\beta$ ) and murine eggs. J Biol Chem 275:11576-11584

Boerboom D, Pilon N, Behdjani R, Silversides DW, Sirois J 2000 Expression and regulation of transcripts encoding two members of the NR5A nuclear receptor subfamily of orphan nuclear receptors, steroidogenic factor-1 and NR5A2, in equine ovarian cells during the ovulatory process. Endocrinology 141:4647-4656

Brisken C, Heineman A, Chavarria T, Elenbaas B, Tan J, Dey SK, McMahon JA, McMahon AP, Weinberg RA 2000 Essential function of Wnt-4 in mammary gland development downstream of progesterone signaling. Genes Dev 14:650-654

Brownawell AM, Kops GJPL, Macara IG, Burgering BMT 2001 Inhibition of nuclear import by protein kinase B (Akt) regulates the subcellular distribution and activity of the forkhead transcription factor AFX. Mol Cell Biol 21:3534-3546

Brunet A, Bonni A, Zigmond MJ, Lin MZ, Juo P, Hu LS, Anderson MJ, Arden KC, Blenis J, Greenberg ME 1999 Akt promotes cell survival by phosphorylating and inhibiting a forkhead transcription factor. Cell 96:857-868

Brunet A, Park J, Tran H, Hu LS, Hemmings BA, Greenberg ME 2001 Protein kinase SGK mediates survival signals by phosphorylating the forkhead transcription factor FKHRL1 (FOXO3a). Mol Cell Biol 21:952-965

Burks DJ, de Mora JF, Schubert M, Withers DJ, Myers MG, Towery HH, Altamuro SL, Flint CL, White MF 2000 IRS-2 pathways integrate female reproduction and energy homeostasis. Nature 407:377-382 
Burns KH, Yan C, Kumar TR, Matzuk MM 2001 Analysis of ovarian gene expression in follicle-stimulating hormone $\beta$ knockout mice. Endocrinology 142:2742-2751

Cadigan KM, Nusse R 1997 Wnt signaling: a common theme in animal development. Genes Dev 11:3286-3305

Carlone DL, Richards JS 1997 Functional interactions, phosphorylation, and levels of 3'5'-cyclic adenosine monophosphate-regulatory element binding protein and steroidogenic factor-1 mediate hormone-regulated and constitutive expression of aromatase in gonadal cells. Mol Endocrinol 11:292-304

Chapman SC, Woodruff TK 2001 Modulation of activin signal transduction by inhibin B and inhibin-binding protein (InhBP). Mol Endocrinol 15:668

Chen L, Zhang H, Powers RW, Russell PT, Larsen WJ 1996 Covalent linkage between proteins of the inter-a-inhibitor family and hyaluronic acid is mediated by a factor produced by granulosa cells. J Biol Chem 271:19409-19414

Clemens JW, Lala D, Parker KL, Richards JS 1994 Steroidogenic factor-1 binding and transcriptional activity of the cholesterol side-chain cleavage promoter in rat granulosa cells. Endocrinology 134:1499-1508

Clemens JW, Kraus WL, Katzenellenbogen BS, Richards JS 1998 Hormone induction of progesterone receptor (PR) mRNA and activation of PR promoter regions in ovarian granulosa cells: evidence for a role of cAMP but not estradiol. Mol Endocrinol 12:1201-1214

Coleman-Krnacik S, Rosen JM 1994 Differential temporal and spatial gene expression of fibroblast growth factor family members during mouse mammary gland development. Mol Endocrinol $8: 218-229$

Colvin JS, Green RP, Schmahl J, Capel B, Ornitz DM 2001 Male-to-female sex reversal in mice lacking fibroblast growth factor 9. Cell 104:875-889

Conneely OM, Mulac-Jericevic B, Lydon JP, DeMayo FJ 2001 Reproductive functions of the progesterone receptor isoforms: lessons from knockout mice. Mol Cell Endocrinol 179:97103

Couse JF, Korach KS 1999 Estrogen receptor null mice: what have we learned and where will they lead us? Endocr Rev 20:358-417

Couse JF, Bunch DO, Lindzey J, Schomberg DW, Korach KS 1999 Prevention of the polycystic ovarian phenotype and characterization of ovulatory capacity in the estrogen receptor- $\alpha$ knockout mouse. Endocrinology 140:5855-5894

Davis BJ, Lennard DE, Lee CA, Tiano HF, Morham SG, Wetsel WC, Langenbach R 1999 Anovulation in cyclo-oxygenase-2-deficient mice is restored by prostaglandin E2 and interleukin-1 $\beta$. Endocrinology 140:2685-2696

de Rooij J, Zwartkruis FJT, Verheijen MHG, Cool RH, Nijman SMB, Wittinghofer A, Bos JL 1998 Epac is a Rap1 guanine-nucleotide-exchange factor directly activated by cAMP. Nature 396:474-477

de Rooij J, Rehmann H, van Triest M, Cool RH, Wittinghofer A, Bos JL 2000 Mechanism of regulation of the Epac family of cAMP-dependent RapGEFs. J Biol Chem 275:20829-20836

Dinchuk JE, Car BD, Focht RJ, Johnston JJ, Jaffee BD, Covington MB, Contel NR, Eng VM, Collins RJ, Czerniak PM, Gorry SA, Trzaskos J 1995 Renal abnormalities and an altered inflammatory response in mice lacking cyclooxygenase II. Nature 378:406-409

Elvin JA, Clark AT, Wang P, Wolfman NM, Matzuk MM 1999 Paracrine actions of growth differentiation factor-9 in the mammalian ovary. Mol Endocrinol 13:1035-1048

Eppig JJ 1991 Intercommunication between the mammalian oocytes and companion somatic cells. Bioessays 13:569-574

Espey LL, Ujoka T, Russell DL, Skelsey M, Vladu B, Robker RL, Okamura H, Richards JS 2000a Induction of early growth response protein-1 (Egr-1) gene expression in the rat ovary in response to an ovulatory dose of hCG. Endocrinology 141:2385-2391 
Espey LL, Yoshioka S, Russell DL, Robker RL, Fujii S, Richards JS 2000b Ovarian expression of a disintegrin metalloproteinase with thrombospondin motifs during ovulation in the gonadotropin-primed immature rat. Biol Reprod 62:1090-1095

Fitzpatrick SL, Richards JS 1994 Identification of a cyclic adenosine $3^{\prime}, 5^{\prime}$-monophosphate response element in the rat aromatase promoter that is required for transcriptional activation in rat granulosa cells and R2C Leydig cells. Mol Endocrinol 8:1309-1319

Fulop C, Kamath RV, Li Y, Otto JM, Salustri A, Olsen BR, Glant TT, Hascall VC 1997 Coding sequence, exon-intron structure and chromosomal localization of murine TNF-stimulated gene 6 that is specifically expressed by expanding cumulus cell-oocyte complexes. Gene 202:95-102

Gonzalez-Robayna IJ, Alliston TN, Buse P, Firestone GL, Richards JS 1999 Functional and subcellular changes in the A-kinase signaling pathway: relation to aromatase and Sgk expression during the transition of granulosa cells to luteal cells. Mol Endocrinol 13:13181337

Gonzalez-Robayna IJ, Falender AE, Ochsner S, Firestone GL, Richards JS 2000 FSH stimulates phosphorylation and activation of protein kinase B (PKB/Akt) and serum and glucocorticoidinduced kinase (Sgk): evidence for A-kinase independent signaling in granulosa cells. Mol Endocrinol 14:1283-1300

Gras S, Hannibal J, Fahrenkrug J 1999 Pituitary adenylate cyclase-activating polypeptide is an auto/paracrine stimulator of acute progesterone accumulation and subsequent luteinization in cultured periovulatory granulosa/lutein cells. Endocrinology 140:2199-2205

Guarente L, Kenyon C 2000 Genetic pathways that regulate ageing in model organisms. Nature 408:255-262

Guidice LC 2000 IGFs in the female reproductive system. In Rosenfeld R, Roberts C, eds. Contemporary Endocrinology: The IGF System. Totowa, NJ: Humana Press; 379-404

He TC, Sparks AB, Rago C, Hermeking H, Zawel L, da Costa LT, Morin PJ, Vogelstein B, Kinzler KW 1998 Identification of c-MYC as a target of the APC pathway. Science 281:1509-1512

Heckert LL 2000 Activation of the rat follicle-stimulating hormone receptor promoter by steroidogenic factor-1 is blocked by protein kinase A and requires upstream stimulatory factor binding to a proximal E box element. Mol Endocrinol 15:704-715

Hess KA, Chen L, Larsen WJ 1999 Inter- $\alpha$-inhibitor binding to hyaluronan in the cumulus extracellular matrix is required for optimal ovulation and development of mouse oocytes. Biol Reprod 61:436-443

Hizaki H, Segi E, Sugimoto Y, Hirose M, Saji T, Ushikubi F, Matsuoka T, Noda Y, Tanaka T, Yoshida N, Narumiya S, Ichikawa A 1999 Abortive expansion of the cumulus and impaired fertility in mice lacking the prostaglandin E receptor subtype EP2. Proc Natl Acad Sci USA 96:10501-10506

Hsieh M, Johnson MA, Greenberg NM, Richards JS 2002 Regulated expression of Wnts and Frizzleds at specific stages of follicular development in the rodent ovary. Endocrinology, in press

Hsueh AJW, Billig H, Tsafriri A 1994 Ovarian follicle atresia: a hormonally controlled apoptotic process. Endocr Rev 15:707-724

Ishidoh K, Kominami E 1998 Gene regulation and extracellular functions of procathepsin L. J Biol Chem 379:131-135

Ishiguro K, Kojima T, Taguchi O, Saito H, Muramatsu T, Kadomatsu K 1999 Syndecan-4 expression is associated with follicular atresia in mouse ovary. Histochem Cell Biol 112: $25-33$

Ishikawa T, Tamai Y, Zorn AM 2001 Mouse Wnt receptor Frd5 is essential for yolk sac and placental angiogenesis. Development 128:25-33 
Ito M, Park Y, Mayo KF, Jameson JL 2000 Synergistic activation of the inhibin alpha-promoter by steroidogenic factor-1 and cyclic adenosine $3^{\prime}, 5^{\prime}$-monophosphate. Mol Endocrinol 14: 66-81

Jaffe RC, Donnelly KM, Mavrogianis PA, Verhage HG 1989 Molecular cloning and characterization of a progesterone-dependent cat endometrial secretory protein complementary deoxyribonucleic acid. Mol Endocrinol 3:1807-1814

Jordan BK, Mohammed M, Ching ST, Delot E, Chen XN, Dewing P, Swain A, Rao PN, Eljalde BR, Vilain E 2001 Up-regulation of WNT-4 signaling and dosage-sensitive sex reversal in humans. Am J Hum Genet 68:1102-1109

Joyce IM, Pendola FL, O'Brien M, Eppig JJ 2001 Regulation of prostaglandin-endoperoxide synthase 2 ribonucleic acid in mouse granulosa cells during ovulation. Endocrinology 142:3187-3197

Kaestner KH, Knochel W, Martinex DE 2000 Unified nomenclature for the winged helix/forkhead transcription factors. Genes Dev 14:142-146

Kawasaki H, Springett GM, Mochizuki N, Toki S, Nakaya M, Matsuda M, Housman DE, Graybiel AM 1998 A family of cAMP-binding proteins that directly activate Rap1. Science 282:2275-2279

Kispert A, Vanio S, McMahon AP 1998 Wnt-4 is a mesenchymal signal for epithelial transformation of metanephric mesenchyme in the developing kidney. Development 125:4225-4234

Ko C, Park-Sarge OK 2000 Progesterone receptor activation mediates LH-induced type-I pituitary adenylate cyclase activating polypeptide receptor (PAC(1)) gene expression in rat granulosa cells. Biochem Biophys Res Commun 277:270-279

Ko C, In YH, Park-Sarge OK 1999 Role of progesterone receptor activation in pituitary adenylatecyclase activating polypeptide gene expression in rat ovary. Endocrinology 140:5185-5194

Kops GJPL, de Ruiter ND, De Vries-Smits AMM, Powell DR, Bos JL, Burgering BMT 1999 Direct control of the Forkhead transcription factor AFX by protein kinase B. Nature 398:630-634

Kuhl M, Sheldahl LC, Park M, Miller JR, Moon RT 2000 The Wnt/Ca ${ }^{2+}$ pathway: a new vertebrate Wnt signaling pathway takes shape. Trends Genet 16:279-283

Kumar TR, Wang Y, Lu N, Matzuk MM 1997 Follicle stimulating hormone is required for ovarian follicle maturation but not male fertility. Nature Genet 15:201-204

Kuno K, Terashima Y, Matsushima K 1999 ADAMTS-1 is an active metalloproteinase with the extracellular matrix. J Biol Chem 274:18821-18826

Kuno K, Okada Y, Kawashima H, Nakamura H, Miyasaka M, Ohno H, Matsushima K 2000 ADAMTS-1 cleaves a cartilage proteoglycan, aggrecan. FEBS Lett 478:241-245

Lee TH, Wisiewski H-G, Vilcek J 1992 A novel secretory tumor necrosis factor-inducible protein (TSG-6) is a member of the family of hyaluronate binding proteins, closely related to the adhesion receptor CD44. J Cell Biol 116:545-557

Lei ZM, Mishra S, Zou W, Xu B, Foltz M, Li X, Rao CV 2001 Targeted disruption of luteinizing hormone/human chorionic gonadotropin receptor gene. Mol Endocrinol 15:184-200

Le Naour F, Rubenstein E, Jasmin C, Prenant M, Boucheix C 2000 Severely reduced female fertility in CD9-deficient mice. Science 287:319-324

LeRoith D, Werner H, Beitner-Johnson D, Roberts CT Jr 1995 Molecular and cellular aspects of insulin-like growth factor I receptor. Endocr Rev 16:143-163

Levallet J, Koskimies P, Rahman N, Huhtaniemi I 2001 The promoter of murine folliclestimulating hormone receptor: functional characterization and regulation by transcription factor steroidogenic factor 1. Mol Endocrinol 15:80-92

Lewis KA, Gray PC, Blount AL, MacConell LA, Wiater E, Bilezikjian LM, Vale W 2000 Betaglycan binds inhibin and can mediate functional antagonism of activin signalling. Nature 404:411-414 
Lin K, Hsin H, Libina N, Kenyon C 2001 Regulation of the Caenorhabditis elegans longevity protein DAF-16 by insulin/IGF-1 and germline signaling. Nature Genet 28:139-145

Lin X, Perrimon N 1999 Dally cooperates with Drosophila Frizzled 2 to transduce Wingless signalling. Nature 400:281-284

Liu T, DeCostanzo AJ, Liu X, Wang HY, Hallagan S, Moon RT, Malbon CC 2001 G protein signaling from activated rat frizzled-1 to beta-catenin-Lef-Tcf pathway. Science 292:17181722

Lui K, Wahlberg P, Ny T 1998 Coordinated and cell-specific regulation of membrane type matrix metalloproteinase 1 (MT1-MMP) and its substrate matrix metalloproteinase 2 (MMP-2) by physiological signals during follicular development and ovulation. Endocrinology 139:47354738

Lydon JP, DeMayo F, Funk CR, Mani SK, Hughes AR, Montgomery CA, Shyamala G, Conneely OM, O'Malley BW 1995 Mice lacking progesterone receptor exhibit reproductive abnormalities. Genes Dev 9:2266-2278

MacArthur ME, Irving-Rodgers HF, Byers S, Rodgers RJ 2000 Identification and immunolocalization of decorin, versican, perlecan, nidogen, and chondroitin sulfate proteoglycans in bovine small-antral ovarian follicles. Biol Reprod 63:913-924

Maizels ET, Mukherjee A, Sithanandam G, Peters CA, Cottom J, Mayo KE, Hunzicker-Dunn M 2001 Developmental regulation of mitogen-activated protein kinase-activated kinases-2 and -3 (MAPKAPK-2/3) in vivo during corpus luteum formation in the rat. Mol Endocrinol 15:716-733

Mao J, Wang J, Liu B, Pann W, Farr GH, Flynn C, Yuan H, Takada S, Kimelman D, Li L, Wu D 2001 Low-density lipoprotein related-receptor protein-5 binds to Axin and regulates the canonical Wnt signaling pathway. Mol Cell 7:801-809

Medema RH, Kops GJP, Bos JL, Burgering BMT 2000 AFX-like forkhead transcription factors mediate cell cycle regulation by Ras and PKB through p27 $7^{\mathrm{KIP} 1}$. Nature 404:782-787

Miller JR, Hocking AM, Brown JD, Moon RT 1999 Mechanism and function of signal transduction by Wnt/beta-catenin and $\mathrm{Wnt} / \mathrm{Ca}^{2+}$ pathways. Oncogene 18:7860-7872

Monkley SJ, Delaney SJ, Pennisi DJ, Christiansen JH, Wainwright BJ 1996 Targeted disruption of the Wnt2 gene results in placental defects. Development 122:3343-3353

Morham SG, Langenback R, Loftin CD, Tiano HF, Vouloumanos N, Jennette JC, Mahler JF, Kluckman KD, Ledford A, Lee CA, Smithies O 1995 Prostaglandin synthase 2 gene disruption causes severe renal pathology in the mouse. Cell 83:473-482

Morohashi K, Yoshioka H, Kawajiri K 2001 Organogenesis of gonads and transcription factors. In: Program \& Abstracts of the 83rd Annual Meeting of The Endocrine Society, June 20-23, Denver, Colorado; 22 (Abstract TP1-1)

Mulac-Jericevic B, Mullinax RA, DeMayo FJ, Lydon JP, Conneely OM 2000 Subgroup of reproductive functions of progesterone mediated by receptor-B isoform. Science 289:17511754

Nakae J, Barr V, Accili D 2000 Differential regulation of gene expression by insulin and IGF-1 receptors correlates with phosphorylation of a single amino acid residue in the forkhead transcription factor FKHR. EMBO J 19:989-996

Nakamura H, Fujii Y, Inoka I, Kazuhiko S, Tanzawa K, Matsuki H, Miura R, Yamaguchi Y, Okada Y 2000 Brevican is degraded by matrix metalloproteinases and aggrecanase-1 (ADAMTS-4) at different sites. J Biol Chem 275:38885-38890

Natraj U, Richards JS 1993 Hormonal regulation, localization and functional activity of the progesterone receptor in granulosa cells of rat preovulatory follicles. Endocrinology 133:761769 
Ochsner SA, Russell DL, Richards JS 2001 Absence of TSG-6 expression in cumulus cells of the cyclooxygenase-2 knockout mouse. In: Program \& Abstracts of the $83^{\text {rd }}$ Annual Meeting of The Endocrine Society, June 20-23, Denver, CO; 372 (Abstract P2-370)

Orly J 2000 Molecular events defining follicular developments and steroidogenesis in the ovary. In Shupnik MA, ed. Gene Engineering in Endocrinology. Totowa, NJ: Humana Press; 239-275

Otsuka F, Moore RK, Shimasaki S 2001 Biological effects and cellular mechanisms of bone morphogenetic protein-6 in the ovary. J Biol Chem 276:32889-32895

Pall M, Mikuni M, Mitsube K, Brannstrom M 2000 Time-dependent ovulation inhibition of a selective progesterone-receptor antagonist (Org 31710) and effect on ovulatory mediators in the in vitro perfused rat ovary. Biol Reprod 63:1642-1647

Park J-II, Kim W-J, Wang L, Park H-J, Lee J, Park J-H, Kwon H-B, Tsafriri A, Chun S-Y 2000 Involvement of progesterone in gonadotropin-induced pituitary adenylate cyclase-activating polypeptide gene expression in preovulatory follicles of rat ovary. Mol Human Reprod 6:238-245

Park O-K, Mayo K 1991 Transient expression of progesterone receptor messenger RNA in ovarian granulosa cells after the preovulatory luteinizing hormone surge. Mol Endocrinol 5:967-978

Park PW, Reizes O, Bernfield M 2000 Cell surface heparan sulfate proteoglycans: selective regulators of ligand-receptor encounters. J Biol Chem 275:29923-29926

Parr BZ, McMahon AP 1998 Sexually dimorphic development of the mammalian reproductive tract requires Wnt 7a. Nature 395:707-710

Phippard DJ, Weber-Hall SJ, Sharpe PT, Naylor MS, Jayatalake H, Maas R, Woo I, Roberts-Clark D, Francis-West PH, Liu YH, Maxson R, Hill RE, Dale TC 1996 Regulation of Msx-1, Msx-2, Bmp-2 and Bmp-4 during foetal and postnatal mammary gland development. Development 122:2729-2737

Pinson KI, Brennan J, Monkley S, Avery BJ, Skarnes WC 2000 An LDL-receptor-related protein mediates Wnt signaling in mice. Nature 407:535-538

Porter DA, Vickers SL, Cowan RG, Huber SC, Quirk SM 2000 Expression and function of Fas antigen in bovine granulosa cells and theca cells during ovarian follicular development and atresia. Biol Reprod 62:62-66

Pru JK, Tilly JL 2001 Programmed cell death in the ovary: insight and future prospects using genetic technologies. Mol Endocrinol 15:845-853

Rao MC, Midgley AR, Richards JS 1978 Hormonal regulation of ovarian cellular proliferation. Cell 14:71-78

Rattner A, Hsieh JC, Smallwood PM, Gilbert DJ, Copeland NG, Jenkins NA, Nathans J 1997 A family of secreted proteins contains homology to the cysteine-rich ligand binding domain of frizzled receptors. Proc Natl Acad Sci USA 94:2859-2863

Richards JS 1994 Hormonal control of gene expression in the ovary. Endocr Rev 15:725-751

Richards JS 2001a New signaling pathways for hormones and cyclic adenosine 3',5'-monophosphate action in endocrine cells. Mol Endocrinol 15:209-218

Richards JS 2001b The ovarian follicle - a perspective in 2001. Endocrinology 142:1-10

Richards JS, Russell DL, Robker RL, Dajee M, Alliston TN 1998 Molecular mechanisms of ovulation and luteinization. Mol Cell Endocrinol 145:47-54

Richards JS, Robker RL, Russell D, Sharma CS, Espey LE, Lydon J, O'Malley BW 2000 Ovulation: a multi-gene, multi-step process. Steroids 65:559-570

Richards JS, Russell DL, Ochsner S, Espey LL 2002a Ovulation: new dimensions and new regulators of the inflammatory-like response. Annu Rev Physiol 64, in press

Richards JS, Sharma SC, Falender AE, Lo YH 2002b Expression of FKHR, FKHRL1 and AFX genes in the rodent ovary: evidence for regulation by IGF-1, estrogen and the gonadotropins. Mol Endocrinol, in press 
Robker RL, Richards JS 1998a Hormonal control of the cell cycle in ovarian cells: proliferation versus differentiation. Biol Reprod 59:476-482

Robker RL, Richards JS 1998b Hormone-induced proliferation and differentiation of granulosa cells: a coordinated balance of the cell cycle regulators cyclin D2 and p27KIP1. Mol Endocrinol 12:924-940

Robker RL, Russell DL, Espey LL, Lydon JP, O’Malley BW, Richards JS 2000 Progesteroneregulated genes in the ovulation process: ADAMTS-1 and cathepsin L proteases. Proc Natl Acad Sci USA 97:4689-4694

Rose UM, Hanssen RGJM, Kloosterboer HJ 1999 Development and characterization of an in vitro ovulation model using mouse ovarian follicles. Biol Reprod 61:503-511

Salustri A, Camaioni A, Di Giacomo M, Fulop C, Hascall VC 1999 Hyaluronan and proteoglycans in ovarian follicles. Hum Reprod Update 5:293-301

Sambrano GR, Huang W, Faruqi T, Mahrus S, Craik C, Coughlin SR 2000 Cathepsin G activates protease-activated receptor-4 in human platelets. J Biol Chem 275:6819-6823

Sato H, Kajikawa S, Kuroda S, Horisawa Y, Nakamura N, Kaga N, Kakinuma C, Kato K, Morishita H, Niwa H, Miyazaki J-I 2001 Impaired fertility in female mice lacking urinary trypsin inhibitor. Biochem Biophys Res Commun 281:1154-1160

Schuur ER, Loktev AV, Sharma M, Sun Z, Roth RA, Weigel RJ 2001 Ligand-dependent interaction of estrogen receptor- $\alpha$ with FKHR. J Biol Chem 276:33554-33560

Sharma CS, Richards JS 2000 Regulation of AP1 (Jun/Fos) factor expression and activation in ovarian granulosa cells: relation of JunD and Fra2 to terminal differentiation. J Biol Chem 275:33718-33728

Shen W-H, Moore CCD, Ikeda Y, Parker K, Ingraham HA 1994 Nuclear receptor steroidogenic factor 1 regulates the Mullerian inhibiting substance gene: a link to the sex determination cascade. Cell 77:651-661

Sirois J, Richards JS 1993 Transcriptional regulation of the rat prostaglandin endoperoxide synthase 2 gene in granulosa cells. J Biol Chem 268:21931-21938

Sirois J, Simmons DL, Richards JS 1992 Hormonal regulation of messenger ribonucleic acid encoding a novel isoform of prostaglandin endoperoxide $\mathrm{H}$ synthase in rat preovulatory follicles. J Biol Chem 267:11586-11592

Slusarski DC, Corces VG, Moon RT 1997 Interaction of Wnt and a Frizzled homologue triggers G-protein-linked phosphatidylinositol signalling. Nature 390:410-413

Swain A, Zanaria E, Hacker A, Lovell-Badge R, Camerino G 1996 Mouse Dax-1 expression is consistent with a role in sex determination as well as adrenal and hypothalamic function. Nature Genet 12:404-409

Tamai K, Semenov M, Kato Y, Spokony R, Liu C, Katsuyama Y, Hess F, Saint-Jeannet J-P, He X 2000 LDL-receptor-related proteins in Wnt signal transduction. Nature 407:530-535

Tanaka M, Kirito K, Kashii Y, Uchida M, Watanabe T, Endo H, Endoh T, Ozawa K, Komatsu N 2001 Forkhead family transcription factor FKHRL1 is expressed in human megakaryocytes. Regulation of cell cycling as a downstream molecule of thrombopoietin signaling. J Biol Chem 276:15082-15089

Tilley SL, Audoly LP, Hicks EH, Kim H-S, Flannery PJ, Coffman TM, Koller BH 1999 Reproductive failure and reduced blood pressure in mice lacking the EP2 prostaglandin E2 receptor. J Clin Invest 103:1539-1545

Tortorella MD, Pratta M, Liu R-Q, Austin J, Ross OH, Abbaszade I, Burn T, Arner E 2000 Sites of aggrecan cleavage by recombinant human aggrecanase-1 (ADAMTS-4). J Biol Chem 275:18566-18573

Tsuda M, Kamimura K, Nakato H, Archer M, Staatz W, Fox B, Humphrey M, Olson S, Futch T, Kaluza V, Siegfried E, Stam L, Selleck SB 1999 The cell-surface proteoglycan Dally regulates Wingless signalling in Drosophila. Nature 400:276-280 
Vainio S, Heikkila M, Kispert A, Chin N, McMahon AP 1999 Female development in mammals is regulated by Wnt-4 signalling. Nature 397:405-409

Vanhaesebroeck B, Alessi DR 2000 The PI3K-PDK1 connection: more than just a road to PKB. Biochem J 346:561-576

Vazquez F, Hastings G, Ortega M-A, Lane TF, Oikemus S, Lombardo M, Iruela-Arispe ML 1999 METH-1, a human ortholog of ADAMTS-1, and METH-2 are members of a new family of proteins with angio-inhibitory activity. J Biol Chem 274:23349-23357

Wakefield LM, Piek E, Bottinger EP 2001 TGF-beta signaling in mammary gland development and tumorigenesis. J Mammary Gland Biol Neoplasia 6:67-82

Watanabe K, Clarke TR, Lane AH, Wang X, Donahoe PK 2000 Endogenous expression of Mullerian inhibiting substance in early postnatal rat Sertoli cells requires multiple steroidogenic factor-1 and GATA-4-binding sites. Proc Natl Acad Sci USA 97:1624-1629

Weber-Hall SJ, Phippard DJ, Niemeyer CC, Dale TC 1994 Developmental and hormonal regulation of Wnt gene expression in the mouse mammary gland. Differentiation 57:205-214

Wehrli M, Dougan ST, Caldwell K, O'Keefe L, SchwartZ S, Vaizel-Ohayon D, Schejter E, Tomlinson A, DiNardo S 2000 Arrow encodes an LDL-receptor-related protein essential for Wingless signaling. Nature 407:527-530

Weigel PH, Hascall VC, Tammi M 1997 Hyaluronan synthase. J Biol Chem 272:13997-14000

Wu JY, Gonzalez-Robayna IJ, Richards JS, Means AR 2000 Female fertility is reduced in mice lacking $\mathrm{Ca}^{+2} /$ calmodulin-dependent kinase IV. Endocrinology 141:4777-4784

Yoshioka S, Ochsner S, Russell DL, Ujioka T, Fujii S, Richards JS, Espey LL 2000 Expression of tumor necrosis factor-stimulated gene- 6 in the rat ovary in response to an ovulatory dose of gonadotropin. Endocrinology 141:4114-4119

Yu RN, Ito M, Saunders TL, Camper S, Jameson JL 1998 Role of Ahch in gonadal development and gametogenesis. Nature Genet 20:353-357

Zaczek D, Service D, Bartke A, Coschigano K, Suen L, Wandji S, Hammond J 2001 Ovarian follicles are reduced in number but not function in GH receptor (GHR) knockout (KO) mice. In Program \& Abstracts of the 83rd Annual Meeting of The Endocrine Society, June 20-23, Denver, CO; 377 (Abstract P2-394)

Zang P, Mellon SH 1996 The orphan nuclear receptor steroidogenic factor-1 regulates the cyclic adenosine 3',5'-monophosphate-mediated transcriptional activation of rat cytochrome P450c17 (17alpha-hydroxylase/c17-20 lyase). Mol Endocrinol 10:147-158

Zhao HH, Herrera RE, Coronado-Heinsohn E, Yang MC, Ludes-Meyers JH, Seybold-Tilson KJ, Nawaz Z, Yee C, Barr FG, Diab SG, Brown PH, Fuqua SAW, Osborne CK 2001 FKHR functions as a bifunctional nuclear receptor interacting protein with both coactivator and corepressor functions. J Biol Chem 276:27907-27912

Zhou J, Refuerzo J, Bondy C 1995 Granulosa cell DNA synthesis is strictly correlated with the presence of insulin-like growth factor I and absence of c-fos/c-jun expression. Mol Endocrinol 9:924-931

Zhou J, Kumar TR, Matzuk MM, Bondy C 1997 Insulin-like growth factor I regulates gonadotropin responsiveness in the murine ovary. Mol Endocrinol 11:1924-1933

Zhou L, Yoneda M, Zhao M, Yingsung W, Yoshida N, Kitagawa Y, Kawamura K, Suzuki T, Kimata K 2001 Defect in SHAP-hyaluronan complex causes severe female infertility: a study by inaction of the bikunin gene in mice. J Biol Chem 276:7693-7696 


\title{
Transcription Factors Underlying the Development and Endocrine Functions of the Placenta
}

\author{
James C. Cross, ${ }^{* \dagger}$ Lynn Anson-Cartwright, ${ }^{\dagger}$ and Ian C. Scott ${ }^{\dagger}$ \\ *Department of Biochemistry \& Molecular Biology, University of Calgary Faculty of Medicine, \\ Calgary, Alberta T2N 4 N1 Canada; '`Samuel Lunenfeld Research Institute, Mount Sinai Hospital, \\ Toronto, Ontario M5G 1 X5 Canada
}

\begin{abstract}
The placenta has been the subject of extensive basic research efforts in two distinct fields. The developmental biology of placenta has been studied because it is the first organ to develop during embryogenesis and because a number of different gene mutations in mice result in embryonic lethality due to placental defects. The trophoblast cell lineage is relatively simple such that only two major, terminally differentiated cell types appear: an "invasive trophoblast" cell subtype such as extravillous cytotrophoblast cells in humans and trophoblast giant cells in mice, and a "transport trophoblast" cell subtype that is a syncytium (syncytiotrophoblast) in humans and mice. These two cell types also have been the focus of endocrinologists because they are the source of major placental hormones. Understanding the transcriptional regulation of placental hormone genes has given insights into the control of specificity of gene expression. Because most placental hormones are produced by very specific trophoblast cell subtypes, the transcriptional details promise to give insights into cell-subtype specification. The fields of developmental biology and molecular endocrinology appear to be meeting on this common ground with the recent discovery of key transcription factors. Specifically, the basic helix-loop-helix (bHLH) transcription factor Hand1 is essential for differentiation of trophoblast giant cells in mice and also regulates the promoter for the giant cell-specific hormone, placental lactogen I gene $(P l 1)$. In contrast, formation of syncytiotrophoblast cells in mice is controlled by a distinct genetic pathway that is governed by the Gcm1 transcription factor, a homologue of the Drosophila glial cells missing gene. Human GCM1 has been shown to regulate the activity of the placental-specific enhancer of the aromatase gene (CYP19), which is specifically expressed in syncytiotrophoblast. Together, these findings imply that some key transcription factors have the dual functions of controlling both critical cell fate decisions in the trophoblast cell lineage and later the transcription of cell subtype-specific genes unrelated to development.
\end{abstract}

\section{Introduction}

Although we leave it behind at birth, the placenta is an amazing organ that is essential for intrauterine development. It allows the embryo to implant into the uterus and transports the nutrients and oxygen necessary for fetal growth. In addition, it has a major endocrine function that helps to subvert and orchestrate several maternal physiological systems that, together, further promote fetal 
growth and survival. These functions include promoting the growth of maternal blood vessels to the implantation site and their dilation, suppression of the local immune system, promoting mammary gland development and continued production of progesterone from the corpus luteum (Linzer and Fisher, 1999; Cross et $a l$, in press). Failures in any one of these functions are associated with a range of complications of human pregnancy, including missed abortion, miscarriage, intrauterine growth restriction, and pre-eclampsia (Cross, 1996; Kingdom and Kaufmann, 1997; Cross, 1999). Aside from these specific functional aspects, study of the placenta gives insights into general aspects of developmental biology that are applicable to other systems. Indeed, because of the relative simplicity of the placental cell lineages, combined with the fact that the placenta is so sensitive to genetic perturbation, the placenta is an attractive model system for understanding the control of stem cells, cell lineage, and cell-cell interactions (Cross, 2000; Hemberger and Cross, 2001).

The placenta is derived from two major cell lineages (Cross et al., 1994; Cross, 2000). Trophectoderm of the blastocyst is the precursor to the trophoblast cell lineage that will give rise to the epithelial parts of the placenta. Extraembryonic mesoderm gives rise to the stromal cells and blood vessels of the placenta. In rodents and primates, the outer layer of the mature fetal placenta consists of cells that are inherently invasive and associate with maternal blood vessels (called extravillous cytotrophoblast cells in primates and trophoblast giant cells in rodents). The innermost layer of the placenta consists of villous, tree-like branches that provide a large surface area for nutrient and gas exchange (called chorionic villi in primates and "the labyrinth" in rodents). The villi are covered with multinucleated syncytiotrophoblast (two layers in rodents) and also contain trophoblast stem cells, stromal cells, and blood vessels. The middle layer of the rodent and primate placenta consists of densely packed trophoblast cells called cytotrophoblast cell columns in primates and the spongiotrophoblast layer in rodents. Its function may simply be structural to support the underlying villi, although the spongiotrophoblast cells in rodents secrete a number of polypeptide hormones (Soares et al., 1996,1998; Linzer and Fisher, 1999). In addition, the cells may represent a reserve of precursors of the invasive trophoblast population. This idea is based on the observation, made in both human (Damsky et al., 1992,1994) and murine (Carney et al., 1993) systems, that while trophoblast stem cells spontaneously differentiate into their invasive derivatives in vitro, along the way, they progress through a stage typical of the intermediate cell layer.

The differentiation of trophoblast stem cells to either "invasive trophoblast" or syncytiotrophoblast represents the fundamental, alternative cell fate decision in the trophoblast lineage (Figure 1). Along the way to forming these two different cell types, different genetic programs are initiated and include differences in hormone gene expression. One approach to understanding differences in cell-differentiation programs at a genetic level has been to study the transcrip- 


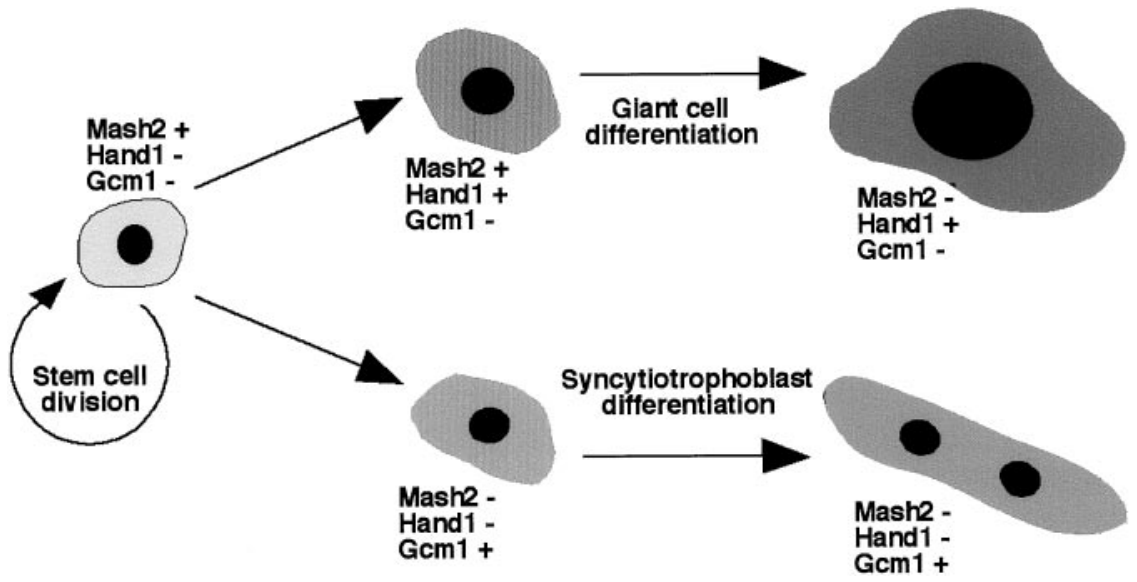

FIG. 1. Summary of the trophoblast cell lineage outlining the alternative differentiated trophoblast cell fates and the expression of key regulatory transcription factors. + indicates that the gene is expressed, whereas - indicates that the gene is not expressed.

tional mechanisms that regulate these trophoblast cell subtype-specific hormones. This approach has been very successful in other systems. An example is Pit-1, which was discovered for its ability to regulate pituitary hormone gene expression and later turned out to be essential for organ development as well (Andersen and Rosenfeld, 2001). Several different hormone genes have been intensively studied. For example, placental lactogen (Pll and $P l 2)$ and several prolactinrelated protein genes are expressed exclusively by the trophoblast giant cell subtype in rodents (Linzer and Fisher, 1999). Their promoters have been intensively characterized in both transfected cells and transgenic mice (Shida et al., 1992,1993; Vuille et al., 1993; Ma et al., 1997; Lin and Linzer, 1998; Sun and Duckworth, 1999; Ma and Linzer, 2000). The Cyp19 gene, which encodes the aromatase enzyme involved in estrogen biosynthesis, is an example of a gene that is exclusively expressed in the syncytiotrophoblast cell subtype (Hinshelwood et al., 1995; Yamada et al., 1995,1999; Kamat et al., 1998,1999). Curiously, while estrogen is produced by syncytiotrophoblast in the human placenta, the mouse placenta does not make estrogen or express Cyp19. However, when transgenic mice are made using the human gene promoter, the transgene is expressed in syncytiotrophoblast cells of the labyrinth (Kamat et al., 1999). This implies that the mouse cells must have the transcription factors that are necessary to regulate the gene appropriately. Why the endogenous mouse Cyp19 gene is not expressed likely reflects differences with the human gene in the gene sequence itself. 
The alternative approach to finding transcription factors necessary for development has been to simply clone new members of transcription factor families that have an "evolutionary history" of regulating cell fate decisions during development. Some of these gene families were first identified in lower organisms such as Drosophila melanogaster. Such families include homeobox and basic helix-loop-helix (bHLH) genes that encode "master" regulators of development. We have taken this latter approach and have, as a result, identified a transcription factor that underlies the development of trophoblast giant cells (Hand1) and another critical for syncytiotrophoblast development $(\mathrm{Gcm} 1)$ in mice.

\section{Molecular Basis of Placental Trophoblast Cell Development}

\section{A. TROPHOBLAST GIANT CELLS}

Considerable genetic evidence indicates that bHLH transcription factors function as cell-lineage determinants in a variety of cell lineages. This was first demonstrated by studies of skeletal muscle development in mammals (MyoD, Myogenin, Mrf4, Myf5), and in mesoderm (twist) and neuronal cell differentiation in Drosophila (achaete-scute) (Olson, 1990; Jan and Jan, 1993; Olson and Klein, 1994). Members of the bHLH family are thought to function as heterodimers, typically between the cell subtype-specific factors and the widely expressed $\mathrm{E}$ proteins, such as E12/E47 (which are products of the E2A gene) (Murre et al., 1991), HEB (Hu et al., 1992), and ITF2 (Henthorn et al., 1990). The ability of cell subtype-specific factors to heterodimerize with $\mathrm{E}$ factors provided a functional means of cloning new members of the family. Cloning of genes based on such functional characteristics was revolutionized 10 years ago with the development of two techniques that relied on protein-protein interaction properties of gene products. Yeast two-hybrid screening relies on interactions of proteins in transformed yeast cells. An alternative method based on phage expression of target proteins, called "interaction cloning," uses a labeled bait protein to screen the library (Blanar and Rutter, 1992). The advantage of the latter is that the approach could be used with existing $\lambda$ gt11 or $\lambda$-based cDNA libraries.

The Handl cDNA was cloned independently by three different groups and was originally given the different names Hxt, eHand, and Thingl (Cross et al., 1995; Cserjesi et al., 1995; Hollenberg et al., 1995). Using an interaction cloning approach with an E47 bait protein, Handl was isolated from a blastocyst stage cDNA library (Cross et al., 1995). Later, it was shown that Handl mRNA is expressed in trophoblast cells of the placenta and also in several structures of the embryo proper (Cross et al., 1995; Cserjesi et al., 1995; Hollenberg et al., 1995). The original blastocyst library was made using sheep blastocysts, yet a mouse homologue was found to be expressed in mouse blastocysts and later the placenta 
(Cross et al., 1995). The choice of library was partly convenience, as a similar mouse library was not available at the time. However, given the differences in placental morphology among mammalian species (Wooding and Flint, 1994), the selection of a bHLH factor that was expressed in both mouse and sheep increased the level of stringency of the screen for factors important for fundamentally conserved aspects of placental development. Most of the subsequent work has concentrated on dissecting the functions of Handl using the mouse as a model system.

The initial evidence that Handl might regulate the differentiation of trophoblast giant cells came from the expression pattern of the gene during early mouse development. At early post-implantation stages, Handl mRNA is not detectable by in situ hybridization in trophoblast stem cells of the chorion layer. It appears to be upregulated in the ectoplacental cone (the precursor to the spongiotrophoblast) and is most highly expressed in the trophoblast giant cell layer surrounding the implanted conceptus (Cross et al., 1995; Scott et al., 2000). This early pattern persists through later stages of development such that Handl mRNA is detectable in spongiotrophoblast and trophoblast giant cells. To test the function of Handl, the gene was overexpressed in Rcho- 1 cells, a rat trophoblast tumor (choriocarcinoma) cell line. It has the interesting property that the dividing cells will spontaneously differentiate into postmitotic cells that eventually take on the morphological, cell-cycle, and gene-expression profile characteristics of giant cells (Faria and Soares, 1991; Hamlin et al., 1994; Cross et al., 1995; MacAuley et al., 1998). Because the rate of giant cell transformation is normally relatively low when the cells are maintained under growth conditions, it affords the opportunity to detect the effect of factors that promote giant cell differentiation. Overexpression of Handl promotes cell proliferation arrest and giant cell differentiation in transfected Rcho-1 cells (Cross et al., 1995). A similar effect has been observed in primary trophoblast stem cell lines (I.C. Scott and J.C. Cross, unpublished data).

The real test of Handl function was the generation of Handl-deficient mice through gene targeting. Embryos that are homozygous for a Handl null mutation do not survive beyond embryonic day (E) 8.5-9.0 and, in terms of their overall size, do not progress beyond E7.5-8.0 (Firulli et al., 1998; Riley et al., 1998). The mutants are recognizable at E8.5 because of their overall reduced size, a failure of the embryo proper to undergo its characteristic turning and blebbing of the yolk sac. In the placenta, the ectoplacental cone is smaller than normal (Riley et al., 1998). The outer layer of trophoblast cells, which should contain trophoblast giant cells, has several defects in the Handl mutants (Figure 2). First, the outer layer has many fewer cells than normal and, as a result, the overall conceptus is within a much smaller sac (Riley et al., 1998; Scott et al., 2000). The number of cells present is not significantly different than the number of trophectoderm cells present at the blastocyst stage. Normally, this number increases as new giant cells differentiate at the edge of the ectoplacental cone. Differences in 


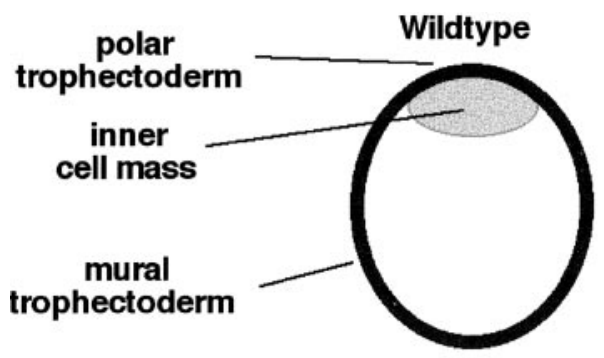

E4.5

Hand1 Mutant
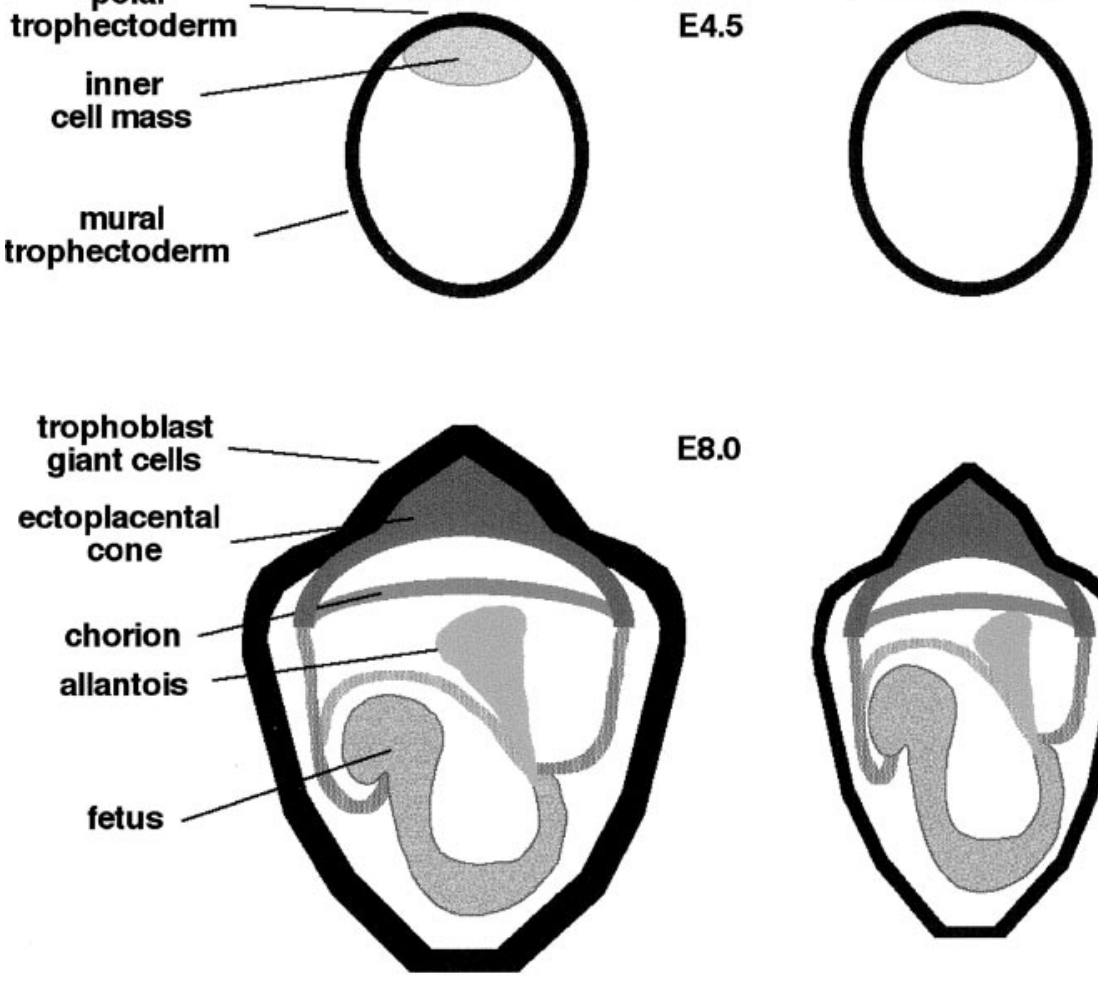

E8.0

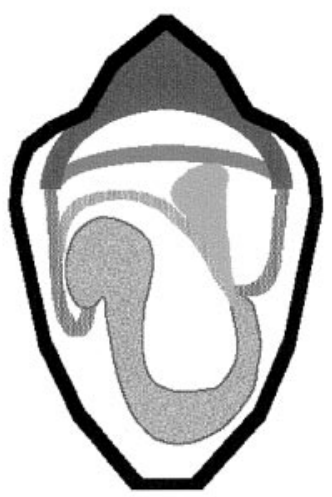

FIG. 2. Summary of the development of Handl null mutant conceptuses at embryonic day (E) 4.5 and 8.0

gene-expression patterns are consistent with the conclusion that this secondary differentiation does not occur (Riley et al., 1998). The second major difference is that the trophoblast cells in the outer layer of the placenta, which are present in the Handl mutants, failed to undergo the characteristic morphological giant transformation (Riley et al., 1998; Scott et al., 2000). Therefore, Handl is essential for trophoblast giant cell differentiation.

While Handl promotes differentiation of trophoblast giant cells, another bHLH gene has the opposite effect. Mash2 is required for the maintenance of giant cell precursors (Guillemot et al., 1994) and its overexpression in Rcho-1 cells prevents giant cell differentiation (Cross et al., 1995; Kraut et al., 1998; Scott et al., 2000). Handl and Mash2 mRNA expression overlaps in the ectoplacental cone and spongiotrophoblast, layers of the placenta that contain giant cell precursors (Scott et al., 2000). This observation implies that the 
opposing activities of Hand 1 and Mash 2 must be coordinated. Handl function is required to turn off Mash 2 mRNA expression coincident with trophoblast giant cell differentiation (Riley et al., 1998). In addition, the Hand1 protein can compete with Mash2 for binding to E proteins (Scott et al., 2000). Because Mash2 must dimerize with an E protein in order to bind DNA and therefore regulate transcription, this competition can titrate away Mash2 function. However, the Handl null mutant phenotype is not solely explained by ectopic activity of Mash2, as the Handl mutant phenotype is not altered by further mutation of Mash2 (Scott et al., 2000). Although the Hand1 protein dimerizes with E proteins in vitro, the latter do not appear to be expressed in trophoblast giant cells (Scott et al., 2000). This suggests that Hand1 likely dimerizes with some other factor(s) in these cells. Candidates include Hand1 itself, since Hand1 can homodimerize at least in vitro (Firulli et al., 2000; Scott et al., 2000). Another bHLH protein encoded by the Stral3 gene is expressed in trophoblast giant cells (Boudjelal et al., 1997) but its ability to interact with Hand1 has not been tested. The complexity of the multiple potential bHLH protein interactions involving Hand1 has been one factor that has slowed the identification of Hand1 DNA recognition site(s) and target genes. One likely scenario is that Hand1 complexed with different partner proteins has different DNA binding specificities.

\section{B. PLACENTAL VILLI AND SYNCYTIOTROPHOBLAST}

While bHLH genes regulate the formation of trophoblast giant cells, no bHLH genes have been discovered that directly regulate the differentiation of syncytiotrophoblast cells or the villous structures of the labyrinth. The Mash2 and Handl genes are expressed focally within the labyrinth layer in mice but neither pattern of expression is obviously localized to syncytiotrophoblast. While we have found other bHLH factors that are expressed in the labyrinth (I.C. Scott, K. Dawson, J.C. Cross, unpublished observations), none have shown syncytiotrophoblast-specific expression.

In looking for other transcription factors that control cell-fate decisions, we became interested in the Gcm (for glial cells missing) gene family. In Drosophila, Gcm mutants show a cell fate change in the nervous system such that precursor cells that normally divide to give rise to neural and glial derivatives instead only give rise to neurons (Wegner and Riethmacher, 2001). The gene name is slightly misleading because the mutants also show defects in hemocyte development, implying that $\mathrm{Gcm}$ regulates cell fate in general rather than specifies glial cell fate. The first reports describing attempts to identify mammalian homologues of $\mathrm{Gcm}$ revealed two genes in both mice and humans that were called $G \mathrm{cml} / \mathrm{Gcm}-a$ and $G \mathrm{~cm} 2 / G \mathrm{~cm}-b$ (Akiyama et al., 1996; Altshuller et al., 1996; Kim et al., 1998). The initial published report indicating that $\mathrm{Gcml} \mathrm{mRNA}$ was only detected in the placenta, based on northern blotting (Altshuller et al., 1996), prompted further investigation. It soon was discov- 
ered that expression of $\mathrm{Gcml}$ in the mouse placenta is confined to the labyrinth layer (Kim et al., 1998; Basyuk et al., 1999), including syncytiotrophoblast cells (Basyuk et al., 1999). Expression initiates at the time that the labyrinth first begins to form at E8.5 and persists as long as the labyrinth grows through repeated branching morphogenesis (Basyuk et al., 1999).

Detailed mRNA expression analysis has shown that $\mathrm{Gcml}$ gene expression is initiated around E8.0-8.5 in small clusters of cells within the chorion layer that otherwise contains trophoblast stem cells (Anson-Cartwright et al., 2000). These $\mathrm{Gcml}$ expressing cells are found at sites where the chorionic plate folds to form branches, regions where syncytiotrophoblast differentiation also begins. Both the initiation of chorioallantoic branching (Figure 3) and the formation of syncytiotrophoblast are blocked completely when Gcml function is ablated (Anson-Cartwright et al., 2000). As a result, the labyrinth layer of the placenta does not form in Gcml null mutants and the chorion layer persists (AnsonCartwright et al., 2000; Schreiber et al., 2000). Without the labyrinth, the embryos die around E10. Given that Gcm in the Drosophila nervous system

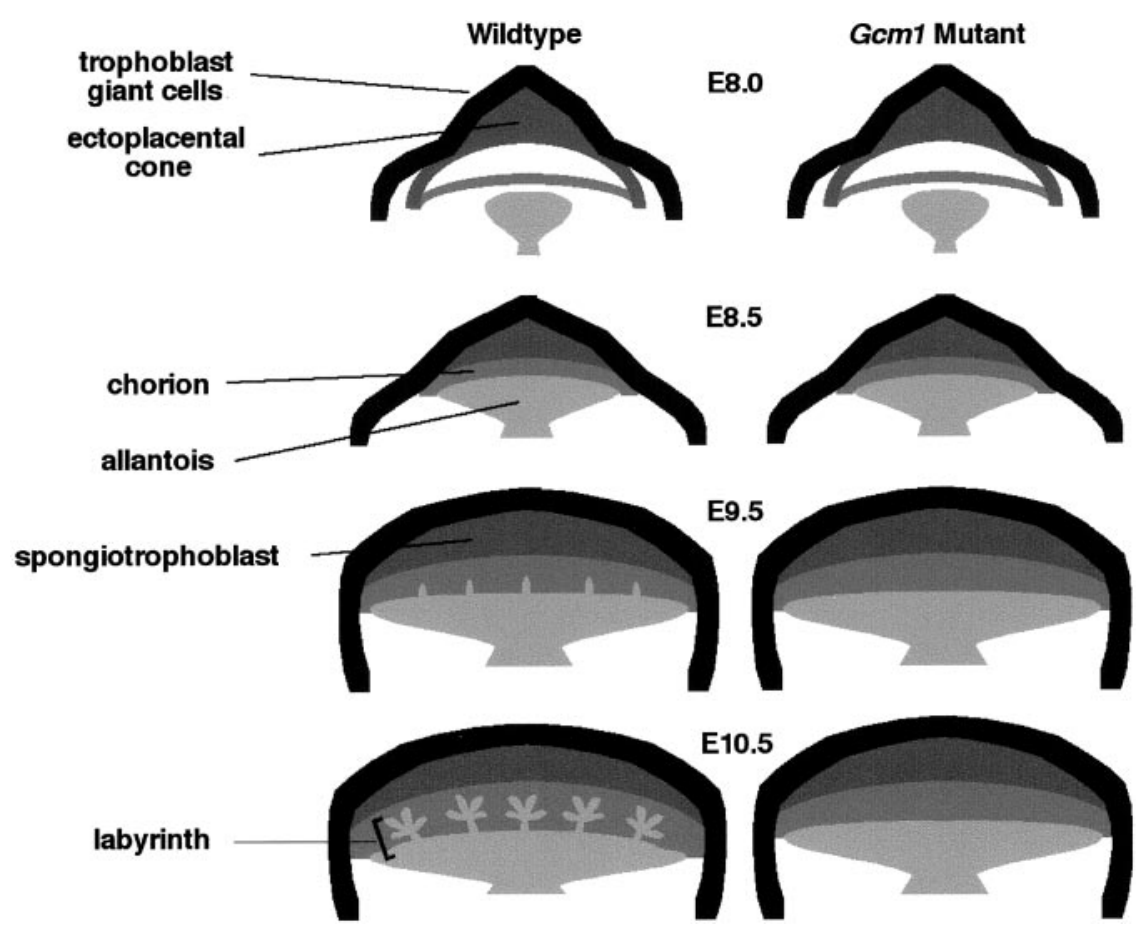

FIG. 3. Summary of the development of $G \mathrm{~cm} 1$ null mutant conceptuses between embryonic day (E) 8.0 and 10.5 . 
regulates a cell-fate change, it is interesting to consider what happens in the Gcml-deficient mice and whether the phenotype can be interpreted in a similar way. Because the chorionic trophoblast cell lineage has not been described in detail, this is a matter for speculation only. However, a reasonable hypothesis is that $G \mathrm{cml}$ is required for trophoblast cells to "choose" a syncytiotrophoblast fate rather than remain as stem cells.

\section{Transcription Factors Controlling Development Also Regulate Placental Hormone Production}

Both Hand1 and Gcm1 were discovered as orphan transcription factors in the sense that, although their DNA-binding specificities were learned early on, their biologically important target genes remain largely unknown. It is safe to say that this is true for the vast majority of transcription factors that have been described. The criteria that prove that a transcription factor is an important and direct regulator of a gene are 1) the transcription regulatory regions of the gene should contain binding sites for the factor, 2) selective mutation of the binding site should alter gene expression, and 3) gene expression should be similarly reduced in mutants lacking the transcription factor. Trophoblast subtype-specific hormone genes fulfill some of these criteria and suggest them as target genes of Hand1 and Gcm1.

The mouse $P l l$ gene (encoding the prolactin-like hormone, placental lactogen I) is specifically expressed in trophoblast giant cells (Faria et al., 1991). The expression of $P l l$ mRNA is dramatically reduced in Handl mutants, indicating that $P l l$ is genetically downstream of Handl (Firulli et al., 1998; Riley et al., 1998). Because Handl mutants show other abnormalities in giant cell differentiation, the reduced expression in the mutants could be indirect. However, the mouse Pll promoter contains a variant E-box-like element (Figure 4) that conforms to the consensus site to which Hand1/E47 dimers can bind (Hollenberg et al., 1995). Although a point mutation of this site has not been tested, deletion of a 86-bp region of the promoter (between -274 and -188 relative to the transcription start site) that encompasses this element results in a reduction in overall promoter activity in transfected Rcho-1 trophoblast giant cells (Figure 4) (Shida et al., 1993). Co-transfection of Handl expression vector with a Pll promoter/reporter gene construct results in dose-dependent transactivation (Figure 4) (Cross et al., 1995; Scott et al., 2000). Deletion of the promoter between -274 and -188 prevents transactivation by Hand1 (Cross et al., 1995). These data suggest that Hand 1 likely regulates $P l l$ gene promoter activity directly. It is important to note, however, that Hand1 must not provide cell-type specificity to $P l l$ transcription on its own because the Handl gene is expressed in other cells and tissues where Pll is not. For example, in the trophoblast lineage, Handl is expressed in the ectoplacental cone/spongiotrophoblast (Cross et al., 1995; Scott et al., 2000). It is likely that Hand 1 interactions with other trophoblast subtype- 


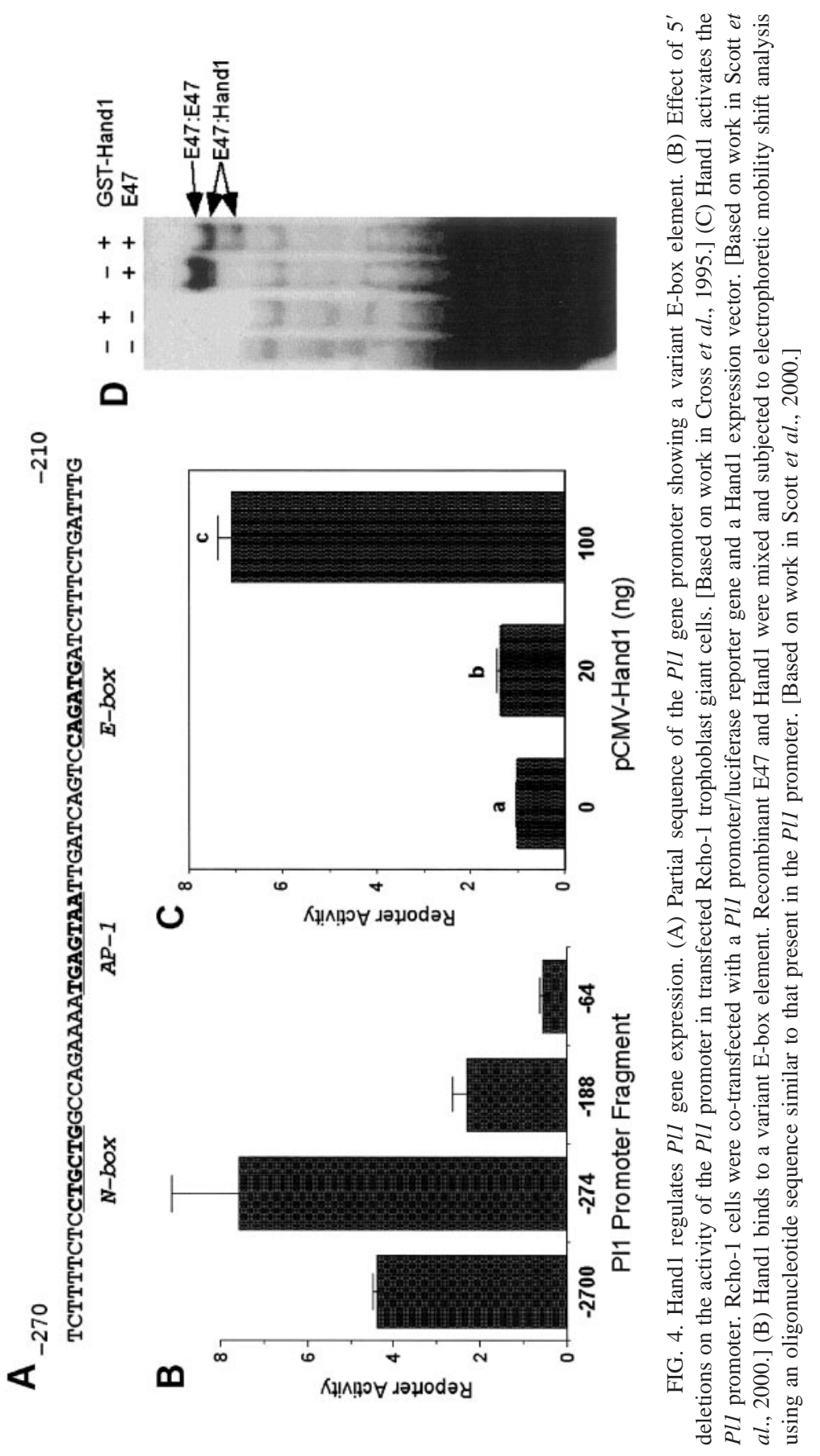


specific transcription factors together provide the specificity to $P l l$ gene expression. These other factors could include Gata factors (Ng et al., 1994) and/or the bHLH factor Stra13 (Boudjelal et al., 1997).

In contrast to the directed studies aimed at making a connection between Hand1 and Pll transcription, the $\mathrm{Gcm} 1$ transcription factor has been associated with regulation of the Cyp19 gene but by a more indirect route. In characterizing the Cyp19 promoter by conventional molecular and biochemical means, Yamada and colleagues localized a critical regulatory element to a short region of DNA designated TSE2 (Yamada et al., 1999). The TSE2 element was used in a one-hybrid screen in which DNA sequences are employed as the bait to identify transcription factors able to bind it. This screen identified a single cDNA clone from a human placental cDNA library that turned out to encode GCM1. In retrospect, the critical regulatory element fit the known consensus for a Gcm1/GCM1-binding element (Schreiber et al., 1997,1998). Yamada also showed that a similar DNA sequence was present in the placental enhancer of the leptin gene (Yamada et al., 1999). What is obviously missing from the analysis is proof that Gcm1/GCM1 is critical for transcriptional regulation of Cyp19 and Leptin but the data thus far appear quite compelling.

\section{Conclusions}

How do we interpret the observations that, while the Hand1 and $\mathrm{Gcm} 1$ transcription factors are critical for development of distinct trophoblast cell subtypes, they also appear to regulate cell subtype-specific genes? In the case of the hormone genes implicated to date, it seems unlikely that their function underlies the developmental roles of their transcriptional regulators. A more likely explanation is simply that what has been observed in other systems holds true in trophoblast cells as well: transcription factors controlling cellular development have ongoing effects in the same cells to regulate the transcription of genes whose function is peculiar to those cells. Not so many years ago, it was argued that studying the promoters of cell type-specific genes, as a means of getting insights into transcription factors that control cell fate, would be an endless road. As regulators would be identified, the search would turn to "the regulators of the regulators," and so on. If the Pll and Cyp19 genes are any indication, however, the procession from key transcription factor to cell type-specific gene may be much less complex and, indeed, can occur in a single step without need for a cascade of gene-induction events. With the discovery that the human genome may contain many fewer genes than originally thought, in retrospect, it may make sense that regulatory networks would involve fewer steps.

\section{ACKNOWLEDGMENTS}

Supported by operating grants from the Canadian Institutes of Health Research (CIHR). J.C.C. is a CIHR Investigator and a Senior Scholar of the Alberta Heritage Foundation for Medical Research. 


\section{REFERENCES}

Akiyama Y, Hosoya T, Poole AM, Hotta Y 1996 The Gcm-motif: a novel DNA-binding motif conserved in Drosophila and mammals. Proc Natl Acad Sci USA 93:14912-14916

Altshuller Y, Copeland NG, Gilbert DJ, Jenkins NA, Frohman MA $1996 \mathrm{Gcml}$, a mammalian homolog of Drosophila Glial Cells Missing. FEBS Lett 393:201-204

Andersen B, Rosenfeld MG 2001 POU domain factors in the neuroendocrine system: lessons from developmental biology provide insights into human disease. Endocr Rev 22:2-35.

Anson-Cartwright L, Dawson K, Holmyard D, Fisher SJ, Lazzarini RA, Cross JC 2000 The glial cells missing-1 protein is essential for branching morphogenesis in the chorioallantoic placenta. Nature Genet 25:311-314

Basyuk E, Cross JC, Corbin J, Nakayama H, Hunter PJ, Nait-Oumesmar B, Lazzarini RA 1999 The murine $\mathrm{Gcm} 1$ gene is expressed in a subset of placental trophoblast cells. Dev Dynam 214:303-311

Blanar MA, Rutter WJ 1992 Interaction cloning: identification of a helix-loop-helix zipper protein that interacts with c-Fos. Science 256:1014-1018

Boudjelal M, Taneja R, Matsubara S, Bouillet P, Dolle P, Chambon P 1997 Overexpression of Stra13, a novel retinoic acid-inducible gene of the basic helix-loop-helix family, inhibits mesodermal and promotes neuronal differentiation of P19 cells. Genes Dev 11:2052-2065

Carney EW, Prideaux V, Lye SJ, Rossant J 1993 Progressive expression of trophoblast-specific genes during formation of mouse trophoblast giant cells in vitro. Mol Reprod Dev 34:357-368

Cross JC 1996 Trophoblast function in normal and preeclamptic pregnancy. Fetal Matern Med Rev 8:57-66

Cross JC 1999 Molecular regulation of placental development. In: Baker PN, Kingdom JCP, eds. Intrauterine Growth Restriction. London: Springer-Verlag; 387-400

Cross JC 2000 Genetic insights into trophoblast differentiation and placental morphogenesis. Semin Cell Dev Biol 11:105-113

Cross JC, Werb Z, Fisher SJ 1994 Implantation and the placenta: key pieces of the development puzzle. Science 266:1508-1518

Cross JC, Flannery ML, Blanar MA, Steingrimsson E, Jenkins NA, Copeland NG, Rutter WJ, Werb Z 1995 Hxt encodes a basic helix-loop-helix transcription factor that regulates trophoblast cell development. Development 121:2513-2523

Cross JC, Hemberger M, Lu Y, Nozaki T, Whiteley K, Masutani M, Adamson SL 2002 Trophoblast functions, angiogenesis and remodeling of the maternal vasculature in the placenta. Mol Cell Endocrinol, in press

Cserjesi P, Brown D, Lyons GE, Olson EN 1995 Expression of the novel basic helix-loop-helix gene $e H A N D$ in neural crest derivatives and extraembryonic membranes during mouse development. Dev Biol 170:664-678

Damsky CH, Fitzgerald ML, Fisher SJ 1992 Distribution patterns of extracellular matrix components and adhesion receptors are intricately modulated during first trimester cytotrophoblast differentiation along the invasive pathway in vivo. J Clin Invest 89:210-222

Damsky CH, Librach C, Lim KH, Fitzgerald ML, McMaster MT, Janatpour M, Zhou Y, Logan SK, Fisher SJ 1994 Integrin switching regulates normal trophoblast invasion. Development 120:3657-3666

Faria TN, Soares MJ 1991 Trophoblast cell differentiation: establishment, characterization, and modulation of a rat trophoblast cell line expressing members of the placental prolactin family. Endocrinology 129:2895-2906

Faria TN, Ogren L, Talamantes F, Linzer DIH, Soares MJ 1991 Localization of placental lactogen-I in trophoblast giant cells of the mouse placenta. Biol Reprod 44:327-331 
Firulli AB, McFadden DG, Lin Q, Srivastava D, Olson EN 1998 Heart and extra-embryonic mesodermal defects in mouse embryos lacking the bHLH transcription factor Hand1. Nature Genet 18:266-270

Firulli BA, Hadzic DB, McDaid JR, Firulli AB 2000 The basic helix-loop-helix transcription factors dHAND and eHAND exhibit dimerization characteristics that suggest complex regulation of function. J Biol Chem 275:33567-33573

Guillemot F, Nagy A, Auerbach A, Rossant J, Joyner AL 1994 Essential role of Mash-2 in extraembryonic development. Nature 371:333-336

Hamlin GP, Lu XJ, Roby KF, Soares MJ 1994 Recapitulation of the pathway for trophoblast giant cell differentiation in vitro: stage-specific expression of members of the prolactin gene family. Endocrinology 134:2390-2396

Hemberger M, Cross JC 2001 Genes governing placental development. Trends Endocrinol Metab 12:162-168

Henthorn P, Kiledjian M, Kadesch T 1990 Two distinct transcription factors that bind the immunoglobulin enhancer uE5/kE2 motif. Science 247:467-470

Hinshelwood MM, Liu Z, Conley AJ, Simpson ER 1995 Demonstration of tissue-specific promoters in nonprimate species that express aromatase P450 in placentae. Biol Reprod 53:1151-1159

Hollenberg SM, Sternglanz R, Cheng PF, Weintraub H 1995 Identification of a new family of tissue-specific basic helix-loop- helix proteins with a two-hybrid system. Mol Cell Biol 15:3813-3822

Hu JS, Olson EN, Kingston RE 1992 HEB, a helix-loop-helix protein related to E2A and ITF2 that can modulate the DNA-binding ability of myogenic regulatory factors. Mol Cell Biol 12:1031-1042

Jan YN, Jan LY 1993 Functional gene cassettes in development. Proc Natl Acad Sci USA 90:8305-8307

Kamat A, Alcorn JL, Kunczt C, Mendelson CR 1998 Characterization of the regulatory regions of the human aromatase (P450arom) gene involved in placenta-specific expression. Mol Endocrinol 12:1764-1777

Kamat A, Graves KH, Smith ME, Richardson JA, Mendelson CR 1999 A 500-bp region, approximately $40 \mathrm{~kb}$ upstream of the human CYP19 (aromatase) gene, mediates placentaspecific expression in transgenic mice. Proc Natl Acad Sci USA 96:4575-4580

Kim J, Jones BW, Zock C, Chen Z, Wang H, Goodman CS, Anderson DJ 1998 Isolation and characterization of mammalian homologs of the Drosophila gene glial cells missing. Proc Natl Acad Sci USA 95:12364-12369

Kingdom JCP, Kaufmann P 1997 Oxygen and placental villous development: origins of fetal hypoxia. Placenta 18:613-621

Kraut N, Snider L, Chen C, Tapscott SJ, Groudine M 1998 Requirement of the mouse I-mfa gene for placental development and skeletal patterning. EMBO J 17:6276-6288

Lin J, Linzer DI 1998 Identification of trophoblast-specific regulatory elements in the mouse placental lactogen II gene. Mol Endocrinol 12:418-427

Linzer DI, Fisher SJ 1999 The placenta and the prolactin family of hormones: regulation of the physiology of pregnancy. Mol Endocrinol 13:837-840

Ma GT, Roth ME, Groskopf JC, Tsai FY, Orkin SH, Grosveld F, Engel JD, Linzer DI 1997 GATA-2 and GATA-3 regulate trophoblast-specific gene expression in vivo. Development 124:907-914

Ma GT, Linzer DI 2000 GATA-2 restricts prolactin-like protein A expression to secondary trophoblast giant cells in the mouse. Biol Reprod 63:570-574

MacAuley A, Cross JC, Werb Z 1998 Reprogramming the cell cycle for endoreduplication in rodent trophoblast cells. Mol Biol Cell 9:795-807 
Murre C, Voronova A, Baltimore D 1991 B-cell- and myocyte-specific E2-box-binding factors contain E12/E47-like subunits. Mol Cell Biol 11:1156-1160

Ng YK, George KM, Engel JD, Linzer DI 1994 GATA factor activity is required for the trophoblast-specific transcriptional regulation of the mouse placental lactogen I gene. Development 120:3257-3266

Olson EN 1990 MyoD family: a paradigm for development. Genes Dev 4:1454-1461

Olson EN, Klein WH 1994 bHLH factors in muscle development: dead lines and commitments, what to leave in and what to leave out. Genes Dev 8:1-8

Riley P, Anson-Cartwright L, Cross JC 1998 The Hand1 bHLH transcription factor is essential for placentation and cardiac morphogenesis. Nature Genet 18:271-275

Schreiber J, Sock E, Wegner M 1997 The regulator of early gliogenesis glial cells missing is a transcription factor with a novel type of DNA-binding domain. Proc Natl Acad Sci USA 94:4739-4744

Schreiber J, Enderich J, Wegner M 1998 Structural requirements for DNA binding of GCM proteins. Nucleic Acids Res 26:2337-2343

Schreiber J, Riethmacher-Sonnenberg E, Riethmacher D, Tuerk EE, Enderich J, Bosl MR, Wegner M 2000 Placental failure in mice lacking the mammalian homolog of glial cells missing, GCMa. Mol Cell Biol 20:2466-2474

Scott IC, Anson-Cartwright L, Riley P, Reda D, Cross JC 2000 The Hand1 basic helix-loop-helix transcription factor regulates trophoblast giant cell differentiation via multiple mechanisms. Mol Cell Biol 20:530-541

Shida MM, Jackson-Grusby LL, Ross SR, Linzer DI 1992 Placental-specific expression from the mouse placental lactogen II gene promoter. Proc Natl Acad Sci USA 89:3864-3868

Shida MM, Ng YK, Soares MJ, Linzer DI 1993 Trophoblast-specific transcription from the mouse placental lactogen-I gene promoter. Mol Endocrinol 7:181-188

Soares MJ, Chapman BM, Rasmussen CA, Dai G, Kamei T, Orwig KE 1996 Differentiation of trophoblast endocrine cells. Placenta 17:277-289

Soares MJ, Muller H, Orwig KE, Peters TJ, Dai G 1998 The uteroplacental prolactin family and pregnancy. Biol Reprod 58:273-284

Sun Y, Duckworth ML 1999 Identification of a placental-specific enhancer in the rat placental lactogen II gene that contains binding sites for members of the Ets and AP-1 (activator protein 1) families of transcription factors. Mol Endocrinol 13:385-399

Vuille J-C, Cattini PA, Bock ME, Verstuyf A, Schroedter IC, Duckworth ML, Friesen HG 1993 Rat prolactin-like protein A partial gene and promoter structure: promoter activity in placental and pituitary cells. Mol Cell Endocrinol 96:91-98

Wegner M, Riethmacher D 2001 Chronicles of a switch hunt: gcm genes in development. Trends Genet 17:286-290

Wooding FBP, Flint APF 1994 Placentation. In: Lamming GE, ed. Marshall's Physiology of Reproduction, 4th edit. New York: Chapman and Hall; 233-460

Yamada K, Harada N, Honda S, Takagi Y 1995 Regulation of placenta-specific expression of the aromatase cytochrome P-450 gene. Involvement of the trophoblast-specific element binding protein. J Biol Chem 270:25064-25069

Yamada K, Ogawa H, Honda S, Harada N, Okazaki T 1999 A GCM motif protein is involved in placenta-specific expression of human aromatase gene. J Biol Chem 274:32279-32286 


\title{
Neuroendocrine Modulation and Repercussions of Female Reproductive Aging
}

\author{
Phyllis M. Wise, Matthew J. Smith, Dena B. Dubal, Melinda E. Wilson, \\ Shane W. Rau, Adrienne B. Cashion, Martina Böttner, \\ AND KATHERINE L. Rosewell \\ Department of Physiology, University of Kentucky College of Medicine, \\ Lexington, Kentucky 40536-0298
}

\begin{abstract}
The menopause marks the end of a woman's reproductive life. During the postmenopausal period, plasma estrogen concentrations decrease dramatically and remain low for the rest of her life, unless she chooses to take hormone replacement therapy. During the past 20 years, we have learned that changes in the central nervous system are associated with and may influence the timing of the menopause in women. Recently, it has become clear that estrogens act on more than just the hypothalamus, pituitary, ovary, and other reproductive organs. In fact, they play roles in a wide variety of nonreproductive functions. With the increasing life span of humans from approximately 50 to 80 years and the relatively fixed age of the menopause, a larger number of women will spend over one third of their lives in the postmenopausal state. It is not surprising that interest has increased in factors that govern the timing of the menopause and the repercussions of the lack of estrogen on multiple aspects of women's health. We have used animal models to better understand the complex interactions between the ovary and the brain that lead to the menopause and the repercussions of the hypoestrogenic state. Our results show that when rats reach middle age, the patterns and synchrony of multiple neurochemical events that are critical to the preovulatory gonadotropin-releasing hormone $(\mathrm{GnRH})$ surge undergo subtle changes. The precision of rhythmic pattern of neurotransmitter dynamics depends on the presence of estradiol. Responsiveness to this hormone decreases in middle-aged rats. The lack of precision in the coordination in the output of neural signals leads to a delay and attenuation of the luteinizing hormone surge, which lead to irregular estrous cyclicity and, ultimately, to the cessation of reproductive cycles. We also have examined the impact of the lack of estrogen on the vulnerability of the brain to injury. Our work establishes that the absence of estradiol increases the extent of cell death after stroke-like injury and that treatment with low physiological levels of estradiol are profoundly neuroprotective. We have begun to explore the cellular and molecular mechanisms that underlie this novel nonreproductive action of estrogens. In summary, our studies show that age-related changes in the ability of estradiol to coordinate the neuroendocrine events that lead to regular preovulatory GnRH surges contribute to the onset of irregular estrous cycles and eventually to acyclicity. Furthermore, we have shown that the lack of estradiol increases the vulnerability of the brain to injury and neurodegeneration.
\end{abstract}

\section{Introduction}

Women undergo the menopause at approximately 51 years of age. The timing of this dramatic physiological change has remained essentially constant 
since records have been kept. The menopause occurs at the time of the exhaustion of the ovarian follicular reserve. Since the ovarian follicles are not only the source of germ cells but also are the primary source of estrogens, plasma estrogen concentrations drop precipitously during the postmenopausal years and remain low for the remainder of a woman's life, unless she chooses to take hormone replacement therapy (HRT). Thus, the end of the reproductive life has farreaching implications for women because they become permanently hypoestrogenic at this time. In recent years, we have come to appreciate that estrogens are not only reproductive hormones but also pleiotropic hormones that play roles in a wide variety of nonreproductive functions as disparate as bone and mineral metabolism (Lindsay, 1996; Manolagas, 2000; Compston, 2001), memory and cognition (Fillit, 1994; Erkkola, 1996; Sherwin, 1996,1999), cardiovascular function (Wild, 1996; Matthews et al., 2000; Mendelsohn, 2000; Stevenson, 2000; Losordo and Isner, 2001), and the immune system (Jansson and Holmdahl, 1998; Ahmed and Hissong, 1999). With the substantial increase in the average life span of humans from approximately 50 to 80 years that occurred during the last century, and the relatively fixed age of the menopause, the number of women who will spend over one third of their lives in the postmenopausal state has increased dramatically. It is not surprising, then, that an increasing number of clinical and basic science researchers have focused their efforts on understanding reproductive aging, since it has become increasingly important to understand the factors that drive the menopausal transition. A number of excellent reviews that discuss the role of the brain in female reproductive aging have appeared during the past 10 years (Lu et al., 1994; Wise et al., 1997; Rubin, 2000; Lapolt and Lu, 2001). This review focuses primarily on our work; however, we have benefited immensely from the studies of our colleagues and will cite them in appropriate places. It should be noted that a better understanding of the mechanisms regulating female reproductive aging will be important to gerontologists because the female reproductive system undergoes senescence relatively early during the aging process, in the absence of pathological changes that often confound gerontological studies. Therefore, we hope that concepts derived from our understanding of the aging reproductive system may shed light on the process of the biology of aging of other systems (Kirkwood, 1998).

\section{The Central Nervous System Plays an Important Role in Female Reproductive Aging}

Our studies have focused on the influence of changes in the central nervous system (CNS) on female reproductive aging and the repercussions of hypoestrogenicity on the brain. For many years, it was accepted that reproductive aging and, in particular, the menopause in women - resulted simply from the depletion of the postmitotic pool of ovarian follicles that is set down during embryonic 
development (vom Saal et al., 1994). It was thought that CNS changes that accompany the menopause were merely a consequence of declining ovarian function. More recently, investigators have realized that the brain plays an important role in the sequence of events leading to reproductive senescence. It appears that the temporal patterns of neural signals undergo subtle but important changes during middle age in both women and animal models, before the cessation of reproductive cycles, and that these changes may accelerate the loss of follicles leading to the menopause.

These conclusions are based predominantly upon studies performed in rodent models. Therefore, one must ask whether experimental results using animal models of reproductive senescence will improve our understanding of the human menopause. This remains controversial, with advocates on both sides of this lively discussion. On the one hand, since rodents do not undergo a real menstrual cycle, by definition, they do not undergo a true 'meno-pause.' Arguments that rodents are not good models of the human menopause are based on two important observations. First, in postmenopausal women, plasma gonadotropin levels are high; whereas in aged acyclic, repeatedly pseudopregnant rats, they remain relatively normal, despite substantial decreases in estradiol in both species (for a review, see Wise, 2000). This suggests that decreased estrogen secretion is paramount to the postmenopausal state and hypothalamic influences may not be as critical in the human female. In contrast, hypothalamic influences are paramount to the postreproductive state in rodents: ovaries of young rodents transplanted into old hosts fail to cycle normally, whereas ovaries of old rodents transplanted into young hosts respond to neuroendocrine signals and reproductive cyclicity is restored. However, the regularity of the cycle is not totally restored (Peng and Huang, 1972; Felicio et al., 1983). Second, it has been suggested that the temporal dynamics of loss of the ovarian follicular reserve are fundamentally different in women, compared to rodents. However, we would contend that thorough study and careful analysis of the rate of loss of primordial follicles have not been performed in rodents the way they have been in humans (Faddy et al., 1983; Richardson et al., 1987).

Despite these differences between older postmenopausal women and older acyclic rats, striking parallels exists between middle-aged female rats and preand perimenopausal women. First, the sentinel event that heralds impending reproductive decline in both humans and rats is a rise in follicle-stimulating hormone (FSH) concentrations (DePaolo, 1987; Klein et al., 1996). In humans, the change is prominent during the periovulatory phase of the menstrual cycle. In a similar manner, middle-aged rats exhibit elevated FSH levels during estrous afternoon. Second, the pattern of luteinizing hormone (LH) secretion changes in both perimenopausal women and middle-aged rats as they enter the transition to acyclicity. Controversy exists as to the changes in pulsatile LH release in pre- and perimenopausal women. A report by Matt et al. (1998) shows that, in regularly 
cycling, middle-aged women, the duration of LH pulses increases and the frequency of pulses decreases. To our knowledge, the work of Matt and colleagues is the only study performed in regularly cycling, premenopausal women. None of the other studies in humans have controlled for changing cycle length during the perimenopausal period when they monitored LH pulses. This may underlie the discrepancy among studies in humans. These results mirror the changes that we observed in middle-aged, regularly cycling rats (Scarbrough and Wise, 1990). Third, menstrual cycle length in women and estrous cycle length in rats become highly variable (Sherman et al., 1976; Fitzgerald et al., 1994). Cycles of increased and decreased length have been reported in women between the ages of 37-45 years, as they enter the perimenopausal transition. Likewise, rats exhibit highly variable estrous cycles, with prolonged periods of estrus or diestrus between each preovulatory LH surge. Fourth, although the postmenopausal period is characterized by extremely low levels of estradiol, several studies (Klein et al., 1996; Santoro et al., 1996) have shown that estradiol concentrations do not decrease during the pre- and perimenopausal period but, in fact, remain normal or are elevated. These new findings are strikingly similar to what has been observed in middle-aged rats as they enter the transition to irregular cyclicity (Butcher and Page, 1981; Lu, 1983). Finally, the ability of estradiol to induce LH surges is attenuated in both perimenopausal women and middle-aged rats. Van Look and colleagues (1977) showed that estradiol was able to induce LH surges of attenuated amplitude in only a small portion of the women studied. This parallels precisely the changes that we observed in middleaged rats (Wise, 1984). For all these reasons, we believe that rodents serve as excellent models in which to examine the factors that initiate the process of reproductive aging during middle age. We assume that information gained from these species can be extrapolated to humans and will allow us to uncover and explore concepts that can be generalized to human reproductive aging.

\section{Changes in the Pattern of Gonadotropin Secretion Reflect Changes in Hypothalamic GnRH Input}

We (Wise, 1982a) and other investigators (van der Schoot, 1976; Lu, 1983; Nass et al., 1984) established that one of the earliest changes that occurs during middle age is in the secretion pattern of the preovulatory LH and FSH surge. In rats that had not exhibited any change in estrous cycle length and still maintained normal, regular, 4-day estrous cycles, we observed a consistent delay in the onset of the LH surge and attenuation in peak concentrations (Figure 1). These changes occur at an age when we could not detect any changes in the responsiveness of the pituitary gland to GnRH. Therefore, we asked: do GnRH neurons change during the middle-aged period, leading to alterations in the ability to drive LH secretion? Methods to directly quantitate changes in the secretion pattern of 


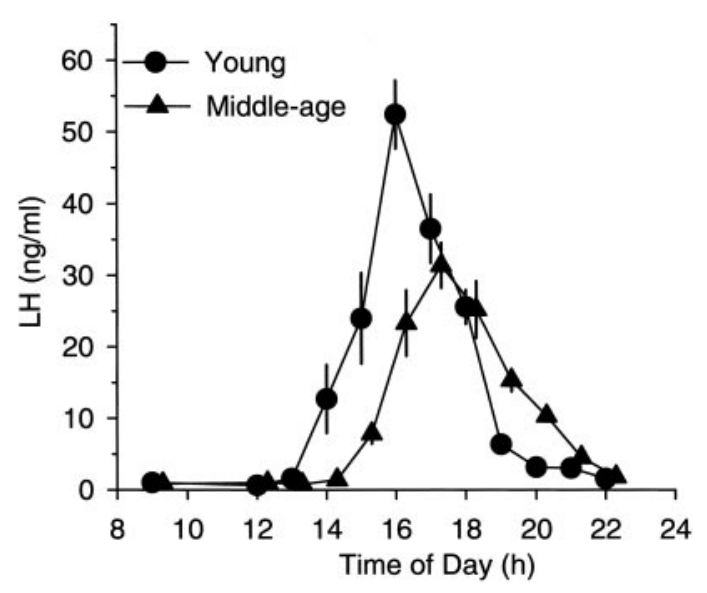

FIG. 1. The luteinizing hormone (LH) surge is blunted and delayed in middle-aged, compared to young, rats. Young and middle-aged regularly cycling rats were sequentially bled from right atrial cannulae during the day of proestrus. Plasma was radioimmunoassayed for LH. The first significant increase in LH was delayed by 1 hour and attenuated significantly in middle-aged rats.

GnRH have been problematic, since the hormone is not detectable in peripheral blood, GnRH neurons are few in number, and are distributed diffusely in the anterior preoptic region of the hypothalamus. Instead, investigators have used immunocytochemical methods to determine the number of GnRH neurons and the percentage that are activated. Expression of immediate early genes (e.g., Fos) by individual neurons has been used as a marker of increased neuronal activity (Hoffman et al., 1993). Therefore, we used this method to test whether alterations in the timing and amplitude of the proestrous LH surge involve alterations in the activation of GnRH neurons. In these studies, we have never detected any age-related change in the number of GnRH neurons (Lloyd et al., 1994; Krajnak et al., 2001). However, the number of GnRH neurons that express Fos during the proestrous LH surge decreased dramatically in middle-aged, regularly cycling rats (Figure 2). These results have been confirmed and extended using threedimensional reconstructions of the forebrain populations of $\mathrm{GnRH}$ neurons in young and middle-aged rats (Rubin et al., 1995). Our studies strongly suggest that the pattern of afferent input to GnRH neurons changes during the early stages of reproductive aging. It should be noted that additional changes in GnRH activity have been reported by investigators who have utilized rats that have reached more advanced stages of reproductive senescence, particularly during times of increased demand on the population of GnRH neurons. These changes include decreased in vivo output of GnRH in conjunction with the steroidinduced LH surge using push-pull perfusion (Rubin and Bridges, 1989) and decreased LH pulse frequency and amplitude in ovariectomized rats (Scarbrough 


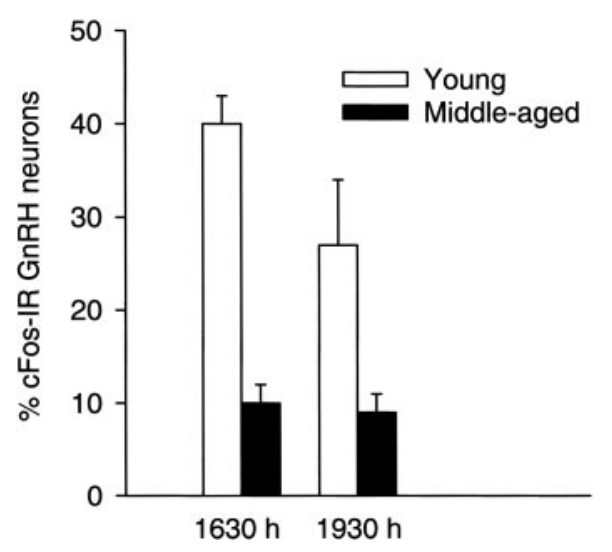

FIG. 2. Percent of gonadotropin-releasing hormone (GnRH) neurons that express Fos during the proestrous LH surge decreases with age. Young and middle-aged rats regularly cycling rats were perfused with paraformaldehyde and their brains sectioned for dual immunocytochemical localization of GnRH and Fos. Age significantly decreased the level of activation of GnRH neurons. [Reprinted with permission from Lloyd JM, Hoffman GE, Wise PM 1994 Decline in immediate early gene expression in gonadotropin-releasing hormone neurons during proestrus in regularly cycling, middleaged rats. Endocrinology 134:1800-1805. Copyright The Endocrine Society.]

and Wise, 1990). In addition, investigators have observed a decline in pituitary GnRH receptor mRNA (Rubin and Jimenez-Linan, 1999) and binding (Marchetti and Cioni, 1988), which are regulated by the pattern of pulsatile GnRH secretion. These age-related receptor changes are likely to lead to decreased responsiveness to GnRH (Smith et al., 1982; Hogg et al., 1992; Brito et al., 1994).

\section{Changes in the Temporal Pattern and Synchrony of Neurotransmitter Input May Alter the Pattern of GnRH Secretion}

The pattern of preovulatory GnRH secretion is determined by afferent input from multiple neurotransmitters and neuropeptides. Over the past 50 years, researchers have come to recognize the complexity of the signaling system that ultimately leads to the GnRH surge. We continue to add to the list of neurotransmitters and neuropeptides that modulate the pattern of GnRH secretion. The hierarchy of modulators is still unclear. Indeed, it is not clear whether a single neurotransmitter is essential or whether there is plasticity in the repertoire of neurotransmitters that can participate or substitute for one another in the generation of the GnRH surge. Data favor the latter possibility: there is remarkable redundancy and plasticity in the ensemble of factors that influences GnRH secretion. Together, they insure the maintenance or reappearance of LH surges when one of the players is disrupted. Thus, Clifton and Sawyer (1979) found that 
disruption of the catecholaminergic input to GnRH neurons halts estrous cyclicity but only temporarily, suggesting that, when necessary, other inputs are able to replace the important role that catecholamines normally play in $\mathrm{GnRH}$ secretion. It is clear that the synchrony, timing, and interplay among the multiple neural signals are required to insure that the proper timing and amplitude of preovulatory LH surges is maintained.

In an elegant and seminal series of studies beginning over 50 years ago, Everett and Sawyer (Everett et al., 1949; Sawyer et al., 1949; Everett and Sawyer, 1950,1953) showed that if the neural signals that regulate the LH surge are delayed by even 2 hours, the surge is delayed by an entire day, occurring at the proper time 24 hours later. Thus, these studies implicated that a circadian pacemaker regulates the precise timing of the events leading to the LH surge and the exact timing of the surge itself. Thus, the daily rhythmicity in the activity of neural events serves as a foundation for the orderly timing of the GnRH surge and hence the preovulatory release of LH. Over the past several years, we have examined whether the diurnal rhythm in various aspects of neural activity is altered in middle-aged rats as they begin the transition to reproductive senescence. We measured the diurnal rhythm of monoamine turnover rates (Wise, 1982b; Cohen and Wise, 1988), neurotransmitter receptor densities (Weiland and Wise, 1990), and neuropeptide mRNAs (Weiland et al., 1992; Cai and Wise, 1996; Krajnak et al., 1998; M.J. Smith, A.B. Cashion, L. Jennes and P.M. Wise, unpublished observations). The clear theme that emerges is that virtually all of the rhythms are dampened or altered when female rats are middle aged and begin the transition to irregular estrous cycles. Often, the attenuation in rhythmicity was progressive and changes were more exaggerated as animals aged. The change in rhythmicity of any single neurotransmitter must be considered subtle, since the overall average often did not change. Investigators who measure these endpoints at only one time of day would be unlikely to detect a significant age-related change. Yet, together, disruption of the synchrony and coordination of multiple neural signals that govern the precise timing of GnRH secretion may ultimately lead to profound changes in the ability of rats to maintain regular reproductive cyclicity.

Until very recently, we thought that deterioration in the integrity of the circadian biological clock, which is located in the suprachiasmatic nuclei (SCN) in mammals, may underlie the desynchronization of multiple rhythms (Wise et al., 1988,1997). However, our newest findings suggest that we must modify our thinking. We tested our hypothesis by measuring the rhythm of key neuropeptides in the SCN. We reasoned that if aging involves a change in the integrity of the SCN itself and all its essential elements (i.e., inputs, oscillators, outputs), the pattern of expression of all critical neuropeptides of the SCN would be affected. On the other hand, if only some of the components of the clock are affected with age, we might observe differential effects on these neuropeptides. We measured 
the rhythm of gene expression of two key functionally critical neuropeptides heavily expressed in the SCN: vasoactive intestinal polypeptide (VIP) and arginine vasopressin (AVP) (Krajnak et al., 1998). VIP neurons are located primarily in the ventrolateral aspect of the $\mathrm{SCN}$, where they receive direct retinal input (Ibata et al., 1989). The rhythmic expression of VIP mRNA and protein depends upon exposure to the light/dark cycle (Albers et al., 1990). Therefore, this neuropeptide is likely to convey time-of-day information to efferent targets in different regions of the brain. In contrast, AVP neurons are predominantly located in the dorsomedial portion of the SCN. The 24-hour rhythm in its gene and peptide expression is endogenous and does not depend upon the light/dark cycle for its existence. The AVP rhythm, therefore, serves as a marker of the integrity of the SCN (Gillette and Reppert, 1987). Both VIP and AVP neurons relay circadian information to several regions of the brain by sending efferent projections to diverse regions of the brain, including the rostral preoptic area, where they may influence GnRH neurons or other neurotransmitters that regulate GnRH secretion (Harney et al., 1996; van der Beek et al., 1999; Smith et al., 2000; Krajnak et al., 2001). Our results were clear - and surprising. As expected, VIP and AVP mRNA levels exhibited a 24-hour rhythm in young females. Furthermore, the rhythm in VIP mRNA disappeared by the time animals were middle aged (Figure 3). In marked contrast, the AVP mRNA rhythm was totally unaffected with age: the rhythm and overall level of mRNA were the same in

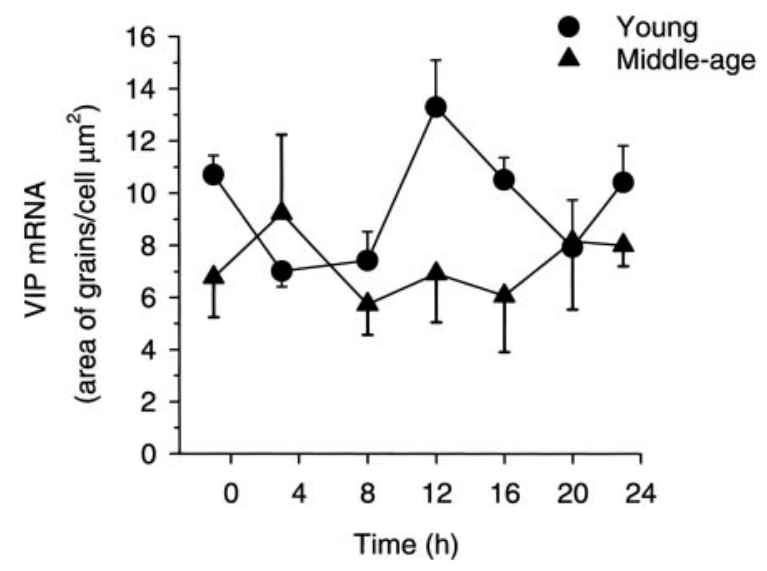

FIG. 3. VIP mRNA levels/cell in young and middle-aged ovariectomized, estradiol-treated rats as measured by in situ hybridization exhibits age-related changes in rhythmicity. Young, middleaged, and old rats were killed at 7 times of day over a 24-hour period. Young rats exhibited a diurnal rhythm in gene expression. The rhythm was no longer detectable in middle-aged or old rats. [Reprinted from Krajnak K, Kashon ML, Rosewell KL, Wise PM 1998 Aging alters the rhythmic expression of vasoactive intestinal polypeptide mRNA, but not arginine vasopressin mRNA in the suprachiasmatic nuclei of female rats. J Neurosci 18:4767-4774.] 
young, middle-aged, and old rats, both in terms of the amount of mRNA/cell (Figure 4) and the number of cells expressing AVP mRNA. This was the first time that we had observed the preservation of a neural rhythm in middle-aged rats. Based on these results, we concluded that the integrity of the entire biological clock does not deteriorate in a unified manner; instead, age differentially influences various components of the SCN.

The disappearance of the rhythm in VIP expression in the SCN is particularly intriguing, since this neuropeptide may play a uniquely important role in conveying time-of-day information directly to GnRH neurons. We hypothesized that the decrease in VIP levels in the SCN and the disappearance of its rhythm may lead to the delay in the timing of the LH surge and the attenuation in its amplitude. To test this possibility, we suppressed the level and rhythm of VIP by administering antisense oligonucleotides to VIP directed at the SCN of ovariectomized, estradiol-treated rats and assessed the effect on the LH surge (Harney et al., 1996). Peak LH concentrations during the surge were delayed and attenuated in antisense-treated animals, compared to random, oligo-treated control rats, in a manner that was strikingly similar to that observed previously in middle-aged rats (Figure 5). Similar results have been obtained using administration of VIP antisera intracerebroventricularly to ovariectomized, estradiol-treated rats (van der Beek et al., 1999). More recently, we determined whether aging alters the innervation of GnRH neurons by VIP and/or the ability of VIP to activate GnRH

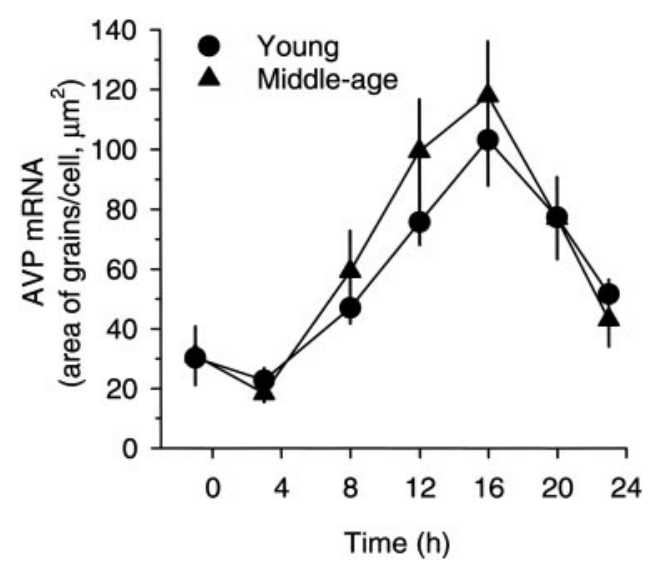

FIG. 4. Arginine vasopressin (AVP) mRNA levels/cell in young and middle-aged ovariectomized, estradiol-treated rats as measured by in situ hybridization exhibits age-related changes in rhythmicity. Brain sections from the same young, middle-aged, and old rats were used to measure AVP and vasoactive intestinal peptide (VIP) (see Figure 3). The diurnal rhythm was identical in all age groups. [Reprinted from Krajnak K, Kashon ML, Rosewell KL, Wise PM 1998 Aging alters the rhythmic expression of vasoactive intestinal polypeptide mRNA, but not arginine vasopressin mRNA in the suprachiasmatic nuclei of female rats. J Neurosci 18:4767-4774.] 


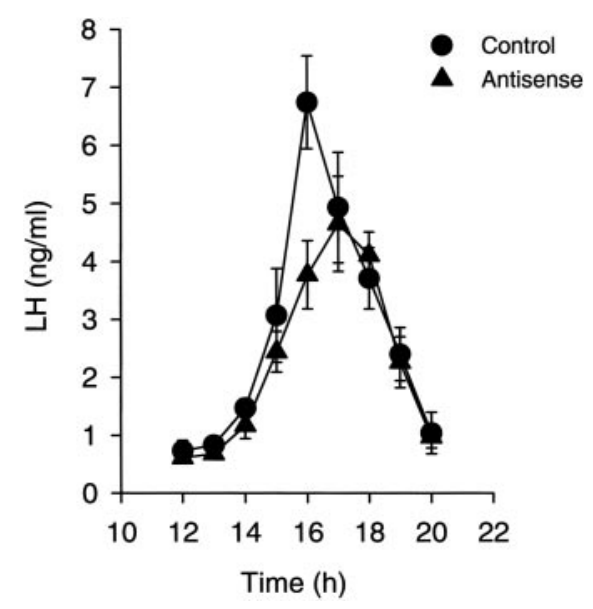

FIG. 5. The steroid-induced LH is blunted and delayed in rats that were treated with antisense oligos to VIP or control scrambled oligos directly at the suprachiasmatic nucleus (SCN). Ovariectomized, estradiol-treated young rats were administered antisense or scrambled oligos and sequentially bled. The steroid-induced surge of LH exhibited changes that are remarkably like those observed during aging. [Reprinted with permission from Harney JP, Scarbrough K, Rosewell KL, Wise PM 1996 In vivo antisense antagonism of vasoactive intestinal peptide in the suprachiasmatic nucleus causes aging-like changes in the estradiol-induced LH and prolactin surge. Endocrinology 137:3696-3701. Copyright The Endocrine Society.]

neurons by examining the effects of aging on the number of GnRH neurons apposed by VIP fibers and the number of GnRH neurons that receive VIP input that express Fos. Using triple-label immunocytochemistry for GnRH, VIP, and Fos in young and and middle-aged females, we quantified the number of GnRH neurons, GnRH neurons apposed by VIP fibers, and the number of GnRH neurons that express Fos that are apposed by VIP fibers. Our results clearly demonstrate that aging does not alter the number of GnRH neurons that receive VIP innervation. However, the number of GnRH neurons that receive VIP innervation and co-express Fos decreases significantly (Krajnak et al., 2001) (Figure 6). Immunocytochemical methods and light microscopy did not allow us to evaluate whether VIP acted directly upon GnRH neurons or through another neurotransmitter. However, several lines of evidence suggest that the communication between VIP and GnRH neurons is direct. 1) Using triple-label immunofluorescence to simultaneously localize GnRH, VIP, and VIP 2 receptor protein, we showed that about $40 \%$ of all GnRH neurons analyzed contain $\mathrm{VIP}_{2}$ receptor immunoreactivity and that VIP-containing processes were seen in close apposition to a significant number of $\mathrm{VIP}_{2}$ receptor-positive GnRH neurons (Smith et al., 2000). 2) Horvath and colleagues demonstrated synaptic contacts between VIP- and GnRH-containing neurons (Horvath et al., 1998). 3) Lesions of the 
A

B
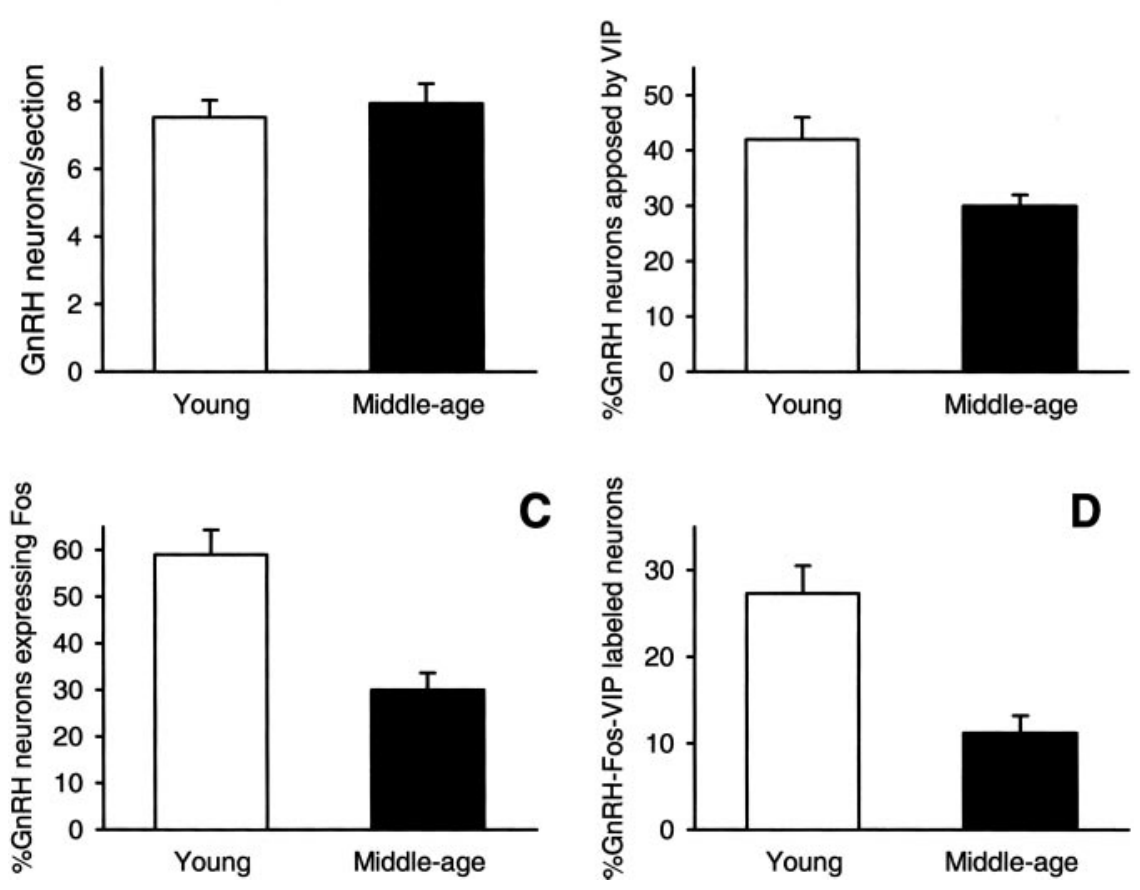

FIG. 6. (A) Number of GnRH-immunopositive neurons per section; (B) percent of GnRH and VIP-immunopositive neurons; (C) percent of GnRH and Fos-immunopositive neurons; and (D) percent of GnRH, Fos, and VIP immunoreactive neurons in the preoptic area of young and middle-aged females during the peak of a steroid-induced LH surge exhibit age-related changes. Aging is associated with no change in the number of GnRH immunopositive neurons or the percent of GnRH and VIP immunopositive neurons. However, percent of activated GnRH neurons and the percent of activated GnRH that were closely apposed to VIP neurons decreased with age. [Reprinted from Krajnak K, Rosewell KL, Wise PM 2001 Fos-induction in gonadotropin-releasing hormone neurons receiving vasoactive intestinal polypeptide innervation is reduced in middle-aged female rats. Biol Reprod 64:1160-1164.]

SCN indicate the presence of direct, VIP-containing projections to GnRH neurons (van der Beek et al., 1993). Together, these findings provide further support for a direct, VIP-containing pathway from the SCN to GnRH neurons and indicate that VIP can communicate directly with GnRH neurons. Furthermore, it appears that the age-related delay in the timing of the LH surge is not due to a change in VIP innervation of GnRH neurons but instead may result from a decreased sensitivity of GnRH neurons to VIP input. 


\section{Aging Influences the Balance Between Stimulatory and Inhibitory Neural Inputs to GnRH Neurons}

The ultimate pattern of GnRH release is governed by the orchestration of stimulatory and inhibitory inputs. Most work has focused on stimulatory side of the balance sheet, since, clearly, this is critical to the GnRH surge. Most recently, investigators have focused attention on the possible role of decreased glutamate input to GnRH neurons in aging rats (Zuo et al., 1996; Gore et al., 2000a,b), since glutamate is a neurotransmitter that exerts important direct stimulatory effects on GnRH neurons (Brann, 1995; Eyigor and Jennes, 1996). However, we are beginning to appreciate more deeply that the amplitude and timing of the preovulatory LH surge depends upon a decrease in inhibitory tone (Akabori and Barraclough, 1986a,b; Smith and Gallo, 1997). Opioid peptides and gamma aminobutyric acid (GABA) are critical inhibitory neurotransmitters that normally restrain $\mathrm{GnRH}$ secretion during the estrous cycle. A decrease in their activity normally occurs on proestrous afternoon, permitting stimulatory factors to maximally influence GnRH neurons. Researchers have found that, unless the inhibitory inputs to GnRH neurons are suppressed, the effects of norepinephrine and other stimulatory factors do not result in LH surges of normal amplitude or timing (for a review, see Kalra and Kalra, 1984). In addition, pharmacological blockade of the inhibitory tone early on proestrus results in a premature LH surge. We currently are examining the diurnal rhythm of preprodynorphin mRNA levels in young and middle-aged proestrous rats to determine whether the rhythm of this neuropeptide is altered and whether its activity increases in middle-aged rats and may contribute to the diminished LH surge (M.J. Smith, A.B. Cashion, L. Jennes, and P.M. Wise, unpublished observations). Preliminary data (Figure 7) demonstrate that, in young proestrous rats, preprodynorphin mRNA levels decrease prior to the LH surge, decreasing inhibitory tone and possibly allowing stimulatory factors to be maximally effective. In middle-aged rats, the diurnal rhythm is no longer detectable and overall mRNA levels are higher, compared to young controls. Thus, it is possible that the inhibitory tone that dynorphin communicates to GnRH neurons is amplified in middle-aged rats and does not subside to allow stimulatory factors to act. This could contribute to the attenuated and delayed preovulatory surge observed in middle-aged animals. We currently are examining whether GABAergic tone also may increase with age. This is another important inhibitory neuropeptide that communicates directly with GnRH neurons (Petersen et al., 1993) and exerts an important inhibitory tone on GnRH secretion. The role of this inhibitory neurotransmitter may be particularly important, since estradiol's actions on GABA may regulate cyclic morphological changes in astrocytes (Tranque et al., 1987; Parducz et al., 1993; Mong et al., 1999). These changes in the stellation of astrocytes on proestrus may then affect ensheathment of neurons, including possibly GnRH 


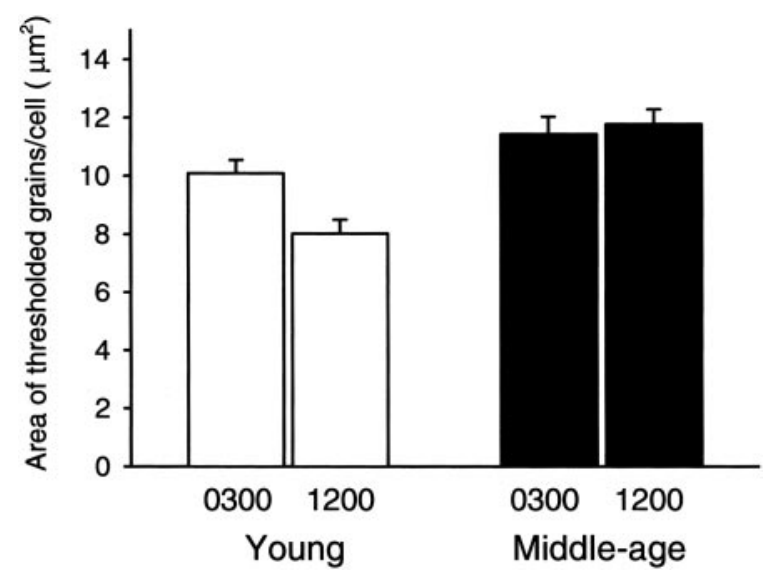

FIG. 7. Preprodynorphin mRNA levels are higher and lack rhythmicity in middle-aged compared to young rats on proestrus. Young and middle-aged rats were killed at 0300 and $1200 \mathrm{~h}$ on proestrus. Brains were prepared for in situ hybridization. Preprodynorphin mRNA was analyzed in the anteroventral periventricular nucleus (AVPV). Overall levels of gene expression are elevated and the rhythm is no longer detectable by the time rats reach middle age.

neurons (Cashion et al., 2001), leading to changes in the ability of neurotransmitters to communicate with each other to coordinate the GnRH surge.

\section{Estradiol Is a Neuroprotective Factor}

Recently, many researchers have focused their attention on the nonreproductive protective actions of estrogen. Aging and the menopause involve the gradual depletion of the ovarian follicular reserve and, with it, a decrease in plasma levels of estradiol. Since women will be spending a considerable portion of their lives in a hypoestrogenic state, the potential that women will be more vulnerable to neurodegenerative diseases and injury, due to the lack of estrogen, becomes even more important to understand. Numerous recent reviews provide excellent documentation of the many experimental and clinical circumstances in which estrogens provide profound protection against neuronal cell death (Green and Simpkins, 2000; Hurn and Macrae, 2000; Roof and Hall, 2000; Garcia Segura et al., 2001; Wise et al., 2001a,b). We will focus on our studies in this emerging area of interest.

We reported that low physiological levels of estradiol replacement dramatically decrease the degree of brain injury and cell death in an animal model of cerebrovascular stroke (Dubal et al., 1998). When we occlude the middle cerebral artery (MCAO) and permanently decrease blood flow to approximately $50 \%$ of normal, both the cerebral cortex and striatum undergo cell death. 
Ovariectomized rats are particularly vulnerable to injury and exhibit progressive infarction that evolves over a 24-hour period. Replacement with estradiol, to levels that mimic those which normally occur during the estrous cycle, results in profound protection of the cortex but not the striatum. Interestingly, middle-aged rats were equally responsive to the protective actions of estradiol (Dubal and Wise, 2000) (Figure 8). Our findings that estradiol replacement exerts equivalent neuroprotection in young and middle-aged female rats were unexpected because responsiveness of the hypothalamus to estradiol, as measured by a variety of endpoints - such as estradiol-induced activation of GnRH neurons that leads to LH surges (Wise, 1982b,1984; Lloyd et al., 1994), organization of diurnal rhythmicity in the hypothalamic neurotransmitter activity (Wise, 1982b; Cohen and Wise, 1988) or gene expression (Weiland et al., 1992; Krajnak et al., 1998), and stimulation of progesterone receptor binding (Wise et al., 1984) - diminishes with age. Therefore, we had hypothesized that estradiol would be less able to protect the brains of older animals against ischemic brain injury.

We have begun to investigate the cellular and molecular mechanisms that mediate the protective actions of estradiol. Several of our observations lead us to believe that low physiological levels of estradiol act through estrogen receptor alpha $(\mathrm{ER} \alpha)$-dependent mechanisms, leading to changes in gene expression that favor cell survival and suppress apoptotic cell death. First, we found that estradiol slows the progression, rate, and extent of cell death in the brain. Hormone treatment does not influence the extent of cell death that occurs immediately after stroke injury. Instead, its effects are confined to protecting against delayed cell death that occurs during the later phases of injury. Thus, it appears that estradiol protects specifically against apoptotic cell death (Dubal et

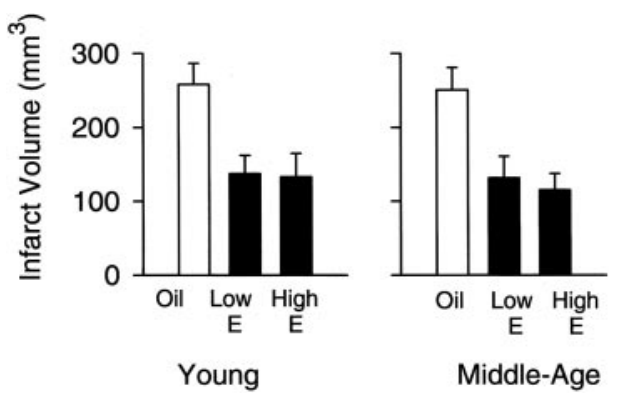

FIG. 8. Estradiol protects against middle cerebral artery occlusion (MCAO) in young and middle-aged rats. Low and high physiological levels of estradiol decreased total injury as measured by staining of brain sections with $2 \%$ triphenyltetrazolium chloride and measurement of infarct size using a computer-assisted imaging system and NIH Image. [Reprinted with permission from Dubal DB, Wise PM 2000 Neuroprotective effects of estradiol in middle-aged female rats. Endocrinology 142:43-48. Copyright The Endocrine Society.] 
al., 2001a; Rau et al., 2001) but does not protect against immediate necrotic cell death. Second, we (Dubal et al., 1999a) reported that, within 24 hours of $\mathrm{MCAO}, \mathrm{ER} \alpha$ mRNA is dramatically upregulated and estradiol pretreatment prevents injury-induced downregulation of ER $\beta$ in the cerebral cortex (Figure 9). These data suggest that brain injury may influence responsiveness of the injured cerebral cortex to estradiol and induce differential actions that are mediated by each receptor subtype (Nilsen et al., 2000; Patrone et al., 2000). It is important to note that $\mathrm{ER} \alpha$ is not usually detectable in the cerebral cortex of the adult rat and is only transiently expressed in this brain region during neonatal development when the cortex undergoes dramatic neurogenesis, neuritogenesis, and differentiation. It is intriguing to speculate that the dramatic reappearance of $\mathrm{ER} \alpha$ in the cerebral cortex may allow a recapitulation of the developmental actions of estradiol in promoting neurogenesis and re-differentiation of the cortex. Several studies support the concept that, following stroke injury, specific features of brain function revert to those seen during early stages of development, with the process of recovery recapitulating ontogeny (reviewed in Cramer and Chopp, 2000). To test whether ER $\alpha$ is a critical functional link in estradiol-mediated neuroprotection, we performed parallel studies in ER $\alpha$ knockout mice (Dubal et al., 2001b). We found that deletion of $\operatorname{ER} \alpha$ completely abolishes the protective actions of estradiol in all regions of the brain, whereas estradiol's ability to protect against
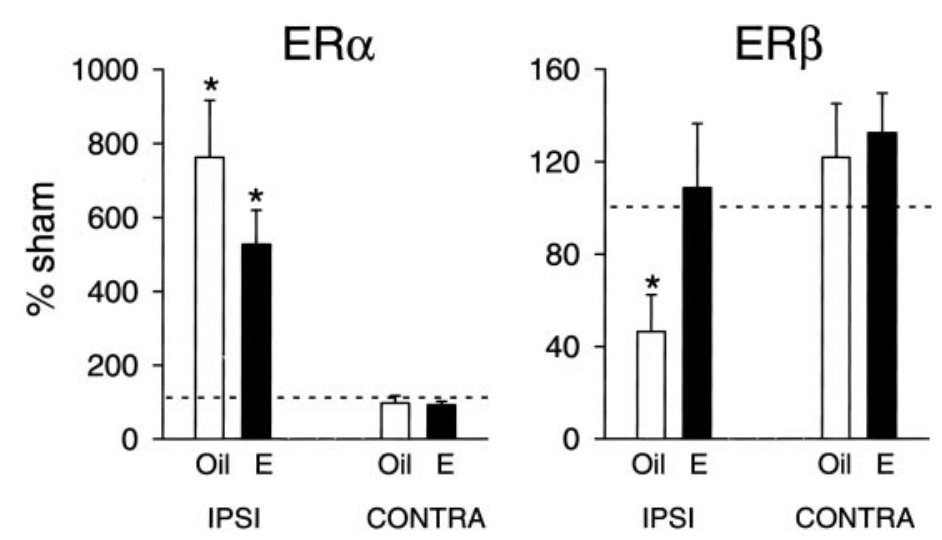

FIG. 9. Estrogen receptor alpha $(\mathrm{ER} \alpha)$ and estrogen receptor beta $(\mathrm{ER} \beta)$ gene expression are differentially modulated after MCAO. (Left panel) ER $\alpha$ mRNA was dramatically upregulated in the ipsilateral cortex of oil (vehicle)- and estradiol-treated rats, compared to the contralateral cortex and compared to constitutive levels in both oil- and estradiol-treated sham controls. (Right panel) Estradiol treatment prevented the injury-induced downregulation of ER $\beta$ mRNA in the ipsilateral cortex. In the absence of estradiol, ER $\beta$ gene expression declined significantly after injury below sham control constitutive levels. [Reprinted from Dubal DB, Shughrue PJ, Wilson ME, Merchenthaler I, Wise PM 1999 Estradiol modulates Bcl-2 in cerebral ischemia: a potential role for estrogen receptors. J Neurosci 19:6385-6393.] 
brain injury is totally preserved in the absence of $\operatorname{ER} \beta$ (Figure 10). These results firmly establish ER $\alpha$ as the critical mechanistic link that mediates the neuroprotective effects of physiological levels of estradiol. Finally, we have found that estradiol treatment alters the expression of multiple genes that have been implicated in the balance between cell survival and cell death, including bcl-2 (Dubal et al., 1999a), c-fos (Rau et al., 2000), galanin (Dubal et al., 1999b), and activin (Böttner et al., 2001). Whether any or all of these genes are critical functional mediators of estradiol's protective actions is not clear at present. Much more work must be done to understand the roles of each of these factors in protecting the brain against injury and cell death.

We have used in vitro methods to assess the protective actions of estradiol because this methodology provides invaluable tools that complement in vivo approaches. Multiple manipulations can be performed in vitro that are not technically or financially feasible using in vivo models. In particular, organotypic explants provide a powerful way to manipulate cellular environments in vitro, while maintaining interneurons, spatial relationships, local synaptic connections, and interactions with the local glial environment. Using explant cultures of the neonatal cerebral cortex, we (Wilson et al., 2000) have shown that low concentrations of estradiol protect against cell death. Our studies strongly suggest that ERs are critical, since the protection was not observed using $17 \alpha$-estradiol and was blocked by co-incubation with ICI 182,780, an ER antagonist (Figure 11).
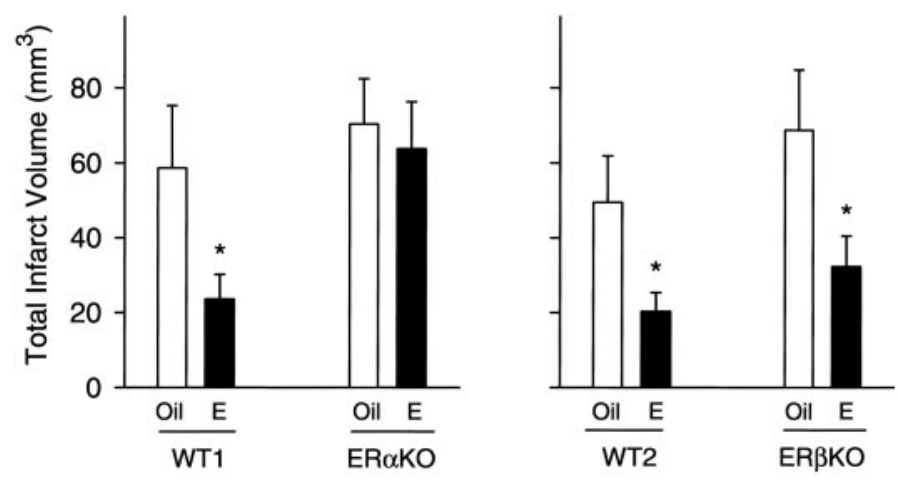

FIG. 10. Estradiol protects against MCAO in wild-type mice of both genetic backgrounds and in $\mathrm{ER} \beta \mathrm{KO}$ mice but not in $\mathrm{ER} \alpha \mathrm{KO}$ mice. (Left panel) Estradiol significantly decreased infarct volume in wild-type compared with oil (vehicle)-treated controls. In contrast, in ER $\alpha \mathrm{KO}$ mice, estradiol did not exert any protective effect. (Right panel) Estradiol significantly decreased infarct volume in wild-type and ER $\beta \mathrm{KO}$ mice. Brain sections were stained with hematoylin and eosin and the volume of the infarct was quantified with a computer-assisted imaging system using NIH Image. [Reprinted from Dubal DB, Zhu B, Yu B, Rau SW, Shughrue PJ, Merchenthaler I, Kindy MS, Wise PM 2001 Estrogen receptor- $\alpha$, not $-\beta$, is a critical link in estradiol-mediated protection against brain injury. Proc Natl Acad Sci USA 98:1952-1957. Copyright National Academy of Sciences.] 


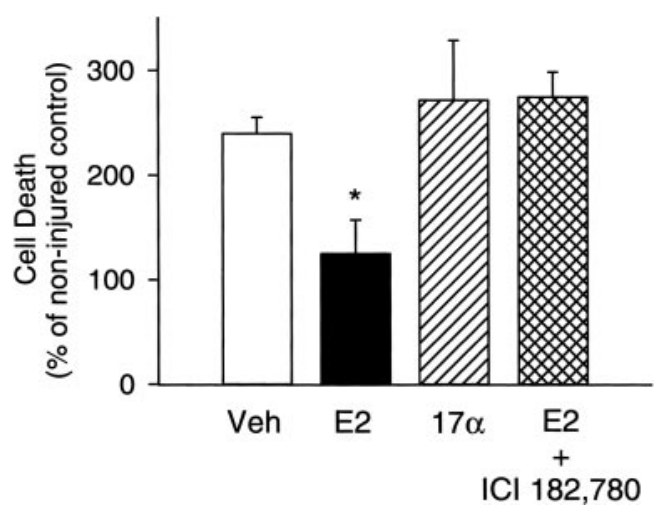

FIG. 11. Estradiol protects against ischemic injury-induced cell death in explant cultures of the cerebral cortex. Cell death, as measured by lactose dehydrogenase (LDH) release, is significantly decreased when explants were cultured in the presence of $17 \beta$-estradiol. However, $17 \alpha$-estradiol failed to protect and ICI182,780, an ER antagonist, blocked the protective actions of $17 \beta$-estradiol. [Reprinted from Wilson ME, Dubal DB, Wise PM 2000 Estradiol protects against injury-induced cell death in cortical explant cultures: a role for estrogen receptors. Brain Res 873:235-242.]

These findings complement those of Gollapudi and Oblinger (1999a,b), who showed that PC12 cells transfected with full-length rat ER $\alpha$ respond to the protective effects of estradiol, whereas cells transfected with vector DNA alone are not protected by estradiol. Other investigators have found that pharmacological levels of estradiol protect, even in the absence of the ER (for reviews, see Green and Simpkins, 2000; Hurn and Macrae, 2000; Roof and Hall, 2000; Garcia Segura et al., 2001; Wise et al., 2001a,b). The cellular and molecular mechanisms that underlie these receptor-independent protective actions are likely to result from estrogen's antioxidant, scavenging, immune-suppressing, and vascular actions. Together, these studies emphasize the breadth of the repertoire of mechanisms that estrogens use to protect against injury and cell death.

\section{Summary}

In summary, our understanding of the role of the brain in reproductive aging - and, conversely, the impact of reproductive aging on the brain - has increased dramatically during the past 20 years. Subtle changes in hypothalamic function and the ability of estradiol to influence the secretion of GnRH begin early during the aging process, ultimately leading to reproductive acyclicity. We are increasingly aware that the permanent hypoestrogenic state has major repercussions on multiple organs and physiological systems. Much attention is focused on the possible mechanisms through which estrogens may act as protective factors. 


\section{REFERENCES}

Ahmed, Hissong SA 1999 Gender and risk of autoimmune diseases: possible role of estrogenic compounds. Envir Health Perspect Supp 107:681

Akabori A, Barraclough CA 1986a Effects of morphine on luteinizing hormone secretion and catecholamine turnover in the hypothalamus of estrogen-treated rats. Brain Res 362:221-226

Akabori A, Barraclough CA 1986b Gonadotropin responses to naloxone may depend upon spontaneous activity in noradrenergic neurons at the time of treatment. Brain Res 362:55-62

Albers HE, Stopa EG, Zoeller RT, Kauer JS, King JC, Fink JS, Mobtaker H, Wolfe H 1990 Day-night variation in prepro vasoactive intestinal peptide/peptide histidine isoleucine mRNA within the rat suprachiasmatic nucleus. Mol Brain Res 7:85-89

Böttner M, Dubal DB, Rau SW, Wise PM 2001 Activin gene expression increases after stroke injury: modulation by estradiol. Soc Neurosci Abstr 27:1165

Brann DW 1995 Glutamate: a major excitatory transmitter in neuroendocrine regulation. Neuroendocrinology 61:213-225

Brito AN, Sayles TE, Krieg RJ Jr, Matt DW 1994 Relation of attenuated proestrous luteinizing hormone surges in middle-aged female rats to in vitro pituitary gonadotropin-releasing hormone responsiveness. Eur J Endocrinol 130:540-544

Butcher RL, Page RD 1981 Role of the aging ovary in cessation of reproduction. In Schwartz NB, Hunzicker-Dunn M, eds. Dynamics of Ovarian Function. New York: Raven Press; 253-271

Cai A, Wise PM 1996 Age-related changes in the diurnal rhythm of CRH gene expression in the paraventricular nuclei. Am J Physiol 270:E238-E243

Cashion AB, Smith MJ, Wise PM 2001 Astrocyte morphology in the rostral preoptic area (rPOA) exhibits a diurnal rhythm on proestrus: relationship to the LH surge and effects of age. Soc Neurosci Abstr 27:1940

Clifton DK, Sawyer CH 1979 LH release and ovulation in the rat following depletion of hypothalamic norepinephrine: chronic vs. acute effects. Neuroendocrinology 28:442-449

Cohen IR, Wise PM 1988 Age-related changes in the diurnal rhythm of serotonin turnover in microdissected brain areas of estradiol-treated ovariectomized rats. Endocrinology 122:26262633

Compston JE 2001 Sex steroids and bone. Physiol Rev 81:419-447

Cramer SC, Chopp M 2000 Recovery recapitulates ontogeny. Trends Neurosci 23:265-271

DePaolo LV 1987 Age-associated increases in serum follicle-stimulating hormone levels on estrus are accompanied by a reduction in the ovarian secretion of inhibin. Exptl Aging Res 13:3-7

Dubal DB, Wise PM 2000 Neuroprotective effects of estradiol in middle-aged female rats. Endocrinology 142:43-48

Dubal DB, Kashon ML, Pettigrew LC, Ren JM, Finklestein SP, Rau SW, Wise PM 1998 Estradiol protects against ischemic injury. J Cereb Blood Flow Metab 18:1253-1258

Dubal DB, Shughrue PJ, Wilson ME, Merchenthaler I, Wise PM 1999a Estradiol modulates Bcl-2 in cerebral ischemia: a potential role for estrogen receptors. J Neurosci 19:6385-6393

Dubal DB, Wilson, ME, Shughrue PJ, Merchenthaler I, Wise PM 1999b Induction of galanin gene expression in estradiol-mediated neuroprotection against cerebral ischemia. Soc Neurosci Abstr 25:1449

Dubal DB, Rau SW, Wise PM 2001a Estradiol protects against delayed cell death in stroke injury. Soc Neurosci Abstr 27:1164

Dubal DB, Zhu B, Yu B, Rau SW, Shughrue PJ, Merchenthaler I, Kindy MS, Wise PM 2001b Estrogen receptor- $\alpha$, not $-\beta$, is a critical link in estradiol-mediated protection against brain injury. Proc Natl Acad Sci USA 98:1952-1957

Erkkola R 1996 Female menopause, hormone replacement therapy, and cognitive processes. Maturitas 23:S27-S30 
Everett JW, Sawyer CH 1950 A 24-hour periodicity in the 'LH-release apparatus' of female rats, disclosed by barbiturate sedation. Endocrinology 47:198-218

Everett JW, Sawyer CH 1953 Estimated duration of the spontaneous activation which causes release of ovulating hormone from the rat hypophysis. Endocrinology 52:83-92

Everett JW, Sawyer CH, Markee JE 1949 A neurogenic timing factor in control of the ovulatory discharge of luteinizing hormone in the cyclic rat. Endocrinology 44:234-250

Eyigor O, Jennes L 1996 Identification of glutamate receptor subtype mRNAs in gonadotropinreleasing hormone neurons in rat brain. Endocrine 4:133-139

Faddy MJ, Gosden RG, Edwards RG 1983 Ovarian follicle dynamics in mice: a comparative study of three inbred strains and an F1 hybrid. J Endocrinol 96:23-33

Felicio LS, Nelson JF, Gosden RG, Finch CE 1983 Restoration of ovulatory cycles by young ovarian grafts in aging mice: potentiation by long-term ovariectomy decreases with age. Proc Natl Acad Sci USA 80:6076-6080

Fillit H 1994 Estrogens in the pathogenesis and treatment of Alzheimer's disease in postmenopausal women. Ann NY Acad Sci 743:233-239

Fitzgerald CT, Seif MW, Killick SR, Bennett DA 1994 Age-related changes in the female reproductive cycle. Br J Obstet Gynaecol 101:229-223

Garcia Segura LM, Azcoitia I, DonCarlos LL 2001 Neuroprotection by estradiol. Progr Neurobiol 63:29-60

Gillette MU, Reppert SM 1987 The hypothalamic suprachiasmatic nuclei: circadian patterns of vasopressin secretion and neuronal activity in vitro. Brain Res Bull 19:135-139

Gollapudi L, Oblinger MM 1999a Estrogen and NGF synergistically protect terminally differentiated, ER $\alpha$-transfected PC12 cells from apoptosis. J Neurosci Res 56: 471-481

Gollapudi L, Oblinger MM 1999b Stable transfection of PC12 cells with estrogen receptor (ER $\alpha$ ): protective effects of estrogen on cell survival after serum deprivation. J Neurosci Res 56:99-108

Gore AC, Oung T, Yung S, Flagg RA, Woller MJ 2000a Neuroendocrine mechanisms for reproductive senescence in the female rat: gonadotropin-releasing hormone neurons. Endocrine 13:315-323

Gore AC, Yeung G, Morrison JH, Oung T 2000b Neuroendocrine aging in the female rat: the changing relationship of hypothalamic GnRH neurons and NMDA receptors. Endocrinology 141:4757-4767

Green PS, Simpkins JW 2000 Neuroprotective effects of estrogens: potential mechanisms of action. Intl J Dev Neurosci 18:347-358

Harney JP, Scarbrough K, Rosewell KL, Wise PM 1996 In vivo antisense antagonism of vasoactive intestinal peptide in the suprachiasmatic nucleus causes aging-like changes in the estradiol-induced LH and prolactin surge. Endocrinology 137:3696-3701

Hoffman GE, Smith MS, Verbalis JG 1993 c-Fos and related immediate early gene products as markers of activity in neuroendocrine systems. Front Neuroendocrinol 14:173-213

Hogg BB, Matt DW, Sayles TE 1992 Attenuated proestrous luteinizing hormone surges in middle-aged rats are associated with decreased pituitary luteinizing hormone-beta messenger ribonucleic acid expression. Am J Obstet Gynecol 167:303-307

Horvath TL, Cela V, van der Beek EM 1998 Gender-specific apposition between vasoactive intestinal peptide-containing and gonadotropin-reeasing hormone producing neurons in the rat. Brain Res 795:277-281

Hurn PD, Macrae IM 2000 Estrogen as a neuroprotectant in stroke. J Cereb Blood Flow Metab 20:631-652

Ibata Y, Yakahaski Y, Okamura H, Kawakami F, Terubayashi H, Kubo T, Yanaihara N 1989 Vasoactive intestinal peptide (VIP)-like immunoreactive neurons located in the rat suprachiasmatic nucleus receive a direct retinal projection. Neurosci Lett 97:1-5 
Jansson L, Holmdahl R 1998 Estrogen-mediated immunosuppression in autoimmune diseases. Inflamm Res 47:290-301

Kalra SP, Kalra PS 1984 Opioid-adrenergic-steroid connection in regulation of luteinizing hormone secretion in the rat. Neuroendocrinology 38:418-426

Kirkwood TBL 1998 Ovarian ageing and the general biology of senescence. Maturitas 30:105-111

Klein NA, Illingworth PJ, Groome NP, McNeilly AS, Battaglia DE, Soules MR 1996 Decreased inhibin B secretion is associated with the monotropic FSH rise in older, ovulatory women: a study of serum and follicular fluid levels of dimeric inhibin A and B in spontaneous menstrual cycles. J Clin Endocrinol Metab 81:2742-2745

Krajnak K, Kashon ML, Rosewell KL, Wise PM 1998 Aging alters the rhythmic expression of vasoactive intestinal polypeptide mRNA, but not arginine vasopressin mRNA in the suprachiasmatic nuclei of female rats. J Neurosci 18:4767-4774

Krajnak K, Rosewell KL, Wise PM 2001 Fos-induction in gonadotropin-releasing hormone neurons receiving vasoactive intestinal polypeptide innervation is reduced in middle-aged female rats. Biol Reprod 64:1160-1164

Lapolt PS, Lu JKH 2001 Factors influencing the onset of female reproductive senescence. In Hof PR, Mobbs CV, eds. Functional Neurobiology of Aging. San Diego: Academic Press; 761-768

Lindsay R 1996 The menopause and osteoporosis. Obstet Gynecol 87:16S-19S

Lloyd JM, Hoffman GE, Wise PM 1994 Decline in immediate early gene expression in gonadotropin-releasing hormone neurons during proestrus in regularly cycling, middle-aged rats. Endocrinology 134:1800-1805

Losordo DW, Isner JM 2001 Estrogen and angiogenesis. Arterioscler Thromb Vasc Biol 21:6-12

Lu JK, Anzalone CK, Lapolt PS 1994 Relation of neuroendocrine function to reproductive decline during aging in the female rat. Neurobiol Aging 15:541-544

Lu JKH 1983 Changes in ovarian function and gonadotropin and prolactin secretion in aging female rats. In Meites J, ed. Neuroendocrinology of Aging. New York: Plenum Press; 103-122

Manolagas SC 2000 Cellular and molecular mechanisms of postmenopausal osteoporosis. In Bellino FL, ed. Proceedings of the International Symposium on the Biology of the Menopause. Norwell, MA: Springer; 134-146

Marchetti B, Cioni M 1988 Opposite changes of pituitary and ovarian receptors for LHRH in ageing rats: further evidence for a direct neural control of ovarian LHRH receptor activity. Neuroendocrinology 48:242-251

Matt DW, Kauma SW, Pincus SM, Veldhuis JD, Evans WS 1998 Characteristics of luteinizing hormone secretion in younger versus older premenopausal women. Am J Obstet Gynecol 178:504-510

Matthews KA, Kuller LH, Sutton-Tyrrell K 2000 Changes in cardiovascular risk factors during the peri- and postmenopausal years. In Bellino FL, ed. Proceedings of the International Symposium on the Biology of the Menopause. Norwell, MA: Springer; 147-158

Mendelsohn ME 2000 Mechanisms of estrogen action in the cardiovascular system. J Steroid Biochem Molec Biol 74:337-343

Mong JA, Glaser E, McCarthy MM 1999 Gonadal steroids promote glial differentiation and alter neuronal morphology in the developing hypothalamus in a regionally specific manner. J Neurosci 19:1464-1472

Nass TE, Lapolt PS, Judd HL, Lu JKH 1984 Alterations in ovarian steroid and gonadotrophin secretion preceding the cessation of regular oestrous cycles in ageing female rats. J Endocrinol 100:43-50

Nilsen J, Mor G, Naftolin F 2000 Estrogen-regulated developmental neuronal apoptosis is determined by estrogen receptor subtype and the fas/fas ligand system. J Neurobiol 43:64-78

Parducz A, Perez J, Garcia-Segura LM 1993 Estradiol induces plasticity of GABAergic synapses in the hypothalamus. Neuroscience 53:395-401 
Patrone C, Pollio G, Vegeto E, Enmark E, de Curtis I, Gustafsson J-A, Maggi A 2000 Estradiol induces differential neuronal phenotypes by activating estrogen receptor $\alpha$ or $\beta$. Endocrinology 141:1839-1845

Peng M-T, Huang H-H 1972 Aging of hypothalamic-pituitary-ovarian function in the rat. Fertil Steril 23:535-542

Petersen SL, McCrone S, Coy D, Adelman JP, Mahan LC 1993 GABA $_{\mathrm{A}}$ receptor subunit mRNAs in cells of the preoptic area: colocalization with LHRH mRNA using dual-label in situ hybridization histochemistry. Endocr J 1:29-34

Rau SW, Dubal DB, Wise PM 2000 Immediate early gene regulation by estradiol in ischemic injury. Soc Neurosci Abstr 26:778

Rau SW, Dubal DB, Wise PM 2001 Estradiol protects against apoptotic cell death in stroke injury: possible mechanisms. Soc Neurosci Abstr 27:1165

Richardson SJ, Senikas V, Nelson JF 1987 Follicular depletion during the menopausal transition: evidence for accelerated loss and ultimate exhaustion. J Clin Endocrinol Metab 65:1231-123.

Roof RL, Hall ED 2000 Gender differences in acute CNS trauma and stroke: neuroprotective effects of estrogen and progesterone. J Neurotrauma 17:367-388

Rubin BS 2000 Hypothalamic alterations and reproductive aging in female rats: evidence of altered LHRH neuronal function. Biol Reprod 63:968-976

Rubin BS, Bridges RS 1989 Alterations in luteinizing hormone-releasing hormone release from the mediobasal hypothalamus of ovariectomized, steroid-primed middle-aged rats as measured by push-pull perfusion. Neuroendocrinology 49:225-232

Rubin BS, Jimenez-Linan M 1999 LHRH receptor mRNA levels and gonadotropin subunit mRNA levels are reduced in the pituitaries of middle-aged rats on the day of a steroid-induced $\mathrm{LH}$ surge. Soc Neurosci Abstr 25:1960

Rubin BS, Mitchell S, Lee CE, King JC 1995 Reconstructions of populations of LHRH neurons in young and middle-aged rats reveal progressive increases in subgroups expressing fos protein on proestrus and age-related deficits. Endocrinology 136:3823-3830

Santoro N, Brown JR, Adel T, Skurnick JH 1996 Characterization of reproductive hormonal dynamics in the perimenopause. J Clin Endocrinol Metab 81:1495-1501

Sawyer CH, Everett JW, Markee JE 1949 A neural factor in the mechanism by which estrogen induces the release of luteinizing hormone in the rat. Endocrinology 44:218-233

Scarbrough K, Wise PM 1990 Age-related changes in the pulsatile pattern of LH release precede the transition to estrous acyclicity and depend upon estrous cycle history. Endocrinology 126:884-890

Sherman BM, West JH, Korenman SG 1976 The menopausal transition: analysis of LH, FSH, estradiol, and progesterone concentrations during menstrual cycles of older women. J Clin Endocrinol Metab 42:629-636

Sherwin BB 1996 Hormones, mood, and cognitive functioning in postmenopausal women. Obstet Gynecol 87:20S-26S

Sherwin BB 1999 Can estrogen keep you smart? Evidence from clinical studies. J Psychiat Neurosci 24:315-321

Smith MJ, Gallo RV 1997 The effect of blockade of $\kappa$-opioid receptors in the medial preoptic area on the luteinizing hormone surge in the proestrous rat. Brain Res 768:111-119

Smith MJ, Jennes L, Wise PM 2000 Localization of the VIP2 receptor protein on GnRH neurons in the female rat. Endocrinology 141:4317-4319

Smith WA, Cooper RL, Conn PM 1982 Altered pituitary responsiveness to gonadotropin-releasing hormone in middle-aged rats with 4-day estrous cycles. Endocrinology 111:1843-1848

Stevenson JC 2000 Cardiovascular effects of oestrogens. J Steroid Biochem Molec Biol 74:387-393

Tranque PA, Suarez I, Olmos G, Fernandez B, Garcia-Segura LM 1987 Estradiol-induced redistribution of glial fibrillary acidic protein immunoreactivity in the rat brain. Brain Res 406:348-351 
van der Beek EM, Wiegant VM, van der Donk HA, van den Hurk R, Buijs RM 1993 Lesions of the suprachiasmatic nucleus indicate the presence of a direct vasoactive intestinal polypeptide-containing projection to gonadotropin-releasing hormone neurons in the female rat. J Neuroendocrinol 5:137-144

van der Beek EM, Swarts HJ, Wiegant VM 1999 Central administration of antiserum to vasoactive intestinal peptide delays and reduces luteinizing hormone and prolactin surges in estrogentreated rats. Neuroendocrinology 69:227-237

van der Schoot $\mathbf{P} 1976$ Changing pro-oestrous surges of luteinizing hormone in ageing 5-day cyclic rats. J Endocrinol 69:287-288

Van Look PFA, Lothian H, Hunter WM, Michie EA, Baird DT 1977 Hypothalamic-pituitaryovarian function in perimenopausal women. Clin Endocrinol 7:13-31

vom Saal FS, Finch CE, Nelson JF 1994 Natural history and mechanisms of reproductive aging in humans, laboratory rodents, and other selected vertebrates. In Knobil E, Neill JD, eds. The Physiology of Reproduction. New York: Raven Press; 1213-1314

Weiland NG, Wise PM 1990 Aging progressively decreases the densities and alters the diurnal rhythm of alpha-1-adrenergic receptors in selected hypothalamic regions. Endocrinology 126:2392-2397

Weiland NG, Scarbrough K, Wise PM 1992 Aging abolishes the estradiol-induced suppression and diurnal rhythm of proopiomelanocortin gene expression in the arcuate nucleus. Endocrinology 131:2959-2964

Wild RA 1996 Estrogen: effects on the cardiovascular tree. Obstet Gynecol 87:27S-35S

Wilson ME, Dubal DB, Wise PM 2000 Estradiol protects against injury-induced cell death in cortical explant cultures: a role for estrogen receptors. Brain Res 873:235-242

Wise PM 1982a Alterations in proestrous LH, FSH, and prolactin surges in middle-aged rats. Proc Soc Exp Biol Med 169:348-354

Wise PM 1982b Norepinephrine and dopamine activity in microdissected brain areas of the middle-aged and young rat on proestrus. Biol Reprod 27:562-574

Wise PM 1984 Estradiol-induced daily luteinizing hormone and prolactin surges in young and middle-aged rats: correlations with age-related changes in pituitary responsiveness and catecholamine turnover rates in microdissected brain areas. Endocrinology 115:801-809

Wise PM 2000 New understanding of the complexity of the menopause and challenges for the future. In Bellino FL, ed. Proceedings of the International Symposium on the Biology of Menopause. Norwell, MA: Springer; 1-8

Wise PM, McEwen BS, Parsons B, Rainbow TC 1984 Age-related changes in cytoplasmic estradiol receptor concentrations in microdissected brain nuclei: correlations with changes in steroid-induced sexual behavior. Brain Res 321:119-126

Wise PM, Cohen IR, Weiland NG, London ED 1988 Aging alters the circadian rhythm of glucose utilization in the suprachiasmatic nucleus. Proc Natl Acad Sci USA 85:5305-5309

Wise PM, Kashon ML, Krajnak KM, Rosewell KL, Cai A, Scarbrough K, Harney JP, McShane T, Lloyd J, Weiland NG 1997 Aging of the female reproductive system: a window into brain aging. Rec Progr Hormone Res 52:279-305

Wise PM, Dubal DB, Wilson ME, Rau SW, Böttner M 2001a Minireview: neuroprotective effects of estrogen - new insights into mechanisms of action. Endocrinology 142:969-973

Wise PM, Dubal DB, Wilson ME, Rau SW, Liu Y 2001b Estrogens: trophic and protective factors in the adult brain. Front Neuroendocrinol 22:33-66

Zuo Z, Mahesh VB, Zamorano PL, Brann DW 1996 Decreased gonadotropin-releasing hormone neurosecretory response to glutamate agonists in middle-aged female rats on proestrus afternoon: a possible role in reproductive aging? Endocrinology 137:2334-2338 


\title{
Hormonal Changes in the Menopause Transition
}

\author{
Henry G. Burger*, Emma C. Dudley ${ }^{\dagger}$, David M. Robertson*, and \\ LORRAINE DENNERSTEIN ${ }^{\dagger}$
}

*Prince Henry's Institute of Medical Research at Monash Medical Centre, Clayton, Victoria, Australia; 'Office for Gender and Health, Royal Melbourne Hospital, Parkville, Victoria, Australia

\begin{abstract}
The menopause is the permanent cessation of menstruation resulting from the loss of ovarian follicular activity. It is heralded by the menopausal transition, a period when the endocrine, biological, and clinical features of approaching menopause begin. A common initial marker is the onset of menstrual irregularity. The biology underlying the transition to menopause includes central neuroendocrine changes as well as changes within the ovary, the most striking of which is a profound decline in follicle numbers. Follicle-stimulating hormone (FSH) is an established indirect marker of follicular activity. In studies of groups of women, its concentration, particularly in the early follicular phase of the menstrual cycle, begins to increase some years before there are any clinical indications of approaching menopause. The rise in FSH is the result of declining levels of inhibin B (INH-B), a dimeric protein that reflects the fall in ovarian follicle numbers, with or without any change in the ability of the lining granulosa cells to secrete INH-B. Estradiol levels remain relatively unchanged or tend to rise with age until the onset of the transition and are usually well preserved until the late perimenopause, presumably in response to the elevated FSH levels. During the transition, hormone levels frequently vary markedly - hence, measures of FSH and estradiol are unreliable guides to menopausal status. Concentrations of testosterone have been reported to fall by about $50 \%$ during reproductive life, between the ages of 20 and 40. They change little during the transition and, after menopause, may even rise. Dehydroepiandrosterone (DHEA) and DHEAS, its sulphate, on the other hand, decline with age, without any specific influence of the menopause. Symptoms of the menopause can be interpreted as resulting primarily from the profound fall in estradiol, occurring over a 3- to 4-year period around final menses, a fall that presumably contributes importantly to the beginning, in the late perimenopause, of loss of bone mineral density.
\end{abstract}

\section{Introduction and Definitions}

The World Health Organization has defined the menopause as the permanent cessation of menstruation resulting from loss of ovarian follicular activity (World Health Organization, 1996). The perimenopause commences when the first features of approaching menopause begin until at least 1 year after final menstrual period (FMP). The term "menopausal transition" has been applied to that portion of the perimenopause that ends with the FMP. The median age of 
onset has been reported as between 45.5 and 47.5 years; the average duration is 4 years (Treloar, 1981; McKinlay et al., 1992). The incidence of dysfunctional uterine bleeding and hysterectomy are maximal during the menopausal transition and the incidence of symptoms is similar to that in early postmenopausal women (McKinlay et al., 1992; Dennerstein et al., 1993).

It is strongly recommended that the term perimenopause be used as indicated here and not applied loosely to women in their 40s and early 50s who continue to have regular menstrual cycles and no symptoms of approaching menopause. Studies of the hormonal changes during the perimenopause have been based on various experimental designs and definitions. In some instances, they have been recorded as a function of age with little attention to menstrual cycle status (Sherman et al., 1976). In the few longitudinal studies reported, the FMP has been used as a reference point, with hormonal changes described in terms of time intervals before and after that point (Metcalf et al., 1981; Longcope et al., 1986; Rannevik et al., 1986,1995). A few studies have reported hormone changes in relation to changes in menstrual cycle characteristics, such as the first selfreported change in the amount of menstrual flow, in the frequency of menstruation, or in the combination of changes in flow and frequency, an approach that has been adopted in the Melbourne Women's Midlife Health Project (Burger et al., 1995).

\section{Changes in the Pituitary-Ovarian Axis with Age in Regularly Cycling Women}

Although the changes that occur in the circulating concentrations of folliclestimulating hormone $(\mathrm{FSH})$, luteinizing hormone $(\mathrm{LH})$, estradiol $\left(\mathrm{E}_{2}\right)$, and progesterone $(\mathrm{P})$ during the normal menstrual cycle have been well known for several decades, it is only within the last 10 years that the changes in circulating inhibins have been documented (Groome et al., 1996). Relatively stable and low values for both $\mathrm{E}_{2}$ and inhibin A (INH-A) are found during the first half of the follicular phase of the cycle, followed by a rise to midcycle peaks approximately 24 hours before the ovulatory LH surge. The concentrations then fall and rise again to secondary peaks parallel to the peak in $\mathrm{P}$ secretion during the luteal phase. There is a subsequent fall in the circulating concentrations of all three hormones prior to the onset of menses. Inhibin B (INH-B), on the other hand, rises and falls closely parallel to the changes in FSH during the luteal-follicular transition and the early follicular phase. There is a midcycle peak but, during the luteal phase, INH-B concentrations decline to their lowest points in the cycle (Figure 1). The normal pattern of $\mathrm{E}_{2}$ and INH-A secretion is preserved when their concentrations are examined as a function of increasing age in regularly cycling women. For example, in a large cross-sectional study of women who ranged in age from 24-50 years (Lee et al., 1988), early follicular-phase concentrations of 

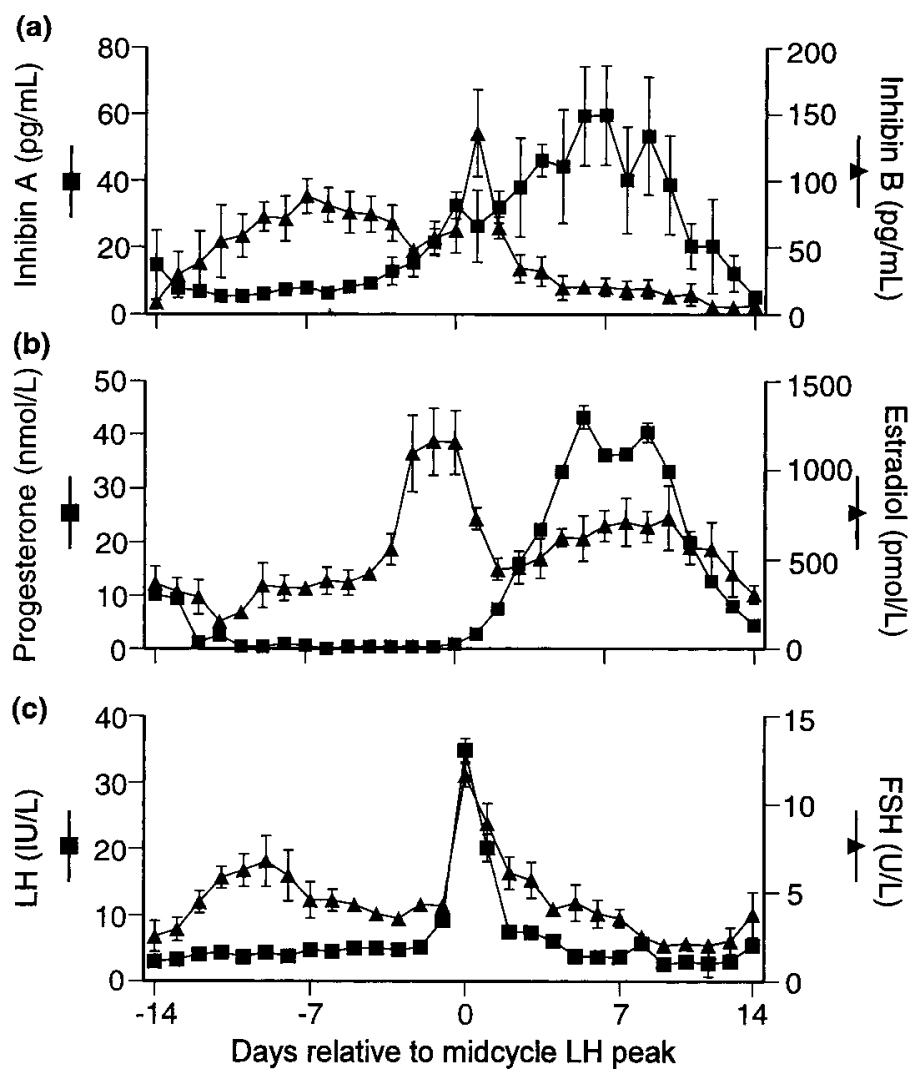

FIG. 1. Plasma concentrations of (a) inhibin A and inhibin B, (b) estradiol and progesterone, and (c) luteinizing hormone (LH) and follicle-stimulating hormone (FSH) during the menstrual cycle. Data displayed with respect to day of midcycle LH peak. [Adapted with permission from Groome NP, Illingworth PJ, O’Brien M, Rodger PAL, Rodger FE, Mather JR, McNeilly AS 1996 Measurement of dimeric inhibin B throughout the human menstrual cycle. J Clin Endocrinol Metab 81:1401-1405. Copyright The Endocrine Society.]

$\mathrm{E}_{2}$ tended to rise slightly in the oldest group of subjects, despite a progressive rise in FSH concentrations. In another study, older women were compared with a young control group and showed increased levels of urinary estrogens and a fall in urinary pregnandiol excretion in the luteal phase of the cycle, despite continued regular cyclicity (Santoro et al., 1996). The rise in serum FSH was accompanied by minimal, if any, changes in circulating LH. The most-plausible explanation for the monotropic rise in FSH was a decline in circulating INH concentrations with age. This was first indicated in a study by MacNaughton et al. (1992) in which single samples were taken in the early follicular phase of 
regularly cycling subjects and showed a rise in $\mathrm{FSH}$, no significant change in $\mathrm{LH}$, and a decline in the circulating concentrations of immunoreactive INH. That the rise in FSH actually was specifically related to a fall in the circulating concentrations of INH-B was shown by Klein et al. (1996), who compared a group of older ovulatory women, selected to have increased circulating concentrations of FSH, with a group of young, regularly cycling subjects. Serum FSH was significantly elevated as expected and INH-B levels were significantly lower in the older women than in the younger women, while INH-A was unchanged and $\mathrm{E}_{2}$ was actually higher in the older women (Figure 2).

The present authors have more-recently confirmed the progressive increase in circulating FSH levels with increasing age in regularly cycling women, though the rise in FSH was seen predominantly in some women over the age of 40 (Burger et al., 2000b) (Figure 3). A clear inverse relationship between INH-B and FSH in women over the age of 40 was demonstrated in that study (Figure 4).

Substantial evidence exists that the circulating concentrations of INH-A and INH-B are under differential control. INH-A, together with $\mathrm{E}_{2}$, is derived
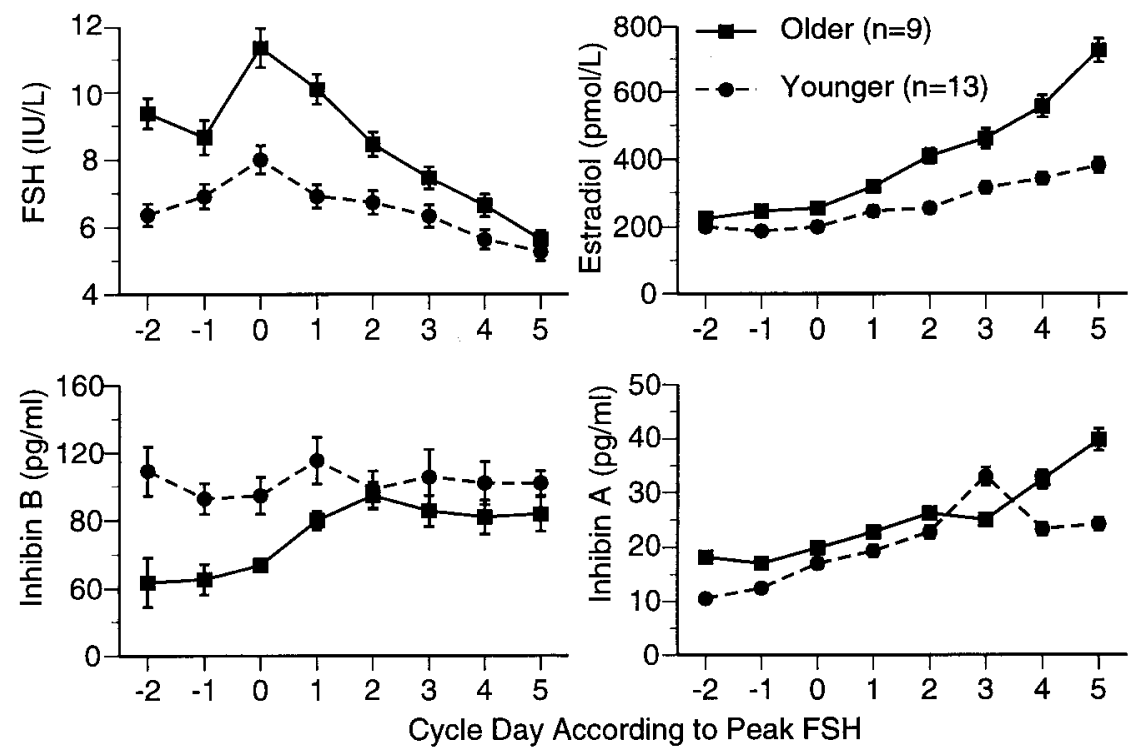

FIG. 2. Mean \pm SEM concentrations of (clockwise from top left) FSH (p $<0.01), \mathrm{E}_{2}(\mathrm{p}<$ $0.01)$, inhibin $\mathrm{A}(\mathrm{p}=0.61)$, and inhibin $\mathrm{B}(\mathrm{p}=0.04)$, according to the day of maximal FSH concentration (peak FSH = day 0). [Reprinted with permission from Klein NA, Illingworth PJ, Groome NP, McNeilly AS, Battaglia DE, Soules MR 1996 Decreased inhibin B secretion is associated with the monotropic rise of FSH in older, ovulatory women: a study of serum and follicular fluid levels of dimeric inhibin A and B in spontaneous menstrual cycles. J Clin Endocrinol Metab 81:2742-2745. Copyright The Endocrine Society.] 


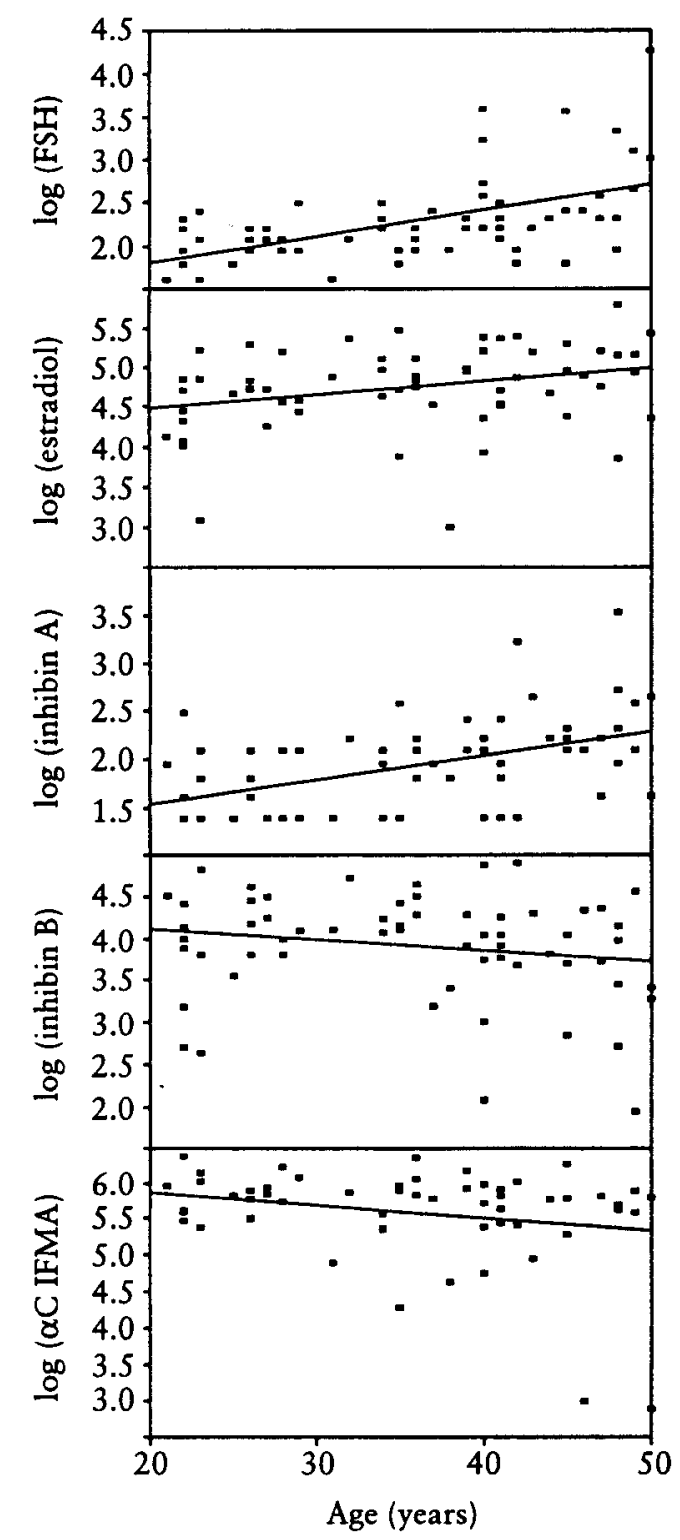

FIG. 3. Log-transformed concentrations of serum FSH, estradiol, inhibin A, inhibin B, and $\alpha$-subunit immunoactivity as functions of age $(n=59-63)$. C-terminal segment of $\alpha$ subunit; IFMA, immunofluorometric assay. [Reprinted with permission from Burger HG, Dudley EC, Mamers P, Groome N, Robertson DM 2000 Early follicular phase serum FSH as a function of age:the roles of inhibin B, inhibin A and estradiol. Climacteric 3:17-24. Copyright Parthenon Publishing Group.] 


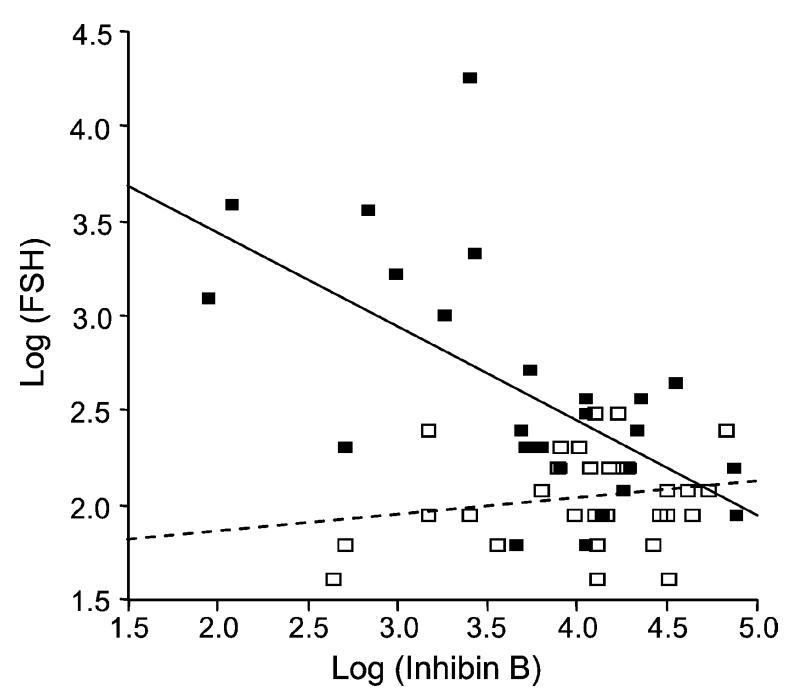

FIG. 4. Relationships between $\log (\mathrm{FSH})$ and $\log$ (inhibin B) in two age groups, 20-39 years ( $\square$ ) and 40-50 years $(\square)$. There was no significant correlation between the two hormones in the younger age group ( $r=0.19$, not significant) (dashed line) but, in the 40- to 50-year group, there was a significant inverse correlation $(r=-0.61, p<0.0001)$ solid line. FSH, follicle-stimulating hormone. [Reprinted with permission from Burger HG, Dudley EC, Mamers P, Groome N, Robertson DM 2000 Early follicular phase serum FSH as a function of age:the roles of inhibin B, inhibin A and estradiol. Climacteric 3:17-24. Copyright Parthenon Publishing Group.]

particularly from the dominant follicle and the ensuing corpus luteum (Roberts $e t$ al., 1993; Groome et al., 1996). INH-B, on the other hand, is a secretory product of small antral follicles (Roberts et al., 1993; Groome et al., 1996). Its circulating concentrations may reflect the number of follicles recruited from the primordial pool, the size of which decreases with increasing age (Richardson et al., 1987). Dominant follicles of older women continue to produce adequate amounts of $\mathrm{E}_{2}$ and INH-A and may do so as a result of the differential fall in INH-B, which allows an increased drive from the elevated concentrations of FSH.

Preservation of circulating $\mathrm{E}_{2}$ concentrations until late in female reproductive life could be hypothesised to be desirable for the preservation of overall health, including vascular and bone function.

\section{Hormonal Changes in Perimenopausal Women}

\section{A. FSH AND ESTRADIOL}

The most-noteworthy characteristic of the perimenopause is significant hormonal variability. A landmark study was performed by Sherman and Koren- 
man (1975), who reported on 50 complete menstrual cycles in 37 women. Ten women, aged 18 to 30 years with a history of regular cycles, served as controls. Six cycles were examined in regularly cycling women aged 46 to 51 , in which it was noted that the follicular phase was shorter than in younger women and that $\mathrm{E}_{2}$ concentrations were significantly lower than those observed in younger women. FSH was strikingly increased throughout the cycle, despite $\mathrm{E}_{2}$ concentrations that might have been expected to suppress its secretion. Daily hormone concentrations were measured in two women, one aged 49 and one aged 50, both clearly in the menopausal transition. Two of the cycles studied were anovulatory but were characterised by increasing $\mathrm{E}_{2}$ and initially postmenopausal values of LH and FSH, which subsequently fell with the rise of $\mathrm{E}_{2}$. An anovulatory cycle was followed by a cycle that demonstrated evidence of follicular maturation.

Metcalf and colleagues $(1979,1981)$ examined the excretion of FSH, LH, estrogens, and pregnanediol in weekly urine samples collected for 14 to 87 weeks from 31 perimenopausal women aged 36 to 55 years. Their study concentrated particularly on gonadotropin changes, although wide fluctuations in estrogen excretion were noted. These authors stated that "about the only conclusion that can be made with confidence concerning pituitary-ovarian function in individual perimenopausal women, is that it is unsafe to generalize." Metcalf (1988) subsequently concluded that "in older women, a good menstrual history is probably the single most useful measure of ovarian status." Hee et al. (1993) confirmed the variability of perimenopausal $\mathrm{E}_{2}$ concentrations and added data on immunoreactive INH in a small longitudinal study of three volunteer women who had developed irregular cycles at age 45 to 46 . Abrupt decreases in $E_{2}$ and INH into the postmenopausal range were followed by values characteristic of reproductive-aged women. It was striking that, depending on when during a cycle hormone values were determined, FSH, for example, could be at normal reproductive age levels, intermediately elevated levels, or levels characteristic of the postmenopause (Figure 5).

\section{B. STUDIES INCLUDING INHIBINS}

The Melbourne Women's Midlife Health Project was based on a crosssectional survey of a randomly selected population of 2001 Melbourne women, all Australian born, aged between 45 and 55 years at the time of initial interview (Dennerstein et al., 1993). A longitudinal study of 438 women was undertaken to examine many aspects of the menopausal transition. The data from the first year of this study were subjected to cross-sectional analysis in terms of menstrual cycle history (Burger et al., 1995). Of the subjects, 27\% reported no change in menstrual frequency or flow (Group I), 23\% a change in flow with no change in frequency (Group II), 9\% a change in frequency without change in flow (Group III), and $28 \%$ a change in both frequency and flow (Group V). By the time of 


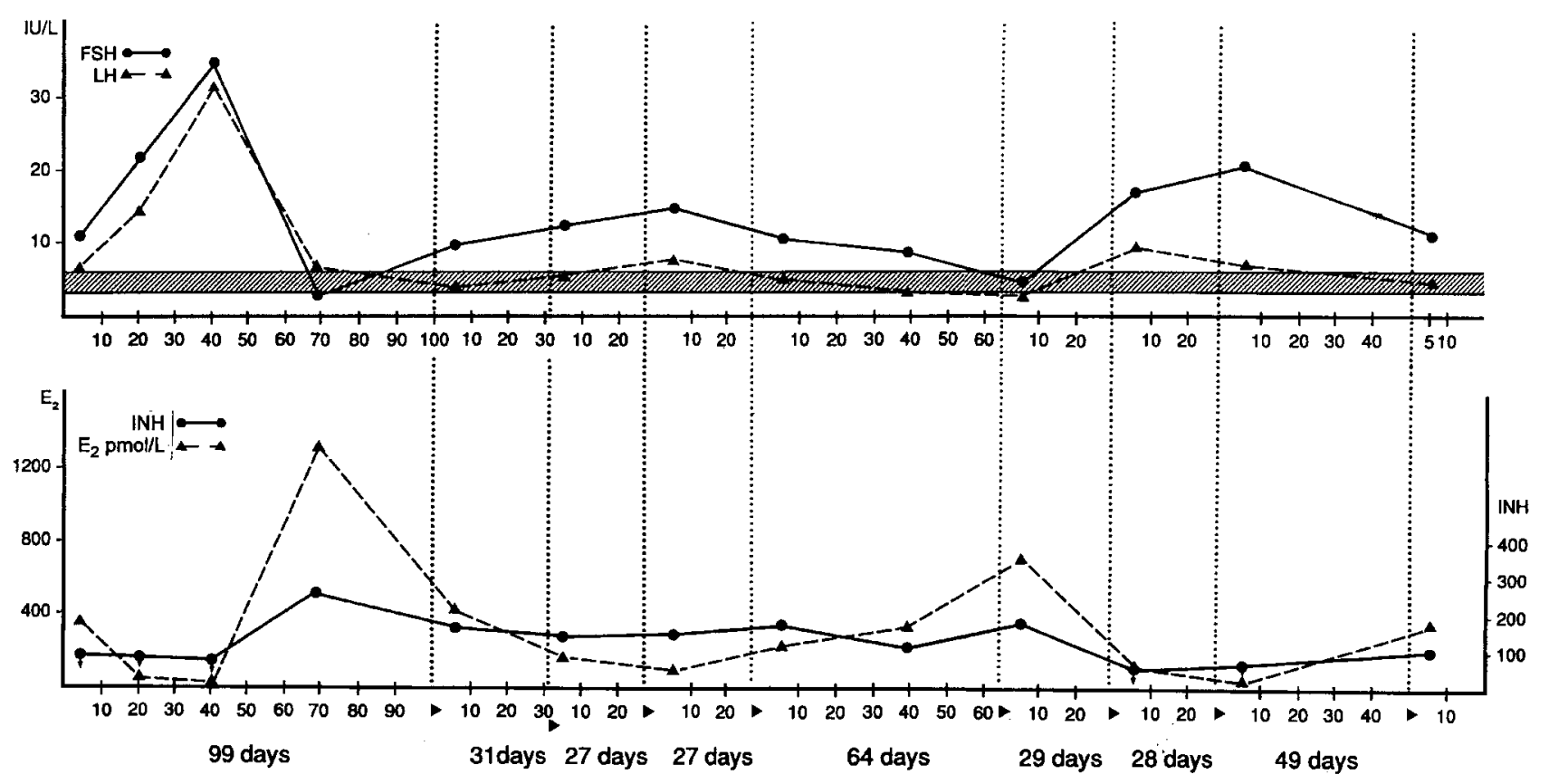

FIG. 5. Concentrations of FSH, LH, $\mathrm{E}_{2}$, and INH in a volunteer studied at the onset of the menopausal transition. The vertical dotted lines represent times of menses. The horizontal shaded bar represents the young-normal range for FSH. Note the marked fluctuations in hormone levels. [Reprinted from Hee J, MacNaughton J, Bangah M, Burger HG, Perimenopausal patterns of gonadotropins, immunoreactive inhibin, estradiol and progesterone. Maturitas 18:9-20. Copyright 1993, with permission of Elsevier Science.] 
blood sampling, $13 \%$ noted a lapse of at least 3 months since their last menstrual period. Mean age increased from 48.5 years in the first group to 51.4 in the last. Geometric mean serum FSH was similar in Groups I and II, slightly increased in Groups III and IV, and markedly increased in Group V (Figure 6). The marked variability in FSH levels between subjects in each group is noteworthy.

Although unadjusted $\mathrm{E}_{2}$ values were slightly lower in the groups experiencing a change in frequency, or those with changing frequency and flow (88 and $82 \%$ of those without any change), the only statistically significant decline in $\mathrm{E}_{2}$ occurred in those who had had no menses for at least 3 months (Group V), in whom geometric mean $\mathrm{E}_{2}$ concentration was $42 \%$ of that observed in the first group (Figure 7). After adjustment of $\mathrm{E}_{2}$ for age and body mass index (BMI), the only significant change was in the group with 3 months or more of amenorrhea, the $\mathrm{E}_{2}$ geometric mean being $54 \%$ of Group I women without change in menses. There was a very broad spread of $E_{2}$ values, some being greater than $1500 \mathrm{pmol} / \mathrm{l}$. These may reflect hyperstimulation of granulosa cells by elevated FSH levels and could give rise to symptoms of breast fullness and fluid retention.

Immunoreactive INH concentrations were significantly lower (71\% of those in the first group) in those experiencing a change in frequency and flow and had

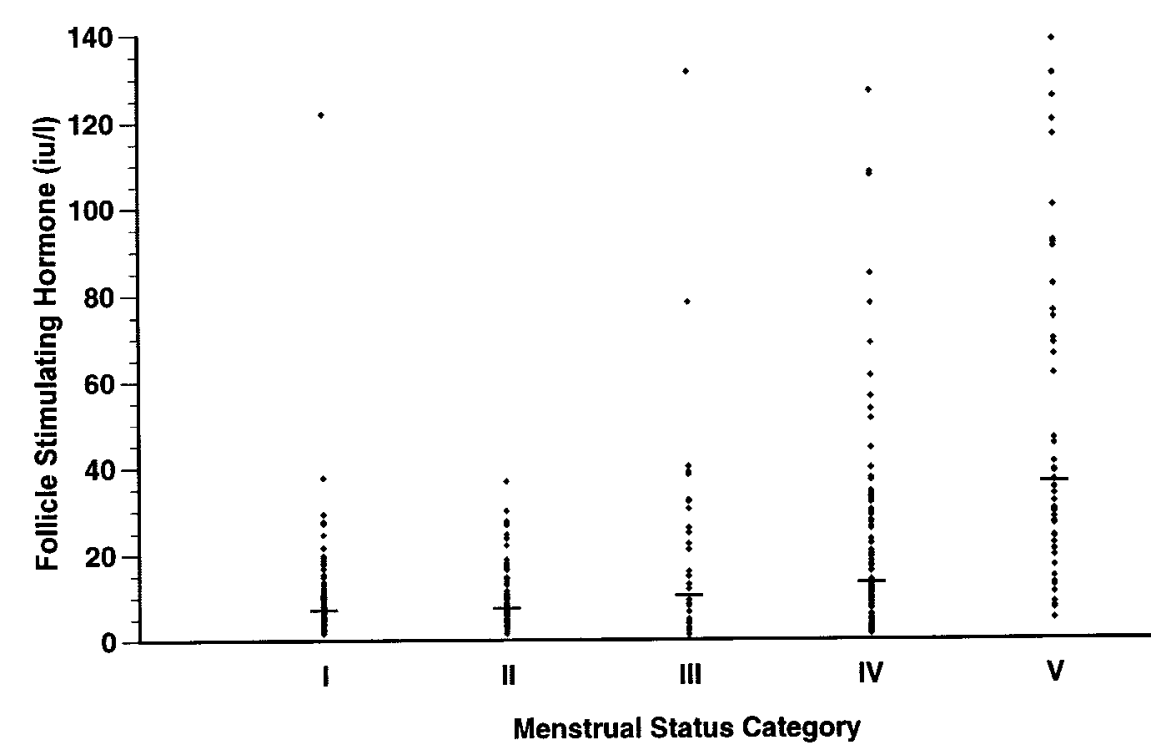

FIG. 6. Scatterplot of FSH levels versus menstrual status categories I to V. Geometric mean levels are shown by the horizontal bars. [Reprinted from Burger HG, Dudley EC, Hopper JL, Shelley JM, Green A, Smith A, Dennerstein L, Morce C 1995 The endocrinology of the menopausal transition: a cross-sectional study of a population-based sample. J Clin Endocrinol Metab 80:35373545. Copyright The Endocrine Society.] 


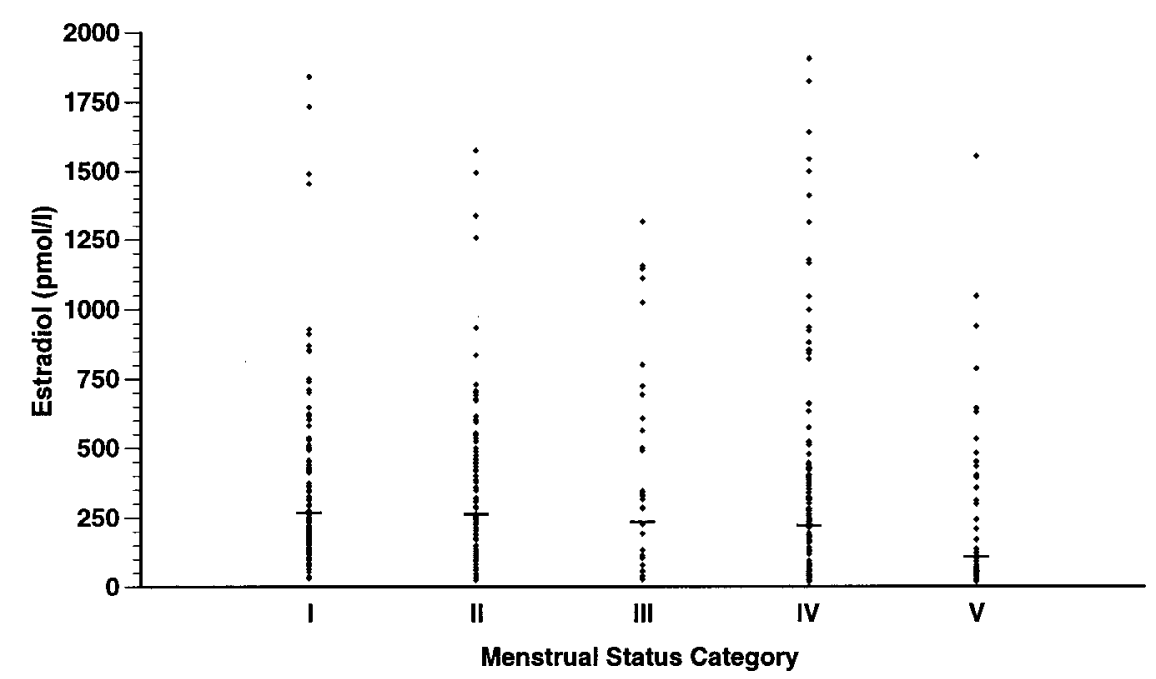

FIG. 7. Scatterplot of $E_{2}$ levels versus menstrual status categories I to V. Geometric mean levels are shown by the horizontal bars. [Reprinted with permission from Burger HG, Dudley EC, Hopper JL, Shelley JM, Green A, Smith A, Dennerstein L, Morce C 1995 The endocrinology of the menopausal transition: a cross-sectional study of a population-based sample. J Clin Endocrinol Metab 80:3537-3545. Copyright The Endocrine Society.]

fallen to $38 \%$ in those with 3 months or more of amenorrhea (Figure 8). After adjustment for age and BMI, only the change in the final group was significant, with a geometric mean $53 \%$ of that in Group I. These data suggested that decreases in inhibin occurred before decreases in $\mathrm{E}_{2}$, consistent with the hypothesis that declining concentrations of inhibin provide a mechanism for allowing FSH to rise, to maintain early follicular-phase $\mathrm{E}_{2}$ levels relatively intact.

A cross-sectional analysis of year 3 data was reported in 100 subjects aged 48 to 59 (Burger et al., 1998). Subjects were divided into those called premenopausal, without any change in menstrual cycle pattern (Group I); early perimenopausal, with a reported change in cycle frequency but experiencing menses in the preceding 3 months (Group 2); late perimenopausal, with no menses in the preceding 3-11 months (Group 3); and postmenopausal, with no menses for more than 12 months (Group 4). The hormone concentrations in the premenopausal subjects were used for reference. Early perimenopausal subjects had significantly lower levels of INH-B (13.5 ng/l, compared with $48 \mathrm{ng} / \mathrm{l})$ in the presence of a small, statistically nonsignificant rise in FSH (21.4, compared with $13.5 \mathrm{Iu} / \mathrm{l})$. There were no significant changes in $\mathrm{E}_{2}$ and $\mathrm{INH}-\mathrm{A}$. In late perimenopausal subjects, INH-A had fallen, whereas INH-B had not changed further and FSH had risen significantly to $72.2 \mathrm{Iu} / \mathrm{l}$. $\mathrm{E}_{2}$ fell to $89 \mathrm{pmol} / \mathrm{l}$ (compared with $306 \mathrm{pmol} / \mathrm{l}$ in the premenopausal group). The postmenopausal subjects showed no further 


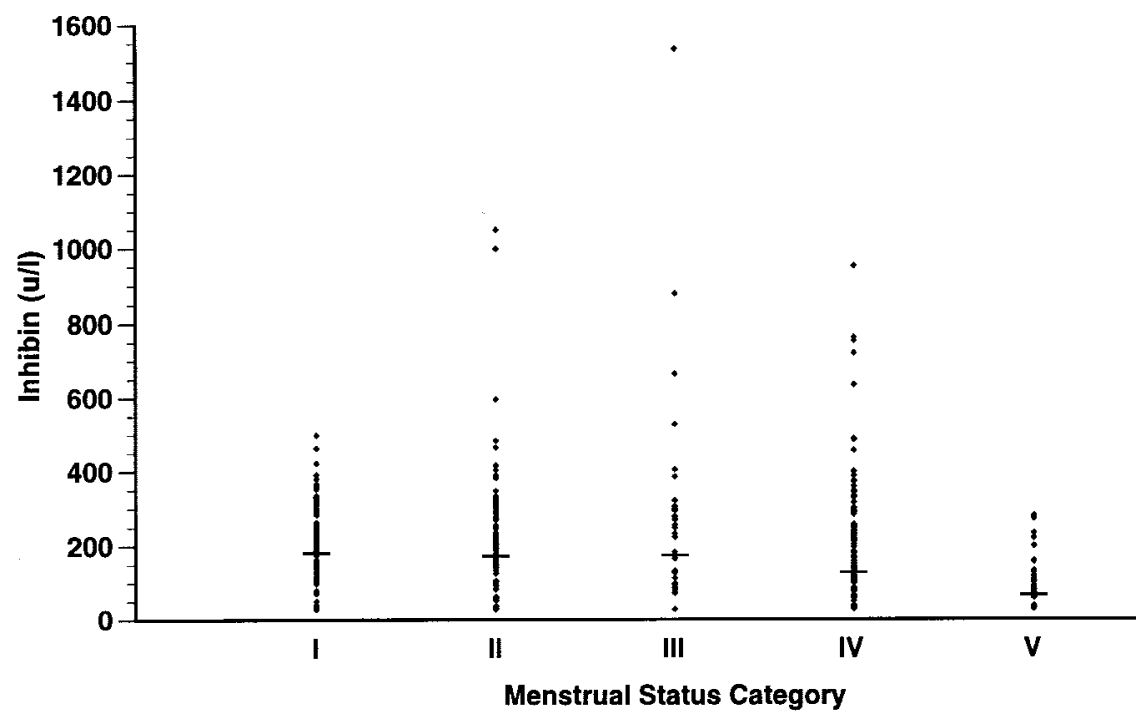

FIG. 8. Scatterplot of INH levels versus menstrual status categories I to V. Geometric mean levels are shown by the horizontal bars. [Reprinted with permission from Burger HG, Dudley EC, Hopper JL, Shelley JM, Green A, Smith A, Dennerstein L, Morce C 1995 The endocrinology of the menopausal transition: a cross-sectional study of a population-based sample. J Clin Endocrinol Metab 80:3537-3545. Copyright The Endocrine Society.]

significant changes in the peptide hormones or in $\mathrm{FSH}$. $\mathrm{E}_{2}$ fell further to 41 $\mathrm{pmol} / \mathrm{l}$ (Figure 9). There was a significant inverse correlation between FSH and $\mathrm{E}_{2}$, FSH and INH-A, and FSH and INH-B. Thus, it was concluded that the earliest endocrine change marking entry into the menopausal transition was a major fall in INH-B, presumably reflecting the attainment of a critically low number of ovarian follicles.

Overall, circulating $\mathrm{E}_{2}$ and inhibin concentrations may fluctuate widely in individual women during the menopausal transition. Grouped data show that mean changes in hormone levels become significant around the FMP, with a decrease in INH-B concentration in early perimenopausal women being the most important and significant initial endocrine event at that time. A subsequent report (Burger et al., 1999) analysed the data from the Melbourne Women's Midlife Health Project in relation to the date of final menses, rather than in relation to menstrual cycle status based on self report. It was noted that mean FSH levels began to increase starting about 2 years before FMP, increased most rapidly 10 months before FMP, and had plateaued by 2 years after FMP. $E_{2}$ levels began to decline about 2 years before FMP, decreased most rapidly around that time, and had plateaued 2 years later. There was, again, marked intersubject variability in hormone levels, 


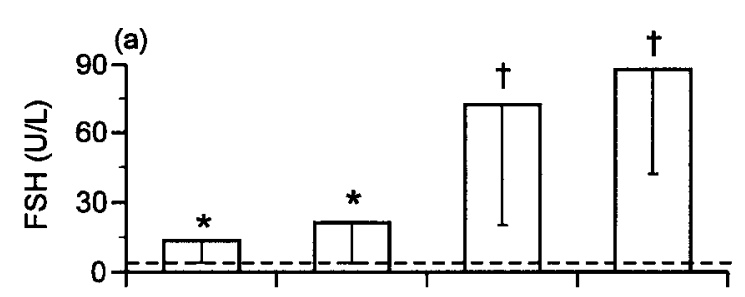

(b)

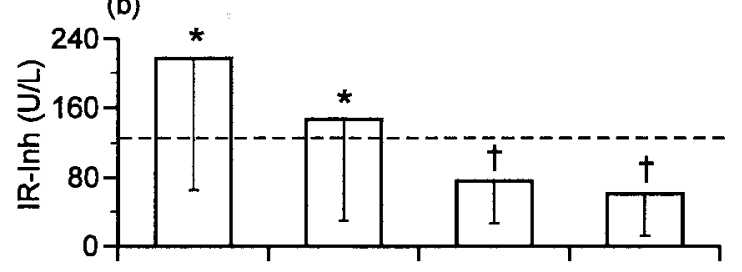

(c)

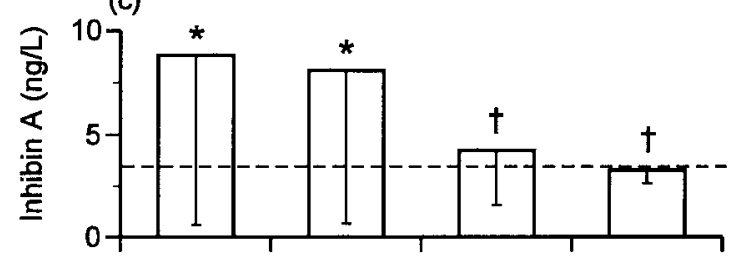

(d)

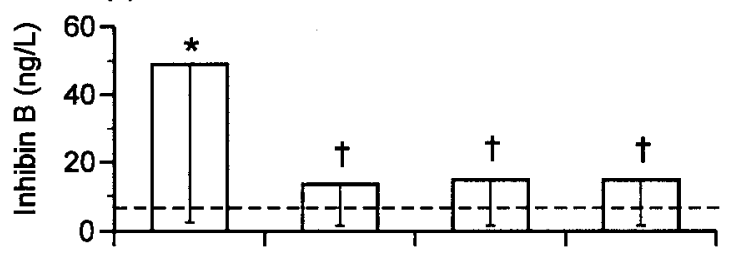

(e)

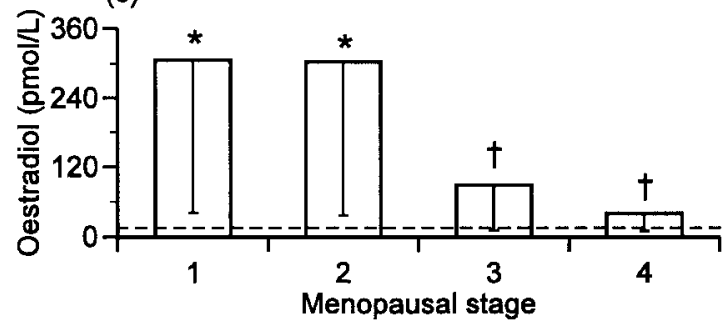

FIG. 9. Geometric mean levels (with lower 95\% confidence intervals) of (a) FSH, (b) IR-INH, (c) INH-A, (d) INH-B, and (e) $\mathrm{E}_{2}$ as a function of menopausal status. Menopausal stages are given in text. Values with the same superscript (* or $\dagger$ ) are not statistically different; values with differing superscripts differ, $\mathrm{P}<0.05$. [Reprinted with permission from Burger HG, Cahir N, Robertson DM, Groome NP, Green A, Dennerstein L 1998 Serum inhibins A and B fall differentially as FSH rises in perimenopausal women. Clin Endocrinol 48:809-813. Copyright Blackwell Science Ltd.] 
regardless of time in relation to FMP. Levels of both INH-A and INH-B decreased in the years before the FMP and were undetectable in the majority of women by the time of the FMP and almost all by 4 years post-FMP (Figure 10). The specificity and sensitivity of FSH and $\mathrm{E}_{2}$ measurements as markers of menopausal status were examined. The levels of serum FSH and $E_{2}$ at the

\section{A}

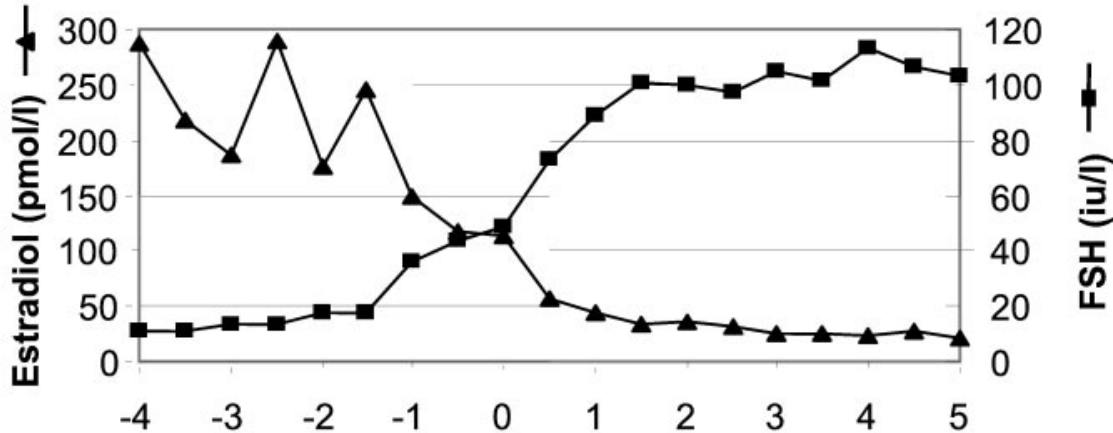

B

\section{Years around Menopause}

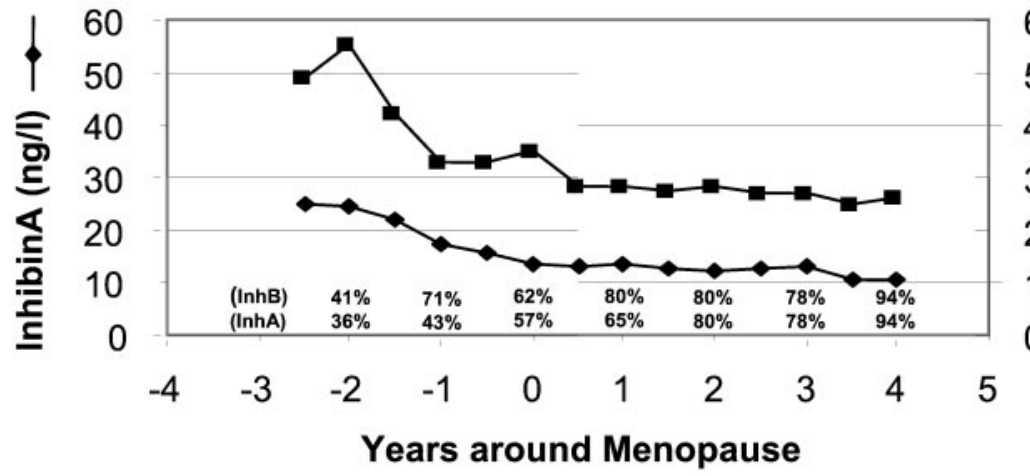

FIG. 10. Geometric means of (A) FSH and $\mathrm{E}_{2}$ and (B) INH-A and INH-B, in relation to the FMP. The horizontal axis represents time (yr) with respect to the FMP (0); negative (positive) numbers indicate time before (after) the FMP. The parentheses above the time scale (B) indicate the percentage of measured INH-A and INH-B at or below the assay sensitivity. [Reprinted with permission from Burger HG, Dudley EC, Hopper JL, Groome N, Guthrie JR, Green A, Dennerstein L 1999 Prospectively measured levels of serum FSH, estradiol and the dimeric inhibins during the menopausal transition in a population-based cohort of women. J Clin Endocrinol Metab 84:40254030. Copyright The Endocrine Society.] 
intersection of the fitted longitudinal curves for the geometric mean data with the date of final menses were calculated and found to be $108 \mathrm{Iu} / 1$ and 88.5 $\mathrm{pmol} / \mathrm{l}$, respectively. Seventy-six percent of premenopausal women had levels of FSH below this concentration and $67 \%$ had levels of $E_{2}$ above this concentration, whereas in postmenopausal women $85 \%$ had levels above that concentration of FSH and $84 \%$ levels of $\mathrm{E}_{2}$ below it.

It was concluded that substantial changes in reproductive hormone levels occurred within 1-2 years on each side of the FMP; that falling concentrations of $\mathrm{E}_{2}$ and the inhibins contribute to the rise in concentrations of FSH; and that there is no single, reliable hormonal marker of menopausal status for an individual woman.

\section{PROGESTERONE}

It is well known from studies in which basal body temperature had been used as a marker of ovulatory function that anovulatory cycles become more prevalent with increasing age. A large study of luteal-phase P concentrations (Trevoux et al., 1986) noted that the frequency of nondetectable P increased as FMP approached. The lack of a luteal-phase rise in $\mathrm{P}$ is a striking feature of the postmenopause, compared with the reproductive period. Rannevik et al. (1995) reported that the frequency of cycles with $\mathrm{P}$ values indicative of ovulation decreased from $60 \%$ to less than $10 \%$ during the 6 years preceding the FMP. Ovulatory P concentrations were found in $62.2 \%$ of women, $72-61$ months premenopausal and in $4.8 \%$ who were 6-0 months premenopausal, whereas all serum $\mathrm{P}$ measurements were less than $2 \mathrm{nmol} / \mathrm{l}$ postmenopausally.

\section{ANDROGENS}

Variable findings have been reported in regard to the change in circulating androgens in relation to the FMP. Rannevik et al. (1995) reported a small but significant decline in testosterone (T), androstenedione (A), and sex hormone-binding globulin (SHBG) during the 2 years around menopause. Longcope et al. (1986) did not see any change in T and A over 80 months from FMP but noted that the mean concentrations of $\mathrm{T}$ in all their subjects, including those still having cyclic menses, were significantly less than those of a group of normal young women sampled on days 5-7 of the cycle. They suggested that there is a decrease in the ovarian secretion of $\mathrm{T}$ prior to menopause. Zumoff et al. (1995), in fact, found that there was a steep decline in total serum $\mathrm{T}$ with age, such that levels in a woman aged 40 were approximately $50 \%$ of those in a woman aged 21 . Free $\mathrm{T}$ concentrations showed a similar decline. Other studies have suggested that total $\mathrm{T}$ levels decrease by approximately $20 \%$ and A by approximately $50 \%$ with natural menopause (Judd, 1976). Vermeulen et al. (1976) showed that postmeno- 
pausal women aged 51 to 65 years had lower mean levels of $\mathrm{T}, \mathrm{A}$, and dihydrotestosterone (DHT) than women aged 18 to 25 , not inconsistent with the observation of Zumoff et al. (1995).

In the Melbourne Women's Midlife Health Project, there was no significant change seen in total $\mathrm{T}$ or in the T:SHBG ratio (the free androgen index, FAI) as a function of changing menopausal status when assessed crosssectionally (Burger et al., 1995). More recently, serum androgen levels have been reported in relation to final menses using longitudinal data from the same project (Burger et al., 2000a). Mean T levels did not vary with time relative to FMP and were independent of age and BMI. FAI increased by $80 \%$ from 4 years before FMP to 2 years after because of a concomitant decrease in SHBG levels across this time period (Figures 11-13). DHEAS levels were not related to FMP but declined slowly with age. It was concluded that SHBG and FAI levels change at the time of menopause, at least partially due to the decline in $\mathrm{E}_{2}$. DHEAS decreases as a function of age but not relative to FMP. $\mathrm{T}$ remained unchanged through the menopausal transition and the early postmenopause.

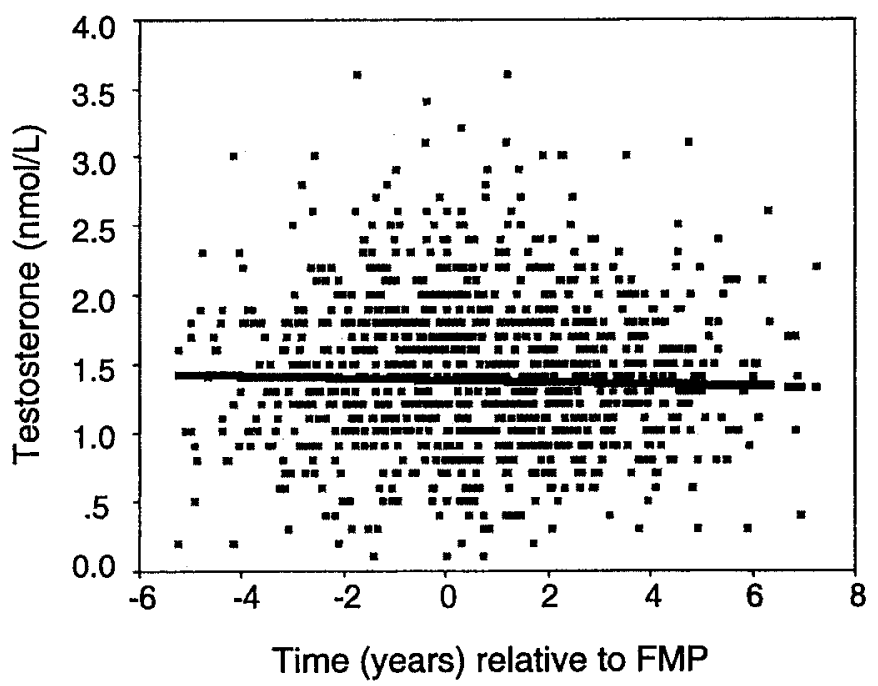

FIG. 11. Linear regression model: observed $\mathrm{T}$ and fitted levels of mean $\mathrm{T}$ across the menopausal transition. The horizontal axis represents time (years) with respect to FMP (0); negative (positive) numbers indicate time before (after) FMP. [Reprinted with permission from Burger HG, Dudley EC, Cui J, Dennerstein L, Hopper JL 2000 A prospective longitudinal study of serum testosterone dehydroepiandrosterone sulphate and sex hormone binding globulin levels through the menopause transition. J Clin Endocrinol Metab 85:2832-2938. Copyright The Endocrine Society.] 


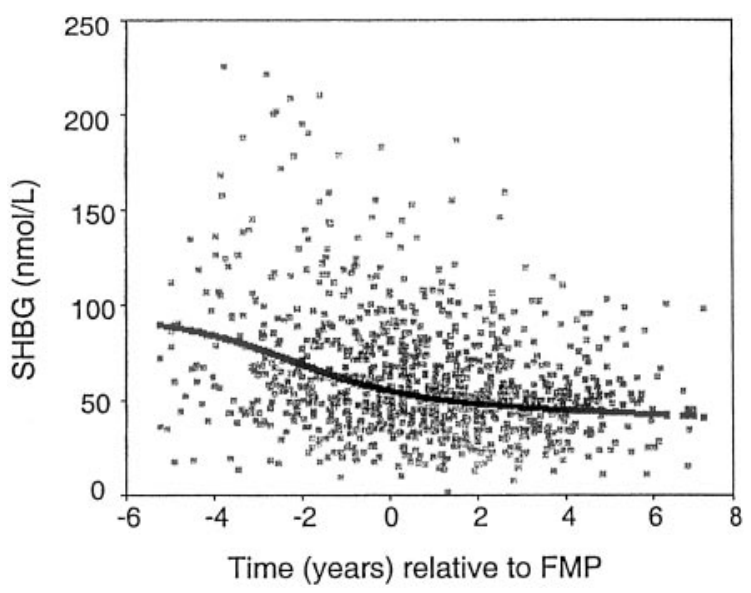

FIG. 12. Double logistic model: observed SHBG and fitted levels of mean SHBG across the menopausal transition. The horizontal axis represents time (years) with respect to FMP (0); negative (positive) numbers indicate time before (after) FMP. [Reprinted with permission from Burger HG, Dudley EC, Cui J, Dennerstein L, Hopper JL 2000 A prospective longitudinal study of serum testosterone dehydroepiandrosterone sulphate and sex hormone binding globulin levels through the menopause transition. J Clin Endocrinol Metab 85:2832-2938. Copyright The Endocrine Society.]

\section{Conclusions}

The perimenopause is a time of markedly fluctuating hormone levels. Attempts to define menopausal status purely on the basis of single measurements of FSH or $\mathrm{E}_{2}$ are unlikely to yield useful information. Though $\mathrm{E}_{2}$ concentrations appear to be preserved in regularly cycling women, at least until around age 50, INH-B declines and FSH rises. The establishment of menstrual irregularity is marked by a decrease in follicular-phase concentrations of INH-B, an increase in FSH, but relative preservation of $\mathrm{E}_{2}$ and INH-A until FMP. The frequency of anovulatory cycles increases markedly as FMP approaches. It is difficult to demonstrate substantial changes in androgen concentrations in the immediate perimenopausal period, though levels postmenopausally appear to be lower than those of young, regularly cycling women, perhaps as a function of increasing age during the reproductive years rather than menopausal status. Hormonal measurements are of little diagnostic value during the perimenopause, other than for purposes of physiological study. The issue of the appropriate reference points for the study of the perimenopause remains unclear, although the staging system adopted in the Melbourne Women's Mid-Life Health Project appears to be potentially useful because of the clear-cut hormonal (and symptomatic) differences between early and late perimenopausal women. 


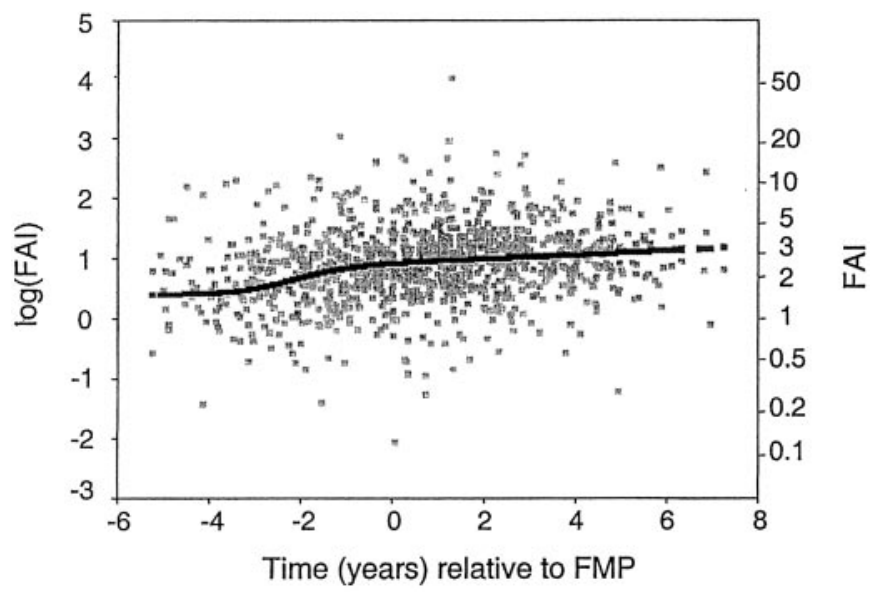

FIG. 13. Double logistic model: observed FAI and fitted levels of mean FAI across the menopausal transition. The left and right axes show FAI levels on the $\log$ and antilog scales, respectively. The horizontal axis represents time (years) with respect to FMP (0); negative (positive) numbers indicate time before (after) FMP. [Reprinted with permission from Burger HG, Dudley EC, Cui J, Dennerstein L, Hopper JL 2000 A prospective longitudinal study of serum testosterone dehydroepiandrosterone sulphate and sex hormone binding globulin levels through the menopause transition. J Clin Endocrinol Metab 85:2832-2938. Copyright The Endocrine Society.]

\section{ACKNOWLEDGMENTS}

The authors acknowledge with gratitude grants received from the Victorian Health Promotion Foundation and the National Health and Medical Research Council. Support for hormone assays was provided by an unrestricted grant from Organon (Australia) Pty Ltd. The assistance of Mr. N. Balazs from the Monash Medical Centre, Department of Chemical Pathology, for gonadotropin and steroid assays is gratefully recognised. Professor Nigel Groome (Oxford Brookes University, Oxford, U.K.) generously provided reagents for inhibin A and B assays. We thank Mrs. Sue Elger and Ms. Sue Panckridge for their assistance in preparing the manuscript.

\section{REFERENCES}

Burger HG, Dudley EC, Hopper JL, Shelley JM, Green A, Smith A, Dennerstein L, Morce C 1995 The endocrinology of the menopausal transition: a cross-sectional study of a populationbased sample. J Clin Endocrinol Metab 80:3537-3545

Burger HG, Cahir N, Robertson DM, Groome NP, Green A, Dennerstein L 1998 Serum inhibins $\mathrm{A}$ and $\mathrm{B}$ fall differentially as FSH rises in perimenopausal women. Clin Endocrinol 48:809-813

Burger HG, Dudley EC, Hopper JL, Groome N, Guthrie JR, Green A, Dennerstein L 1999 Prospectively measured levels of serum FSH, estradiol and the dimeric inhibins during the menopausal transition in a population-based cohort of women. J Clin Endocrinol Metab 84:4025-4030 
Burger HG, Dudley EC, Cui J, Dennerstein L, Hopper JL 2000a A prospective longitudinal study of serum testosterone dehydroepiandrosterone sulphate and sex hormone binding globulin levels through the menopause transition. J Clin Endocrinol Metab 85:2832-2938

Burger HG, Dudley EC, Cui J, Mamers P, Groome N, Robertson DM 2000b Early follicular phase serum FSH as a function of age:the roles of inhibin B, inhibin A and estradiol. Climacteric 3:17-24

Dennerstein L, Smith AM, Morse C, Burger HG, Green A, Hopper J, Ryan M 1993 Menopausal symptomatology: the experience of Australian women. Med J Aust 159:232-236

Groome NP, Illingworth PJ, O'Brien M, Rodger PAL, Rodger FE, Mather JF, McNeilly AS 1996 Measurement of dimeric inhibin B throughout the human menstrual cycle. J Clin Endocrinol Metab 81:1401-1405

Hee J, MacNaughton J, Bangah M, Burger HG 1993 Perimenopausal patterns of gonadotrophins, immunoreactive inhibin, estradiol and progesterone. Maturitas 18:9-20

Judd HL 1976 Hormonal dynamics associated with the menopause. Clin Obstet Gynecol 19:775-788

Klein NA, Illingworth PJ, Groome NP, McNeilly AS, Battaglia DE, Soules MR 1996 Decreased inhibin B secretion is associated with the monotropic rise of FSH in older, ovulatory women: a study of serum and follicular fluid levels of dimeric inhibin A and B in spontaneous menstrual cycles. J Clin Endocrinol Metab 81:2742-2745

Lee SJ, Lenton EA, Sexton L, Cooke ID 1988 The effect of age on the cyclical patterns of plasma LH, FSH, estradiol and progesterone in women with regular menstrual cycles. Hum Reprod 3:851-855

Longcope C, Franz C, Morello C, Baker R, Johnston C Jr 1986 Steroid and gonadotropin levels in women during the perimenopausal years. Maturitas 8:189-196

MacNaughton J, Bangah M, McCloud P, Hee J, Burger HG 1992 Age related changes in follicle stimulating hormone, luteinizing hormone, estradiol and immunoreactive inhibin in women of reproductive age. Clin Endocrinol 36:339-345

McKinlay SM, Brambilla DJ, Posner JG 1992 The normal menopausal transition. Maturitas 14:103-115

Metcalf MG 1988 The approach of menopause: a New Zealand Study. NZ Med J 101:103-106

Metcalf MG, Donald RA 1979 Fluctuating ovarian function in a permenopausal woman. NZ Med J 89:45-47

Metcalf, MG, Donald RA, Livesey JH 1981 Pituitary ovarian function in normal women during the menopausal transition. Clin Endocrinol 14:234-255

Rannevik G, Caristrom K, Jeppsson S, Bjerre B, Svanberg L 1986 A prospective long-term study in women from premenopause to postmenopause: changing profiles of gonadotrophins, oestrogens and androgens. Maturitas 8:297-307

Rannevik G, Jeppsson S, Johnell O, Bjerre B, Laurell-Boruli Y, Svanberg L 1995 A longitudinal study of the perimenopausal transition: altered profiles of steroid and pituitary hormones, SHBG and bone mineral density. Maturitas 21:103-113

Richardson SJ, Senikas V, Nelson JF 1987 Follicular depletion during the menopausal transition: evidence for accelerated loss and ultimate exhaustion. J Clin Endocrinol Metab 65:1231-1237

Roberts VJ, Barth S, El-Roeiy A, Yen SSC 1993 Expression of inhibin/activin subunits and follistatin messenger ribonucleic acids and proteins in ovarian follicles and the corpus luteum during the human menstrual cycle. J Clin Endocrinol Metab 77:1402-1410

Santoro N, Brown JR, Adel T, Skurnick JH 1996 Characterization of reproductive hormonal dynamics in the perimenopause. J Clin Endocrinol Metab 81:1495-1501

Sherman BM, Korenman SG 1975 Hormonal chacteristics of the human menstrual cycle throughout reproductive life. J Clin Invest 55:699-706 
Sherman BM, West JH, Korenman SG 1976 The menopausal transition: analysis of LH, FSH, estradiol, and progesterone concentrations during menstrual cycles of older women. J Clin Endocrinol Metab 42:629-636

Treloar AE 1981 Menstrual cyclicity and the pre-menopause. Maturitas 3:249-264

Trevoux R, De Brux J, Castanier M, Nahoul K, Soule J-P, Scholler R 1986 Endometrium and plasma hormone profile in the peri-menopause and postmenopause. Maturitas 8:309-326

Vermeulen A 1976 The hormonal activity of the postmenopausal ovary. J Clin Endocrinol Metab 42:247-253

World Health Organization 1996 Research on the Menopause in the 1990s. Technical report Scr 866, Geneva, Switzerland

Zumoff B, Strain GW, Miller LK, Rosner W 1995 Twenty-four hour mean plasma testosterone concentration declines with age in normal premenopausal women. J Clin Endocrinol Metab 80:1429-1430 



\title{
Role of Hormonal and Reproductive Factors in the Etiology and Treatment of Uterine Leiomyoma
}

\author{
CHERYL L. WALKeR \\ The University of Texas M.D. Anderson Cancer Center, Department of Carcinogenesis, \\ Science Park-Research Division, Smithville, Texas 78957
}

\begin{abstract}
Uterine leiomyomas are the most common gynecologic neoplasm in reproductive-age women. While it is clear that hormonal factors play a prominent role in this disease, how steroid hormones contribute to disease etiology or may be utilized as targets for intervention are currently areas of active scientific investigation. To study the impact of hormones on uterine leiomyomas, the Eker rat has been developed as an in vivo/in vitro animal model system for these tumors. Spontaneous leiomyomas arise in intact Eker rats with a high frequency and leiomyoma-derived cell lines from these animals maintain the biochemical and physiological characteristics of the tumors from which they were obtained. Using this animal model system, it has been established that tumor development is absolutely dependent on steroid hormones and that sensitivity/responsiveness to estrogen is enhanced in tumors and tumor-derived cell lines. Modulation of hormonal milieu, such as that which naturally occurs during pregnancy, can effectively inhibit tumor development. The hormone responsiveness of these tumors makes them good candidates for hormonal therapy. Selective estrogen receptor modulators (SERMs) tamoxifen and raloxifene hold promise as potential therapeutic agents for this disease. SERMs inhibit proliferation of leiomyoma-derived cell lines in vitro, repress the growth of these lines in nude mice, and, when administered over a 2- to 4-month course of treatment to Eker rats, reduce tumor incidence by more than $50 \%$. In addition to endogenous hormones, xenoestrogens in our environment (e.g., phytoestrogens, organochlorine pesticides, pharmacologic compounds) are of potential concern with regards to their impact on this disease. These environmental estrogens have been shown to promote the growth of leiomyoma cells in vitro and in vivo. Further elucidation of the role of these and other hormonal and reproductive factors in the development of uterine leiomyoma will be invaluable for increasing our understanding of the etiology of this disease and developing new therapeutic strategies to help to reduce the negative impact of uterine leiomyomas on women's health.
\end{abstract}

\section{Introduction}

Uterine leiomyomas, commonly referred to as "fibroids," are benign tumors arising from the myometrial compartment of the uterus. They are typically well differentiated, have a relatively low mitotic index, and retain their smooth muscle phenotype. Uterine leiomyomas are the most common gynecologic neoplasm, occurring with a remarkable frequency in more than $70 \%$ of reproductive age 
women (Cramer and Patel, 1990). Tumors can become quite large and are usually multiple. When symptomatic, they are associated with infertility, menorrhagia, and spontaneous abortion and are the leading indication for hysterectomy in premenopausal women (Buttram and Reiter, 1981).

\section{The Eker Rat Model for Uterine Leiomyoma}

The Eker rat has been extensively characterized as an in vitro/in vivo animal model for uterine leiomyoma (Everitt et al., 1995; Howe et al., 1995a). Female Eker rats carrying a germline mutation in the tuberous sclerosis 2 (Tsc-2) tumor suppressor gene develop uterine leiomyomas by 12-16 months of age with a frequency of $\approx 65 \%$ (Everitt et al., 1995). The predisposing genetic alteration in these animals is an insertion of an endogenous retrovirus between exons 30 and 31 of the Tsc- 2 gene, which inactivates the tumor suppressor protein encoded by this gene (Yeung et al., 1994; Kobayashi et al., 1995). The uterus is a site of high expression of tuberin, the product of the Tsc-2 gene, and tumors that arise in heterozygous animals show loss of tuberin function. At the DNA level, loss of heterozygosity $(\mathrm{LOH})$ at the Tsc-2 locus is commonly observed in these tumors, with loss of tuberin function occurring by several mechanisms, including loss of chromosome 10 on which the wild-type Tsc-2 gene is located (either monosomy or chromosome nondisjunction with retention of two copies of chromosome 10 containing the mutant allele), gene silencing, and point mutations (Yeung et al., 1995).

Uterine leiomyomas that arise in the Eker rat share many phenotypic characteristics with their cognate human disease. Tumors occur spontaneously with a high frequency in intact cycling females. They are often multiple and resemble human leiomyomas histologically (Everitt et al., 1995). Eker rat leiomyomas are benign and their malignant counterparts, uterine leiomyosarcomas, are rarely seen. The similarity in pathogenesis of uterine leiomyoma in Eker rats and women has made these animals useful as a model system to experimentally address questions related to the role of hormones in tumor development and hormonal and reproductive factors that can modulate or prevent the development of this disease.

\section{Eker Leiomyoma Tumor-derived Cell Lines}

Several cell lines have been developed from Eker rat uterine leiomyomas (Howe et al., 1995a). These cell lines have been given the designation ELT (for Eker leiomyoma tumor-derived) and, to date, five such lines have been established and characterized (Table I). All five cell lines are positive for expression of smooth muscle $\alpha$ and $\gamma$ actins and desmin by northern analysis and immunocytochemistry. As shown in Table I, the cell lines vary with respect to steroid 
TABLE I

Characteristics of ELT Cell Lines

\begin{tabular}{llllll}
\hline & ELT-3 & ELT-4 & ELT-6 & ELT-9 & ELT-10 \\
\hline $\begin{array}{l}\text { Smooth } \\
\text { muscle actin }\end{array}$ & Positive & Positive & Positive & Positive & Positive \\
$\begin{array}{l}\text { Desmin } \\
\text { Estrogen }\end{array}$ & Positive & Positive & Positive & Positive & Positive \\
receptor & Positive & Positive & Negative & Positive & Positive \\
$\begin{array}{l}\text { Progesterone } \\
\text { receptor }\end{array}$ & $\begin{array}{l}\text { Positive } \\
(\mathrm{Kd}=1.3 \mathrm{nM})\end{array}$ & Positive & Negative & Positive & Positive \\
$\begin{array}{l}\text { Tumorigenicity } \\
\text { in nude mice }\end{array}$ & Tumorigenic & $\begin{array}{l}\text { Nontumori- } \\
\text { genic }\end{array}$ & $\begin{array}{l}\text { Nontumori- } \\
\text { genic }\end{array}$ & $\begin{array}{l}\text { Nontumori- } \\
\text { genic }\end{array}$ & $\begin{array}{l}\text { Nontumori- } \\
\text { genic }\end{array}$ \\
\hline
\end{tabular}

hormone receptor expression and tumorigenicity, although, in general, these lines continue to express both estrogen and progesterone receptors in culture and are nontumorigenic in nude mice.

\section{Hormone-responsive Phenotype of Uterine Leiomyoma}

A substantial body of evidence from human clinical and epidemiological observations and experimental data obtained using the Eker rat model point to the hormone responsiveness of uterine leiomyomas. These tumors are most symptomatic in pre- and perimenopausal women and often change dramatically in size during pregnancy (Rossi and Diamond, 1992; Strobelt et al., 1994). The indication for hysterectomy due to symptomatic fibroids reaches a maximum incidence at 45 years of age, then declines dramatically, coinciding with the onset of menopause (Cramer, 1992). Uterine leiomyomas express estrogen receptors (ERs) and progesterone receptors (PRs) at levels that have been reported to be the same or higher than normal myometrium (Rein and Nowak, 1992; Brandon et al., 1993,1995; Viville et al., 1997; Englund et al., 1998; Nisolle et al., 1999). Treatment with gonadotropin-releasing hormone $(\mathrm{GnRH})$ agonists is a commonly used adjuvant therapy for these tumors. The hypoestrogenic state induced by these compounds effectively shrinks tumor volume (Andreyko et al., 1987; Adamson, 1992). However, the inhibition of steroid hormone production by GnRH agonists can cause a significant loss of bone mineral density (Dawood et al., 1989), limiting the course of treatment to less than 6 months. Moreover, cessation of therapy results in regrowth of these tumors, often rapidly (Friedman et al., 1990).

In the Eker rat model, the dependence of these tumors on ovarian hormones has been demonstrated unequivocally. Ovariectomy at 4 months of age virtually 
ablates tumor development, whereas animals receiving sham surgeries have a tumor incidence at 16 months of $\approx 65 \%$ (Walker et al., 2000). Similarly, analysis of cell proliferation in normal myometrium and leiomyomas of Eker rats suggests that these tumors have an enhanced responsiveness to the mitogenic effects of estrogens (Burroughs et al., 2000). As shown in Figure 1, in young animals, myometrial cell proliferation correlates well with estrogen levels, being highest when estrogen levels peak during proestrus. As the animal matures, however, myometrial cells become refractory to the mitogenic stimulus of estrogen. This phenomenon appears to be specific for mesenchymal cells of the uterus. While both smooth muscle myometrial cells and endometrial stomal cells become refractory to the proliferative effects of estrogen with age, this is not observed for either luminal or glandular epithelial cells of the endometrium (Burroughs et al., 2000). In contrast, leiomyomas exhibit a significantly elevated proliferative index relative to age-matched normal myometrium, suggesting an enhanced sensitivity to the mitogenic effects of steroid hormones in these tumors (Burroughs et al., 2000). This conclusion is supported by data using human leiomyoma explants, which demonstrated that tumor cells displayed an enhanced sensitivity to estro-

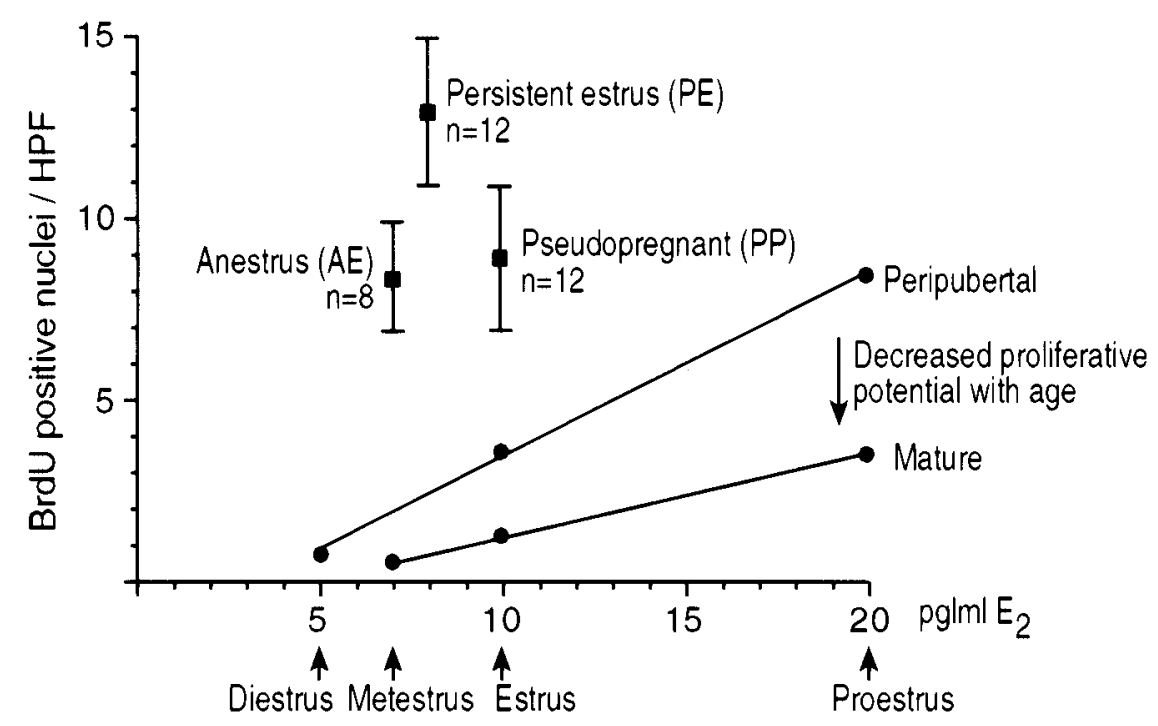

FIG. 1. Cell proliferation in normal myometrium and leiomyomas. Cell proliferation was measured by quantitation of BrdU incorporation into DNA followed by identification of BrdUpositive nuclei by immunohistochemistry using antibodies directed against BrdU. Cell proliferation positively correlated with serum estrogen levels but an overall decrease in the mitogenic response to estrogen occurred in the myometrium with age. Tumors exhibited an increased sensitivity/responsiveness to estrogen relative to normal myometrium. 
gen at the transcriptional level, relative to normal myometrial cells (Andersen et al., 1995).

It is important to appreciate that there are species-specific differences in how levels of estrogen and progesterone change during the menstrual cycle of women and the estrus cycle of rodents. In women, estrogen levels exhibit two peaks: one during late-follicular/proliferative phase of the cycle and a second, broader surge during the luteal/secretory phase. In contrast, progesterone levels are elevated only during the luteal phase of the cycle (Figure 2). Thus, while the luteal phase of the menstrual cycle is often considered to be "progesterone driven," it is important to appreciate that total exposure to estrogen during this phase of the cycle is higher or equal to that of the follicular phase in cycling women. In rodents, circulating estrogen levels peak in proestrus and drop precipitously during estrus following ovulation. Although estrogen levels begin to rise again during diestrus, these levels remain very much attenuated relative to the surge in estrogen that occurs during proestrus. In contrast, progesterone levels show a dramatic bimodal pattern. Peaks in progesterone levels occur during both early proestrus and metestrus (Figure 2), so that significant exposure to progesterone occurs in both early and late phases of the cycle. Therefore, in women, highest coincident estrogen and progesterone exposure occurs during the luteal phase of the cycle, whereas, in rodents, the highest coincident estrogen and progesterone levels occur in proestrus.

The biology of leiomyomas in rats and humans reflects this difference in physiology. For example, in leiomyomas, both cell proliferation and apoptosis vary as a function of the menstrual/estrus cycle. In humans, although the data are limited, leiomyomas appear to be most proliferative during the luteal phase of the cycle (Kawaguchi et al., 1989). Subsequent reports tend to support this earlier data (Nisolle et al., 1999; Wu and Somlo, 2000), although in one of these studies, the difference between proliferation occurring in the two phases did not reach statistical significance (Nisolle et al., 1999). In the Eker rat, empirical data have recently been obtained that demonstrate that cell proliferation in these tumors is maximal during proestrus, where hormone levels correspond to the high estrogen and progesterone levels that occur during the luteal phase in women. Conversely, in the normal myometrium, apoptosis is maximal during estrus, when steroid hormones are approaching their nadir. In addition, leiomyomas in the Eker rat model have been demonstrated to have a defective apoptotic program, relative to age-matched normal myometrium (Burroughs et al., 2000). Human data on apoptotic rates in leiomyoma are extremely limited, although there is some suggestion of such a defect from clinical data as well (Huang et al., 1997; Matsuo et al., 1997).

Work with ELT cell lines in vitro further supports the contention that leiomyomas have an enhanced sensitivity/responsiveness to steroid hormones. These cells retain estrogen and progesterone receptors and proliferate in response 

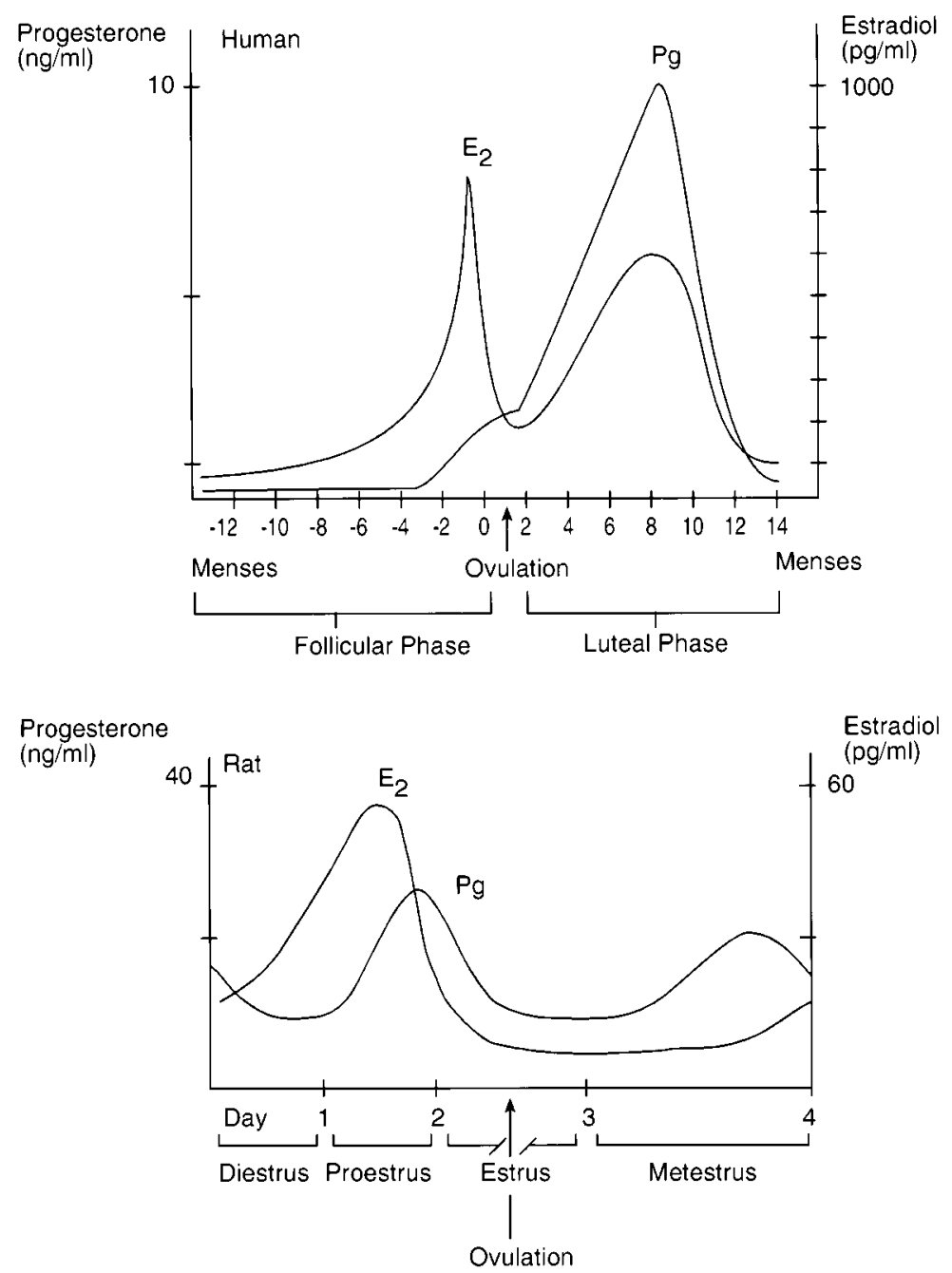

FIG. 2. Comparison of cycling hormone levels during the menstrual and estrus cycles. Species-specific differences occur in the cyclical levels of steroid hormones throughout the menstrual (human) and estrus (rodent) cycles. Estrogen exhibits a bimodal pattern during the menstrual cycle, whereas only a single peak in the level of this hormone occurs during the estrus cycle in rats. Progesterone levels also show differences between the two species, exhibiting a single peak during the menstrual cycle but exhibiting a bimodal pattern during the rat estrus cycle. [Data adapted from Speroff L, Vande Wiele RL 1971 Regulation of the human menstrual cycle. Am J Obstet Gynecol 109:234-247; and adapted with permission from Smith M, Freeman M, Neill J 1975 The control of progesterone secretion during the estrous cycle and early pseudopregnancy in the rat:prolactin, gonadotropin and steroid levels associated with rescue of the corpus luteum of pseudopregnancy. Endocrinology 96:219-226. Copyright The Endocrine Society.] 
to $10^{-6}$ to $10^{-10} \mathrm{M} 17-\beta$-estradiol (Howe et al., 1995b). This is fortuitous and contrasts with human myometrial and leiomyoma cells, which rapidly lose their receptors in tissue culture (Severino et al., 1996). In addition to responding to estrogen in vitro, leiomyoma cells also respond to this hormone in vivo. When ELT cells are injected subcutaneously into nude mice, they form tumors and grow as xenografts. With estrogen supplementation, mean tumor volume of these xenografts is increased two-fold (Howe et al., 1995b), again demonstrating the estrogen responsiveness of these tumor cells. Although hormone responsive, ELT cells do not require estrogen for cell growth, probably due to the insulin-like growth factor 1 (IGF-I) autocrine loop present in these cells (Howe et al., 1996). However, when deprived of growth factors via serum starvation, ELT cells will growth arrest and undergo apoptosis. Treatment of serum-starved cells with estrogen increases cell number. This rescue is mediated by enhanced cell proliferation rather than inhibition of apoptosis, which occurs at the same rate in the presence and absence of estrogen (Burroughs et al., 1997). Thus, estrogen appears to be a mitogen for leiomyomas that can modulate cell proliferation but has little effect on the apoptotic program of these tumors.

\section{Hormonal and Reproductive Factors That Modulate Tumor Development}

Several endogenous or environmental factors that modulate risk for developing uterine leiomyoma affirm the hormone responsive nature of this disease. Obesity and age at menarche have been linked to an increased risk for uterine leiomyoma, while cigarette smoking, use of oral contraceptives containing progesterone, and parity have been identified as protective factors (Ross et al., 1986; Parazzini et al., 1988,1992,1996a,b; Kjerulff et al., 1996, Marshall et al., 1997,1998a,b; Chiaffarino et al., 1999a,b). Obesity, particularly in peri- and postmenopausal women, increases levels of circulating estrogen through aromatization of fat stores, while early menarche increases overall exposure to circulating ovarian hormones. Cigarette smoking induces enzymes that can promote estrogen metabolism. The progesterone present in estrogen + progesterone oral contraceptives opposes the action of estrogen present in these formulations. In the case of pregnancy, the risk of uterine leiomyoma in parous women is approximately half that of nulliparous women. The risk of developing this disease decreases significantly with increased number of pregnancies (Ross et al., 1986; Parazzini et al., 1988,1996a,b). However, epidemiological data have been subject to interpretation as to whether pregnancy per se is protective or, as leiomyomas are a major cause of infertility, women that develop these tumors are less fertile and thus have lower pregnancy rates. Although the mechanism by which parity acts as a protective factor is not yet clear, additional experimental 
data obtained in the Eker rat model suggest that the hormonal milieu associated with pregnancy may help prevent tumor development (Table II).

Nulliparous, virgin female Eker rats develop leiomyomas with a frequency of $65 \%$. When these animals are bred with fertile males, a dramatic shift in tumor incidence and presentation is observed. As shown in Table II, in females allowed to have a single litter to confirm fertility, tumor incidence was $71 \%$ for gross and microscopic lesions combined, whereas in animals undergoing multiple rounds of pregnancy (average five litters/animal), tumor incidence was reduced to $\approx 10 \%$. This protective effect was particularly pronounced for tumors of the uterine cervix, the primary site of tumor development in fertile/nulliparous animals $(\approx 80 \%$ ), whereas less than $15 \%$ of the tumors arose in this location in animals undergoing multiple pregnancies (Walker et al., in press) (Figure 3).

Pregnancy has been shown to modulate the incidence of other types of hormone-dependent tumors, although the mechanisms of this protective effect appear to operate at different levels in different cell types. In breast cancer, early pregnancy is the most protective, suggesting an effect on the normal target cell population from which these tumors arise (Colditz, 1993; Hulka, 1996; Kelsey and Bernstein, 1996; Adami et al., 1998). Experimental animal studies have confirmed this hypothesis and suggest that differentiation of ductal epithelial

TABLE II

Impact of Hormonal and Reproductive Factors on Uterine Leiomyoma

\begin{tabular}{|c|c|c|c|c|}
\hline Condition & Ovariectomy & $\begin{array}{l}\text { SERM treatment } \\
\text { (antiestrogen) }\end{array}$ & $\begin{array}{l}\text { Targertin treatment } \\
\text { (RXR ligand, } \\
\text { antiestrogen) }\end{array}$ & Pregnancy \\
\hline $\begin{array}{l}\text { Expected tumor } \\
\text { incidence }\end{array}$ & $\begin{array}{l}\text { Gross }+ \text { micro } \\
23 / 34=68 \% \\
\text { (sham surgery) }\end{array}$ & $\begin{array}{l}\text { Gross }+ \text { micro } \\
26 / 31=84 \% \\
\text { (placebo) } 23 / 37 \\
=62 \% \text { (vehicle) }\end{array}$ & $\begin{array}{l}\text { Gross }=8 / 24 \\
\text { Micro }=1 / 24 \\
\text { (vehicle) }\end{array}$ & $\begin{array}{l}\text { Gross }+ \text { micro } \\
12 / 17=71 \% \\
\text { (single-litter } \\
\text { fertile females) }\end{array}$ \\
\hline $\begin{array}{l}\text { Observed tumor } \\
\text { incidence }\end{array}$ & $\begin{array}{l}\text { Gross }+ \text { micro } \\
1 / 31=3 \%\end{array}$ & $\begin{array}{l}\text { Gross }+ \text { micro } \\
10 / 29=35 \% \\
\text { (tamoxifen) } \\
11 / 30=37 \% \\
(\text { LY 326315) }\end{array}$ & $\begin{array}{l}\text { Gross }=4 / 38 \\
\text { Micro }=9 / 38\end{array}$ & $\begin{array}{l}\text { Gross }+ \text { micro } \\
\text { (in parous rats } \\
\text { having } 4-5 \\
\text { litters) } \\
6 / 58=10 \%\end{array}$ \\
\hline Modulation & $\begin{array}{l}95 \% \text { decrease in } \\
\text { tumor incidence } \\
(\mathrm{p} \leq 0.001)\end{array}$ & $\begin{array}{l}40-60 \% \\
\text { decrease in } \\
\text { tumor incidence } \\
(p \leq 0.05)\end{array}$ & $\begin{array}{l}65 \% \text { decrease in } \\
\text { macroscopic } \\
\text { lesions } \\
(\mathrm{p} \leq 0.05)\end{array}$ & $\begin{array}{l}86 \% \text { decrease in } \\
\text { tumor incidence } \\
(p \leq 0.001)\end{array}$ \\
\hline Reference & $\begin{array}{l}\text { Walker et al., } \\
2000\end{array}$ & $\begin{array}{l}\text { Walker et al., } \\
2000\end{array}$ & $\begin{array}{l}\text { Gamage et al. } \\
2000\end{array}$ & $\begin{array}{l}\text { Walker et al., in } \\
\text { press }\end{array}$ \\
\hline
\end{tabular}



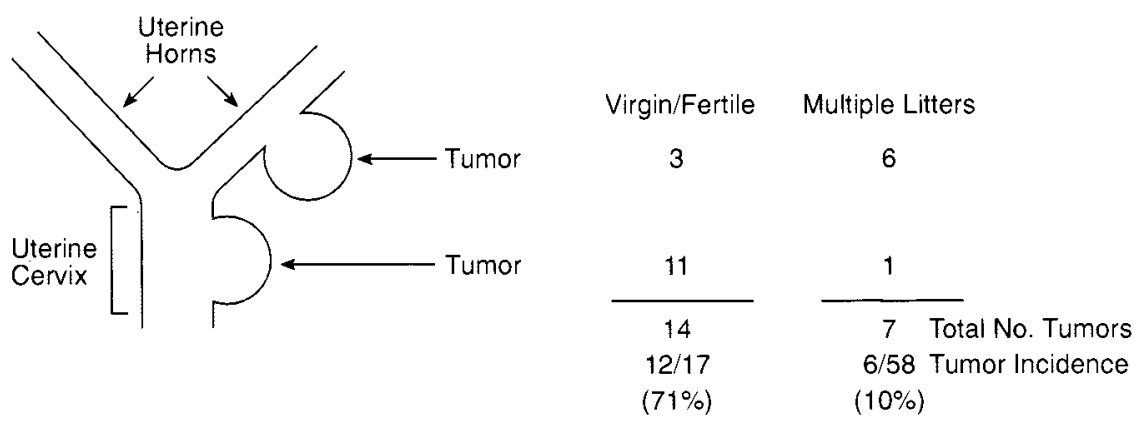

FIG. 3. Presentation of leiomyomas as a function of parity. In addition to reducing tumor incidence, pregnancy changes the spatial distribution of tumors. Virgin animals and those having a single litter (to confirm fertility) primarily develop tumors within the uterine cervix, whereas those undergoing multiple pregnancies develop tumors primarily on the uterine horns.

cells in the mammary gland mediated by the hormones of pregnancy contributes significantly to the protective effect of this condition (Abrams et al., 1998; Russo and Russo, 1998; Sivaraman et al., 1998; Guzman et al., 1999). In contrast, in the endometrium, time of last pregnancy appears to be a more important determinant. In women with only a single pregnancy, the most protection is afforded to women having a late rather than early pregnancy. This suggests that the protective effect is acting against the nascent neoplastic/preneoplastic cell population (Albrektsen et al., 1995; Hirose et al., 1996; Franchesschi, 1998; Lambe et al., 1999; Parslov et al., 2000). The fact that no protection against the development of uterine leiomyoma was afforded female Eker rats undergoing a single early pregnancy would suggest that the protective mechanisms of pregnancy in the myometrium are more similar to the endometrium (i.e., acting against nascent tumor cells) than the breast (protective differentiation).

Anderson and Barbieri first suggested an interesting hypothesis in which leiomyoma cells were described as resembling a myometrial cell of pregnancy rather than a typical myometrial cell under the influence of the menstrual cycle (Andersen et al., 1995). These similarities include increased levels of steroid hormone receptors, expression of IGF-I, and production of extracellular matrix proteins such as collagens type I and II. Furthermore, leiomyomas constitutively express high levels of connexin 43, the predominant connexin found in the myometrium during pregnancy (Andersen et al., 1993). Connexin 43 expression is negligible in myometrial cells during the normal menstrual cycle and early pregnancy; however, at term, connexin 43 becomes abundantly expressed under the influence of estrogen (Chow and Lye, 1994). This confirms that leiomyomas not only share the characteristics of myometrial cells during pregnancy but more specifically have the phenotype of these cells at parturition. At parturition, myometrial smooth muscle cells have a unique phenotype that differentiates 
them from myometrial cells at other stages of pregnancy. High levels of expression of a number of contraction-associated proteins (e.g., connexin 43, oxytocin receptors, cycloxygenase and its associated prostaglandins) are unique to the parturient myometrial cell. Leiomyoma cells share this phenotype but differ from the parturient myometrium in that they are neoplastic. Key biochemical and molecular differences must exist between a myometrial cell at parturition and a leiomyoma cell to account for this difference. An example of one such fundamental difference between myometrial cells and leiomyomas is their ability or inability, respectively, to undergo the biological program responsible for remodeling and involution of the uterus that occurs in a pregnant myometrial cell following parturition. In leiomyoma cells, this deficiency may be due to their hypersensitivity to steroid hormones, which may prevent them from triggering the normal dedifferentiation or apoptotic program of a parturient myometrial cell and returning to a nongravid, quiescent state (Cesen-Cummings et al., 2000).

Selective estrogen receptor modulators (SERMs) are a new class of synthetic compounds that bind to the estrogen receptor and exhibit tissue-specific agonist or antagonist activity (Jordan and Furr, 2001). Compounds such as tamoxifen and raloxifene are two prototypical SERMs currently in clinical use for prevention/ treatment of breast cancer and osteoporosis (Nayfield et al., 1991; Jordan, 1992; Fuchs-Young, 2001). Tamoxifen is a nonsteroidal triphenylethylene that is metabolized in vivo to several intermediates, the principal one being N-desmethyltamoxifen. Raloxifene is a benzothiophene derivative that binds to the estrogen receptor with nanomolar affinity and, like tamoxifen, acts as an estrogen agonist or antagonist, depending on cellular context. The tissue specificity observed for these compounds has been exploited to provide antagonism of estrogen action in the breast, while avoiding adverse side effects in other hormone-responsive tissues. Unfortunately, in the uterus, tamoxifen acts as an agonist in the endometrium and can promote the development of endometrial carcinoma (Hardell, 1988; Fornander et al., 1989; Kedar et al., 1994; Hulka, 1997). However, until recently, the effect of these or other SERMs on the myometrium and on leiomyomas had not been investigated. Preclinical data obtained from the Eker rat model now suggest that this class of compounds may be effective therapeutic agents for leiomyoma.

In culture, ELT cell lines proliferate in response to estrogen. Both basal and estrogen-induced cell proliferation are inhibited by 4-OH tamoxifen (a biologically active metabolite of tamoxifen) and raloxifene (Howe et al., 1995b,1996; Fuchs-Young et al., 1996). When these cells are grown as xenografts in nude mice, estrogen treatment increases tumor size, whereas tamoxifen treatment decreases tumor size and increases tumor latency (Howe et al., 1995b). Furthermore, in vivo studies in which Eker rats were treated with either tamoxifen or raloxifene for 2-4 months demonstrated that SERMs reduced tumor incidence by $\approx 50 \%$ and significantly reduced the size of the remaining tumors (Walker $e t$ 
al., 2000) (Table II). Importantly, sectioning of uteri and examination of microscopic lesions in treated rats confirmed a reduction in the absolute number of persistent lesions. This contrasts with the clinical experience with GnRH agonists, which primarily reduce tumor size but do not decrease tumor cellularity (Cohen et al., 1994). These preclinical data in the Eker rat suggest that, in contrast to GnRH agonists, SERMs such as tamoxifen and raloxifene, which both protect the bone and cardiovascular system and ablate leiomyomas, may have efficacy as medicinal therapeutic agents for leiomyoma. In support of the potential efficacy of SERMs, raloxifene recently has been reported to shrink leiomyoma volume in postmenopausal women (Palomba et al., 2001).

An interesting aspect of the preclinical studies with SERMs was the finding that while tumors from treated animals were significantly smaller and had a reduced mitotic index, there was no change in the apoptotic indices of the treated tumors relative to vehicle-treated controls (Walker et al., 2000). SERMs acting as anti-estrogens in the myometrium and leiomyomas were able to inhibit cell proliferation but were ineffective at inducing apoptosis. These in vivo data are consistent with the description of leiomyoma cells as having an apoptotic defect that is refractory to modulation by estrogen. However, in another preclinical study, the retinoid X receptor (RXR) ligand targretin (LGD 1069) was shown to be effective at inducing apoptosis in these cells (Table II) (Gamage et al., 2000). Targretin previously has been demonstrated to act as an estrogen antagonist and to have efficacy as a chemopreventive agent for hormone-dependent mammary cancer (Gottardis et al., 1996; Fitzgerald et al., 1997). Treatment of tumorbearing Eker rats for 4 months with targretin decreased the number of grossly observable tumors and significantly increased tumor apoptotic indices (Gamage et al., 2000). These data suggest that, while refractory to the influence of traditional anti-estrogens, the apoptotic program of leiomyomas may be modulated via other signaling cascades, such as that induced via RXRs.

\section{Potential Impact of Environmental Estrogens}

Xenoestrogens with endocrine-disrupting activity have been associated with the dysregulation of reproductive function and promotion of malignancies in experimental animals and human populations. With the recognition of the ubiquitous presence of many of these xenoestrogens in our environment, the high incidence of uterine leiomyomas in women has called into question the potential influence of xenoestrogens in the pathogenesis of these tumors. Using ELT cell lines, several in vitro assays have been developed to assess the estrogen-like agonist activity of potential endocrine disruptors on leiomyoma cells. These assays demonstrate that compounds from three major classes of xenoestrogens can mimic the effect of estrogen on leiomyoma cells and act as ER agonists: phytoestrogens, organochlorine pesticides, and pharmacologic agents. These 
compounds can stimulate proliferation of ELT cells in culture and transactivate expression of an ERE-reporter construct and an endogenous estrogen-responsive gene, the progesterone receptor, in leiomyoma cells (Hunter et al., 1999; Hodges et al., 2000). Diethylstilbestrol, the phytoestrogens coumesterol, genistein, and narigenin, and the organochlorine pesticides endosulfan, kepone, and HPTE (the active metabolite of metoxychlor) are all able to stimulate cell proliferation in estrogen-deficient medium. Additionally, the organochlorine pesticides methoxychlor, dieldrin, and toxaphene, while unable to stimulate cell proliferation, were able to act as agonists at the molecular level to transactivate the ER to induce expression of both ERE reporter genes and transcription of the PR. Interestingly, agonist activity by these compounds in leiomyoma cells requires activation of both AF1 and AF2 functions of the ER. Compounds that activate AF1 but fail to activate $\mathrm{AF} 2,4-\mathrm{OH}$ tamoxifen, and two raloxifene analogs act as antagonists in these cells (Hunter et al., 1999).

Significantly elevated levels of organochlorine pesticides previously have been detected in tumors and blood samples of women with leiomyoma (Saxena et al., 1987). These studies suggest a possible link between organochlorine pesticide exposure and the development of uterine leiomyomas. Organochlorine pesticides tend to bioaccumulate in fat stores and to be released during lactation and times of stress such as fasting. Under these conditions, exposure levels may be substantially higher within the body than those originally encountered in the environment. Furthermore, windows of susceptibility may exist at times when circulating estrogen levels are low. Exposure to even relatively low levels of xenoestrogens during windows of susceptibility, such as during the neonatal period, could produce deleterious effects on the reproductive system and potentially contribute to the development of leiomyomas. Several studies have implicated exposure to organochlorine pesticides such as dieldrin with an increased incidence of breast cancer (Wolff et al., 1993; Hoyer et al., 1998), although these data are controversial and recently have been called into question (Hunter et al., 1999).

\section{Summary}

It is clear that hormonal milieu has a tremendous impact on the growth and development of uterine leiomyoma. Using experimental animal models such as the Eker rat, it is possible to dissect these hormonal factors and determine their influence on specific aspects of tumor etiology and treatment in ways that are difficult or impossible to approach in humans. As shown in Figure 4, using the Eker rat model, it has been possible to clearly demonstrate that the development of leiomyoma is dependent on steroid hormones and this process can be modulated by several hormonal and reproductive factors. Tumor development is promoted by steroid hormones, and possibly by xenoestrogens, while other 


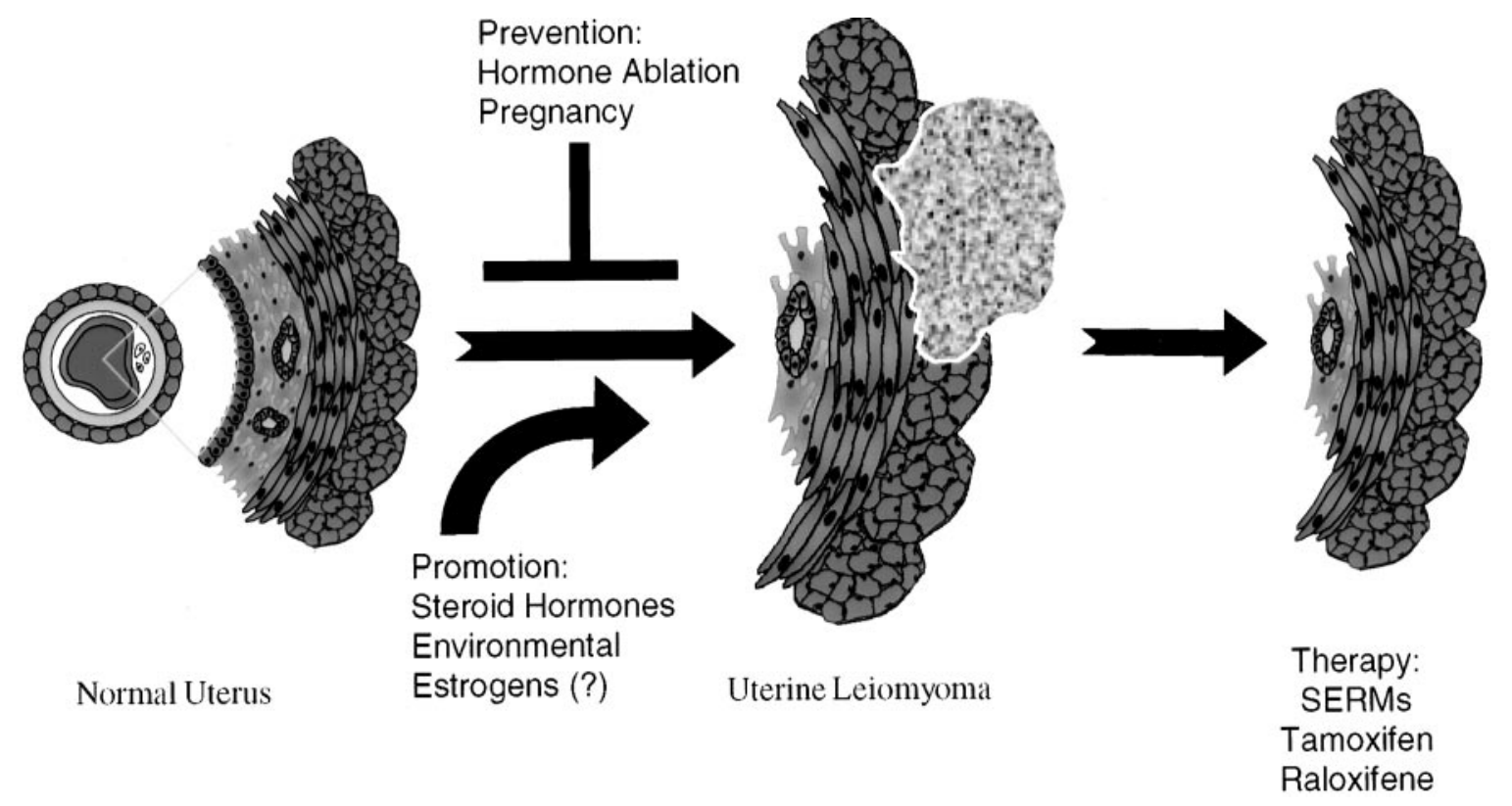

FIG. 4. Hormonal and reproductive factors that impact uterine leiomyomas. Uterine leiomyomas are hormone-dependent tumors and the development of this disease can be promoted by estrogens and inhibited by hormone ablation and pregnancy. SERMs have been demonstrated in preclinical studies to be effective at inhibiting tumor growth and offer promise as new therapeutic agents for this disease. 
alterations in a women's hormonal milieu, such as that which occurs during pregnancy, may prevent the development of these tumors. Because leiomyomas retain their hormone-responsive phenotype, they are amenable to hormonal therapy. Traditional adjuvant therapy with GnRH agonists has been demonstrated to reduce tumor burden and symptoms but induces serious side effects associated with the generalized hypoestrogenic milieu these drugs induce. Preclinical studies in the Eker rat model indicate that SERMs, which act as estrogen antagonists in leiomyomas while acting as estrogen agonists in the bone and cardiovascular system, hold promise as new candidate therapeutic agents for this disease.

\section{ACKNOWLEDGMENTS}

These studies were sponsored in part by funding from the National Institutes of Health grant ES08263 and CA72253 (C.L.W.), ES07784 and CA16672. We thank M. Gardiner for assistance with manuscript preparation and D. Hunter for comments and critical review of the manuscript.

\section{REFERENCES}

Abrams TJ, Guzman RC, Swanson SM, Thordarson G, Talamantes F, Nandi S 1998 Changes in the parous rat mammary gland environment are involved in parity-associated protection against mammary carcinogenesis. Anticancer Res 18:4115-4121

Adami HO, Signorello LB, Trichopoulos D 1998 Towards an understanding of breast cancer etiology. Semin Cancer Biol 8:255-262

Adamson GD 1992 Treatment of uterine fibroids: current findings with gonadotropin- releasing hormone agonists. Am J Obstet Gynecol 166:746-751

Albrektsen G, Heuch I, Tretli S, Kvale G 1995 Is the risk of cancer of the corpus uteri reduced by a recent pregnancy? A prospective study of 765,756 Norwegian women. Intl J Cancer 61:485-490

Andersen J, Grine E, Eng CL, Zhao K, Barbieri RL, Chumas JC, Brink PR 1993 Expression of connexin-43 in human myometrium and leiomyoma. Am J Obstet Gynecol 169:1266-1276

Andersen J, DyReyes VM, Barbieri RL, Coachman DM, Miksicek RJ 1995 Leiomyoma primary cultures have elevated transcriptional response to estrogen compared with autologous myometrial cultures. J Soc Gynecol Invest 2:542-551

Andreyko JL, Marshall LA, Dumesic DA, Jaffee RB 1987 Therapeutic uses of gonadotropinreleasing hormone analogs. Obstet Gynecol Surv 42:1-21

Brandon DD, Bethea CL, Strawn EY, Novy MJ, Burry KA, Harrington MS, Erickson TE, Warner C, Keenan EJ, Clinton GM 1993 Progesterone receptor messenger ribonucleic acid and protein are overexpressed in human uterine leiomyomas. Am J Obstet Gynecol 169: $78-85$

Brandon DD, Erickson TE, Keenan EJ, Strawn EY, Novy MJ, Burry KA, Warner C, Clinton GM 1995 Estrogen receptor gene expression in human uterine leiomyomata. J Clin Endocrinol Metab 80:1876-1881

Burroughs KD, Kiguchi K, Howe SR, Fuchs-Young R, Trono D, Barrett JC, Walker C 1997 Regulation of apoptosis in uterine leiomyomata. Endocrinology 138:3056-3064

Burroughs KD, Fuchs-Young R, Davis B, Walker CL 2000 Altered hormonal responsiveness of proliferation and apoptosis during myometrial maturation and the development of uterine leiomyomas in the rat. Biol Reprod 63:1322-1330 
Buttram V, Reiter R 1981 Uterine leiomyomata: etiology, symptomatology, and management. Fertil Steril 36:433-445

Cesen-Cummings K, Copland JA, Barrett JC, Walker CL, Davis BJ 2000 Pregnancy, parturition, and prostaglandins: defining uterine leiomyomas. Envir Health Perspect 108(suppl 5):817820

Chiaffarino F, Parazzini F, La Vecchia C, Chatenoud L, Di Cintio E, Marsico S 1999a Diet and uterine myomas. Obstet Gynecol 94:395-398

Chiaffarino F, Parazzini F, La Vecchia C, Marsico S, Surace M, Ricci E 1999b Use of oral contraceptives and uterine fibroids: results from a case-control study. Br J Obstet Gynaecol 106:857-860

Chow L, Lye SJ 1994 Expression of the gap junction protein connexin-43 is increased in the human myometrium toward term and with the onset of labor. Am J Obstet Gynecol 170:788-795

Cohen D, Mazur MT, Jozefczyk MA, Badawy SZA 1994 Hyalinization and cellular changes in uterine leiomyomata after gonadotropin releasing hormone agonist therapy. J Reprod Med 194:337-380

Colditz GA 1993 Epidemiology of breast cancer. Findings from the nurses' health study. Cancer 71:1480-1489

Cramer D 1992 Epidemiology of myomas. Semin Repro Endocrinol 10:320-324

Cramer S, Patel B 1990 The frequency of uterine leiomyomas. Am J Clin Pathol 94:435-438

Dawood MY, Lewis V, Ramos J 1989 Cortical and trabecular bone mineral content in women with endometriosis: effect of gonadotropin-releasing hormone agonist and danazol. Fertil Steril 52:21-26

Englund K, Blanck A, Gustavsson I, Lundkvist U, Sjoblom P, Norgren A, Lindblom B 1998 Sex steroid receptors in human myometrium and fibroids: changes during the menstrual cycle and gonadotropin-releasing hormone treatment. J Clin Endocrinol Metab 83:4092-4096

Everitt JI, Wolf DC, Howe SR, Goldsworthy TL, Walker C 1995 Rodent model of reproductive tract leiomyomata. Clinical and pathological features. Am J Pathol 146:1556-1567

Fitzgerald P, Teng M, Chandraratna RA, Heyman RA, Allegretto EA 1997 Retinoic acid receptor alpha expression correlates with retinoid-induced growth inhibition of human breast cancer cells regardless of estrogen receptor status. Cancer Res 57:2642-2650

Fornander T, Cedermark B, Mattsson A, Skoog L, Theve T, Askergren J, Rutqvist LE, Glas U, Silfversward C, Somell A, Wilking N, Hjalmar M-L 1989 Adjuvant tamoxifen in early breast cancer: occurrence of new primary cancers. Lancet 1:117-120

Franchesschi S 1998 Role of reproductive factors on the risk of endometrial cancer. Int J Cancer 76:784-786

Friedman AJ, Lobel SM, Rein MS, Barbieri RL 1990 Efficacy and safety considerations in women with uterine leiomyomas treated with gonadotropin-releasing hormone agonists: the estrogen threshold hypothesis. Am J Obstet Gynecol 163:1114-1119

Fuchs-Young R 2002 Preclinical studies of raloxifene and related compounds. In Jordan VC, Furr W, eds. Antiestrogens and Antiandrogens. Totowa, NJ: Humana Press, in press

Fuchs-Young R, Howe S, Hale L, Miles R, Walker C 1996 Inhibition of estrogen-stimulated growth of uterine leiomyomas by selective estrogen receptor modulators. Mol Carcinog 17:151-159

Gamage SD, Bischoff ED, Burroughs KD, Lamph WW, Gottardis MM, Walker CL, FuchsYoung R 2000 Efficacy of LGD1069 (Targretin), a retinoid X receptor-selective ligand, for treatment of uterine leiomyoma. J Pharmacol Exp Ther 295:677-681

Gottardis MM, Bischoff ED, Shirley MA, Wagoner MA, Lamph WW, Heyman RA 1996 Chemoprevention of mammary carcinoma by LGD1069 (Targretin): an RXR-selective ligand. Cancer Res 56:5566-5570 
Guzman RC, Yang J, Rajkumar L, Thordarson G, Chen X, Nandi S 1999 Hormonal prevention of breast cancer: mimicking the protective effect of pregnancy. Proc Natl Acad Sci USA 96:2520-2525

Hardell L 1988 Tamoxifen as risk factor for carcinoma of corpus uteri. Lancet 563(2):563

Hirose K, Tajima K, Hamajima N, Takezaki T, Inoue M, Kuroishi T, Kuzuya K, Nakamura S, Tokudome S 1996 Subsite (cervix/endometrium)-specific risk and protective factors in uterus cancer. Jpn J Cancer Res 87:1001-1009

Hodges LC, Bergerson JS, Hunter DS, Walker CL 2000 Estrogenic effects of organochlorine pesticides on uterine leiomyoma cells in vitro. Toxicol Sci 54:355-364

Howe S, Gottardis M, Everitt J, Goldsworthy T, Wolf D, Walker C 1995a Rodent model of reproductive tract leiomyomata: establishment and characterization of tumor-derived cell lines. Am J Pathol 146:1568-1579

Howe SR, Gottardis MM, Everitt JI, Walker C 1995b Estrogen stimulation and tamoxifen inhibition of leiomyoma cell growth in vitro and in vivo. Endocrinology 136:4996-5003

Howe SR, Pass HI, Ethier SP, Matthews WJ, Walker C 1996 Presence of an insulin-like growth factor I autocrine loop predicts uterine fibroid responsiveness to tamoxifen. Cancer Res 56:4049-4055

Hoyer AP, Grandjean P, Jorgensen T, Brock JW, Hartvig HB 1998 Organochlorine exposure and risk of breast cancer. Lancet 352:1816-1820

Huang SC, Chou CY, Lin YS, Tsai YC, Hsu KF, Liu CH, Huang KE 1997 Enhanced deoxyribonucleic acid damage and repair but unchanged apoptosis in uterine leiomyomas treated with gonadotropin-releasing hormone agonist. Am J Obstet Gynecol 177:417-424

Hulka BS 1996 Epidemiology of susceptibility to breast cancer. Prog Clin Biol Res 395:159-174

Hulka BS 1997 Epidemiologic analysis of breast and gynecologic cancers. Prog Clin Biol Res 396:17-29

Hunter DS, Hodges LC, Vonier PM, Fuchs-Young R, Gottardis MM, Walker CL 1999 Estrogen receptor activation via activation function 2 predicts agonism of xenoestrogens in normal and neoplastic cells of the uterine myometrium. Cancer Res 59:3090-3099

Jordan VC 1992 The role of tamoxifen in the treatment and prevention of breast cancer. Curr Prob Cancer 16:129-176

Jordan VC, Furr W, eds. 2002 Antiestrogens and Antiandrogens. Totowa, NJ: Humana Press

Kawaguchi K, Fuji S, Konishi I, Nanbu Y, Nonogaki H, Mori T 1989 Mitotic activity in uterine leiomyomas during the menstrual cycle. Am J Obstet Gynecol 160:637-641

Kedar RP, Bourne TH, Powles TJ, Collins WP, Ashley SE, Cosgrove DO, Campbell S 1994 Effects of tamoxifen on uterus and ovaries of postmenopausal women in a randomised breast cancer prevention trial. Lancet 343:1318-1321

Kelsey JL, Bernstein L 1996 Epidemiology and prevention of breast cancer. Annu Rev Public Health 17:47-67

Kjerulff KH, Langenberg P, Seidman JD, Stolley PD, Guzinski GM 1996 Uterine leiomyomas. Racial differences in severity, symptoms and age at diagnosis. J Reprod Med 41:483-490

Kobayashi T, Hirayama Y, Kobayashi E, Kubo Y, Hino O 1995 A germline insertion in the tuberous sclerosis (Tsc2) gene gives rise to the Eker rat model of dominantly inherited cancer. Nature Genet 9:70-74

Lambe M, Wuu J, Weiderpass E, Hsieh CC 1999 Childbearing at older age and endometrial cancer risk (Sweden). Cancer Causes Control 10:43-49

Marshall LM, Spiegelman D, Barbieri RL, Goldman MB, Manson JE, Colditz GA, Willett WC, Hunter DJ 1997 Variation in the incidence of uterine leiomyoma among premenopausal women by age and race. Obstet Gynecol 90:967-973 
Marshall LM, Spiegelman D, Goldman MB, Manson JE, Colditz GA, Barbieri RL, Stampfer MJ, Hunter DJ 1998a A prospective study of reproductive factors and oral contraceptive use in relation to the risk of uterine leiomyomata. Fertil Steril 70:432-439

Marshall LM, Spiegelman D, Manson JE, Goldman MB, Barbieri RL, Stampfer MJ, Willett WC, Hunter DJ 1998b Risk of uterine leiomyomata among premenopausal women in relation to body size and cigarette smoking. Epidemiology 9:511-517

Matsuo H, Maruo T, Samoto T 1997 Increased expression of Bc1-2 protein in human uterine leiomyoma and its upregulation by progesterone. J Clin Endocrinol Metab 82:293-299

Nayfield SG, Karp JE, Ford LG, Dorr A, Kramer BS 1991 Potential role of tamoxifen in prevention of breast cancer. J Natl Cancer Inst 83:1450-1459

Nisolle M, Gillerot S, Casanas-Roux F, Squifflet J, Berliere M, Donnez J 1999 Immunohistochemical study of the proliferation index, oestrogen receptors and progesterone receptors A and $\mathrm{B}$ in leiomyomata and normal myometrium during the menstrual cycle and under gonadotrophin-releasing hormone agonist therapy. Hum Reprod 14:2844-2850

Palomba S, Sammartino A, Di Carlo C, Affinito P, Zullo F, Nappi C 2001 Effects of raloxifene treatment on uterine leiomyomas in postmenopausal women. Fertil Steril 76:38-43

Parazzini F, Negri E, La Vecchia C, Fedele L, Rabaiotti M, Luchini L 1992 Oral contraceptive use and risk of uterine fibroids. Obstet Gynecol 79:430-433

Parazzini F, Negri E, La Vecchia C, Chatenoud L, Ricci E, Guarnerio P 1996a Reproductive factors and risk of uterine fibroids. Epidemiology 7:440-442

Parazzini F, Negri E, La Vecchia C, Rabaiotti M, Luchini L, Villa A, Fedele L 1996b Uterine myomas and smoking. Results from an Italian study. J Reprod Med 41:316-320

Parazzini F, La Vecchia C, Negri E, Cecchetti G, Fedele L 1988 Epidemiologic characteristics of women with uterine fibroids: a case-control study. Obstet Gynecol 72:853-857

Parslov M, Lidegaard O, Klintorp S, Pedersen B, Jonsson L, Eriksen PS, Ottesen B 2000 Risk factors among young women with endometrial cancer: a Danish case-control study. Am J Obstet Gynecol 182:23-29

Rein M, Nowak R 1992 Biology of uterine myomas and myometrium in vitro. Semin Reprod Endocrinol 10:310-319

Ross RK, Pike MC, Vessey MP, Bull D, Yeates D, Casagrande JT 1986 Risk factors for uterine fibroids: reduced risk associated with oral contraceptives. Br Med J (Clin Res Ed) 293: $359-362$

Rossi G, Diamond MP 1992 Myomas, reproductive function, and pregnancy. Semin Reprod Endocrinol 10:332-338

Russo IH, Russo J 1998 Role of hormones in mammary cancer initiation and progression. J Mammary Gland Biol Neoplasia 3:49-61

Saxena SP, Khare C, Farooq A, Murugesan K, Buckshee K, Chandra J 1987 DDT and its metabolites in leiomyomatous and normal human uterine tissue. Arch Toxicol 59:453-455

Severino MF, Murray MJ, Brandon DD, Clinton GM, Burry KA, Novy MJ 1996 Rapid loss of oestrogen and progesterone receptors in human leiomyoma and myometrial explant cultures. Mol Hum Reprod 2:823-828

Sivaraman L, Stephens LC, Markaverich BM, Clark JA, Krnacik S, Conneely OM, O’Malley BW, Medina D 1998 Hormone-induced refractoriness to mammary carcinogenesis in Wistar-Furth rats. Carcinogenesis 19:1573-1581

Smith M, Freeman M, Neill J 1975 The control of progesterone secretion during the estrous cycle and early pseudopregnancy in the rat: prolactin, gonadotropin and steroid levels associated with rescue of the corpus luteum of pseudopregnancy. Endocrinology 96:219-226

Speroff L, Vande Wiele RL 1971 Regulation of the human menstrual cycle. Am J Obstet Gynecol 109:234-247 
Strobelt N, Ghidini A, Cavallone M, Pensabene I, Ceruti P, Vergani P 1994 Natural history of uterine leiomyomas in pregnancy. J Ultrasound Med 13:399-401

Viville B, Charnock-Jones DS, Sharkey AM, Wetzka B, Smith SK 1997 Distribution of the A and $\mathrm{B}$ forms of the progesterone receptor messenger ribonucleic acid and protein in uterine leiomyomata and adjacent myometrium. Hum Reprod 12:815-822

Walker CL, Burroughs KD, Davis B, Sowell K, Everitt JI, Fuchs-Young R 2000 Preclinical evidence for therapeutic efficacy of selective estrogen receptor modulators for uterine leiomyoma. J Soc Gynecol Investig 7:249-256

Walker CL, Cesen-Cummings K, Houle C, Baird D, Barrett JC, Davis B 2002 Protective effect of pregnancy for development of uterine leiomyoma. Carcinogenesis, in press

Wolff MS, Toniolo PG, Lee EW, Rivera M, Dubin N 1993 Blood levels of organochlorine residues and risk of breast cancer. J Natl Cancer Inst 85:648-652

Wu G, Somlo S 2000 Molecular genetics and mechanism of autosomal dominant polycystic kidney disease. Mol Genet Metab 69:1-15

Yeung RS, Xiao GH, Jin F, Lee WC, Testa JR, Knudson AG 1994 Predisposition to renal carcinoma in the Eker rat is determined by germ-line mutation of the tuberous sclerosis 2 (TSC2) gene. Proc Natl Acad Sci USA 91:11413-11416

Yeung RS, Xiao GH, Everitt JI, Jin F, Walker CL 1995 Allelic loss at the tuberous sclerosis 2 locus in spontaneous tumors in the Eker rat. Mol Carcinog 14:28-36 


\title{
Definition of the Molecular and Cellular Mechanisms Underlying the Tissue-selective Agonist/Antagonist Activities of Selective Estrogen Receptor Modulators
}

\author{
Donald P. McDonnell, Caroline E. Connor, Ashini Wijayaratne, \\ ChING-YI Chang, AND John D. NORRIS \\ Department of Pharmacology and Cancer Biology, Duke University Medical Center, \\ Durham, North Carolina 27710
}

\begin{abstract}
The term selective estrogen receptor modulators describes a group of pharmaceuticals that function as estrogen receptor (ER) agonists in some tissues but that oppose estrogen action in others. Although the name for this class of drugs has been adopted only recently, the concept is not new, as compounds exhibiting tissue-selective ER agonist/antagonist properties have been around for nearly 40 years. What $i s$ new is the idea that it may be possible to capitalize on the paradoxical activities of these drugs and develop them as target organ-selective ER agonists for the treatment of osteoporosis and other estrogenopathies. This realization has provided the impetus for research in this area, the progress of which is discussed in this review.
\end{abstract}

\section{Introduction}

Selective estrogen receptor modulators (SERMs) are a class of estrogen receptor (ER) ligands that, depending on the cell and tissue in which they operate, can function as agonists or antagonists (McDonnell, 1999). Initially classified as partial agonists, the realization that the relative agonist/antagonist activities of SERMs can differ between cells has indicated that they constitute a pharmacologically distinct class of compounds. This misclassification was apparent as early as 1967 when the properties of the "anti-estrogen" tamoxifen were first described. Specifically, it was observed that this compound could function as an antagonist in the reproductive systems of mice, a partial agonist in rats, and a pure antagonist in chickens (Harper and Walpole, 1967). The true significance of these findings - which indicated that tamoxifen could oppose estrogen action in some environments but mimic the action of the hormone in others - was not realized until much later, although, in retrospect, it was clear that the SERM concept was already developing (Kurl and Borthwick, 1980; Beall et al., 1984; Turner et al., 1987). It was not until a randomized clinical study, aimed at evaluating the impact of tamoxifen chemotherapy on skeletal integrity in breast 
cancer patients, was performed that the beneficial effects of SERM action became clear (Love et al., 1992). Unexpectedly, it was found that although tamoxifen functioned as an anti-estrogen in breast, it exhibited significant estrogenic activity in the skeletal and cardiovascular systems. These compelling data suggested that SERMs might have utility outside the realm of oncology as treatments for osteoporosis, cardiovascular disease, and other conditions associated with hypoestrogenicity. Indeed, were it not for the fact that tamoxifen can function as an ER agonist in the uterus and elevates a woman's risk of getting uterine cancer, it is possible that this drug could have been developed as an antiresorbtive therapy for osteoporosis (Ismail, 1994). Fortunately, other compounds were available that had more favorable therapeutic profiles. One drug in particular, keoxifene (subsequently renamed raloxifene), was found to function similarly to tamoxifen (as an anti-estrogen) in breast tumors but functioned as a pure antagonist in the uterus (Clemens et al., 1983; Poulin et al., 1989). This drug emerged from a discovery program aimed at developing an anti-estrogen that could be used for the treatment of tamoxifen-refractory breast cancers. Whereas it was not found to be effective as a breast cancer chemotherapeutic, its ability to function as an agonist in bone, but not uterus, led to the approval of this drug for the treatment and prevention of osteoporosis (Turner et al., 1994; Black et al., 1994). Thus, the SERM concept was established and the search for improved drugs of this class took off and continues today at a frantic pace.

\section{The Unmet Medical Need for SERMs with Improved Therapeutic Profiles}

The therapeutic profile of raloxifene makes it appealing to women who do not want to take classical estrogen-containing medicines. However, it is clearly not a substitute for established estrogen replacement therapies (Delmas et al., 1997; Ettinger et al., 1999). On the positive side, it was determined that raloxifene was about $30 \%$ as effective as estrogen in bone, had insignificant uterotrophic activity, and lowered low-density lipoprotein-cholesterol without elevating triglycerides (Delmas et al., 1997). Unlike estrogen, it was unable to suppress hot flashes and exacerbated other climacteric conditions associated with menopause (Delmas et al., 1997). However, the main impediments to a wider acceptance of the existing SERMs are the unknowns. Estrogens have been shown to have beneficial effects in the cardiovascular and central nervous system (CNS) (Zumoff, 1993; Yaffe et al., 1998). However, since we do not know the mechanism(s) underlying these positive activities of estrogens, there is a possibility that administration of a SERM, which has the potential to manifest agonist or antagonist activity, may lead to a deterioration of function in these systems. These limitations not withstanding, it is the initial data that demonstrated that breast cancer incidence could be reduced by up to $72 \%$ in postmenopausal 
women taking raloxifene that suggested that SERMs will become a mainstream medicine (Cummings et al., 1999). Although it remains to be seen if this initial suppression of breast cancer incidence translates into improvements in survival, this finding has encouraged pharmaceutical manufacturers to launch major efforts to search for the perfect SERM (Willson et al., 1997; Ke et al., 1998). Clearly, despite the beneficial effects of raloxifene, it is generally held that better SERMs remain to be identified. From what is known about the next generation of SERMs now under development, it appears that they will display an advantage over raloxifene with respect to potency (Ke et al., 1998; Neven, 2000). Apart from improved pharmaceutical properties, however, it is not clear if secondgeneration SERMs will have a therapeutic profile that is better than raloxifene. It is likely that, as we begin to understand the molecular mechanisms that determine the relative agonist/antagonist activities of these first- and secondgeneration SERMs, we will be in a position to develop mechanism-based screens for compounds with improved therapeutic profiles. Since millions of years of evolution have enabled cell-signaling systems to optimally recognize the ERestradiol complex, it is unlikely that a compound can be developed that will possess all of the positive and none of the negative attributes of estradiol. However, given what we have learned thus far about estrogen action, it does appear that we can improve over current SERMs and that, in the future, we may have an expanded family of such compounds that may have different target site specificities. How - and in what direction - this field develops is now in the hands of the basic scientist. It is unlikely that truly novel therapies can be developed until the mechanisms of action of estrogens and SERMs in bone, the CNS, or the cardiovascular system are known and this information is incorporated into drug screens.

\section{Lessons in SERM Action from Studying Tamoxifen Resistance}

Until recently, tamoxifen was considered to have a relatively simple mechanism of action, functioning as a competitive antagonist that opposed the binding of estrogen to its receptor (Clark and Peck, 1979; Clark and Markaverich, 1988). Not surprisingly therefore, much of the past research on this drug has focused on defining how it functioned as an anti-estrogen. Now, research interests have broadened to probe the mechanism(s) by which the SERM activity of tamoxifen is manifest. Some investigators have focused on determining how resistance to tamoxifen arises in breast tumors, whereas others are interested in how it (and related compounds) can function as tissue-selective agonists/antagonists. We believe that these two apparently dichotomous actions of tamoxifen are, in fact, related. Thus, several years ago, we considered that insights into SERM action could be gained from studying the mechanism(s) by which breast cancer cells escape the antagonist actions of tamoxifen. 
Tamoxifen was initially approved as an anti-estrogen in 1978 for the treatment of metastatic breast cancer and as an adjuvant chemotherapeutic in noninvasive disease (Osborne, 1998). A large number of studies have demonstrated a significant impact of this drug on both disease-free and overall survival (Gockerman et al., 1986; Fisher et al., 1996,2001; Early Breast Cancer Trialists' Collaborative Group, 1998). However, in the metastatic setting, tamoxifen failure (resistance) eventually arises within the first 5 years of treatment (Touchette, 1992; Tonetti and Jordan, 1995). Initially, this was considered to reflect classical resistance where receptor mutations, changes in tamoxifen metabolism, or multiple drug resistance systems were considered to be the primary mechanism(s) responsible (Osborne et al., 1991,1992; Sluyser, 1992; Karnik et al., 1994; Tonetti and Jordan, 1995). However, several pieces of evidence have suggested that it may not be as simple as initially suspected and that failure actually may reflect a change in the biocharacter of tamoxifen in which it switches from being recognized by cells as an antagonist to an agonist (LegaultPoisson et al., 1979; Canney et al., 1987; Hu et al., 1993; Norris et al., 1999; Connor et al., 2001). This contention is clearly supported by the fact that a significant number of patients who initially respond to tamoxifen, and who subsequently progress, demonstrate a secondary response upon discontinuation of therapy (Legault-Poisson et al., 1979; Stein et al., 1983; Canney et al., 1987; Belani et al., 1989). Another more recent, and alarming, finding came from the NSABP-B14 adjuvant chemotherapy trial performed in ER-positive, node-negative breast cancer patients (Fisher et al., 1996,2001). In this study, it was demonstrated that disease-free survival in patients receiving tamoxifen improved by over 50\%. However, patients who received tamoxifen for longer than 5 years did no better, and possibly worse, than patients receiving tamoxifen for only 5 years (Fisher et al., 1996,2001). These findings suggested that tamoxifen did not simply fail but that it may be responsible for harm in some patients, possibly due to an antagonist/agonist switch. Given these findings, and the observation that tamoxifen could function as an agonist in the uterus, bone, and the cardiovascular system, we considered that resistance to this drug may arise as a consequence of 1) positive selection of a population of cells that is already poised to recognize tamoxifen as an agonist or 2) a drug-facilitated change in the processes that enable cells to distinguish between agonists and antagonists. It appeared, therefore, that a definition of the mechanism(s) by which tamoxifen agonist activity was manifest in some circumstances would help us to understand the molecular basis of resistance and may also shed light on SERM action.

\section{Definition of the Processes That Permit Cells to Distinguish Between Different ER-Ligand Complexes}

In established models of ER action, the role of estrogens was considered to be that of a switch that converted the transcriptionally inactive ER within the nuclei of target cells into a form that was capable of interacting with the 
regulatory regions within target genes and positively or negatively regulating transcription (McDonnell, 1999). Within the confines of this model, it was hard to understand how tamoxifen could manifest SERM activity, since it would predict that compounds were able to function as estrogens and activate or bind to the receptor and competitively inhibit estrogen binding and thus function as antagonists. Clearly, this was an oversimplification of what was occurring in the cell. Indeed, several major discoveries have occurred over the past decade that have revealed additional complexity in ER action and help to explain the pharmacology of SERMs such as tamoxifen. The most important of these are 1) the discovery of a second estrogen receptor, $\mathrm{ER} \beta ; 2$ ) the observation that the conformation of the two receptor subtypes is influenced by the nature of the bound ligand; and 3) the identification of receptor-associated comodulators, proteins that can enhance (coactivators) or repress (co-repressors) receptor transcriptional activity (Beekman et al., 1993; McDonnell et al., 1995; Onate et al., 1995; Kuiper et al., 1996; Mosselman et al., 1996; Brzozowski et al., 1997; Shiau et al., 1998; Pike et al., 1999,2001; McKenna et al., 1999; McKenna and O'Malley, 2000). All three of these activities have been shown to be important in SERM pharmacology and have been studied in great detail. For a more complete description of these particular advances, the reader is referred to several excellent reviews that have been published recently (McKenna et al., 1999; McKenna and O'Malley, 2000). However, the roles that these processes play in determining the agonist/antagonist activities of SERMs are highlighted below.

\section{The Role of ER $\beta$ in SERM Action}

Exploitation of differential expression of receptor isoforms, or subtypes, is a common mechanism by which tissue-selective drugs can be developed. Thus, with the identification of a second, genetically distinct ER, ER $\beta$, and the demonstration that its expression pattern was not identical to that of the previously identified receptor $\mathrm{ER} \alpha$, a potentially simple explanation for SERM pharmacology was anticipated (Kuiper et al., 1997). However, to date, we know very little about the role of ER $\beta$ in estrogen action. mRNA expression studies have demonstrated that ER $\beta$ is expressed in a wide variety of tissues (Shughrue et al., 1996; Kuiper et al., 1997). However, confirmation of the existence of significant levels of receptor protein in all mRNA-positive tissues has been more difficult and needs further investigation. Compounding this problem is the difficulty in defining the phenotypes in $\operatorname{ER} \beta$ knockouts where no consensus among investigators is yet apparent (Couse and Korach, 1999). Studies performed in vitro using reconstituted transcription systems indicate that, on ERE-containing promoters, both $\mathrm{ER} \alpha$ and $\mathrm{ER} \beta$ can activate transcription in response to agonists such as estradiol (McInerney et al., 1998; Hall and McDonnell, 1999). With one documented exception, however, it appears as if 
$\mathrm{ER} \alpha$ is a significantly more efficient activator of transcription than $\mathrm{ER} \beta$ (Harris et al., 2001). In cells where both subtypes are expressed, ER $\beta$ can dampen ER $\alpha$ activity and decrease overall sensitivity to agonists (Hall and McDonnell, 1999). The mechanism by which this occurs is not known but could represent competition between the receptors for the same DNA response element and/or heterodimerization of the weaker $\operatorname{ER} \beta$ with $\operatorname{ER} \alpha$. The inability to ascribe specific functions to $\mathrm{ER} \beta$ in vivo has limited our analysis of the role of this isoform on ER pharmacology to studies performed in vitro. In transfected cell systems, on classical ERE-containing promoters, it appears as if tamoxifen is always an ER $\beta$ antagonist (Hall and McDonnell, 1999). In contrast, some of these same cells are capable of supporting tamoxifen agonist activity when $\mathrm{ER} \alpha$ alone is expressed in an ectopic manner (Tzukerman et al., 1994). Interestingly, in cells where tamoxifen activates ER $\alpha$-mediated transcriptional activity, coexpression of ER $\beta$ completely suppresses this activity (Hall and McDonnell, 1999). Thus, it is possible that, whereas $\mathrm{ER} \alpha$ is required for tamoxifen partial agonist activity, the expression level of ER $\beta$ can regulate the magnitude of this activity. When analogous studies are performed on AP- 1 element-containing promoters, where ER binds indirectly to the promoter by contact with a prebound fos/jun complex, it was observed that the pharmacology of estrogens and SERMs is not the same as that observed on classical ERE-containing promoters (Paech et al., 1997). Notably, in AP1/ER $\alpha$ systems, estrogens activate and SERMs display a range of activities from partial to full agonists. However, when ER $\beta$ is studied in the same manner, it is noticed that estrogens antagonize and SERMs (all tested) activate transcription. The physiological significance of these results awaits the demonstration that the ER-AP1 interaction occurs in vivo. Considering what we now know, it appears as if tamoxifen can inhibit estradiol-activated transcriptional regulation by both $\mathrm{ER} \alpha$ and $\mathrm{ER} \beta$. Importantly, however, in some cell and promoter contexts, the tamoxifen-ER $\alpha$ complex can manifest partial agonist activity. Thus, it remains to be explained how the same ER $\alpha$-ligand complex can be recognized differently in different cells.

\section{Receptor Conformation and ER Pharmacology}

The ability of tamoxifen to manifest agonist activity in a cell-selective manner suggested that tamoxifen was not merely freezing ER in an apo-state, as the initial models proposed, but rather it must do something to the receptor that enables its unique pharmacological activities. Data to support this idea came from studies that used protease digestion assays to map the surfaces presented on ER $\alpha$ when occupied by different ligands (Beekman et al., 1993; McDonnell et al., 1995). Although this is not a sensitive technique, the conclusions of these studies were that tamoxifen was able to induce a change in ER $\alpha$ conformation that distinguished this receptor-ligand complex from apo-ER and that observed in 
the presence of estradiol or the pure anti-estrogens such as ICI182,780 (Beekman et al., 1993; McDonnell et al., 1995; Kraichely et al., 2000). Identical changes in conformation were observed when other SERMs were evaluated in this assay. Thus, it was not clear why tamoxifen and other SERMs such as raloxifene and nafoxidine were functionally distinct when assayed in vivo (McDonnell et al., 1995). Nevertheless, these early studies indicated that there was a link between the structure of the receptor-ligand complex and function. The general findings of these initial protease digestion studies were confirmed by subsequent crystallographic analysis of different ER $\alpha$-ligand complexes (Brzozowski et al., 1997; Shiau et al., 1998; Pike et al., 1999, 2001). As with the protease digestion experiments, crystallography has not yet been able to provide an obvious explanation for the observed functional differences between tamoxifen and other SERMs like raloxifene (Brzozowski et al., 1997; Pike et al., 1999). Regardless, these studies were able to demonstrate that ER can exist in states other than "off" and "on" and that receptor conformation was a key regulator of receptor pharmacology.

\section{Receptor Coactivators and Co-repressors}

The true significance of the finding that ER $\alpha$ could adopt different conformations in the presence of agonists, SERMs, and antagonists came with the discovery of receptor coactivators and co-repressors. The coactivator proteins, among them the p160 class members steroid receptor coactivator (SRC-1) and glucocorticoid receptor interacting protein (GRIP-1), interact with agonist-activated ER (Onate et al., 1995; Hong et al., 1996; Norris et al., 1998). This permits the ligand-activated receptor to contact the transcriptional apparatus and stabilize the preinitiation complex. In addition, some of these coactivators possess histone acetyl transferase (HAT) activity and recruit additional proteins with HAT activity like CBP and pCAF to the ligand-receptor complex (Spencer et al., 1997; McKenna et al., 1999). One major function of the DNA-bound ER-ligand complex, therefore, is to facilitate the acetylation of histones at target gene promoters, thus permitting a local decondensation of chromatin. Transcriptional corepressors (e.g., NcoR, SMRT), on the other hand, oppose the activity of coactivators by interacting with apo-ER, or that activated by antagonists, facilitating the assembly of large protein complexes that can deacetylate histones (McKenna et al., 1999). As a consequence, a localized condensation of chromatin and subsequent diminution of ER-mediated transcriptional activity are effected. With respect to drug-selective biological activities, the identification of a large number of different coactivators whose level of expression can differ between cells is important, as it suggests that differential cofactor recruitment may be a primary determinant of the tissue-selective actions of SERMs. This hypothesis is generally supported by recent studies that have tried to link the 
differential pharmacology of ER ligands to coactivator availability (Smith et al., 1997; Jepsen et al., 2000; Kraichely et al., 2000). Notwithstanding the successes to date, however, a final resolution of this issue, ascribing a given function to a specific coactivator, is likely to be several years away.

\section{Linking Receptor Conformation, Receptor Interacting Proteins, and Pharmacology}

When the crystal structure of estradiol-activated $\operatorname{ER} \alpha$, complexed with a fragment of GRIP-1, encompassing the nuclear receptor interacting domain (NR-box), was solved, a clear understanding of how agonists activate and antagonists inhibit ER transcriptional activity emerged (Shiau et al., 1998). Upon binding an agonist, it was demonstrated that there was a realignment of five of the $12 \alpha$-helices in the ER $\alpha$-ligand-binding domain such that a hydrophobic pocket on the receptor surface formed that enabled coactivator docking. It was also shown that tamoxifen and other antagonists induce distinctly different receptor conformations and prevent the formation of the hydrophobic coactivator-binding pocket (Brzozowski et al., 1997). These findings, which have been confirmed by a variety of biochemical approaches, suggest that the mechanism by which tamoxifen and estrogen manifest agonist activity are not the same. However, since one of the obligate steps in ER action appears to be the recruitment of a coactivator, it is likely that the coactivators with which ER-estradiol and ER-tamoxifen complexes interact are different or that the same coactivator can interact with ER in more than one way. As a first step in addressing this hypothesis, we used combinatorial phage display of random peptides to identify probes that could be used to map potential protein-protein interaction surfaces on ER $\alpha$ in the presence of tamoxifen and/or estradiol (Norris et al., 1999; Paige et al., 1999; Wijayaratne et al., 1999). Specifically, we screened large libraries of bacteriophage that expressed random peptides (11-19 amino acids in length) and identified a series of phage that expressed peptides that interacted specifically with ligand-activated $\operatorname{ER} \alpha$. The interaction of the peptides expressed by these phage with $\mathrm{ER} \alpha$ was assessed using a mammalian two-hybrid assay wherein the ability of a Gal4-peptide fusion to recruit an $\mathrm{ER} \alpha$-VP16 protein to a GAL4-responsive promoter was assessed (Figure 1A). The results of a typical analysis are shown in Figure 1B. One class of peptide, $\alpha / \beta$ I, interacted only with agonist-activated ER $\alpha$. Not surprisingly, when sequenced, all members of this class of peptides were found to encode peptides that contain an LXXLL motif, a sequence that has been shown to constitute the docking module on the p160 class of coactivators (Heery et al., 1997). Another of the peptides identified, $\alpha \mathrm{II}$, interacted with $\mathrm{ER} \alpha$ in the presence of either estradiol or tamoxifen. However, the most exciting finding was the identification of the peptides $\alpha / \beta \mathrm{III}$ and $\alpha / \beta \mathrm{V}$ that interacted only with tamoxifen-activated 
A

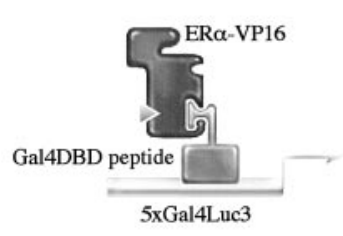

B

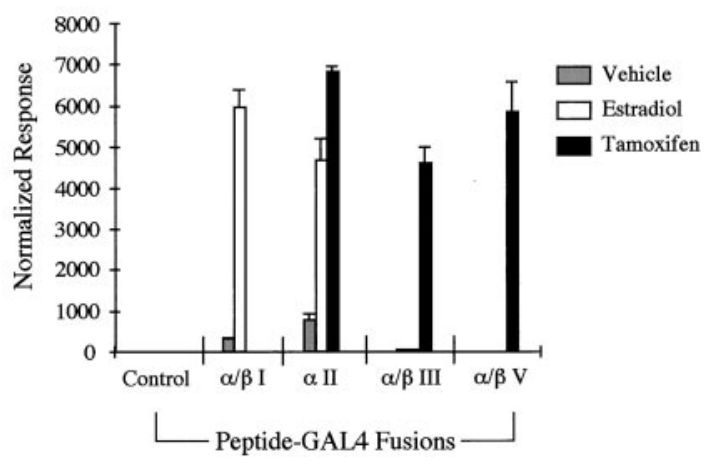

FIG. 1. Identification of peptides whose ability to interact with $\mathrm{ER} \alpha$ is influenced by the nature of the bound ligand. (A) A modified mammalian two-hybrid assay was used to assess the interaction of the peptides identified by phage display to interact with $\mathrm{ER} \alpha$-VP16 in the presence of the indicated ligands. (B) Each peptide was expressed as a fusion with the GAL4-DBD and its ability to interact with the ER $\alpha$-VP16 chimera was measured in transfected HepG2 cells on a Gal4-luciferase reporter, as described previously. All data were normalized to that of a hormone-unresponsive CMV- $\beta$-galactosidase reporter. [Reprinted from Norris JD, Paige LA, Christensen DJ, Chang C-Y, Huacani MR, Fan D, Hamilton PT, Fowlkes DM, McDonnell DP 1999 Peptide antagonists of the human estrogen receptor. Science 285:744-746. Copyright American Association for the Advancement of Science.]

$\mathrm{ER} \alpha$. These data confirmed that the structure of the ER $\alpha$-tamoxifen and $\operatorname{ER} \alpha$ estradiol complexes were not the same and suggested that unique protein-protein interaction surfaces were presented on $\mathrm{ER} \alpha$ following its interaction with these two compounds.

\section{The Agonist Activity of Tamoxifen and Estrogen Are Not Manifest in the Same Manner}

The primary objective of the phage-display experiments was to identify peptides that could be used to probe $\mathrm{ER} \alpha$ structure in the presence of different ligands (Paige et al., 1999). However, a quick review of a large number of studies that have used phage display in a similar manner on other targets indicated that the peptides that are identified in these types of studies usually bind to surfaces on proteins responsible for protein-protein interactions (Sparks et al., 1996; Kay et al., 1998). In light of this observation, we tested whether expression of the tamoxifen or estradiol-specific peptides in intact cells would have an impact on $\mathrm{ER} \alpha$ transcriptional activity (Norris et al., 1999). To address this issue, we took advantage of the fact that, in cultured liver hepatocellular carcinoma cells (HepG2), tamoxifen and $17 \beta$-estradiol both manifest agonist activity (Figure 
A

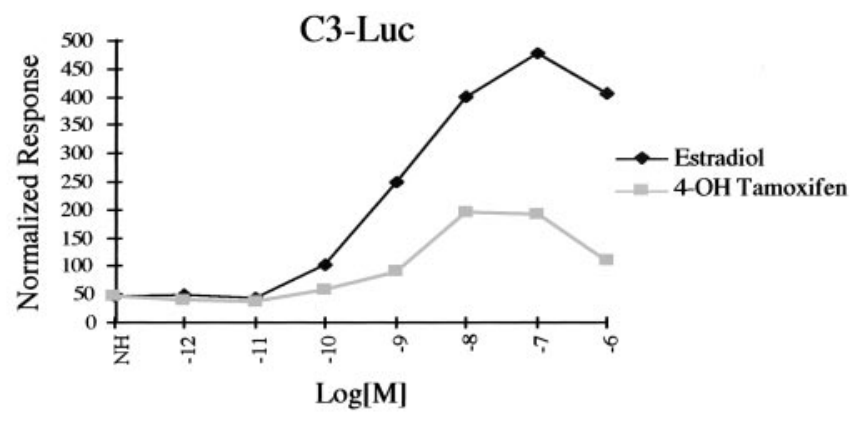

B

Estradiol

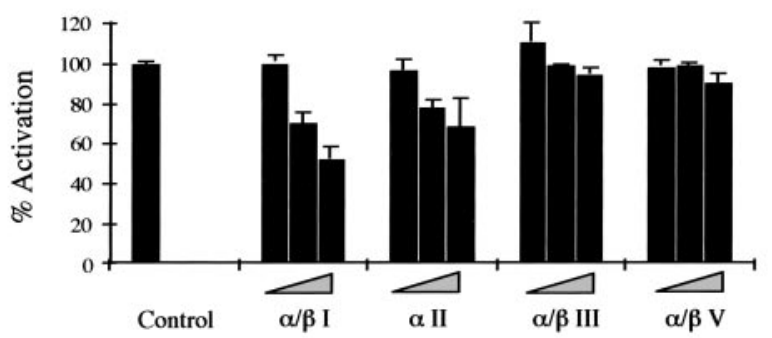

$\mathrm{C}$

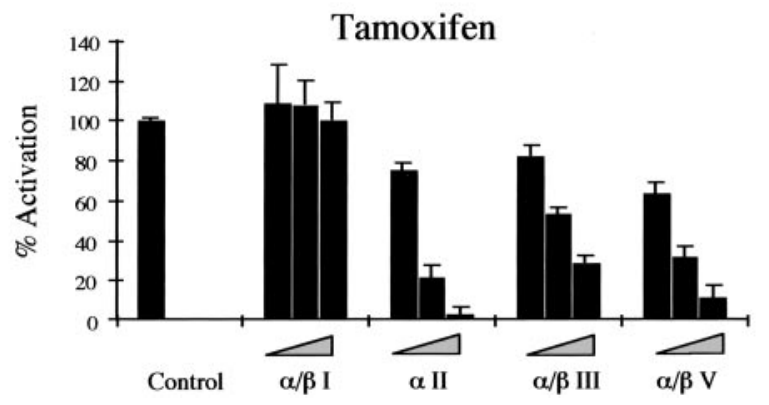

FIG. 2. Disruption of ER $\alpha$ /ERE-mediated transcriptional activity. (A) HepG2 cells were transfected with the estrogen-responsive C3-Luc reporter gene along with an ER $\alpha$ expression vector. Cells were induced with either estradiol or tamoxifen as indicated. NH, no hormone. (B) HepG2 cells were transfected as in (A), except that expression vectors for peptide-Gal4 fusions were included, as indicated. Control represents the transcriptional activity of estradiol $(10 \mathrm{nM})$-activated ER $\alpha$ in the presence of the Ga14DBD alone and is set at 100\% activity. Increasing amounts of input plasmid for each Gal4 peptide fusion is also shown (triangle), with the resulting transcriptional activity presented as percent activation of control. (C) As (B), except that 4-OH tamoxifen (10 nM) was used to activate the receptor. [Reprinted with permission from Norris JD, Paige LA, Christensen DJ, Chang C-Y, Huacani MR, Fan D, Hamilton PT, Fowlkes DM, McDonnell DP 1999 Peptide antagonists of the human estrogen receptor. Science 285:744-746. Copyright 1999 American Association for the Advancement of Science.] 
2A). Specifically, in HepG2 cells transfected with ER $\alpha$ and the complement 3-luciferase reporter, we were able to show that tamoxifen manifests partial agonist activity demonstrating 30\% the efficacy of estradiol (Norris et al., 1996,1999). When this experiment was performed in the presence of a vector expressing the $\alpha / \beta \mathrm{I}$ peptide, it was observed that estradiol, but not tamoxifen, agonist activity was inhibited (Figure 2B). Overexpression of the peptides that interacted specifically with tamoxifen-activated ER $\alpha$ inhibited tamoxifen agonist activity (Figure 2C), while having no effect on estradiol activity. Clearly, the mechanisms by which tamoxifen and estradiol manifest agonist activity in this model system were different. Furthermore, these latter findings suggest that the surface(s) with which the tamoxifen-specific peptides interact enables ER $\alpha$ to interact with a cofactor that facilitates its agonist activity. Since tamoxifen is a synthetic ligand, it must be assumed that this interaction is not physiological but pharmacologically induced and that agonist activity occurs as a consequence of an ectopic interaction of $\mathrm{ER} \alpha$ with some cofactor that is expressed in a cell-specific manner (Figure 3).

\section{The Surfaces Presented on ER Upon Binding Different SERMs Are Not Identical}

The concept that tamoxifen can facilitate an ectopic interaction of $\mathrm{ER} \alpha$ with a transcriptional coactivator was unanticipated. Ultimately, proof that this hypothesis is correct will require the identification of the coactivators that interact with the ER $\alpha$-tamoxifen complex and demonstration that their expression is sufficient to confer upon a cell the ability to recognize tamoxifen as an agonist. Studies aimed at identifying this elusive coactivator(s) are underway. In the meantime, however, we considered that it might be possible to test the relationship between receptor structure, coactivator recruitment, and pharmacology in an indirect manner. If the conformation of $\mathrm{ER} \alpha$ is an important determinant of how it interacts with coactivators and co-repressors, then we believed that it may be possible to regulate these interactions with compounds that enable ER $\alpha$ to adopt a conformation that is distinct from that formed in the presence of tamoxifen or $17 \beta$-estradiol. To test this hypothesis, a series of ER $\alpha$ agonists and antagonists were examined. Previous studies had determined them to have functional activities distinct from tamoxifen and estradiol, for their ability to facilitate the interaction of the ER-interacting phage described above (Willson et al., 1994,1997; Connor et al., 2001). This analysis was performed using an enzymelinked immunosorbent assay (ELISA) to assess the interaction of a representative phage from each class with different ER $\alpha$-ligand complexes (Figure 4). The results of this analysis indicated that these SERMs and antagonists induce a broad spectrum of conformational changes in ER $\alpha$ structure (Paige et al., 1999; Norris et al., 1999; Wijayaratne et al., 1999). Notably, the peptides $\alpha / \beta I I I$ and 


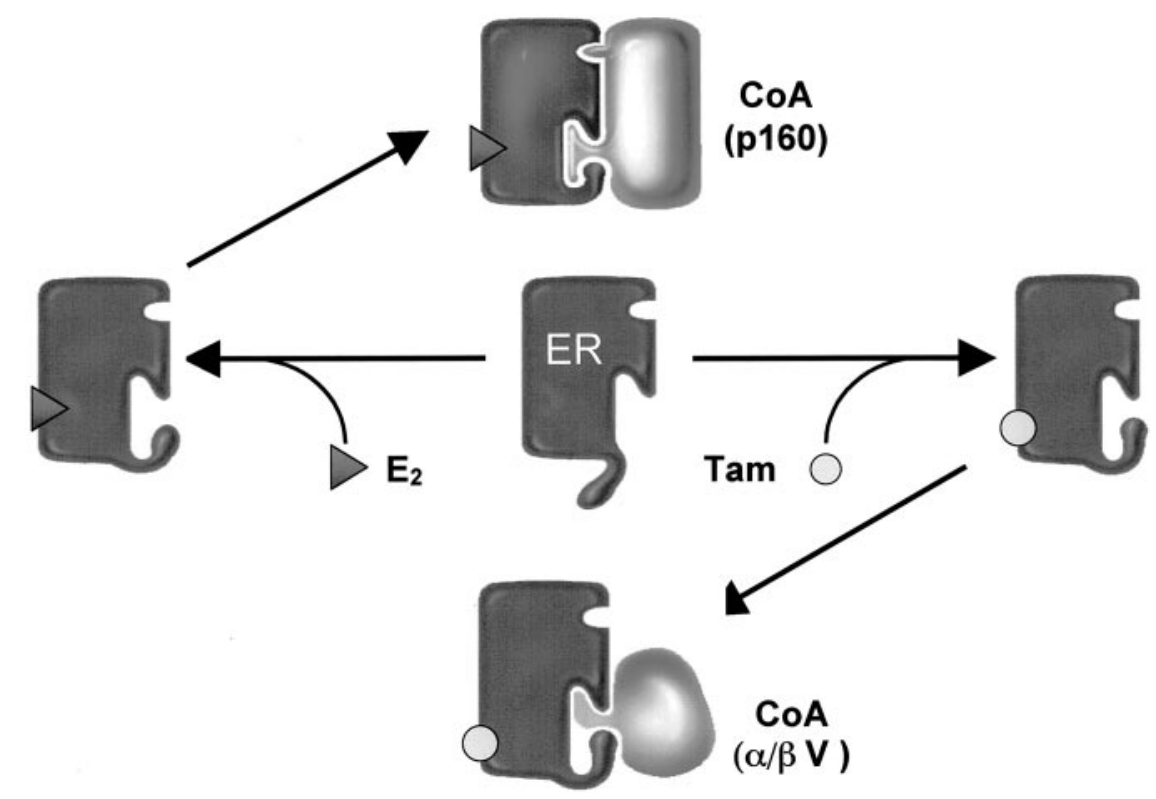

FIG. 3. Using peptide antagonists that inhibit specific protein-protein interactions, it has been demonstrated that the mechanisms by which estradiol and tamoxifen manifest agonist activity are dissimilar. Estradiol binding enables ER $\alpha$ to adopt a conformation that is compatible with the binding of a coactivator of the p160 class (i.e., SRC-1). Tamoxifen binding, on the other hand, induces a unique alteration in receptor structure that permits an ectopic interaction of the receptor with an as-yetunidentified coactivator that shares the binding characteristics of the $\alpha / \beta \mathrm{V}$ peptide. The existence of this coactivator is supported by the fact that peptides of the $\alpha / \beta \mathrm{V}$ class will inhibit tamoxifen, but not estradiol-mediated transcriptional activity, when expressed in target cells (see Figure 2).

$\alpha / \beta \mathrm{V}$ (tamoxifen specific) did not interact with $\mathrm{ER} \alpha$ when activated with ICI182,780 or GW5638 (or its 4-OH metabolite GW7604), compounds that have been shown previously to be biologically distinguishable from tamoxifen. Some binding of the "tamoxifen-specific" peptide $\alpha / \beta I I I$ to raloxifene-activated ER $\alpha$ was observed (Figure 4). Given what is known about the pharmacological activities of the ligands studied, it is likely that the conformational changes observed are functionally important. For instance, with the exception of their actions in the uterus, raloxifene and tamoxifen (the only compounds that can interact with the $\alpha / \beta \mathrm{V}$ peptide) have been shown to have similar biological activities (Sato et al., 1996). Consequently, it is not surprising that, given their similar mechanism of action, raloxifene was found to be ineffective as a treatment for tamoxifen-refractory breast cancer (Gradishar et al., 2000). In contrast, however, ICI182,780, a compound that our studies and more recent crystallographic analysis have indicated to induce a novel conformational change 

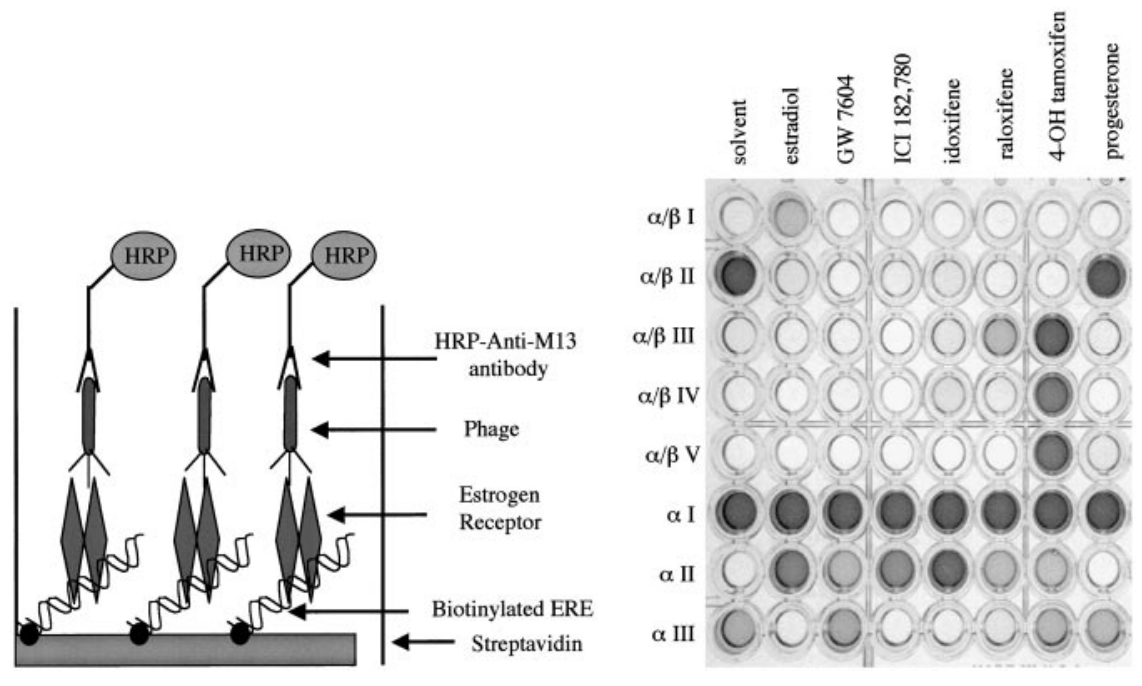

FIG. 4. Effect of ligands on ER $\alpha$ conformation. (Left panel) Phage ELISA assay: a biotinylated vitellogenin ERE was immobilized on 96-well plates coated with streptavidin. Following this step, $\mathrm{ER} \alpha$ was immobilized on the ERE and incubated in the presence of the indicated ligand. Subsequently, bacteriophage expressing the indicated peptides were added. Phage were incubated for 30 minutes at room temperature and washed five times to remove unbound phage. The bound phage were detected using an anti-M13 antibody coupled to horseradish peroxidase (HRP). (Right panel) The binding specificity of a series of peptide-expressing phage to ER $\alpha$ was evaluated in the presence of estradiol, SERMs, and the pure antagonist ICI182,780. Binding in the presence of progesterone was assessed for control purposes. The details of the screens that yielded the specific phage used in this analysis have been described previously (Paige et al., 1999). [Reprinted with permission from Wijayaratne AL, Nagel SC, Paige LA, Christensen DJ, Norris JD, Fowlkes DM, McDonnell DP 1999 Comparative analyses of the mechanistic differences among antiestrogens. Endocrinology 140:58285840. Copyright The Endocrine Society.]

in $\mathrm{ER} \alpha$, was found to be effective as a treatment for tamoxifen-refractory breast cancers (Howell et al., 1995; Norris et al., 1999; Paige et al., 1999; Wijayaratne et al., 1999; Pike et al., 2001). Taken together, these data are consistent with the hypothesis that novel cofactor binding surfaces are presented on ER $\alpha$ upon binding tamoxifen and other SERMs and that the availability of coactivators capable of interacting with the surfaces presented are the primary determinants of pharmacology.

\section{Confirmation of the Link Between ER Structure and Function in Vivo}

The in vitro studies performed over the past 10 years on ER pharmacology have enabled the derivation of molecular models with which to explain the 
different biological activities of ER ligands (McDonnell, 1999). However, the true test of whether these findings are physiologically relevant requires that the in vivo activity of a compound can be predicted based on its in vitro properties. In the past, studies have been done to show that ER ligands that functioned differently in vivo could subsequently be distinguished in vitro. However, to our knowledge, the reverse was never demonstrated with an ER ligand. Considering the usefulness of predictive in vitro assays, we decided to test whether compounds that were mechanistically distinct from tamoxifen were capable of inhibiting tamoxifen-refractory tumors. For these studies, we developed a tamoxifen-resistant MCF-7 breast cancer xenograft model (Connor et al., 2001). This was accomplished by growing MCF-7 cells in estrogenized athymic nude mice, then suppressing estrogen-stimulated growth by simultaneous treatment with tamoxifen. As expected, tamoxifen was initially found to be an effective inhibitor of tumor growth. However, after several months, the tumors resumed growing, despite the continued presence of tamoxifen. Indeed, we were able to demonstrate that these tumors had changed in such a way as to require tamoxifen for growth (Figure 5). This phenomenon has been observed by others using similar systems. Thus, it appears that it is relatively easy for breast cancer cells to switch from recognizing tamoxifen as an antagonist to seeing it as an agonist (Gottardis and Jordan, 1988; Osborne et al., 1992). As with any model system, it is always difficult to prove that it truly reflects the clinical situation and, in our case, that the mechanism by which MCF-7 cells become resistant to tamoxifen in mice is similar to how resistance arises in humans. However, given the clinical observations that 1) tamoxifen can function as an agonist in some organs, 2) a significant number of patients with tamoxifen-refractory breast cancer respond favorably to tamoxifen withdrawal, and 3) a tendency towards harm has been noted in breast cancer patients receiving tamoxifen for periods longer than 5 years, we are confident in the utility of the MCF-7 xenograft model. We propose, therefore, that the ability of tamoxifen to manifest agonist activity and resistance are integrally linked.

Our studies indicate that specific surfaces on tamoxifen-activated ER $\alpha$ enable it to manifest agonist activity by recruiting coactivators in some cells. Thus, we believed that a compound that bound ER but did not enable the presentation of the tamoxifen-specific surfaces would be an effective inhibitor of the growth of tamoxifen-refractory breast tumors. To test this hypothesis, we examined the ability of GW5638 (a compound that permitted ER $\alpha$ to adopt a conformational state distinct from that induced by tamoxifen and did not manifest agonist activity in cell systems where tamoxifen was a robust agonist) to inhibit the growth of tamoxifen-refractory breast tumors in athymic nude mice (Connor et al., 2001). The key results of this study, shown in Figure 6, are entirely consistent with our hypothesis. Specifically, it was demonstrated that tamoxifen-stimulated growth of these particular tumors was inhibited when 

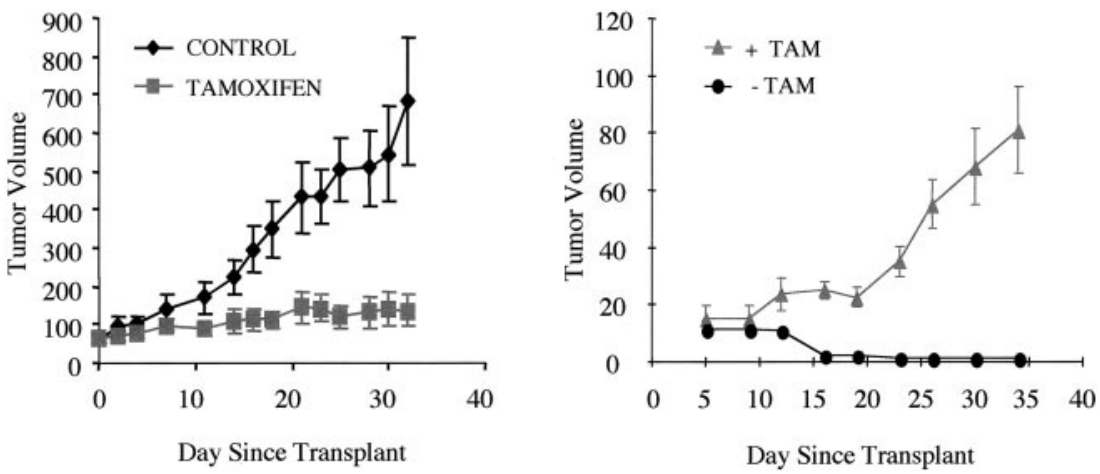

FIG. 5. MCF-7 cell xenografts switch from recognizing tamoxifen as an antagonist to an agonist. (Left panel) The growth of ER-positive MCF-7 cell xenografts is stimulated by estradiol and inhibited by tamoxifen. In this study, xenografts were implanted into estrogenized, ovariectomized mice. After tumors were established, mice were randomized and given vehicle (diamonds) or tamoxifen (squares) three times weekly. Day 0 represents the first day of treatment, approximately 4 weeks after tumor implantation. Data are expressed as mean tumor volumes ( $n=8-9$ mice/group). Animals that died during the experiment (five total) were included for the calculation of mean tumor volumes until their death. Eventually, the tamoxifen-inhibited tumors began to grow and appeared to be resistant. (Right panel) Putative tamoxifen-resistant tumors were implanted into both flanks of estrogenized, ovariectomized, athymic nude mice. One half of the animals in each group were left untreated, whereas one half received tamoxifen $(T A M)$ three times a week. Tamoxifen was administered as a 1.0-mg injection in $0.1 \mathrm{ml}$ of corn oil. Day 0 corresponds to the day of tumor implantation; treatment began immediately. Data are expressed as mean tumor volume. Bars, SE. [Reprinted with permission from Connor CE, Norris JD, Broadwater G, Willson TM, Gottardis MM, Dewhirst MW, McDonnell DP 2001 Circumventing tamoxifen resistance in breast cancers using antiestrogens that induce unique conformational changes in the estrogen receptor. Cancer Res 61:2917-2922. Copyright The American Association for Cancer Research.]

GW5638 was coadministered. In addition, although some growth of tumors persisted in the presence of GW5638 alone, it was significantly less than that which occurred in the presence of tamoxifen. Based on these findings, we propose a revised model to explain how cells are able to distinguish between different SERMs (Figure 7). Specifically, we propose that, in the presence of tamoxifen, ER undergoes a conformational change that enables it to interact in an ectopic manner with a coactivator that shares the binding characteristics of the $\alpha / \beta \mathrm{V}$ peptide described above (Figures 1 and 2). Thus, the presence or absence of this $\alpha / \beta \mathrm{V}$-like protein in a particular cell determines whether tamoxifen functions as an agonist or an antagonist. Upon binding a compound such as GW5638, ER adopts a conformational state that is distinct from that observed in the presence of tamoxifen. This receptor conformation does not permit the interaction of the $\alpha / \beta V$-like coactivator and, thus, no agonist activity in this environment is observed. The findings from the ongoing clinical trial of GW5638 


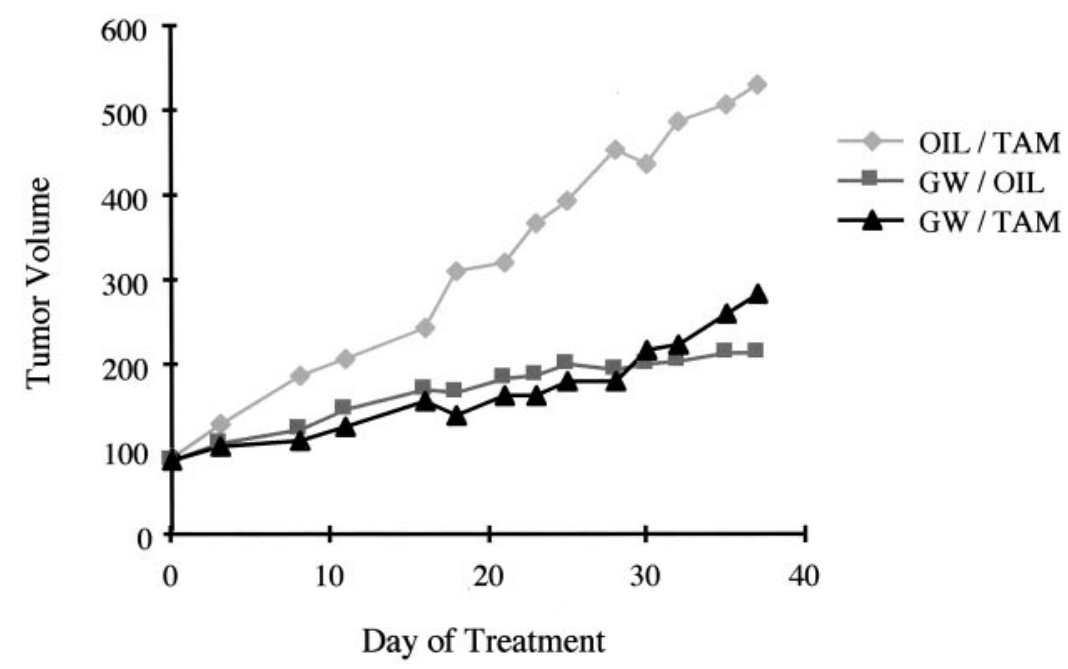

FIG. 6. Inhibition of tamoxifen-refractory tumors by the SERM GW5638. MCF-7 ${ }_{\mathrm{DU}} / \mathrm{TAM}$ tumors were implanted into athymic ovariectomized mice; tamoxifen was administered to promote tumor growth. After tumors were measurable, animals were randomized by tumor volume into treatment groups as follows: tamoxifen (diamonds), GW5638 (squares), and tamoxifen + GW5638 (triangles). Data are expressed as mean tumor volumes, $n=8-10$ mice/group. Tumor measurements of two animals that died randomly during the study were included in the mean volumes until the animal died. [Reprinted with permission from Connor CE, Norris JD, Broadwater G, Willson TM, Gottardis MM, Dewhirst MW, McDonnell DP 2001 Circumventing tamoxifen resistance in breast cancers using antiestrogens that induce unique conformational changes in the estrogen receptor. Cancer Res 61:2917-2922. Copyright The American Association for Cancer Research.]

in metastatic, tamoxifen-refractory disease will help to test the validity of this model.

\section{Final Comments}

In this review, the major processes that have been shown to be involved in modulating ER pharmacology have been considered. When considered together, it is now apparent that different ligands can induce different alterations in the structure of the ER and, by virtue of differential cofactor expression, cells can distinguish between the resultant receptor-ligand complexes. It is clear that the models presented here will continue to evolve as additional new processes that interface with ER are discovered and their physiological relevance established. For instance, it has been shown that receptor activity and response to ligands is modulated by signaling pathways initiated at the cell membrane that directly impinge on ER or modulate its activity by modifying the biochemical properties 


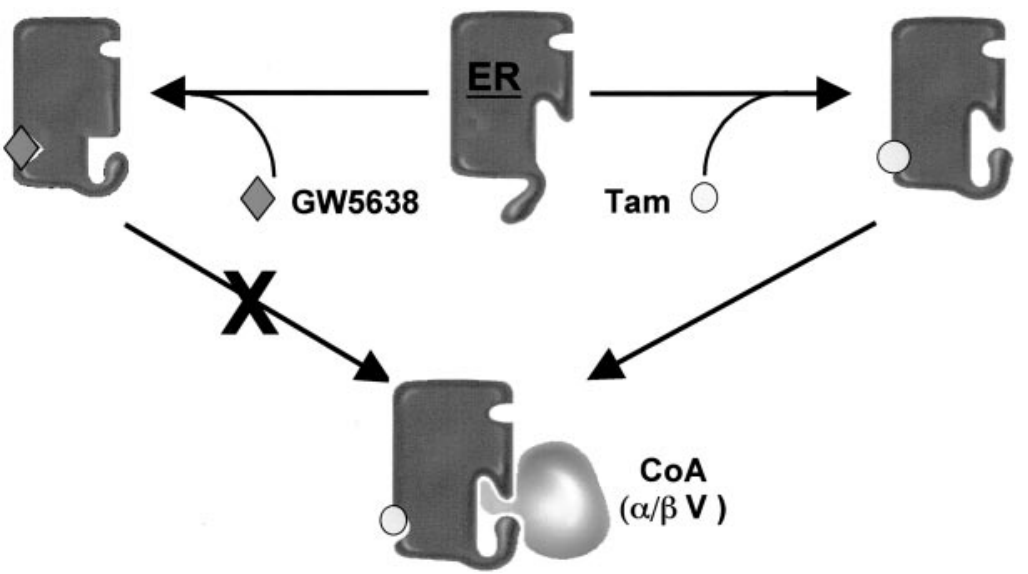

FIG. 7. Linking SERM action and receptor conformation. The studies that we have done on the mechanism of tamoxifen resistance have led us to believe that resistance and the ability of tamoxifen to manifest partial agonist activity in some contexts are related. Specifically, as mentioned above, it has been demonstrated that upon binding tamoxifen, ER adopts a conformation that does not allow it to interact with the p160 class of coactivators but with a coactivator whose binding properties resemble that of the $\alpha / \beta \mathrm{V}$ peptide. We have shown that this peptide (and presumably the corresponding coactivator) does not interact with the GW5638-activated ER, as the alteration in receptor structure induced by this particular ligand is not compatible with its interaction. Thus, a strong case is made for the treatment of tamoxifen-refractory breast tumors with SERMs/anti-estrogens whose mechanism of action is distinct from tamoxifen.

of coactivators and/or co-repressors (Power et al., 1991a,b; Ignar-Trowbridge et al., 1993,1996; Aronica et al., 1994; Mora and Brown, 2000; Rowan et al., 2000). In addition, it has been demonstrated in vitro that estrogens and antiestrogens can manifest nongenomic, ER-dependent activities in cultured cells. For instance, it has been shown that estrogens and some SERMs can activate mitogen-activated protein kinase (MAP-K) and phosphatidylinositol-3 kinase (PI3-K) in cultured cells (Improta-Brears et al., 1999). The physiological relevance of these latter activities is not clear, since they are only observed in cells that have been serum starved for extended periods, a situation that is not duplicated in vivo. Regardless, these findings demonstrate just how complex ER pharmacology is and suggest new avenues for intervention with new classes of pharmaceuticals.

\section{ACKNOWLEDGMENTS}

The author would like to thank Ms. Trena Martelon for help with the preparation of this manuscript. This work was supported by National Institutes of Health grants DK 48807 and CA90645 


\section{REFERENCES}

Aronica SM, Kraus WL, Katzenellenbogen BS 1994 Estrogen action via the cAMP signaling pathway: stimulation of adenylate cyclase and cAMP-regulated gene transcription. Proc Natl Acad Sci USA 91:8517-8521

Beall PT, Misra LK, Young RL, Spjut HJ, Evans HJ, LeBlanc A 1984 Clomiphene protects against osteoporosis in the mature ovariectomized rat. Calcif Tiss Int 36:123-125

Beekman JM, Allan GF, Tsai SY, Tsai M-J, O'Malley BW 1993 Transcriptional activation by the estrogen receptor requires a conformational change in the ligand binding domain. Mol Endocrinol 7:1266-1274

Belani CP, Pearl P, Whitley NO, Aisner J 1989 Tamoxifen withdrawal response. Report of a case. Arch Int Med 149:449-450

Black LJ, Sato M, Rowley ER, Magee DE, Bekele A, Williams DC, Cullinan GJ, Bendele R, Kauffman RF, Bensch WR, Frolik CA, Termine JD, Bryant HU 1994 Raloxifene (LY139481 HCI) prevents bone loss and reduces serum cholesterol without causing uterine hypertrophy in ovariectomized rats. J Clin Invest 93:63-69

Brzozowski AM, Pike AC, Dauter Z, Hubbard RE, Bonn T, Engstrom O, Ohman L, Greene GL, Gustafsson JA, Carlquist M 1997 Molecular basis of agonism and antagonism in the oestrogen receptor. Nature 389:753-758

Canney PA, Griffiths T, Latief TN, Priestman TJ 1987 Clinical significance of tamoxifen withdrawal response. Lancet 1:36

Clark JH, Markaverich BM 1988 Actions of ovarian steroid hormones. In: Knobil E, Neill J, eds. The Physiology of Reproduction. New York: Raven Press; 675-724

Clark JH, Peck EJ 1979 Female sex steroids:receptors and function. Monographs Endocrinol 14:1-245

Clemens JA, Bennet DR, Black LJ, Jones CD 1983 Effects of a new anti-estrogen, keoxifene (LY156758), on growth of carcinogen-induced mammary tumors and on LH and prolactin secretion. Life Sci 32:2869-2875

Connor CE, Norris JD, Broadwater G, Willson TM, Gottardis MM, Dewhirst MW, McDonnell DP 2001 Circumventing tamoxifen resistance in breast cancers using antiestrogens that induce unique conformational changes in the estrogen receptor. Cancer Res 61:2917-2922

Couse JF, Korach KS 1999 Estrogen receptor null mice: what have we learned and where will they lead us? Endocr Rev 20:358-417

Cummings SR, Eckert S, Krueger KA, Grady D, Powles TJ, Cauley JA, Norton L, Nickelsen T, Bjarnason NH, Morrow M, Lippman ME, Black D, Glusman JE, Costa A, Jordan VC 1999 The effect of raloxifene on risk of breast cancer in postmenopausal women: results from the MORE randomized trial. JAMA 281:2189-2197

Delmas PD, Bjarnason NH, Mitlak BH, Ravoux AC, Shah AS, Huster WJ, Draper M, Christiansen C 1997 Effects of raloxifene on bone mineral density, serum cholesterol concentrations, and uterine endometrium in postmenopausal women. N Engl J Med 337: 1641-1647

Early Breast Cancer Trialists' Collaborative Group 1998 Tamoxifen for early breast cancer: an overview of the randomised trials. Lancet 351:1451-1467

Ettinger B, Black DM, Mitlak BH, Knickerbocker RK, Nickelsen T, Genant HK, Christiansen C, Delmas PD, Zanchetta JR, Stakkestad J, Gluer CC, Krueger K, Cohen FJ, Eckert S, Ensrud KE, Avioli LV, Lips P, Cummings SR 1999 Reduction of vertebral risk in postmenopausal women with osteoporosis treated with raloxifene: results from a 3-year randomized clinical trial. Multiple Outcomes of Raloxifene Evaluation (MORE) Investigators. JAMA 282:637-645 
Fisher B, Dignam J, Bryant J, DeCillis A, Wickerham DL, Wolmark N, Costantino J, Redmond C, Fisher ER, Bowman DM, Deschenes L, Dimitrov NV, Margolese RG, Robidoux A, Shibata H, Terz J, Paterson AH, Feldman MI, Farrar W, Evans J, Lickley HL 1996 Five versus more than five years of tamoxifen therapy for breast cancer patients with negative lymph nodes and estrogen receptor-positive tumors. J Natl Cancer Inst 88:1529-1542

Fisher B, Dignam J, Bryant J, Wolmark N 2001 Five versus more than five years of tamoxifen for lymph node-negative breast cancer: updated findings from the National Surgical Adjuvant Breast and Bowel Project B-14 randomized trial. J Natl Cancer Inst 93:684-690

Gockerman JP, Spremulli EN, Raney M, Logan T 1986 Randomized comparison of tamoxifen versus diethylstilbestrol in estrogen receptor-positive or -unknown metastatic breast cancer: a southeastern cancer study group trial. Cancer Treatment Rep 70:1199-203

Gottardis MM, Jordan VC 1988 Development of tamoxifen-stimulated growth of MCF-7 tumors in athymic mice after long-term antiestrogen administration. Cancer Res 48:5183-5187

Gradishar W, Glusman J, Lu Y, Vogel C, Cohen FJ, Sledge GW Jr 2000 Effects of high dose raloxifene in selected patients with advanced breast carcinoma. Cancer 88:2047-2053

Hall JM, McDonnell DP 1999 The estrogen receptor $\beta$-isoform (ER $\beta$ ) of the human estrogen receptor modulates ER $\alpha$ transcriptional activity and is a key regulator of the cellular response to estrogens and antiestrogens. Endocrinology 140:5566-5578

Harper MJK, Walpole AL 1967 A new derivative of triphenylethylene: effect on implantation and mode of action in rats. J Reprod Fert 13:101-119

Harris HA, Henderson RA, Bhat RA, Komm BS 2001 Regulation of metallothionein II messenger ribonucleic acid measures exogenous estrogen receptor- $\beta$ activity in SAOS-2 and LNCaPLN3 cells. Endocrinology 142:645-652

Heery DM, Kalkhoven E, Hoare S, Parker MG 1997 A signature motif in transcriptional co-activators mediates binding to nuclear receptors. Nature 387:733-736

Hong H, Kohli K, Trivedi A, Johnson DL, Stallcup MR 1996 GRIP1, a novel mouse protein that serves as a transcriptional coactivator in yeast for the hormone binding domains of steroid receptors. Proc Natl Acad Sci USA 93:4948-4952

Howell A, DeFriend D, Robertson J, Blamey R, Walton P 1995 Response to a specific antiestrogen (ICI182,780) in tamoxifen-resistant breast cancer. Lancet 345:29-30

Hu XF, Veroni M, Luise Md, Wakeling A, Sutherland R, Watts CKW, Zalcberg JR 1993 Circumvention of tamoxifen resistance by the pure antiestrogen ICI 182,780. Intl J Cancer 55:873-876

Ignar-Trowbridge DM, Teng CT, Ross KA, Parker MG, Korach KS, McLachlan JA 1993 Peptide growth factors elicit estrogen receptor-dependent transcriptional activation of an estrogen-responsive element. Mol Endocrinol 7:992-998

Ignar-Trowbridge DM, Pimentel M, Parker MG, McLachlan JA, Korach KS 1996 Peptide growth factor cross-talk with the estrogen receptor requires the A/B domain and occurs independently of protein kinase C or estradiol. Endocrinology 137:1735-1744

Improta-Brears T, Whorton AR, Codazzi F, York JD, Meyer T 1999 Estrogen-induced activation of mitogen-activated protein kinase requires mobilization of intracellular calcium. Proc Natl Acad Sci USA 96:4686-4691

Ismail SM 1994 Effects of tamoxifen on uterus. Lancet 344:622-624

Jepsen K, Harmanson O, Onami TM, Gleiberman AS, Lunyak V, McEvilly RJ, Kurokawa R, Kumar V, Liu F, Seto E, Hedrick SM, Mandel G, Glass CK, Rose DW, Rosenfeld MG 2000 Combinatorial roles of the nuclear receptor corepressor in transcription and development. Cell 102:753-763

Karnik PS, Kulkarni S, Liu X-P, Budd GT, Bukowski RM 1994 Estrogen receptor mutations in tamoxifen-resistant breast cancer. Cancer Res 54:349-353 
Kay BK, Kurakin AV, Hyde-DeRuyscher R 1998 From peptides to drugs via phage display. DDT 3:370-378

Ke HZ, Paralkar VM, Grasser WA, Crawford DT, Qi H, Simmons HA, Pirie CM, ChidseyFrink KL, Owen TA, Smock SL, Chen HK, Jee WS, Cameron KO, Rosati RL, Brown TA, Dasilva-Jardine P, Thompson DD 1998 Effects of CP-336, 156, a new, nonsteroidal estrogen agonist/antagonist, on bone, serum cholesterol, uterus and body composition in rat models. Endocrinology 139:2068-2076

Kraichely DM, Sun J, Katzenellenbogen JA, Katzenellenbogen BS 2000 Conformational changes and coactivator recruitment by novel ligands for estrogen receptor- $\alpha$ and estrogen receptor- $\beta$ : correlations with biological character and distinct differences among SRC coactivator family members. Endocrinology 141:3534-3545

Kuiper GGJM, Enmark E, Pelto-Huikko M, Nilsson S, Gustafsson J-A 1996 Cloning of a novel estrogen receptor expressed in rat prostate and ovary. Proc Natl Acad Sci USA 93:5925-5930

Kuiper GGJM, Carlsson B, Grandien K, Enmark E, Häggblad J, Nilsson S, Gustafsson J-A 1997 Comparison of the ligand binding specificity and transcript tissue distribution of estrogen receptors $\alpha$ and $\beta$. Endocrinology 138:863-870

Kurl RN, Borthwick NM 1980 Clomiphene and tamoxifen action in the rat uterus. J Endocrinol 85:519-524

Legault-Poisson S, Jolivet J, Poisson R, Beretta-Piccoli M, Band PR 1979 Tamoxifen-induced tumor stumulation and withdrawal response. Cancer Treatment Rep 63:1839-1841

Love RR, Mazess RB, Barden HS, Epstein S, Newcomb PA, Jordan VC, Carbone PP, DeMets DL 1992 Effects of tamoxifen on bone mineral density in postmenopausal women with breast cancer. N Engl J Med 326:852-856

McDonnell DP 1999 The molecular pharmacology of SERMs. Trends Endocrinol Metab 10:301-311

McDonnell DP, Clemm DL, Hermann T, Goldman ME, Pike JW 1995 Analysis of estrogen receptor function in vitro reveals three distinct classes of antiestrogens. Mol Endocrinol 9:659-668

McInerney EM, Weis KE, Sun J, Mosselman S, Katzenellenbogen BS 1998 Transcription activation by the human estrogen receptor subtype $\beta(\mathrm{ER} \beta)$ studied with $\operatorname{ER} \beta$ and $\operatorname{ER} \alpha$ receptor chimeras. Endocrinology 139:4513-4522

McKenna NJ, Lanz RB, O’Malley BW 1999 Nuclear receptor coregulators: cellular and molecular biology. Endocr Rev 20:321-344

McKenna NJ, O'Malley BW 2000 An issue of tissues: divining the split personalities of selective estrogen receptor modulators. Nature Med 6:960-962

Mora JF, Brown M 2000 AIB1 is a conduit for kinase-mediated growth factor signaling to the estrogen receptor. Mol Cell Biol 20:5041-5047

Mosselman S, Polman J, Dijkema R 1996 ER $\beta$ : identification and characterization of a novel human estrogen receptor. FEBS Lett 392:49-53

Neven P 2000 Other selective oestrogen receptor modulators (SERMs) in development. Eur J Cancer 36:S65-S67

Norris JD, Fan D, McDonnell DP 1996 Identification of the sequences within the human complement 3 promoter required for estrogen responsiveness provides insight into the mechanism of tamoxifen mixed agonist activity. Mol Endocrinol 10:1605-1616

Norris JD, Fan D, Stallcup MR, McDonnell DP 1998 Enhancement of estrogen receptor transcriptionl activity by the coactivator GRIP-1 highlights the role of activation function 2 in determining estrogen receptor pharmacology. J Biol Chem 273:6679-6688

Norris JD, Paige LA, Christensen DJ, Chang C-Y, Huacani MR, Fan D, Hamilton PT, Fowlkes DM, McDonnell DP 1999 Peptide antagonists of the human estrogen receptor. Science 285:744-746 
Onate SA, Tsai S, Tsai M-J, O’Malley BW 1995 Sequence and characterization of a coactivator for the steroid hormone receptor superfamily. Science 270:1354-1357

Osborne CK 1998 Tamoxifen in the treatment of breast cancer. N Engl J Med 339:1609-1617

Osborne CK, Coronado E, Allred DC, Wiebe V, DeGregorio M 1991 Acquired tamoxifen resistance: correlation with reduced breast tumor levels of tamoxifen and isomerization of trans-4-hydroxytamoxifen. J Natl Cancer Inst 83:1477-1482

Osborne CK, Wiebe BJ, McGuire WL, Ciocca DR, DeGregorio MW 1992 Tamoxifen and the isomers of 4-hydroxytamoxifen in tamoxifen-resistant tumors from breast cancer patients. J Clin Oncol 10:304-310

Paech K, Webb P, Kuiper GGJM, Nilsson S, Gustafsson J-A, Kushner PJ, Scanlan TS 1997 Differential ligand activation of estrogen receptors ER $\alpha$ and ER $\beta$ at AP1 sites. Science 277:1508-1510

Paige LA, Christensen DJ, Grøn H, Norris JD, Gottlin EB, Padilla KM, Chang C-Y, Ballas LM, Hamilton PT, McDonnell DP 1999 Estrogen receptor (ER) modulators each induce distinct conformational changes in ER $\alpha$ and ER $\beta$. Proc Natl Acad Sci USA 96:3999-4004

Pike ACW, Brzozowski AM, Hubbard RE, Bonn T, Thorsell A-G, Engström W, Ljunggren J, Gustafsson J-Å, Carlquist M 1999 Structure of the ligand-binding domain of oestrogen receptor beta in the presence of a partial agonist and a full antagonist. EMBO J 18:4608-4618

Pike ACW, Brzozowski AM, Walton J, Hubbard RE, Thorsell A-G, Li Y-L, Gustafsson J-Å, Carlquist M 2001 Structural insights into the mode of action of a pure antiestrogen. Structure 9:145-153

Poulin R, Merand Y, Poirier D, Levesque C, Dufour JM, Labrie F 1989 Antiestrogenic properties of keoxifene, trans-4-hydroxytamoxifen and ICI164,384, a new steroidal antiestrogen, in ZR-75-1 human breast cancer cells. Breast Cancer Res Treatment 14:65-76

Power RF, Lydon JP, Conneely OM, O’Malley BW 1991a Dopamine activation of an orphan of the steroid receptor superfamily. Science 252:1546-1547

Power RF, Mani SK, Codina J, Conneely OM, O'Malley BW 1991b Dopaminergic and ligand-independent activation of steroid hormone receptors. Science 254:1636-1639

Rowan BG, Weigel NL, O'Malley BW 2000 Phosphorylation of steroid receptor coactivator-1: Identification of the phosphorylation sites and phosphorylation through the mitogen-activated protein kinase pathway. J Biol Chem 275:4475-4483

Sato M, Rippy MK, Bryant HU 1996 Raloxifene, tamoxifen, nafoxidine, or estrogen effects on reproductive and nonreproductive tissues in ovariectomized rats. FASEB J 905-912

Shiau AK, Barstad D, Loria PM, Cheng L, Kushner PJ, Agard DA, Greene GL 1998 The structural basis of estrogen receptor/coactivator recognition and the antagonism of this interaction by tamoxifen. Cell 95:927-937

Shughrue PJ, Komm B, Merchenthaler I 1996 The distribution of estrogen receptor- $\beta$ mRNA in the rat hypothalamus. Steroids 61:678-681

Sluyser M 1992 Role of estrogen receptor variants in the development of hormone resistance in breast cancer. Clin Biochem 25:407-414

Smith CL, Nawaz Z, O'Malley BW 1997 Coactivator and corepressor regulation of the agonist/ antagonist activity of the mixed antiestrogen, 4-hydroxytamoxifen. Mol Endocrinol 11:657666

Sparks AB, Hoffman NG, McConnell SJ, Fowlkes DM, Kay BK 1996 Cloning of ligand targets: Systematic isolation of SH3 domain-containing proteins. Nature Biotechnol 14:741-744

Spencer TE, Jenster G, Burcin MM, Allis CD, Zhou J, Mizzen CA, McKenna NJ, Onate SA, Tsai SY, Tsai M-J, O'Malley BW 1997 Steroid receptor coactivator-1 is a histone acetyltransferase. Nature 389:194-198

Stein W, Hortobagyi GN, Blumenschein GR 1983 Response of metastatic breast cancer to tamoxifen withdrawal: report of a case. J Surg Oncol 22:45-46 
Tonetti DA, Jordan VC 1995 Possible mechanisms in the emergence of tamoxifen-resistant breast cancer. Anti-Cancer Drugs 6:498-507

Touchette N 1992 Tamoxifen resistance in breast cancer. J NIH Res 4:67-72

Turner CH, Sato M, Bryant HU 1994 Raloxifene preserves bone strength and bone mass in ovariectomized rats. Endocrinology 135:2001-2005

Turner RT, Wakley GK, Hannon KS, Bell NH 1987 Tamoxifen prevents the skeletal effects of ovarian hormone deficiency in rats. J Bone Miner Res 2:449-456

Tzukerman MT, Esty A, Santiso-Mere D, Danielian P, Parker MG, Stein RB, Pike JW, McDonnell DP 1994 Human estrogen receptor transactivational capacity is determined by both cellular and promoter context and mediated by two functionally distinct intramolecular regions. Mol Endocrinol 8:21-30

Wijayaratne AL, Nagel SC, Paige LA, Christensen DJ, Norris JD, Fowlkes DM, McDonnell DP 1999 Comparative analyses of the mechanistic differences among antiestrogens. Endocrinology 140:5828-5840

Willson TM, Henke BR, Momtahen TM, Charifson PS, Batchelor KW, Lubahn DB, Moore LB, Oliver BB, Sauls HR, Triantafillou JA, Wolfe SG, Baer PG 1994 3-[4-(1,2-diphenylbut1-enyl)phenyl]acrylic acid: a non-steroidal estrogen with functional selectivity for bone over uterus in rats. J Med Chem 37:1550-1552

Willson TM, Norris JD, Wagner BL, Asplin I, Baer P, Brown HR, Jones SA, Henke B, Sauls H, Wolfe S, Morris DC, McDonnell DP 1997 Dissection of the molecular mechanism of action of GW5638, a novel estrogen receptor ligand, provides insights into the role of ER in bone. Endocrinology 138:3901-3911

Yaffe K, Sawaya G, Lieberburg I, Grady D 1998 Estrogen therapy in postmenopausal women: effects on cognitive function and dementia. JAMA 279:688-695

Zumoff B 1993 Hormone replacement and cardiovascular risk factors. N Engl J Med 329:1041-1043 


\title{
Aromatase and Its Inhibitors: Significance for Breast Cancer Therapy
}

\author{
Evan R. Simpson* and Mitch DowsetT ${ }^{\dagger}$ \\ *Prince Henry's Institute of Medical Research, Monash Medical Centre, Clayton, \\ Victoria 3168, Australia; 'Department of Biochemistry, Royal Marsden Hospital, \\ London SW3 6JJ, United Kingdom
}

\begin{abstract}
Endocrine adjuvant therapy for breast cancer in recent years has focussed primarily on the use of tamoxifen to inhibit the action of estrogen in the breast. The use of aromatase inhibitors has found much less favor due to poor efficacy and unsustainable side effects. Now, however, the situation is changing rapidly with the introduction of the so-called phase III inhibitors, which display high affinity and specificity towards aromatase. These compounds have been tested in a number of clinical settings and, almost without exception, are proving to be more effective than tamoxifen. They are being approved as first-line therapy for elderly women with advanced disease. In the future, they may well be used not only to treat young, postmenopausal women with early-onset disease but also in the chemoprevention setting. However, since these compounds inhibit the catalytic activity of aromatase, in principle, they will inhibit estrogen biosynthesis in every tissue location of aromatase, leading to fears of bone loss and possibly loss of cognitive function in these younger women. The concept of tissue-specific inhibition of aromatase expression is made possible by the fact that, in postmenopausal women when the ovaries cease to produce estrogen, estrogen functions primarily as a local paracrine and intracrine factor. Furthermore, due to the unique organization of tissue-specific promoters, regulation in each tissue site of expression is controlled by a unique set of regulatory factors. These factors are potential targets for the design of selective aromatase modulators, which could selectively inhibit aromatase expression in breast with the same efficacy as the phase III inhibitors of activity but leave expression in other local sites such as bone and brain untouched.
\end{abstract}

\section{Aromatase and Its Gene}

Estrogen biosynthesis is catalyzed by a microsomal member of the cytochrome P450 superfamily, namely, aromatase cytochrome P450 (P450arom, the product of the CYP19 gene). The P450 gene superfamily is a very large one, containing (as of 1996) over 480 members in 74 families, of which cytochrome P450arom is the sole member of family 19 (Nelson et al., 1996). This heme protein is responsible for binding of the $\mathrm{C}_{19}$ androgenic steroid substrate and catalyzing the series of reactions leading to formation of the phenolic A ring characteristic of estrogens. 
The human CYP19 gene was cloned some years ago (Means et al., 1989; Harada et al., 1990; Toda et al., 1990), when it was shown that coding region spans nine exons, beginning with exon II. Upstream of exon II are a number of alternative first exons that are spliced into the $5^{\prime}$-untranslated region of the transcript in a tissue-specific fashion (Figure 1). For example, placental transcripts contain at their $5^{\prime}$-end a distal exon, I.1. This is because placental expression is driven by a powerful distal promoter upstream of exon I.1 (Means et al., 1991). Examination of Human Genome Project data reveals that exon I.1 is $89 \mathrm{~kb}$ upstream of exon II (Sebastian and Bulun, 2001). On the other hand, transcripts in ovary and testes contain at their $5^{\prime}$-end sequence that is immediately upstream of the translational start site. This is because expression of the gene in the gonads utilizes a proximal promoter, promoter II. By contrast, transcripts in cells of mesenchymal origin (e.g., adipose stromal cells, osteoblasts) contain yet another distal exon (I.4) located $20 \mathrm{~kb}$ downstream of exon I.1 (Mahendroo et al., 1993). Adipose tissue transcripts also contain promoter II-specific exonic sequences that are undetectable in bone (Shozu and Simpson, 1998).

Splicing of these untranslated exons to form the mature transcript occurs at a common 3 '-splice junction upstream of the translational start site. This means that although transcripts in different tissues have different $5^{\prime}$-termini, the coding region - and thus the protein expressed in these various tissue sites - is always the same. However, the promoter regions upstream of each of the several untranslated first exons have different cohorts of response elements, so regulation of aromatase expression in each tissue differs. Thus, the gonadal promoter (II) binds the transcription factors cyclic AMP (cAMP) response binding protein (CREB) and steroidogenic factor 1 (SF1), so aromatase expression in gonads is regulated by cAMP and gonadotrophins. In adipose tissue, promoter II-mediated expression is stimulated by prostaglandin $\mathrm{E}_{2}$. On the other hand, promoter I.4 is regulated by class I cytokines such as interleukin (IL)-6, IL-11, and oncostatin M as well as by tumour necrosis factor alpha $(\mathrm{TNF} \alpha)$. Thus, the regulation of estrogen biosynthesis in each tissue site of expression is unique (reviewed in Simpson et al., 1997). This situation leads to a complex physiological situation that makes, for example, interpretation of circulating estrogen levels as a marker of aromatase activity in specific tissues or in response to specific stimuli very difficult.

\section{The Concept of Local Estrogen Biosynthesis}

In premenopausal women, the ovaries are the principal source of estrogen, which functions as a circulating hormone to act on distal target tissues. However, this is no longer the case in postmenopausal women, when the ovaries cease to produce estrogens, and in men. Under these circumstances, estradiol is no longer 

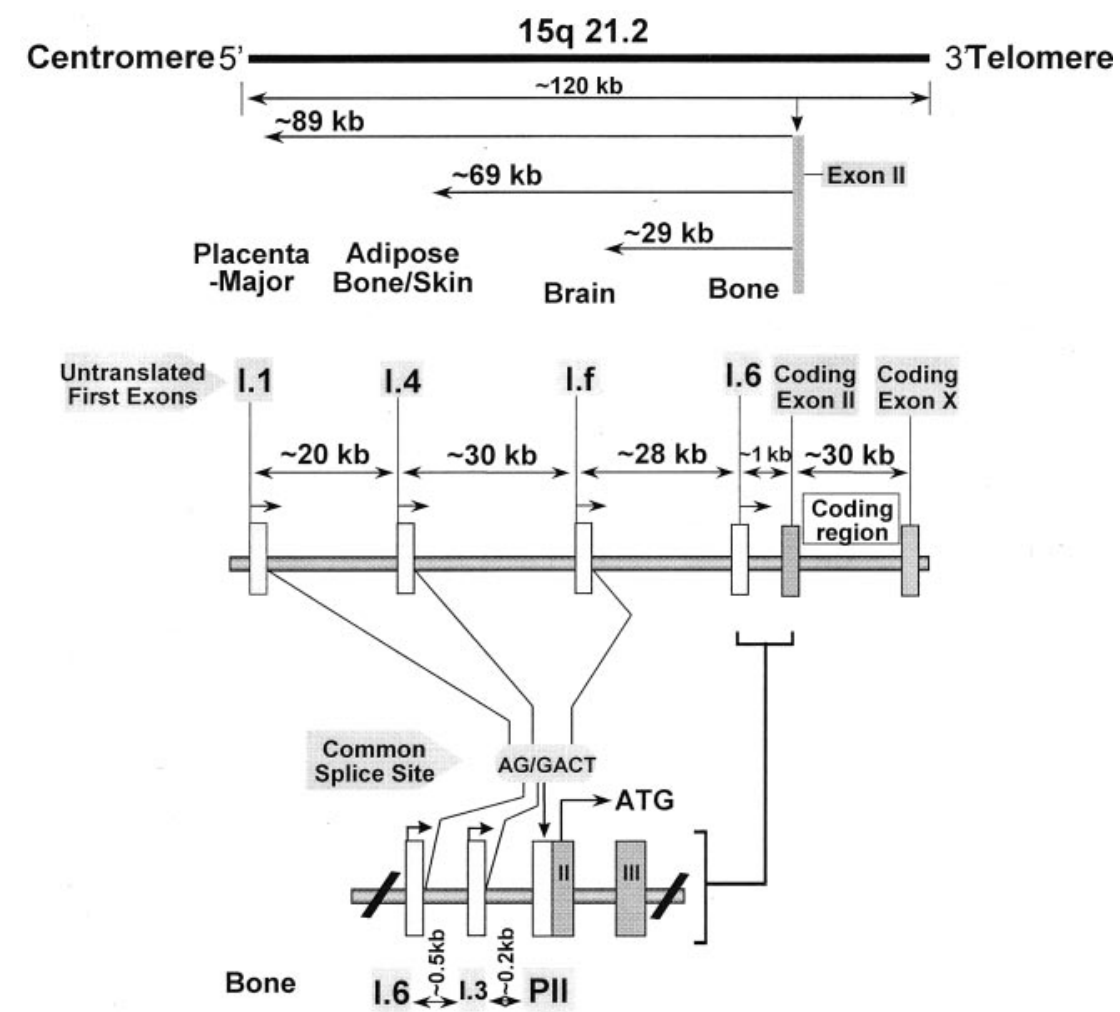

\section{Adiposel Breast Cancer

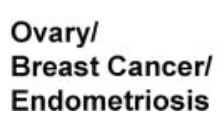

FIG. 1. Genomic organization of the human CYP19 gene. BLAST searches of various promoters and coding region revealed alignment to distinct locations in two overlapping BAC clones of chromosome 15q 21.2 region. The distance of each promoter with respect to the first coding exon (exon II) also was determined. The major placental promoter I.1 is the most distally located (approximately $89 \mathrm{~kb}$ ). Even though each tissue expresses a unique untranslated first exon $5^{\prime}$ untranslated region (UTR), by splicing into a highly promiscuous splice acceptor site (AG/A\ACT) of exon II, the coding region and the translated protein is identical in all tissue sites of expression. [Adapted with permission from Sebastian S, Bulun SE 2001 A highly complex organization of the regulatory region of the human CYP19 (aromatase) gene revealed by the human genome project. J Clin Endocrinol Metab 86:4600-4602. Copyright The Endocrine Society.]

solely an endocrine factor. Instead, it is produced in a number of extragonadal sites where it acts locally, including the mesenchymal cells of adipose tissue, osteoblasts and chondrocytes of bone, the vascular endothelium and aortic 
smooth muscle cells, and numerous sites in the brain. Thus, circulating levels of estrogens in postmenopausal women and in men are not the sole drivers of estrogen action: they may be predominantly reactive rather than proactive. This is because circulating estrogen in this situation originates in extragonadal sites, where it acts locally. If it escapes local metabolism, it enters the circulation. Therefore, circulating levels in large part reflect, rather than direct, estrogen action in postmenopausal women and in men.

Extragonadal sites of estrogen biosynthesis possess several fundamental features that differ from those of the ovaries. First, the estrogen synthesized within these compartments acts predominantly at the local tissue level in a paracrine or intracrine fashion (Labrie et al., 1997,1998) (Figure 2). The total amount of estrogen synthesized by these extragonadal sites may be small but the local tissue concentrations achieved are probably high and exert biological influence locally. As a consequence, extragonadal estrogen biosynthesis plays an important but hitherto largely unrecognised physiological and pathophysiological role.

The power of local estrogen biosynthesis is illustrated in the case of postmenopausal breast cancer (Pasqualini et al., 1996). It has been determined that the concentration of estradiol present in breast tumours of postmenopausal women is at least 20-fold greater than that present in the plasma. With aromatase inhibitor therapy, intratumoural concentrations of estradiol and estrone drop precipitously, together with a corresponding loss of intratumoural aromatase activity, consistent with this activity being within the tumour and the surrounding

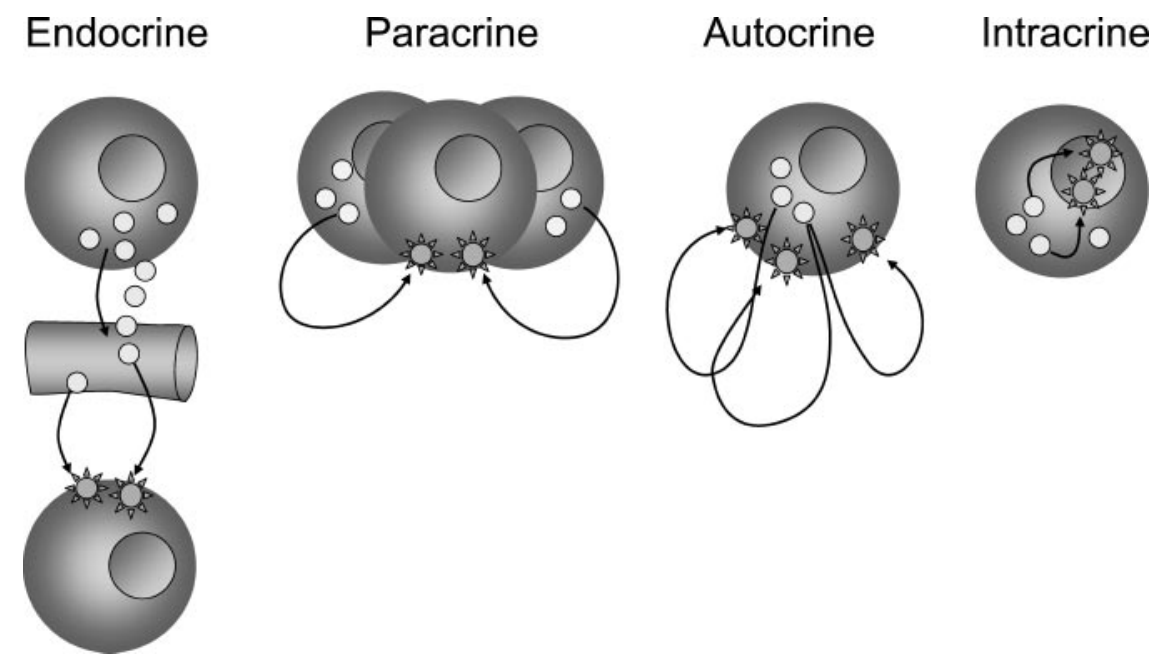

FIG. 2. Modes of action of endocrine, paracrine, autocrine, and intracrine factors. 
breast adipose tissue and being responsible for these high tissue concentrations (DeJong et al., 1997). Studies to assess directly the proportion of estrogen that is synthesized in breast tumours as opposed to reaching them from the blood are technically difficult but particularly instructive. The studies of Reed and colleagues as well as of Miller (Reed et al., 1989; Miller, 1999) indicate that the majority of breast tumours generate estrogen through the intratumoural route. However, this varies substantially between tumours, and overall, about half of the estrogen is produced locally within the tumour or surrounding tissue (Miller, 1999).

In bone, aromatase is expressed primarily in osteoblasts and chondrocytes (Sasano et al., 1997). Aromatase activity in cultured osteoblasts is comparable to that present in adipose stromal cells (Shozu and Simpson, 1998). Thus, it appears that in bone also, local aromatase expression is a major source of estrogen responsible for the maintenance of mineralization (although this is extremely difficult to prove due to sampling problems). Hence, for both breast tumours and bone, it is likely that circulating estrogen levels are only partly responsible for the relatively high endogenous tissue estrogen levels. The circulating levels reflect the sum of local formation in its various sites. This is a fundamental concept for interpreting relationships between circulating estrogen levels in postmenopausal women and estrogen insufficiency in specific tissues.

The second important point is that estrogen production in these extragonadal sites is dependent on an external source of $\mathrm{C}_{19}$ androgenic precursors, since these extragonadal tissues are incapable of converting cholesterol to the $\mathrm{C}_{19}$ steroids (Labrie et al., 1997a,1998). As a consequence, circulating levels of testosterone and androstenedione as well as dehydroepiandrosterone (DHEA) and DHEA sulfate (DHEAS) become extremely important in terms of providing adequate substrate for estrogen biosynthesis in these sites.

It should be pointed out that, in the postmenopausal woman, circulating testosterone and androstenedione levels are an order of magnitude greater than circulating estradiol and estrone levels. Differences in the levels of circulating androgens are likely to be important determinants for maintenance of local estrogen levels in extragonadal sites. Moreover, in men, circulating testosterone levels are an order of magnitude greater than those in postmenopausal women. In postmenopausal women, the ovaries secrete $25-35 \%$ of the circulating testosterone. The remainder is formed peripherally from androstenedione and DHEA produced in the ovaries and from androstenedione, DHEA, and DHEAS secreted by the adrenals. However, the secretion of these steroids and their plasma concentrations decrease markedly with advancing age (Labrie et al., 1997b).

In this context, it is appropriate to consider why osteoporosis is more common in women than in men and why it affects women at a younger age in terms of fracture incidence. We have suggested that uninterrupted sufficiency of circulating testosterone in men throughout life supports the local production of 
estradiol by aromatization of testosterone in estrogen-dependent tissues. This affords ongoing protection against the so-called estrogen deficiency diseases. This appears to be important in terms of protecting the bones of men against mineral loss and also may contribute to the maintenance of cognitive function and prevention of Alzheimer's disease (Simpson et al., 2000).

\section{Aromatase Inhibitors}

\section{A. BACKGROUND}

A large number of aromatase inhibitors have been developed and utilised in clinical studies over the last 20 years. The most successful are now being licensed mainly for breast cancer treatment (Buzdar and Howell, 2001; Goss and Strasser, 2001).

This development was prompted by the recognition that the cytochrome P450 inhibitor aminoglutethimide is an aromatase inhibitor (Thompson and Siiteri, 1974) and exerts its therapeutic effectiveness in postmenopausal women with advanced breast cancer via the inhibition of aromatase (Santen et al., 1978; Stuart-Harris et al., 1984). This recognition validated aromatase as a new target for treatment of breast cancer patients with hormone-dependent disease. The widespread acceptance that aminoglutethimide was anything but a perfect drug (e.g., significant clinical side effects, incomplete inhibition of aromatase, poor selectivity for aromatase leading to adrenal suppression) and the need for combination with glucocorticoid led to the development of numerous new drugs. These have generally been categorised as first-, second-, and third-generation inhibitors. Figure 3 categorises the structures of a selection of the most prominent. Aminoglutethimide is recognised as the dominant first-generation compound. The second generation showed improved potency but either metabolic or symptomatic side effects limited the dose at which these inhibitors could be used. Therefore, overall pharmacological efficacy of the compounds was no greater than that of aminoglutethimide itself (Boeddinghaus and Dowsett, 2001). In contrast, the third-generation compounds have been found to be highly specific and well tolerated such that they have been usable at dosages that effectively obliterate the activity of aromatase.

Many pathological states at least partly depend on continued estrogen stimulation and, thus, in principle, might be expected to be good targets for aromatase inhibition. However, most of these diseases (e.g., endometriosis, fibroids) are almost entirely limited to premenopausal women and are not subject to targeting with aromatase inhibitors alone. Thus, the clinical application of inhibitors has been confined almost entirely to the main estrogen-dependent disease in postmenopausal women (i.e., breast cancer). 


\section{Steroidal Inhibitors}

Formestane<smiles>CC12CC[C@H](O)C(O)=C1CCC1C2CCC2(C)C(O)CCC12</smiles>

\section{Exemestane}<smiles>C=C1CC2C(CCC3(C)C(=O)CCC23)C2(C)C=CC(=O)C=C12</smiles>

\section{Non-steroidal Inhibitors}

\section{Aminoglutethimide}<smiles>CCC1(c2ccc(N)cc2)CCC(=O)NC1=O</smiles>

\section{Fadrozole}<smiles>N#Cc1ccc(C2CCCc3cncn32)cc1</smiles>

Anastrozole

Vorozole

Letrozole<smiles>CC(C)(C)c1cc(Cn2cncn2)cc(C(C)(C)C#N)c1</smiles><smiles>Cn1nnc2ccc(Cn3cncn3)cc21</smiles><smiles>Cc1ccc(C(c2ccc(C#N)cc2)n2cncn2)cc1</smiles>

FIG. 3. Chemical structures of the most widely used aromatase inhibitors.

\section{B. STRUCTURE AND BASIS OF INHIBITION}

There are two main structural types of aromatase inhibitor: 1) steroidal, substrate analogs such as 4-hydroxyandrostenedione (formestane) and exemestane, and 2) nonsteroidal compounds that operate by virtue of their interaction with the cytochrome $\mathrm{P} 450$ heme prosthetic group of the aromatase enzyme (Kao et al., 1996). These are known as type I and type II inhibitors, respectively. While the steroidal group includes inhibitors that act in a competitive manner, those of 
greatest contemporary interest and clinical importance act as enzyme inactivators or suicide inhibitors (Brodie et al., 1981; Lonning, 2000). This activity requires that the enzyme itself converts the inhibitor to a chemically reactive intermediate that binds irreversibly and covalently to the protein structure of the enzymesubstrate binding site. Thus, the individual enzyme molecule is irreversibly inactivated and the inhibitor molecule is no longer available to interact with other enzyme molecules. These types of inhibitors have the potential for exquisite selectivity for the enzyme target and long-term effectiveness, since the recovery of enzyme activity depends on the re-synthesis of enzyme as well as on the pharmacokinetics of the drug. However, such steroidal structures also have the potential for hormonal activity. The potential advantage of long-term effectiveness is of little importance when the turnover of enzyme is rapid. It is notable that plasma estradiol levels rise relatively rapidly after cessation of orally administered formestane (Dowsett et al., 1987). This suggests that the aromatase enzyme is replenished in peripheral tissues within about 24 hours. Thus, this inactivation type of mechanism has yet to be demonstrated to be of significant clinical advantage (see below).

All type 2 inhibitors have a basic nitrogen atom that allows them to interact with the iron atom of the heme prosthetic group of the enzyme (Kao et al., 1996). Their specificity for inhibition of the aromatase enzyme (as opposed to the very large number of other cytochrome P450 enzymes) is determined by the other structural aspects of the drugs and the way that these allow a close fit to the substrate-binding site of aromatase. This results in high-affinity binding and limits the fit into the substrate-binding site of other enzymes. A full understanding of these molecular interactions has been restricted by the unavailability of a crystallized aromatase preparation for structural analysis of the inhibitor-enzyme interaction. Thus, computer-generated models have depended largely on the structural analogies that can be surmised between aromatase and the few cytochrome P450 enzymes whose structure has been determined (GrahamLorence et al., 1995; Graham-Lorence and Peterson, 1996). Use of such models has illustrated the much-better fit to the substrate-binding site of aromatase by the triazole compounds anastrozole, letrozole, and vorozole than by aminoglutethimide, with letrozole and vorozole apparently having a somewhat more complete space-filling effect (Kao et al., 1996).

The potency of these drugs generally has been assessed in vitro using human placental microsomal aromatase preparations. In this type of assay, fadrozole is one of the most potent drugs known, having an IC50 of $5 \mathrm{nM}$. However, its potency in vivo has been compromised by very rapid metabolism such that its vitro activity has not been matched in in vivo studies (Bhatnagar et al., 2001). Letrozole and anastrozole appear to have relatively similar IC50s to each other. But when these compounds have been tested on intact cells - including hamster ovarian tissue, human breast fibroblasts, and aromatase-transfected human breast 
cancer cells - a 10- to 30-fold difference in effectiveness has been found, with letrozole being the more potent. The explanation for the difference in the measurements made in intact cell and cell-free systems is not clear but may be related to uptake of the respective compounds. It might be expected that the intact cell systems would more accurately predict the likely effectiveness of these compounds in the in vivo setting.

\section{PRECLINICAL MODEL SYSTEMS}

Until recently, preclinical modelling of the use of aromatase inhibitors in rodents has been largely limited to premenopausal systems (i.e., those with intact ovarian function) because rodents appear to have little peripheral aromatase activity (in contrast to postmenopausal women, where these drugs have found their greatest application). In these models, the aromatase inhibitors generally have been shown to have good antitumour activity on carcinogen-induced mammary tumours (DeCoster et al., 1992; Brodie et al., 1983). However, the compounds also have had a marked effect on ovarian morphology, with the induction of multiple follicles due to the increase in gonadal stimulation from loss of estrogen feedback on the hypothalamic-pituitary axis (Shetty et al., 1997).

More recently, model systems have focused on the use of aromatasetransfected human MCF7 breast cancer cells in a xenograft model employing athymic nude mice (Lee et al., 1995; Lu et al., 1998). These are more representative of the situation in postmenopausal women and rely on intratumoural aromatase as their primary source of estrogen. Using such models, it has been possible to show the effectiveness of contemporary aromatase inhibitors and compare them with tamoxifen. In general, the inhibitors show greater efficacy than tamoxifen ( $\mathrm{Lu}$ et al., 1998). As always, the interpretation of these data depends on the comparative pharmacology of the compounds in the mouse and human and the degree to which the experimental tumour represents the range of biological characteristics of human breast cancer.

\section{PHARMACOLOGICAL EFFECTIVENESS}

\section{Peripheral Effects}

Two methodologies have been used to estimate the clinical pharmacological effectiveness of aromatase inhibitors. Most studies have accumulated data on the effects of the compounds on plasma estrogen levels. This methodology, however, suffers from a number of deficits. First, it cannot distinguish between effects on production and those that changes in clearance may have. However, a more important issue is the limited sensitivity of plasma estrogen assays: in effect, the maximum degree of suppression that can be shown of primary estrogens (estrone and estradiol) levels using the most sensitive immunoassays available is about 
$85 \%$. Many assays lack the sensitivity to show even this degree of efficacy. Thus, comparing results between different studies and approaches has little validity.

Of substantially greater value has been application of the more complicated methodology used to measure aromatase activity directly. This involves the injection of $\left[{ }^{3} \mathrm{H}\right]$-androstenedione and $\left[{ }^{14} \mathrm{C}\right]$-estrone before and during the treatment of women with the respective inhibitor (Jacobs et al., 1991). Collecting urine over a 72-hour period and establishing the $\left[{ }^{3} \mathrm{H}\right]:\left[{ }^{14} \mathrm{C}\right]$ ratio in the purified estrogen fractions allow calculation of the peripheral aromatase activity in the patient and the degree of inhibition exerted. An advantage of this methodology is that the inclusion of $\left[{ }^{14} \mathrm{C}\right]$-estrone provides an internal standard that permits better comparability of results between studies and over time.

Table I compares the degree of aromatase inhibition achieved among drugs of contemporary importance. This demonstrates that while aminoglutethimide and the second-generation inhibitors suppress aromatase by little more than $90 \%$ at their clinically used dosages, the new third-generation compounds approach complete ablation of aromatase activity. In a recent study, letrozole was found to inhibit by greater than $99 \%$ in all 12 patients (Geisler et al., 2001).

In contrast to the numerous effects of aminoglutethimide on adrenal steroidal function and other cytochrome P450-dependent processes (e.g., prostaglandin and thyroxine synthesis), the newer aromatase inhibitors essentially are completely specific at clinical dosages. Exemestane causes minor reduction in sex hormone-binding globulin (SHBG) levels, probably due to its androgenic nature. Letrozole has been noted to exert a statistically significant effect on corticotropin (ACTH)-stimulated adrenal function (Bajetta et al., 1999). However, these effects are unlikely to be of clinical significance.

TABLE I

Degree of Whole-body Aromatase Inhibition by Drugs Used in Breast Cancer Clinical Efficacy

\begin{tabular}{llcl}
\hline \multicolumn{1}{c}{ Drug } & \multicolumn{1}{c}{ Dose } & $\begin{array}{c}\text { Mean percentage } \\
\text { inhibition }\end{array}$ & \multicolumn{1}{c}{ Reference } \\
\hline $\begin{array}{l}\text { Aminoglutethimide } \\
(+ \text { hydrocortisone })\end{array}$ & $1000 \mathrm{mg}(+40 \mathrm{mg}) / \mathrm{d}$ & 90.6 & MacNeill et al., 1992 \\
Formestane & $250 \mathrm{mg} / 2 \mathrm{w}(\mathrm{im})$ & 84.8 & Jones et al., 1992 \\
Fadrozole & $2 \mathrm{mg} / \mathrm{d}$ & 82.4 & Lonning et al., 1991 \\
Vorozole & $1 \mathrm{mg} / \mathrm{d}$ & 93.0 & Van der Wall et al., 1993 \\
Letrozole & $2.5 \mathrm{mg} / \mathrm{d}$ & $>99.1$ & Geisler et al., 2001 \\
Anastrozole & $1 \mathrm{mg} / \mathrm{d}$ & 97.3 & Geisler et al., 2001 \\
Exemestane & $25 \mathrm{mg} / \mathrm{d}$ & 97.9 & Geisler et al., 1998 \\
\hline
\end{tabular}

[All analyses, other than Van der Wall et al. (1993) were conducted in the Dowsett laboratory.] 


\section{Premenopausal Women}

The observation that formation of multiple ovarian follicles occurs upon administration of aromatase inhibitors in animal model systems has discouraged the widespread investigation of these compounds in premenopausal women (Shetty et al., 1997). Studies with aminoglutethimide generally reveal that estrogen synthesis is largely unaffected by the aromatase inhibitor, although increases in gonadotrophins indicated a degree of compensated inhibition. Application of the more potent steroidal aromatase inhibitor, 4-hydroxyandrostenedione, even at the high dose of $500 \mathrm{mg} / \mathrm{week}$, had no significant impact on premenopausal estrogen levels. The only data from use of a potent nonsteroidal aromatase inhibitor in premenopausal women are from a single-dose study with vorozole. It showed some degree of suppression but duration with repeated doses is unknown. Thus, for the moment, application of aromatase inhibitors to treatment of premenopausal women with breast cancer is limited to their combined usage with gonadotropinreleasing hormone (GnRH) agonists such as goserelin. However, successful use of letrozole in ovulation induction in women with anovulatory infertility recently was reported (Mitwally and Casper, 2001). This is an area that merits substantial further investigation.

\section{Intratumoural Effects}

The observation that the aromatase enzyme is expressed in both normal and malignant breast has led to substantial investigation of the significance of enzyme source. In postmenopausal women, estrogen that is produced locally likely would cause at least as much stimulation of breast tissues as that by estrogen derived from the circulation. It is clear that aromatase inhibitors effectively suppress estrogen synthesis within the breast in almost all cases (DeJong et al., 1997; Miller, 1999). The degree of residual aromatase activity in tumours is very difficult to estimate, however.

Studies by Miller's group have indicated that a significant association may exist between the presence of intratumoural aromatase and the lesion's response to aromatase inhibitors (Miller and O'Neill, 1987). However, expanding these studies to the large scale required to establish any clinical utility of this observation has been limited by the relatively large mass of fresh tissue required to make the measurements. It is hoped that, in the near future, sensitive and specific antibodies will permit the characterisation of aromatase expression in breast carcinomas using immunohistochemical techniques. This will allow the relationship between aromatase and the clinical benefit derived from aromatase inhibitors to be established more conclusively. 


\section{Application of Aromatase Inhibitors in Breast Cancer}

Most data on the effectiveness of aromatase inhibitors in breast cancer are from studies of locally advanced or metastatic disease. Comparative clinical trials have established that the third-generation compounds letrozole and vorozole are more effective than aminoglutethimide and that letrozole is more effective than fadrozole (Bergh et al., 1997; Gershanovich et al., 1998; Tominaga et al., 1998). These data are important because they establish that drugs that achieve essentially complete inhibition of aromatase are more efficacious than those that suppress aromatase by approximately $90 \%$. Thus, over this range of suppression, a clinical response:dose relationship with aromatase inhibition seems to exist.

All of the aromatase inhibitors have shown some benefit over megestrol acetate, the previously standard second-line agent for advanced breast cancer treatment (Buzdar et al., 1996; Dombernowsky et al., 1998; Kaufmann et al., 2000). Aromatase inhibitors have supplanted this agent as the second-line agent of choice.

The most important clinical data, some of the most important in the hormonal treatment of breast cancer for the last decade, have matured over the last couple of years. For over 20 years, tamoxifen was the most widely used agent in breast cancer treatment because of its efficacy and excellent tolerability. Comparative studies of tamoxifen with aminoglutethimide and the secondgeneration inhibitors, 4-hydroxyandrostenedione and fadrozole, showed no improvement in efficacy of these inhibitors over tamoxifen (Perez Carrion et al., 1994; Thurlimann et al., 1996) in advanced beast cancer. However, large clinical trials of tamoxifen versus the third-generation nonsteroidal aromatase inhibitors, anastrozole and letrozole, have reported greater efficacy of the aromatase inhibitors over the antiestrogen (Nabholtz et al., 1999; Bonneterre et al., 2000; Mouridsen et al., 2001). Thus, in patients with estrogen receptor- and/or progesterone receptor-positive advanced breast cancer, an aromatase inhibitor will now be the first agent of choice.

\section{Neoadjuvant Therapy}

The conventional approach to the management of operable breast cancer is the immediate removal of the primary lesion and the subsequent delivery of adjuvant medical treatment. Neoadjuvant therapy reverses this process such that medical treatment is given prior to surgery. A series of small studies suggested that aromatase inhibitors might be more effective than tamoxifen in this context (Dixon et al., 1999), although this was not confirmed in a relatively small study of vorozole versus tamoxifen (Harper-Wynne et al., 2001). Most importantly, however, a large, double-blind, randomized study has confirmed that letrozole is a substantially more effective agent in primary breast cancer therapy than is tamoxifen, thus confirming the enhanced effectiveness of this compound in 
advanced disease (Ellis et al., 2001). Neoadjuvant studies have the advantage of allowing correlation between the presence of specific biomarkers in the disease before treatment and the clinical response in the same lesion immediately afterwards. Particularly provocative data (Ellis et al., 2001) indicate that letrozole is more effective in steroid-receptor positive tumours, which are also either epidermal growth factor (EGF) receptor or HER-2 positive, than in those that are growth factor receptor negative (Figure 4). While this observation concurs with some preclinical studies (Kurokawa et al., 2000), further clinical evidence is required to confirm these potentially highly important data.

\section{Adjuvant Therapy}

Survival benefit rarely is shown in comparative advanced breast cancer studies. Medical agents generally need to be applied immediately after surgery as adjuvant therapy to achieve marked improvements in survival. It is well established that in estrogen receptor-positive disease, tamoxifen reduces the odds of death by about $30 \%$ after 5 years of use (Early Breast Cancer Trialists'

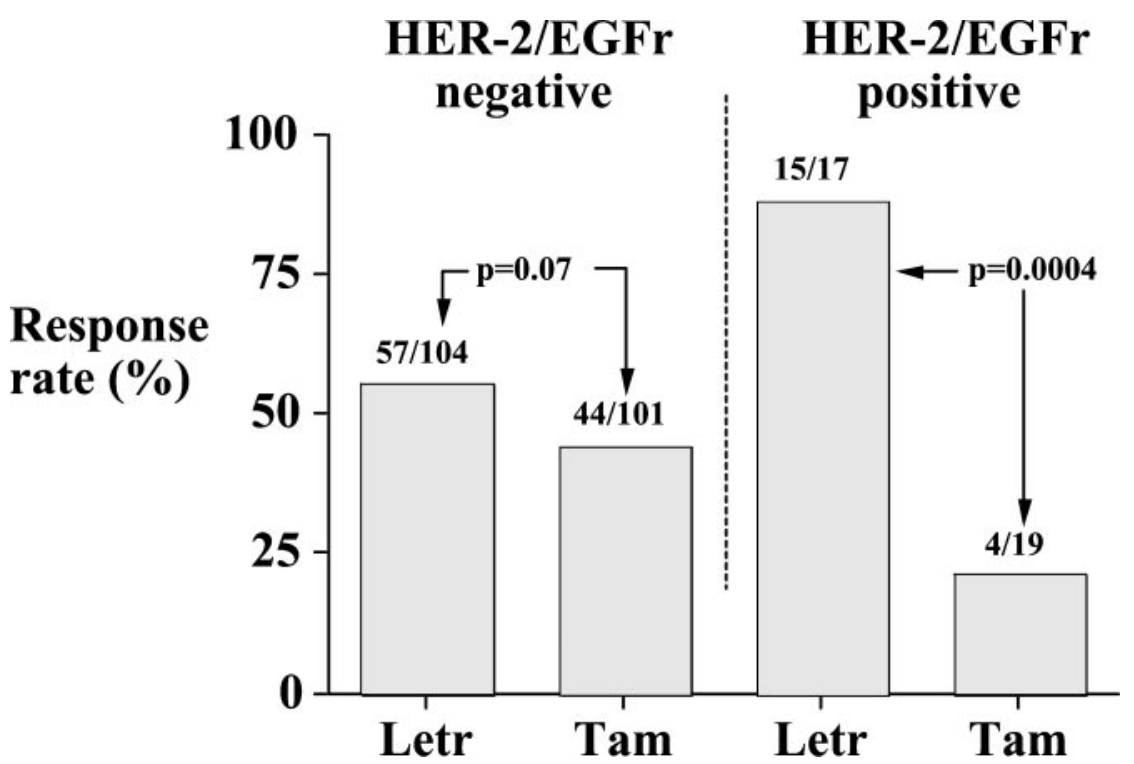

FIG. 4. Response rates to letrozole and tamoxifen according to HER2/EGFr studies in a neoadjuvant clinical trial in primary breast cancer. All patients were either EDR or PgR positive. [Data from Ellis MJ, Coop A, Singh B, Mauriac L, Llombert-Cussac A, Janicke F, Miller WR, Evans DB, Dugan M, Brady C, Quebe-Fehling E, Borgs M 2001 Letrozole is more effective neoadjuvant endocrine therapy than tamoxifen for ErbB-1- and/or ErbB-2-positive, estrogen receptor-positive primary breast cancer: evidence from a phase III randomized trial. J Clin Oncol 19:3808-3816.] 
Collaborative Group, 1998). The very encouraging data from comparisons between tamoxifen and aromatase inhibitors in advanced breast cancer and neoadjuvant therapy prompt the hope that the inhibitors can improve upon the survival seen with tamoxifen in adjuvant therapy. Thus, several very large comparative trials of aromatase inhibitors versus tamoxifen and others will assess the application of these agents in sequence in patients with no apparent metastatic disease. It is important to note that long-term side effects may be as important as efficacy data in this population of patients. It is clear that estrogen deprivation might have adverse effects on the integrity of bone in postmenopausal women (Harper-Wynne et al., 2001). Possible effects on cardiovascular and cognitive function could adversely influence the applicability of inhibitors in this context. Thus, each of the ongoing, large studies is systematically collecting detailed information on these issues of tolerability.

\section{Prospects for Preventative Use}

The involvement of estrogens as promoters of breast cancer is very well established, being based on a wealth of indirect epidemiological evidence (Henderson, 1989). For example, early menarche and late menopause, which extend the exposure of the breast to cyclical estrogenic stimuli, both increase risk of breast cancer. Obesity in postmenopausal women, which increases the plasma concentrations of estrogens, and the application of hormone replacement therapy also enhance breast cancer risk. Of particular note is a series of prospective studies that have collected plasma from women many years before they developed breast cancer. This study has consistently indicated that increased plasma levels of estrogen are associated with increased breast cancer incidence (Thomas et al., 1997). These observations have encouraged the experimental application of agents that can attenuate the estrogenic stimulation of the breast as agents for the prophylaxis of breast cancer. The observations that tamoxifen can reduce the incidence of breast cancer in women at increased risk by approximately $50 \%$ over a 4-year period (Fisher et al., 1998; Cauley et al., 2001) and that the selective estrogen receptor modulator (SERM) raloxifene reduces the incidence of breast cancer in women at high osteoporotic risk have both provided support to this concept and have led aromatase inhibitors being considered as potential preventative agents. The observation that fadrozole can markedly reduce the incidence of sporadic mammary tumours in Sprague-Dawley rats over their lifetime (Gunson et al., 1995) provides compelling support for this concept.

If the effects of tamoxifen and raloxifene are dependent on their antagonising estrogen signalling, the aromatase inhibitors would be expected to be at least as effective in preventing breast cancer. An additional advantage of the inhibitors over the SERMs is that the aromatase inhibitors would be expected to reduce development of genotoxic DNA adducts that may result from the chemical 
reactivity of catecholestrogen metabolites and have a theoretical basis for being involved with breast cancer development (Cavalieri et al., 1997). However, in the chemopreventive setting, side effects are of particular importance, especially in women at only moderate risk. SERMs such as tamoxifen and raloxifene may have an advantage over aromatase inhibitors in this regard. The former have been found to reduce the incidence of osteoporotic fractures (Fisher et al., 1998; Ettinger et al., 1999) and to have effects on lipids that might be expected to have a beneficial effect on cardiac disease (Powles et al., 1989; Johnston et al., 2000). In contrast, the SERMs have deleterious effects on incidence of thrombo-embolic events and tamoxifen enhances the incidence of endometrial cancer (Fisher et al., 1998). Any studies of aromatase inhibitors in the chemopreventative setting will need to consider these issues and compare the effectiveness and side effects of aromatase inhibitors with those of tamoxifen.

One approach to reducing the possible impact of aromatase inhibitors on normal tissues would be to target the therapy at those women with the highest exposure to endogenous estrogens. These could, for example, be identified by plasma estrogen measurements or, alternatively, by assessment of bone mineral density (BMD). BMD tends to reflect long-term estrogen exposure and is itself linked to breast cancer incidence. Rather than obliterating the residual estrogen in these postmenopausal women, a partial reduction in estrogen levels to those in women with low risk of breast cancer is an attractive concept. However, this may be difficult to achieve with low-dose aromatase inhibitors because of variable intersubject pharmacokinetics and pharmacodynamics.

\section{Selective Aromatase Modulators}

Another approach to reducing the risk of side effects is to develop tissuespecific inhibitors of aromatase expression, rather than inhibitors of the catalytic activity. Third-generation aromatase inhibitors are finding utility in the treatment of estrogen-dependent diseases such as breast cancer and, more recently, endometriosis (Zeitoun et al., 1999). However, a disadvantage is that they inhibit aromatase activity in a global fashion and thus could adversely impact sites where estrogen is required for normal function (e.g., maintenance of bone mineralization, prevention of hepatic steatosis and loss of cognitive function). The concept of selective aromatase modulators (SAMs) is made possible by three considerations. The first is that, in postmenopausal women and in men, estrogen largely acts at a local level in sites where it is produced in a paracrine or even intracrine fashion. Second, aromatase expression in these different tissue sites of expression is regulated by tissue-specific promoters. Third, various tissuespecific aromatase promoters employ different signalling pathways and thus different cohorts of transcription factors. Therefore, it is possible to envision 
tissue-specific inhibition of aromatase expression in a similar fashion to the concept of tissue-specific regulation of estrogen action (the concept of SERMs).

As indicated previously, when a breast tumour is present, aromatase activity within the tumour and its surrounding adipose tissue is such that intratumoural estradiol levels are at least an order of magnitude greater than those in circulating plasma of postmenopausal women. (This may be one reason why HRT carries relatively little increased risk of breast cancer.) This is because the tumour produces factors that stimulate aromatase expression locally. This stimulation is associated with switching of the aromatase gene promoter from I.4 to promoter II, the gonadal-type promoter (Harada et al., 1993; Agarwal et al., 1996; Zhou et al., 1997) (Figure 5). Thus, drugs that target promoter II-driven expression of aromatase would be most useful. In postmenopausal women, this promoter would appear to be exclusively utilized in tumour-containing breast tissue (and

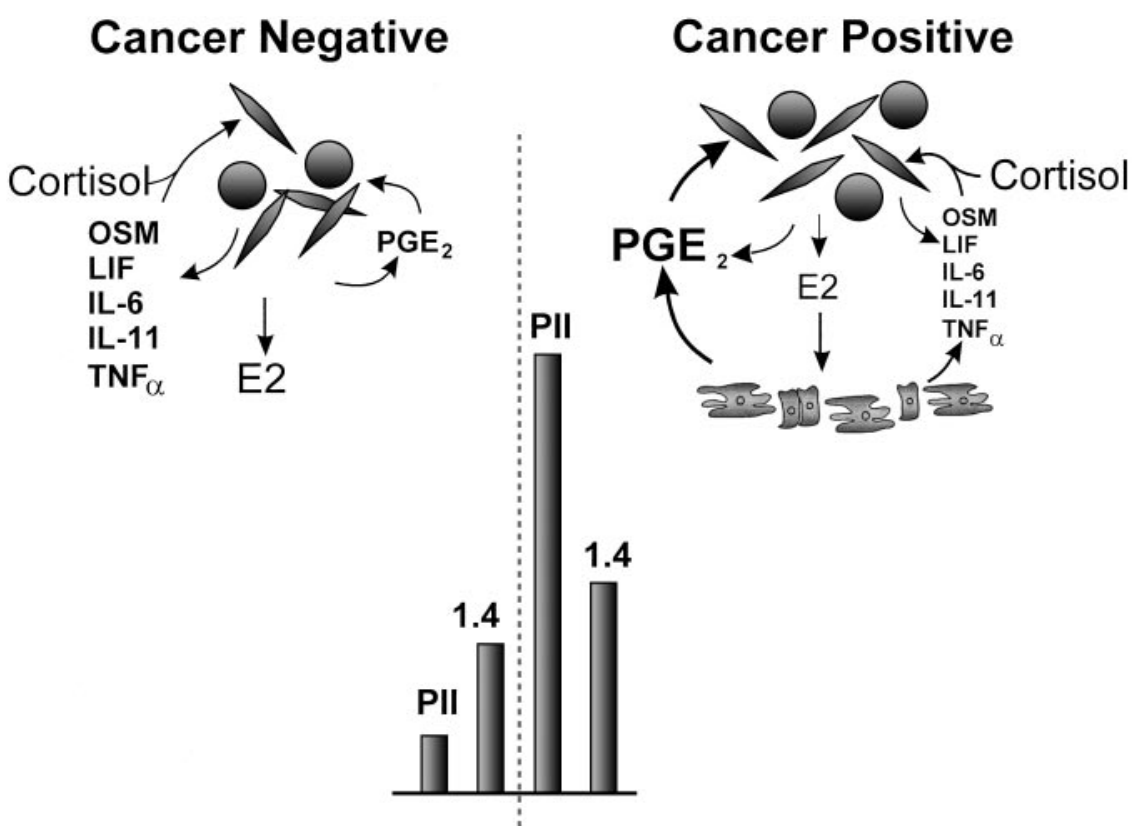

FIG. 5. Proposed regulation of aromatase gene expression in breast adipose tissue from cancer-free individuals and from those with breast cancer. In the former case, expression is stimulated primarily by class I cytokines and tumour necrosis factor alpha produced locally, in the presence of systemic glucocorticoids. As a consequence, promoter I.4-specific transcripts of aromatase predominate. In the latter case, aromatase expression is increased and promoter II-specific transcripts of aromatase predominate, suggesting a major role for prostaglandin E2 $\left(\mathrm{PGE}_{2}\right)$ in aromatase expression. $\mathrm{PGE}_{2}$ in this case could be derived from the tumourous epithelium, tumour-derived fibroblasts, and/or macrophages recruited to the tumour site. 
in endometriotic plaques) (Agarwal et al., 1996; Zeitoun et al., 1999). Thus, bone in particular, which does not express promoter-II specific transcripts (Shozu and Simpson, 1998), would be spared.

Tumour-derived factors include prostaglandin E (PGE) $)_{2}$ (Schrey and Patel, 1995), which stimulates adenylate cyclase in adipose stromal cells, and promoter II, which is regulated by cAMP. It was found that $\mathrm{PGE}_{2}$ is a powerful stimulator of aromatase expression in these cells via promoter II (Zhao et al., 1996). Moreover, expression of the CYP19 gene was correlated with COX-1 and COX-2 expression in human breast cancer and normal tissue specimens (Brueggemeier et al., 1999). A case-control study published some years ago indicated that daily use of nonsteroidal, anti-inflammatory drugs such as ibuprofen reduced the incidence of breast tumours by up to $40 \%$ (Harris et al., 1996). More recently, it has been shown that the COX-2 inhibitor, celecoxib, has strong chemopreventive activity against mammary carcinoma in rats (Harris et al., 2000). From these considerations, it appears likely that inhibition of aromatase expression selectively in breast tissue could play an important role in this chemopreventive action of cyclo-oxygenase inhibitors, which therefore might qualify as the first generation of SAMs. However, ongoing research on regulation of aromatase expression in the breast is likely to uncover other candidates in the not-too-distant future.

\section{ACKNOWLEDGMENTS}

The authors gratefully acknowledge the work of their colleagues and collaborators, in particular, Colin Clyne, Serdar Bulun, Susan Davis, Per Lonning, Ian Smith, and Steve Johnston. The work from the Simpson lab was supported by a grant from the Victorian Breast Cancer Research Consortium Inc. and by U.S. Public Health Service Grant \#R37 AG08174. Sue Elger provided skilled editorial assistance.

\section{REFERENCES}

Agarwal VR, Bulun SE, Leitch M, Rohrich R, Simpson ER 1996 Use of alternative promoters to express the aromatase cytochrome P450 (CYP19) gene in breast adipose tissues of cancer-free and breast cancer patients. J Clin Endocrinol Metab 81:3843-3849

Bajetta E, Zilembo N, Dowsett M, Guillevin L, Di Leo A, Celio L, Martinetti A, Marchiano A, Pozzi P, Stani S, Bichisao E 1999 Double-blind, randomised, multicentre endocrine trial comparing two letrozole doses, in postmenopausal breast cancer patients. Eur J Cancer 35:208-213

Bergh J, Bonneterre J, Illiger HJ, Murray R, Nortier J, Paridaens R, Rubens RD, Samonigg H, van Zyl J, for the Vorozole Study Group 1997 Vorozole versus aminoglutethimide in the treatment of postmenopausal breast cancer relapsing after tamoxifen. Proc Am Soc Clin Oncol $16: 1551$

Bhatnagar AS, Brodie AM, Long BJ, Evans DB, Miller WR 2001 Intracellular aromatase and its relevance to the pharmacological efficacy of aromatase inhibitors. J Steroid Biochem Mol Biol 76:199-202 
Boeddinghaus I, Dowsett, M 2002 Comparative clinical pharmacology and pharmacokinetic interactions of aromatase inhibitors. J Steroid Biochem Mol Biol, in press

Bonneterre J, Thurlimann B, Robertson JF, Krzakowski M, Mauriac L, Koralewski P, Vergote I, Webster A, Steinberg M, von Euler M 2000 Anastrozole versus tamoxifen as first-line therapy for advanced breast cancer in 668 postmenopausal women: results of the Tamoxifen or Arimidex Randomized Group Efficacy and Tolerability study. J Clin Oncol 18:3748-3757

Brodie AM, Garrett WM, Hendrickson JR, Tsai-Morris CH, Williams JG 1983 1. Estrogen antagonists. Aromatase inhibitors, their pharmacology and application. J Steroid Biochem 19:53-58

Brodie AMH, Garrett WM, Hendrickson JR, Tsai-Morris CH, Marcotte PA, Robinson CH 1981 Inactivation of aromatase in vitro by 4-hydroxy-4androstene-3,17-dione and 4-acetoxy4-androstene-3,17-dione and sustained effects in vivo. Steroids 38:693-702

Brueggemeier RW, Quinn AL, Parrett ML, Joarder FS, Harris RE, Robertson FM 1999 Correlation of aromatase and cyclo-oxygenase gene expression in human breast cancer specimens. Cancer Lett 140:27-35

Buzdar A, Howell A 2001 Advances in aromatase inhibition: clinical efficacy and tolerability in the treatment of breast cancer. Clin Cancer Res 7:2620-2635

Buzdar A, Jonat W, Howell A, Jones SE, Blomqvist C, Vogel CL, Eiermann W, Wolter JM, Azab M, Webster A, Plourde PV 1996 Anastrozole, a potent and selective aromatase inhibitor, versus megestrol acetate in postmenopausal women with advanced breast cancer: results of overview analysis of two phase III trials. Arimidex Study Group. J Clin Oncol 14:2000-2011

Cauley JA, Norton L, Lippman ME, Eckert S, Krueger KA, Purdie DW, Farrerons J, Karasik A, Mellstrom D, Ng KW, Stepan JJ, Powles TJ, Morrow M, Costa A, Silfen SL, Walls EL, Schmitt H, Muchmore DB, Jordan VC 2001 Continued breast cancer risk reduction in postmenopausal women treated with raloxifene: 4-year results from the MORE trial. Multiple outcomes of raloxifene evaluation. Breast Cancer Res Treat 65:125-134

Cavalieri EL, Stack DE, Devanesan PD, Todorovic R, Dwivedy I, Higginbotham S, Johansson SL, Patil KD, Gross ML, Gooden JK, Ramanathan R, Cerny RL, Rogan EG 1997 Molecular origin of cancer: catechol estrogen-3,4-quinone as endogenous tumor initiators. Proc Natl Acad Sci USA 94:10937-10942

De Coster R, Van Ginckel RF, Callens MJ, Goeminne NK, Janssens BL 1992 Antitumoral and endocrine effects of $(+)$-vorozole in rats bearing dimethylbenzanthracene-induced mammary tumors. Cancer Res 52:1240-1244

DeJong PC, ven de Ven J, Nortier HW, Maitimu-Sneede I, Danker TH, Thijssen JH, Slee PH, Blankenstein RA 1997 Inhibition of breast cancer tissue aromatase activity and estrogen concentrations by the third-generation aromatase inhibitor vorozole. Cancer Res 57:21092111

Dixon JM, Love CD, Renshaw L, Bellamy C, Cameron DA, Miller WR, Leonard RC 1999 Lessons from the use of aromatase inhibitors in the neoadjuvant setting. Endocr Relat Cancer 6:227-230

Dombernowsky P, Smith I, Falkson G, Leonard R, Panasci L, Bellmunt J, Bezwoda W, Gardin G, Gudgeon A, Morgan M, Fornasiero A, Hoffmann W, Michel J, Hatschek T, Tjabbes T, Chaudri HA, Hornberger U, Trunet PF 1998 Letrozole, a new oral aromatase inhibitor for advanced breast cancer: double-blind randomized trial showing a dose effect and improved efficacy and tolerability compared with megestrol acetate. J Clin Oncol 16:453-461

Dowsett M, Goss PE, Powles TJ, Hutchinson G, Brodie AM, Jeffcoate SL, Coombes RC 1987 Use of the aromatase inhibitor 4-hydroxyandrostenedione in postmenopausal breast cancer: optimization of therapeutic dose and route. Cancer Res 47:1957-1961 
Early Breast Cancer Trialists' Collaborative Group 1998 Tamoxifen for early breast cancer: an overview of the randomised trials. Lancet 351:1451-1467

Ellis MJ, Coop A, Singh B, Mauriac L, Llombert-Cussac A, Janicke F, Miller WR, Evans DB, Dugan M, Brady C, Quebe-Fehling E, Borgs M 2001 Letrozole is more effective neoadjuvant endocrine therapy than tamoxifen for ErbB-1- and/or ErbB-2-positive, estrogen receptor-positive primary breast cancer: evidence from a phase III randomized trial. J Clin Oncol 19:3808-3816

Ettinger B, Black DM, Mitlak BH, Knickerbocker RK, Nickelsen T, Genant HK, Christiansen C, Delmas PD, Zanchetta JR, Stakkestad J, Gluer CC, Krueger K, Cohen FJ, Eckert S, Ensrud KE, Avioli LV, Lips P, Cummings SR 1999 Reduction of vertebral fracture risk in postmenopausal women with osteoporosis treated with raloxifene: results from a 3-year randomized clinical trial. Multiple Outcome of Raloxifene Evaluation (MORE) Investigators. JAMA 282:637-645

Fisher B, Costantino JP, Wickerham DL, Redmond CK, Kavanah M, Cronin WM, Vogel V, Robidoux A, Dimitrov N, Atkins J, Daly M, Wieand S, Tan-Chiu E, Ford L, Wolmark N 1998 Tamoxifen for prevention of breasst cancer: report of the National Surgical Adjuvant Breast and Bowel Project P-1 Study. J Natl Cancer Inst 90:1371-1388

Geisler J, King N, Anker G, Ornati G, Di Salls E, Lonning PE, Dowsett M 1998 In vivo inhibition of aromatization by exemestane, a novel irreversible aromatase inhibitor, in postmenopausal breast cancer patients. Clin Cancer Res 4:2089-2093

Geisler J, Haynes B, Anker G, Dowsett M, Lonning PE 2002 Influence of letrozole and anastrozole on total body aromatization and plasma estrogen levels in postmenopausal breast cancer patients evaluated in a randomized cross-over-designed study. J Clin Oncol, in press

Gershanovich M, Chaudri HA, Campos D, Lurie H, Bonaventura A, Jeffrey M, Buzzi F, Bodrogi I, Ludwig H, Reichardt P, O'Higgins N, Romieu G, Friederich P, Lassus M 1998 Letrozole, a new oral aromatase inhibitor: randomised trial comparing $2.5 \mathrm{mg}$ daily, 0.5 $\mathrm{mg}$ daily and aminoglutehimide in postmenopausal women with advanced breast cancer. Letrozole International Trial Group (AR/BC3). Ann Oncol 9:639-645

Goss PE, Strasser K 2001 Aromatase inhibitors. J Clin Oncol 19:881-894

Graham-Lorence S, Amarneh B, White RE, Peterson JA, Simpson ER 1995 A three-dimensional model of aromatase cytochrome P450. Protein Sci 4:1065-1080

Graham-Lorence S, Peterson JA 1996 P450s: structural similarities and functional differences. FASEB J 10:206-214

Gunson DE, Steele RE, Chau RY 1995 Prevention of spontaneous tumours in female rats by fadrozole hydrochloride, an aromatase inhibitor. Br J Cancer 72:72-75

Harada N, Utsume T, Takagi Y 1993 Tissue-specific expression of the human aromatase cytochrome P450 gene by alternative use of multiple exons 1 and promoters, and switching of tissue-specific exons 1 in carcinogenesis. Proc Natl Acad Sci USA 90:11312-11316

Harada N, Yamada K, Saito K, Kibe N, Dohmae S, Takagi Y 1990 Structural characterization of the human estrogen synthetase (aromatase gene). Biochem Biophys Res Commun 166: $365-372$

Harper-Wynne C, Sacks NP, Shenton K, MacNeill FA, Sauven P, Laidlaw IJ, Rayter Z, Miall S, Salter J, Hills MJ, Lowe FM, A’Hern RA, Nasiri N, Doody D, Iqbal J, Dowsett M 2002 Comparison of the systemic and intratumoural effects of tamoxifen and the aromatase inhibitor vorozole in postmenopausal patients with breast cancer. J Clin Oncol, in press

Harris RE, Alshafie GA, Abou-Issa H, Seibert K 2000 Chemoprevention of breast cancer in rats by celecoxib, a cycloxygenase 2 inhibitor. Cancer Res 60:2101-2113

Harris RE, Namboodiri KK, Farrar WB 1996 Non-steroidal anti-inflammatory drugs and breast cancer. Epidemiology 7:203-205 
Henderson BE 1989 Endogenous and exogenous endocrine factors. Hematol Oncol Clin N Am 3:577-598

Jacobs S, Lonning PE, Haynes B, Griggs L, Dowsett M 1991 Measurement of aromatisation by a urine technique suitable for the evaluation of aromatase inhibitors in vivo. J Enzyme Inhib 4:4315-4325

Johnston CC Jr, Bjarnason NH, Cohen FJ, Shah A, Lindsay R, Mitlak BH, Huster W, Draper MW, Harper KD, Heath H III, Gennari C, Christiansen C, Arnaud CD, Delmas PD 2000 Long-term effects of raloxifene on bone mineral density, bone turnover, and serum lipid levels in early postmenopausal women: three-year data from 2 double-blind, randomized, placebocontrolled trials. Arch Intern Med 160:3444-3450

Jones AL, MacNeill F, Jacobs S, Lonning PE, Dowsett M, Powles TJ 1992 The influence of intramuscular 4-hydroxyandrostenedione on peripheral aromatisation in breast cancer patients. Eur J Cancer 28A:1712-1716

Kao YC, Cam LL, Laughton CA, Zhou D, Chen S 1996 Binding characteristics of seven inhibitors of human aromatase: a site-directed mutagenesis study. Cancer Res 56:3451-3460

Kaufmann M, Bajetta E, Dirix LY, Fein LE, Jones SE, Zilembo N, Dugardyn JL, Nasurdi C, Mennel RG, Cervek J, Fowst C, Polli A, di Salle E, Arkhipov A, Piscitelli G, Miller LL, Massimini G 2000 Exemestane is superior to megestrol acetate after tamoxifen failure in postmenopausal women with advanced breast cancer: results of a phase III randomized double-blind trial. The Exemestane Study Group. J Clin Oncol 18:1399-1411

Kurokawa H, Lenferink AE, Simpson JF, Pisacane PI, Sliwkowski MX, Forbes JT, Arteaga CL 2000 Inhibition of HER2/neu (erbB-2) and mitogen-activated protein kinases enhances tamoxifen action against HER2-overexpressing, tamoxifen-resistant breast cancer cells. Cancer Res 60:5887-5894

Labrie F, Belanger A, Cusan L, Candas B 1997a Physiological changes in dehydroepiandrosterone are not reflected by serum levels of active androgens and estrogen but of their metabolites: intracrinology. J Clin Endocrinol Metab 82:2403-2409

Labrie F, Belanger A, Cusan L, Gomez JL, Candas B 1997b Marked decline in serum concentrations of adrenal C19 sex steroid precursor. J Clin Endocrinol Metab 82: 2396-2402

Labrie F, Belanger A, Luu-The V, Labrie C, Simond J, Cusan L, Gomez JL, Candas B 1998 DHEA and the intracrine formation of androgens and estrogens in peripheral target tissues: its role during aging. Steroids 63:322-328

Lee K, Macaulay VM, Nicholls JE, Detre S, Ashworth A, Dowsett M 1995 An in vivo model of intratumoural aromatase using aromatase-transfected MCF7 human breast cancer cells. Intl J Cancer 62:297-302

Lonning PE 2000 Clinico-pharmacological aspects of different hormone treatments. Eur J Cancer 36:S81-S82

Lonning PE, Jacobs S, Jones A, Haynes B, Powles T, Dowsett M 1991 The influence of CGS 16949A on peripheral aromatisation in breast cancer patients. Br J Cancer 63:789-793

Lu Q, Yue W, Wang J, Liu Y, Long B, Brodie A 1998 The effects of aromatase inhibitors and antiestrogens in the nude mouse model. Breast Cancer Res Treat 50:63-71

MacNeill FA, Jones AL, Jacobs S, Lonning PE, Powles TJ, Dowsett M 1992 The influence of aminoglutethimide and its analogue rogletimide on peripheral aromatisation in breast cancer. Br J Cancer 66:692-697

Mahendroo, MS, Mendelson, CR, Simpson ER 1993 Tissue-specific and hormonally controlled alternative promoters regulate aromatase cytochrome $\mathrm{P} 450$ gene expression in human adipose tissue. J Biol Chem 268:19463-19470

Means GD, Kilgore MW, Mahendroo MS, Mendelson CR, Simpson ER 1991 Tissue-specific promoters regulate aromatase cytochrome P450 gene expression in human ovary and fetal tissues. Mol Endocrinol 5:2005-2013 
Means GD, Mahendroo M, Corbin CJ, Mathis JM, Powell FE, Mendelson CR, Simpson ER 1989 Structural analysis of the gene encoding human aromatase cytochrome P-450, the enzyme responsible for estrogen biosynthesis. J Biol Chem 264:19385-19391

Miller WR 1999 Biology of aromatase inhibitors: pharmacology/endocrinology within the breast. Endocr Relat Cancer 6:187-195

Miller WR, O'Neill J 1987 The importance of local synthesis of estrogen within the breast. Steroids 50:537-548

Mitwally MF, Casper RF 2001 Use of an aromatase inhibitor for induction of ovulation in patients with an inadequate response to clomiphene citrate. Fertil Steril 75:305-309

Mouridsen H, Gershanovich M, Sun Y, Perez-Carrion R, Boni C, Monnier A, Apffelstaedt J, Smith R, Sleeboom HP, Janicke F, Pluzanska A, Dank M, Becquart D, Bapsy PP, Salminen E, Snyder R, Lassus M, Verbeek JA, Staffler B, Chaudri-Ross HA, Dugan M 2001 Superior efficacy of letrozole versus tamoxifen as first-line therapy for postmenopausal women with advanced breast cancer: results of a phase III study of the International Letrozole Breast Cancer Group. J Clin Oncol 19:2596-2606

Nabholtz JM, Buzdar A, Pollak M, Harwin W, Burton G, Mangalik A, Steinberg M, Webster A, von Euler M 2000 Anastrozole is superior to tamoxifen as first-line therapy for advanced breast cancer in postmenopausal women: results of a North American multicenter randomized trial. Arimidex Study Group. J Clin Oncol 18:3758-3767

Nelson DR, Koymans L, Kamataki T, Stegeman JJ, Feyereisen R, Waxman DJ, Waterman MR, Gotoh O, Coon MJ, Estabrook RW, Gunsalus IC, Nebert DW 1996 P450 superfamily: update on new sequences, gene mapping, accession numbers and nomenclature. Pharmacogenetics $6: 1-42$

Pasqualini JR, Chetrite G, Blacker C, Feinstein MC, Delalonde L, Talbi M, Maloche C 1996 Concentrations of estrone, estradiol, and estrone sulfate and evaluation of sulfatase and aromatase activities in pre and postmenopausal breast cancer patients. J Clin Endocrinol Metab 81:1460-1464

Perez Carrion R, Alberola Candel V, Calabresi F, Michel RT, Santos R, Delozier T, Goss P, Mauriac L, Feuilhade F, Freue M 1994 Comparison of the selective aromatase inhibitor formestane with tamoxifen as first-line hormonal therapy in postmenopausal women with advanced breast cancer. Ann Oncol 7:S19-S24

Powles TJ, Hardy JR, Ashley SE, Farrington GM, Cosgrove D, Davey JB, Dowsett M, McKinna JA, Nash AG, Sinnett HD, Tillyer CR, Treleaven JG 1989 A pilot trial to evaluate the acute toxicity and feasibility of tamoxifen for prevention of breast cancer. Br J Cancer 60:126-131

Reed MJ, Owen AM, Lai LC, Coldham NG, Ghilchik MW, Shaikh NA, James VH 1989 In situ oestrone synthesis in normal breast and breast tumour tissues: effect of treatment with 4-hydroxyandrostenedione. Intl J Cancer 44:233-237

Santen RJ, Santner S, Davis R, Veldhuis J, Samojlik E, Ruby E 1978 Aminoglutehimide inhibits extraglandular estrogen production in postmenopausal women with breast carcinoma. J Clin Endocrinol Metab 47:1257-1265

Sasano H, Uzuki M, Sawai T, Nagura H, Matsunaga G, Kashimoto O, Harada N 1997 Aromatase in human bone tissue. J Bone Miner Res 12:1416-1423

Schrey MP, Patel KV 1995 Prostaglandin E2 production and metabolism in human breast cancer cells and breast fibroblasts. Regulation by inflammatory mediators. Br J Cancer 72:1412-1419

Sebastian S, Bulun SE 2001 A highly complex organization of the regulatory region of the human CYP19 (aromatase) gene revealed by the human genome project. J Clin Endocrinol Metab $86: 4600-4602$

Shetty G, Krishnamurthy H, Krishnamurthy HN, Bhatnagar AS, Mondgal RN 1997 Effect of estrogen deprivation on the reproductive physiology of male and female primates. J Steroid Biochem Mol Biol 61:157-166 
Shozu M, Simpson ER 1998 Aromatase expression of human osteoblast-like cells. Mol Cell Endocrinol 139:117-129

Simpson ER, Rubin G, Clyne C, Robertson K, O'Donnell L, Jones M, Davis S 2000 The role of local estrogen biosynthesis in males and females. Trends Endocrinol 5:184-188

Simpson ER, Zhao Y, Agarwal VR, Michael, MD, Bulun SE, Hinshelwood MM, GrahamLorence S, Sun T, Fisher CR, Qin K, Mendelson CR 1997 Aromatase expression in health and disease. Rec Progr Horm Res 52:185-214

Stuart-Harris R, Dowsett M, Bozek T, McKinna JA, Gazet JC, Jeffcoate SL, Kurkure A, Carr L, Smith IE 1984 Low-dose aminoglutehimide in treatment of advanced breast cancer. Lancet 2:604-607

Thomas HV, Reeves GK, Key TJ 1997 Endogenous estrogen and postmenopausal breast cancer: quantitative review. Cancer Causes Control 8:922-928

Thompson EA Jr, Siiteri PK 1974 Utilization of oxygen and reduced nicotinamide adeninedinucleotide phosphate by human placental microsomes during aromatization of androstenedione. J Biol Chem 249:5364-5372

Thurlimann B, Beretta K, Bacchi M, Castiglione-Gertsch M, Goldhirsch A, Jungi WF, Cavalli F, Senn HJ, Fey M, Lohnert T 1996 First-line fadrozole HCI (CGS 16949A) versus tamoxifen in postmenopausal women with advanced breast cancer. Prospective randomised trial of the Swiss Group for Clinical Cancer Research SAKK 20/88. Ann Oncol 7:471-479

Toda K, Terashima M, Kamamoto T, Sumimoto H, Yamamoto Y, Sagara Y, Ikeda H, Shizuta Y 1990 Structural and functional characterization of human aromatase. Eur J Biochem 193:559-565

Tominaga T, Morimoto T, Ohasi Y, for the Japan Letrozole Study Group 2000 A pivotal double-blind trial in Japan of an aromatase inhibitor Letrozole (third generation) vs. its predecessor fadrozole hydrochloride (second generation). Ann Oncol 11 (suppl):25

van der Wall E, Donker TH, de Frankrijker E, Nortier HW, Thijssen JH, Blankenstein MA 1993 Inhibition of the in vivo conversion of androstenedione to estrone by the aromatase inhibitor vorozole in healthy postmenopausal women. Cancer Res 53:4563-4566

Zeitoun K, Takayama K, Michael MD, Bulun SE 1999 Stimulation of aromatase P450 promoter (II) activity in endometriosis and its inhibition in endometrium are regulated by competitive binding of steroidogenic factor-1 and chicken ovalbumin upstream promoter transcription factor to the same cis-acting element. Mol Endocrinol 13:239-253

Zhao Y, Agarwal VR, Mendelson CR, Simpson ER 1996 Estrogen biosynthesis proximal to a breast tumor is stimulated by PGE2 via cyclic AMP, leading to activation of promoter II of the CYP19 (aromatase) gene. Endocrinology 137:5739-5742

Zhou J, Zhou C, Chen S 1997 Gene regulation studies of aromatase expression in breast cancer and adipose stromal cells. J Steroid Biochem Mol Biol 61:273-280 


\title{
Reproductive Functions of Progesterone Receptors
}

\author{
Orla M. Conneely, Biserka Mulac-Jericevic, Francesco DeMayo, \\ John P. Lydon, ANd Bert W. O’MALLEy \\ Department of Molecular and Cellular Biology, Baylor College of Medicine, \\ Houston, Texas 77030
}

\begin{abstract}
The steroid hormone progesterone plays a central role in the reproductive events associated with pregnancy establishment and maintenance. Physiological effects of progesterone are mediated by interaction of the hormone with specific intracellular progesterone receptors (PRs) that are expressed as two protein isoforms, PR-A and PR-B. Both proteins arise from the same gene and are members of the nuclear receptor superfamily of transcription factors. Since these two isoforms were identified in the early 1970s, extensive controversy has existed regarding the selective contributions of the individual PR proteins to the physiological functions of progesterone. During the past decade, significant progress has been made in this regard using two complimentary approaches. First, analysis of the structural and functional relationships of each isoform using in vitro systems has generated compelling evidence to support the conclusion that PR-A and PR-B have different transcription activation properties when liganded to progesterone. Second, the advent of gene-targeting approaches to introduce subtle mutations into the mouse genome has facilitated the evaluation of the significance of observations made in vitro in a physiological context. Selective ablation of PR-A and PR-B proteins in mice using these technologies has allowed us to address the spatiotemporal expression and contribution of the individual PR isoforms to the pleiotropic reproductive activities of progesterone. Analysis of the phenotypic consequences of these mutations on female reproductive function has provided proof of concept that the distinct transcriptional responses to PR-A and PR-B observed in cell-based transactivation assays are, indeed, reflected in an ability of the individual isoforms to elicit distinct, physiological responses to progesterone. In PR-A knockout mice, in which the expression of the PR-A isoform is selectively ablated (PRAKO), the PR-B isoform functions in a tissue-specific manner to mediate a subset of the reproductive functions of PRs. Ablation of PR-A does not affect responses of the mammary gland or thymus to progesterone but instead results in severe abnormalities in ovarian and uterine function, leading to female infertility. These tissue-selective activities of PR-B are due to this isoform's ability to regulate a subset of progesterone-responsive target genes in reproductive tissues rather than to differences in its spatiotemporal expression relative to the PR-A isoform. More recent studies using PR-B knockout (PRBKO) mice have shown that ablation of PR-B does not affect ovarian, uterine, or thymic responses to progesterone but rather results in reduced mammary ductal morphogenesis. Thus, PR-A is both necessary and sufficient to elicit the progesterone-dependent reproductive responses necessary for female fertility, while PR-B is required to elicit normal proliferative responses of the mammary gland to progesterone. This chapter will summarize recent progress in our understanding of the selective contribution of the two PR isoforms to progesterone action.
\end{abstract}




\section{Progesterone Receptor (PR) Isoforms}

Receptors for progesterone are expressed as two distinct isoforms, PR-A and PR-B, that arise from a single gene (Conneely et al., 1989; Kastner et al., 1990). The expression of both isoforms is conserved in rodent and humans and overlaps spatiotemporally in female reproductive tissues. However, the ratios of the individual isoforms vary in reproductive tissues as a consequence of developmental (Shyamala et al., 1990) and hormonal status (Duffy et al., 1997) and during carcinogenesis (Brandon et al., 1993; Graham et al., 1996).

PRs have a modular protein structure consisting of distinct, functional domains capable of binding steroidal ligand, dimerizing liganded receptors, interacting with hormone-responsive DNA elements, and interacting with coregulator proteins required for bridging receptors to the transcriptional apparatus (Guiochon-Mantel et al., 1989; Tsai and O'Malley, 1994; Giangrande and McDonnell, 1999; McKenna et al., 1999). Binding of progestin agonists induces conformational changes in receptor structure that promote interaction of coactivator proteins with distinct activation domains (AFs) located within both the amino- and carboxy-terminal regions of the receptor. Such coactivators promote chromatin remodeling and bridging with general transcription factors, resulting in the formation of productive transcription initiation complexes at the receptorresponsive promoter. In contrast, binding of receptor antagonist compounds induces receptor conformational changes that render AFs nonpermissive to coactivator binding and instead promote interaction with co-repressor proteins that inhibit the receptor's transcriptional activity. The ability of PRs to interact with a variety of coactivator and co-repressor proteins, together with the differing expression of co-regulators, illustrates a key role of these proteins in mediating different tissue-specific responses of progesterone receptors to steroidal ligand. Importantly, progesterone receptors also can be activated in the absence of steroidal ligand by phosphorylation pathways that modulate their interactions with co-regulator proteins (Rowan and O'Malley, 2000; Rowan et al., 2000).

PR-A and PR-B isoforms differ in that the PR-B protein contains an additional sequence of amino acids at its amino terminus. This PR-B-specific domain encodes a third transactivation function (AF3) that is absent from PR-A (Sartorius et al., 1994a; Wen et al., 1994). Recent evidence has demonstrated that AF3 allows binding of a subset of coactivators to PR-B that is not efficiently recruited by progestin-bound PR-A (Giangrande et al., 2000). Thus, when expressed individually in cultured cells, PR-A and PR-B display different transactivation properties that are specific to both cell type and target gene promoter context (Tora et al., 1988; Meyer et al., 1992; Vegeto et al., 1993; Hovland et al., 1998) and are associated with the differential ability of PR-A and PR-B to recruit specific co-regulator proteins (Giangrande et al., 2000). Agonistbound PR-B functions as a strong activator of transcription of several PR- 
dependent promoters and in a variety of cell types in which PR-A is inactive. Furthermore, when both isoforms are coexpressed in cultured cells, in cell and promoter contexts in which agonist-bound PR-A is inactive, PR-A can repress the activity of PR-B. PR-A's repressor capability extends to other steroid receptors, including estrogen receptor alpha (ER $\alpha)$ (McDonnell et al., 1994). Finally, PR-A and PR-B respond differently to progesterone antagonists (reviewed in Giangrande and McDonnell, 1999). While antagonist-bound PR-A is inactive, antagonist-bound PR-B can be converted to a strongly active transcription factor by modulating intracellular phosphorylation pathways (Beck et al., 1993; Musgrove et al., 1993; Sartorius et al., 1994b). Although the ligandbinding domain sequences of PR-A and PR-B are identical, the ability of different ligands to induce different conformational changes in PR, together with the synergistic activity of the amino- and carboxy-terminal activation domains (Tetel et al., 1999), predicts that PR-A- or PR-B-selective transcriptional regulation can be achieved by manipulating ligand interactions with the carboxy terminus.

\section{Physiological Role of PRs}

Null mutation of the PR gene encoding both isoforms has provided evidence of an essential role of PRs in a variety of female reproductive and nonreproductive activities. Female mice lacking both PRs exhibit impaired sexual behavior, neuroendocrine gonadotrophin regulation, anovulation, uterine dysfunction, and impaired ductal branching morphogenesis and lobuloalveolar differentiation of the mammary gland (Lydon et al., 1995; Mani et al., 1996; Chappell et al., 1997,1999). PRs also play an essential role in the regulation of thymic involution during pregnancy and in the cardiovascular system through regulation of endothelial and vascular smooth muscle cell proliferation and response to vascular injury (Tibbetts et al., 1999; Vazquez et al., 1999). Progesterone receptors have been identified in the central nervous system and bone, where progesterone has been implicated in cognitive function and bone maintenance. However, the essential role of PRs in these regions has not been confirmed.

\section{Generation of Mouse Models to Examine Selective Physiological Functions of PR-A and PR-B Proteins}

Differences in transcriptional activities and co-regulator interactions between PR-A and PR-B observed in vitro predicted that these proteins may mediate different physiological responses to progesterone. In addition, the selective ability of PR-A to inhibit transcriptional responses induced by both PR-B and ERs suggested that PR-A has the capacity to diminish overall progesterone responsiveness in certain tissues as well as to contribute to anties- 
trogenic activities of progesterone previously observed in the uterus. However, physiological validation of the functional differences between PR-A and PR-B isoforms has been hampered by a lack of information on the specific cell types that express each isoform in vivo and a dearth of appropriate animal models to determine their selective functions. The observation that PR-A and PR-B are produced by translation at two distinct AUG signals encoded by a single gene (Conneely et al., 1987; Kastner et al., 1990) predicted that mutation of either ATG codon in the PR gene would result in selective ablation of expression of a single isoform in vivo. Thus, we have used the CRE-loxP gene-targeting approach in embryonic stem cells to introduce a point mutation into the PR gene at the ATG codon encoding Met 1 (M1A) to specifically ablate expression of the PR-B protein and at the ATG encoding Met 166 (M166A) to ablate expression of PR-A. Consistent with previous findings in vitro (Conneely et al., 1987; Giangrande et al., 1997), we have demonstrated that these mutations are sufficient to ablate expression of the PR-B (PRBKO) or PR-A isoforms of PR (PRAKO) in mice (McDonnell, 1995) (Figure 1). The strategy provides a powerful model system to examine the selective expression of each isoform in situ as well as to assess the selective contributions of PR-A and PR-B in their normal cellular context to the physiological functions of progesterone.

\section{PRs and Ovarian Function}

Evidence that ovary-derived progesterone may participate in autocrine regulation of ovarian function first emerged when it was demonstrated that

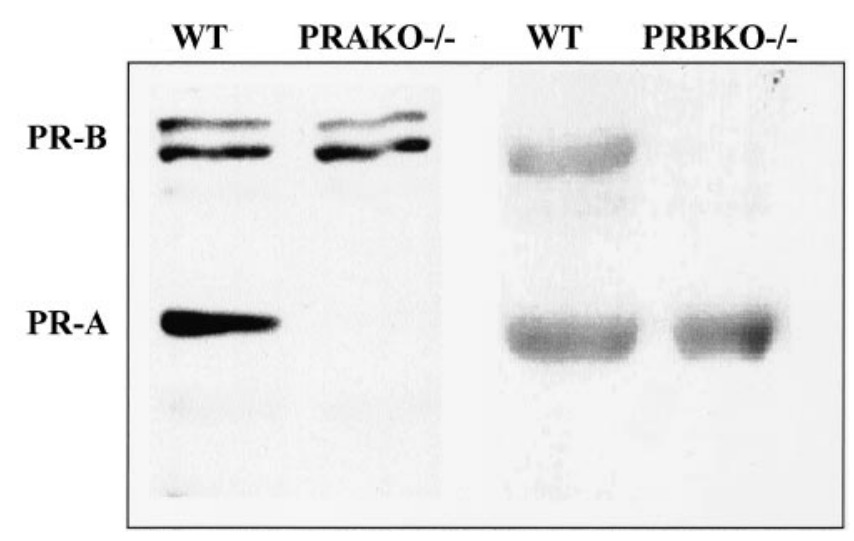

FIG. 1. Western immunoblot analysis to detect progesterone receptor (PR) isoform expression in uterine tissue extracts derived from wild-type (WT), PR-A knockout (PRAKO), and PR-B knockout (PRBKO) mice. 
luteinizing hormone ( $\mathrm{LH})$, the primary signal for rupture of preovulatory ovarian follicles leading to ovulation, can stimulate transient expression of PR mRNA and protein in granulosa cells isolated from preovulatory follicles (Park and Mayo, 1991; Natraj and Richards, 1993; Park-Sarge and Mayo, 1994) and that the antiprogestin, RU486, can inhibit ovulation (Loutradis et al., 1991). Definitive proof that PRs are essential mediators of ovulation has been provided through analysis of the ovarian phenotype of the PRKO mouse. Despite exposure to superovulatory levels of gonadotropins, PRKO mice fail to ovulate. Analysis of the histology of these mice has revealed normal development of intraovarian follicles to the tertiary follicular stage (Lydon et al., 1995). The follicles contain a mature oocyte that is fully functional when isolated and fertilized in vitro. However, follicular rupture is effectively eliminated. Despite the ovulatory block, the preovulatory granulosa cells within these follicles can differentiate into a luteal phenotype and express the luteal marker, P450 side-chain cleavage enzyme (Robker et al., 2000). Thus, PR is required specifically for LH-dependent follicular rupture leading to ovulation but not for differentiation of granulosa cells to form a corpus luteum (luteinization). Follicular rupture requires induction of a prostaglandin-mediated inflammatory response to $\mathrm{LH}$ as well as tissue degradation at the apex of the preovulatory follicle, an event that is mediated by matrix-digesting proteinases (Espey, 1994). Recent investigations to examine the molecular events associated with ovulation that are mediated by PRs have shown that PRs are induced specifically in the mural granulosa cells of the mature tertiary follicle and are absent from the cumulus granulosa cells that surround the oocyte (Robker et al., 2000). Analysis of potential ovulation mediator expression in PRKO mice has demonstrated that LH-induced regulation of Cox-2, an enzyme that catalyzes prostaglandin production, is unaffected (Robker et al., 2000). Cox-2 is required for ovulation and is expressed by cumulus granulosa cells (Dinchuk et al., 1995; Morham et al., 1995). In contrast, the expression of two metalloproteinases, ADAMTS-1 (a desintegrin and metalloproteinase with thrombospondin motifs) and cathepsin-L (a lysosomal cysteine protease) is inhibited in granulosa cells of mature follicles (Robker et al., 2000) in PRKO mice. ADAMTS-1 recently was shown to play an essential role in ovulation (Shindo et al., 2000), suggesting that this protein may be a critical mediator of the progesterone-induced ovulatory event.

Previous studies have indicated that both the PR-A and PR-B proteins are induced in preovulatory follicles in response to LH stimulation (Natraj and Richards, 1993). Therefore, to examine the selective roles of the individual PR isoforms in mediating the ovulatory function of progesterone, we have analyzed the ovulatory phenotype of mice in which expression of either the PRAKO or PRBKO isoform is selectively ablated. Stimulation of immature PRAKO mice with gonadotropins indicated that superovulation is severely impaired in these mice relative to their wild-type counterparts but, unlike PRKO mice, is not 
completely absent (Figure 2). In contrast, superovulation was unaffected in PRBKO mice expressing only the PR-A protein. Thus, PR-A expression is both necessary and sufficient to mediate the ovulatory response to progesterone.

Histological analysis of the ovaries of PRAKO mice (Figure 2) showed numerous mature anovulatory follicles that contained an intact oocyte and were arrested at a similar stage to that previously observed in PRKO mice. Most surprisingly, however, in contrast to PRKO mice, progesterone's spatiotemporal regulation of ADAMTS-1 and cathepsin-L was unaffected. Thus, despite its inability to mediate follicular rupture, PR-B is functional in the ovary and capable of regulating a subset of progesterone-responsive target genes (B. Mulac-Jericevic and O.M. Conneely, unpublished data). The observation that the PR-A and PR-B proteins are not functionally redundant in the ovary provides physiological validation of previous studies in tissue culture demonstrating that these transcription factors have different functional activities. From a mechanistic standpoint, the observation that PR-A alone is sufficient to support normal ovulation indicates that heterodimeric interactions between PR-A and PR-B proteins are not required to regulate essential progestin-responsive target genes associated with ovulation. Using differential gene array approaches to identify differentially expressed genes in PRAKO and PRBKO mice should facilitate isolation of PR-A-selective target genes essential for ovulation as well as provide important new information on the molecular mechanisms of progesteroneinduced follicular rupture.

A

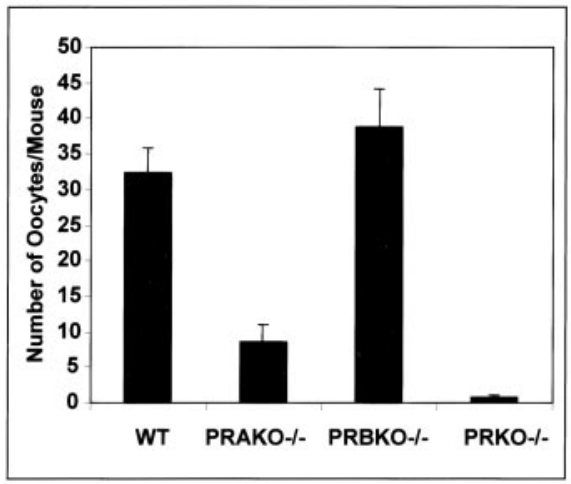

B

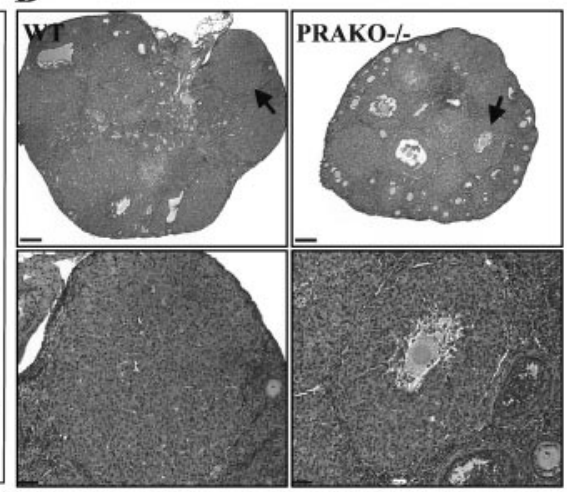

FIG. 2. Ovulation is critically dependent on PR-A function. (A) Average number of oocytes ( \pm SEM) released per mouse after superovulation in WT, PRAKO-/-, PRBKO-/-, and PRKO-/- mice; $\mathrm{n}=8$ per test group. (B) Hematoxylin- and eosin-stained sections of ovaries from superovulated WT and PRAKO-/- mice. Scale bars $=200 \mu \mathrm{m}$. Higher magnifications of the ovarian structures indicated by the arrow are presented in the lower panels. Scale bars $=50 \mu \mathrm{m}$ in the lower panels. 


\section{PR Isoforms and Uterine Implantation}

Female infertility in PRKO mice also is associated with defective uterine implantation and a lack of decidualization of uterine stromal cells in response to progesterone (Lydon et al., 1995). Consistent with these findings, wildtype embryos failed to implant when transferred into uteri of pseudopregnant PRKO females. Similarly, mating attempts between superovulated PRAKO females and wild-type males failed to result in successful pregnancies, despite the release of low numbers of oocytes from PRAKO females. To determine whether the PR-A protein is required for uterine decidualization, we treated ovariectomized mice with progesterone and estrogen, followed by mechanical stimulation of the left uterine horn of each animal (Ledford et al., 1976), to induce decidualization of stromal cells. Decidualization is linked with a marked increase in uterine weight and a characteristic histological appearance associated with the differentiation of stromal cells into decidual cells. Both responses were inhibited in PRAKO mice, indicating that expression of PR-A in the uterus is required to mediate the decidualization response to progesterone (Figure 3).

To determine whether the decidualization defect in PRAKO mice was linked to aberrant regulation of progesterone-responsive target genes associated with implantation, we analyzed the expression of several implantationspecific uterine epithelial target genes. Specifically, we examined regulation of three genes: calcitonin (CT), histidine decarboxylase (HDC), and amphiregulin (AR) whose expression is increased in the uterine epithelium in response to progesterone in association with uterine receptivity (Das et al., 1995; Paria et al., 1998; Zhu et al., 1998) and the epithelial secretory glycoprotein, lactoferrin (LF). LF expression is induced by estrogen and inhibited by progesterone (McMaster et al., 1992). Progesterone-dependent regulation of each of these genes is abolished in PRKO mice. Ablation of PR-A, however, resulted in loss of expression of CT and AR, while the regulation of HDC and LF was fully retained (Mulac-Jericevic et al., 2000). These findings indicated that defective implantation in PRAKO uteri is associated with loss of progesterone-regulated expression of a subset of genes involved with uterine epithelial receptivity. Importantly, this differential target gene regulation by PR-B was not due to differences in spatiotemporal expression of PR-B relative to PR-A. The expression of PR-B in PRAKO mice showed the same pattern of intrauterine expression and regulation by estrogen as that observed in wild-type mice (Mulac-Jericevic et al., 2000). Thus, the uterine defects observed in these mice are due to differences in PR-B transcription factor activity rather than to differences in expression of the protein relative to PR-A. 
A

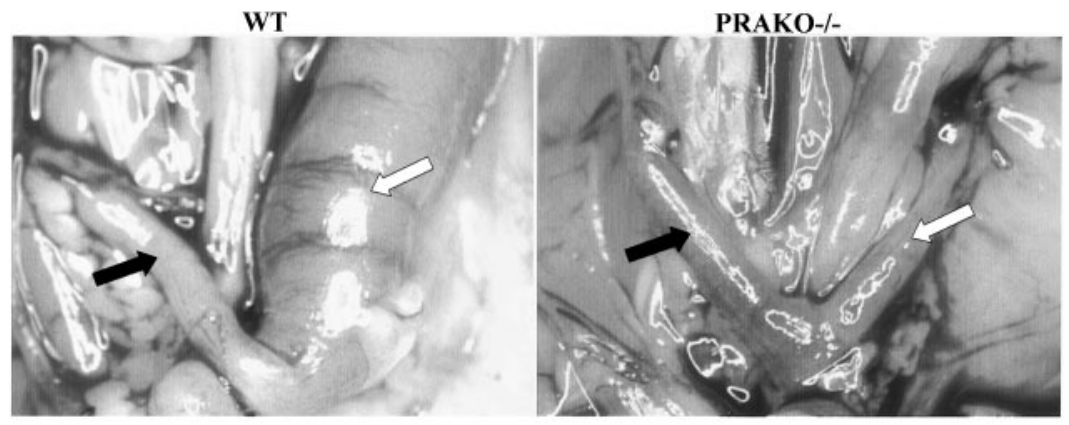

B

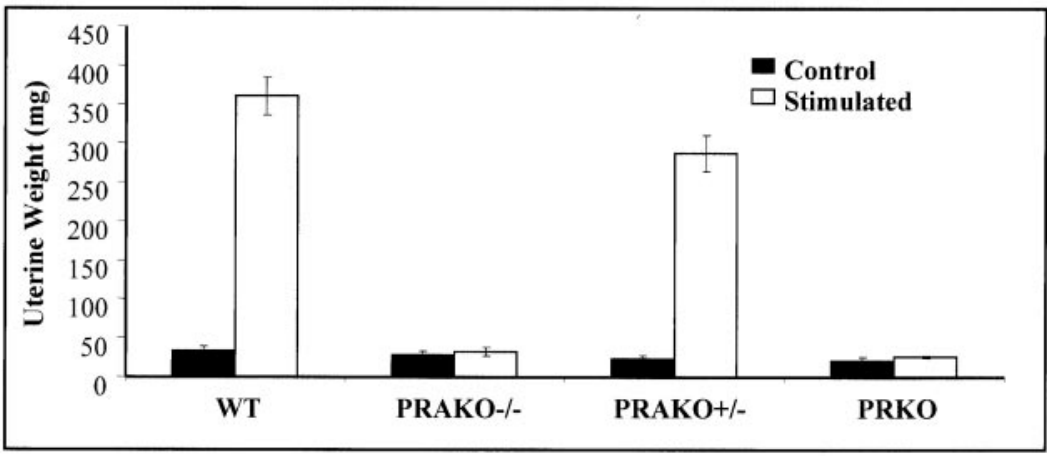

FIG. 3. The PRAKO uterus is unable to undergo decidualization. (A) Responses of the WT and PRAKO-/- uteri to mechanical decidualization. Black arrows indicate the unstimulated left uterine horn; white arrows denote the right uterine horn following stimulation. (B) Uterine weights following decidualization shown as means $\pm \mathrm{SEM} ; \mathrm{n}=6$ per test group.

\section{Opposing Functions of PR-A and PR-B in the Regulation of Uterine Epithelial Proliferation}

Estrogen is the primary proliferative stimulus for uterine epithelium. Its effects are inhibited by progesterone (Clarke and Sutherland, 1990; Lydon et al., 1995). Ablation of both the PR-A and PR-B isoforms in PRKO mice results in marked hyperplasia of the lumenal and glandular epithelial tissue due to the unopposed action of estrogen (Lydon et al., 1995). Selective ablation of PR-A, however, revealed an unexpected capacity of PR-B to contribute to rather than inhibit epithelial cell proliferation. Treatment of PRAKO mice with estrogen induced epithelial hyperplasia in a manner similar to that observed in PRKO and wild-type mice. However, progesterone plus estrogen resulted in a marked increase in proliferation over that observed with estrogen alone, a response that was not observed in PRKO mice (Figure 4). These results indicate that expression of the PR-B protein alone in the uterus results in a gain of proliferative 


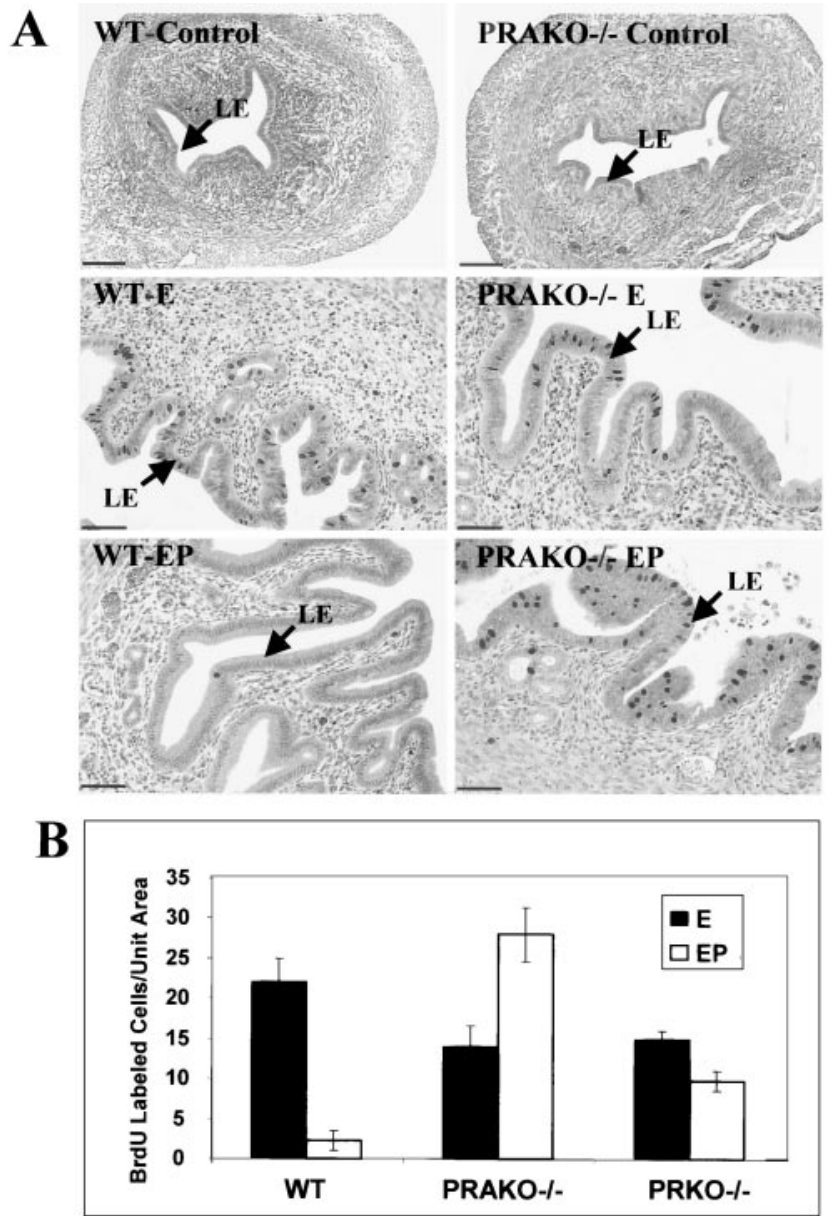

FIG. 4. PRAKO uterine epithelium exhibits abnormal proliferative responses to estrogen (E) and progesterone (P) treatment. (A) Bromodeoxyuridine immunolabeling of proliferating epithelial cells in uteri from ovariectomized WT and PRAKO-/- mice treated with sesame oil (control), E, or $\mathrm{E}+\mathrm{P}(\mathrm{EP})$. Scale bars $=200 \mu \mathrm{m}$ (controls) and $50 \mu \mathrm{m}$ (hormone treated). LE, lumenal epithelium. (B) Quantitations of BrdU-positive uterine LE cells in WT, PRAKO-/-, and PRKO mice. The results presented are mean values \pm SEM; $n=6$ (WT and PRAKO-/-); $n=4$ (PRKO).

activity (Mulac-Jericevic et al., 2000). This acquisition of a proliferative activity of progesterone represents a PR-B-dependent gain of function not previously observed in the uterus and indicates that uterine expression of the PR-A isoform is required not only to oppose estrogen-induced proliferation but also that induced by progesterone acting through the PR-B protein. This dual role of PR-A 
is of particular significance in light of previous studies carried out in vitro. Such studies have demonstrated that PR-A, when bound to progestin agonists, can inhibit target gene activation by both PR-B and ER $\alpha$ when tested in transfection assays using cultured cell lines in which agonist-bound PR-A alone is transcriptionally inactive (Vegeto et al., 1993; Kraus et al., 1995). These data predicted that PR-A has the capacity to diminish overall progesterone as well as estrogen responsiveness. Importantly, the inhibitory activity of PR-A observed in the uterus is tissue specific and is not observed in the mammary gland, where both estrogen and progesterone receptors contribute to ductal epithelial proliferation in the presence of PR-A.

The discovery that PR-B can contribute to rather than inhibit uterine epithelial cell proliferation is likely to have important clinical implications with regard to hormonal management of uterine endometrial dysplasias. Clearly, the relative expression of PR isoforms under these conditions will be an important determinant of the effectiveness of progestin therapy. Our results predict that progestin agonists selective for the PR-A protein should improve the effectiveness of progestin therapy in these conditions.

\section{Tissue-selective Regulation of Mammary Epithelial Branching Morphogenesis and Differentiation of Alveolar Lobules by the PR-B Isoform}

Estrogen and progesterone are essential to maintain postnatal developmental plasticity of the mammary gland. Both hormones play a key role in mammary tumorigenesis. Null mutation of both PR isoforms in PRKO mice has demonstrated that PRs are specifically required for pregnancy-associated ductal proliferation and lobuloalveolar differentiation of the mammary epithelium. The mammary glands of PRKO mice failed to develop the pregnancy-associated side branching of the ductal epithelium with attendant lobular alveolar differentiation, despite normal postpubertal mammary gland morphogenesis of the virgin mice. Ablation of PR expression in these mice also resulted in a significantly reduced incidence of mammary tumor growth in response to carcinogen challenge (Lydon et al., 1999). These observations underscore a specific role of PRs (as distinct from ERs) as obligate mediators of the intracellular signaling pathways essential for the initiation of murine mammary tumors induced by carcinogens.

Use of PRKO mice in combination with mammary gland transplantation techniques has provided important insights into the mechanisms underlying progesterone-dependent mammary gland morphogenesis. Throughout postpubertal mammary gland development, PRs are expressed exclusively in the epithelium (Seagroves et al., 2000; Sivaraman et al., 2001). Consistent with these observations, tissue transplantation approaches using wild-type and PRKO mouse tissue to produce mammary gland recombinants that were devoid of PR 
in either the stromal or epithelial compartments have provided strong support for the functional involvement of epithelial rather than stromal PRs in mediating mammary gland morphogenic responses to progesterone (Brisken et al., 1998). PR expression is localized to a scattered subset of epithelial cells throughout the ductal epithelium, most of which appear segregated from proliferating epithelial cells (Lydon et al., 2000; Seagroves et al., 2000). The hierarchical organization of these receptors and their segregation from proliferating cells are conserved features in rodent and human mammary tissue (Clarke et al., 1997; Seagroves et al., 2000; Sivaraman et al., 2001). Such an expression pattern predicted that regulation of epithelial cell proliferation by progesterone may occur through a paracrine mechanism, whereby PRs residing in nonproliferating cells induce expression of a signal that promotes proliferation of neighboring receptornegative cells. While PRKO mammary epithelium cannot undergo side branching, mixing experiments with PRKO and wild-type epithelial cells demonstrate that branching and differentiation defects can be overcome when PRKO cells are placed in close contact with PR-positive cells (Brisken et al., 1998). Thus, although lacking PR-positive cells, the PRKO mammary epithelium retains those PR-negative cells that are responsive to PR-mediated paracrine signaling. Recent attempts to uncover downstream mediators of the progesterone response have identified the secreted glycoprotein, Wnt-4, as a potential PR target that is coexpressed in PR-positive cells, is regulated by progesterone, and is essential for regulating ductal branching via paracrine regulation of proliferation (Brisken et al., 2000). Identification of additional paracrine mediators of the progesterone response using differential gene array analysis of PRKO and wild-type mammary glands is an imminently achievable goal.

In contrast to paracrine signaling pathways operative in the normal gland, in many breast tumors, the majority of ER- and PR-positive cells undergo proliferation (Clarke et al., 1997). This observation would suggest that a switch in steroid-dependent regulation from a paracrine to autocrine mechanism may be an important part of the tumorigenic process. Consistent with this hypothesis, we recently observed that one of the earliest responses to carcinogen challenge in the mammary gland is the emergence of a population of proliferating cells that score positively for the expression of steroid receptors. This pattern is clearly at odds with the paracrine signaling pathways operative in the normal gland (Sivaraman et al., 2001).

Both PR isoforms are expressed in the mammary gland of the virgin mouse (Shyamala et al., 1990) and during pregnancy (Fantl et al., 1999), although levels of PR-A exceed those of PR-B by at least a 2:1 ratio. To examine the selective contributions of each isoform to the morphogenic responses of the mammary epithelium to progesterone, we compared the morphology of mammary glands of ovariectomized, wild-type PRAKO and PRBKO mice after exposure to estrogen and progesterone. Ablation of PR-A in PRAKO mice did not affect the ability of 
PR-B to elicit normal progesterone responsiveness in the mammary gland. The morphological changes in ductal side branching and lobular alveolar development in these glands were similar to those observed in wild-type mice (Figure 5) (Mulac-Jericevic et al., 2000). Thus, the PR-B isoform is sufficient to elicit normal proliferation and differentiation of the mammary epithelium in response to progesterone. Neither process appears to require functional expression of PR-A. In contrast, more recent analysis of the mammary glands of PRBKO mice under similar conditions has shown markedly reduced ductal side branching and lobular alveolar differentiation (B. Mulac-Jericevic and O.M. Conneely, in preparation).

The PR isoform-selective morphogenic responses of the mammary gland observed in our studies do not phenocopy those previously observed when disruption of PR isoform ratios was achieved by overexpression of either PR-A or PR-B in the mammary glands of transgenic mice (Shyamala et al., 1998,2000). PR-A overexpression caused mammary glands to exhibit increased ductal branching and hyperplasia and, most interestingly, abnormal disruption of basement membrane organization and decreased cell-cell adhesion (Shyamala et al., 1998). In contrast, PR-B overexpression resulted in limited ductal elongation and branching, while alveolar growth was unaffected (Shyamala et al., 2000). The striking differences in mammary defects observed in isoform-selective knockout mice relative to PR-A and PR-B transgenic mice could be explained by inappropriate targeting in transgenic mice of PR-A and PR-B expression to epithelial subtypes that normally would not express PR but may be competent to proliferate. Thus, indiscriminate targeting of these receptor isoforms to the mammary gland would breach the cellular segregation rules that apply to normal epithelial cell growth, resulting in a scenario reminiscent of the inappropriate colocalization of steroid receptor expression and proliferation observed in cells of breast tumors. Despite the phenotypic differences observed between the isoformselective knockout and transgenic models, both approaches provide strong evidence that a regulated expression of both PR isoforms is critical for the mammary gland to respond appropriately to progesterone.

\section{Summary}

Over the past decade, significant progress has been made in understanding the collective and selective contributions of PR isoforms to the signaling pathways controlled by progesterone. Molecular dissection of progesterone signaling mechanisms using in vitro systems has demonstrated that the PR-A and PR-B proteins can respond to the same steroid ligand to induce both overlapping and distinct transcriptional responses that are promoter and cell context dependent. The use of genetically manipulated mouse models in which one or both of the PR isoforms is ablated has been pivotal in defining the physiological 
A

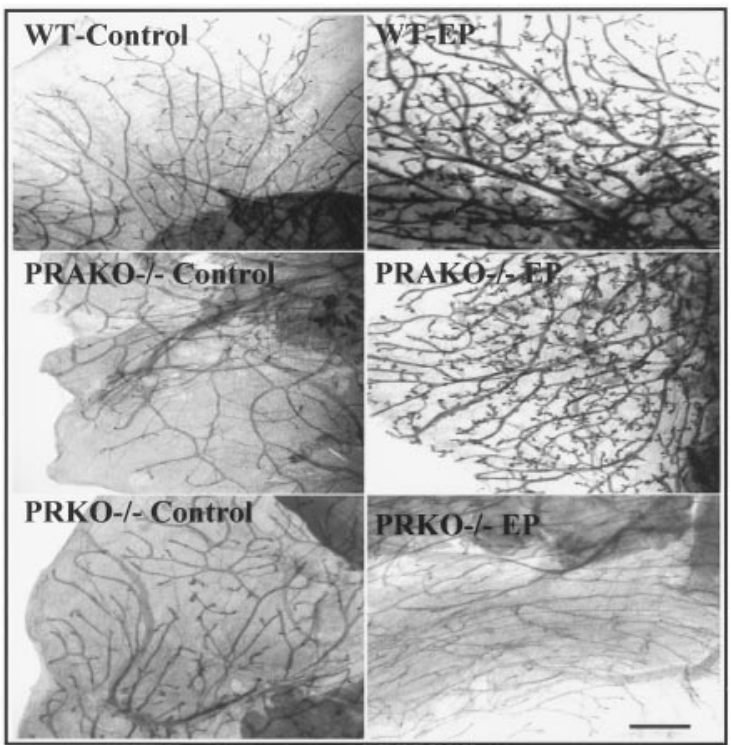

B

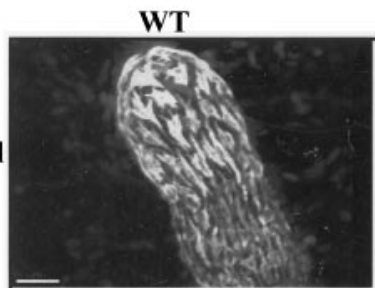

PRAKO-/-

EP
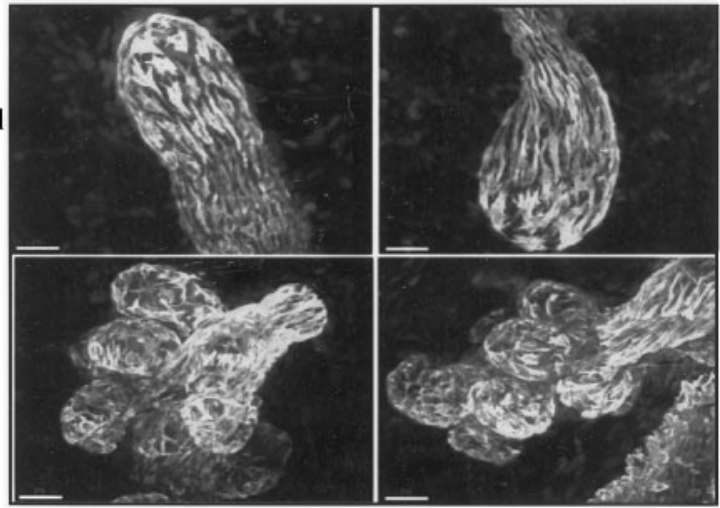

FIG. 5. The PR-B isoform mediates tissue-selective mammary gland tertiary ductal side branching and alveologenesis. (A) Thoracic mammary gland whole mounts of untreated (control) or $\mathrm{E}+\mathrm{P}(\mathrm{EP})$ treated WT, PRAKO-/-, and PRKO mice. Scale bar $=500 \mu \mathrm{m}$. (B) Terminal ductal and alveolar structures visualized in the WT and PRAKO-/- mice using cytokeratin-14 immunofluorescence that specifically labels myepithelial cells. Scale bar $=20 \mu \mathrm{m}$.

spectrum of PR action as well as the contribution of individual protein isoforms to the pleiotropic activities of the hormone. These approaches have provided compelling evidence that the differences in transactivation properties of the PR 
isoforms observed in vitro are reflected in a differential capacity to regulate the tissue-selective reproductive activities of progesterone. From a mechanistic standpoint, differences in physiological activities observed between PR-A and PR-B isoforms illustrate the key role played by amino-terminal AF domains in distinguishing tissue-specific responses to steroidal ligand. The results demonstrate that the inclusion or deletion of the N-terminal AF3 transactivation domain in PR is sufficient to alter tissue-specific responses to progesterone. Studies to date have selectively addressed the role of PR isoforms in mediating reproductive activities of progesterone. Genetic mouse models will provide valuable tools to address the physiological significance of ligand-independent pathways of receptor activation as well as the contribution of specific receptor subtypes to the activities of tissue-selective receptor modulators. In this regard, our observations predict that isoform-specific modulators of PR action should facilitate the identification of novel, tissue-selective modulators of PR-dependent reproductive functions.

\section{REFERENCES}

Beck CA, Weigel NL, Moyer ML, Nordeen SK, Edwards DP 1993 The progesterone antagonist RU486 acquires agonist activity upon stimulation of cAMP signaling pathways. Proc Natl Acad Sci USA 90:4441-4445

Brandon DD, Bethea CL, Strawn EY, Novy MJ, Burry KA, Harrington MS, Erickson TE, Warner C, Keenan EJ, Clinton GM 1993 Progesterone receptor messenger ribonucleic acid and protein are overexpressed in human uterine leiomyomas. Am J Obstet Gynecol 169: $78-85$

Brisken C, Park S, Vass T, Lydon JP, O'Malley BW, Weinberg RA 1998 A paracrine role for the epithelial progesterone receptor in mammary gland development. Proc Natl Acad Sci USA 95:5076-5081

Brisken C, Heineman A, Chavarria T, Elenbaas B, Tan J, Dey SK, McMahon JA, McMahon AP, Weinberg RA 2000 Essential function of Wnt-4 in mammary gland development downstream of progesterone signaling. Genes Dev 14:650-654

Chappell PE, Lydon JP, Conneely OM, O'Malley BW, Levine JE 1997 Endocrine defects in mice carrying a null mutation for the progesterone receptor gene. Endocrinology 138:4147-4152

Chappell PE, Schneider JS, Kim P, Xu M, Lydon JP, O'Malley BW, Levine JE 1999 Absence of gonadotropin surges and gonadotropin-releasing hormone self-priming in ovariectomized (OVX), estrogen (E2)-treated, progesterone receptor knockout (PRKO) mice. Endocrinology 140:3653-3658

Clarke CL, Sutherland RL 1990 Progestin regulation of cellular proliferation. Endocr Rev 11:266-301

Clarke RB, Howell A, Potten CS, Anderson E 1997 Dissociation between steroid receptor expression and cell proliferation in the human breast. Cancer Res 57:4987-4991

Conneely OM, Maxwell BL, Toft DO, Schrader WT, O'Malley BW 1987 The A and B forms of the chicken progesterone receptor arise by alternate initiation of translation of a unique mRNA. Biochem Biophys Res Commun 149:493-501

Conneely OM, Kettelberger DM, Tsai MJ, Schrader WT, O'Malley BW 1989 The chicken progesterone receptor $\mathrm{A}$ and $\mathrm{B}$ isoforms are products of an alternate translation initiation event. J Biol Chem 264:14062-14064 
Das SK, Chakraborty I, Paria BC, Wang XN, Plowman G, Dey SK 1995 Amphiregulin is an implantation-specific and progesterone-regulated gene in the mouse uterus. Mol Endocrinol 9:691-705

Dinchuk JE, Car BD, Focht RJ, Johnston JJ, Jaffee BD, Covington MB, Contel NR, Eng VM, Collins RJ, Czerniak PM, Gorry PM, Trzaskos JM 1995 Renal abnormalities and an altered inflammatory response in mice lacking cyclooxygenase II. Nature 378:406-409

Duffy DM, Wells TR, Haluska GJ, Stouffer RL 1997 The ratio of progesterone receptor isoforms changes in the monkey corpus luteum during the luteal phase of the menstrual cycle. Biol Reprod 57:693-699

Espey LL, Lipner, H 1994 Ovulation. In Knobil E, Neill JD, eds. The Physiology of Reproduction. New York: Raven Press; 725-780

Fantl V, Edwards PA, Steel JH, Vonderhaar BK, Dickson C 1999 Impaired mammary gland development in Cyl-1(-/-) mice during pregnancy and lactation is epithelial cell autonomous. Dev Biol 212:1-11

Giangrande PH, McDonnell DP 1999 The A and B isoforms of the human progesterone receptor: two functionally different transcription factors encoded by a single gene. Recent Progr Horm Res 54:291-313

Giangrande PH, Pollio G, McDonnell DP 1997 Mapping and characterization of the functional domains responsible for the differential activity of the A and B isoforms of the human progesterone receptor. J Biol Chem 272:32889-32900

Giangrande PH, Kimbrel EA, Edwards DP, McDonnell DP 2000 The opposing transcriptional activities of the two isoforms of the human progesterone receptor are due to differential cofactor binding. Mol Cell Biol 20:3102-3215

Graham JD, Yeates C, Balleine RL, Harvey SS, Milliken JS, Bilous AM, Clarke CL 1996 Progesterone receptor A and B protein expression in human breast cancer. J Steroid Biochem Mol Biol 56:93-98

Guiochon-Mantel A, Loosfelt H, Lescop P, Sar S, Atger M, Perrot-Applanat M, Milgrom E 1989 Mechanisms of nuclear localization of the progesterone receptor: evidence for interaction between monomers. Cell 57:1147-1154

Hovland AR, Powell RL, Takimoto GS, Tung L, Horwitz KB 1998 An N-terminal inhibitory function, IF, suppresses transcription by the A-isoform but not the B-isoform of human progesterone receptors. J Biol Chem 273:5455-5460

Kastner P, Krust A, Turcotte B, Stropp U, Tora L, Gronemeyer H, Chambon P 1990 Two distinct estrogen-regulated promoters generate transcripts encoding the two functionally different human progesterone receptor forms A and B. EMBO J 9:1603-1614

Kraus WL, Weis KE, Katzenellenbogen BS 1995 Inhibitory cross-talk between steroid hormone receptors: differential targeting of estrogen receptor in the repression of its transcriptional activity by agonist- and antagonist-occupied progestin receptors. Mol Cell Biol 15:1847-1857

Ledford BE, Rankin JC, Markwald RR, Baggett B 1976 Biochemical and morphological changes following artificially stimulated decidualization in the mouse uterus. Biol Reprod 15:529-535

Loutradis D, Bletsa R, Aravantinos L, Kallianidis K, Michalas S, Psychoyos A 1991 Preovulatory effects of the progesterone antagonist mifepristone (RU486) in mice. Hum Reprod 6:12381240

Lydon JP, DeMayo FJ, Funk CR, Mani SK, Hughes AR, Montgomery CA Jr, Shyamala G, Conneely OM, O'Malley BW 1995 Mice lacking progesterone receptor exhibit pleiotropic reproductive abnormalities. Genes Dev 9:2266-2278

Lydon JP, Ge G, Kittrell FS, Medina D, O'Malley BW 1999 Murine mammary gland carcinogenesis is critically dependent on progesterone receptor function. Cancer Res 59:4276-4284

Lydon JP, Sivaraman L, Conneely OM 2000 A reappraisal of progesterone signaling in the mammary gland. J Mammary Gland Biol Neoplasia 5:325-338 
Mani SK, Allen JM, Lydon JP, Mulac-Jericevic B, Blaustein JD, DeMayo FJ, Conneely O, O’Malley BW 1996 Dopamine requires the unoccupied progesterone receptor to induce sexual neurobehavior in mice. Mol Endocrinol 10:1728-1737

McDonnell DP 1995 Unraveling the human progesterone receptor signal transduction pathway: insights into antiprogestin action. Trends Endocrinol Metab 6:133-138

McDonnell DP, Shahbaz MM, Vegeto E, Goldman ME 1994 The human progesterone receptor A-form functions as a transcriptional modulator of mineralocorticoid receptor transcriptional activity. J Steroid Biochem Mol Biol 48:425-432

McKenna NJ, Xu J, Nawaz Z, Tsai SY, Tsai MJ, O’Malley BW 1999 Nuclear receptor coactivators: multiple enzymes, multiple complexes, multiple functions. J Steroid Biochem Mol Biol 69:3-12

McMaster MT, Teng CT, Dey SK, Andrews GK 1992 Lactoferrin in the mouse uterus: analyses of the preimplantation period and regulation by ovarian steroids. Mol Endocrinol 6:101-111

Meyer ME, Quirin-Stricker C, Lerouge T, Bocquel MT, Gronemeyer H 1992 A limiting factor mediates the differential activation of promoters by the human progesterone receptor isoforms. J Biol Chem 267:10882-10887

Morham SG, Langenbach R, Loftin CD, Tiano HF, Vouloumanos N, Jennette JC, Mahler JF, Kluckman KD, Ledford A, Lee CA, Smithies O 1995 Prostaglandin synthase 2 gene disruption causes severe renal pathology in the mouse. Cell 83:473-482

Mulac-Jericevic B, Mullinax RA, DeMayo FJ, Lydon JP, Conneely OM 2000 Subgroup of reproductive functions of progesterone mediated by progesterone receptor-B isoform. Science 289:1751-1754

Musgrove EA, Hamilton JA, Lee CS, Sweeney KJ, Watts CK, Sutherland RL 1993 Growth factor, steroid, and steroid antagonist regulation of cyclin gene expression associated with changes in T-47D human breast cancer cell cycle progression. Mol Cell Biol 13:3577-3587

Natraj U, Richards JS 1993 Hormonal regulation, localization, and functional activity of the progesterone receptor in granulosa cells of rat preovulatory follicles. Endocrinology 133:761769

Paria BC, Das N, Das SK, Zhao X, Dileepan KN, Dey SK 1998 Histidine decarboxylase gene in the mouse uterus is regulated by progesterone and correlates with uterine differentiation for blastocyst implantation. Endocrinology 139:3958-3966

Park OK, Mayo KE 1991 Transient expression of progesterone receptor messenger RNA in ovarian granulosa cells after the preovulatory luteinizing hormone surge. Mol Endocrinol 5:967-978

Park-Sarge OK, Mayo KE 1994 Regulation of the progesterone receptor gene by gonadotropins and cyclic adenosine $3^{\prime}, 5^{\prime}$-monophosphate in rat granulosa cells. Endocrinology 134:709-718

Robker RL, Russell DL, Espey LL, Lydon JP, O'Malley BW, Richards JS 2000 Progesteroneregulated genes in the ovulation process: ADAMTS-1 and cathepsin L proteases. Proc Natl Acad Sci USA 97:4689-4694

Rowan BG, O’Malley BW 2000 Progesterone receptor coactivators. Steroids 65:545-549

Rowan BG, Garrison N, Weigel NL, O'Malley BW 2000 8-Bromo-cyclic AMP induces phosphorylation of two sites in SRC-1 that facilitate ligand-independent activation of the chicken progesterone receptor and are critical for functional cooperation between SRC-1 and CREB binding protein. Mol Cell Biol 20:8720-8730

Sartorius CA, Melville MY, Hovland AR, Tung L, Takimoto GS, Horwitz KB 1994a A third transactivation function (AF3) of human progesterone receptors located in the unique N-terminal segment of the B-isoform. Mol Endocrinol 8:1347-1360

Sartorius CA, Groshong SD, Miller LA, Powell RL, Tung L, Takimoto GS, Horwitz KB 1994b New T47D breast cancer cell lines for the independent study of progesterone B- and A-receptors: only antiprogestin-occupied B-receptors are switched to transcriptional agonists by cAMP. Cancer Res 54:3868-3877 
Seagroves TN, Lydon JP, Hovey RC, Vonderhaar BK, Rosen JM 2000 C/EBPbeta (CCAAT/ enhancer binding protein) controls cell fate determination during mammary gland development. Mol Endocrinol 14:359-368

Shindo T, Kurihara H, Kuno K, Yokoyama H, Wada T, Kurihara Y, Imai T, Wang Y, Ogata M, Nishimatsu H, Moriyama N, Oh-hashi Y, Morita H, Ishikawa T, Nagai R, Yazaki Y, Matsushima K 2000 ADAMTS-1: a metalloproteinase-disintegrin essential for normal growth, fertility, and organ morphology and function [see comments]. J Clin Invest 105: $1345-1352$

Shyamala G, Schneider W, Schott D 1990 Developmental regulation of murine mammary progesterone receptor gene expression. Endocrinology 126:2882-2889

Shyamala G, Yang X, Silberstein G, Barcellos-Hoff MH, Dale E 1998 Transgenic mice carrying an imbalance in the native ratio of A to B forms of progesterone receptor exhibit developmental abnormalities in mammary glands. Proc Natl Acad Sci USA 95:696-701

Shyamala G, Yang X, Cardiff RD, Dale E 2000 Impact of progesterone receptor on cell-fate decisions during mammary gland development. Proc Natl Acad Sci USA 97:3044-3049

Sivaraman L, Hilsenbeck SG, Zhong L, Gay J, Conneely OM, Medina D, O'Malley BW 2001 Early exposure of the rat mammary gland to estrogen and progesterone blocks co-localization of estrogen receptor expression and proliferation. J Endocrinol 171:75-83

Tetel MJ, Giangrande PH, Leonhardt SA, McDonnell DP, Edwards DP 1999 Hormonedependent interaction between the amino- and carboxyl-terminal domains of progesterone receptor in vitro and in vivo. Mol Endocrinol 13:910-924

Tibbetts TA, DeMayo F, Rich S, Conneely OM, O’Malley BW 1999 Progesterone receptors in the thymus are required for thymic involution during pregnancy and for normal fertility. Proc Natl Acad Sci USA 96:12021-12026

Tora L, Gronemeyer H, Turcotte B, Gaub MP, Chambon P 1988 The N-terminal region of the chicken progesterone receptor specifies target gene activation. Nature 333:185-188

Tsai MJ, O'Malley BW 1994 Molecular mechanisms of action of steroid/thyroid receptor superfamily members. Annu Rev Biochem 63:451-486

Vazquez F, Rodriguez-Manzaneque JC, Lydon JP, Edwards DP, O'Malley BW, Iruela-Arispe ML 1999 Progesterone regulates proliferation of endothelial cells. J Biol Chem 274:21852192

Vegeto E, Shahbaz MM, Wen DX, Goldman ME, O’Malley BW, McDonnell DP 1993 Human progesterone receptor A form is a cell- and promoter-specific repressor of human progesterone receptor B function. Mol Endocrinol 7:1244-1255

Wen DX, Xu YF, Mais DE, Goldman ME, McDonnell DP 1994 The A and B isoforms of the human progesterone receptor operate through distinct signaling pathways within target cells. Mol Cell Biol 14:8356-8364

Zhu LJ, Cullinan-Bove K, Polihronis M, Bagchi MK, Bagchi IC 1998 Calcitonin is a progesterone-regulated marker that forecasts the receptive state of endometrium during implantation. Endocrinology 139:3923-3934 



\title{
Estrogen Actions Throughout the Brain
}

\author{
BRUCE McEwen \\ Harold and Margaret Milliken Hatch Laboratory of Neuroendocrinology, \\ The Rockefeller University, New York, New York 10021
}

\begin{abstract}
Besides affecting the hypothalamus and other brain areas related to reproduction, ovarian steroids have widespread effects throughout the brain, on serotonin pathways, catecholaminergic neurons, and the basal forebrain cholinergic system as well as the hippocampal formation, a brain region involved in spatial and declarative memory. Thus, ovarian steroids have measurable effects on affective state as well as cognition, with implications for dementia. Two actions are discussed in this review; both appear to involve a combination of genomic and nongenomic actions of ovarian hormones. First, regulation of the serotonergic system appears to be linked to the presence of estrogen- and progestin-sensitive neurons in the midbrain raphe as well as possibly nongenomic actions in brain areas to which serotonin neurons project their axons. Second, ovarian hormones regulate synapse turnover in the CA1 region of the hippocampus during the 4- to 5-day estrous cycle of the female rat. Formation of new excitatory synapses is induced by estradiol and involves $\mathrm{N}$-methyl-D-aspartate (NMDA) receptors, whereas downregulation of these synapses involves intracellular progestin receptors. A new, rapid method of radioimmunocytochemistry has made possible the demonstration of synapse formation by labeling and quantifying the specific synaptic and dendritic molecules involved. Although NMDA receptor activation is required for synapse formation, inhibitory interneurons may play a pivotal role as they express nuclear estrogen receptor-alpha (ER $\alpha$ ). It is also likely that estrogens may locally regulate events at the sites of synaptic contact in the excitatory pyramidal neurons where the synapses form. Indeed, recent ultrastructural data reveal extranuclear ER $\alpha$ immunoreactivity within select dendritic spines on hippocampal principal cells, axons, axon terminals, and glial processes. In particular, the presence of ER in dendrites is consistent with a model for synapse formation in which filopodia from dendrites grow out to find new synaptic contacts and estrogens regulate local, post-transcriptional events via second messenger systems.
\end{abstract}

\section{Introduction}

Most studies of estrogen action in brain have focused on reproduction. The majority of these studies have dealt with animal models in which evidence accumulating over more than 30 years has shown that estrogens target the brain of experimental animals (Pfaff, 1980). Now, medical science has recognized that the brain is one of the organs of the body that suffers from the loss of estrogen after surgical or natural menopause and that damage from stroke and neurodegeneration in dementia may be retarded by estrogen actions. Indeed, with 
increasing life expectancy during the 20th century, women are likely to live a substantial part of their lives in a state of estrogen deficiency. Although hot flushes are, for many women, the most-dramatic and noticeable consequence of loss of ovarian hormones, some women experience, at surgical or natural menopause, difficulties in remembering names and other information important for daily life as well as deficits in fine motor coordination and reaction times and feelings of depression and anxiety (McEwen and Alves, 1999). Loss of bone calcium and osteoporosis are other consequences that have led many women to take estrogen replacement therapy (ERT) at the menopause. Likewise, the loss of protection of the coronary arteries, leading postmenopausal women to increased risk for cardiovascular disease, is another result of estrogen deficiency that has reinforced the value of ERT. Thus, the somewhat-delayed recognition of the brain as an important estrogen target provides another compelling reason to investigate the value of ERT for states of estrogen deficiency.

Although most of the animal studies cited above focused on estrogen actions on the hypothalamus affecting ovulation and reproductive behavior, it is now apparent in animal models and clinical studies that estrogens exert many actions outside of reproductive function, including actions on brain areas that are important for learning and memory and for emotions and affective state as well as motor coordination and pain sensitivity. These effects reflect the actions of estrogens on a large number of brain areas outside of the hypothalamus, such as the midbrain and brain stem neurons that produce serotonin and catecholamines, spinal cord, cerebral cortex, and hippocampus. The problem in these brain regions has been to recognize the receptors and mechanisms by which estrogens produce their effects.

This review will focus on two aspects. First, the cellular and molecular mechanisms by which estrogens produce their diverse effects on the brain. Second, the brain regions and cell types in which estrogens produce their effects, emphasizing new knowledge regarding estrogen actions outside of the hypothalamus and pituitary gland. The serotonin system will be discussed as an example as well as the hippocampus. We will discuss the actions of estrogens in regulating synapse formation in hippocampus because they reflect a novel, nonreproductive action of estrogens related to cognitive function that is relevant for postmenopausal changes. Furthermore, these actions also illustrate the emerging evidence that estrogens act via mechanisms other than the classical cell nuclear estrogen receptors (ERs).

\section{Intracellular Mechanisms of Estrogen Action in the Central Nervous System}

\section{A. INTRACELLULAR ESTROGEN RECEPTORS}

In the early 1960s, ERs were identified that bind to DNA and regulate gene expression (Jensen and Jacobson, 1962). Radioactive estrogens were used to 
identify ERs inside of the cell nuclei. The identification and mapping of cells that contain ERs were extended from the uterus and mammary glands to the brain and pituitary gland (Pfaff, 1980). At first, only ERs in the hypothalamus and pituitary gland were studied because they were the most obvious and also the most obviously related to estrogen actions on reproduction. Eventually, however, nerve cells containing ERs were recognized in brain regions like the hippocampus, cerebral cortex, midbrain, and brainstem. We are now aware of two types of intracellular ER, ER $\alpha$ and ER $\beta$ (see McEwen and Alves, 1999, for summary).

Measurements of mRNA for $\operatorname{ER} \alpha$ and $\operatorname{ER} \beta$ reveal distributions in the body that differ quite markedly from each other, with moderate to high expression of $\mathrm{ER} \alpha$ in pituitary, kidney, epididymus, and adrenal; moderate to high expression of ER $\beta$ in prostate, lung, bladder, and brain; and overlapping high expression in ovary, testis, and uterus (Kuiper et al., 1998). Isoforms of ER $\beta$ are now being identified (see McEwen and Alves, 1999, for summary). The best characterized of these splice variants is ER $\beta 2$, as opposed to the originally identified isoform, $\mathrm{ER} \beta 1$. ER $\beta 2$ appears to have a lower affinity for estrogens than ER $\beta 1$, presumably due to an 18 amino acid insertion in the ligand-binding domain (Maruyama et al., 1998). There are other splice variants of $\mathrm{ER} \beta$ with differential expression in brain and other tissues, including a variant missing Exon 4 that does not bind estradiol in hippocampus (Price et al., 2000).

In brain, the distribution of $\mathrm{ER} \alpha$ is fairly well established but there is less certainty surrounding the localization of ER $\beta$. Autoradiographic maps of ${ }^{3} \mathrm{H}$ estradiol uptake and retention in brain (Stumpf and Sar, 1976; Pfaff, 1980) are presumed to reflect binding to all forms of the ER, particularly the ER $\alpha$ and ER $\beta 1$ isoforms, which have similar, high affinities for $17 \beta$ estradiol (Kuiper $e t$ al., 1998). In situ hybridization data indicate widespread distribution of $\mathrm{ER} \beta$ mRNA throughout much of the brain, including olfactory bulbs, cerebellum, and cerebral cortex (Kuiper et al., 1997,1998). Results from immunocytochemical studies for $\mathrm{ER} \beta$ indicate a more-restricted localization of detectable protein, although the antisera that are currently available do not always provide specific signals in some brain areas (see McEwen and Alves, 1999, for discussion). Recent evidence shows good agreement between ER $\beta$ mRNA levels and ER $\beta$ immunoreactivity with a polyclonal antibody to the C terminus of ER $\beta$ (Shughrue and Merchenthaler, 2001). In particular, colocalization of ER $\beta$ mRNA with cell nuclear ER $\beta$ immunoreactivity was demonstrated in the rat cerebral cortex, paraventricular nuclei of the hypothalamus, and hypothalamic preoptic area (Shughrue and Merchenthaler, 2001). Other brain regions remain to be studied for such co-localization.

The introduction of ${ }^{125}$ I estrogen to label ER with a higher specific radioactivity has revealed the presence of binding sites not previously detected using ${ }^{3} \mathrm{H}$ estradiol (Shughrue et al., 1999; Shughrue and Merchenthaler, 2000). In hippocampus, labeling was evident in the interneurons previously seen to contain 
$\mathrm{ER} \alpha$ by other methods, with higher concentrations in ventral hippocampus (Shughrue and Merchenthaler, 2000). However, the most important difference from ${ }^{125} \mathrm{I}$ estrogen labeling of ER was the detection of label in pyramidal cells of CA1-CA3 in the ventral hippocampus. In parallel studies of ER $\alpha$ and $-\beta$ mRNA, there was a similar dorsal-to-ventral gradient as that seen for ${ }^{125} \mathrm{I}$ estrogen binding and the $\operatorname{ER} \alpha$ signal appeared to be stronger than that for $\operatorname{ER} \beta$ (Shughrue and Merchenthaler, 2000). Thus, the greater sensitivity of ${ }^{125} \mathrm{I}$ estrogen labeling of ER reveals sites that may indicate locations of estrogen actions in hippocampal pyramidal cells, particularly in pyramidal cell nuclei of the ventral hippocampus.

$\mathrm{ER} \alpha$ and $\operatorname{ER} \beta$ are similar not only in affinity for a number of estrogens and estrogen antagonists (Kuiper et al., 1998) but also in their ability to regulate genes in which the estrogen response element (ERE) is the primary site of interaction (Paech et al., 1997). The major differences between $\operatorname{ER} \alpha$ and $\operatorname{ER} \beta$ concern their ability to regulate transcription via the AP-1 response element. With AP-1, estradiol $17 \beta$ activated transcription with $\mathrm{ER} \alpha$, whereas it failed to activate transcription with $\mathrm{ER} \beta$ and was able to cause inhibition. In contrast, with AP-1, nonsteroidal estrogen antagonists such as tamoxifen activated transcription with ER $\beta$ and did so to a lesser extent with ER $\alpha$ (Paech et al., 1997).

$\mathrm{ER} \alpha$ and $\operatorname{ER} \beta$ can form heterodimers when expressed in the same cells, thus giving rise to additional possible variants as far as gene regulation (Pettersson $e t$ al., 1997). Thus far, endogenous colocalization of $\operatorname{ER} \alpha$ and $\operatorname{ER} \beta$ has been demonstrated in the hypothalamic preoptic area, bed nucleus of the stria terminalis, and medial amygdaloid nucleus (Hrabovszky et al., 1998) and probably exists in other brain regions, including midbrain raphe (see below).

Nonsteroidal estrogen antagonists exert agonist-like effects on some neurochemical or functional endpoints in the rat brain and antagonistic effects on others. Antagonistic effects for CI-628, a tamoxifen-like estrogen antagonist, were seen in terms of blockade of estrogen-induced progestin receptor induction and lordosis behavior (Roy et al., 1979; Meisel et al., 1987). Agonist-like effects of CI-628 were seen for induction of choline acetyltransferase in basal forebrain and repression of monoamine oxidase A in amygdala (Luine and McEwen, 1977). Recently, CI-628 was shown to block estrogen-induced synapse formation in the hippocampus without having any agonist-like effects (McEwen et al., 1999b; Brake et al., 2001).

One important implication of these findings concerning the mixed agonist and antagonist role of some estrogen antagonists is that nonsteroidal antiestrogens, like CI-628 and possibly also tamoxifen and raloxifene, will not have uniformly agonistic or antagonistic effects on the diversity of actions that estrogens normally produce in the brain. This has implications for the therapeutic applications of such agents and requires a separate study of the actions of these agents on each endpoint of estrogen action. 
Unfortunately, the molecular basis of these effects is not clear. Although anti-estrogens function as antagonists for $\operatorname{ER} \alpha$ and $\operatorname{ER} \beta$ via the ERE, these same anti-estrogens worked through the AP-1 response element to activate transcription via $\operatorname{ER} \beta$ also, to a lesser extent, via $\mathrm{ER} \alpha$ (Paech et al., 1997). Yet, with AP-1, estradiol $17 \beta$ activated transcription with $\mathrm{ER} \alpha$, whereas it failed to activate transcription with $\operatorname{ER} \beta$ and was able to cause inhibition (Paech et al., 1997). Thus, to account for parallel agonist-like actions of estrogen antagonists, such as we have demonstrated for choline acetyltransferase and monoamine oxidase, it is necessary to postulate that $\mathrm{ER} \alpha$ is the receptor involved and that it uses the AP-1 response element where both estradiol and CI-628 can have agonistic-like actions.

\section{B. NOVEL ESTROGEN ACTIONS VIA NON-NUCLEAR RECEPTORS}

Rapid estrogen effects on neuronal excitability (Kelly et al., 1977; Nabekura et al., 1986) have been known for a number of years. Yet, only recently has this topic emerged in full force as an alternative aspect of estrogen action that involves interactions of ERs with second messenger systems and potentially novel types of ERs (Kelly and Wagner, 1999; Brinton, 2001; Kelly and Levin, 2001; Lee and McEwen, 2001).

The variety of nongenomic estrogen effects includes 1) rapid actions on excitability of neuronal and pituitary cells; 2) the activation by estrogens of cyclic AMP and mitogen-activated protein kinase (MAP kinase) pathways that affect activity of such targets as kainate and insulin-like growth factor-1 (IGF-1) receptors; 3 ) estrogen actions that involve modulation of $\mathrm{G}$ protein coupling and affect calcium currents and gonadotropin-releasing hormone $(\mathrm{GnRH})$ release; 4) effects on calcium channels and calcium ion entry; and 5) protection of neurons from damage by excitotoxins and free radicals (Kelly and Wagner, 1999; Brinton, 2001; Kelly and Levin, 2001; Lee and McEwen, 2001).

For estrogen actions on some aspects of calcium homeostasis, certain aspects of second messenger systems, and some features of neuroprotection, a novel receptor mechanism is implicated in which stereospecificity for $17 \beta$ over $17 \alpha$ estradiol is replaced by a broader specificity for the 3 hydroxyl group on the A ring ( McEwen and Alves, 1999; Lee and McEwen, 2001). On the other hand, there is also evidence that $\operatorname{ER} \alpha$ and $\operatorname{ER} \beta$ are capable of participating in second messenger cascades involving second messenger activation and $\mathrm{G}$ protein coupling (Razandi et al., 1999; Kelly and Levin, 2001). Besides $\mathrm{ER} \alpha$ and $\mathrm{ER} \beta$, membrane ERs have been reported on pituitary, uterine, ovarian granulosa cell, spermatozoa, testes, and liver cell membranes. However, these have been only partially characterized in binding studies and only in a few cases have been shown to be linked to signal 
transduction mechanisms (for a review, see McEwen and Alves, 1999; Kelly and Levin, 2001; Lee and McEwen, 2001).

Finally, estrogenic compounds protect nerve cells from damage by excitotoxins and free radicals in novel ways (McEwen and Alves, 1999; Lee and McEwen, 2001). In this realm are neuroprotective effects that appear to be mediated via classical genomic receptors, based upon the fact that they can be blocked by estrogen antagonists. But other actions are not blocked by these antagonists and appear to involve a novel mechanism in which estradiol $17 \alpha$ is as potent as estradiol 17 $\beta$ (Green et al., 1997; Lee and McEwen, 2001). These actions of estrogens, albeit in 100-nM to micromolar concentrations, reduce the production of or actions of free radicals in causing cell damage and promoting cell death through apoptosis. Mitochondria are major targets of estrogen action. Estrogen effects stabilize mitochondrial membrane potentials, prevent adenosine triphosphate (ATP) depletion, and reduce the generation of oxygen free radicals (Mattson et al., 1997; Wang et al., 2001).

\section{Estrogen Actions Throughout the Central Nervous System}

We now know that ovarian steroids have numerous effects on the brain throughout the lifespan, beginning during gestation and continuing on into senescence. Estrogens participate in the sexual differentiation of the brain during early embryonic or neonatal life. These effects undoubtedly involve the classical intracellular ERs (McEwen, 1983; Auger et al., 2000). The process of sexual differentiation involves the secretion of testosterone in fetal or early neonatal life and the actions of testosterone either through androgen receptors or via aromatization to estrogen in the defeminization and masculinization of brain structures and function (Goy and McEwen, 1980; Naftolin, 1994). Although initially believed to be confined to the hypothalamus, structural and functional sex differences have been found in higher cognitive centers and in sensory and autonomic ganglia as well as structures of the limbic system of the brain and the midbrain, brainstem, and basal forebrain structures (for a review, see McEwen and Alves, 1999).

Estrogens affect areas of the brain that are not primarily involved in reproduction, such as the basal forebrain cholinergic system, the hippocampus and cerebral cortex, the caudate-putamen, midbrain raphe and brainstem locus coeruleus, and the spinal cord. These systems are involved in a variety of estrogen actions on mood, locomotor activity, pain sensitivity, vulnerability to epilepsy, and attentional mechanisms and cognition (for a review, see McEwen and Alves, 1999).

Functional or structural sex differences exist in a number of these brain regions (Fischette et al., 1984; Kimura, 1992; Bazzett and Becker, 1994; Witelson et al., 1995). For example, developmentally programmed sex 
differences in hippocampal structure may help explain differences in the strategies that male and female rats use to solve spatial navigation problems (Williams and Meck, 1991). A similar sex difference in spatial problemsolving is reported in humans (Kimura, 1992). During the period of development when testosterone is elevated in the male, aromatase activity and ERs are transiently expressed in hippocampus. Recent data on behavior and synapse induction strongly suggest that this pathway is involved in the masculinization or defeminization of hippocampal structure and function.

In spite of the paucity of $\mathrm{ER} \alpha$ outside the hypothalamus, hypothalamic preoptic region, and amygdala, estrogens have effects on many other brain regions and neurochemical systems involved in a host of nonreproductive brain functions. The expression of ER $\beta$ mRNA in many of these brain regions has raised the possibility of functional ERs in these brain areas. At the same time, the presence of a few ER $\alpha$-containing nerve cells has led to the discovery, for example in the hippocampus, that these few nerve cells can have powerful trans-synaptic effects on neighboring neurons. As will be discussed in detail below, treatment of ovariectomized rats with estradiol $17 \beta$ induces certain hippocampal neurons to form new synaptic connections with other nerve cells. These estrogen effects appear to be attributable, at least in part, to intracellular ER $\alpha$ in inhibitory interneurons (see below). In addition, the rapidity and structure-activity profile of some of these effects have raised questions about the possible "nontraditional" and even nongenomic actions of estrogens in some brain regions. For example, actions of estrogens on dopaminergic activity in the corpus striatum and nucleus accumbens appear to be mediated by membrane actions in the absence of any documented expression of either ER $\alpha$ or $\mathrm{ER} \beta$ in cell nuclei of these brain regions (Maus et al., 1990; Mermelstein et al., 1996).

Estrogen actions upon cholinergic, noradrenergic, serotonergic, and hypothalamic dopaminergic systems, on the other hand, are likely to be mediated by known nuclear intracellular ER $\alpha$ or ER $\beta$ (see McEwen and Alves, 1999, for summary). This will be decribed for the serotonin system. The spinal cord also has intracellular $\operatorname{ER} \alpha$ and $\operatorname{ER} \beta$ but the reported effects on nociception and analgesia do not directly relate to those receptor sites in enkephalin-expressing spinal neurons. Moreover, endothelial cells and at least some glial cells must be considered as targets for estrogen action that affect glucose uptake and mechanisms that support the replenishment of cell membranes and possibly also synaptogenesis and other forms of structural plasticity (see McEwen and Alves, 1999; McEwen et al., 2001, for summary). We now will examine two systems involving actions of estrogens on brain areas that are involved in many functions beyond those specifically concerned with reproduction, namely, the midbrain serotonin system and the hippocampus. 


\section{Estrogen Actions on Midbrain Raphe and Serotonin Receptors in Forebrain}

The serotonin system projects widely to many brain regions and is concerned with the regulation of many aspects of brain function, ranging from autonomic nervous system reactivity to mood, aggression, and cognitive function (Jacobs, 1994; Higley and Linnoila, 1997; Rubinow et al., 1998; Bethea et al., 1999). Ovarian steroids regulate the serotonin system of rodents and primates (Alves et al., 1998; Bethea et al., 1999), but important differences exist in the relationship between detectable intracellular ERs and the serotonergic neurons.

In primates, both $\mathrm{ER} \alpha$ and $\mathrm{ER} \beta$ are found in midbrain 5HT neurons and estrogens regulate tryptophan hydroxylase as well as progestin receptor expression (Bethea et al., 1999). In rats, $\mathrm{ER} \alpha$ is found in non-5HT neurons, where estrogen regulates expression of progestin receptors (Alves et al., 1998) but not expression of tryptophan hydroxylase (S.E. Alves, unpublished data). Sex differences are found in the ability of estrogen treatment to induce progestin receptors (Alves et al., 1998). In mice, both $\operatorname{ER} \alpha$ and $\operatorname{ER} \beta$ are present and functional in the midbrain. ER $\alpha$ is expressed in 5HT neurons that also express progestin receptors (Alves et al., 2000). Yet, the $\alpha$ ERKO mouse also shows estrogen induction of progestin receptors in midbrain raphe, implying that another ER, most likely ER $\beta$, is involved (Alves et al., 2000) (Figure 1).

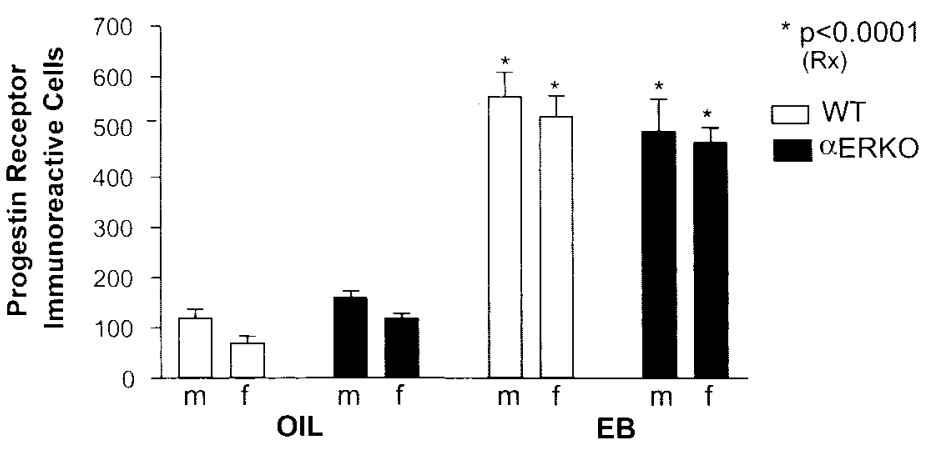

FIG. 1. Number of progestin receptor (PR) immunoreactive cells counted in the dorsal raphe nucleus (A) and median raphe nucleus (B) of wild-type (WT) and $\alpha$ ERKO mice of both sexes. Estradiol benzoate (EB) treatment induces PR in both sexes of both genotypes. Although EB induces PR in both genotypes, the significant interaction between treatment and genotype in both dorsal $(\mathrm{F}(1,16)=4.73, \mathrm{p}<.045)$ and median $(\mathrm{F}(1,16)=9.33, \mathrm{p}<.008)$ raphe nuclei indicates that the magnitude of PR induction by EB over baseline levels was less in the $\alpha$ ERKO animals. No significant gender differences were found. We conclude that another ER besides $\mathrm{ER} \alpha$, most likely $\mathrm{ER} \beta$, mediates the effects of $\mathrm{EB}$ in the raphe nuclei. [Reprinted with permission from Alves SE, McEwen BS, Hayashi S, Korach KS, Pfaff DW, Ogawa S 2000 Estrogen-regulated progestin receptors are found in the midbrain raphe but not hippocampus of estrogen receptor alpha (ER $\alpha$ ) gene-disrupted mice. J Comp Neurol 427:185-195. Copyright Wiley-Liss, Inc., a subsidiary of John Wiley \& Sons, Inc.] 
Estrogens also regulate other components of the serotonin system besides tryptophan hydroxylase. In midbrain raphe of primates, estrogen treatment decreased serotonin transporter mRNA expression (Bethea et al., 1999). In macaque hypothalamus, estrogen treatment decreased expression of the 5HT2C receptor in a number of hypothalamic nuclei (Gundlah et al., 1999). In rat brain, 32-hour estrogen treatment increased levels of 5HT2A mRNA in dorsal raphe and 5HT2A receptor binding in frontal, cingulate, and primary olfactory cortex as well as in nucleus accumbens (Sumner and Fink, 1998) (Figure 2). There were no sex differences in this induction (Sumner and Fink, 1998). Another study on rats reported that a 24-hour estrogen treatment increased 5HT2A mRNA levels in amygdala, hippocampus, accumbens, and a number of cortical areas, while

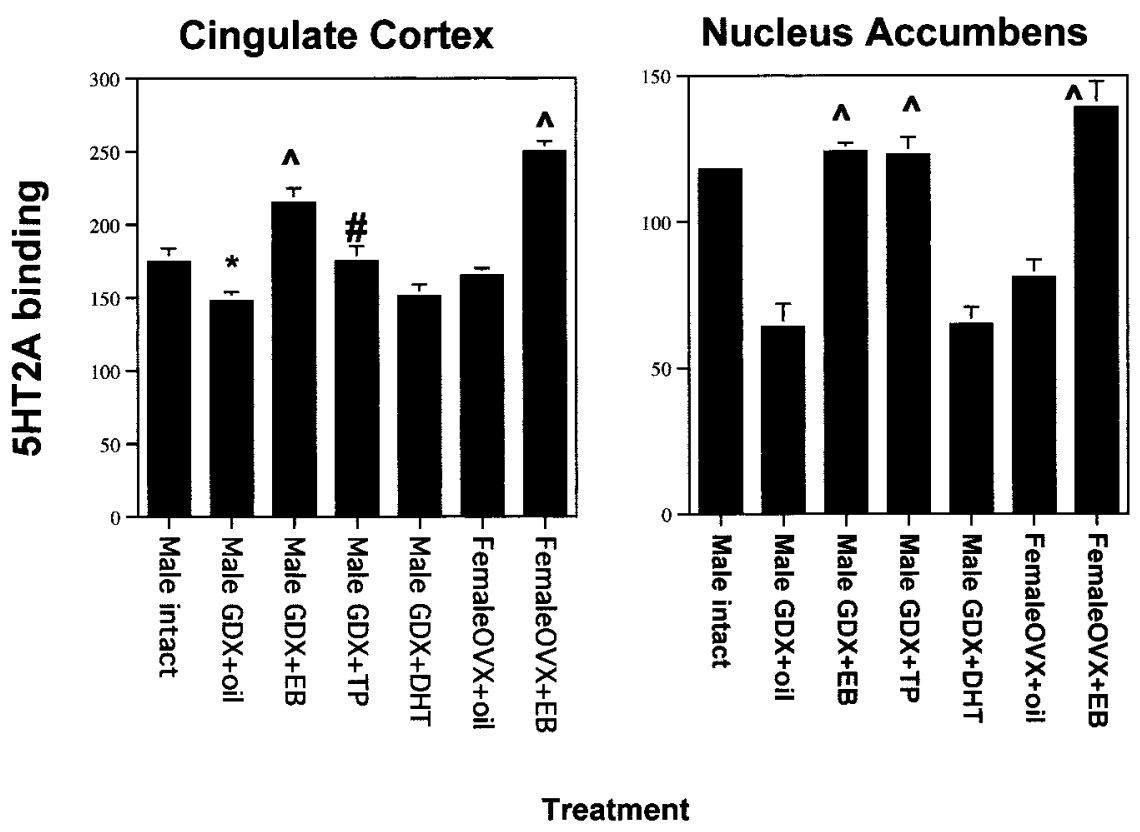

FIG. 2. Serotonin 5HT2A receptor binding in the cingulate cortex and nucleus accumbens of male and female rats after gonadectomy and treatment with either oil vehicle, estradiol benzoate (EB), testosterone propionate (TP), or dihydrotestosterone (DHT). These two brain areas are not normally considered to be estrogen or androgen target areas but the post-synaptic 5HT2A receptor levels are elevated in both males and females by $\mathrm{EB}(\wedge \mathrm{p}<.005)$ and in males by TP $(\# \mathrm{p}<.05$ for cingulate; $\wedge \mathrm{p}<.005$ for accumbens) but not by DHT. There is a significant effect of gonadectomy in males $(\mathrm{p}<.05)$. This suggests that estrogens work equally in both sexes and the aromatase pathway may be involved in the TP effects, although the nature of the ERs that mediate these effects remains uncertain. [Redrawn from Sumner BEH, Fink G 1998 Testosterone as well as estrogen increases serotonin ${ }_{2 \mathrm{~A}}$ receptor mRNA and binding site densities in the male rat brain. Mol Brain Res 59:205-214.] 
decreasing 5HT1A mRNA levels in many of the same brain regions of several strains of rats differing in anxiety-related behaviors (Osterlund et al., 1999). Chronic (2-week) estrogen treatment also decreased 5HT1A receptor binding in amygdala, hippocampus, and cerebral cortex (Osterlund et al., 2000). Yet, in this study, the effects of estrogen on 5HT1A mRNA levels, evident after acute estrogen treatment, disappeared with the chronic treatment that decreased 5HT1A receptor binding.

The actions of estrogen on the 5HT1A receptor system illustrate the complexities of distinguishing between traditional genomic effects of estrogens and those involving a non-nuclear action. Estrogen treatment causes a rapid decrease in coupling to $\mathrm{G}$ proteins that reduces the inhibitory effect of 5HT1A agonists on lordosis behavior, hyperphagia, and oxytocin and corticotropin (ACTH) responses (Raap et al., 2000; Mize et al., 2001). The rapid, estrogeninduced decrease in 5HT1A efficacy has been assessed by measuring radiolabeled GTP $\gamma$ S binding (Mize et al., 2001) after treatment with estrogen in homogenates of hippocampus and frontal cortex. Estradiol $17 \beta(E C 50=25 \mathrm{nM})$ showed a dose-dependent ability to decrease GTP $\gamma \mathrm{S}$ binding. This effect was mimicked by diethylstilbestrol but not by the less-potent estrogens, estradiol $17 \alpha$ and estriol, and was blocked by the estrogen antagonist, ICI 182780 (Mize et al., 2001). These results are consistent with the involvement of a non-nuclear form of $\mathrm{ER} \alpha$ or $\mathrm{ER} \beta$, as discussed above.

\section{Estrogen Actions in Hippocampus re: Cognitive Function and Memory Processes}

The hippocampus is a brain region that is involved in episodic, declarative, contextual, and spatial learning and memory as well as serving as a component in the control of autonomic and vegetative functions such as ACTH secretion (Jacobson and Sapolsky, 1991; Eichenbaum and Otto, 1992; Phillips and LeDoux, 1992). The hippocampus is also vulnerable to damage by stroke and head trauma and susceptible to damage during aging and repeated stress (Sapolsky, 1992). Among the most novel and fascinating effects of estrogen are those on cognitive function. Estrogen effects on memory have been reported in animal models and in studies on humans (McEwen and Alves, 1999). The memories affected are ones in which the hippocampus plays a role along with the basal forebrain cholinergic system and other neurochemical systems. Rather than one estrogen-regulated process, many types of estrogen actions on a number of neurochemical and neuroanatomical substrates and a number of molecular mechanisms are likely to underlie the actions of estrogens on cognition and other aspects of behavior such as mood, pain perception, and nociception.

One of the processes regulated by ovarian hormones is the cyclic formation and breakdown of excitatory synapses in the hippocampus (Woolley et al., 1990). 
This finding was surprising because the hippocampus is a brain region in which cell nuclear ERs are present in scattered inhibitory interneurons but not in principal neurons where synapse formation occurs (Weiland et al., 1997). Yet, the effects of ovarian hormones on synaptic turnover were as impressive in the hippocampus as those in the ventromedial hypothalamus (Carrer and Aoki, 1982; Frankfurt et al., 1990; Calizo and Flanagan-Cato, 2000), a classic estrogen target area of the brain for female sexual behavior (Pfaff, 1980).

\section{A. MECHANISM OF EXCITATORY SYNAPSE FORMATION IN THE HIPPOCAMPUS}

Estrogen treatment increases dendritic spine density on CA1 pyramidal neurons. As observed by electron microscopy, treatment of ovariectomized adult rats with estrogen also induces new synapses on spines and not on dendritic shafts of CA1 neurons (Woolley and McEwen, 1992). Estrogen did not effect dendritic length or branching (Gould et al., 1990; Woolley et al., 1990; Woolley and McEwen, 1992). Progesterone treatment acutely enhances spine formation. But, over a 12- to 24-hour period, progesterone caused the downregulation of estrogen-induced synapses (Gould et al., 1990; Woolley and McEwen, 1993).

Estrogens do not act alone and, in fact, ongoing excitatory neurotransmission is required for synapse induction, as shown by the finding that antagonists of NMDA receptors block estrogen-induced synaptogenesis on dendritic spines in ovariectomized female rats (Woolley and McEwen, 1994). Because estrogen treatment increases the density of NMDA receptors in the CA1 region of the hippocampus (Weiland, 1992; Woolley et al., 1997), the activation of NMDA receptors by glutamate is an essential factor in causing new excitatory synapses to develop.

Spines are occupied by asymmetric, excitatory synapses and are sites of $\mathrm{Ca}^{2+}$ ion accumulation and contain NMDA receptors (Horner, 1993). NMDA receptors are expressed in large amounts in CA1 pyramidal neurons and can be imaged by conventional immunocytochemistry as well as by confocal imaging, in which individual dendrites and spines can be studied for co-localization with other markers (Gazzaley et al., 1996). Confocal microscopic imaging showed that estrogen treatment upregulates immunoreactivity for the largest NMDA receptor subunit, NR1, on dendrites and cell bodies of CA1 pyramidal neurons, whereas NR1 mRNA levels did not change after estrogen treatment that induces new synapses (Gazzaley et al., 1996). This suggests the possibility that NR1 expression is regulated post-transcriptionally by estrogen.

Recent evidence indicates that in young female rats, estrogen induction of NR1 is proportional to the induction of new spines, so that NMDA receptor density per spine is not increased; however, in the aging female rat, there is NR1 induction without an increase in dendritic spines (Adams et al., 2001) (Figure 3). 


\section{YOUNG}

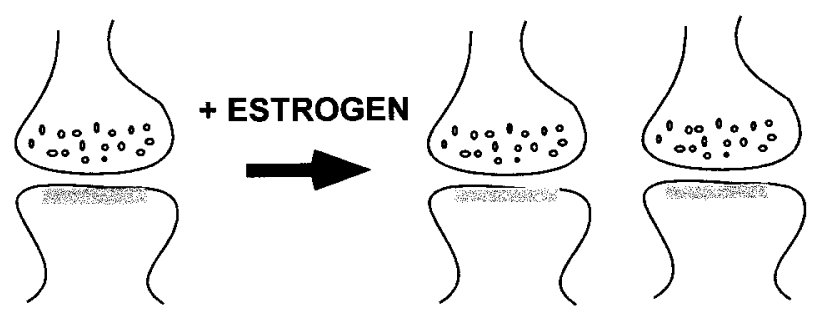

\section{AGED}

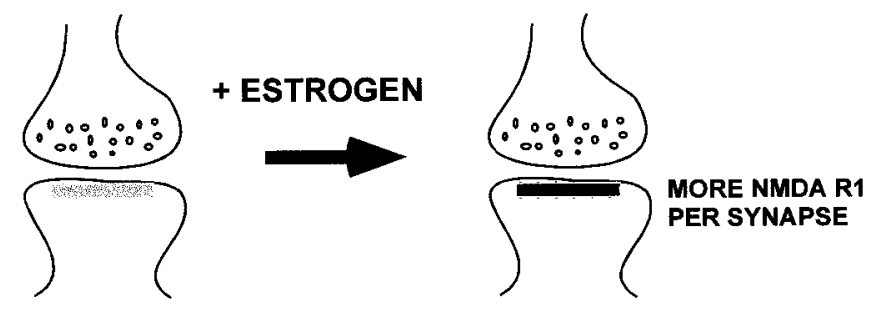

FIG. 3. Young adult female rats show upregulation of both NMDA receptors and dendritic spines on CA1 pyramidal neurons in hippocampus, with a proportional increase of NMDA receptors and spines. In contrast, the aging rat hippocampus responds to estrogen by upregulating NMDA receptor R1 subunit expression but not by increasing the number of spine synapses. [Redrawn from Adams MM, Shah RA, Janssen WGM, Morrison JH 2001 Different modes of hippocampal plasticity in response to estrogen in young and aged female rats. Proc Natl Acad Sci USA 98:8071-8076. Copyright 2001 National Academy of Sciences, USA.]

This might make the aging hippocampus more vulnerable to excitotoxic damage, for example, by stroke or seizures.

\section{B. CELLULAR AND MOLECULAR EVENTS ASSOCIATED WITH SYNAPSE FORMATION}

The estrogen-induced increase in dendritic spines on CA1 neurons parallels an increase in synapse density on spines without any decrease in shaft synapses (Woolley and McEwen, 1992), implying that new spine synapses are formed. Whereas synapse formation during development is considered to be a collaborative process involving in-growth of a presynaptic element on a site where a postsynaptic spine is either present or ready to form (Horner, 1993), the story for estrogen-regulated hippocampal synaptogenesis is somewhat different. Estrogens induce increased numbers of synapses on multiple synaptic boutons between neurons not previously connected (Yankova et al., 2001) (Figure 4). This is 

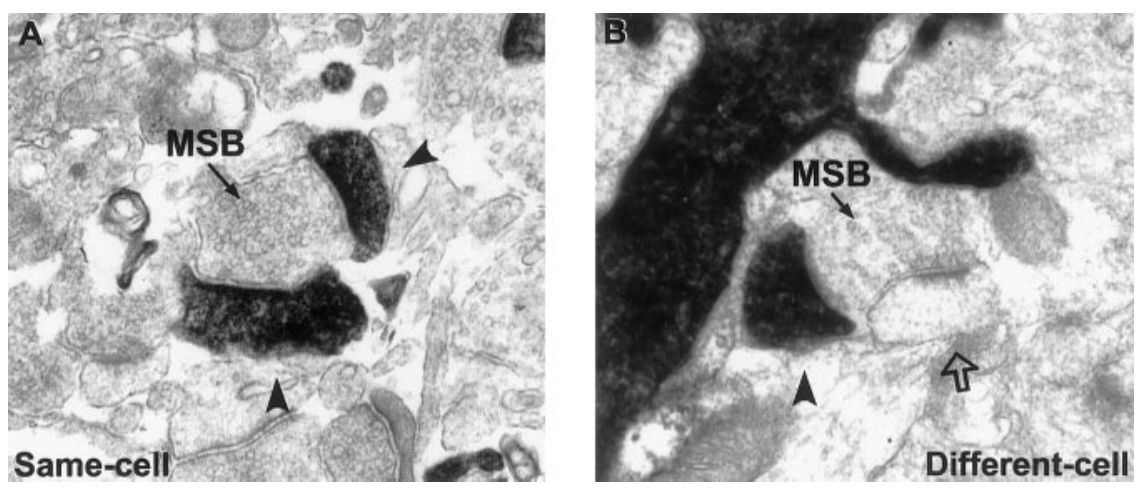

FIG. 4. Estrogen treatment of young adult female rats increases the number of dendritic spines on CA1 pyramidal neurons that are associated with multiple synaptic boutons (MSB), (i.e., more than one spine contacting a presynaptic terminal). Two examples are shown. On the left is a same cell bouton receiving two spines from the same cell that had been filled with dye to make it electron dense in the electron micrograph. On the right is a different cell bouton, where two different cells send spines to contact the same presynaptic terminal. Estrogen treatment increases preferentially the number of different cell MSBs. [Reprinted with permission from Yankova M, Hart SA, Woolley CS 2001 Estrogen increases synaptic connectivity between single presynaptic inputs and multiple postsynaptic CA1 pyramidal cells: a serial electron-microscopic study. Proc Natl Acad Sci USA 98:3525-3530. Copyright 2001 National Academy of Sciences, USA.]

reminiscent of the finding in cultured hippocampal cells studied by time-lapse photography that filopodia extend from dendrites and reach out to establish contact with nearby axons (Ziv and Smith, 1996). This implies an active role for the dendrite in forming synaptic contacts.

Gene products characterizing dendritic spines include spinophilin, a protein that helps to bundle actin filaments in the dendritic spine and regulates many of the properties of spines (Allen et al., 1997; Feng et al., 2000). As reviewed in McEwen et al. (2001), the calcium-calmodulin kinase II (CaMKII) is a major protein of the postsynaptic density that plays an important role in long-term potentiation (LTP) and synaptic differentiation. Recent evidence indicates that CaMKII plays a key role in the formation of synapses and localization of receptors in synapses. Glutamatergic synapses contain other key proteins in the postsynaptic density besides CaMKII; these include PSD-95, densin-180, and citron, a rac/rho effector protein. PSD-95 plays a key role in anchoring the NMDA receptor within the synapse. The NMDA R1 (NR1) receptor subunit is one of those proteins that may be translated from mRNA located in the dendrites (Gazzaley et al., 1997).

There are a number of presynaptic molecular markers of mature synapses that can be used in studies of synapse formation (McEwen et al., 2001). GAP43 is a marker of the growth cone and has been shown to increase in the 
hypothalamus after estrogen treatment; however, no studies of this type have been done on the hippocampus. SNAP-25 is a marker of synaptic vesicles, as are syntaxin, synaptotagmins, synaptoporin, synaptophysin, and the synapsins. Although mRNAs for these proteins are most likely found in neuron cell bodies, growth cones of hippocampal neurons in culture have been reported to have mRNAs for proteins such as GAP43 and Arc and perhaps other presynaptic proteins; these can be translated in the growth cone (Crino and Eberwine, 1996).

\section{APPLICATION OF RADIOIMMUNOCYTOCHEMISTRY TO STUDY SYNAPSE FORMATION AND MATURATION}

The methods used to assess synapse formation (i.e., Golgi staining, dye filling of cells, electron microscopy) are all labor intensive and time consuming and do not provide information about the underlying molecular events. Radioimmunocytochemistry is a method for assessing the locally expressed levels of synaptic and spine proteins using a primary antibody and a radioactive secondary antibody, then assessing levels of radioactivity using quantitative autoradiography. This procedure has not only confirmed the anatomical methods for assessing spine synapse formation but has also added a new dimension by providing insights into estrogen-induced increases in proteins that characterize presynaptic terminals and spines.

Figure 5 shows the results of the first study, using synaptophysin and syntaxin as presynaptic markers and spinophilin as a spine marker (Brake et al., 2001). Estrogen treatment was conducted exactly as in previous studies using the Golgi method and the estrogen antagonist, CI 628, was used to block estrogen actions (McEwen et al., 1999a). Estrogen treatment induced both pre- and postsynaptic markers in the stratum oriens and stratum radiatum of the CA1 region, location of the spine synapses (Brake et al., 2001). The magnitude of the increases corresponded very well to the magnitude of the changes in spine density seen with the Golgi method (Woolley et al., 1997; McEwen et al., 1999a). These effects were blocked by CI-628, which had no agonist effects by itself, and this finding agreed with the Golgi results (McEwen et al., 1999a). One unique finding with radioimmunocytochemistry is the estrogen-induced increase in spinophilin immunoreactivity in the hilus of the dentate gyrus and in the stratum lucidum of CA3, neither of which is accompanied by increases in the presynaptic markers, synaptophysin, and syntaxin (Brake et al., 2001). This suggests that there may be a process of synapse maturation taking place that reflects expression of spinophilin in the absence of any estrogen-induced changes in presynaptic proteins. Further studies are needed to confirm this interpretation.

\section{CELL NUCLEAR ESTROGEN RECEPTORS IN THE HIPPOCAMPUS}

Adult CA1 pyramidal cells of the dorsal hippocampus do not express detectable cell nuclear ER by tritium autoradiography and light microscopic 


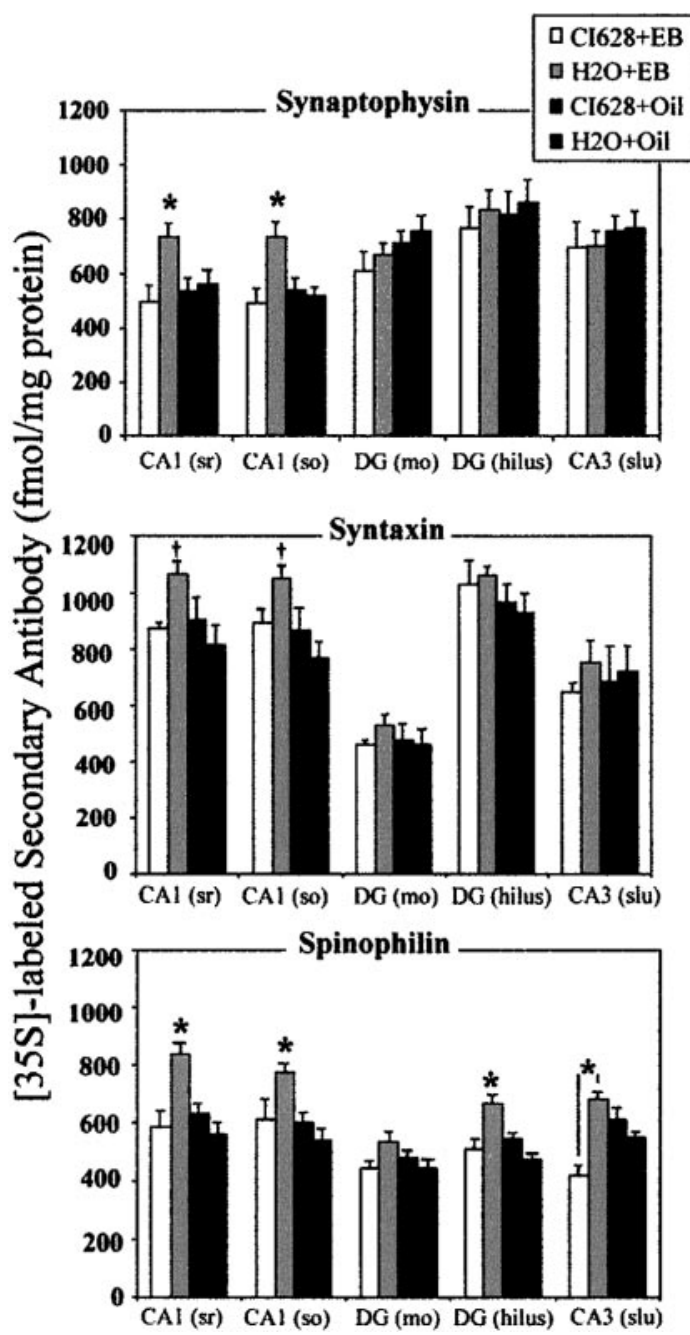

FIG. 5. Radioimmunocytochemistry for two presynaptic (synaptophysin and syntaxin) and one spine-specific (spinophilin) marker reveals the same magnitude of up-regulation by estrogen treatment as has been shown by morphological techniques for dendritic spines on CA1 neurons of the female rat hippocampus. ${ }^{35}$ S-labeled secondary antibody was used for quantitative autoradiography of the distribution and intensity of labeling of synaptic proteins by specific primary antibodies. Treatment with the nonsteroidal estrogen antagonist, CI 628, blocks the estrogen effect and does not produce an agonist-like effect of its own. These findings are very similar to a study using the Golgi method to reveal dendritic spines (McEwen et al., 1999a). [Reprinted with permission from Brake WG, Alves SE, Dunlop JC, Lee SJ, Bulloch K, Allen PB, Greengard P, McEwen BS 2001 Novel target sites for estrogen action in the dorsal hippocampus: an examination of synaptic proteins. Endocrinology 142:1284-1289. Copyright The Endocrine Society.] 
immunocytochemistry (Weiland et al., 1997), whereas they express low levels of $\mathrm{ER} \alpha$ and $\mathrm{ER} \beta$ mRNA by in situ hybridization (Shughrue and Merchenthaler, 2000; McEwen et al., 2001). Instead, immunocytochemistry for ER $\alpha$ showed cell nuclear ER in sparsely distributed interneurons in the CA1 region as well as other regions of Ammon's horn and dentate gyrus, with greater density in the ventral than dorsal hippocampus (McEwen and Alves, 1999). As far as ER $\beta$ immunoreactivity, an antibody generated at the C-terminal end of the receptor revealed only weak labeling of cell nuclei, particularly in the ventral hippocampus, and some staining of dendrites of pyramidal cells (Shughrue and Merchenthaler, 2001).

Autoradiography with ${ }^{125}$ I estrogen to label ER with a higher-specific radioactivity showed binding sites not previously detected in hippocampus using ${ }^{3} \mathrm{H}$ estradiol (Shughrue and Merchenthaler, 2000). Besides the interneurons previously seen to contain ER $\alpha$ by other methods, labeling with ${ }^{125} \mathrm{I}$ estrogen was found in CA1-CA3 pyramidal cell nuclei in ventral hippocampus. A similar dorsal-to-ventral gradient was seen for ER $\alpha$ and ER $\beta$ mRNA and ${ }^{125}$ I estrogen binding. The ER $\alpha$ signal appeared to be stronger than that for $\operatorname{ER} \beta$ (Shughrue and Merchenthaler, 2000). Thus, the greater sensitivity of ${ }^{125}$ I estrogen labeling of ER reveals sites that may indicate locations of estrogen actions in hippocampal pyramidal cells, particularly in the ventral hippocampus. A recent developmental study of $\mathrm{ER} \alpha$ in the rat hippocampus is consistent with this and suggests that, early in development, more pyramidal cells may have nuclear ER $\alpha$ than in the adult (Solum and Handa, 2001). Recent data with radioimmunocytochemistry have shown much stronger estrogen effects on synapse and spine protein levels in ventral, compared to dorsal hippocampus (Bulloch et al., 2000).

\section{E. CELL-CULTURE MODEL OF SYNAPSE FORMATION}

It has been possible to study estrogen-induced synapse formation in cell cultures of hippocampal neurons. In this model, estrogen induces spines on dendrites of dissociated hippocampal neurons in cell culture by a process that is blocked by an NMDA receptor antagonist and not by an AMPA (alpha-amino3-hydroxy-5-methylisoxasole proprionic acid)/kainate receptor blocker (Murphy and Segal, 1996). Furthermore, estrogen treatment was found to increase expression of phosphorylated cyclic AMP response binding protein (CREB). A specific antisense to CREB prevented both the formation of dendritic spines and the elevation in phosphoCREB immunoreactivity (IR) (Murphy and Segal, 1997).

The cellular location of $\mathrm{ER} \alpha$ in the cultures, resembling the in vivo localization, was in putative inhibitory interneurons (i.e., glutamic acid decarboxylase (GAD)-immunoreactive cells) that constituted around $20 \%$ of total neuronal population. Estrogen treatment caused decreases in GAD content and the number of neurons expressing GAD. Mimicking this decrease with an 
inhibitor of gamma aminobutyric acid (GABA) synthesis, mercaptopropionic acid, caused an upregulation of dendritic spine density, paralleling the effects of estrogen (Murphy et al., 1998a). Thus, estrogen-induced synapse formation may involve the suppression of GABA inhibitory input to the pyramidal neurons where the synapses are being generated (Figure 6).

An additional factor in the formation of dendritic spines in the in vitro cell culture model is the neurotrophin, brain-derived neurotrophic factor (BDNF) (Murphy et al., 1998b). Besides downregulating GABA expression in inhibitory interneurons, estrogen treatment also reduced BDNF by $60 \%$ within 24 hours (Murphy et al., 1998b). Exogenous BDNF blocked estrogen induction of dendritic spines; furthermore, BDNF depletion either with an antisense or blockade with BDNF antibodies mimicked estrogen in inducing spine density. Thus, $\mathrm{BDNF}$ is also an important player in regulating dendritic spines in hippocampal neurons (Murphy et al., 1998b). In this connection, neurotrophins such as BDNF and neurotrophin-3 (NT-3) also increase the function of inhibitory and excitatory

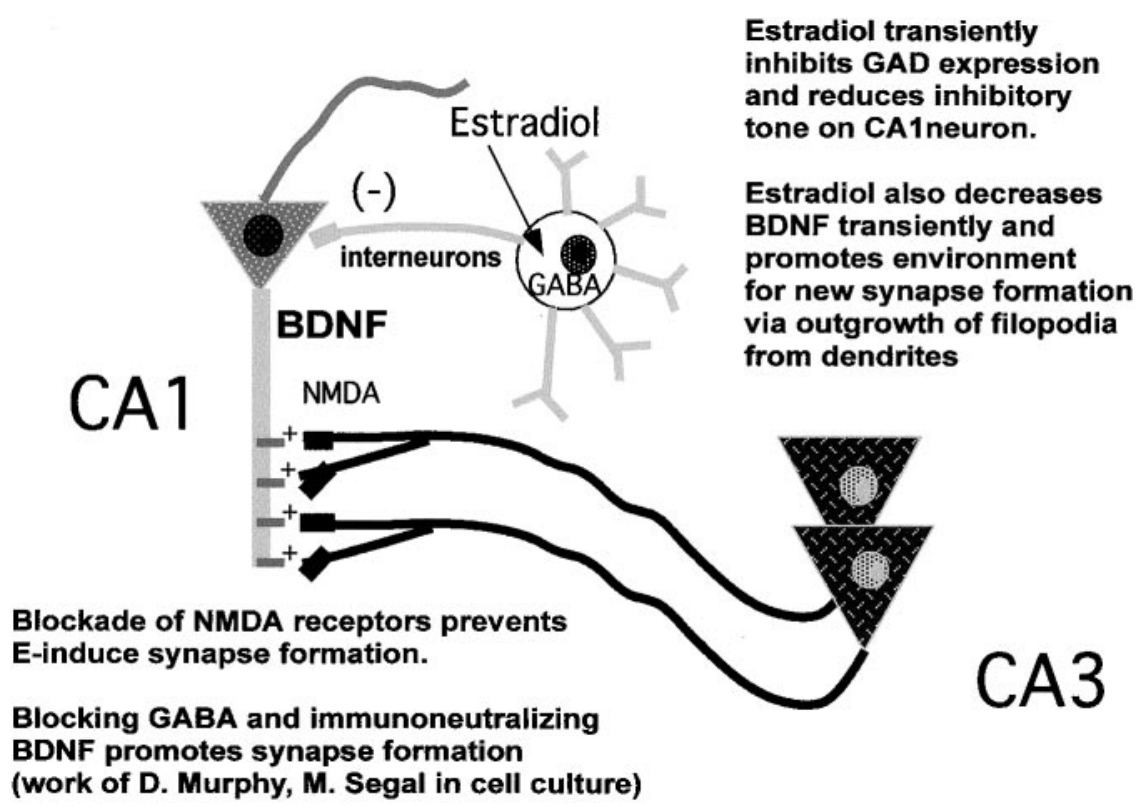

Fig. 6. Because of the prominent presence of $\mathrm{ER} \alpha$ in some of the inhibitory interneurons in the hippocampus and the presence of similar ER $\alpha$-positive GABA cells in hippocampal cell culture, Murphy and Segal proposed a model of the control of spine synapse formation in which estrogen treatment suppresses the inhibitory tone upon CA1 pyramidal neurons and creates an environment conducive to the formation of new excitatory spine synapses. Blocking GABA inhibition and immunoneutralizing the neurotrophin, BDNF increased spine synapse formation in cell culture. [See text for details and references.] 
synapses in hippocampal cell cultures; moreover, BDNF causes an increase in axonal branching and length of GABAergic interneurons (Vicario-Abejon et al., 1998). Hence, BDNF appears to favor the growth of inhibitory interneurons and the functional activation of synaptic transmission in excitatory and inhibitory cells, while suppressing the formation of the type of excitatory synaptic connections induced by estradiol (Figure 6).

\section{F. NON-NUCLEAR ESTROGEN RECEPTORS}

As compelling as the role of estrogen-regulated GABA input appears to be to the mechanism of synapse formation, we were not satisfied that there was no additional role for estrogen actions in the pyramidal neurons themselves. Given the increasing evidence for such a mechanism, it seemed plausible that, besides the indirect, trans-synaptic mechanism described above, local signaling by estrogen might be involved. This hypothesis was stimulated by a seminal study that $\operatorname{ER} \alpha$ and $\operatorname{ER} \beta$ into Chinese hamster ovarian cells found that both ERs are expressed in a form that couples to second messenger systems that are stimulated by estrogen and blocked at least partially by nonsteroidal estrogen antagonists (Razandi et al., 1999). Previous studies had indicated that non-nuclear ERs can be seen at the light microscopic level in cultured cells (Clarke et al., 2000) and also at the electron microscopic (EM) level in hypothalamus (Blaustein et al., 1992). The proliferation of articles on non-nuclear actions of estrogen via membrane ER and membrane-associated ER (Kelly and Levin, 2001) has reinforced the importance of investigating non-nuclear actions of estrogens in the hippocampus.

Electron microscopy was used to examine ER $\alpha$ localization in rat hippocampal formation (Milner et al., 2001) utilizing four antibodies to different parts of the $\operatorname{ER} \alpha$ structure, two polyclonal and two monoclonal. The specificity of these antibodies was determined by preabsorption with the full-length ER protein, which abolished labeling in all sites examined, both nuclear and non-nuclear. We were able to see at the EM level the cell nuclear labeling seen by light microscopy in some GABA interneurons. In addition, some pyramidal and granule neuron perikarya have small amounts of $\operatorname{ER} \alpha$ IR in the nuclear membrane, which is consistent with a recent report that ${ }^{125} \mathrm{I}$ estradiol labels a small number of estrogen-binding sites in cell nuclei of hippocampal principal cells (Shughrue and Merchenthaler, 2000).

In stratum radiatum of CA1, around $50 \%$ of the ER $\alpha$-IR profiles were found in unmyelinated axons and axon terminals containing small synaptic vesicles. This is of potential functional relevance, given findings that estrogen can influence neurotransmitter release (see McEwen et al., 2001, for references). The synaptic $\operatorname{ER} \alpha$-IR was found in terminals that formed both asymmetric and symmetric synapses on dendritic shafts and spines, suggesting that both excita- 
tory and inhibitory transmitter systems are associated with $\mathrm{ER} \alpha$ (Milner et al., 2001).

Around $25 \%$ of the ER $\alpha$ IR was found in dendritic spines of principal cells, where it often was associated with spine apparati and/or post-synaptic densities, suggesting that estrogen might act locally to regulate calcium availability, phosphorylation, or protein synthesis. Finally, the remaining $25 \%$ of ER $\alpha$ IR was found in astrocytic profiles, often located near the spines of principal cells.

While these findings corroborate existing evidence for an indirect GABAergic mediation of estrogen actions (Murphy et al., 1998a; Rudick and Woolley, 2000), the close association between the $\operatorname{ER} \alpha$-IR and dendritic spines supports a possible local, nongenomic role for this ER in regulation of dendritic spine density via second messenger systems. Initial in vivo and in vitro studies in hippocampus of one second messenger pathway, the phosphorylation of CREB, have indicated that estrogen has rapid effects that are evident within as little as 15 minutes to increase phosphoCREB immunoreactivity in cell nuclei of hippocampal pyramidal neurons (S. Lee, S. Alves, B. McEwen, unpublished data). One pathway by which CREB phosphorylation may occur involves the phosphoinositol-3 (PI3) kinase, or Akt, system (Datta et al., 1999). Studies are underway to try to connect these events together in the early actions of estrogen on hippocampal neurons that precede the induction of synapse formation. We next consider some of the cellular and molecular events associated with the formation of synapses in which estrogen actions may be involved.

\section{G. DENDRITIC MRNA TRANSPORT AND PROTEIN SYNTHESIS}

The finding of non-nuclear $\mathrm{ER} \alpha$ in dendritic spines compelled us to consider sites and mechanisms whereby estrogens could regulate the process of synapse formation via post-transcriptional mechanisms. Protein synthesis is likely to be an essential component of de novo synapse formation. Neurons have at least three strategies for activity-dependent regulation of protein synthesis and targeting of those proteins to pre- and postsynaptic sites (Tiedge et al., 2001). First, there is translation of mRNA in the cell soma and trafficking of proteins to "tagged" synapses. Second is transport of mRNA into the dendrites or growth cones and local translation into protein on polyribosomal clusters such as are found at the base of spines. The third strategy is local regulation of the translation of transported mRNAs (Tiedge et al., 2001).

Dendrites contain transported mRNAs for gene products such as MAP-2, CaMKII, NMDA R1 subunit, Arc, GAP-43, and BC1 (Gao, 1998). One feature of the regulation of translation is that the dendritic mRNAs are deficient in poly A; therefore, the regulation of polyadenylation by cytoplasmic polyadenylation element binding protein (CPEP) is able to rapidly activate translation (Wells et al., 2000). Moreover, other regulatory points such as the initiation of the 
translation process via regulatory factors are subject to second messengerstimulated phosphorylation (Gingras et al., 1999). These are currently under investigation in our laboratory as far as possible regulation by estrogen.

\section{H. A MODEL OF ESTROGEN ACTION INVOLVING GENOMIC AND NONGENOMIC ERs}

The results summarized above have led us to propose a testable, working model (Figure 7) that delineates possible sites of estrogen action in relation to the location of nuclear and non-nuclear ERs. Although this model pertains to ER $\alpha$ because we know more about its distribution, further studies of ER $\beta$ may reveal that it is present in non-nuclear as well as cell nuclear sites within the hippocampus and may participate in some of the processes outlined in Figure 7. According to our model, ER in the dendritic spine may be associated with the activation of mRNA translation from polyribosomes (Tiedge et al., 2001) or endomembrane structures found in spines (Pierce et al., 2000). In addition, other second messenger signaling effects might include the phosphorylation of neurotransmitter receptors or ion channels. ERs in certain presynaptic terminals might modulate neurotransmitter release or reuptake (see McEwen et al., 2001, for references). Moreover, ER-mediated activation of second messenger systems in dendritic spines and presynaptic endings might lead to retrograde signal transduction back to the cell nucleus, perhaps via Akt or CREB, providing another pathway through which estrogen could regulate gene expression. As indicated at the bottom right of Figure 7, we consider that these postulated actions of estrogen operate synergistically with the actions of estrogen via nuclear receptors in interneurons that modulate the inhibitory tone upon the CA1 pyramidal neurons where synapse formation occurs.

\section{Possible Functional Significance}

The functional significance of estrogen actions in the hippocampal CA1 region is evident from electrophysiological studies indicating that estrogen treatment of ovariectomized rats produces a delayed facilitation of synaptic transmission in CA1 neurons that is NMDA mediated and leads to an enhancement of voltage-gated $\mathrm{Ca}^{2+}$ currents (Wong and Moss, 1992). Using biocytin injection after recording from CA1 pyramidal neurons in order to visualize estrogen induction of dendritic spines, Woolley and coworkers (1997) found that spine density correlated negatively with input resistance. Input/output curves showed an increased slope under conditions where NMDA receptor-mediated currents predominated, whereas there was no increased slope where AMPA receptor currents predominated. Other studies have shown that LTP sensitivity peaks on the afternoon of proestrus in intact female rats at exactly the time when 


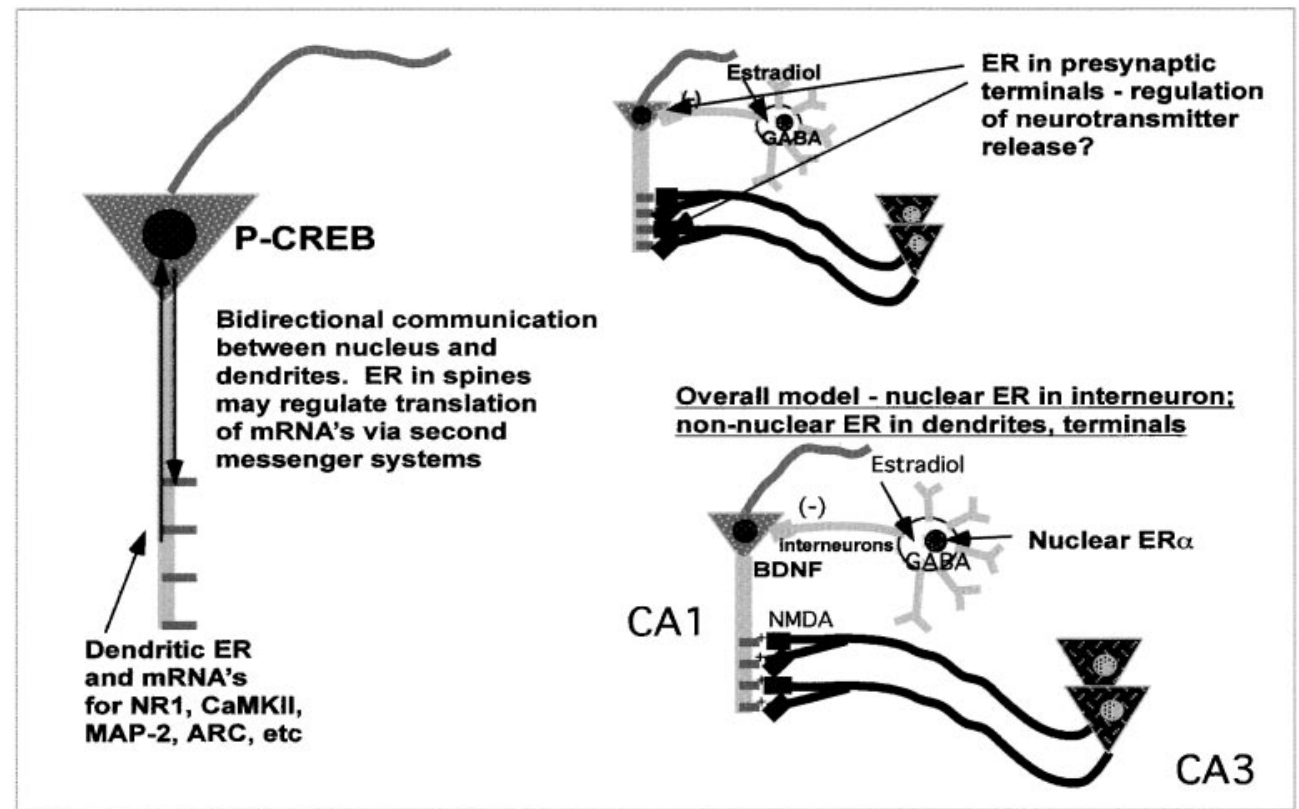

FIG. 7. Schematic depiction of an overall model of estrogen (E) regulation of spine synapse formation, based upon the model shown in Figure 6, combined with ER localization in non-nuclear sites in CA1 pyramidal neurons that respond to E with synapse formation. As summarized in the text, ER $\alpha$ immunoreactivity is found in dendrites, presynaptic terminals, glia, and the nuclear envelope of some principal cells as well as in cell nuclei of inhibitory interneurons (Milner et al., 2001). Glia may be involved in synapse formation and/or removal. The left side of the figure highlights the fact that dendrites are sites of protein synthesis on polyribosomes and at endomembrane structures using RNAs transported from the cell body (see text). Non-nuclear ER may be involved in other E effects linked to second messenger activation on processes such as neurotransmitter release (top of figure) and phosphorylation of neurotransmitter receptors and ion channels. Second messenger activation by E in nerve terminals, dendrites, and glial cell processes may result in retrograde second messenger signals, such as P-CREB and P-Akt, that return to signal the genome. These actions synergize with the transient decrease in GABA-inhibitory tone produced by estrogen actions on ER $\alpha$-containing inhibitory interneurons based upon the model in Figure 6. 
excitatory synapse density has reached its peak (Warren et al., 1995). Proestrus is also the time of the estrous cycle when seizure thresholds in dorsal hippocampus are the lowest (Terasawa and Timiras, 1968).

Besides affecting neuronal activity in hippocampus, estrogen treatment affects hippocampal-dependent learning and memory. In the natural estrous cycle of the female rat, a recent study has utilized a delayed matching-to-place task in female rats to show a close parallel between the temporal conditions by which estrogen improves memory and the conditions for it to induce new excitatory synaptic connections in the hippocampus (McEwen et al., 2001). Moreover, estrogen treatment of ovariectomized female rats has been reported to improve acquisition on a radial maze task as well as in a reinforced T-maze alternation task (Fader et al., 1998; Daniel et al., 1999). Furthermore, sustained treatment is reported to improve performance in a working memory task (O'Neal et al., 1996) as well as in the radial-arm maze (Luine et al., 1998; Daniel et al., 1999). Finally, the effects of estrogen replacement in rats are reminiscent of the effects of treatment in women whose estrogen levels have been suppressed by a gonadotrophin-releasing hormone agonist used to shrink the size of fibroids prior to surgery (Sherwin and Tulandi, 1996).

In addition to the delayed effects of estrogens in hippocampus, estrogens and some form of ER are involved in local signaling within neurons. Among the possible targets of local signaling is the translation of RNAs found in dendrites of hippocampal and other neurons. There are at least three other targets for local signaling by estrogens. They are 1) the rapid activation of kainate-induced ion currents via a $\mathrm{G}$ protein-coupled ER that is present in $\alpha$ ERKO mice and is insensitive to nonsteroidal estrogen antagonists (Gu et al., 1999); 2) the suppression of calcium ion currents that is mimicked by a nonsteroidal estrogen antagonist (Mermelstein et al., 1996); and 3) rapid actions of estradiol on NMDA receptor activity and LTP (Teyler et al., 1980; Foy et al., 1999).

\section{Conclusions}

It is abundantly clear that estrogen actions in brain and other tissues involve an array of cellular and molecular mechanisms and that estrogens influence many behavioral and physiological processes that go beyond their traditional role in reproduction. Brain regions such as the hippocampus are affected by estrogens, even though they have a paucity of cell nuclear ERs. These actions may be explained by the powerful trans-synaptic influence of a small number of ERcontaining cells upon other neurons (e.g., GABA inhibitory neurons depicted in Figure 6) or by non-nuclear ERs and second messenger activation, or a combination of both. Elsewhere in the nervous system, cell nuclear ER $\beta$ may well help explain estrogen actions in other brain areas that do not express ER $\alpha$. What all of this means is that, besides the classical genomic actions of estradiol that have 
been studied for over 40 years, the molecular mechanisms of estrogen action are likely to involve many pathways not ordinarily considered, in which indirect genomic activation may occur as a consequence of second messenger activation.

\section{REFERENCES}

Adams MM, Shah RA, Janssen WGM, Morrison JH 2001 Different modes of hippocampal plasticity in response to estrogen in young and aged female rats. Proc Natl Acad Sci USA 98:8071-8076

Allen PB, Ouimet CC, Greengard P 1997 Spinophilin, a novel protein phosphatase 1 binding protein localized to dendritic spines. Proc Natl Acad Sci USA 94:9956-9961

Alves S, Weiland NG, Hayashi S, McEwen B 1998 Immunocytochemical localization of nuclear estrogen receptors and progestin receptors within the dorsal raphe nucleus. J Comp Neurol 391:322-334

Alves SE, McEwen BS, Hayashi S, Korach KS, Pfaff DW, Ogawa S 2000 Estrogen-regulated progestin receptors are found in the midbrain raphe but not hippocampus of estrogen receptor alpha (ER $\alpha$ ) gene-disrupted mice. J Comp Neurol 427:185-195

Auger AP, Tetel MJ, McCarthy MM 2000 Steroid receptor coactivator-1 (SRC-1) mediates the development of sex-specific brain morphology and behavior. Proc Natl Acad Sci USA 97:7551-7555

Bazzett TJ, Becker JB 1994 Sex differences in the rapid and acute effects of estrogen on striatal D dopamine receptor binding. Brain Res 637:163-172

Bethea CL, Pecins-Thompson M, Schutzer WE, Gundlah CLZN 1999 Ovarian steroids and serotonin neural function. Mol Neurobiol 18:87-123

Blaustein JD, Lehman MN, Turcotte JC, Greene G 1992 Estrogen receptors in dendrites and axon terminals in guinea pig hypothalamus. Endocrinology 131:281-290

Brake WG, Alves SE, Dunlop JC, Lee SJ, Bulloch K, Allen PB, Greengard P, McEwen BS 2001 Novel target sites for estrogen action in the dorsal hippocampus: an examination of synaptic proteins. Endocrinology 142:1284-1289

Brinton RD 2001 Cellular and molecular mechanisms of estrogen regulation of memory function and neuroprotection against Alzheimer's disease: Recent insights and remaining challenges. Learn Memory 8:121-133

Bulloch K, Brake WG, Lee SJ, Patel A, Wolf OT, McEwen BS 2000 Estrogen increases synaptic protein immunoreactivity in the dorsal and ventral hippocampus of ovariectomized rats. Abstr Soc Neurosci 26(7.1):4 (Abstract)

Calizo LH, Flanagan-Cato LM 2000 Estrogen selectively regulates spine density within the dendritic arbor of rat ventromedial hypothalamic neurons. J Neurosci 20:1589-1596

Carrer H, Aoki A 1982 Ultrastructural changes in the hypothalamic ventromedial nucleus of ovariectomized rats after estrogen treatment. Brain Res 240:221-233

Clarke CH, Norfleet AM, Clarke MSF, Watson CS, Cunningham KA, Thomas ML 2000 Perimembrane localization of the estrogen receptor $\alpha$ protein in neuronal processes of cultured hippocampal neurons. Neuroendocrinology 71:34-42

Crino PB, Eberwine J 1996 Molecular characterization of the dendritic growth cone: regulated mRNA transport and local protein synthesis. Neuron 17:1173-1187

Daniel JM, Roberts SL, Dohanich GP 1999 Effects of ovarian hormones and environment on radial maze and water maze performance of female rats. Physiol Behav 66:11-20

Datta SR, Brunet A, Greenberg ME 1999 Cellular survival: a play in three Akts. Genes Dev 13:2905-2927

Eichenbaum H, Otto T 1992 The hippocampus - what does it do? Behav Neural Biol 57:2-36 
Fader AJ, Hendricson AW, Dohanich GP 1998 Estrogen improves performance of reinforced T-maze alternation and prevents the amnestic effects of scopolamine administered systemically or intrahippocampally. Neurobiol Learn Memory 69:225-240

Feng J, Yan Z, Ferreira A, Tomizawa K, Liauw JA, Zhuo M, Allen PB, Ouimet CC, Greengard P 2000 Spinophilin regulates the formation and function of dendritic spines. Proc Natl Acad Sci USA 97:9287-9292

Fischette C, Biegon A, McEwen BS 1984 Sex steroid modulation of the serotonin behavioral syndrome. Life Sci 35:1197-1206

Foy MR, Xu J, Xie X, Brinton RD, Thompson RF, Berger TW $199917 \beta$-estradiol enhances NMDA receptor-mediated EPSPs and long-term potentiation. J Neurophysiol 81:925-929

Frankfurt M, Gould E, Wolley C, McEwen BS 1990 Gonadal steroids modify dendritic spine density in ventromedial hypothalamic neurons: a Golgi study in the adult rat. Neuroendocrinology 51:530-535

Gao F-B 1998 Messenger RNAs in dendrites: localization, stability, and implications for neuronal function. BioEssays 20:70-78

Gazzaley AH, Weiland NG, McEwen BS, Morrison JH 1996 Differential regulation of NMDAR1 mRNA and protein by estradiol in the rat hippocampus. J Neurosci 16:6830-6838.

Gazzaley AH, Benson DL, Huntley GW, Morrison JH 1997 Differential subcellular regulation of NMDAR1protein and mRNA in dendrites of dentate gyrus granule cells after perforant path transection. J Neurosci 17:2006-2017

Gingras A-C, Raught B, Sonenberg N 1999 eIF4 initiation factors: Effectors of mRNA recruitment to ribosomes and regulators of translation. Annu Rev Biochem 68:913-963

Gould E, Woolley C, Frankfurt M, McEwen BS 1990 Gonadal steroids regulate dendritic spine density in hippocampal pyramidal cells in adulthood. J Neurosci 10:1286-1291

Goy R, McEwen BS 1980 Sexual Differentiation of the Brain. Cambridge: MIT Press

Green PS, Bishop J, Simpkins JW 1997 17 $\beta$-Estradiol exerts neuroprotective effects on SK-N-SH cells. J Neurosci 17:511-515

Gu Q, Korach KS, Moss RL 1999 Rapid action of 17 $\beta$-estradiol on kainate-induced currents in hippocampal neurons lacking intracellular estrogen receptors. Endocrinology 140:660-666

Gundlah C, Pecins-Thompson M, Schutzer WE, Bethea CL 1999 Ovarian steroid effects on serotonin $1 \mathrm{~A}, 2 \mathrm{~A}$ and $2 \mathrm{C}$ receptor mRNA in macaque hypothalamus. Mol Brain Res 63:325-339

Higley JD, Linnoila M 1997 Low central nervous system serotonergic activity is traitlike and correlates with impulsive behavior. Ann NY Acad Sci 836:39-56

Horner CH 1993 Plasticity of the dendritic spine. Progr Neurobiol 41:281-321

Hrabovszky E, Kallo I, Hajszan T, Shughrue PJ, Merchenthaler I, Liposits Z 1998 Expression of estrogen receptor- $\beta$ messenger ribonucleic acid in oxytocin and vasopressin neurons of the rat supraoptic and paraventricular nuclei. Endocrinology 139:2600-2604

Jacobs BL 1994 Serotonin, motor activity and depression-related disorders. Am Sci 82:456-463

Jacobson L, Sapolsky R 1991 The role of the hippocampus in feedback regulation of the hypothalamic-pituitary-adrenocortical axis. Endocr Rev 12:118-134

Jensen E, Jacobson H 1962 Basic guides to the mechanism of estrogen action. Rec Progr Horm Res 18:387-408

Kelly MJ, Levin ER 2001 Rapid actions of plasma membrane estrogen receptors. Trends Endocrinol Metab 12:152-156

Kelly MJ, Wagner EJ 1999 Estrogen modulation of G-protein-coupled receptors. Trends Endocrinol Metab 10:369-374

Kelly M, Moss R, Dudley C 1977 The effects of microelectrophoretically applied estrogen, cortisol and acetylcholine on medial preoptic-septal unit activity throughout the estrous cycle of the female rat. Exp Brain Res 30:53-64 
Kimura D 1992 Sex differences in the brain. Sci Am 267:119-125

Kuiper GGJM, Carlsson B, Grandien K, Enmark E, Haggblad J, Nilsson S, Gustafsson J-A 1997 Comparison of the ligand binding specificity and transcript tissue distribution of estrogen receptors $\alpha$ and $\beta$. Endocrinology 138:863-870

Kuiper GGJM, Shughrue PJ, Merchenthaler I, Gustafsson J-A 1998 The estrogen receptor $\beta$ subtype: a novel mediator of estrogen action in neuroendocrine systems. Front Neuroendocrinol 19:253-286

Lee SJ, McEwen BS 2001 Neurotrophic and neuroprotective actions of estrogens and their therapeutic implications. Annu Rev Pharmacol Toxicol 41:569-591

Luine V, McEwen BS 1977 Effects of an estrogen antagonist on enzyme activities and 3H estradiol nuclear binding in uterus, pituitary and brain. Endocrinology 100:903-910

Luine VN, Richards ST, Wu VY, Beck KD 1998 Estradiol enhances learning and memory in a spatial memory task and effects levels of monoaminergic neurotransmitters. Horm Behav 34:149-162

Maruyama K, Endoh H, Sasaki-Iwaoka H, Kanou H, Shimaya E, Hashimoto S, Kato S, Kawashima H 1998 A novel isoform of rat estrogen receptor beta with 18 amino acid insertion in the ligand binding domain as a putative dominant negative regular of estrogen action. Biochem Biophys Res Commun 246:142-147

Mattson MP, Robinson N, Guo Q 1997 Estrogens stabilize mitochondrial function and protect neural cells against the pro-apoptotic action of mutant presenilin-1. NeuroReport 8:38173821

Maus M, Homburger V, Bockaert J, Glowinski J, Premont J 1990 Pretreatment of mouse striatal neurons in primary culture with $17 \beta$-estradiol enhances the pertussis toxin-catalyzed ADPribosylation of $\mathrm{G} \alpha_{\mathrm{o}, \mathrm{i}}$ protein subunits. J Neurochem 55:1244-1251

McEwen BS 1983 Gonadal steroid influences on brain development and sexual differentiation. In: Greep R, ed. Reproductive Physiology IV. University Park, MD: University Park Press; $99-145$

McEwen BS, Alves SH 1999 Estrogen actions in the central nervous system. Endocr Rev 20:279_ 307

McEwen BS, Tanapat P, Weiland NG 1999a Inhibition of dendritic spine induction on hippocampal CA1 pyramidal neurons by a non-steroidal estrogen antagonist in female rats. Endocrinology 140:1044-1047

McEwen BS, Tanapat P, Weiland NG 1999b Inhibition of dendritic spine induction on hippocampal CA1 pyramidal neurons by a nonsteroidal estrogen antagonist in female rats. Endocrinology 140:1044-1047

McEwen BS, Akama K, Alves S, Brake WG, Bulloch K, Lee S, Li C, Yuen G, Milner TA 2001 Tracking the estrogen receptor in neurons: Implications for estrogen-induced synapse formation. Proc Natl Acad Sci USA 98:7093-7100

Meisel R, Dohanich G, McEwen BS, Pfaff DW 1987 Antagonism of sexual behavior in female rats by ventromedial hypothalamic implants of antiestrogen. Neuroendocrinology 45:201-207

Mermelstein PG, Becker JB, Surmeier DJ 1996 Estradiol reduces calcium currents in rat neostriatal neurons via a membrane receptor. J Neurosci 16:595-604

Milner TA, McEwen BS, Hayashi S, Li CJ, Reagen L, Alves SE 2001 Ultrastructural evidence that hippocampal alpha estrogen receptors are located at extranuclear sites. J Comp Neurol 429:355-371

Mize AL, Poisner AM, Alper RH 2001 Estrogens act in rat hippocampus and frontal cortex to produce rapid, receptor-mediated decreases in serotonin $5-\mathrm{HT}_{1 \mathrm{~A}}$ receptor function. Neuroendocrinology 73:166-174

Murphy DD, Segal M 1996 Regulation of dendritic spine density in cultured rat hippocampal neurons by steroid hormones. J Neurosci 16:4059-4068 
Murphy DD, Segal M 1997 Morphological plasticity of dendritic spines in central neurons is mediated by activation of cAMP response element binding protein. Proc Natl Acad Sci USA 94:1482-1487

Murphy DD, Cole NB, Greenberger V, Segal M 1998a Estradiol increases dendritic spine density by reducing GABA neurotransmission in hippocampal neurons. J Neurosci 18:2550-2559

Murphy DD, Cole NB, Segal M 1998b Brain-derived neurotrophic factor mediates estradiol-induced dendritic spine formation in hippocampal neurons. Proc Natl Acad Sci USA 95:11412-11417

Nabekura J, Oomura Y, Minami T, Mizuno Y, Fukuda A 1986 Mechanism of the rapid effect of $17 \beta$-estradiol on medial amygdala neurons. Science 233:226-228

Naftolin F 1994 Brain aromatization of androgens. J Reprod Med 39:257-261

O'Neal MF, Means LW, Poole MC, Hamm RJ 1996 Estrogen affects performance of ovariectomized rats in a two-choice water-escape working memory task. Psychoneuroendocrinology 21:51-65

Osterlund MK, Overstreet DH, Hurd YL 1999 The flinders sensitive line rats, a genetic model of depression, show abnormal serotonin receptor mRNA expression in the brain that is reversed by $17 \beta$-estradiol. Mol Brain Res 74:158-166

Osterlund MK, Halldin C, Hurd YL 2000 Effects of chronic 17 $\beta$-estradiol treatment on the serotonin 5- $\mathrm{HT}_{1 \mathrm{~A}}$ receptor mRNA and binding levels in the rat brain. Synapse 35:39-44

Paech K, Webb P, Kuiper GGJM, Nilsson S, Gustafsson J-A, Kushner PJ, Scanlan TS 1997 Differential ligand activation of estrogen receptors ER $\alpha$ and ER $\beta$ at AP1 sites. Science 277:1508-1510

Pettersson K, Grandien K, Kuiper GGJM, Gustafsson J-A 1997 Mouse estrogen receptor $\beta$ forms estrogen response element-binding heterodimers with estrogen receptor $\alpha$. Mol Endocrinol 11:1486-1496

Pfaff DW 1980 Estrogens and Brain Function. New York: Springer-Verlag

Phillips RG, LeDoux JE 1992 Differential contribution of amygdala and hippocampus to cued and contextual fear conditioning. Behav Neurosci 106:274-285

Pierce JP, van Leyen K, McCarthy JB 2000 Translocation machinery for synthesis of integral membrane and secretory proteins in dendritic spines. Nature Neurosci 3:311-313

Price RH Jr, Lorenzon N, Handa RJ 2000 Differential expression of estrogen receptor beta splice variants in rat brain: identification and characterization of a novel variant missing exon $4^{1}$. Mol Brain Res 80:260-268

Raap DK, DonCarlos L, Garcia F, Muma NA, Wolf WA, Battaglia G, Van de Kar LD 2000 Estrogen desensitizes 5-HT 1 A receptors and reduces levels of $\mathrm{G}_{\mathrm{z}}, \mathrm{G}_{\mathrm{i} 1}$ and $\mathrm{G}_{\mathrm{i} 3}$ proteins in the hypothalamus. Neuropharmacology 39:1823-1832

Razandi M, Pedram A, Greene GL, Levin ER 1999 Cell membrane and nuclear estrogen receptors (ERs) originate from a single transcript: studies of ER $\alpha$ and $\mathrm{ER} \beta$ expressed in Chinese hamster ovary cells. Mol Endocrinol 13:307-319

Roy E, MacLusky N, McEwen BS 1979 Antiestrogen inhibits the induction of progestin receptors by estradiol in the hypothalamus, pituitary and uterus. Endocrinology 104:1333-1336

Rubinow DR, Schmidt PJ, Roca CA 1998 Estrogen-serotonin interactions: Implications for affective regulation. Biol Psychiat 44:839-850

Rudick CN, Woolley CS 2000 Estradiol induces a phasic Fos response in the hippocampal CA1 and CA3 regions of adult female rats. Hippocampus 10:274-283

Sapolsky R 1992 Stress, the aging brain and the mechanisms of neuron death. Cambridge: MIT Press; $1-423$

Sherwin BB, Tulandi T 1996 "Add-back" estrogen reverses cognitive deficits induced by a gonadotropin-releasing hormone agonist in women with leiomyomata uteri. J Clin Endocrinol Metab 81:2545-2549 
Shughrue PJ, Merchenthaler I 2000 Evidence for novel estrogen binding sites in the rat hippocampus. Neuroscience 99:605-612

Shughrue PJ, Merchenthaler I 2001 Distribution of estrogen receptor $\alpha$ immunoreactivity in the rat central nervous system. J Comp Neurol 43:64-81

Shughrue PJ, Lane MV, Merchenthaler I 1999 Biologically active estrogen receptor- $\alpha$ : evidence from in vivo autoradiographic studies with estrogen receptor $\alpha$-knockout mice. Endocrinology 140:2613-2620

Solum DT, Handa RJ 2001 Localization of estrogen receptor alpha (ER $\alpha$ ) in pyramidal neurons of the developing rat hippocampus. Devel Brain Res 128:165-175

Stumpf WE, Sar M 1976 Steroid hormone target sites in the brain: the differential distribution of estrogen, progestin, androgen and glucocorticosteroid. J Steroid Biochem 7:1163-1170

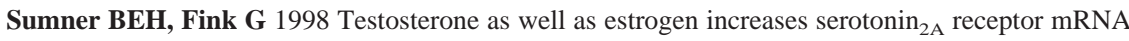
and binding site densities in the male rat brain. Mol Brain Res 59:205-214

Terasawa E, Timiras P 1968 Electrical activity during the estrous cycle of the rat: cyclic changes in limbic structures. Endocrinology 83:207-216

Teyler T, Vardaris R, Lewis D, Rawitch A 1980 Gonadal steroids: effects on excitability of hippocampal pyramidal cells. Science 209:1017-1019

Tiedge H, Bloom FE, Richter D 2001 Colloquium: molecular kinesis in cellular function and plasticity. Proc Natl Acad Sci USA 98:6997-7106

Vicario-Abejon C, Collin C, McKay RDG, Segal M 1998 Neurotrophins induce formation of functional excitatory and inhibitory synapses between cultured hippocampal neurons. J Neurosci 18:7256-7271

Wang J, Green PS, Simpkins JW 2001 Estradiol protects against ATP depletion, mitochondrial membrane potential decline and the generation of reactive oxygen species induced by 3-nitroproprionic acid in SK-N-SH human neuroblastoma cells. J Neurochem 77:804-811

Warren SG, Humphreys AG, Juraska JM, Greenough WT 1995 LTP varies across the estrous cycle: enhanced synaptic plasticity in proestrus rats. Brain Res 703:26-30

Weiland NG 1992 Estradiol selectively regulates agonist binding sites on the $N$-methyl- $D$-aspartate receptor complex in the CA1 region of the hippocampus. Endocrinology 131:662-668

Weiland NG, Orikasa C, Hayashi S, McEwen BS 1997 Distribution and hormone regulation of estrogen receptor immunoreactive cells in the hippocampus of male and female rats. J Comp Neurol 388:603-612

Wells DG, Richter JD, Fallon JR 2000 Molecular mechanisms for activity-regulated protein synthesis in the synapto-dendritic compartment. Curr Opin Neurobiol 10:132-137

Williams CL, Meck WH 1991 The organizational effects of gonadal steroids on sexually dimorphic spatial ability. Psychoneuroendocrinology 16:155-176

Witelson SF, Glezer II, Kigar DL 1995 Women have greater density of neurons in posterior temporal cortex. J Neurosci 15:3418-3428

Wong M, Moss RL 1992 Long-term and short-term electrophysiological effects of estrogen on the synaptic properties of hippocampal CA1 neurons. J Neurosci 12:3217-3225

Woolley C, McEwen BS 1992 Estradiol mediates fluctuation in hippocampal synapse density during the estrous cycle in the adult rat. J Neurosci 12:2549-2554

Woolley C, McEwen BS 1993 Roles of estradiol and progesterone in regulation of hippocampal dendritic spine density during the estrous cycle in the rat. J Comp Neurol 336:293-306

Woolley C, McEwen BS 1994 Estradiol regulates hippocampal dendritic spine density via an N-methyl-D-aspartate receptor dependent mechanism. J Neurosci 14:7680-7687

Woolley C, Gould E, Frankfurt M, McEwen BS 1990 Naturally occurring fluctuation in dendritic spine density on adult hippocampal pyramidal neurons. J Neurosci 10:4035-4039 
Woolley CS, Weiland NG, McEwen BS, Schwartzkroin PA 1997 Estradiol increases the sensitivity of hippocampal CA1 pyramidal cells to NMDA receptor-mediated synaptic input: correlation with dendritic spine density. J Neurosci 17:1848-1859

Yankova M, Hart SA, Woolley CS 2001 Estrogen increases synaptic connectivity between single presynaptic inputs and multiple postsynaptic CA1 pyramidal cells: a serial electron-microscopic study. Proc Natl Acad Sci USA 98:3525-3530

Ziv NE, Smith SJ 1996 Evidence for a role of dendritic filopodia in synaptogenesis and spine formation. Neuron 17:91-102 
Sex Steroids and Bone

\author{
S.C. Manolagas, S. Kousteni, and R.L. Jilka
}

Division of Endocrinology and Metabolism, Center for Osteoporosis and Metabolic Bone

Diseases, University of Arkansas for Medical Sciences, and the Central Arkansas Veterans Health

Care System, Little Rock, Arkansas 72205

\begin{abstract}
The adult skeleton is periodically remodeled by temporary anatomic structures that comprise juxtaposed osteoclast and osteoblast teams and replace old bone with new. Estrogens and androgens slow the rate of bone remodeling and protect against bone loss. Conversely, loss of estrogen leads to increased rate of remodeling and tilts the balance between bone resorption and formation in favor of the former. Studies from our group during the last 10 years have elucidated that estrogens and androgens decrease the number of remodeling cycles by attenuating the birth rate of osteoclasts and osteoblasts from their respective progenitors. These effects result, in part, from the transcriptional regulation of genes responsible for osteoclastogenesis and mesenchymal cell replication and/or differentiation and are exerted through interactions of the ligand-activated receptors with other transcription factors. However, increased remodeling alone cannot explain why loss of sex steroids tilts the balance of resorption and formation in favor of the former. Estrogens and androgens also exert effects on the lifespan of mature bone cells: pro-apoptotic effects on osteoclasts but anti-apoptotic effects on osteoblasts and osteocytes. These latter effects stem from a heretofore unexpected function of the classical "nuclear" sex steroid receptors outside the nucleus and result from activation of a Src/Shc/extracellular signal-regulated kinase signal transduction pathway probably within preassembled scaffolds called caveolae. Strikingly, estrogen receptor (ER) alpha or beta or the androgen receptor can transmit anti-apoptotic signals with similar efficiency, irrespective of whether the ligand is an estrogen or an androgen. More importantly, these nongenotropic, sex-nonspecific actions are mediated by the ligand-binding domain of the receptor and can be functionally dissociated from transcriptional activity with synthetic ligands. Taken together, these lines of evidence strongly suggest that, in sex steroid deficiency, loss of transcriptional effects may be responsible for the increased osteoclastogenesis and osteoblastogenesis and thereby the increased rate of bone remodeling. Loss of nongenotropic anti-apoptotic effects on mature osteoblasts and osteocytes, in combination with an opposite effect on the lifespan of mature osteoclasts, may be responsible for the imbalance between formation and resorption and the progressive loss of bone mass and strength. Elucidation of the dual function of sex steroid receptors has important pathophysiologic and pharmacologic implications. Specifically, synthetic ligands of the ER that can evoke the nongenotropic but not the genotropic signal may be bone anabolic agents, as opposed to natural estrogens or selective estrogen receptor modulators that are antiresorptive agents. The same ligands may also circumvent the side effects associated with conventional hormone replacement therapy.
\end{abstract}




\section{Introduction}

Loss of ovarian function at menopause represents the most important factor for the development of osteoporosis, the metabolic bone disease that affects millions worldwide and contributes considerably to the morbidity and mortality among elderly women. Loss of androgens in males from chemical or surgical castration or an age-associated decline of androgen levels - albeit not as universal or abrupt as menopause - has the same adverse effect on the skeleton. Appreciation of the adverse effects of estrogen and androgen deficiency on bone in the 1940s by Fuller Albright (Albright and Reisfenstein, 1948) launched a quest for the elucidation of the mechanism of the skeletal actions of sex steroids and the pathogenesis of the bone loss resulting from sex steroid deficiency.

The purpose of this review is to provide a personal perspective of our group's contribution to the advancement of understanding of the cellular and molecular mechanisms by which estrogens and androgens influence adult bone homeostasis and, by extension, the pathogenetic mechanisms responsible for the development of osteoporosis following sex steroid deficiency. This chapter also will point out new pharmacotherapeutic opportunities brought about with the elucidation of the molecular mechanisms of the anti-osteoporotic effects of sex steroids.

\section{A. THE WORKING HYPOTHESIS}

The adult skeleton regenerates continuously in the form of a periodic replacement of old bone with new. This process, called remodeling, is accomplished by temporary anatomic structures comprising osteoclasts in the front, which excavate old bone, and osteoblasts in the rear, which fill the fresh cavities with new bone. These so-called basic multicellular units (BMUs) arise from progenitor cells residing in the bone marrow. After the BMUs complete their task, usually in a period of 6 to 9 months in humans, they disappear without trace due to the apoptotic death of the executive cells of the unit. The rate of remodeling depends on the number of cycles but the effects on bone mass depend on the focal balance within each cycle. Sex steroid deficiency, as well as old age and glucocorticoid excess, do not cause loss of bone mass by turning on a completely new process. Instead, they cause a derangement in the normal process of bone regeneration.

Until the 1990s, research on the pathophysiology of osteoporosis had focused on the functions of differentiated cells: the rates and/or total amounts of bone resorbed or formed by individual osteoclasts or osteoblasts. But these cells have relatively short active lifespans that are within the range of lifespans of the other cells that originate in the bone marrow. Such cells must be continually replaced; the number present depends both on the birth rate, which reflects the frequency of cell division of the appropriate precursor cell, and on the lifespan, 
which most likely reflects the timing of death by apoptosis. The importance of the distinction between cell number and individual cell function often has been obscured by using the vague term "activity" (Manolagas, 1999). The essential hypothesis tested by our work is that the balance between bone resorption and bone formation depends more on the number of cells carrying out these processes than on their individual capacities. Since these cells originate from precursor cells within the bone marrow, our research has placed particular attention to relationships between the different components of this complex tissue and the birth of osteoblasts and osteoclasts. Recognition of the fact that osteoblasts and osteoclasts disappear without a trace guided us more recently to the study of cell death that, for both cell types, is as important as cell birth in determining overall cell numbers.

\section{B. DISTINCTION BETWEEN GROWING AND NONGROWING SKELETON}

In addition to their effects on the maintenance of the homeostasis of the adult skeleton during its continuous regeneration by the process of bone remodeling, estrogens and androgens play a very profound role in skeletal growth and perhaps the sexual dimorphism of the skeleton. Bone mass later in life is a function of the bone accrued during the second decade and the amount of bone lost after menopause. Bone mass accrual during puberty depends more on sexual maturation than chronological age. Hence, the effects of sex steroids on the growing skeleton are relevant to the development of osteoporosis later in life. However, the stimulating effects of estrogens on bone growth during puberty are different from their effects on the maintenance of the mature skeleton and prevention of bone loss. To place the significance of the evidence we will cover later in the appropriate perspective, we will first discuss important distinctions between growing and nongrowing skeleton vis-a-vis estrogen action.

Gender is a major determinant of the size and shape of the skeleton, reflecting the need of the female pelvis to accommodate gestation and delivery of the offspring. Sexual skeletal dimorphism is most likely determined by genetic programs, which have yet to be elucidated. Nonetheless, estrogens or androgens may contribute to such dimorphism by actions exerted during fetal development (Couse and Korach, 1999). Evidence that short-term exposure to exogenous estrogen during fetal life affects bone growth and development in postnatal life suggests the existence of an imprinting mechanism that acts on bone cell programming early in skeletal development (Migliaccio et al., 1996). Sexual skeletal dimorphism is also influenced by the well-known effects of sex steroids on the initiation of the pubertal growth spurt and the closure of the epiphyses at the end of puberty (Frank, 2000; Parfitt, in press). The cellular and molecular mechanisms responsible for these latter effects are also unknown. Linear skeletal 
growth is governed by the chondrocytes of the growth plate. Therefore, the effects of sex steroids on pubertal growth and epiphysial closure result from direct actions of these hormones on chondrocytes. Consistent with this contention, chondrocytes are direct targets for sex steroid action, as they express classical receptors for estrogens and androgens. Thus, the target cells of the effects of sex steroids on bone growth are different from the target cells of their effects on the maintenance of the mature skeleton.

True bone mineral density (i.e., the amount of mineral per volume of bone $\left.\left(\mathrm{gr} / \mathrm{cm}^{3}\right)\right)$ does not appear to increase with size or age. The apparent changes in areal bone mineral density, the popular measurement performed with dual-energy $\mathrm{x}$-ray absorptiometry (DEXA) - the so-called bone mineral density (BMD) that is expressed in $\mathrm{gr} / \mathrm{cm}^{2}$ - is a reflection of growth and an increase in size rather than an increase in bone mineral per unit volume. Hence, the purported changes of BMD during growth are predominantly due to changes on bone volume, not to changes in true bone density. As we reviewed recently elsewhere (Manolagas et al., in press), most of the 40- to 50-fold increase in bone mass from birth to maturity is the result of growth. During growth, bone mass and density may change in opposite directions. In contrast, in the mature skeleton, the change in bone size with time is trivial and mass and density always change in the same direction. Furthermore, the growing and nongrowing skeleton differ profoundly in the spatial and temporal organization of bone cell function. These differences were encapsulated several years ago by Frost in his definitions of modeling as a more-or-less continuous process of bone redistribution and remodeling as a relatively infrequent process of bone replacement (Frost, 1973; Parfitt, 1997). Very importantly, the changes in cortical bone surface location due to modeling can, in some bones, amount to more than $1 \mathrm{~cm}$ from birth to maturity or about $2 \mu \mathrm{m} /$ day. By contrast, changes in cancellous bone surface location due to remodeling are expansion of about $2 \mu \mathrm{m} /$ year during growth (Parfitt et al., 2000) and contraction of about $1 \mu \mathrm{m} /$ year after age 50 (Parfitt, 2000). During cortical bone growth, large numbers of new cells are needed over an extended area every day for many years. In this situation, cell recruitment is not rate limiting and the lifespan of an individual cell is relatively unimportant. But, during adult cancellous bone remodeling, a 5- $\mu \mathrm{m}$ increase in the depth of a resorption cavity (about $10 \%$ ), due to corresponding prolongation of osteoclast lifespan, or a 5- $\mu \mathrm{m}$ decrease in the thickness of new bone deposited, due to corresponding shortening of osteoblast lifespan, can have a large effect on the rate of bone loss, which in a few years may determine whether or not a vertebral fracture occurs.

Unfortunately, lack of appreciation of these facts - and, even more seriously, the incorrect use of BMD measurements by DEXA in growing children - has led to the mistaken conclusion that genetic disorders of the estrogen receptor (ER) or the aromatase gene (Smith et al., 1994; Carani et al., 1997; Bilezikian et al., 1998) cause osteoporosis. Lack of estrogen action in the growing skeleton 
prolongs the duration of growth, leading to increases in height and in long bone length (Simpson, 1998; Grumbach and Auchus, 1999). Hence, in contrast to osteoporosis, the longer (eunochoid) bones of these individuals suggest that, if anything, they accrued more bone than normal. Furthermore, the increased rate of remodeling in these subjects is due to the fact that they did not reach skeletal maturity. Indeed, increased rate of remodeling is physiologic and appropriate for the rapid growth and sculpting of bones during puberty. On the other hand, increased remodeling of the mature skeleton caused by loss of sex steroids - or, for this matter, other conditions - is always pathologic and inappropriate.

\section{Effects of Sex Steroids on Osteoclastogenesis: The IL-6 Paradigm Shift}

Classical receptors for estrogens (ER $\alpha$ and $\mathrm{ER} \beta$ ) or androgens (AR) are present in chondrocytes, bone marrow stromal cells, osteoblasts, and osteoclasts and their progenitors (Eriksen et al., 1988; Komm et al., 1988; Benz et al., 1991a; Bellido et al., 1995; Couse and Korach, 1999), indicating that the effects of sex steroids on bone are mediated, at least in part, directly. The level of receptor expression in osteoblastic and osteoclastic cells is low (at least 10-fold), compared to reproductive sites of sex steroid action. The distribution of these receptors in bone cells does not vary by gender, as similar levels of ER and AR have been found in bone cells from males and females (Benz et al., 1991a,b; Braidman et al., 2000). However, in spite of the demonstration of receptors, convincing evidence that estrogens or androgens modulate the biosynthetic activity of osteoblasts and osteoclasts in a manner that can explain the welldocumented skeletal effects of estrogen loss in vivo remained elusive until the critical role of the bone marrow in bone remodeling was appreciated about a decade ago (Scheven et al., 1986; Kurihara et al., 1989).

Osteoclasts are derived from hematopoietic progenitors of the myeloid lineage, colony-forming unit-granulocyte/macrophage (CFU-GM) and CFU-M. Osteoblasts, as well as the hematopoiesis-supporting stromal cells and adipocytes of the bone marrow, are derived from mesenchymal stem cells. More important, the development of osteoclasts depends on a network of autocrine and paracrine factors produced by stromal and osteoblastic cells. With this knowledge in hand, we proposed the hypothesis that estrogens act to regulate the production of osteoclastogenic cytokines by bone marrow stromal cells and osteoblasts (Manolagas and Jilka, 1992).

Interleukin-6 (IL-6) is a paradigm of a cytokine important for osteoclastogenesis (Manolagas et al., 1996). IL-6 is a member of a family of structurally related cytokines that use the gp130 signal transducer in their receptor complex. Besides IL-6, the family includes IL-11, oncostatin M (OSM), leukemia inhibitory factor (LIF), and cardiotropin 1. Binding of IL-6 to its specific cell surface 
receptor (gp80) causes recruitment and dimerization of gp130, which is then tyrosine phosphorylated by members of the JAK family of tyrosine kinases. This event results in tyrosine phosphorylation of several downstream signaling molecules, including members of the signal transducers and activators of transcription (STAT) family of transcription factors. Phosphorylated STATs, in turn, undergo homo- and hetero-dimerization and translocate to the nucleus, where they activate cytokine-responsive gene transcription. The gp 80 subunit of the IL-6 receptor also exists in a soluble form (sIL-6R) but, unlike most soluble cytokine receptors, it functions as an agonist by binding to IL-6 and then interacting with membrane-associated gp130 to stimulate JAK/STAT signaling.

We found that both estrogens and androgens suppress the production of IL-6 as well as the expression of the two subunits of the IL-6 receptor, IL-6R $\alpha$ and gp130, in cells of the bone marrow stromal/osteoblastic lineage (Girasole et al., 1992; Passeri et al., 1993; Manolagas et al., 1994; Bellido et al., 1995; Jilka et al., 1995; Manolagas and Jilka, 1995; Lin et al., 1997; Manolagas, 1998). Similar results were obtained subsequently by others in rats as well as in humans, in the bone marrow and in the peripheral blood (Manolagas, 2000a; Scheidt-Nave et al., 2001). Importantly, we and others showed that neutralization of IL-6 with antibodies or knockout of the IL-6 gene in mice prevents the upregulation of CFU-GM in the marrow, and the expected increase of osteoclast numbers in trabecular bone sections; and also protects the loss of bone following loss of sex steroids (Jilka et al., 1992; Poli et al., 1994; Bellido et al., 1995). In support of the pathogenetic role of IL- 6 in the bone loss caused by loss of sex steroids, IL-6 seems to play a similar role in several other conditions associated with increased bone resorption, as evidenced by increased local or systemic production of IL-6 and the IL-6 receptor in patients with multiple myeloma, Paget's disease, rheumatoid arthritis, Gorham-Stout or disappearing bone disease, hyperthyroidism, primary and secondary hyperparathyroidism, as well as McCune Albright syndrome (Papanicolaou et al., 1998; Manolagas, 2000a). Nonetheless, IL-6 does not seem to be required for osteoclastogenesis in vivo under normal physiologic conditions. In fact, osteoclast formation is unaffected in sex steroid-replete mice treated with a neutralizing anti-IL-6 antibody or in IL-6-deficient mice (Jilka et al., 1992; Poli et al., 1994). This situation probably reflects the fact that the expression of the gp80 subunit of the IL-6 receptor in bone is a limiting factor for the effects of the cytokine.

At the molecular level, we determined that the suppressive effects of estrogens or androgens on IL-6 production were mediated via the classical receptors and resulted from an indirect effect of the receptor protein on the transcriptional activity on the proximal 225-bp sequence of the human IL-6 gene promoter (Pottratz et al., 1994; Bellido et al., 1995). Moreover, whereas both androgens and estrogens could inhibit IL-6 transcription, their effects were strictly dependent on the expression of their respective receptor and could not be 
due to actions of androgens mediated by the ER, or vice versa (Figure 1). In support of these findings, others established that suppression of IL-6 production by estrogen, or selective estrogen receptor modulators (SERMs) such as raloxifene, is due to protein-protein interaction between the ER and transcription factors such as nuclear factor kappa beta $(\mathrm{NF}-\kappa \beta)$ and CCAAT/enhancer binding protein (C/EBP) (McDonnell and Norris, 1997).

We also found that receptors for IL-6-type cytokines are expressed by stromal/osteoblastic cells and that binding of the ligands to these receptors induces progression toward a more-mature osteoblast phenotype (Bellido et al., 1996,1997; Taguchi et al., 1998) and upregulation of the gp130 promoter (O'Brien and Manolagas, 1997). As in other cell types, the actions of IL-6-type cytokines on osteoblastic cells involve activation of both the JAK/STAT and the mitogen-activated protein kinase (MAP-K) pathway (Bellido et al., 1997) and probably are mediated by the cyclin-dependent kinase (CDK) inhibitor p21 ${ }^{\mathrm{WAF} 1, \mathrm{CIP} 1, \mathrm{SD} 11}$ - a downstream effector of gp130/Stat3 activation (Bellido et al., 1998).

The paradigm of IL- 6 as a target gene for sex steroid action in bone - and, in particular, the suppressive effects of these hormones on osteoclastogenesis -

$E R \alpha$

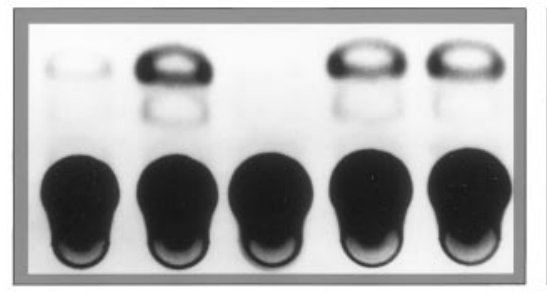

TPA

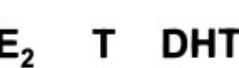

AR

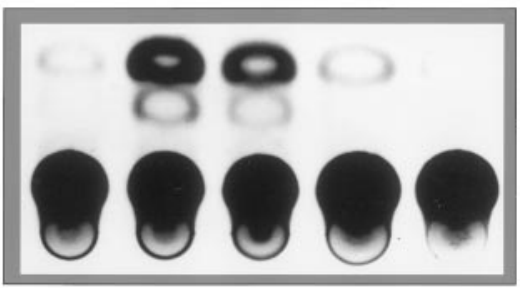

-

FIG. 1. Sex-specific inhibition of interleukin-6 (IL-6) transcription by estrogens or androgens. Hela cells transiently transfected with either estrogen receptor-alpha (ER $\alpha$ ) or androgen receptor (AR) and a proximal 225-bp sequence of the human IL-6 gene promoter driving the expression of chloramphenicol acetyl transferase (CAT). Cells were maintained for 24 hours in medium without or with tetradecanoyl phorbol acetate (TPA) in the presence or absence of estrogen (E2), testosterone (T), or dihydrotestosterone (DHT). Cells were extracted and the CAT products visualized after thin-layer chromatography. [Adapted with permission from Pottratz ST, Bellido T, Mocharla H, Crabb D, Manolagas SC 1994 17 $\beta$-estradiol inhibits expression of human interleukin-6 promoterreporter constructs by a receptor-dependent mechanism. J Clin Invest 93:944-950; and Bellido T, Jilka RL, Boyce BF, Girasole G, Broxmeyer H, Dalrymple SA, Murray R, Manolagas SC 1995 Regulation of interleukin-6, osteoclastogenesis and bone mass by androgens: the role of the androgen receptor. J Clin Invest 95:2886-2895.] 
has been since extended to include tumor necrosis factor (TNF) and macrophagecolony-stimulating factor (M-CSF) (Srivastava et al., 1998,1999). In addition, there has been evidence that estrogens stimulate the production of osteoprotegerin (OPG), a potent anti-osteoclastogenic factor. OPG acts as a decoy, blocking the binding of the receptor activator of NF- $\kappa \beta$ (RANK), expressed in osteoclast progenitors, to RANK-ligand (RANKL) which is expressed in committed preosteoblastic cells (Hofbauer et al., 1999). The high-affinity binding of RANKL to RANK is evidently essential and, together with M-CSF, sufficient for osteoclastogenesis. IL-6, IL-11, IL-1, and TNF as well as parathyroid hormone (PTH) and 1,25-dihydroxyvitamin D3 seem to exert at least part of their osteoclastogenic effects by stimulating the expression of RANKL. PTH and 1,25-dihydroxyvitamin D3 also inhibit the production of OPG (Hofbauer et al., 2000). IL-6 and IL-11 may influence osteoclastogenesis by stimulating the self-renewal and inhibiting the apoptosis of osteoclast progenitors (Girasole et al., 1994; Jilka, 1998). Estrogen loss also may increase the sensitivity of osteoclasts to IL-1 by increasing the ratio of the IL-1RI over the IL-1 decoy receptor (IL-RII) (Sunyer et al., 1999) or increasing the number of TNF-synthesizing lymphocytes (Cenci et al., 2000). As in the case of IL-6, the effects of estrogen on TNF and M-CSF are mediated via protein-protein interactions between the ER and other transcription factors (Table I). Because of the interdependent nature of the production of IL-1, IL-6, and TNF, a significant increase in one of them may amplify, in a cascade fashion, the effect of the others (Jilka, 1998).

TABLE I

Estrogens Regulate Cytokines via Estrogen Response Element (ERE)-independent Mechanisms

\begin{tabular}{ll}
\hline Cytokine & Mechanism of action \\
\hline Interleukin (IL)-6 & $\begin{array}{c}\text { Interference with nuclear factor } \kappa \text { B } \\
\text { binding to promoter }\end{array}$ \\
Tumor necrosis factor & $\begin{array}{c}\text { Interference with jun kinase, jun, } \\
\text { AP-1 actions on the promoter }\end{array}$ \\
IL-1RI/IL-1RII & $?$ \\
Macrophage-colony-stimulating factor & Interference with ckII, egr-1, and \\
Transforming growth factor $\beta$ & Sp1 activity \\
Osteoprotegerin & $?$ \\
\hline
\end{tabular}




\section{Effects of Sex Steroids on Osteoblastogenesis}

Loss of sex steroids increases not only the resorption but also the formation of bone. Based on evidence that increased bone resorption after loss of sex steroids increases the number of osteoclast progenitors in the murine bone marrow, we hypothesized that estrogen loss also stimulates osteoblastogenesis. In support of this hypothesis, we found that, indeed, the number of osteoblast progenitors, CFU-OB, was increased after ovariectomy, in parallel with an increase in the circulating levels of the bone formation marker osteocalcin (Jilka et al., 1998a). The temporal pattern of these changes was very similar to the increase in osteoclastogenesis and the rate of bone loss. In a unique mouse model of osteopenia due to defective osteoblastogenesis, we also found defective osteoclastogenesis (Jilka et al., 1996). The osteoclastogenic defect was secondary to impaired osteoblast formation, as evidenced by the fact that osteoclastogenesis in ex vivo cultures of the bone marrow of these mice could be restored by addition of osteoblastic cells from normal mice. Moreover, unlike control mice, ovariectomy or orchidectomy in these mice failed to increase osteoclastogenesis. These lines of evidence led us to the idea that stimulation of mesenchymal cell differentiation towards the osteoblastic lineage following sex steroid loss may be the first event that ensues following the hormonal change. Increased osteoclastogenesis and bone loss might be downstream consequences of this change. In support of this contention, we found that the rate of remodeling and the expected bone loss following gonadectomy were attenuated in the mice with the defective osteoblastogenesis (Weinstein et al., 1997).

The defining property of stem cell progenitors in regenerating tissues is their ability to self-renew (Loeffler and Potten, 1997). Indeed, such progenitors may divide to produce two identical daughter cells (self-renewal with amplification), or one identical daughter cell and one cell with more differentiated characteristics (self-renewal without amplification), or two cells with a more differentiated phenotype. The decision to self-renew or to differentiate may be stochastic or may be governed by factors in the local microenvironment. Rapid increases in executive cell production can thus be achieved by stimulating the self-renewal of early transit amplifying progenitors. Based on this understanding and our earlier results showing that loss of estrogens upregulates the production of $\mathrm{CFU}-\mathrm{OB}$, we examined the mechanism by which estrogen may regulate CFU-OB production. We found that most CFU-OB are dividing early transit amplifying cells and that $17 \beta$-estradiol suppresses their self-renewal via the $\alpha$ form of the estrogen receptor (Di Gregiorio et al., 2001) (Figure 2). A similar inhibitory effect of estrogens has been demonstrated in self-renewing progenitors that give rise to cells of the inner root sheath and the mature hair fiber (Chanda et al., 2000). Although not shown directly, it is very likely that a similar mechanism might be operating in the actions of estrogen on the closure of the growth plate at the end 


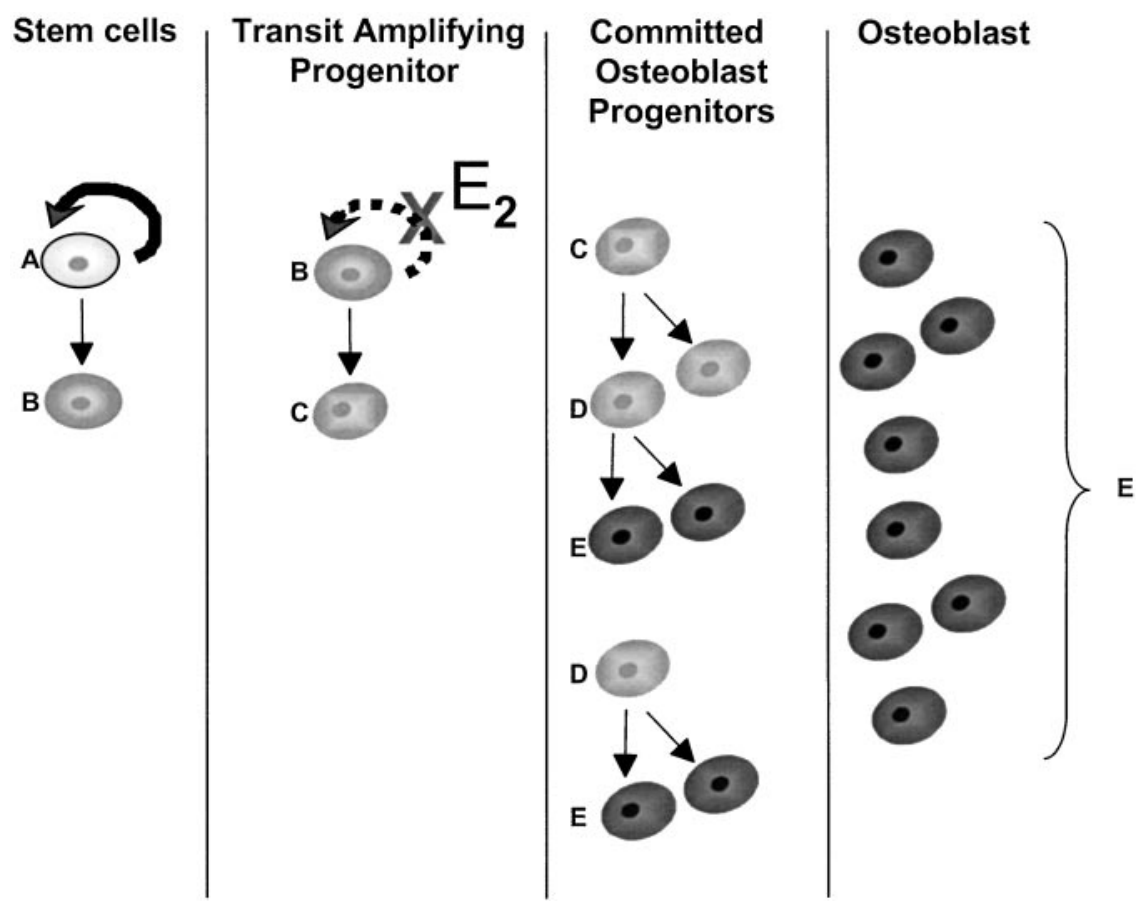

FIG. 2. Attenuation of early mesenchymal osteoblast progenitor (CFU-OB) self-renewal by estrogens via $\mathrm{ER} \alpha$. Mesenchymal stem cells (A) with high self-renewal capacity (solid arrow) give rise to early-transit amplifying osteoblast progenitors (B) with limited self-renewal capacity (dashed arrow). The latter differentiate into late-transit amplifying progenitors lacking self-renewal capability (C). Subsequently, late-transit amplifying cells develop into committed osteoblast progenitors (D) that eventually give rise to fully differentiated osteoblasts (E). Estrogens (E2) suppress the selfrenewal of the early-transit amplifying progenitors. [Adapted with permission from Di Gregorio GB, Yamamoto M, Ali AA, Abe E, Roberson P, Manolagas SC, Jilka RL 2001 Attenuation of the self-renewal of transit-amplifying osteoblast progenitors in the murine bone marrow by $17 \beta$-estradiol. J Clin Invest 107:803-812.]

of puberty, as estrogens have been shown to inhibit prehypertrophic chondroblast proliferation (Tajima et al., 1998). Unpublished studies of ours show that the inhibitory effect of estrogen on CFU-OB self-renewal may be critical for their bone-protective effects. Thus, the mouse model of osteopenia displays blunted osteoblastogenic and osteoclastogenic response, blunted bone loss following loss of estrogens, as well as low CFU-OB self-renewal, compared to control mice (DiGregorio-Taguchi et al., 2001).

Attenuation of CFU-OB self-renewal and osteoclastogenic cytokine production by estrogens, together with the evidence for the molecular dependency of osteoclastogenesis on mesenchymal cell differentiation (through RANK/RANKL 
interaction), provides a strong mechanistic basis for explaining the antiremodeling properties of these hormones. Moreover, in view of the fact that both osteoblasts and the stromal/osteoblastic cells that are required for osteoclast development are derived from CFU-OB, suppression of the self-renewal of this common progenitor may, in fact, represent the key mechanism of the antiremodeling effects of estrogens.

\section{Effects of Sex Steroids on Osteoblast and Osteocyte Apoptosis}

Increased remodeling, resulting from upregulation of osteoblastogenesis and osteoclastogenesis, alone can cause a transient loss of bone because, during remodeling-based regeneration, resorption always precedes (and is faster) than bone formation, creating a temporary deficit, otherwise referred to as expanded remodeling space. However, whereas turnover depends on the number of cycles of remodeling, in the long-term, bone mass depends on the focal balance between formation and resorption within each cycle.

Following loss of sex steroids, osteoclasts erode deeper than normal cavities, leading to the removal of entire cancellous elements and loss of connection between the remaining ones (Parfitt et al., 1996; Eriksen et al., 1999). This phenomenon is most likely due to the removal of pro-apoptotic effects of estrogens on osteoclasts (Hughes et al., 1996). Based on our studies indicating that the majority of osteoblasts die by apoptosis (Jilka et al., 1998b), we have turned our attention to the effects of estrogens as well as androgens on osteoblast and osteocyte apoptosis.

We have found that ovariectomy or orchidectomy causes a dramatic increase in the prevalence of osteoblast and osteocyte apoptosis in mice. Addition of estrogens or androgens suppresses osteoblast and osteocyte apoptosis induced by a variety of pro-apoptotic stimuli in vitro (Kousteni et al., 2001) (Figure 3). We undertook an extensive effort to investigate the molecular mechanism of these anti-apoptotic effects, using primary cultures of murine osteoblastic cells as well as an osteocytic cell line, mouse embryonic fibroblasts, and HeLa cells. We determined that both estrogens and androgens increase the phosphorylation of extracellular signal-regulated kinases (ERKs) in osteoblasts and osteocytes, with a peak at 5 minutes and return to baseline by 15 minutes (Kousteni et al., 2001). ERKs are one of the three subfamilies of MAPK. The other two subfamilies are the jun N-terminal kinases (JNKs), also called stress-activated protein kinases (SAPK), and the p38 kinases. MAPKs are serine/threonine kinases that transduce chemical and physical signals from the cell surface to the nucleus, thereby controlling proliferation, differentiation, and survival (Chang and Karin, 2001). The transduction of the signal from the cell surface to the MAPKs begins with phosphorylation and recruitment of accessory proteins such as Ras, son of sevenless (SOS), Src, or Shc (Figure 4). This event is followed by a three-step 

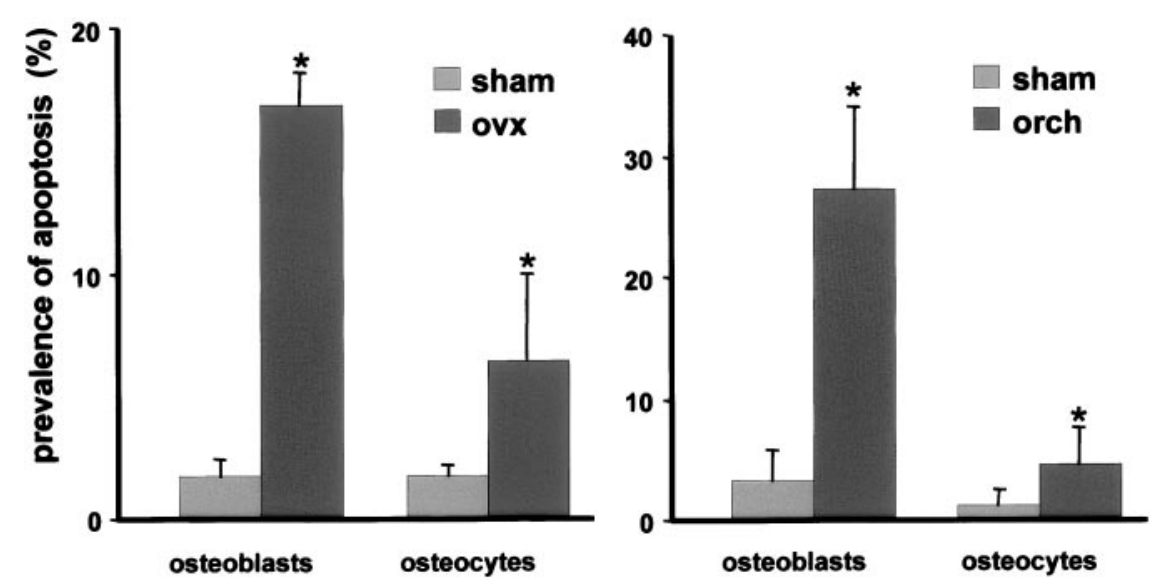

FIG. 3. Control of osteoblast and osteocyte apoptosis by sex steroids in vivo. Prevalence of osteoblast and osteocyte apoptosis in cancellous bone of mice at 4 and 3 weeks after ovariectomy or orchidectomy, respectively. Bars are means \pm SD of four to five animals per group. ${ }^{*} p<0.05$ vs. sham by Student's t-test. [Adapted from Cell 104, Kousteni S, Bellido T, Plotkin LI, O'Brien CA, Bodenner DL, Han L, Han K, Di Gregorio GB, Katzenellenbogen JA, Katzenellenbogen BS, Roberson PK, Weinstein RS, Jilka RL, Manolagas SC, Non-genotropic, sex non-specific signaling through the estrogen or androgen receptors: dissociation from transcriptional activity, 719-730, Copyright 2001, with permission from Elsevier Science.]

cascade leading to MAPK activation by two intermediate kinases: a MAPK kinase (e.g., MAPKK, MKK, or MEK) and a MAPKK kinase or MEK kinase (e.g., MAPKKK or MEKK). Activation of ERKs has been associated with cell survival in a variety of cell types and can result in phosphorylation - and thereby inactivation - of the pro-apoptotic protein Bad, a member of the Bcl-2 family of proteins that controls release of caspase-activating factors from the mitochondria. Pro-apoptotic members of this family form heterodimers with anti-apoptotic members like Bcl-2 and $\mathrm{Bcl}^{-}{ }^{\mathrm{XL}}$ (Chao and Korsmeyer, 1998). Phosphorylation and inactivation of $\mathrm{Bad}$ frees $\mathrm{Bcl}-2$ to form homodimers that prevent the release of caspase activators.

We have found that ERK activation and the protective effect of sex steroids on apoptosis can be blocked by specific inhibitors of Src and MEKK. Moreover, the effects of sex steroids on ERK activation and anti-apoptosis are mediated via the classical ERs (ER $\alpha$ or ER $\beta$ ) or the androgen receptor (AR) and require the activation of a $\mathrm{Src} / \mathrm{Shc} / \mathrm{ERK}$ signaling cascade. This effect is absent in cells deficient in Src or in cells overexpressing a kinase-deficient Src mutant. Further, the anti-apoptotic effect of either class of sex steroids can be abrogated with Src mutants lacking the $\mathrm{SH} 2$ and $\mathrm{SH} 3$ domains or with Shc mutants in which the primary sites of phosphorylation by Src kinases are substituted by alanine. 


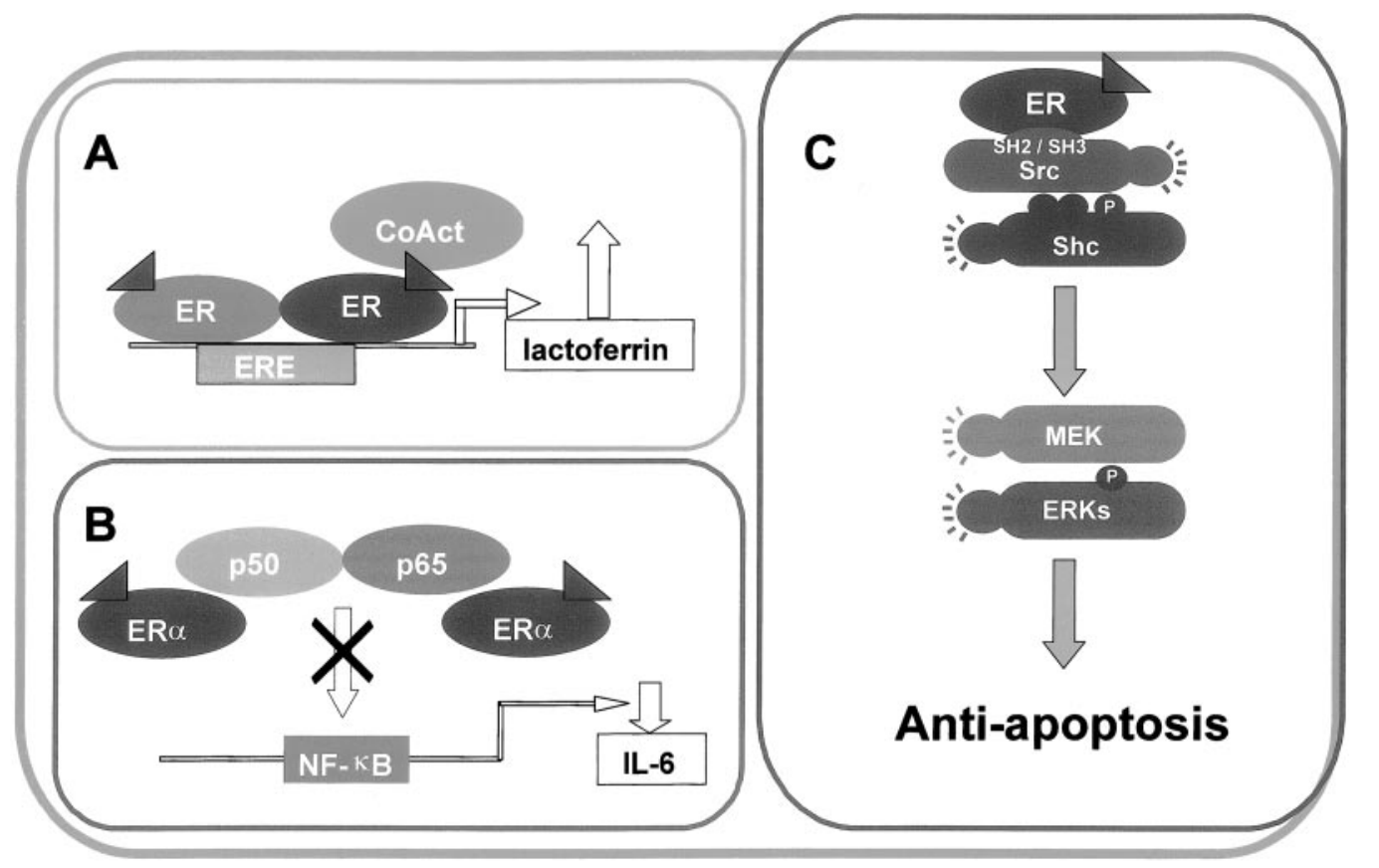

FIG. 4. Paradigms of genotropic and nongenotropic actions of the ER. Cartoon A depicts the protein-DNA interaction of the ER in the case of the transcriptional regulation of an ERE-containing gene (e.g., lactoferrin). Cartoon B depicts the protein-protein interaction responsible for the transcriptional regulation of a gene that does not contain an ERE (e.g., IL-6). Cartoon C depicts a nongenotropic action involving the interaction of a membrane-associated form of the ER with the Src/Shc/ERK signaling pathway. Although in this third model, the ligand-activated receptor protein is depicted as a monomer, it may well be that, in fact, the membrane-associated receptor, as it is the case with the one localized in the nucleus, interacts with components of the signal transduction pathway as a dimer. [Reprinted with permission from Manolagas SC, Kousteni S 2001 Perspective: nonreproductive sites of action of reproductive hormones. Endocrinology 142:2200-2204. Copyright The Endocrine Society.] 
Using the ER $\alpha$ as a paradigm, we also found that activation of the Src/Shc/ ERK pathway by estrogens or androgens is nongenotropic and mediated via a region of the classical ER that is distinct from the one responsible for the genotropic actions of the ligand-activated protein (Kousteni et al., 2001). Indeed, this effect requires only the ligand-binding domain. Furthermore, targeting this domain to the plasma membrane reproduces fully the ERK-mediated antiapoptotic function of the full-length $\operatorname{ER} \alpha$. In contrast, targeting this domain exclusively to the cell nucleus results in complete loss of its anti-apoptotic activity. The Src kinase physically associates with the ER or AR proteins (Migliaccio et al., 2000). As in the case of the Src/Shc/ERK pathway, estrogens can activate the PI3 kinase (PI3K). Activation of PI3K results from direct interaction of the ER with the p85 regulatory subunit of the PI3K and is independent of the transcriptional activity of the receptor (Simoncini et al., 2000).

Several members of the MAPK signaling pathway, including Src, Shc and ERKs, are clustered in caveolae - specialized membrane invaginations that are enriched in the scaffolding protein caveolin-1 and compartmentalize signal transduction (Okamoto et al., 1998). Caveolae are found in a variety of cell types, including neuronal, endothelial, and osteoblastic cells (Toran-Allerand et al., 1999; Solomon et al., 2000). ER $\alpha$ co-immunoprecipitates with caveolin-1 (Schlegel et al., 1999). Furthermore, a subpopulation of $\operatorname{ER} \alpha$ has been co-localized in caveolae with both caveolin and endogenous nitric oxide synthase (eNOS) in human endothelial cells (Chambliss et al., 2000). These lines of evidence, taken together with the finding that the anti-apoptotic signal of the ligand-binding domain of the ER is preserved when targeting this protein to the membrane but is lost when targeting it to the nucleus, suggest strongly that nongenotropic activation of signaling pathways by sex steroids is mediated via the classical receptors, or perhaps a shortened spliced variant that is localized in the membrane and, in particular, within caveolae. In support of this notion, both $\operatorname{ER} \alpha$ and $\operatorname{ER} \beta$ can be detected in the cell membrane. The membrane receptors appear to be derived from the same transcripts as the nuclear ones (Razandi et al., 1999). A membrane-impermeable form of estrogen rapidly activates the p38 MAPK and protects endothelial cells from hypoxia-induced apoptosis (Razandi et al., 1999,2000). Membrane-impermeable forms of estrogen or testosterone activate ERKs in osteoblasts and osteocytes and protect them from apoptosis (Manolagas, 2000a).

In contrast to reproductive tissues where development, growth, maintenance, and function depend on estrogens in females and androgens in males, in nonreproductive tissues like bone and the cardiovascular and central nervous systems, this specificity is greatly relaxed (Manolagas and Kousteni, 2001). For example, closure of the epiphyses of long bones is estrogen dependent in both sexes (Grumbach and Auchus, 1999; Frank, 2000). On the other hand, estrogens or nonaromatizable androgens prevent osteoblast and promote osteoclast apop- 
tosis or activate eNOS in endothelial cells in vitro, irrespective of the gender of the cell donor (Chen et al., 2001) (P. Shaul, personal communication). Furthermore, estrogens restore bone mass in males with aromatase deficiency (Bilezikian et al., 1998), while nonaromatizable androgens can protect the female skeleton against the adverse effects of estrogen deficiency (Tobias et al., 1994; Coxam et al., 1996). Some of these paradoxical effects may be explained by the fact that the distribution of the receptors for sex steroids does not vary by gender. In other words, similar levels of ER and AR have been found in bone, endothelial, and neuronal cells from males and females. Hence, estrogens could very well exert some of their effects in males through ERs residing in male cells and vice versa for androgens.

We have determined that the nongenotropic Src/Shc/ERK-mediated antiapoptotic effect of estrogens or androgens on osteoblasts and osteocytes is transmitted by the $\mathrm{ER} \alpha, \mathrm{ER} \beta$, or the AR with similar efficiency, irrespective of whether the ligand is an estrogen or an androgen (Kousteni et al., 2001). Gender-independent signaling through the ERs or ARs may be operating in the neuroprotective effects of estrogen in males and androgen in females (Roof and Hall, 2000). A great deal more research will be required to establish the significance of the phenomenon in vivo. But it is theoretically plausible that it may provide an additional mechanistic basis for the relaxed gender specificity of the effects described above (Gee and Katzenellenbogen, 2001; Kousteni et al., 2001).

One of the most striking and unexpected results of the elucidation of the nongenotropic regulation of kinases by the classical sex steroid receptors was the discovery that ERK activation - and the resulting prevention of apoptosis - can be dissociated from the transcriptional activity of the nuclear receptors with synthetic ligands (Kousteni et al., 2001). Indeed, by comparing the effects of various ligands on the transcription of the Complement 3 gene, which contains an ERE in its promoter and is regulated by estrogen in a classical manner (or on the transcriptional activation of an ERE-driven promoter or the IL-6 promoter), with their anti-apoptotic effect on primary calvaria cells, we have demonstrated that an estren, with no transcriptional activity, exhibits potent anti-apoptotic efficacy. On the other hand, a pyrazole with potent transcriptional activity has minimal anti-apoptotic efficacy. The relative anti-apoptotic potency of these two compounds correlates with their ability to induce ERK phosphorylation.

Extension of the working life of the bone-resorbing cells and simultaneous shortening of the working life of the bone-forming cells can explain the imbalance between bone resorption and formation that ensues following loss of sex steroids. In agreement with our findings, others have demonstrated an increase in osteocyte apoptosis following loss of estrogen in rats as well as in humans (Tomkinson et al., 1997,1998). The increase in osteocyte apoptosis could further weaken the skeleton by impairment of the osteocyte-canalicular 
mechanosensory network. The increase in bone remodeling that occurs with estrogen deficiency would partly replace some of the nonviable osteocytes in cancellous bone. However, cortical apoptotic osteocytes might accumulate because of their anatomic isolation from scavenger cells and the need for extensive degradation to small molecules to dispose of the osteocytes through the narrow canaliculi. Hence, the accumulation of apoptotic osteocytes caused by loss of estrogen could increase bone fragility even before significant loss of bone mass because of the impaired detection of microdamage and repair of substandard bone.

\section{Pharmocotherapeutic Implications of the Anti-apoptotic Effects of Sex Steroids: the ANGELS Concept}

Besides the critical role of sex steroid-controlled osteoblast and osteocyte apoptosis for the pathogenesis of osteoporosis caused by loss of sex steroids, several additional studies from our group have demonstrated that osteoblast and osteocyte apoptosis are key pathogenetic mechanisms in other forms of osteoporosis. Moreover, control of osteoblast and osteocyte apoptosis may explain the antiosteoporotic efficacy of the most commonly used drugs.

Specifically, we have found that in rodents as well as in humans increased apoptosis of osteoblast and osteocytes is a key pathogenetic mechanism of the adverse effects of glucocorticoid excess on bone (Weinstein et al., 1998; Manolagas and Weinstein, 1999). Osteocyte apoptosis also might be the cause of the so-called osteonecrosis of the hip (Weinstein et al., 2000). Moreover, osteocyte apoptosis might explain the results of a large epidemiological study showing a rapid onset of fracture incidence following the initiation of glucocorticosteroid treatment and, most unexpectedly, a rapid offset of the incidence of fractures following discontinuation of steroid treatment (Manolagas, 2000b).

It has long been known that intermittent administration of PTH exerts an anabolic effect on the skeleton, which is opposite from the better-known catabolic effect of chronic elevation of PTH in primary hyperparathyroidism. To address the mechanism of this anabolic effect, we examined the effect of daily PTH injections in mice over a 1-month period (Jilka et al., 1999). We found a dramatic increase in the number of osteoblasts, bone formation rate, and BMD. All these changes could be accounted for by a dramatic decrease in the prevalence of osteoblast apoptosis, not by increasing the generation of new osteoblasts or the proliferation of existing ones. Importantly, in other species, including humans, the increased bone formation caused by daily PTH injections is also associated with increased osteoblast numbers without change in osteoblast proliferation. The only other agent known to increase bone mass by rebuilding bone, prostaglandin E, also increases the lifespan of mature osteoblasts by reducing the prevalence of their apoptosis (Machwate et al., 1998). Our findings 
provided for the first time proof of principle of the concept that increased work performed by a cell population by suppressing apoptosis can augment bone mass. They also offered a rational explanation for the efficacy of daily PTH injections in various forms of osteoporosis, including the one induced by glucorticoid excess (Manolagas et al., 1998).

All currently approved drugs for the prevention and treatment of osteoporosis - estrogens, bisphosphonates, the SERM raloxifene (McDonnell, 1999), and calcitonin - are "anti-resorptive" agents. The first two stimulate osteoclast apoptosis. Through their pro-apoptotic mechanism of action alone (as in the case of bisphosphonates) or together with the ability to decrease the development of osteoclast progenitors (estrogens and SERMS) or the recruitment and function of osteoclasts (calcitonin), all these agents slow the rate of bone remodeling (Manolagas, 2000a). In most instances, this is sufficient for preventing or slowing the rate of bone loss that is due to the decrease of estrogens after the menopause.

We have demonstrated that like sex steroids, bisphosphonates and calcitonin attenuate osteoblast and osteocyte apoptosis in vitro and in vivo (Plotkin et al., 1999). In the case of bisphosphonates, attenuation of apoptosis is mediated via ERK activation. The anti-apoptotic effect of these two drugs on osteocyte apoptosis provides a potential explanation for their disproportional effects on BMD and antifracture efficacy. Hence, estrogens, PTH, and bisphosphonates exert their anti-apoptotic effects on osteoblasts/osteocytes by stimulating signaling pathways at sites upstream from the common executioners of the apoptosis program (Figure 5). As discussed above, the effect of estrogens (and androgens for this matter) is mediated by a novel paradigm of sex steroid action that requires only the ligand-binding domain of the receptor, extranuclear localization of the protein, and sex nonspecificity (Kousteni et al., 2001). Importantly, the effect of bisphosphonates is exerted via connexin43 hemichannels - a novel gap junction - independent action of connexin (Plotkin et al., 2001). PTH, on the other hand, inhibits apoptosis via cyclic AMP (cAMP)-dependent activation of several survival pathways mediated by protein kinase A (Bellido et al., 2001). Taken together, these discoveries and the demonstration of the principle that increased work output of a cell population by suppressing apoptosis can augment tissue mass, are pointing to an entirely new approach for the treatment of osteoporosis: one that could lead to cure rather than prevention or slowing of the disease process. The results of a recent clinical trial with daily injections of PTH for 18 months support strongly this contention by showing that the anabolic property of PTH restores BMD in the normal range and prevents bone fractures to levels much greater than those seen with antiresorptive agents (Neer et al., 2001).

In the United States, fewer than $7 \%$ of estrogen-deficient women with osteoporosis are receiving therapy. Of those who receive therapy, only $30 \%$ stay on it long enough to enjoy its benefits during their late 60s and 70s when the incidence of the most serious bone fractures (e.g., hip) reaches its zenith 


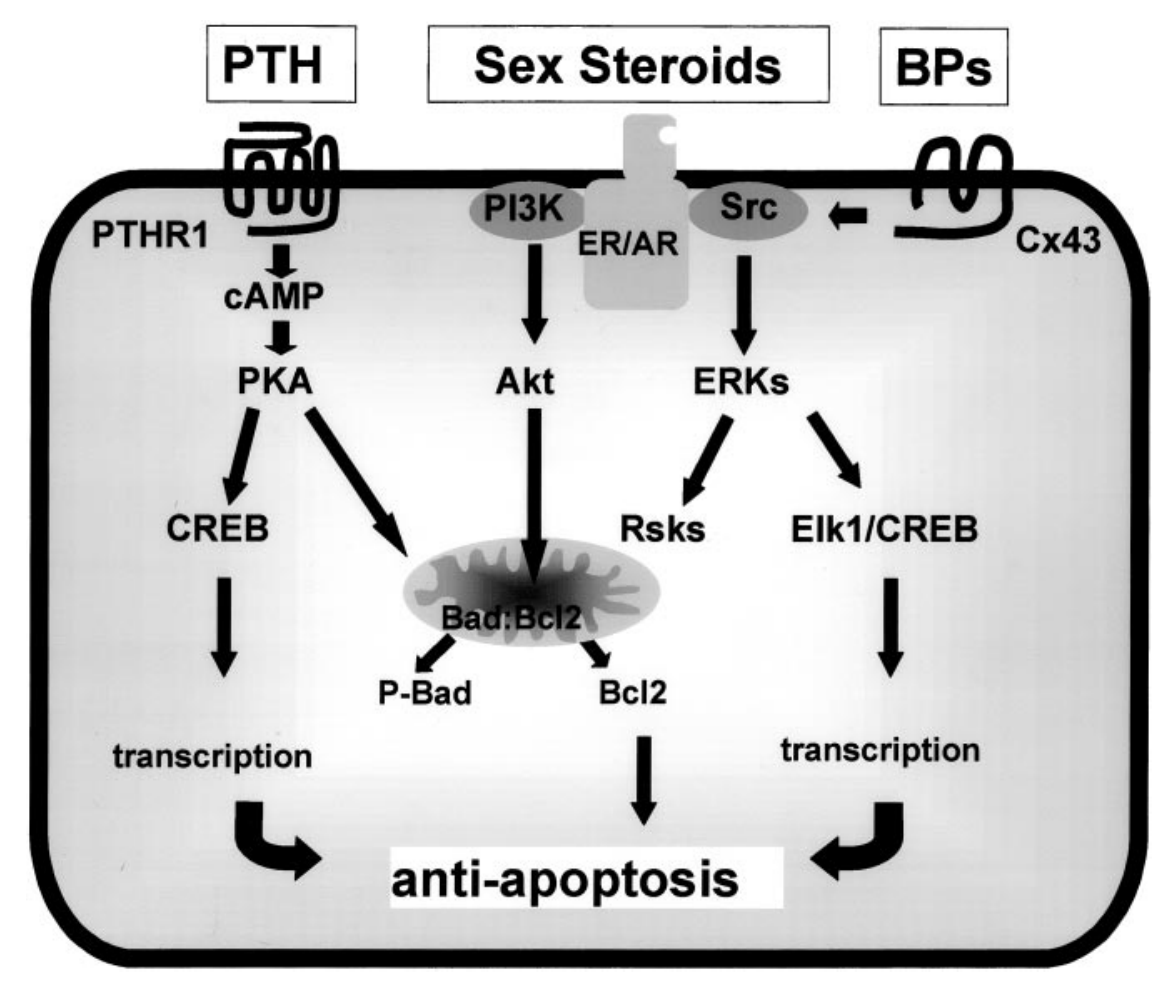

FIG. 5. Survival signaling by PTH, sex steroids, and bisphosphonates. The G proteincoupled receptor for PTH (PTHR1), the membrane-associated ERs or ARs depicted in physical contact with the phosphatidyl inositol 3 kinase (PI3K) and the Src kinase, and the connexin43 (Cx43) hemichannels can all trigger anti-apoptotic signals. The principal downstream kinases of these signaling pathways - protein kinase A, Akt, and ERKs - phosphorylate and inactivate the pro-apoptotic Bad and/or activate transcription of genes that suppress apoptosis through effects on trancription factors, such as Elk-1 and CREB. [Reprinted with permission from Manolagas SC 2001 Manipulating programmed cell death for better living! Science's STKE http://www.stke. org/cgi/content/full/OC_sigtrans;2001/87/pe1. Copyright The American Association for the Advancement of Science.]

(Salamone et al., 1996; Centers for Disease Control and Prevention, 1999). Consequently, the optimal therapeutic modality for osteoporosis, especially in patients who have already suffered significant bone loss, is obviously an anabolic agent that can restore bone mass by rebuilding bone within a short period of time. Bisphosphonates, and to a lesser extent estrogens, cause a small increase in apparent BMD, owing to the contraction of the remodeling space (i.e., the temporary deficit of bone created by the fact that in each site undergoing a cycle of remodeling, resorption precedes formation by several weeks). 
Since daily PTH administration or prostaglandins increase bone mass by preventing osteoblast apoptosis without slowing remodeling, we have reasoned that ER ligands with anti-apoptotic but not antiresorptive/antiremodeling properties will expand the pool of mature osteoblasts at sites of new bone formation and allow these cells more time to make bone, to a much greater degree than the antiresorptive agents that also slow remodeling (Manolagas, 2000a). Based on our findings that the nongenotropic activity of the ER can be dissociated from its transcriptional activity with synthetic ligands (Kousteni et al., 2001), we have coined the acronym ANGELS for ER ligands that function as "activators of nongenotropic estrogen-like signaling." These ligands lack, completely or partially, the ability to induce the transcriptional activity of the ER. Several reasons made us suspect that this class of compounds may be a more rational and advantageous approach to the classical hormone replacement therapy or even SERMS, during postreproductive life. First, nonreproductive tissues express lower levels of receptors, compared to reproductive tissues (10- to 50-fold), which may be sufficient for accommodating nongenotropic actions of sex steroids but is insufficient for genotropic actions (Manolagas and Kousteni, 2001). Second, nonreproductive tissues exhibit a lack of variation of the levels of the ERs or ARs in cells from males versus females, which may account, at least in part, for the relaxed gender specificity in the responsiveness of females and males to estrogens or androgens. Hence, nonreproductive tissues might be ideal targets for gender-neutral ligands. Third, and most important, unlike reproductive tissues in which the majority of estrogenic effects are mediated via transcriptional activation, in nonreproductive tissues, the predominant mechanism of ER action is seemingly nongenotropic. In support of these ideas, we have now obtained evidence that synthetic ligands with potent anti-apoptotic but no genotropic activity increase BMD and bone strength significantly more than estrogens, in estrogen-deficient mice, without affecting the breast or the uterus. Future studies will, of course, be required to establish the validity of the ANGELS concept on nonreproductive tissues other than bone. But, if nongenotropic effects of estrogen are as important in other nonreproductive tissues as the evidence suggests, we are optimistic that ANGELS may also retain at least some of the beneficial effects of estrogens on the vasomotor, cardiovascular system, and CNS.

\section{ACKNOWLEDGMENTS}

The authors wish to thank their colleagues Drs. A. Michael Parfitt, Robert S. Weinstein, Teresita Bellido, and Charles A. O'Brien for discussions and research contributions leading to the ideas and concepts described in this article; and Michelle Douglas for her assistance in preparing this manuscript. They also recognize the National Institutes of Health (P01-AG13918, R01 AR46823), the Department of Veterans Affairs (VA Merit Reviews and a Research Enhancement Award Program), and the 1999 Allied Signal Award for Research on Aging, for supporting their research. 


\section{REFERENCES}

Albright F, Reisfenstein EC 1948 Parathyroid Glands and Metabolic Bone Disease. Baltimore: Williams \& Wilkins; $1-311$

Bellido T, Jilka RL, Boyce BF, Girasole G, Broxmeyer H, Dalrymple SA, Murray R, Manolagas SC 1995 Regulation of interleukin-6, osteoclastogenesis and bone mass by androgens: the role of the androgen receptor. J Clin Invest 95:2886-2895

Bellido T, Stahl N, Farruggella TJ, Borba V, Yancopoulos GD, Manolagas SC 1996 Detection of receptors for interleukin-6, interleukin-11, leukemia inhibitory factor, oncostatin $\mathrm{M}$, and ciliary neurotrophic factor in bone marrow stromal/osteoblastic cells. J Clin Invest 97:431437

Bellido T, Borba VZ, Roberson P, Manolagas SC 1997 Activation of the Janus kinase/STAT (signal transducer and activator of transcription) signal transduction pathway by interleukin6-type cytokines promotes osteoblast differentiation. Endocrinology 138:3666-3676

Bellido T, O'Brien CA, Roberson PK, Manolagas SC 1998 Transcriptional activation of the p21 WAFI,CIPI,SDII gene by interleukin-6 type cytokines - a prerequisite for their prodifferentiating and anti-apoptotic effects on human osteoblastic cells. J Biol Chem 273:21137-21144

Bellido T, Plotkin LI, Davis J, Manolagas SC, Jilka RL 2001 Protein kinase A-dependent phosphorylation and inactivation of the pro-apoptotic protein Bad mediates the anit-apoptotic effect of PTH on osteoblastic cells. J Bone Miner Res 16:S203 (Abstract)

Benz DJ, Haussler MR, Komm BS 1991a Estrogen binding and estrogenic responses in normal human osteoblast-like cells. J Bone Miner Res 6:531-541

Benz DJ, Haussler MR, Thomas MA, Speelman B, Komm BS 1991b High-affinity androgen binding and androgenic regulation of $\alpha_{1}(\mathrm{I})$-procollagen and transforming growth factor- $\beta$ steady state messenger ribonucleic acid levels in human osteoblast-like osteosarcoma cells. Endocrinology 128:2723-2730

Bilezikian JP, Morishima A, Bell J, Grumbach MM 1998 Increased bone mass as a result of estrogen therapy in a man with aromatase deficiency. N Engl J Med 339:599-603

Braidman I, Baris C, Wood L, Selby P, Adams J, Freemont A, Hoyland J 2000 Preliminary evidence for impaired estrogen receptor-alpha protein expression in osteoblasts and osteocytes from men with idiopathic osteoporosis. Bone 26:423-427

Carani C, Qin K, Simoni M, Faustini-Fustini M, Serpente S, Boyd J, Korach KS, Simpson ER 1997 Effect of testosterone and estradiol in a man with aromatase deficiency. N Engl J Med 337:91-95

Cenci S, Weitzmann MN, Roggia C, Namba N, Novack D, Woodring J, Pacifici R 2000 Estrogen deficiency induces bone loss by enhancing T-cell production of TNF-alpha. J Clin Invest 106:1229-1237

Centers for Disease Control and Prevention 1999 Osteoporosis among estrogen-deficient women - United States, 1988-1994. JAMA 281:224-226

Chambliss KL, Yuhanna IS, Mineo C, Liu P, German Z, Sherman TS, Mendelsohn ME, Anderson RG, Shaul PW 2000 Estrogen receptor alpha and endothelial nitric oxide synthase are organized into a functional signaling module in caveolae. Circulat Res 87:E44-E52

Chanda S, Robinette CL, Couse JF, Smart RC 2000 17beta-estradiol and ICI-182780 regulate the hair follicle cycle in mice through an estrogen receptor-alpha pathway. Am J Physiol Endocrinol Metab 278:E202-E210

Chang L, Karin M 2001 Mammalian MAP kinase signalling cascades. Nature 410:37-40

Chao DT, Korsmeyer SJ 1998 BCL-2 family: regulators of cell death. Annu Rev Immunol 16:395-419 
Chen JR, Kousteni S, Bellido T, Han L, DiGregorio GB, Jilka RL, Manolagas SC 2001 Gender-independent induction of murine osteoclast apoptosis in vitro by either estrogens or non-aromatizable androgens. J Bone Miner Res 16:S159 (Abstract)

Couse JF, Korach KS 1999 Estrogen receptor null mice: what have we learned and where will they lead us? Endocr Rev 20:358-417

Coxam V, Bowman BM, Mecham M, Roth CM, Miller MA, Miller SC 1996 Effects of dihydrotestosterone alone and combined with estrogen on bone mineral density, bone growth, and formation rates in ovariectomized rats. Bone 19:107-114

Di Gregorio GB, Yamamoto M, Ali AA, Abe E, Roberson P, Manolagas SC, Jilka RL 2001 Attenuation of the self-renewal of transit-amplifying osteoblast progenitors in the murine bone marrow by $17 \beta$-estradiol. J Clin Invest 107:803-812

Di Gregorio-Taguchi GB, Gubrij I, Smith C, Parfitt AM, Manolagas SC, Jilka RL 2001 The transit amplifying mesenchymal progenitor cell compartment, not the stem cell compartment, is the principal site of control of osteoblast production in normal and pathologic bone remodeling. J Bone Miner Res 15:S376

Eriksen EF, Colvard DS, Berg NJ, Graham ML, Mann KG, Spelsberg TC, Riggs BL 1988 Evidence of estrogen receptors in normal human osteoblast-like cells. Science 241:84-86

Eriksen EF, Langdahl B, Vesterby A, Rungby J, Kassem M 1999 Hormone replacement therapy prevents osteoclastic hyperactivity: a histomorphometric study in early postmenopausal women. J Bone Miner Res 14:1217-1221

Frank GR 2000 Growth and estrogen. Growth Genet Horm 16:1-5

Frost HM 1973 Bone Remodeling and Its Relationship to Metabolic Bone Disease. Springfield, MA: Charles C Thomas

Gee AC, Katzenellenbogen JA 2001 Probing conformational changes in the estrogen receptor: evidence for a partially unfolded intermediate facilitating ligand binding and release. Mol Endocrinol 15:421-428

Girasole G, Jilka RL, Passeri G, Boswell S, Boder G, Williams DC, Manolagas SC 1992 $17 \beta$-estradiol inhibits interleukin-6 production by bone marrow-derived stromal cells and osteoblasts in-vitro: a potential mechanism for the antiosteoporotic effect of estrogens. J Clin Invest 89:883-891

Girasole G, Passeri G, Jilka RL, Manolagas SC 1994 Interleukin-11: a new cytokine critical for osteoclast development. J Clin Invest 93:1516-1524

Grumbach MM, Auchus RJ 1999 Estrogen: consequences and implications of human mutations in synthesis and action. J Clin Endocrinol Metab 84:4677-4694

Hofbauer LC, Khosla S, Dunstan CR, Lacey DL, Spelsberg TC, Riggs BL 1999 Estrogen stimulates gene expression and protein production of osteoprotegerin in human osteoblastic cells. Endocrinology 140:4367-4370

Hofbauer LC, Khosla S, Dunstan CR, Lacey DL, Boyle WJ, Riggs BL 2000 The roles of osteoprotegerin and osteoprotegerin ligand in the paracrine regulation of bone resorption. J Bone Miner Res 15:2-12

Hughes DE, Dai A, Tiffee JC, Li HH, Mundy GR, Boyce BF 1996 Estrogen promotes apoptosis of murine osteoclasts mediated by TGF- $\beta$. Nature Med 2:1132-1136

Jilka RL 1998 Cytokines, bone remodeling, and estrogen deficiency: a 1998 update. Bone 23:75-81

Jilka RL, Hangoc G, Girasole G, Passeri G, Williams DC, Abrams JS, Boyce B, Broxmeyer H, Manolagas SC 1992 Increased osteoclast development after estrogen loss: mediation by interleukin-6. Science 257:88-91

Jilka RL, Passeri G, Girasole G, Cooper S, Abrams J, Broxmeyer H, Manolagas SC 1995 Estrogen loss upregulates hematopoiesis in the mouse: a mediating role of interleukin-6. Exp Hematol 23:500-506 
Jilka RL, Weinstein RS, Takahashi K, Parfitt AM, Manolagas SC 1996 Linkage of decreased bone mass with impaired osteoblastogenesis in a murine model of accelerated senescence. J Clin Invest 97:1732-1740

Jilka RL, Takahashi K, Munshi M, Williams DC, Roberson PK, Manolagas SC 1998a Loss of estrogen upregulates osteoblastogenesis in the murine bone marrow: evidence for autonomy from factors released during bone resorption. J Clin Invest 101:1942-1950

Jilka RL, Weinstein RS, Bellido T, Parfitt AM, Manolagas SC 1998b Osteoblast programmed cell death (apoptosis): modulation by growth factors and cytokines. J Bone Miner Res 13:793-802

Jilka RL, Weinstein RS, Bellido T, Roberson P, Parfitt AM, Manolagas SC 1999 Increased bone formation by prevention of osteoblast apoptosis with parathyroid hormone. J Clin Invest 104:439-446

Komm BS, Terpening CM, Benz DJ, Graeme KA, Gallegos A, Korc M, Greene GL, O’Malley BW, Haussler MR 1988 Estrogen binding, receptor mRNA, and biologic response in osteoblast-like osteosarcoma cells. Science 241:81-84

Kousteni S, Bellido T, Plotkin LI, O'Brien CA, Bodenner DL, Han L, Han K, DiGregorio GB, Katzenellenbogen JA, Katzenellenbogen BS, Roberson PK, Weinstein RS, Jilka RL, Manolagas SC 2001 Non-genotropic, sex non-specific signaling through the estrogen or androgen receptors: dissociation from transcriptional activity. Cell 104:719-730

Kurihara N, Suda T, Miura Y, Nakauchi H, Kodama H, Hiura K, Hakeda Y, Kumegawa M 1989 Generation of osteoclasts from isolated hematopoietic progenitor cells. Blood 74:12951302

Lin SC, Yamate T, Taguchi Y, Borba VZ, Girasole G, O'Brien CA, Bellido T, Abe E, Manolagas SC 1997 Regulation of the gp80 and gp130 subunits of the IL-6 receptor by sex steroids in the murine bone marrow. J Clin Invest 100:1980-1990

Loeffler M, Potten CS 1997 Stem cells and cellular pedigrees - a conceptual introduction. In: Potten CS, ed. Stem Cells. San Diego: Academic Press; 1-27

Machwate M, Rodan GA, Harada S 1998 Putative mechanism for PGE2 anabolic effects: indomethacin administered in vivo induces apoptosis in alkaline positive bone marrow cells. Bone 23:S347

Manolagas SC 1998 The role of IL-6 type cytokines and their receptors in bone. Ann NY Acad Sci 840:194-204

Manolagas SC 1999 Cell number versus cell vigor - what really matters to a regenerating skeleton? Endocrinology 140:4377-4381

Manolagas SC 2000a Birth and death of bone cells: basic regulatory mechanisms and implications for the pathogenesis and treatment of osteoporosis. Endocr Rev 21:115-137

Manolagas SC 2000b Corticosteroids and fractures: a close encounter of the third cell kind. J Bone Miner Res 15:1001-1005

Manolagas SC 2001 Manipulating programmed cell death for better living! Science's STKE http://www.stke.org/cgi/content/full/OC_sigtrans;2001/87/pe1

Manolagas SC, Jilka RL 1992 Cytokines, hematopoiesis, osteoclastogenesis, and estrogens. Calcif Tissue Int 50:199-202

Manolagas SC, Jilka RL 1995 Mechanisms of disease: bone marrow, cytokines, and bone remodeling - emerging insights into the pathophysiology of osteoporosis. N Engl J Med 332:305-311

Manolagas SC, Kousteni S 2001 Perspective: nonreproductive sites of action of reproductive hormones. Endocrinology 142:2200-2204

Manolagas SC, Weinstein RS 1999 New developments in the pathogenesis and treatment of steroid-induced osteoporosis. J Bone Miner Res 14:1061-1066 
Manolagas SC, Jilka RL, Girasole G, Passeri G, Bellido T 1994 Estrogens, cytokines, and the pathophysiology of osteoporosis. In: Kohler PO, ed. Current Opinion in Endocrinology and Diabetes. Philadelphia: Current Science Ltd; 275-281

Manolagas SC, Jilka RL, Bellido T, O'Brien CA, Parfitt AM 1996 Interleukin-6-type cytokines and their receptors. In: Bilezikian JP, Raisz LG, and Rodan GA, eds. Principles of Bone Biology. San Diego: Academic Press; 701-713

Manolagas SC, Weinstein RS, Jilka RL, Parfitt AM 1998 Parathyroid hormone and corticosteroidinduced osteoporosis [letter; comment]. Lancet 352:1940

Manolagas SC, Kousteni S, Bellido T 2002 Extranuclear functions of sex steroid receptors, kinases and osteoporosis: leads from improved HRT. Trends Endocrinol Metab, in press

McDonnell DP 1999 The molecular pharmacology of SERMs. Trends Endocrinol Metab 10:301-311

McDonnell DP, Norris JD 1997 Analysis of the molecular pharmacology of estrogen receptor agonists and antagonists provides insights into the mechanism of action of estrogen in bone. Osteopor Intl 7(suppl 1):S29-S34

Migliaccio S, Newbold RR, Bullock BC, Jefferson WJ, Sutton FG Jr, McLachlan JA, Korach KS 1996 Alterations of maternal estrogen levels during gestation affect the skeleton of female offspring. Endocrinology 137:2118-2125

Migliaccio A, Castoria G, Di Domenico M, de Falco A, Bilancio A, Lombardi M, Barone MV, Ametrano D, Zannini MS, Abbondanza C, Auricchio F 2000 Steroid-induced androgen receptor-oestradiol receptor beta-Src complex triggers prostate cancer cell proliferation. EMBO J 19:5406-5417

Neer RM, Arnaud CD, Zanchetta JR, Prince R, Gaich GA, Reginster JY, Hodsman AB, Eriksen EF, Ish-Shalom S, Genant HK, Wang O, Mitlak BH 2001 Effect of parathyroid hormone (1-34) on fractures and bone mineral density in postmenopausal women with osteoporosis. N Engl J Med 344:1434-1441

O'Brien CA, Manolagas SC 1997 Isolation and characterization of the human gp130 promoter regulation by STATs. J Biol Chem 272:15003-15010

Okamoto T, Schlegel A, Scherer PE, Lisanti MP 1998 Caveolins, a family of scaffolding proteins for organizing "preassembled signaling complexes" at the plasma membrane. J Biol Chem 273:5419-5422

Papanicolaou DA, Wilder RL, Manolagas SC, Chrousos GP 1998 The pathophysiologic roles of interleukin-6 in human disease. Ann Int Med 128:127-137

Parfitt AM 1997 Genetic effects on bone mass and turnover-relevance to black/white differences. J Am Coll Nutr 16:325-333

Parfitt AM 2000 Osteoporosis: 50 years of change, mostly in the right direction. In: Compston JRS, ed. Osteoporosis and Bone Biology: the State of the Art. London: International Medical Press; $1-13$

Parfitt AM 2002 Misconceptions (1): epiphyseal fusion causes cessation of growth. Bone, in press

Parfitt AM, Mundy GR, Roodman GD, Hughes DE, Boyce BF 1996 A new model for the regulation of bone resorption, with particular reference to the effects of bisphosphonates. J Bone Miner Res 11:150-159

Parfitt AM, Travers R, Rauch F, Glorieux FH 2000 Structural and cellular changes during bone growth in healthy children. Bone 27:487-494

Passeri G, Girasole G, Jilka RL, Manolagas SC 1993 Increased interleukin-6 production by murine bone marrow and bone cells after estrogen withdrawal. Endocrinology 133:822-828

Plotkin LI, Weinstein RS, Parfitt AM, Roberson PK, Manolagas SC, Bellido T 1999 Prevention of osteocyte and osteoblast apoptosis by bisphosphonates and calcitonin. J Clin Invest 104:1363-1374

Plotkin LI, Manolagas SC, Bellido T 2000 Connexin-43 hemichannel opening: a requirement for bishophonate-mediated prevention of osteocyte apoptosis. J Bone Miner Res 15:S172 
Poli V, Balena R, Fattori E, Markatos A, Yamamoto A, Tanaka H, Ciliberto G, Rodan GA, Costantini F 1994 Interleukin-6 deficient mice are protected from bone loss caused by estrogen depletion. EMBO J 13:1189-1196

Pottratz ST, Bellido T, Mocharla H, Crabb D, Manolagas SC $199417 \beta$-estradiol inhibits expression of human interleukin-6 promoter-reporter constructs by a receptor-dependent mechanism. J Clin Invest 93:944-950

Razandi M, Pedram A, Greene GL, Levin ER 1999 Cell membrane and nuclear estrogen receptors (ERs) originate from a single transcript: studies of ERalpha and ERbeta expressed in Chinese hamster ovary cells. Mol Endocrinol 13:307-319

Razandi M, Pedram A, Levin ER 2000 Estrogen signals to the preservation of endothelial cell form and function. J Biol Chem 275:38540-38546

Roof RL, Hall ED 2000 Gender differences in acute CNS trauma and stroke: neuroprotective effects of estrogen and progesterone. J Neurotrauma 17:367-388

Salamone LM, Pressman AR, Seeley DG, Cauley JA 1996 Estrogen replacement therapy. A survey of older women's attitudes. Arch Int Med 156:1293-1297

Scheidt-Nave C, Bismar H, Leidig-Bruckner G, Woitge H, Seibel MJ, Ziegler R, Pfeilschifter J 2001 Serum interleukin 6 is a major predictor of bone loss in women specific to the first decade past menopause. J Clin Endocrinol Metab 86:2032-2042

Scheven BA, Visser JW, Nijweide PJ 1986 In vitro osteoclast generation from different bone marrow fractions, including a highly enriched haematopoietic stem cell population. Nature 321:79-81

Schlegel A, Wang C, Katzenellenbogen BS, Pestell RG, Lisanti MP 1999 Caveolin-1 potentiates estrogen receptor alpha (ERalpha) signaling. caveolin-1 drives ligand-independent nuclear translocation and activation of ERalpha. J Biol Chem 274:33551-33556

Simoncini T, Hafezi-Moghadam A, Brazil DP, Ley K, Chin WW, Liao JK 2000 Interaction of oestrogen receptor with the regulatory subunit of phosphatidylinositol-3-OH kinase. Nature 407:538-541

Simpson ER 1998 Genetic mutations resulting in estrogen insufficiency in the male. Mol Cell Endocrinol 145:55-59

Smith EP, Boyd J, Frank GR, Takahashi H, Cohen RM, Specker B, Williams TC, Lubahn DB, Korach KS 1994 Estrogen resistance caused by a mutation in the estrogen-receptor gene in a man. N Engl J Med 331:1056-1061

Solomon KR, Danciu TE, Adolphson LD, Hecht LE, Hauschka PV 2000 Caveolin-enriched membrane signaling complexes in human and murine osteoblasts. J Bone Miner Res 15:2380-2390

Srivastava S, Neale WM, Kimble RB, Rizzo M, Zahner M, Milbrandt J, Patrick RF, Pacifici R 1998 Estrogen blocks M-CSF gene expression and osteoclast formation by regulating phosphorylation of egr-1 and its interaction with Sp-1. J Clin Invest 102:1850-1859

Srivastava S, Weitzmann MN, Cenci S, Ross FP, Adler S, Pacifici R 1999 Estrogen decreases TNF gene expression by blocking JNK activity and the resulting production of c-Jun and JunD. J Clin Invest 104:503-513

Sunyer T, Lewis J, Collin-Osdoby P, Osdoby P 1999 Estrogen's bone-protective effects may involve differential IL-1 receptor regulation in human osteoclast-like cells. J Clin Invest 103:1409-1418

Taguchi Y, Yamamoto M, Yamate T, Lin SC, Mocharla H, DeTogni P, Nakayama N, Boyce BF, Abe E, Manolagas SC 1998 Interleukin-6-type cytokines stimulate mesenchymal progenitor differentiation toward the osteoblastic lineage. Proc Assn Am Physicians 110:559-574

Tajima Y, Yokose S, Kawasaki M, Takuma T 1998 Ovariectomy causes cell proliferation and matrix synthesis in the growth plate cartilage of the adult rat. Histochem J 30:467-472 
Tobias JH, Gallagher A, Chambers TJ $19945 \alpha$-dihydrotestosterone partially restores cancellous bone volume in osteopenic ovariectomized rats. Am J Physiol Endocrinol Metab 267:E853E859

Tomkinson A, Reeve J, Shaw RW, Noble BS 1997 The death of osteocytes via apoptosis accompanies estrogen withdrawal in human bone. J Clin Endocrinol Metab 82:3128-3135

Tomkinson A, Gevers EF, Wit JM, Reeve J, Noble BS 1998 The role of estrogen in the control of rat osteocyte apoptosis. J Bone Miner Res 13:1243-1250

Toran-Allerand CD, Singh M, Setalo GJ 1999 Novel mechanisms of estrogen action in the brain: new players in an old story. Front Neuroendocrinol 20:97-121

Weinstein RS, Jilka RL, Parfitt AM, Manolagas SC 1997 The effects of androgen deficiency on murine bone remodeling and bone mineral density are mediated via cells of the osteoblastic lineage. Endocrinology 138:4013-4021

Weinstein RS, Jilka RL, Parfitt AM, Manolagas SC 1998 Inhibition of osteoblastogenesis and promotion of apoptosis of osteoblasts and osteocytes by glucocorticoids: potential mechanisms of their deleterious effects on bone. J Clin Invest 102:274-282

Weinstein RS, Nicholas RW, Manolagas SC 2000 Apoptosis of osteocytes in glucocorticoidinduced osteonecrosis of the hip. J Clin Endocrinol Metab 85:2907-2912 



\title{
Anabolic Steroids
}

\author{
Cynthia M. KuHN \\ Department of Pharmacology and Cancer Biology, Duke University Medical Center, \\ Durham, North Carolina 27710
}

\begin{abstract}
The term "anabolic steroids" refers to testosterone derivatives that are used either clinically or by athletes for their anabolic properties. However, scientists have questioned the anabolic effects of testosterone and its derivatives in normal men for decades. Most scientists concluded that anabolic steroids do not increase muscle size or strength in people with normal gonadal function and have discounted positive results as unduly influenced by positive expectations of athletes, inferior experimental design, or poor data analysis. There has been a tremendous disconnect between the conviction of athletes that these drugs are effective and the conviction of scientists that they aren't. In part, this disconnect results from the completely different dose regimens used by scientists to document the correction of deficiency states and by athletes striving to optimize athletic performance. Recently, careful scientific study of suprapharmacologic doses in clinical settings - including aging, human immunodeficiency virus, and other disease states - supports the efficacy of these regimens. However, the mechanism by which these doses act remains unclear.

"Anabolism" is defined as any state in which nitrogen is differentially retained in lean body mass, either through stimulation of protein synthesis and/or decreased breakdown of protein anywhere in the body. Testosterone, the main gonadal steroid in males, has marked anabolic effects in addition to its effects on reproduction that are easily observed in developing boys and when hypogonadal men receive testosterone as replacement therapy. However, its efficacy in normal men, as during its use in athletes or in clinical situations in which men are eugonadal, has been debated. A growing literature suggests that use of suprapharmacologic doses can, indeed, be anabolic in certain situations; however, the clear identification of these situations and the mechanism by which anabolic effects occur are unclear. Furthermore, the pharmacology of "anabolism" is in its infancy: no drugs currently available are "purely" anabolic but all possess androgenic properties as well. The present review briefly recapitulates the historic literature about the androgenic/anabolic steroids and describes literature supporting the anabolic activity of these drugs in normal people, focusing on the use of suprapharmacologic doses by athletes and clinicians to achieve anabolic effects in normal humans. We will present the emerging literature that is beginning to explore more specific mechanisms that might mediate the effects of suprapharmacologic regimens. The terms anabolic/androgenic steroids will be used throughout to reflect the combined actions of all drugs that are currently available.
\end{abstract}

\section{Use of Suprapharmacologic Doses of Anabolic/Androgenic Steroids (AAS)}

People have been taking testosterone to restore "vitality" since the efficacy of some hormonal component of the testes was first described by Brown-Sequard 
in 1889. He reported the reversal of his own aging by self-injection of a testicular extract, thereby stimulated a flurry of experimentation into the putative anti-aging effects of testicular hormones long before the identity of testosterone was confirmed. The first use to improve athletic performance occurred shortly thereafter, in 1896. A contemporary of Brown-Sequard self-administered testicular extract, then measured his finger strength. Athletes have been using purified testosterone since it was first available (see a review of this early history in Yesalis et al., 2000). The modern use of anabolic steroids in athletic competition dates from the Olympic competitions during the Cold War era. Russian athletes were putatively the first to use anabolic steroids to improve athletic performance in international competitions. Although the International Olympic Committee banned use of anabolic agents in 1964, the practice spread and probably reached its pinnacle in the athletic programs in Germany during the 1970s (Yesalis et al., 2000).

Medical use of testicular extract began in the late 1800s. Clinical use of supraphysiological doses of AAS in eugonadal patients for anabolic benefit started in the 1940s. High-dose AAS regimens have been used to promote muscle deposition after burns, surgery, radiation therapy, and aging-related sarcopenia (muscle wasting). Recent uses include treating wasting in human immunodeficiency virus (HIV) and contraception (Bhasin et al., 1996,1997; Amory and Bremner, 2000).

\section{Anabolic Steroids}

All steroids that are anabolic are derivatives of testosterone and are androgenic as well as anabolic, as they stimulate growth and function of male reproductive tract. Individual drugs vary in their balance of anabolic:androgen activity but none of the currently available drugs are purely anabolic. All the anabolic steroids currently used are derivatives of testosterone or are structural modifications of testosterone that influence its pharmacokinetics, bioavailability, or balance of androgenic to anabolic activity. These include testosterone itself, all of the derivatives that are used clinically, as well as numerous plant products that at least claim to possess anabolic actions.

The testosterone derivatives available in the United States comprise several groups: 1) endogenously produced androgens or their precursors, including testosterone and androstenedione; 2) synthetic derivatives of testosterone with altered metabolic or receptor-binding characteristics; and 3) various uncharacterized plant or animal materials. Testosterone actions represent the combination of several activities. First, it binds to the androgen receptor to exert its androgenic activity. Second, it is $5 \alpha$ reduced in some target tissues (including the male urogenital tract, skin, liver, and sebaceous glands) to dihydrotestosterone (DHT), which also acts on the androgen receptor. Finally, it can be aromatized to 
estradiol and exert estrogenic activities. The latter two actions are highly undesirable in anabolic drugs, $5 \alpha$ reduction because it decreases the ratio of anabolic:androgenic activity and aromatization because of the feminizing side effects.

Structural and pharmacokinetic properties have been reviewed extensively (Wilson, 1988,1996) and are abstracted briefly here (see Figures 1 and 2).

1. Testosterone as an injectable form, a transdermal patch, skin cream, and a micronized oral preparation

2. 17- $\beta$ esters of testosterone:testosterone cypionate, propionate, enanthate, and undecanoate. Esterification at this site renders the steroid more fat soluble and delays absorption into the circulation. All but the undecanoate must be injected. Nandrolone $17-\beta$ esters also exist.

3. 17- $\alpha$ derivatives (methyltestosterone, methandrostenolone, norethandrolone, fluoxymesterone, danazol, oxandrolone, stanozol). These derivatives resist metabolism in the liver, so are orally active. This modification is associated with significant hepatic toxicities.

4. Modifications of the $\mathrm{A}, \mathrm{B}$, or $\mathrm{C}$ rings (mesterolone, nortestosterone, methenolone, fluoxymesterone, methandrostenolone, northandrolone, danazol, nandrolone, stanozol). These modifications achieve a number of goals, including a) slow metabolism; b) enhanced affinity for the androgen receptor (19-nortestosterone); c) resistance to aromatization to estradiol (fluoxymesterone, 19 nortestosterone); and d) decreased binding of metabolites to androgen receptor ( $5 \alpha$-reduced metabolites of 19-nortestosterone, $7 \alpha-19$ - nortestosterone).

Structure:activity modifications that limit either conversion to DHT and/or to estradiol partially target specific testosterone derivatives to specific activities. Agents such as fluoxymesterone and 19-nortestosterone (nandrolone) that resist aromatization lack the feminizing side effects of testosterone. 19-nortestosterone possesses another characteristic that increases its anabolic activity because its

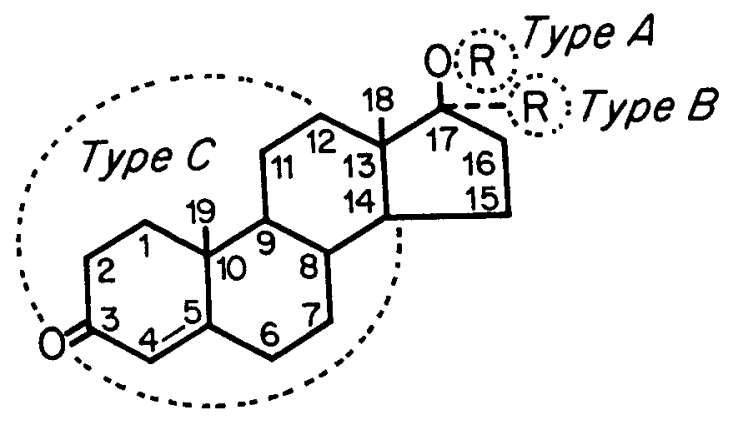

FIG. 1. Model testosterone structure. [Reprinted with permission from Wilson JD 1998 Androgen abuse by athletes. Endocr Rev 9:181-191. Copyright The Endocrine Society.] 


\section{ORALLY ACTIVE AGENTS}

$17 \alpha$-Alkyl Substitutes

(BC)

1-Methyl Derivatives

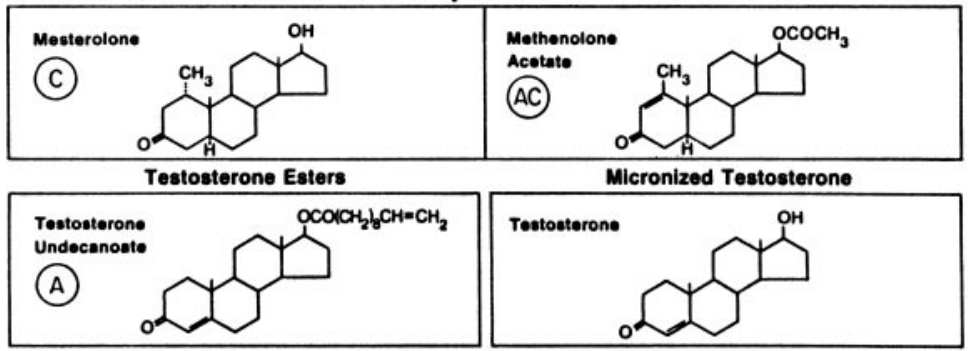

PARENTERAL AGENTS

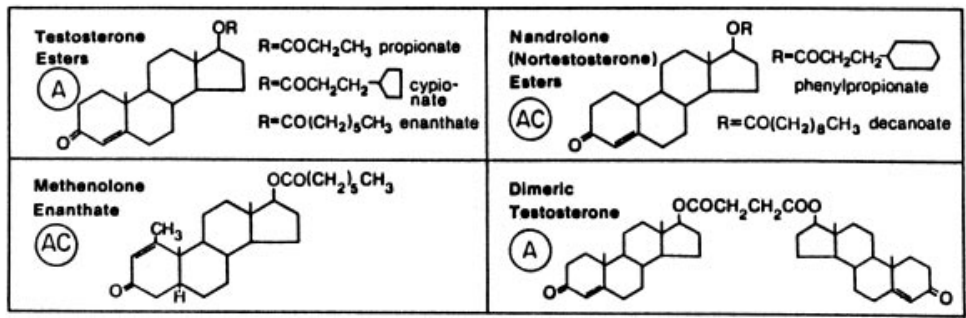

FIG. 2. Structures of several anabolic steroids. [Reprinted with permission from Wilson JD 1998 Androgen abuse by athletes. Endocr Rev 9:181-191. Copyright The Endocrine Society.] 
$5 \alpha$-reduced metabolite has poor affinity for the androgen receptor. Similarly, alpha-methyl-19-nortestosterone is not a substrate for $5 \alpha$ reductase (Sundaram et al., 1995).

Finally, a number of "natural products" that are purported to exhibit anabolic qualities are marketed freely in the United States due to the exemption of "natural products" from U.S. Food and Drug Administration regulation. Most of these are steroid precursors. Androstenedione and norandrostenedione are two widely marketed precursors. Marketers claim that they are converted into testosterone and nortestosterone (nandrolone). While a small percentage is, indeed, converted, the total amount produced is likely far below that which would have any anabolic activity in a eugonadal male. Finally, there are undefined mixtures with catchy names like "Horny Goat Weed" and "Testicular Extract" that are derived from both plant and animal materials and contain absolutely unknown ingredients.

All of the drugs listed above possess both anabolic and androgenic activities; none are absolutely selective. However, this ratio varies across a broad range. Table I shows the approximate anabolic:androgenic ratio of a number of clinically used AAS. The range is fairly narrow by clinical standards. All anabolic steroids are virilizing if administered for long enough at high enough doses.

These values are based on data collected in the 1950s and 1960s from bioassays of varying degrees of specificity and accuracy (see excellent historical review in Kochakian, 1976). Typically, the ability of a test drug to stimulate growth of a skeletal muscle and a reproductive target (prostate gland) was assessed. Two classic methods for establishing anabolic efficacy were the stimulation of growth of the levator ani muscle in the castrated rodent and stimulation of whole-body nitrogen retention in a castrated animal. Neither of these are ideal measures. The levator ani muscle may actually reflect androgenic efficacy of AAS because it can be viewed as part of the reproductive system. Its use as a bioassay for "anabolic" activity has been questioned. While the

TABLE I

Anabolic:Androgenic Ratio for Selected Anabolic Drugs

\begin{tabular}{lc}
\hline Anabolic/androgenic steroid & Anabolic:androgenic ratio \\
\hline Testosterone, methyltestosterone & 1 \\
Methandrostenolone & $2-5$ \\
Oxymetholone & 9 \\
Oxandrolone & 10 \\
Nandrolone & 10 \\
Stanozol & 30 \\
\hline
\end{tabular}


nitrogen-retention assay is better, it provides an extremely indirect measure of muscle deposition.

There has been virtually no investigation of the relative anabolic and androgenic properties of AAS since the mid-1970s and none using more modern tools to assess androgen receptor activity. One major goal of this chapter is to summarize recent developments in the molecular pharmacology of androgen receptors that are opening this area up for pharmaceutical development.

\section{Androstenedione}

In the summer of 1998, baseball player Mark McGwire revealed that he took regular androstenedione supplements during the season that he set a new home run record. The first scientific study of the anabolic efficacy of androstenedione appeared shortly thereafter (King et al., 1999). This study showed that giving modest doses to untrained men who were started on an exercise program increased testosterone only transiently at the higher (i.e., 300-mg) dose but did not improve strength. However, it did increase plasma estradiol levels, a finding that was confirmed in a later study (Leder et al., 2000). That is, this study recapitulated the large number of negative studies in the literature that documented that such combinations of training and AAS were no more effective than training alone. This study has been replicated in older men using a similar design (Broeder et al., 2000). In this case, users took doses recommended by supplement manufacturers while they were engaged in resistance training. The results of both studies were similar: neither younger nor older subjects who received androstenedione showed greater increases in strength than those who received placebo, although circulating lipid profiles changed in the direction of greater cardiovascular risk (low-density to high-density lipoprotein/apolipoprotein A/apolipoprotein $\mathrm{B})$ ratio. Since, at most, $10-15 \%$ of a dose is converted to testosterone, it is unlikely that regimens used by athletes will prove anabolic but the research has not been conducted. No published studies report effective anabolic activity of suprapharmacologic doses of androstenedione.

\section{Do Anabolic Steroids Increase Muscle Size and/or Strength in Eugonadal Men?}

The anabolic effects of restoring normal physiologic levels of testosterone in hypogonadal men are uncontested. The rise in testosterone during puberty contributes to the increase in linear growth as well as muscle deposition at that time. Increased muscle deposition clearly results when hypogonadal men receive testosterone treatment (Kopera, 1985; Wilson, 1996; Bross et al., 1999). AAS also can be anabolic in men who are hypogonadal as a result of disease such as HIV or after burns (Bhasin et al., 1996,1999). 
The anabolic effects of testosterone derivatives in women athletes are similarly explicable, as circulating testosterone levels of women are typically about $10 \%$ of those observed in men (Wilson, 1996). Therefore, raising female testosterone levels to those comparable to males provides supraphysiologic levels. Although there are very few published studies of muscle size and strength after AAS use in women, one report found elevations up to 30-fold of normal levels in women who were self-administering AAS (Malarkey et al., 1991). There are virtually no controlled studies of AAS effects on women for obvious ethical reasons but dramatic evidence of these effects derives from the recently released results of the East German sports program of the 1970s and 1980s (Franke and Berendonk, 1997). Figure 3 shows shotput performance of a female German athlete, with the bars below indicating the periods of AAS administration. Unfortunately, women receiving AAS inevitably experience the androgenization associated with these drugs.

The benefit of anabolic steroid use for eugonadal men is far more controversial.

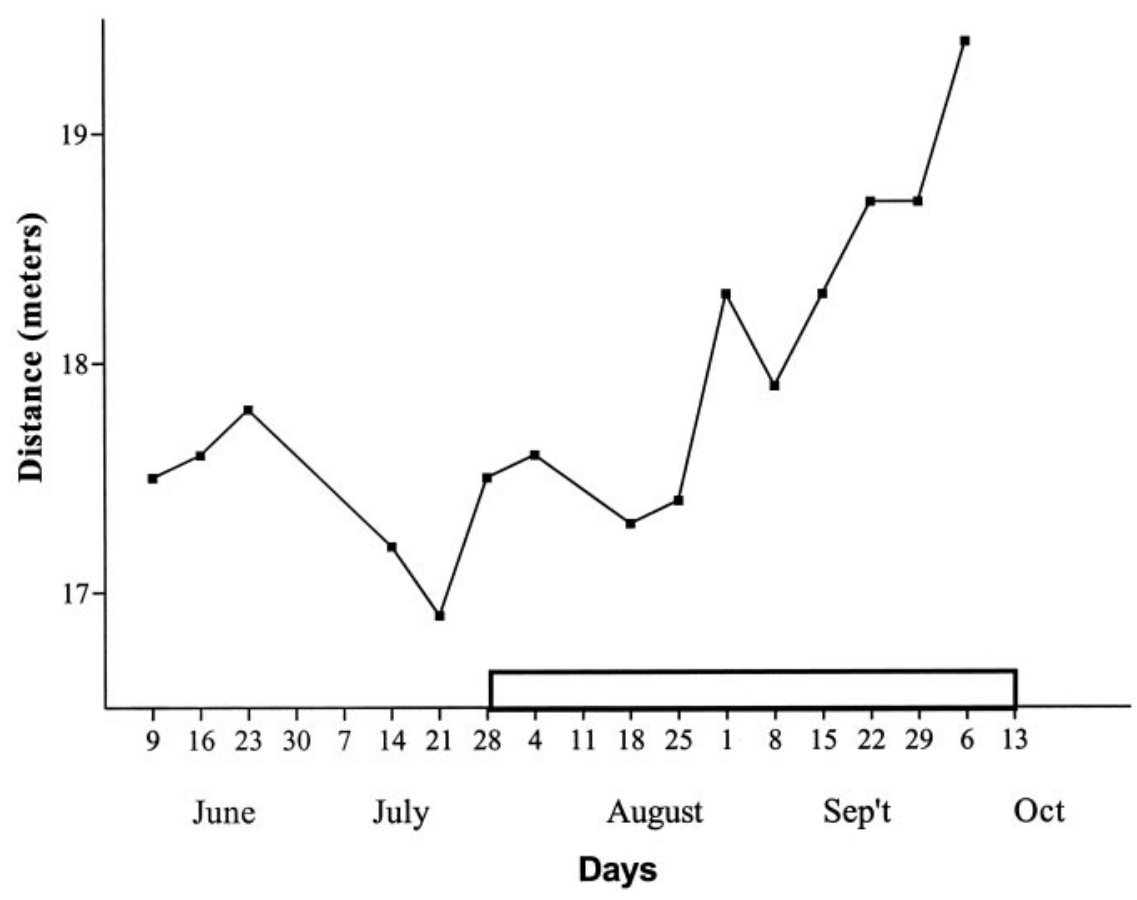

FIG. 3. Shot-put performance of woman athlete from East Germany. [Redrawn from Franke WW, Berendonk B 1997 Hormonal doping and androgenization of athletes: a secret program of the German Democratic Republic government. Clin Chem 43:1262-1279.] 
For decades, scientists argued that anabolic steroids do not increase muscle mass or strength in normal men. This was based first on clinical experience that suggested that the nitrogen-retaining effects of testosterone treatment to normal men were modest and transient (Wilson, 1996). The controversy between those who state that anabolic steroids do not increase muscle mass or strength and those who believe in their effectiveness derives in part from which body of research is cited. Many critics properly cite many negative studies in which addition of testosterone or another AAS to a training regimen failed to improve performance (see reviews in Wilson, 1988; Elashoff et al., 1991; O'Connor and Cicero, 1993; Friedl, 2000). They also critique the poor study design in studies conducted in athletes, including lack of placebo control, nonblinded study conditions, reliance on case studies or small study populations, lack of standardization of dose and training regimen, and the impact that expectation of benefit had on results.

These differences in study design might well play an important part in the different findings. First, nonfit people who are started on a training regimen generally experience such substantial benefit from the training regimen alone that it is difficult to show an additive benefit of AAS. The use of physiologic doses of AAS contributed to this problem. Little benefit of increasing testosterone within the physiologic range has been demonstrated in such studies. Furthermore, the dependent measure chosen to assess muscle strength is critical. Testosterone increases upper body mass differentially, so performance in tasks like weightlifting should improve more than lower-body tasks or tasks in which aerobic capacity rather than strength are assessed. As expected, the task in which increases have been reported most reliably are in the bench press (Fried1, 2000). Finally, the degree of improvement expected in such studies is generally small. Changes in performance of $1-5 \%$ are rarely statistically or clinically significant but they represent the margin of victory for elite athletes. Therefore, scientists, clinicians, and athletes all might interpret data from the same study quite differently.

Controversies about anabolic effects of AAS in animals have been similar but less intense. The same factors have entered into the outcome: gender of animals, conducting the study in trained vs. sedentary animals, dose regimen, and duration of exposure. AAS do effectively increase muscle size, protein content, and contractility in both male and female rats (Exner et al., 1973; Menschikowski et al., 1988; Lewis et al., 1999), although negative findings have been reported (Bates et al., 1987). Efficacy of AAS in trained animals has been established (Lubek et al., 1984; Elashoff et al., 1991; Lewis et al., 1999), although different muscle beds respond differentially and slow twitch muscles improve more than fast twitch (Sachs and Leipheimer, 1988; Lewis et al., 1999; Joumaa and Leoty, 2001). 


\section{Challenging the Conventional Wisdom: AAS Can Increase Muscle Size and Strength in Normal Men}

Studies providing suprapharmacologic doses, using maximally trained athletes and testing performance in tasks like weightlifting, are mainly likely to show an effect of AAS. A recent study showing clear, statistically significant increases in muscle mass and strength after AAS administration in a proper placebo-controlled, blinded study may help put these controversies to rest. This complemented previous studies from the same laboratory demonstrating benefit in hypogonadal, HIV-infected men using the same strategy (Bhasin et al., 1996,1999,2001; Strawford et al., 1999).

Biochemical and anatomical studies show that AAS do significantly influence muscle morphology and biochemistry in humans. Body weight reliably increases after AAS use and part of the increase is in lean body mass, although part also reflects retention of water (see recent review in Friedl, 2000). Muscle biopsies in weightlifters reported that both the number of muscle fibers and average fiber size in the trapezius muscle were greater in AAS users than nonusers (Doumit et al., 1996; Kadi et al., 1999a). Controlled studies show that both the number of muscle fibers and the size of individual fibers increase with AAS treatment in animal models (Joubert and Tobin, 1989). Both of these processes depend upon activation of satellite cells within the muscle. Satellite cells contain androgen receptors (Doumit et al., 1996). AAS action within these cells to stimulate proliferation may represent an important mechanism of AAS action. The specific genes that are regulated by androgens in the muscle are unknown. Muscle biopsies in AAS-using powerlifters, in comparison to drugfree powerlifters, showed increased expression of embryonic forms of myosin and the Leu-19 antigen that is expressed in developing myotubes and newly formed myonuclei. This finding supports the hypothesis that AAS trigger both hypertrophy and hyperplasia but does not elucidate the specific genes that are activated (Kadi et al., 1999a,b,2000).

Increases in strength can also result not from hypertrophy or hyperplasia but from increased expression of specific elements of the contractile apparatus. Again, this has been little studied. However, a recent study (Joumaa and Leoty, 2001) began to address this phenomenon by evaluating potassium and caffeineinduced contractures. Both the magnitude of potassium-induced contractures and the rate of recovery were greater in slow-twitch muscles of animals that received training and nandrolone. The authors speculated that these results suggested changes in both the activation mechanism and recovery mechanisms that sequestrate calcium in the sarcoplasmic reticulum. Enhanced caffeine contractures could reflect enhanced calcium release from the sarcoplasmic reticulum or changes in the calcium sensitivity of the contractile proteins. 


\section{Mechanism of Anabolic Effect in Eugonadal Men}

\section{A. ROLE OF SUPRAPHARMACOLOGIC DOSES}

The mechanism by which AAS increase muscle size and strength is surprisingly confusing. Androgen receptors clearly mediate the increase in muscle size and protein synthesis in hypogonadal men and during puberty. In these situations, androgen increases net nitrogen balance, increases lean body mass, and increases the rate of muscle protein synthesis (see review by Wilson, 1996). However, it often is asserted that comparable effects are not observed in men with normal gonadal function because androgen receptors are saturated at physiologic levels of testosterone. If androgen effects are mediated by androgen receptors, which are saturated at physiologic levels of testosterone, then no additional benefit should result from providing more androgen.

Steroid regimens favored by athletes differ markedly from those used clinically to provide replacement for hypogonadal men. Athletes use suprapharmacologic doses and typically "stack" multiple drugs at total androgen doses that range from 10-100-fold above normal levels (Wilson, 1988). Typically, they take androgens in cycles of weeks, with drug holidays interspersed of weeks or months. Many athletes use "stacking" regimens that involve taking multiple agents simultaneously, and/or a pyramiding dose regimen in which doses are started low, increased, then tapered back down.

A small but expanding literature suggests that suprapharmacologic doses are effective in eugonadal men. The active hormone is probably testosterone, since $5 \alpha$ reductase is not present in muscle (Wilson and Gloyna, 1970). Two older studies (Griggs et al., 1989; Forbes et al., 1992) have been supplemented by several recent findings demonstrating increased lean body mass, muscle protein synthesis, and/or positive nitrogen balance in normal men after high doses of AAS (Bhasin et al., 1996; Ferrando et al., 1998; Sheffield-Moore et al., 1999; Strawford et al., 1999; see also review in Sheffield-Moore, 2000). The most important recent finding is the dose-response study showing that androgenic effects of testosterone saturate at fairly low doses, in contrast to measurable anabolic effects, which require considerably higher doses (Bhasin et al., 1999).

\section{B. ANDROGEN RECEPTOR IN AAS: EFFECTS ON EUGONADAL MEN}

The finding that muscle hypertrophy associated with exercise is blocked by androgen antagonists (Inoue et al., 1994) supports a primary role for androgen receptors in exercise-induced muscle hypertrophy. One androgen receptor has been cloned (see review by Lamb et al., 2001) and while its expression varies quantitatively in muscle and reproductive tissues (Sar et al., 1990; Kimura et al., 1993), it is likely that this receptor mediates AAS effects in the muscle. The one study comparing the binding of a range of AAS to skeletal muscle and prostate 
reported, as expected, that little tissue specificity in binding affinity was observed across a broad range in binding affinities (Saartok et al., 1984). Androgen receptors are present in skeletal muscle of every mammalian species (Sar et al., 1990; Takeda et al., 1990). Levels of expression differ from muscle bed to muscle bed in a manner consistent with reported AAS effects on muscle strength in different tasks. For example, human muscle beds differ from each other, with expression higher in the muscles of the neck and chest girdle, in comparison to the limbs (Kadi et al., 2000).

Recent studies in multiple species show that androgen receptor can be upregulated by exposure to AAS (Bricout et al., 1994; Doumit et al., 1996; Sheffield-Moore et al., 1999; Kadi et al., 2000). The induction reported in humans (Sheffield-Moore et al., 1999) suggests that suprapharmacologic concentrations might be effective because they increase the population of androgen receptors upon which they can act. These findings suggest at least one potential mechanism by which high doses could elicit different effects than physiologic doses.

In summary, two aspects of androgen receptor expression can influence the magnitude of anabolic effects: variations from muscle bed to muscle bed in androgen receptor expression and induction of androgen receptor expression after treatment with AAS. These are shown schematically in Figure 4.

Recent insights into the organization of the steroid hormone receptor:DNA complex suggest an alternative explanation for the varying anabolic:androgenic ratio of AAS. Steroid hormone receptors form a "tripartite" complex between ligand, receptor, and effector that can have varying actions (Katzenellenbogen et al., 1996). When steroid hormones or their analogues bind to their receptor, they form a complex that binds to DNA. However, the receptor:DNA complex also binds a group of adaptor proteins that influence the transcriptional consequences of receptor binding to DNA. These proteins function as coactivators or co-repressors to enhance or prevent activation of transcription by the receptor (Torchia et al., 1998). Each drug that binds steroid hormone receptor induces a particular "shape" in the drug:receptor complex that permits a unique pattern of adapter protein association. The adapter proteins that associate influence the consequence of drug:receptor binding on transcription (Darimont et al., 1998). Selectivity can derive from the drug, the receptor, or the pattern of adaptor protein expression. This model has been exploited successfully in the development of tissue-selective estrogenic compounds. Different estrogenic compounds have specific actions, depending upon the coactivator and/or co-repressor environment (see McDonnell et al., this volume).

A recent commentary (Negro-Vilar, 1999) suggests that a similar approach could be considered for androgens. Up to six different coactivators that are relatively specific for the androgen receptor have been described in the literature (Yeh and Chang, 1996; Fujimoto et al., 1999; Kang et al., 1999; Muller et al., 


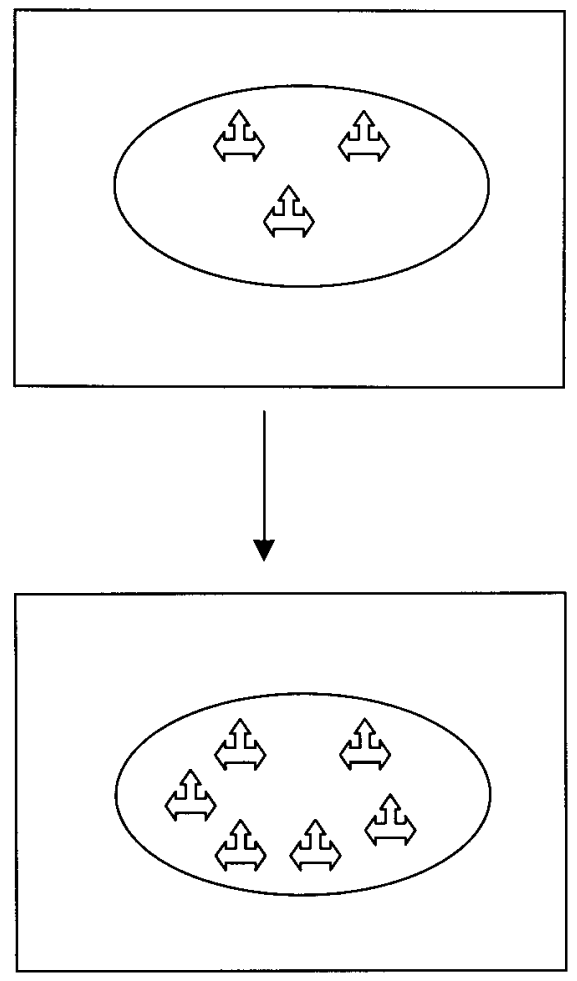

FIG. 4. Model cell containing nucleus with few (top) or more (bottom) androgen receptors. Top represents head and neck muscle relative to leg or normal individual compared to AAS-treated individual.

2000). Although tissue distribution is incompletely described, all are expressed in testis and prostate with widely varying levels of expression in other androgen target tissues. One, FHL2, is expressed highly in heart, slightly in prostate, but not elsewhere (Muller et al., 2000). This coactivator is the first described that is expressed more highly in a nonreproductive tissue than in reproductive tissues. Its existence suggests that tissue-specific distribution of coactivators could theoretically contribute to the ability of different AAS agonists to vary in their ratio of actions in different tissues due to the different tissue distribution of coactivators or co-repressors. Information from a different source supports the possibility that different agonists do induce different conformations of the drug:receptor complex. An NH2-terminal and carboxyl-terminal interaction of the androgen receptor occurs in the presence of agonist binding (Langley et al., 1995). In a co-transfection system, this interaction parallels agonist activity to a degree but weak agonists like medroxyprogesterone possess agonist activity in 
the absence of this interaction (Kemppainen et al., 1999). However, the study of androgen receptor interactions of this type is in its infancy. It suggests that nonselective steroids like testosterone might occupy the androgen receptor in a way that produces a receptor conformation that permits binding of both general and tissue-selective co-activators (Figure 5). Model drugs with selective actions would results in a ligand:receptor conformation that permitted association only of one set of tissue-selective ligands (Figure 6).

\section{ANTICATABOLIC EFFECTS OF ANDROGENS}

There is also evidence to support a role for anticatabolic mechanisms in the anabolic effects of suprapharmacologic AAS regimens. A recent case report of two patients with a point mutation in the androgen receptor that rendered it inactive showed that a suprapharmacologic steroid regimen was anabolic in both individuals (Tincello et al., 1997). Evidence from animal models also supports this possibility. These begin with binding studies that show that androgens can bind, albeit at low affinity, to glucocorticoid receptors (Danheive and Rousseau, 1986,1988). Such low-affinity binding would not be effective, unless extremely

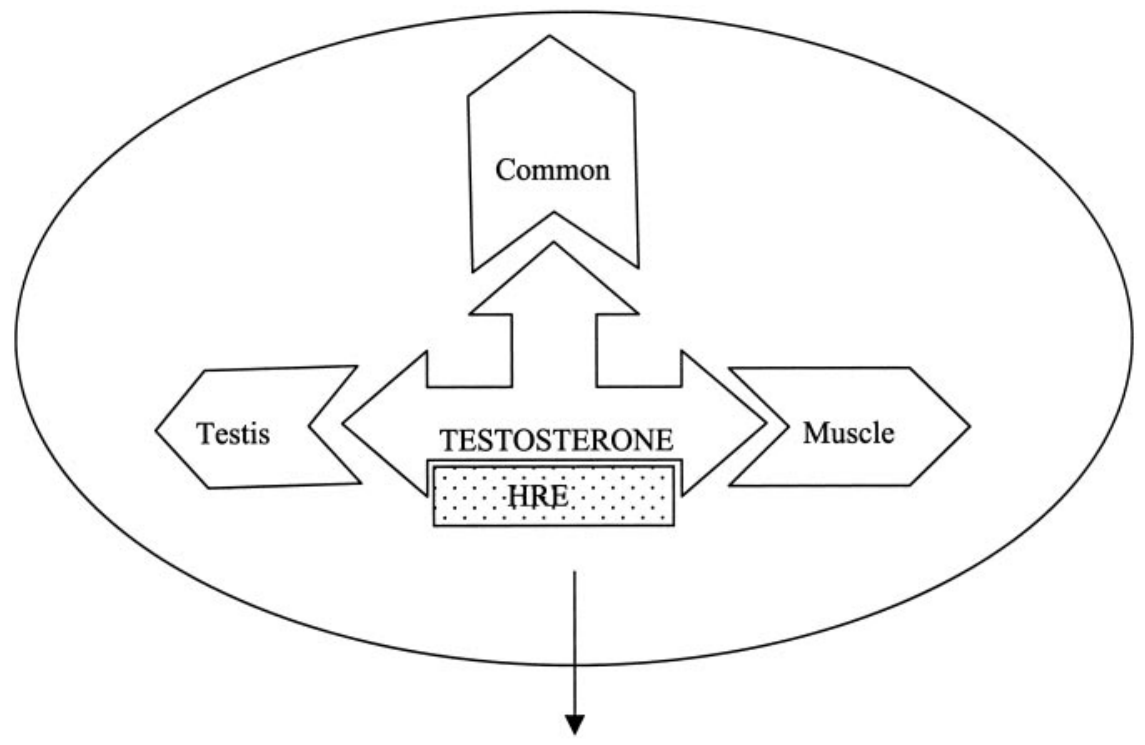

Androgen Regulated Proteins

FIG. 5. Model of testosterone activation of androgen receptor (showing just one of the two ligand:receptor dimers that bind to DNA and activate transcription). HRE $=$ hormone response element. 

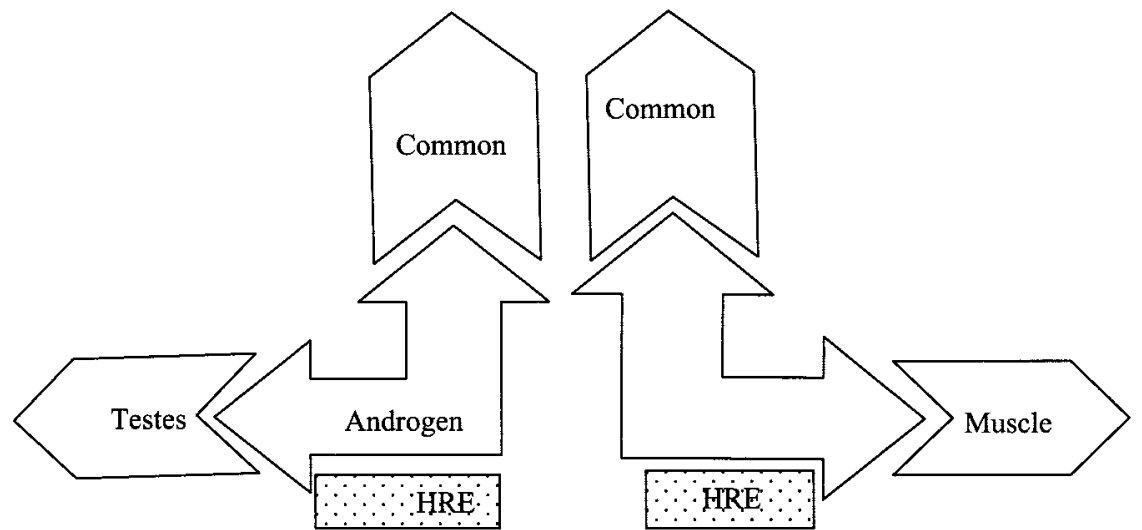

FIG. 6. Hypothetical ligand-occupied androgen receptor conformations that would allow an agonist to recruit coactivators in a tissue-specific way.

high doses of AAS were present. More directly, testosterone can block glucocorticoid-mediated induction of tyrosine amino transferase in liver just like the glucocorticoid antagonist RU486 (Danhaive and Rousseau 1986,1988). However, androgen blockade of glucocorticoid-induced muscle wasting is not observed consistently (see review by Hickson et al., 1990). Furthermore, in healthy young men as well as in burn patients, the anabolic steroid oxandrolone has been shown to increase net protein synthesis without slowing protein degradation (Sheffield-Moore et al., 1999; Hart et al., 2001). Therefore, the specific contribution of glucocorticoid antagonism in AAS-induced anabolic effects has not been demonstrated unequivocally.

\section{COMPLEMENTARY EFFECTS ON GROWTH HORMONE SECRETION AND INSULIN-LIKE GROWTH FACTOR-1 PRODUCTION}

The growth hormone (GH)-insulin-like growth factor-1 (IGF-1) axis is thought to contribute to the anabolic effects of testosterone, both through androgen-induced stimulation of GH secretion and direct stimulation of hepatic production of IGF-1 (Rosenfeld et al., 1994; Veldhuis and Iranmanesh, 1996). IGF-1 can stimulate skeletal muscle formation (Florini et al., 1991). Increases in IGF mRNA have been reported in rats following nandrolone administration and increases in both IGF mRNA and circulating IGF-1 occur in men after testosterone treatment (Urban et al., 1995; Gayan-Ramirez et al., 2000) and decreases in mRNA occur when gonadal function is suppressed (Mauras et al., 1998). However, none of these studies measured IGF-1 directly or established the relationship between IGF-1 and anabolic effects of the drugs. 


\section{E. COMPLEMENTARY EFFECTS OF TRAINING AND AAS}

One of the challenges involved in understanding the effects of AAS in normal athletes is that many of the endpoints (e.g., muscle size, strength) are enhanced by training. Most studies of AAS action involve administration of AAS to sedentary animals, which presents a clear picture of what isolated AAS administration can achieve. Furthermore, comparing the benefits of beginning an exercise regimen and/or AAS in an unfit person provides a model for potential treatment of patient populations such as patients with HIV or the elderly. It does not replicate the environment in which AAS are most often used, which is in a highly trained athlete who is adding AAS to a rigorous exercise regimen. Clearly, exercise increases muscle mass on its own. The prospective, placebo-controlled testosterone trial in eugonadal men by Bhasin and colleagues (1996) that compared placebo, testosterone, exercise, or exercise plus testosterone showed clearly that effects of testosterone and resistance exercise were additive. Another recent study suggests a possible mechanism by which AAS use and exercise might complement each other. Resistance exercise itself increases androgen receptor mRNA and/or binding in both rodent and human muscle (Deschenes et al., 1994; Bamman et al., 2001). If androgen receptor number is induced in muscle by exercise, then more binding sites become available.

\section{F. ROLE OF THE CENTRAL NERVOUS SYSTEM IN AAS EFFECTS ON STRENGTH}

An increased sense of energy and wellbeing is one of the earliest and most frequently documented effects in hypogonadal men. It has been suggested that effects within the central nervous system (CNS) contribute to AAS effects on strength because AAS users feel more energetic and therefore train harder. Case reports, cross-sectional studies, and prospective, longitudinal studies show that AAS use by athletes can be accompanied by increased feelings of energy, aggressiveness, and elevated mood (Bahrke and Yesalis, 1996; Rubinow and Schmidt, 1996; Pope et al., 2000). Effects of AAS typically focus on negative reports of psychotic symptoms and criminal aggressive behavior (Pope et al., 1988,2000; Uzych, 1992; Porcerelli and Sandler, 1998). However, two studies of high-dose androgen administration to normal volunteers reported increases in euphoria, energy, and sexual arousal, as well as several negative mood characteristics, including irritability, mood swings, violent feelings, and hostility (Hannan et al., 1991; Su et al., 1993). However, administration of supraphysiologic levels of testosterone did not change aggression as assessed with the Multi-Dimensional Anger Inventory in normal, eugonadal men (Tricker et al., 1996). These few laboratory studies do not provide definitive answers to this question because they utilize controlled dosing of testosterone (although in the supraphysiologic range), employ a variety of methods for measuring aggression/ 
hostility, and use as subjects eugonadal men in laboratory settings rather than athletes in highly competitive settings who are self-administering even-higher doses of steroids. However, the frequency of case reports of extreme behaviors and positive findings in controlled studies suggest that AAS might influence strength through effects on behavior.

The mechanism by which the psychological effects of androgens occur is unknown. A study of cerebrospinal fluid (CSF) monoamine levels in a controlled study of high-dose methyltestosterone administration reported that levels of the serotonin metabolite 5HIAA were higher and levels of the norepinephrine metabolite MHPG were lower after methyltestosterone treatment. 5HIAA levels correlated negatively in subjects who experienced more negative mood symptoms (e.g., irritability, hostility) and higher 5HIAA in subjects who experienced increased mood symptoms such as euphoria (Daly et al., 2001). The latter findings are consistent with a broad literature supporting an association between low serotonin and aggression/irritability/hostility (Lucki, 1998; Oquendo and Mann, 2000). Another study showed increases in aggression that correlated with changes in CSF dopamine metabolites (Hannan et al., 1991).

Testosterone influences brain function by three mechanisms. It contributes to the differentiation of brain areas that regulate regulation of reproductive hormone secretion, sexual behavior, as well nonreproductive behaviors, including aggression (reviewed by Rubinow and Schmidt, 1996). While these organizational effects establish the anatomical basis for sex-specific behavior patterns, they do not contribute to the acute effects of AAS. Androgens also influence many neural functions through both classical genomic effects and rapid membrane effects. Androgen receptors are distributed (Simerly et al., 1990; Pelletier, 2000) and likely have similar distributions in humans. AAS administration - at least in animal models - can increase androgen receptor number in some brain areas, just as it does in muscle (Lynch and Story, 2000). Effects on many neurotransmitterspecific proteins, including serotonin receptors, choline acetyltransferase, the rate-limiting synthetic enzyme for acetylcholine, and monoamine oxidase have been described (see review in Rubinow and Schmidt, 1996). These likely reflect changes in transcriptional activity but effects of suprapharmacologic doses are virtually unexplored.

Rapid membrane effects also may contribute to behavioral effects of AAS. Suprapharmacologic doses of AAS influence GABA receptor function acutely, over a timeframe that likely reflects rapid membrane rather than genomic effects. In some brain areas and model systems, AAS decrease GABA receptor function, while in others it increases it (Masonis and McCarthy, 1996; Jorge-Rivera et al., 2000). Rapid changes in GABA function theoretically could contribute to disinhibition of behavior and changes in arousal like those reported in AAS users. A single recent report (Schlussman et al., 2000) showed nandrolone caused 
increases in corticotropin (ACTH) and corticosterone secretion acutely as well as protracted effects that were the reverse 24 hours later. This finding indicates that AAS influences at least one neuronal system related to stress and arousal exhibits through what may be both rapid and genomic effects.

Unfortunately, the question remains: do the behavioral effects of AAS influence training intensity and, therefore, muscle strength? Furthermore, although speculations abound that AAS improve neuromuscular function, this hypothesis has not been tested either.

The issue of AAS "dependence" reflects another widely publicized notion based on a small amount of data. Although not directly related to anabolic effects of AAS, a brief discussion is provided because this issue features prominently in discussion of AAS effects on behavior. Several studies report incidence of steroid "dependence" as reflected by psychological symptoms, including depressed mood, fatigue, anorexia, insomnia, restlessness, muscle and joint pain, depression, and desire to take more AAS when athletes stop using (Uzych, 1992; Bahrke and Yesalis, 1996). These reports - and the public perception that AAS use represented a public health crisis - led to the labeling of AAS as "addictive" drugs that were then scheduled by the Drug Enforcement Agency.

There are no clear data supporting the "addictiveness" of AAS use. This may reflect a lack of information or the fact that these drugs are not "addictive" in a neurobiologic sense. "Addictive drugs" must 1) be self-administered by humans and animals, 2) produce positive subjective effects, and 3) produce tolerance and dependence, manifested as a withdrawal syndrome when use stops. Other addictive drugs elicit positive subjective effects by activating the "reward" system in the brain, adaptation of which is thought to produce the gradual dysregulation of drug use (Wise, 1998). In a classical sense, anabolic steroids do not activate the reward system. They are not self-administered by animals and people cannot discriminate an injection of testosterone from placebo (see review in Lukas, 1996). It is impossible to conduct double-blind, placebo-controlled studies of long-term testosterone treatment on mood because users can usually recognize the active drug from the side effects. However, few AAS users fulfill psychiatric criteria for drug dependence (Lukas, 1996).

Nevertheless, some AAS users report positive feelings when they are taking drug and changes in mood when they stop (Su et al., 1993; Lukas 1996). How does one reconcile the clinical reports with the laboratory studies? Genomic effects of AAS are delayed rather than immediate, so they would not be detected in any of the standard models of drug taking. It is possible that AAS do affect reward systems in the brain but in a delayed manner, as would be expected from a gonadal steroid, and so these effects have not been detected. Occasional reports of AAS effects on aspects of dopaminergic transmission, including upregulation of D1 receptors and increases in dopamine turnover, suggest that further explo- 
ration of this possibility is warranted (Thiblin et al., 1999; Kindlundh et al., 2001).

\section{Other Consequences of Suprapharmacologic AAS Regimens}

Androgen receptors are distributed throughout the body. Androgens affect behavior, cardiovascular function, reproduction, and other endocrine functions. Since anabolic actions are not easily dissociated pharmacologically from the other actions of testosterone derivatives, anabolic steroid use by athletes and patients inevitably is accompanied by unwanted side effects that result from the many actions of androgens in the body. During a typical high-dose paradigm, additional AAS effects occur, including 1) feedback inhibition of reproductive function, including decreased production of testosterone and sperm; 2) acne, due to stimulation of sebaceous glands in the skin; and 3) male-pattern hair distribution (Wilson, 1988). In addition, multiple effects on the cardiovascular system occur, including increased blood pressure, change in the ratio of blood lipids (decrease in HDL:LDL ratio), increased blood clotting, increased production of red blood cells, and left ventricular hypertrophy and subsequent decreased left ventricular function (Sullivan et al., 1998). Extended discussion of potential mechanisms for these effects is beyond the scope of this review. However, the regular occurrence of these additional effects contradicts the common argument that AAS cannot be anabolic because androgen receptors are completely saturated at physiologic levels of androgen. The impact of these other systemic effects on the anabolic effects of AAS is unknown. Although increased production of red blood cells should theoretically improve oxygen-carrying capacity of the blood, and so the ability to do sustained work, these effects have not been documented in eugonadal men.

One final note about the use of AAS for their anabolic properties: when used in women, they produce a consistent pattern of virilizing side effects that are predictable, severe, and, in some cases, irreversible. The first published study (Strauss et al., 1985) reported physical changes in the majority of a small group of AAS-using female athletes, including deepening of the voice, clitoral hypertrophy, menstrual irregularities, decreased body fat, and increased facial hair. Behavioral changes included increased libido, aggressiveness, and appetite. About half reported additional changes, including acne, breast size, and body hair distribution. A more recent study (Malarkey et al., 1991) reported a 39\% fall in HDL lipoprotein. All of these effects were reported more recently (Gruber and Pope, 2000) in a study involving a larger group. Some of these effects (e.g., deepening of the voice, clitoral hypertrophy) represent irreversible virilization, while others (e.g., reproductive effects, acne, blood lipids) are reversible. The consistency of these findings argues strongly that clinical trials for AAS use for anabolic purposes, as in burn patients, be conducted with great caution because there is no clinically available AAS that lacks androgenizing effects in women. 


\section{Conclusions}

Studies in AAS-using human subjects as well as experimental model systems have refuted the decades-old assertion that suprapharmacologic dose regimens of AAS are not anabolic in normal men or are only anabolic due to the impact of their CNS effects on motivation to train. The physiopathology of suprapharmacologic doses of AAS is clearly demonstrated and predicted by the beneficial effects on the same systems when AAS are used in hypogonadal men. However, there has been surprisingly little work on the mechanism by which these suprapharmacologic doses exert their actions or on pharmacologic strategies to distinguish beneficial (anabolic) effects from pathologic side effects on brain and heart. The recent demonstration of clinical benefits of suprapharmacologic regimens (Bhasin et al., 1996,1997,1999,2001) suggests that such developments could be clinically beneficial. A recent review proposed the potential value of exploring the possible tissue specificity of protein regulators of androgen receptor function, comparable to those which have been exploited so successfully in the development of selective estrogen receptor modulators (Negro-Vilar, 1999).

\section{REFERENCES}

Amory JK, Bremner WJ 2000 Newer agents for hormonal contraception in the male. Trends Endocrinol Metab 11:61-66

Bahrke MS, Yesalis CE 1996 Psychological and behavioral effects of endogenous testosterone and anabolic-androgenic steroids. Sports Med 22:367-390

Bamman MM, Shipp JR, Jiang J, Gower BA, Hunter GR, Goodman A, McLafferty CL, Urban RJ 2001 Mechanical load increases muscle IGF-1 and androgen receptor mRNA concentrations in humans. Am J Physiol 280:E383-E390

Bates PCL, Chew LF, Millward DJ 1987 Effects of the anabolic steroid stanozol on growth and protein metabolism in the rat. J Endocrinol 114:373-381

Bhasin SW, Storer TW, Berman N, Callegari C, Clevenger B, Phillips J, Bunnell TJ, Rricker R, Shirazi A, Casaburi R 1996 The effects of supraphysiologic doses of testosterone on muscle size and strength in normal men. N Engl J Med 335:1-7

Bhasin S, Storer TW, Berman N, Yarashski K, Cleveneger B, Casaburi R 1997 A replacement dose of testosterone increases fat-free mass and muscle size in hypogonadal men. J Clin Endocrinol Metab 82:407-413

Bhasin S, Storer TW, Javanbakht M, Berman N, Yarasheski KE, Phillips J, Dike M, SinhaHikim I, Shen R, Hays RD, Beall G 1999 Testosterone replacement and resistance exercise in HIV-infected men with weight loss and low testosterone levels. J Am Med Assn 283:763-770

Bhasin S, Woodhouse L, Casaburi R, Singh AB, Bhasin D, Berman N, Yarasheski KE, Magliano L, Dzekov C, Dzekov J, Bross R, Phillips J, Sinha-Hikim I, Sher R, Storer TW 2002 Testosterone dose response relationship in healthy young men. Am J Physiol, in press

Bricout V, Germain P, Serrurier B, Guezennec C 1994 Changes in testosterone muscle receptors: effects of an androgen treatment on physically-trained rat. Cell Mol Biol 40:291-294

Broeder CE, Quindry J, Brittingham K, Panton L, Thomson J, Appakondu S, Breul K, Byrd R, Douglas J, Earnest C, Mitchell C, Olson M, Roy T, Yarlagadda C 2000 The Andro 
project: physiological and hormonal influences of androstenedione supplementation in men 35 to 65 years old participating in a high-intensity resistance training program. Arch Intern Med 160:3093-3104

Bross R, Javanbakht M, Bhasin S 1999 Anabolic interventions for aging-associated sarcopenia. J Clin Endocrinol Metab 84:3420-3430

Daly RC, Su TP, Schmidt PJ, Pickar D, Murphy DL, Rubinow DR 2001 Cerebrospinal fluid and behavioral changes after methyltestosterone administration: preliminary findings. Arch Gen Psych 58:172-177

Danhaive PA, Rousseau GG 1986 Binding of glucocorticoid antagonists to androgen and glucocorticoid hormone receptors in rat skeletal muscle. J Steroid Biochem 24:481-487

Danhaive PA, Rousseau GG 1988 Evidence for sex-dependent anabolic response to androgenic steroids mediated by muscle glucocorticoid receptors in the rat. J Steroid Biochem 29:575581

Darimont BD, Wagner RL, Apriletti JW, Stallcup MR, Kushner PJ, Baxter JD, Fletterick RJ, Yamamoto KR 1998 Structure and specificity of nuclear receptor-coactivator interactions. Genes Dev 12:3343-3356

Deschenes M, Maresh C, Armstrong L, Covault J, Kraemer W, Crivello J 1994 Endurance and resistance exercise induce muscle fiber type specific responses in androgen binding capacity. J Ster Biochem Mol Biol 50:175-179

Doumit ME, Cook DR, Merkel RA 1996 Testosterone up-regulates androgen receptors and decreases differentiation of porcine myogenic satellite cells in vitro. Endocrinology 137: 1385-1394

Elashoff JD, Jacknow AD, Shain SG, Braunstein GD 1991 Effects of metabolic-androgenic steroids on muscular strength. Ann Intern Med 115:387-393

Exner GU, Staudte HW, Pette D 1973 Isometric training of rats: effects on fast and slow muscle and modification by an anabolic hormone (nandrolone decanoate). II. Male rats. Pfluger's Arch 345:15-22

Ferrando AA, Sheffield-Moore M, Wolf SE, Herndon DN, Wolfe RR 2000 Tetosterone normalization in severe burns ameliorates muscle catabolism. FASEB J 14(4):A797

Florini JR, Ewton DZ, Roof SL 1991 IGF-1 stimulates terminal myogenic differentiation by induction of myogenin gene expression. Mol Endocrinol 5:718-724

Forbes GB, Porta CR, Herr BE, Griggs MRC 1992 Sequence of changes in body composition induced by testosterone and reversal of changes after drug is stopped. $\mathrm{J}$ Am Med Assn 267:397-399

Franke WW, Berendonk B 1997 Hormonal doping and androgenization of athletes: a secret program of the German Democratic Republic government. Clin Chem 43:1262-1279

Friedl KE 2000 Effect of anabolic steroid use on body composition and physical performance. In: Yesalis CE, ed. Anabolic Steroids in Sport and Exercise. Champaign, IL: Human Kinetics; $139-174$

Fujimoto N, Yeh S, Kang HY, Inui S, Chang HC, Mizokami A, Chang C 1999 Cloning and charadcterization of androgen receptor co-activator, RA55, in human prostate. J Biol Chem 274:8316-8321

Gayan-Ramirez G, Rollier H, Vanderhoydonc F, Verhoeven G, Gosselink R, Decramer M 2000 Nandrolone decanoate does not enhance training effects but increases IGF-1 mRNA in rat diaphragm. J Appl Physiol 88:26-34

Griggs RC, Kingston W, Jozefowicz RF, Herr BE, Forbest G, Halliday D 1989 Effect of testosterone on muscle mass and muscle protein synthesis. J Appl Physiol 66:498-503

Gruber AJ, Pope HG 2000 Psychiatric and medical effects of anabolic-androgenic steroid use in women. Psychother Psychosom 69:19-26 
Hannan CJ, Friedl KE, Zold A, Kettler TM, Plymate SR 1991 Psychological and serum homovanillic acid changes in men administered androgenic steroids. Psychoneuroendocrinology $16: 335-343$

Hart DW, Wolf SE, Ramzy PI, Chinkes DL, Beauford RB, Ferrando AA, Wolfe RR, Herndon DN 2001 Anabolic effects of oxandrolone after severe burn. Ann Surg 233:556-564

Hickson RC, Czerwinski SM, Falduto MT, Young AP 1990 Glucocorticoid antagonism by exercise and androgenic-anabolic steroids. Med Sci Sports Med 22:331-340

Inoue K, Yamasaki S, Fushiki T, Okada Y, Sugimoto E 1994 Androgen receptor antagonist suppresses exercise-induced hypertrophy of skeletal muscle. Eur J Appl Physiol 69:88-91

Jorge-Rivera JC, McIntyre KL, Henderson LP 2000 Anabolic steroids induce region- and subunit-specific rapid modulation of GABA-A receptor mediated currents in the rat forebrain. J Neurophysiol 83:3299-3309

Joubert Y, Tobin C 1989 Satellite cell proliferation and increase in the number of myonuclei induced by testosterone in the levator ani muscle of the adult female rat. Dev Biol 131:550-557

Joumaa WH, Leoty C 2001 Differential effects of nandrolone decanoate in fast and slow rat skeletal muscle. Med Sci Sports Exerc 33:397-403

Kadi F, Eriksson A, Holmner S, Butler-Browne G, Thornell LE 1999a Cellular adaptation of the trapezius muscle in strength trained athletes. Histochem Cell Biol 111:189-195

Kadi F, Eriksson A, Holmner S, Thornell LE 1999b Effects of anabolic steroids on the muscle cells of strength-trained athletes. Med Sci Sports Exerc 31:1528-1534

Kadi F, Bonnerud P, Eriksson A, Thornell LE 2000 The expression of androgen receptors in human neck and limb muscles: effects of training and self-administration of androgenicanabolic steroids. Histochem Cell Biol 113:25-29

Kang HY, Yeh S, Fujimoto N, Chang C 1999 Cloning and characterization of human prostate coactivator ARA54, a novel protein that associates with the androgen receptor. J Biol Chem 274:8570-8576

Katzenellenbogen JA, O’Malley BW, Katzenellenbogen BS 1996 Tripartite steroid hormone receptor pharmacology: interaction with multiple effector sites as a basis for the cell- and promoter-specific action of these hormones. Mol Endocrinol 10:119-131

Kemppainen JA, Langley E, Wong C, Bobseine K, Kelce WR, Wilson EM 1999 Distinguishing androgen receptor agonists and antagonists: distinct mechanisms of activation by medroxyprogesterone acetate and dihydrotestosterone. Mol Endocrinol 13:440-454

Kimura N, Mizokami MA, Oonuma T, Sasano H, Nagura H 1993 Immunocytochemical localization of androgen receptor with polyclonal antibody in paraffin-embedded human tissues. J Histochem Cytochem 41:671-678

Kindlundh AM, Lindblom J, Bergstromg L, Wikberg JE, Nyberg F 2001 The anabolicandrogenic steroid nandrolone decanoate affects the density of dopamine receptors in the male rat brain. Eur J Neurosci 13:291-296

King DS, Sharp RL, Vukovich MD 1999 Effect of oral androstenedione on serum testosterone and adaptations to resistance training in young men: a randomized controlled trial. J Am Med Assn 281:2020-2028

Kochakian CD 1976 Anabolic-Androgenic Steroids. New York: Springer-Verlag

Kopera H 1985 The history of anabolic steroids and a review of clinical experience with anabolic steroids. Acta Endocrinol (suppl)271:11-18

Lamb DJ, Weigel NL, Marcelli M 2001 Androgen receptors and their biology. Vitam Horm 62:199-230

Langley E, Zhou ZX, Wilson EM 1995 Evidence for an anti-parallel orientation of the ligandactivated human androgen receptor dimmer. J Biol Chem 270:29983-29990 
Leder BZ, Longcope C, Catlin DH, Ahrens B, Shoenfeld DA, Finkelstein JS 2000 Oral androstenedione administration and serum testosterone concentrations in young men. Clin Invest J Am Med Assn 283:779-782

Lewis MI, Fournier M, Yeh AY, Micevych PE, Sieck PM 1999 Alterations in diaphragm contractility after nandrolone administration: an analysis of potential mechanisms. J Appl Physiol 86:985-992

Lubek BM 1984 Contractile responses of rat lateral gastrocnemius and soleus to dianabol (17 beta-hydroxy-17-methyl-1,4androstadien-3-one) and exercise. Steroids 44:485-495

Lucki I 1998 The spectrum of behaviors influenced by serotonin. Biol Psychol 44:151-162

Lukas SE 1996 CNS effects and abuse liability of anabolic-androgenic steroids. Annu Rev Pharmacol Toxicol 36:333-357

Lynch CS, Story AJ 2000 Dihydrotestosterone and estrogen regulation of rat brain androgen receptor immunoreactivity. Physiol Behav 69:445-453

Malarkey WB, Strauss RH, Leizman DJ, Liggett M, Demers LM 1991 Endocrine effects in female weight lifters who self-administer testosterone and anabolic steroids. Am J Obstet Gynecol 165:1385-1390

Masonis AE, McCarthy MP 1996 Effects of the androgenic/anabolic steroid stanozol on GABA A receptor function: GABA-stimulated ${ }^{36} \mathrm{Cl}(-)$ influx and $\left[{ }^{35} \mathrm{~S}\right]$ TBPS binding. J Pharmacol Exp Ther 279:186-193

Mauras N, Hayes V, Welch S, Rini A, Helgeson K, Dokler M, Veldhuis JD, Urban RJ 1998 Testosterone deficiency in young men: marked alterations in whole body protein kinetics, strength, and adiposity. J Clin Endocrinol Metab 83:1886-1892

McDonnell DP, Connor CE, Wijayaratne A, Chang CY, Norris JD 2002 Definition of the molecular and cellular mechanisms underlying the tissue selective agonist/antagonist activities of selective estrogen receptor modulators (SERMs). Rec Progr Horm Res 57:295-316

Menschikowski M, Jung K, Junghans P, Petzke KJ, Albrecht V 1988 The influence of a steroid hormone and physical exercise on protein metabolism in rats. Exp Clin Endocrinol 92:341348

Muller JM, Isele U, Metzger E, Rempel A, Moser M, Pschere A, Breyer T, Holubarsch C, Buettner R, Schule R 2000 FHL2, a novel tissue-specific coactivator of the androgen receptor. EMBO J 19:359-369

Negro-Vilar A 1999 Selective androgen receptor modulators (SARMs): a novel approach to androgen therapy for the new millennium. J Clin Endocrinol Metab 84:3459-3462

O'Connor LH, Cicero TJ 1993 Anabolic steroids: misuse or abuse. In Chulkin JS, ed. Hormonally Induced Changes in Mind and Brain. New York: Academic Press; 129-156

Oquendo MA, Mann JJ 2000 The biology of impulsivity and suicidality. Psychiat Clin N Am 23:11-25

Pelletier G 2000 Localization of androgen and estrogen receptors in rat and primate tissues. Histol Histopathol 15:1261-1270

Pope HG, Katz DL 1988 Affective and psychotic symptoms associated with anabolic steroid use. Am J Psychiat 145:487-490

Pope HG, Kouri EM, Hudson JI 2000 Effects of supraphysiological doses of testosterone on mood and aggression in normal men: a randomized controlled trial. Arch Gen Psychol 57:133-140

Porcerelli JF, Sandler BA 1998 Anabolic:androgenic steroid abuse and psychopathology. Psychiat Clin N Am 21:829-832

Rosenfeld RG, Rosenbloom AL, Guevara-Aguirre J 1994 Growth hormone (GH) insensitivity due to primary GH receptor deficiency. Endocr Rev 15:369-390

Rubinow DR, Schmidt PJ 1996 Androgens, brain and behavior. Am J Psychiat 153:974-984 
Saartok T, Dahlberg E, Gustafsson JA 1984 Relative binding affinity of anabolic-androgenic steroids: comparison of the binding to the androgen receptors in skeletal muscle and in prostate, as well as to sex hormone-binding globulin. Endocrinology 114:2100-2106

Sachs BD, Leipheimer RE 1988 Rapid effect of testosterone on striated muscle activity in rats. Neuroendocrinology 48:453-458

Sar M, Lubahn DB, French FS, Wilson EM 1990 Immunohistochemical localization of the androgen receptor in rat and human tissues. Endocrinology 127:3180-3186

Schlussman SD, Zhou Y, Johansson P, Kiuru A, Ho A, Nyberg F, Kreek MJ 2000 Effects of the androgenic anabolic steroid, nandrolone decanoate, on adrenocorticotropin hormone, corticosterone and proopiomelanocortin, corticotropin releasing factor (CRF) and CRF receptor1 mRNA levels in the hypothalamus, pituitary and amygdala of the rat. Neurosci Lett 284:190-194

Sheffield-Moore M 2000 Androgens and the control of skeletal muscle protein synthesis. Ann Med 32:181-186

Sheffield-Moore M, Urban RJ, Wolf SE, Jiang J, Catlin DH, Herndon DN, Wolfe RR, Ferrando AA 1999 Short-term oxandrolone administration stimulates net muscle protein synthesis in young men. J Clin Endocrinol Metab 84:2705-2711

Simerly RB, Chang C, Muaramatsu M, Swanson LW 1990 Distribution of androgen and estrogen receptor mRNA-containing cells in the rat brain: an in situ hybridization study. J Compar Neurol 294:76-95

Strawford A, Barbieri T, Van Loan M, Parks E, Catlin D, Barton N, Neese R, Christiansen M, King J, Hellerstein MK 1999 Resistance exercise and supraphysiologic androgen therapy in eugonadal men with HIV-related weight loss: a randomized controlled trial. J Am Med Assn 281:1282-1290

Strauss RH, Liggett MT, Lanese RR 1985 Anabolic steroid use and perceived effects in ten weight-trained women athletes. J Am Med Assn 253:2871-2873

Su TP, Pagliaro M, Schmidt PJ, Pickar D, Wolkowitz O, Rubinow DR 1993 Neuropsychiatric effects of anabolic steroids in male normal volunteers. J Am Med Assn 269:2760-2764

Sullivan ML, Martinez CM, Gennis P, Gallagher EJ 1998 The cardiac toxicity of anabolic steroids. Progr Cardiovasc Dis 41:1-15

Sundaram K, Kumar N, Monder C, Bardin CW 1995 Different patterns of metabolism determine the relative anabolic activity of 19-norandrogens. J Steroid Biochem Mol Biol 53:253-257

Takeda H, Chodak G, Mutchnik S, Nakamoto T, Chang C 1990 Immunohistochemical localization of androgen receptors with mono- and polyclonal antibodies to androgen receptor. $\mathrm{J}$ Endocrinol 126:17-25

Thiblin I, Finn A, Ross SB, Stenfors C 1999 Increased dopaminergic and 5-hydroxytryptaminergic activities in male rat brain following long-term treatment with anabolic androgenic steroids. Br J Pharmacol 126:1301-1306

Tincello DG, Saunders PTK, Hodgins MB, Simpson NB, Edwards CRW, Hargreave TB, Wu FCW 1997 Correlation of clinical, endocrine and molecular abnormalities with in vivo responses to high-dose testosterone in patients with partial androgen insensitivity syndrome. Clin Endocrinol 46:497-506

Torchia J, Glass C, Rosenfeld MG 1998 Co-activators and co-repressors in the integration of transcriptional responses. Curr Opin Cell Biol 10:373-383

Tricker R, Casaburi R, Storer TW, Clevenger B, Berman N, Shirazi A, Bhasin S 1996 The effects of supraphysiological doses of testosterone on angry behavior in healthy eugonadal men - a clinical research center study. J Clin Endocrinol Metab 81:3754-3758

Urban RJ, Bodenburg C, Gilkison C, Foxworth J, Coggan AR, Wolfe RR, Ferrando A 1995 Testosterone administration to elderly men increases skeletal muscle strength and protein synthesis. Am J Physiol 269:E820-E826 
Uzych L 1992 Anabolic-androgenic steroids and psychiatric-related effects: a review. Can J Psychiat $37: 23-28$

Veldhuis JD, Iranmanesh A 1996 Physiological regulation of the human growth hormone (GH)insulin-like-growth factor type I (IGF-1) axis: predominant impact of age, obesity, gonadal function and sleep. Sleep 19(10 suppl):S221-S224

Wilson JD 1988 Androgen abuse by athletes. Endocr Rev 9:181-191

Wilson JD 1996 Androgens. In Hardman JG, Limbird LE, Molinoff PB, Ruddon RW, Goodman Gilman A, eds. Goodman Gilman's Experimental Basis of Therapeutics. New York: McGraw-Hill; 1441-1457

Wilson JD, Gloyna E 1970 The intranuclear metabolism of testosterone in the accessory organs of male reproduction. Rec Progr Horm Res 26:309-336

Wise RA 1998 Drug activation of brain reward pathways. Drug Alcoh Depend 51:13-22

Yeh S, Chang C 1996 Cloning and characterization of a specific coactivator, ARA70, for the androgen receptor in human prostate cells. Proc Natl Acad Sci USA 93:5517-5521

Yesalis CE, Courson SP, Wright JE 2000 History of anabolic steroid use in sport and exercise. In: Yesalis CE, ed. Anabolic Steroids in Sport and Exercise. Champaign, IL: Human Kinetics; $51-71$ 


\title{
Prolactin Modulation of Immune and Inflammatory Responses
}

\author{
Li-YuAN Yu-LeE \\ Department of Medicine, Department of Molecular and Cellular Biology, Department of \\ Immunology, and Program in Cell and Molecular Biology, Baylor College of Medicine, Houston, \\ Texas 77030
}

\begin{abstract}
Prolactin (PRL), a pituitary peptide hormone, is known to regulate diverse physiological functions via its effects on cellular processes such as proliferation, differentiation, and cell survival. All these activities are mediated by the PRL receptor (PRL-R), a member of the hematopoietin cytokine receptor superfamily. To understand PRL-dependent mitogenic signaling in $\mathrm{T}$ cells, we cloned PRL, PRL-R, one mediator of PRL signaling, signal transducer and activator of transcription (Stat) $5 \mathrm{~b}$, and a panel of PRL-inducible immediate early-response genes from $\mathrm{T}$ cells. We are employing one of these PRL-inducible genes, the transcription factor interferon regulatory factor- 1 (IRF-1), a multifunctional immune regulator gene, as a tool to understand how PRL modulates T-cell proliferative responses. In investigating regulatory events along the PRL-R/Janus activating kinase (JAK)/Stat/IRF-1 signaling pathway, we show that Stat factors can activate as well as inhibit IRF-1 promoter activity and that cross talk between Stat and nuclear factor $(\mathrm{NF}) \kappa \mathrm{B}$ signaling pathways also regulates IRF-1 expression. In understanding how signaling pathways cross talk at the IRF-1 promoter, we obtained insights into how PRL can modulate immune and inflammatory responses. These findings have much broader implications, not only for cells in the immune system but also for other PRL-responsive cells and tissues.
\end{abstract}

\section{Introduction}

Prolactin (PRL) is a 23-kDa polypeptide that is synthesized primarily in the pituitary. PRL is also synthesized and secreted by many extrapituitary tissues (Ben-Jonathan et al., 1996). Whether endocrine or autocrine, PRL exerts profound effects on a wide range of tissues, with over 300 effects described in vertebrates (Bole-Feysot et al., 1998). PRL regulates the differentiation of secretory glands, including the mammary gland, ovary, prostate, submaxillary and lacrimal glands, pancreas, and liver (for a review, see Horseman, 2001). PRL also regulates proliferation in different cell types, including mammary epithelium, pancreatic beta cells, astrocytes, anterior pituitary cells, adipocytes, and T lymphocytes (Yu-Lee et al., 1990; DeVito et al., 1992; Nanbu-Wakao et al., 2000; Horseman, 2001). We and others have cloned PRL from T lymphocytes, 
where it has been shown to promote proliferation, protect against apoptosis, and enhance cell survival (LaVoie and Witorsch, 1995; Buckley, 2001). Hence, PRL is also known as a T-cell cytokine (Yu-Lee et al., 1998; Montgomery, 2001). How pituitary or extrapituitary PRL modulates target cell function likely depends on the cell type and its stage of differentiation.

As one approach to understanding how PRL modulates T-cell proliferative responses, we cloned a panel of 26 PRL-responsive immediate early-response genes from a rat $\mathrm{T}$-lymphoma cell line, $\mathrm{Nb} 2$, induced to proliferate by PRL (Yu-Lee et al., 1990). Nb2 $\mathrm{T}$ cells express a high number of PRL receptor (PRL-R), which is a member of the hematopoietin/cytokine receptor superfamily and is exquisitely sensitive to PRL for growth (Gout et al., 1980). Several PRL-inducible genes have been extensively characterized (Stevens et al., 1995; Morris et al., 1997). We focus here on the transcription factor interferon regulatory factor-1 (IRF-1), which plays a pivotal role in multiple immune functions. In understanding the signaling pathway to the IRF-1 gene, we elucidated not only positive and negative regulation but also how various cytokine signals compete/cross talk at the IRF-1 promoter. We will highlight controversies concerning PRL's role in mediating immune, autoimmune, and inflammatory responses, then summarize the renewed interest in evaluating PRL as a hormone or cytokine involved in maintaining immune system homeostasis (Dorshkind and Horseman, 2001).

\section{Prolactin and Immune, Autoimmune, and Inflammatory Responses}

\section{A. PROLACTIN AND IMMUNE RESPONSES}

A large body of literature dating from the 1930s suggests a role of PRL and other pituitary hormones in modulating the immune system (Smith, 1930; Kooijman et al., 1996). Clinical, animal, and in vitro studies combine to suggest that PRL exhibits immunostimulatory properties (Yu-Lee, 1997). PRL has been shown to stimulate $\mathrm{T}$ cells, B cells, natural killer (NK) cells, macrophages, neutrophils, CD34+ hematopoietic cells, and antigen-presenting dendritic cells (Kooijman et al., 1996; Dogusan et al., 2001; Matera et al., 2001). However, animals with a targeted disruption of either the PRL (Horseman et al., 1997) or PRL-R (Bouchard et al., 1999) gene (knockout, or KO) suggest that PRL is not essential for normal immune system development or function. The KO animals show normal T-cell, B-cell, and NK-cell development and distribution as well as normal T-cell mitogenic responses, B-cell antibody production, and NK-cellmediated cytotoxicity (Bouchard et al., 1999). A normal immune response to Listeria infection involving innate as well as adaptive immune responses is intact in PRL-R KO mice. However, compensatory actions by other cytokines (redundancy) in these $\mathrm{KO}$ mice have not been examined. 
In Snell dwarf mice that are deficient in anterior pituitary hormones, normal immune responses were observed in animals housed separately from their wild-type littermates (Dorshkind and Horseman, 2000). In contrast, immune defects were observed only in those dwarf animals housed together with their normal littermates, which resulted in a highly stressful environment. The variable housing conditions apparently contributed to conflicting data on the effects of PRL and growth hormone (GH) on immune responses in the dwarf mice. PRL and other pituitary hormones are suggested to act as stress-adaptation molecules important in maintaining immune system homeostasis (Dorshkind and Horseman, 2001). Under stressful conditions, PRL is needed to balance the negative effects of glucocorticoids and other immune or inflammatory mediators to maintain steady-state homeostasis. This interpretation is supported by in vitro studies showing PRL's protective effect in preventing glucocorticoid-induced lymphocyte cell death (apoptosis) (LaVoie and Witorsch, 1995; Buckley, 2001) and by in vivo studies showing that PRL improves macrophage and splenocyte functions following trauma-hemorrhage and infections (Zellweger et al., 1996). A concerted effort by many laboratories is underway to evaluate the immunomodulatory activities of PRL in the context of stress, trauma, injury, inflammation, infection, and various autoimmune diseases (Matera et al., 2000; Richards and Murphy, 2000; Dorshkind and Horseman, 2001; Hooghe et al., 2001).

\section{B. PROLACTIN AND AUTOIMMUNE DISEASES}

Many autoimmune diseases are prevalent in women of childbearing age, most notably, systemic lupus erythematosus (SLE), which occurs more frequently in females than males by a 9:1 ratio. This female gender bias suggests that female hormones (e.g., PRL, estrogen (E2)) may play a role in the pathogenesis of this autoimmune disease. Pituitary PRL expression is under E2 regulation (Couse and Korach, 1999). PRL, in turn, regulates E2 receptor (ER) $\alpha$ and $\mathrm{ER} \beta$ expression in the female reproductive tissues and the mammary gland (Tessier et al., 2000). Thus, a positive regulatory loop exists between PRL and E2 action. PRL levels are higher in women than men. Elevated PRL levels have been reported in patients with SLE, multiple sclerosis, rheumatoid arthritis, psoriatic arthritis, AIDS, and prior to transplant rejection (Kanik and Wilder, 2000; Jacobi et al., 2001; Walker, 2001). Bromocriptine (BRC), a dopamine agonist that inhibits PRL release from the pituitary, can suppress autoimmune uveitis and correct T-cell and NK-cell abnormalities in patients with pathological hyperprolactinemia (Vidaller et al., 1992). BRC also suppresses SLE in some patients and reduces the number of lupus flares (Walker, 2001). Although a clear causal relationship is still lacking, these clinical data suggest that altered PRL levels may exacerbate certain autoimmune diseases. 
A better correlation between PRL and immune regulation is observed in animal models, where circulating PRL levels can be altered by hypophysectomy, BRC treatment, or genetic deletions. Hypophysectomized animals are deficient in mounting various B- and T-cell-mediated immune responses, which are restored by PRL injections. High PRL levels are found in rats with experimentally induced adjuvant arthritis or encephalomyelitis and in the NZB/NZW F1 lupus mice (Kooijman et al., 1996; McMurray, 2001). BRC treatment reduced disease symptoms and delayed lupus-related death, which results primarily from glomerulonephritis (immunoglobulin deposits) in the kidney (McMurray, 2001). Recent animal studies suggest that E2-treated transgenic animals develop a lupus-like phenotype with an expansion in autoreactive B cells (a breakdown of tolerance) and elevation in antibody production (Peeva et al., 2000; Grimaldi et al., 2001). This E2 effect requires the presence of PRL as BRC treatment reduced antibody production (Peeva et al., 2000). Interestingly, both E2 and PRL can upregulate Bcl-2 expression in B cells (Morales et al., 1999; Peeva et al., 2000; Buckley, 2001) and may account for the enhanced survival of autoreactive B cells. Together, these clinical and animal studies support a role of E2 and PRL in modulating lymphocyte functions in the context of various autoimmune diseases.

\section{PROLACTIN AND INFLAMMATORY RESPONSES}

PRL and E2 have been shown to be protective against inflammation in the context of severe trauma (Jarrar et al., 2000; Knoferl et al., 2000b). Trauma is the fourth leading cause of death in the United States (Zhu et al., 1997). Gender seems to play a role in the response to trauma. Female patients survive better than male patients in response to severe trauma (Morris et al., 1990), supporting the notion that female hormones may protect against hemorrhage and/or septic complications. In male trauma patients, a greater susceptibility to infections is correlated with a higher serum level of proinflammatory cytokines such as interleukin-6 (IL-6) (Offner et al., 1999; Oberholzer et al., 2000). In animal models, trauma-hemorrhage is associated with depressed immune functions and increased infection, morbidity, and mortality (Zellweger et al., 1996). Under this condition, PRL as well as E2 protects against trauma-hemorrhage by reducing plasma levels of corticosterone and IL-6, enhancing splenocyte proliferation and function, and increasing survival of animals to septic shock (Zellweger et al., 1996; Knoferl et al., 2000a,b). These studies show that both PRL and E2 protect against inflammation and improve dysfunctional immune responses under conditions of severe stress. A reciprocal relationship is also found between high serum corticosterone versus low PRL levels after a burn injury (Thellin et al., 2001). In this model of burn-induced stress, the low level of PRL is correlated with 
a significant increase in IL-6 production by gut enterocytes, which is accompanied by a loss of gut integrity, bacterial translocation into the circulation, and septic complications (Ogle et al., 2000).

PRL and E2 also inhibit IL-6 gene expression in female reproductive tissues (Deb et al., 1999) and bone (Manolagas, 2000). During pregnancy, IL-6 expression in the decidua is inhibited by E2 and PRL, as increases in IL-6 can lead to termination of pregnancy. Both PRL and E2 downregulate the expression of the gp130 component of the IL-6 receptor complex (Kurebayashi et al., 1997; Deb et al., 1999). In bone, IL-6 is produced by the osteoblast to regulate osteoclast differentiation and bone resorption. IL-6 is thought to contribute to bone loss during menopause (Manolagas, 2000). E2 prevents bone loss in part by inhibiting IL-6 expression in osteoblasts and bone marrow stromal cells (Girasole et al., 1992). E2 also antagonizes IL-6 function by blocking IL-6-inducible signal transducer and activator of transcription (Stat) 3 activity (Yamamoto et al., 2000). Both osteoblasts and bone marrow stromal cells express the PRL-R (McAveney et al., 1996; Goffin et al., 1999), which suggests that PRL may inhibit IL-6 expression in cells in bone marrow. These studies show that PRL and E2 can inhibit IL-6 function at multiple levels, including blocking IL-6 and IL-6 receptor gp130 expression as well as antagonizing IL-6 signaling potential.

Paradoxically, PRL and E2 contribute to hyperplasia and inflammation in the prostate (Tangbanluekal and Robinette, 1993; Leav et al., 1999; van Coppenolle et al., 2001). Transgenic mice overexpressing PRL develop enlarged prostates (Wennbo et al., 1997). Exposure of rats to PRL and E2 results in prostate inflammation, which is characterized by infiltration of lymphocytes and macrophages into the stromal compartment and of neutrophils into the lumen of the dorsolateral lobe of the prostate gland (Tangbanluekal and Robinette, 1993; Stoker et al., 1999; van Coppenolle et al., 2001). The rat dorsolateral prostate is structurally and functionally most similar to the human prostate. PRL appears to be a survival factor (Ahonen et al., 1999) and induces Bcl-2 expression in prostate epithelial cells (van Coppenolle et al., 2001). Interestingly, E2 upregulates IL-6 gene expression (Harris et al., 2000) and IL-6, in turn, induces androgen receptor (AR) gene expression as well as AR function in prostate epithelial cells (Lin et al., 2001). AR is required for PRL expression in the prostate epithelium in vivo (Nevalainen et al., 1997). Thus, a complex positive regulatory loop exists among the hormones, cytokines, and their receptors within the prostate. How these interactions promote prostate inflammation, hyperplasia, and cancer progression remains to be elucidated. Although the mechanisms involved are not known, PRL and E2 can be anti-inflammatory or proinflammatory, depending on the cell type, the tissue, and the physiological state of the organ. 


\section{PROLACTIN AND HEMATOPOIESIS}

PRL and GH play a role in stimulating the hematopoietic system (Bellone et al., 1995; Richards and Murphy, 2000). PRL enhances granulocyte/macrophagecolony stimulating factor (GM-CSF)-mediated maturation of CD34+ human hematopoietic progenitor cells into erythroid precursors in culture (Bellone et al., 1995). Pharmacologic levels of PRL increase the hematopoietic progenitors of the myeloid (colony-forming unit-granulocyte macrophage, or CFU-GM) and erythroid (blast-forming unit-erythocyte, or BFU-E) lineages in the bone marrow and spleen, during myelosuppression following treatment for HIV infection or bone marrow transplantation (Richards and Murphy, 2000). PRL also increases the number of progenitors of other immune cell lineages, including $\mathrm{T}$ cells, B cells, and NK cells (Bellone et al., 1995). In various diseases, PRL antagonizes the immunosuppressive effects of transforming growth factor-beta (TGF- $\beta$ ) (Richards et al., 1998), tumor necrosis factor alpha (TNF $\alpha$ ) (Luo and Yu-Lee, 2000), or corticosterone (LaVoie and Witorsch, 1995; Buckley, 2001) and thus may enhance recovery of the hematopoietic system. In the pregnant maternal uterus, several PRL-like proteins (PLP) interact with immune function cells. The trophoblast-derived PLP-A binds to and inhibits maternal NK cells to ensure successful fetal development (Muller et al., 1999). The placental-derived PLP-E binds to megakaryocytes and promotes their differentiation and maturation, in preparation for accelerated platelet production during pregnancy (Lin and Linzer, 1999). Thus, placental PRL-like hormones play novel roles in regulating hematopoiesis during pregnancy.

In the following section, we will consider some of the mechanisms by which PRL mediates such diverse biological responses. Our studies on PRL signaling to the master immune regulator gene IRF-1 provide some insight into how PRL activates or inhibits gene transcription. These analyses may help to elucidate how PRL can be anti-inflammatory in one tissue but proinflammatory in another or how PRL can exacerbate autoimmune diseases.

\section{Prolactin Receptor Signaling}

\section{A. PRL-R STRUCTURE AND FUNCTION}

The diverse activities of PRL are mediated by the PRL-R, which is expressed on many cell types. Several receptor forms exist, including the long (85-90 kDa) and short (42 kDa) PRL-R, which result from differential splicing of $3^{\prime}$ end cytoplasmic domain exons from a single gene (Goffin and Kelly, 1997). A naturally occurring intermediate PRL-R form $(65 \mathrm{kDa})$ is found in rat $\mathrm{Nb} 2 \mathrm{~T}$ lymphoma cells and results from an in-frame truncation in the cytoplasmic domain. Several intermediate PRL-R forms with varying 
cytoplasmic domains have been reported in human mammary as well as prostate tumors but their functional significance remains unclear (Clevenger et al., 1995). The intermediate Nb2 PRL-R is more potent than the long PRL-R in both mitogenic (Yu-Lee et al., 1998) and lactogenic (Goffin et al., 1999) signaling. The short PRL-R is suggested to modulate the activity of the long or $\mathrm{Nb} 2 \mathrm{PRL}-\mathrm{R}$ by engaging them in heterodimer complex formation, thereby modulating their signaling capacity (O'Neal and Yu-Lee, 1994; Goffin et al., 1999). Whether the short PRL-R has independent functions is unclear. In Nb2 T cells, where PRL-R is abundant (12,000 PRL-R/cell), only $30 \%$ occupancy of surface PRL-R is needed to elicit maximal proliferative response (Gertler, 1997).

\section{Receptor Motifs}

Several motifs in the PRL-R intracellular domain are important for signal transduction. A proline-rich motif (I-F-P-P-V-P-X-P) proximal to the transmembrane domain is critical for interacting with the protein tyrosine kinase (PTK) Janus activating kinase 2 (JAK2) (Goffin et al., 1999). Upon receptor dimerization and JAK2 activation, several receptor tyrosine residues are phosphorylated, presumably by JAK2. Phosphorylated receptor tyrosine residues provide 'docking sites' for the binding of src homology domain 2 (SH2)-containing proteins, including Stat1, -3, and -5; phosphatases; and other adaptor molecules (Shuai, 2000). In the Nb2 PRL-R, the last tyrosine Y382 or its equivalent Y580 in the long PRL-R is important for signaling via Stat5 (Goffin et al., 1999), while both Y309 and Y382 are needed for signaling via Stat1 to an immediate earlyresponse gene IRF-1 (Wang et al., 1997).

\section{PRL-R-interacting Proteins}

Using the intracellular domain of the PRL-R in a genetic screen, we isolated an enzyme, $2^{\prime} 5^{\prime}$-oligodenylate synthetase (OAS), as a PRL-R-interacting protein (McAveney et al., 2000). Interestingly, OAS is acting more as an adaptor molecule than as an enzyme involved in regulating RNA metabolism. In this unconventional capacity, OAS interaction with the PRL-R reduces Stat1 phosphorylation and DNA-binding activity, which leads to a reduction in IRF-1 promoter activity. In contrast, OAS increases Stat5 DNA binding and $\beta$-casein promoter activity (McAveney et al., 2000). Thus, OAS interaction with the PRL-R enhances PRL-mediated differentiated functions. Consistent with this observation, IFN $\tau$-inducible OAS expression is correlated with increased Stat5mediated differentiated functions in the pregnant ovine endometrium (Johnson et al., 2001). 


\section{B. PROLACTIN-INDUCIBLE KINASE PATHWAYS}

\section{JAK/Stat Pathway}

The best-described signaling pathway activated by PRL is the JAK/Stat pathway (Schindler, 1999) that is commonly used by hematopoietin/cytokine receptors. JAK2 is prebound to the inactive PRL-R monomer, in contrast to other cytokine receptors where JAK PTKs are recruited into the receptor complex upon ligand binding (Yu-Lee and Jeay, 2001). Upon PRL binding and PRL-R homodimerization, JAK2 becomes activated and further phosphorylates downstream targets, including tyrosine residues on the PRL-R itself and Stat factors (Goffin et al., 1999). Stat1, -3, and -5 are activated by tyrosine phosphorylation to form homo- (Stat1/1, Stat3/3, Stat5/5) or heteromeric (Stat1/3) complexes, translocate into the nucleus, bind to conserved DNA elements called interferon (IFN) gamma-activated sequence (GAS), and regulate gene transcription. Since all of the components along the JAK/Stat pathway pre-exist in the cytoplasm, PRL-R signaling is initiated within 1-5 minutes by a series of phosphorylation events. PRL-inducible transcription of target genes is detected in the nucleus within 5-10 minutes.

\section{Parallel Kinase Cascades}

Other PTKs are activated by PRL stimulation, including Fyn, Src, Ras, and Raf, as well as serine/threonine kinases such as ZAP-70, PI3 kinase, Akt, mitogen-activated protein kinase (MAPK), jun kinase (JNK), and protein kinase $\mathrm{C}$ (Clevenger and Kline, 2001). Coordination of parallel kinase cascades with the JAK/Stat signaling pathway likely determines specific patterns of gene expression in various PRL-responsive cells and tissues. The pleiotropic actions of PRL on cellular proliferation, differentiation, apoptosis, or cell survival will depend on the interactions among these parallel kinase cascades.

\section{PRL-inducible Signaling Molecules}

\section{A. STAT FACTORS}

Stat factors are a family of latent cytoplasmic transcription factors that mediate signaling from cytokine receptors (Horvath, 2000; Shuai, 2000). Seven mammalian Stat genes (Stat1-4, -5a, -5b, and -6) have been identified, each encoding a protein $\approx 750-800$ amino acids in size with conserved functional domains. These include a coiled-coiled domain; DNA-binding domain; linker domain; SH2, a critical tyrosine residue that is important for dimerization, nuclear translocation, and DNA binding; and a carboxyl-terminus transactivation domain (Horvath, 2000). Additional post-translational modification - such as 
serine phosphorylation (Decker and Kovarik, 1999; Kovarik et al., 2001), methylation (Mowen et al., 2001), and acetylation (Shankaranarayanan et al., 2001) - further contribute to the ability of Stat factors to regulate gene transcription. Stat $1,-3,-5 a,-5 b$, and -6 have naturally occurring splice variants in the carboxyl terminus, generating dominant-negative $\beta$ isoforms that can bind DNA but lack intrinsic transactivation activity (Horvath, 2000). Stat factors utilize various domains to interact/cross talk with a diverse set of proteins, to transduce signals from the cytoplasm into the nucleus and to regulate gene transcription.

\section{B. STATS INTERACT WITH CYTOPLASMIC PROTEINS}

In addition to interacting with components of the cytokine receptor complex, Stats can interact directly with JAK PTK. The coiled-coil domain of Stats (except Stat2) can interact with the cytoplasmic N-myc interacting protein (Nmi) (Zhu et al., 1999), forming a Stat/Nmi complex that enhances Stat transactivation potentials. Other Stat-interacting proteins include Stat3-interacting protein (StIP1), which interacts with both JAK2 and Stat3 (Collum et al., 2000), and protein inhibitor of activated Stats (PIAS) (Shuai, 2000), which downregulates Stat transcriptional activity. Stats also can interact with Src, in one case as an adaptor molecule (Pfeffer et al., 1997) and in another to potentiate Src-mediated cytoskeletal changes in transiently transfected cells (Kabotyanski and Rosen, 2002). Further, in addition to monomers, dimers, and tetramers, Stats can be found in large (i.e., 1- to 4-MDa) cytoplasmic 'statosome' complexes (Sehgal, 2000), which are thought to contain accessory molecules that facilitate Stat recruitment to the receptor complex as well as Stat translocation into the nucleus. Stat1 also interacts in the cytoplasm with the nuclear transport importin $\alpha / \beta$ complex for transport into the nucleus (Sekimoto et al., 1997). Thus, in the cytoplasm, Stats interact with numerous proteins and acquire signal-transducing capability.

\section{STATS INTERACT WITH NUCLEAR PROTEINS}

Activated Stat complexes translocate into the nucleus within minutes (Horvath, 2000). Once in the nucleus, Stats interact with nuclear proteins, bind to cognate DNA elements (interferon-stimulated response elements (ISRE) or GAS), and regulate gene transcription. The transactivation potentials of Stats are modulated by interactions with nuclear proteins such as $\mathrm{p} 48$ (a member of the IRF family), IRF-1, c-jun, Sp1, Src, nuclear hormone receptors, MCM5 and BRCA1 (Chatterjee-Kishore et al., 2000; Horvath, 2000; Shuai, 2000), and with various coactivators (Collingwood et al., 1999). Coactivators not only facilitate interactions of transcription factors with components of the basal transcription machinery but many coactivators also exhibit intrinsic histone acetyltransferase (HAT) activities, which modify histones and remodel chromatin at promoters, 
resulting in transcriptional activation of genes. Stat1 interacts with three regions within the coactivator protein cyclic AMP response binding protein (CBP)/p300 (Horvath, 2000). Interestingly, one of these regions also interacts with Stat5, leading to the speculation that Stat5 competition with Stat1 for binding to $\mathrm{CBP} / \mathrm{p} 300$ forms one basis for competitive interactions between these two Stats at target promoters (Collingwood et al., 1999; Luo and Yu-Lee, 2000). Thus, coactivators can integrate the activities of DNA-binding proteins to activate gene transcription or can be a target of competitive binding between nuclear factors, which may inhibit gene transcription.

\section{Prolactin Regulation of IRF-1 Transcription}

As one approach to understanding PRL action in the immune system, we cloned a panel of immediate early-response genes from a rat T-lymphoma cell line, $\mathrm{Nb} 2$. One of these genes is the transcription factor IRF-1 (Yu-Lee et al., 1990). IRF-1 is an important immune response mediator. Its regulation by PRL may elucidate a role for PRL in modulating the immune response.

\section{A. IRF-1 AND IMMUNITY}

IRF-1 belongs to a small family of nine IRF proteins (Sato et al., 2000). IRF-1 regulates the expression of a number of genes important for mediating antiviral and antibacterial responses, T-helper 1 immune responses, macrophage and dendritic cell function, NK-cell differentiation, cell-cycle progression, and apoptosis (Taniguchi et al., 2001). Thus, IRF-1 plays an important role in mediating host immune defense. In humans, IRF-1 mutations and/or deletions are correlated with a high incidence of leukemias and myelodysplasia (Taniguchi et al., 2001), suggesting that IRF-1 is a tumor suppressor gene. In view of the diverse functions of IRF-1, its unique response to PRL stimulation (Yu-Lee et al., 1990; Stevens et al., 1995), and the ubiquitous expression of the PRL-R on immune function cells (Goffin et al., 1999; Matera et al., 2001), we suggest that PRL, through the JAK/Stat/IRF-1 pathway, modulates the biological activities of many cell types and tissues as well as aspects of the immune response ( $\mathrm{Yu}-\mathrm{Lee}$ et al., 1998).

\section{B. POSITIVE SIGNALING TO IRF-1}

Consistent with its multifunctional role in mediating diverse immunological functions, IRF-1 expression is regulated by a wide variety of signals (Taniguchi et al., 2001). PRL stimulates IRF-1 gene expression in normal rat leukocytes derived from the bone marrow and spleen (Dogusan et al., 2000) and in human granulocytes (Dogusan et al., 2001). In rat Nb2 T cells, PRL stimulates IRF-1 gene transcription in a distinct manner over the cell cycle, with a transient but 
dramatic 25-fold induction during early G1 and a second peak of induction at the G1/S transition (Stevens et al., 1995). PRL-inducible G1 transcriptional response is mediated by at least three factors assembled at the IRF-1 promoter: inducible Stat1 binding to a GAS element at -120 bp (Stevens et al., 1995), constitutive Sp1 binding at $-200 \mathrm{bp}$ (McAlexander and Yu-Lee, 2001b), and protein-protein interaction between Stat 1 and the coactivator CBP/p300 (Luo and Yu-Lee, 2000) (Figure 1A). Our working model is that, upon PRL stimulation, activated Stat1 binds to the IRF-1 GAS. Together with the pre-bound Sp1, it forms an enhanceosome (assembly of transcription factors) (Carey, 1998), which recruits coactivators such as $\mathrm{CBP} / \mathrm{p} 300$ and cofactor required for Sp1 (CRSP) (Ryu et al., 1999), as well as the general transcription machinery for transcriptional activation of the IRF-1 gene. Additionally, chromatin modification has been shown to play an important role in transcriptional regulation. By using chromatin immunoprecipitation (ChIP) assays, more acetylated histone $\mathrm{H} 4$ is found to associate with the IRF-1 promoter, indicating a more 'active' chromatin conformation in response to PRL stimulation, concomitant with the increase in IRF-1 gene transcription during G1 (McAlexander and Yu-Lee, 2001a). Thus, a combination of factors - including PRL-inducible Stat1, constitutively bound Sp1, and coactivators with their associated chromatin remodeling HAT activities - coordinate PRL stimulation of IRF-1 gene transcription in vivo.

\section{NEGATIVE SIGNALING TO IRF-1}

Much less is known about signals that shut off IRF-1 gene transcription. In $\mathrm{Nb} 2 \mathrm{~T}$ cells, PRL also activates Stat5 to bind as a minor component in the G1 PRL-inducible IRF-1 GAS complex (Wang and Yu-Lee, 1996). Surprisingly, the functional consequence of Stat5 interaction at the IRF-1 promoter is one of transcriptional repression rather than transcriptional activation (Luo and $\mathrm{Yu}-\mathrm{Lee}$, 1997,2000). Stat5 does not interact directly with Stat1 (Greenlund et al., 1995) nor does Stat5 compete with Stat1 for binding to the IRF-1 GAS. In transient transfection studies, Stat5 appears to compete with Stat1 for the coactivator p300/CBP via protein/protein interactions to inhibit PRL signaling to the IRF-1 promoter (Figure 1B) (see Section VI). However, the in vivo mechanism involved in Stat5-mediated negative signaling to the IRF-1 gene is unclear. In vivo, Stat5 can act as a transcriptional repressor. In Stat5a/Stat5b double KO mice, 'increased expression' of genes, including the IRF-1 gene (T. Teixeira, unpublished results), has been observed. This suggests that Stat5 normally represses these genes in vivo (Teglund et al., 1998). In the virgin and early pregnant mammary gland, a high level of Stat1 tyrosine phosphorylation (Liu et al., 1996) correlates with elevated IRF-1 gene expression. In contrast, in the late pregnant and fully differentiated lactating gland, a high level of activated Stat5 is correlated with the complete absence of IRF-1 gene expression (T. Teixeira, 
A. Positive signaling to IRF-1

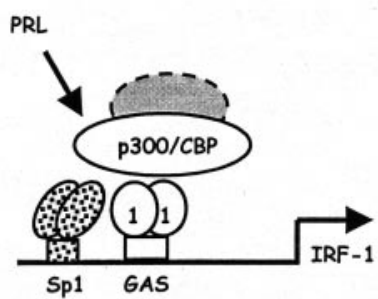

B. Negative signaling to IRF-1

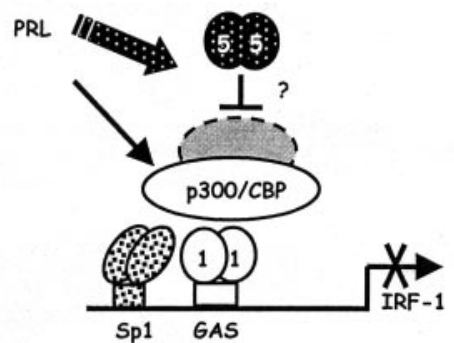

C. Stat1 and NFKB synergy

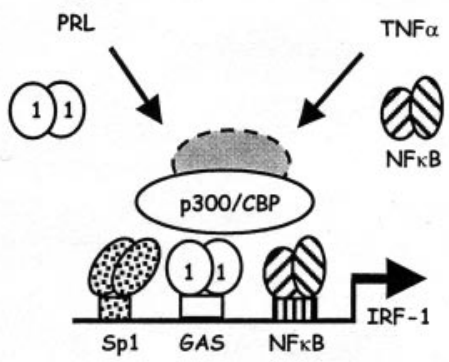

FIG. 1. Model of positive and negative signaling to the interferon regulatory factor-1 (IRF-1) gene. (A) A number of mediators positively regulate prolactin (PRL) stimulation of IRF-1 gene transcription. These include PRL-inducible Stat1, the constitutive factor Sp1, and the coactivator p300/CBP, which enhances Stat1 activation of the IRF-1 promoter. (B) PRLinducible Stat5 inhibits IRF-1 transcription. Although competition for the coactivator p300/CBP appears to be involved (Luo and Yu-Lee, 1997,2000), the in vivo mechanism of inhibition at the IRF-1 promoter is as yet unclear. (C) The IRF-1 promoter can be activated via PRL-inducible Stat 1 as well as by tumor necrosis factor alpha $(\mathrm{TNF} \alpha)$-inducible nuclear factor kappa B $(\mathrm{NF} \kappa \mathrm{B})$ binding to their respective response elements. In this model of positive and negative cytokine signal cross talk, Stat 1 synergizes with $\mathrm{NF} \kappa \mathrm{B}$ but Stat 5 antagonizes NF $\kappa \mathrm{B}$ signaling to the IRF-1 promoter. Additional factors that can be recruited by Stats or $\mathrm{NF} \kappa \mathrm{B}$ to regulate IRF-1 promoter activity are indicated in gray.

unpublished observations). These observations support our model that PRLinducible Stat5 is involved in negative signaling to the IRF-1 promoter. Other mechanisms for transcriptional shutoff may exist, such as PRL-inducible Stat5 activating a repressor to shut off IRF-1 gene transcription. In this regard, the PRL-inducible suppressors of cytokine signaling (SOCS) proteins can bind to the 
PRL-R and turn off signaling at the receptor level in a negative-feedback loop (Naka et al., 1999; Tam et al., 2001).

Interestingly, fewer acetylated histones are associated with the IRF-1 promoter at 4 hours after PRL stimulation, when the transcriptional activity of the IRF-1 gene has returned to baseline (McAlexander and Yu-Lee, 2001a). Thus, a less-active chromatin conformation at the IRF-1 promoter is associated with transcriptional inactivity at the IRF-1 gene. Whether Stat5, co-repressors, and/or histone deacetylase (HDAC) activities are involved in IRF-1 transcriptional shutoff is currently unknown. Our studies show a correlation between the pattern of histone acetylation/deacetylation and biphasic transcription of the IRF-1 gene, implicating histone modification and changes in chromatin structure in PRL regulation of the IRF-1 gene transcription in vivo.

\section{Stat5 and NF $\kappa B$ Cross Talk}

\section{A. NF $\kappa$ B SIGNALING}

The generality of Stat5 acting as a transcriptional inhibitor at the IRF-1 promoter is further illustrated by showing that Stat5 inhibits other signaling molecules that also activate the IRF-1 promoter. One such molecule is NF $\kappa \mathrm{B}$. $\mathrm{NF} \kappa \mathrm{B}$ initially was identified as a nuclear factor that binds to the immunoglobulin kappa light chain gene enhancer in B cells. It is now known to be widely distributed in all cell types (Israel, 2000; Baldwin, 2001). NF $\kappa \mathrm{B}$ was the first transcription factor family shown to reside basally in the cytoplasm but, upon stimulation, translocates into the nucleus to regulate gene transcription. $\mathrm{NF} \kappa \mathrm{B}$ is comprised of several members, including p65/RelA, RelB, c-Rel, p50, and p52. The most abundant form of $\mathrm{NF} \kappa \mathrm{B}$ is a heterodimer of $\mathrm{p} 50 / \mathrm{p} 65$, which is inducible by a wide variety of signals. In unstimulated cells, $\mathrm{NF} \kappa \mathrm{B}$ is sequestered in a complex with its inhibitor $\mathrm{I} \kappa \mathrm{B}$ (Israel, 2000). Upon activation, $\mathrm{NF} \kappa \mathrm{B}$ is released through $\mathrm{I} \kappa \mathrm{B}$ turnover, a process that involves $\mathrm{I} \kappa \mathrm{B}$ phosphorylation, ubiquitination, and degradation via the proteasome pathway. Once in the nucleus, $\mathrm{NF} \kappa \mathrm{B}$ interacts with multiple factors and the basal transcription machinery to regulate gene transcription.

\section{B. STAT5 ANTAGONIZES NFкB SIGNALING}

In addition to the $\mathrm{Sp} 1$ and GAS elements that mediate positive PRL signaling, an NF $\kappa \mathrm{B}$ site mediates TNF $\alpha$ induction of the IRF-1 promoter (Figure 1C). PRL-inducible Stat1 synergizes with $\mathrm{TNF} \alpha$-inducible $\mathrm{NF} \kappa \mathrm{B}$ to activate the IRF-1 promoter (Luo and Yu-Lee, 2000). In contrast, PRL-inducible Stat5 inhibits NF $\kappa \mathrm{B}$-mediated signaling to the IRF-1 promoter. Additionally, PRLinducible Stat5 potently inhibits $\mathrm{NF} \kappa \mathrm{B}$-mediated signaling to promoters that 
contain only $\mathrm{NF} \kappa \mathrm{B}$ binding sites. This observation is significant, as it greatly expands potential targets of Stat5 regulation - in particular, Stat5 inhibition. Interestingly, negative cross talk between Stat5 and $\mathrm{NF} \kappa \mathrm{B}$ is reciprocal in the mammary gland, as $\mathrm{NF} \kappa \mathrm{B}$ inhibits milk protein $\beta$-casein gene expression

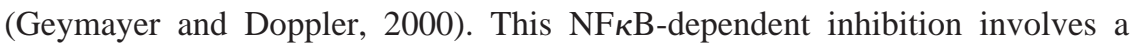
reduction in Stat5 tyrosine phosphorylation in the pregnant gland. We speculate that during mammary gland development, Stat 1 and $\mathrm{NF} \kappa \mathrm{B}$ synergize to activate the IRF-1 gene in the virgin and early pregnant gland, while in the lactating gland, Stat5 coupled with a significant reduction in NF $\kappa \mathrm{B}$ levels prevents IRF-1 expression but maximally induces $\beta$-casein expression. To confirm our model of positive and negative signaling to the IRF-1 promoter, ChIP assays employing antibodies against Stat1, Stat5, p300 coactivator, and perhaps co-repressors will be used to identify which factors are recruited to the IRF-1 promoter in response to PRL stimulation in a temporally distinct manner to regulate IRF-1 gene transcription in vivo.

\section{Positive and Negative Regulation by Stats}

In addition to the well-described functions of Stats as positive mediators of cytokine signaling, several lines of evidence now show that Stats can function as transcriptional repressors. Stat1 has been shown to mediate IFN $\gamma$-dependent activation or repression of target genes (Ramana et al., 2000). In Stat5a/Stat5bdeficient mice, the expression of some Stat 5 target genes is found to be elevated, suggesting a relief of Stat5-mediated repression in vivo (Teglund et al., 1998). These findings support the physiological relevance of our observation that Stat5 acts as a transcriptional repressor at the IRF-1 promoter (Luo and Yu-Lee, 2000). Whether Stat5 is acting directly or through a Stat5-inducible factor to repress IRF-1 gene transcription is yet to be determined. We speculate that negative cross talk between Stat5 and NF $\kappa$ B, Smad, or glutocorticoid receptor (GR) could, in part, explain how PRL antagonizes $\operatorname{TNF} \alpha$, TGF $\beta$, or glucocorticoid signaling, respectively, at target genes. It is now known that conformational changes induced by ligand binding to nuclear hormone receptors, coupled with the levels of coactivators or co-repressors present, determine the biological activities of the receptor complex on target gene transcription (e.g., by changing an estrogen antagonist into an agonist) (Lavinsky et al., 1998; McDonnell, 1999). While the mechanistic details are still unclear, transcriptional regulation by Stats is a complex process. Stats can act as transcriptional activators or transcriptional repressors, depending on the promoter context, the concentrations of available coactivators and co-repressors, the presence of other DNA-binding proteins, and the stage of differentiation of the target cell and tissue. 


\section{Concluding Remarks}

PRL is a versatile neuroendocrine hormone that also works as a locally produced cytokine. In this capacity, PRL regulates a wide range of physiological responses and a correspondingly wide range of target genes. A large panel of PRL-inducible genes in proliferating $\mathrm{Nb} 2 \mathrm{~T}$ cells has been identified (BoleFeysot et al., 2000). A handful of other genes has been studied to understand PRL-mediated differentiative functions and cell survival responses. Interestingly, a recent study shows fine differences in PRL signaling in different populations of blood leukocytes (Dogusan et al., 2001). PRL activates Stat5 and SOCS3 in peripheral blood mononuclear cells, while PRL activates Stat1 and SOCS2 in human granulocytes. Whether this difference in initial signaling components and target gene activation translates into functional differences in PRL action in these two leukocyte populations remains to be determined. At present, the details of what regulates the qualitative differences in PRL signaling are not understood. It is likely that a combination of steady-state levels, availability and activation of Stats, the levels of coactivators and co-repressors, and even the type of SOCS proteins induced by PRL (Tam et al., 2001) contributes to the tissue-specific or cell-type specific responses to PRL. The challenge is to identify and quantify these differences at the gene and protein levels. A further challenge is to analyze these differences in the context of normal versus pathological states. Future studies will employ a wide variety of approaches to fill in this gap in knowledge. These include DNA microarray; ChIP assays; transgenics overexpressing PRL or mice deficient in PRL, PRL-R, Stat5a, Stat5b or Stat5a/Stat5b; and proteomic technologies. Crossing the PRL-R KO with autoimmune disease strains of mice, for example, will better elucidate how PRL functions as a homeostatic molecule in modulating immune, autoimmune, and inflammatory responses.

\section{ACKNOWLEDGMENTS}

I would like to acknowledge the contributions of past and present members of my laboratory and, in particular, Therese Teixeira for the unpublished data discussed in this review, Nelson Horseman for communicating prepublication information, and Jeff Rosen and Sophia Tsai for critical comments. This work is supported by grants from the National Institutes of Health, Linda and Ronald Finger Lupus Research Center, and Women's Fund for Health, Education and Research.

\section{REFERENCES}

Ahonen T, Harkonen PL, Laine J, Rui H, Martikainen PM, Nevalainen MT 1999 Prolactin is a survival factor for androgen-deprived rat dorsal and lateral prostate epithelium in organ culture. Endocrinology 140:5412-5421

Baldwin AS 2001 Control of oncogenesis and cancer therapy resistance by the transcription factor NF-kappaB. J Clin Invest 107:241-246 
Bellone G, Geuna M, Carbone A, Silvestri S, Foa R, Emanuelli G, Matera L 1995 Regulatory action of prolactin on the in vitro growth of $\mathrm{CD} 34+$ ve human hemopoietic progenitor cells. J Cell Physiol 163:221-231

Ben-Jonathan N, Mershon J, Allen D, Steinmetz R 1996 Extrapituitary prolactin: distribution, regulation, functions and clinical aspects. Endocr Rev 17:639-669

Bole-Feysot C, Goffin V, Edery M, Binart N, Kelly PA 1998 Prolactin (PRL) and its receptor: actions, signal transduction pathways and phenotypes observed in PRL receptor knockout mice. Endocr Rev 19:225-268

Bole-Feysot C, Perret E, Roustan P, Bouchard B, Kelly PA 2000 Analysis of prolactin-modulated gene expression profiles during the $\mathrm{Nb} 2$ cell cycle using differential screening techniques. Genome Biol 1:research0008.1-0008.15

Bouchard B, Ormandy CJ, Di Santo JP, Kelly PA 1999 Immune system development and function in prolactin receptor-deficient mice. J Immunol 163:576-582

Buckley AR 2001 Prolactin, a lymphocyte growth and survival factor. Lupus 10:684-690

Carey M 1998 The enhanceosome and transcriptional synergy. Cell 92:5-8

Chatterjee-Kishore M, Wright KL, Ting JPY, Stark GR 2000 How Stat1 mediates constitutive gene expression: a complex of unphosphorylated Stat1 and IRF1 supports transcription of the LMP2 gene. EMBO J 19:4111-4122

Clevenger CV, Kline BJ 2001 Prolactin receptor signal transduction. Lupus 10:706-718

Clevenger CV, Chang WP, Ngo W, Pasha TLM, Montone KT, Tomaszweski JE 1995 Expression of prolactin and prolactin receptor in human breast carcinoma. Am J Pathol 146:695-705

Collingwood TN, Urnov FD, Wolffe AP 1999 Nuclear receptors: coactivators, corepressors and chromatin remodeling in the control of transcription. J Mol Endocrinol 23:255-275

Collum RG, Brutsaer S, Lee G, Schindler C 2000 A Stat3 interacting protein (StIP1) regulates cytokine signal transduction. Proc Natl Acad Sci USA 97:10120-10125

Couse JF, Korach KS 1999 Estrogen receptor null mice: what have we learned and where will they lead us? Endocr Rev 20:358-417

Deb S, Tessier C, Prigent-Tessier A, Barkai U, Ferguson-Gottschall S, Srivastava RK, Faliszek J, Gibori G 1999 The expression of interleukin-6 (IL-6), IL-6 receptor, and gp130 kDa glycoprotein in the rat decidua and a decidual cell line: regulation by $17 \beta$-estradiol and prolactin. Endocrinology 140:4442-4450

Decker T, Kovarik P 1999 Transcription factor activity of Stat proteins: structural requirements and regulation by phosphorylation and interacting proteins. Cell Mol Life Sci 55:1535-1546

DeVito WJ, Okulicz WC, Stone S, Avakian C 1992 Prolactin-stimulated mitogenesis of cultured astrocytes. Endocrinology 130:2549-2556

Dogusan Z, Book ML, Verdood P, Yu-Lee Ly, Hooghe-Peters E 2000 Prolactin activates interferon regulatory factor- 1 expression in normal lympho-hemopoietic cells. Eur Cytokine Netwk 11:435-442

Dogusan Z, Hooghe R, Verdood P, Hooghe-Peters EL 2001 Cytokine-like effects of prolactin in human mononuclear and polymorphonuclear leukocytes. J Neuroimmunol 120:58-66

Dorshkind K, Horseman ND 2000 The roles of prolactin, growth hormone, insulin-like growth factor-I, and thyroid hormones in lymphocyte development and function: insights from genetic models of hormone and hormone receptor deficiency. Endocr Rev 21:292-312

Dorshkind K, Horseman ND 2001 Anterior pituitary hormones, stress, and immune system homeostasis. BioEssays 23:2881793-2941803

Gertler A 1997 Recombinant analogues of prolactin, growth hormone, and placental lactogen: correlations between physical structure, binding characteristics, and activity. J Mamm Gland Biol Neoplasia 2:69-80 
Geymayer S, Doppler W 2000 Activation of $\mathrm{NF}_{\kappa} \mathrm{B}$ p50/p65 is regulated in the developing mammary gland and inhibits Stat5-mediated $\beta$-casein gene expression. FASEB J 14:11591170

Girasole G, Jilka RL, Passeri G, Boswell S, Boder G, Williams DC, Manolagas SC 1992 $17 \beta$-estradiol inhibits interleukin- 6 production by bone marrow-derived stromal cells and osteoblasts in vitro: a potential mechanism for the antiosteoporotic effect of estrogens. J Clin Invest 89:883-891

Goffin V, Kelly PA 1997 The prolactin/growth hormone receptor family: structure/function relationships. J Mamm Gland Biol Neoplasia 2:7-17

Goffin V, Binart N, Clement-Lacroix P, Bouchard B, Bole-Feysot C, Edery M, Lucas BK, Touraine P, Pezet A, Maaskant R, Pichard C, Helloco C, Baran N, Favre H, Bernichtein S, Allamando A, Ormandy C, Kelly PA 1999 From the molecular biology of prolactin and its receptor to the lessons learned from knockout mice models. Genet Anal Biomol Engin 15:189-201

Gout PW, Beer CT, Noble RL 1980 Prolactin-stimulated growth of cell cultures established from malignant $\mathrm{Nb}$ rat lymphomas. Cancer Res 40:2433-2436

Greenlund AC, Morales MO, Viviano BL, Yan H, Krolewski J, Schreiber RD 1995 Stat recruitment by tyrosine-phosphorylated cytokine receptors: an ordered reversible affinitydriven process. Immunity 2:677-687

Grimaldi CM, Michael DJ, Diamond B 2001 Cutting edge: expansion and activation of a population of autoreactive marginal zone B cells in a model of estrogen-induced lupus. J Immunol 167:1886-1890

Harris MT, Feldberg RS, Lau KM, Lazarus NH, Cochrane DE 2000 Expression of proinflammatory genes during estrogen-induced inflammation of the rat prostate. Prostate 44:19-25

Hooghe R, Dogusan Z, Martens N, Velkeniers B, Hooghe-Peters EL 2001 Effects of prolactin on signal transduction and gene expression. Possible relevance for systemic lupus erythematosus. Lupus 10:719-727

Horseman, ND 2001 Prolactin. Boston: Kluwer Academic Publishers

Horseman ND, Zhao W, Montecino-Rodriguez E, Tanaka M, Nakashima K, Eagle SJ, Smith F, Markoff E, Dorshkind K 1997 Defective mammopoiesis, but normal hematopoiesis, in mice with a targeted disruption of the prolactin gene. EMBO J 16:6926-6935

Horvath CM 2000 Stat proteins and transcriptional responses to extracellular signals. Trends Biochem Sci 25:496-502

Israel A 2000 The IKK complex: an integrator of all signals that activate NF-kappaB? Trends Cell Biol 10:129-133

Jacobi AM, Rohde W, Volk HD, Dorner T, Burmester GR, Hiepe F 2001 Prolactin enhances the in vitro production of $\mathrm{IgG}$ in peripheral blood mononuclear cells from patients with systemic lupus erythematosus but not from healthy controls. Ann Rheum Dis 60:242-247

Jarrar D, Wang P, Knoferl MW, Kuebler JF, Cioffi WG, Bland KI, Chaudry IH 2000 Insight into the mechanism by which estradiol improves organ functions after trauma-hemorrhage. Surgery 128:246-252

Johnson AG, Stewart D, Choi Y, Burghardt RC, Yu-Lee Ly, Chebath J, Bazer FW, Spencer TE 2001 Effects of the estrous cycle, pregnancy and interferon $\tau$ on $2^{\prime}, 5^{\prime}$-oligoadenylate synthetase expression in the ovine uterus. Biol Reprod 64:1392-1399

Kabotyanski E, Rosen JM 2002 Signal transduction pathways regulated by prolactin and Src result in different conformations of activated Stat5b. J Biol Chem, in press

Kanik KS, Wilder RL 2000 Hormonal alterations in rheumatoid arthritis, including the effects of pregnancy. Neuroendocr Mech Rheum Dis 26:805-823 
Knoferl MW, Diodato MD, Angele MK, Ayala A, Cioffi WG, Bland KI, Chaudry IH 2000a Do female sex steroid adversely or beneficially affect the depressed immune responses in males after trauma-hemorrhage? Arch Surg 135:425-433

Knoferl MW, Angele MK, Ayala A, Cioffi WG, Bland KI, Chaudry IH 2000b Insight into the mechanism by which metoclopramide improves immune functions after trauma-hemorrhage. Am J Physiol Cell Physiol 279:C72-C80

Kooijman R, Hooghe-Peters EL, Hooghe R 1996 Prolactin, growth hormone, and insulin-like growth factor-1 in the immune system. Adv Immunol 63:377-454

Kovarik P, Mangold M, Ramsauer K, Heidari H, Steinborn R, Zotter A, Levy DE, Muller M, Decker T 2001 Specificity of signaling by Stat 1 depends on SH2 and C-terminal domains that regulate Ser727 phosphorylation, differentially affecting specific target gene expression. EMBO J 20:91-100

Kurebayashi S, Miyashita Y, Hirose T, Kasayama S, Akira S, Kishimoto T 1997 Characterization of mechanisms of interleukin- 6 gene repression by estrogen receptor. J Steroid Biochem Molec Biol 60:11-17

Lavinsky RM, Jepsen K, Heinzel T, Torchia J, Mullen TM, Schiff R, Del-Rio AL, Rocote, Ngo S, Gemsch J, Hilsenbeck SG, Osborne CK, Glass CK, Rosenfeld MG, Rose DW 1998 Diverse signaling pathways modulate nuclear receptor recruitment of N-CoR and SMRT complexes. Proc Natl Acad Sci USA 95:2920-2925

LaVoie HA, Witorsch RJ 1995 Investigation of intracellular signals mediating the anti-apoptotic action of prolactin in Nb2 lymphoma cells. Proc Soc Exp Biol Med 209:257-269

Leav I, Merk FB, Lee KF, Loda M, Mankoki M, McNeal JE, Ho S-M 1999 Prolactin receptor expression in the developing human prostate and in hyperplastic, dysplastic, and neoplastic lesions. Am J Pathol 154:863-870

Lin DL, Whitney MC, Yao Z, Keller ET 2001 Interleukin-6 induces androgen responsiveness in prostate cancer cells through up-regulation of androgen receptor expression. Clin Cancer Res 7:1773-1781

Lin J, Linzer DIH 1999 A novel megakaryocyte differentiation factor from mouse placenta. Trends Cardiovasc Med 9:167-171

Liu X, Robinson GW, Hennighausen L 1996 Activation of Stat5a and Stat5b by tyrosine phosphorylation is tightly linked to mammary gland differentiation. Mol Endocrinol 10: $1496-1506$

Luo G, Yu-Lee Ly 1997 Transcriptional inhibition by Stat5: differential activities at growth-related versus differentiation-specific promoters. J Biol Chem 272:26841-26849

Luo G, Yu-Lee Ly 2000 Stat5 inhibits NF $\kappa$ B-mediating signaling. Mol Endocrinol 14:114-123

Manolagas SC 2000 Birth and death of bone cells: basic regulatory mechanisms and implications for the pathogenesis and treatment of osteoporosis. Endocr Rev 21:115-137

Matera L, Mori M, Geuna M 2000 Prolactin in autoimmunity and antitumor defense. J Neuroimmunol 109:47-55

Matera L, Galetto A, Mori M 2001 Effect of prolactin on the antigen presenting function of monocyte-derived dendritic cells. Lupus 10:728-734

McAlexander MB, Yu-Lee Ly 2001a Prolactin activation of IRF-1 transcription involves changes in histone acetylation. FEBS Lett 488:91-94

McAlexander MB, Yu-Lee Ly 2001b Sp1 is required for prolactin activation of the interferon regulatory factor 1 gene. Mol Cell Endocrinol 184:135-141

McAveney KM, Gimble JM, Yu-Lee Ly 1996 Prolactin receptor expression during adipocyte differentiation of bone marrow stroma. Endocrinology 137:5723-5726

McAveney KM, Book ML, Ling P, Horvath G, Chebath J, Yu-Lee Ly 2000 Association of 2 ',5'-oligoadenylate synthetase with the prolactin receptor: alteration in prolactin-inducible Stat1 signaling to the IRF-1 promoter. Mol Endocrinol 14:295-306 
McDonnell DP 1999 The molecular pharmacology of SERMS. Trends Exp Med 10:301-311

McMurray RW 2001 Estrogen, prolactin, and autoimmunity: actions and interactions. Intl Immunopharmacol 1:995-1008

Montgomery DW 2001 Prolactin production by immune cells. Lupus 10:665-675

Morales P, Carretero M, Geronimo H, Copin SG, Gaspar ML, Marcos MA, Martin-Perez J 1999 Influence of prolactin on the differentiation of mouse B-lymphoid precursors. Cell Growth Diff 10:583-590

Morris JA, MacKenzie EJ, Damiano AM, Bass SM 1990 Mortality in trauma patients: the interaction between host factors and severity. J Trauma 30:1476-1482

Morris SM, Anaya P, Xiang X, Morris NR, May GS, Yu-Lee Ly 1997 A prolactin-inducible T cell gene product is structurally similar to the Aspergillus nidulans nuclear movement protein NUDC. Mol Endocrinol 11:229-236

Mowen KA, Tang J, Zhu W, Schurter BT, Shuai K, Herschman HR, David M 2001 Arginine methylation of STAT1 modulates IFNalpha/beta-induced transcription. Cell 104:731-741

Muller H, Liu, Croy BA, Head JR, Hung JS, Dai G, Soares MJ 1999 Uterine natural killer cells are targets for a trophoblast cell-specific cytokine, prolactin-like protein A. Endocrinology 140:2711-2720

Naka T, Fujimoto M, Kishimoto T 1999 Negative regulation of cytokine signaling: Stat-induced Stat inhibitor. Trends Biochem Sci 24:394-398

Nanbu-Wakao R, Fujitani Y, Masuho Y, Muramatsu M, Wakao H 2000 Prolactin enhances CCAAT enhancer-binding protein-beta (C/EBP beta) and peroxisome proliferator-activated receptor gamma (PPAR gamma) messenger RNA expression and stimulates adipogenic conversion of NIH-3T3 cells. Mol Endocrinol 14:307-316

Nevalainen MT, Valve EM, Ahonen T, Yagi A, Paranko J, Harkonen PL 1997 Androgendependent expression of prolactin in rat prostate epithelium in vivo and in organ culture. FASEB J 11:1297-1307

O'Neal KD, Yu-Lee Ly 1994 Differential signal transduction of the short, Nb2, and long PRL receptor: activation of IRF-1 and cell proliferation. J Biol Chem 269:26076-26082

Oberholzer A, Keel M, Zellweger R, Steckholzer U, Trentz O, Ertel W 2000 Incidence of septic complications and multiple organ failure in severely injured patients is sex specific. J Trauma: Inj Inf Crit Care 48:932-937

Offner PJ, Moore EE, Biffl WL 1999 Male gender is a risk factor for major infections after surgery. Arch Surg 134:935-940

Ogle CK, Kong F, Guo X, Wells DA, Aosasa S, Noel G, Horseman ND 2000 The effect of burn injury on suppressors of cytokine signaling. Shock 14:392-398

Peeva E, Grimaldi C, Spatz L, Diamond B 2000 Bromocryptine restores tolerance in estrogentreated mice. J Clin Invest 106:1373-1379

Pfeffer LM, Mullersman JE, Pfeffer SR, Murti A, Yang CH 1997 Stat3 as an adapter to couple phosphatidylinositol 3-kinase to the IFNAR1 chain of the type I interferon receptor. Science 276:1418-1420

Ramana CV, Chatterjee-Kishore M, Nguyen H, Stark GR 2000 Complex roles of Stat in regulating gene expression. Oncogene 19:2619-2627

Richards SM, Murphy WJ 2000 Use of human prolactin as a therapeutic protein to potentiate immunohematopoietic function. J Neuroimmunol 109:56-62

Richards SM, Garman RD, Keyes L, Kavanagh B, McPherson JM 1998 Prolactin is an antagonist of TGF- $\beta$ activity and promotes proliferation of murine B cell hybridomas. Cell Immunol 184:85-91

Ryu S, Zhou S, Ladurner AG, Tijan R 1999 The transcriptional cofactor complex CRSP is required for activity of the enhancer-binding protein Sp1. Nature 397:446-450 
Sato M, Taniguchi T, Tanaka N 2000 The interferon system and interferon regulatory factor transcription factors - studies from gene knockout mice. Cytokine Growth Factor Rev $12: 133-142$

Schindler C 1999 Cytokine and Jak-Stat signaling. Exp Cell Res 253:7-14

Sehgal PB 2000 Stat-signaling through the cytoplasmic compartment: consideration of a new paradigm. Cell Signal 12:525-535

Sekimoto T, Imamoto N, Makajima K, Hirano T, Yoneda Y 1997 Extracellular signal-dependent nuclear import of Stat 1 is mediated by nuclear pore targeting complex formation with NPI-1, but not Rch1. EMBO J 16:7067-7077

Shankaranarayanan P, Chaitidis P, Kuhn H, Nigam S 2001 Acetylation by histone acetyltransferases $\mathrm{CBP} / \mathrm{p} 300$ of STAT6 is required for the transcriptional activation of the $15-\mathrm{LOX}-1$ gene. J Biol Chem 276:42753-42760

Shuai K 2000 Modulation of Stat signaling by Stat-interacting proteins. Oncogene 19:2638-2644

Smith P 1930 The effect of hypophysectomy upon the involution of the thymus in the rat. Anatom $\operatorname{Rec}$ 47:119

Stevens AM, Wang Y, Sieger KA, Lu H, Yu-Lee Ly 1995 Biphasic transcriptional regulation of the interferon regulatory factor- 1 gene by prolactin: involvement of gamma-interferon activated sequence and Stat-related proteins. Mol Endocrinol 9:513-525

Stoker TE, Robinette CL, Britt BH, Laws SC, Cooper RL 1999 Prepubertal exposure to compounds that increase prolactin secretion in the male rat: effects on the adult prostate. Biol Reprod 61:1636-1643

Tam SP, Lau P, Djiane J, Hilton DJ, Waters MJ 2001 Tissue-specific induction of SOCS gene expression by PRL. Endocrinology 142:5015-5026

Tangbanluekal L, Robinette CL 1993 Prolactin mediates estradiol-induced inflammation in the lateral prostate of Wistar rats. Endocrinology 132:2407-2416

Taniguchi T, Ogasawara K, Takaoka A, Tanaka N 2001 IRF family of transcription factors as regulators of host defense. Annu Rev Immunol 19:623-655

Teglund S, McKay C, Schuetz E, van Deursen JM, Stravopodis D, Wang D, Brown M, Bodner S, Grosveld G, Ihle JN 1998 Stat5a and Stat5b proteins have essential and nonessential, or redundant, roles in cytokine responses. Cell 93:841-850

Tessier C, Deb S, Prigent-Tessier A, Ferguson-Gottschall S, Gibori GB, Shiu RPC, Gibori G 2000 Estrogen receptors $\alpha$ and $\beta$ in rat decidua cells: cell-specific expression and differential regulation by steroid hormones and prolactin. Endocrinology 141:3842-3851

Thellin O, Noel G, Khurana S, Ogle CK, Horseman ND 2001 Stress hormone secretion and gut signal transducer (Stat) proteins after burn injury in rats. Shock 16:393-397

van Coppenolle F, Slomianny C, Carpentier F, Le Bourhis X, Ahidouchi A, Croix D, Legrand G, Dewailly E, Fournier S, Cousse H, Authie D, Raynaud J, Beauvillain JC, Dupouy JP, Prevarskaya N 2001 Effects of hyperprolactinemia on rat prostate growth: evidence of androgeno-dependence. Am J Physiol Endocrinol Metab 280:E120-E129

Vidaller A, Guadarrama F, Llorente L, Mendez JP, Larrea F, Villa AR, Alarcon-Segovia D 1992 Hyperprolactinemia inhibits natural killer (NK) cell function in vivo and its bromocriptine treatment not only corrects it but makes it more efficient. J Clin Immunol 12:210-215

Walker SE 2001 Bromocriptine treatment of systemic lupus erythematosus. Lupus 10:762-768

Wang Y, Yu-Lee Ly 1996 Multiple Stat complexes interact at the IRF-1 GAS in prolactin-stimulated $\mathrm{Nb} 2 \mathrm{~T}$ cells. Mol Cell Endocrinol 121:19-28

Wang Y, O’Neal KD, Yu-Lee Ly 1997 Multiple prolactin receptor cytoplasmic residues and Stat1 mediate prolactin signaling to the IRF-1 promoter. Mol Endocrinol 11:1353-1364

Wennbo H, Kindblom J, Isaksson EGP, Tornell J 1997 Transgenic mice overexpressing the prolactin gene develop dramatic enlargement of the prostate gland. Endocrinology 138:44104415 
Yamamoto T, Matsuda T, Junicho A, Kishi H, Saatcioglu F, Muraguchi A 2000 Cross-talk between signal transducer and activator of transcription 3 and estrogen receptor signaling. FEBS Lett 486:143-148

Yu-Lee Ly 1997 Molecular actions of prolactin in the immune system. Proc Soc Exp Biol Med 215:35-52

Yu-Lee Ly, Jeay S 2002 Prolactin and growth hormone receptors: signal transduction and cross talk. In: Goffin V, Kelly PA (eds) Hormone Signaling. Norwell, MA: Kluwer Academic Publishers; $121-144$

Yu-Lee Ly, Luo G, Moutoussamy S, Finidori J 1998 Prolactin and growth hormone signal transduction in lymphohemopoietic cells. Cell Mol Life Sci 54:1067-1075

Yu-Lee Ly, Hrachovy JA, Stevens AM, Schwarz LA 1990 Interferon-regulatory factor 1 is an immediate-early gene under transcriptional regulation by prolactin in Nb2 T cells. Mol Cell Biol 10:3087-3094

Zellweger R, Zhu X-H, Wichmann MW, Ayala A, DeMaso CM, Chaudry IH 1996 Prolactin administration following hemorrhagic shock improves macrophage cytokine release capacity and decreases mortality from subsequent sepsis. J Immunol 157:5748-5754

Zhu M, John S, Berg M, Leonard WJ 1999 Functional association of Nmi with Stat5 and Stat1 in IL-2- and IFN $\gamma$-mediated signaling. Cell 96:121-130

Zhu X-H, Zellweger R, Wichmann MW, Ayala A, Chaudry IH 1997 Effects of prolactin and metoclopramide on macrophage cytokine gene expression in late sepsis. Cytokine 9:437-446 



\section{AUTHOR INDEX}

A

Anson-Cartwright, Lynn, 221

B

Bakke, Marit, 19

Böttner, Martina, 235

Burger, Henry G., 257

Burkin, Heather, 37

C

Capel, Blanche, 1

Cashion, Adrienne B., 235

Chang, Ching-yi, 295

Conneely, Orla M., 339

Connor, Caroline E., 295

Cross, James C., 221

D

de Kretser, D.M., 149

DeMayo, Francesco, 339

Dennerstein, Lorraine, 257

Dowsett, Mitch, 317

Doyle, Kari H., 195

Dubal, Dena B., 235

Dudley, Emma C., 257
E

Eddy, Edward M., 103

F

Falender, Allison E., 195

Frigeri, Claudia, 19

G

Griswold, Michael D., 129

H

Hanley, Neil A., 19

Heckert, Leslie L., 129

Hsieh, Minnie, 195

I

Ikeda, Yayoi, 19

$\mathbf{J}$

Jilka, R.L., 385 
$\mathbf{K}$

Kousteni, S., 385

Kuhn, Cynthia M., 411

$\mathbf{L}$

Lala, Deepak S., 19

Laurion, Erika, 75

Lele, Karen M., 75

Lo, Yuet K., 195

Luo, Xunrong, 19

Lydon, John P., 339

M

Manolagas, S.C., 385

McDonnell, Donald P., 295

McEwen, Bruce, 357

McLachlan, R.I., 149

McPhaul, Michael J., 181

Meachem, S.J., 149

Miller, David J., 37

Mulac-Jericevic, Biserka, 339

$\mathbf{N}$

Norris, John D., 295

$\mathbf{O}$

O'Donnell, L., 149

O’Malley, Bert W., 339

Ochsner, Scott, 195

$\mathbf{P}$

Parker, Keith L., 19

Pratis, K., 149
$\mathbf{R}$

Rau, Shane W., 235

Rice, Douglas A., 19

Richards, JoAnne S., 195

Robertson, D.M., 149

Robertson, David M., 257

Rosewell, Katherine L., 235

Russell, Darryl L., 195

Schimmer, Bernard P., 19

Scott, Ian C., 221

Sharma, S. Chidananda, 195

Shi, Xudong, 37

Simpson, Evan R., 317

Smith, Matthew J., 235

Stallings, Nancy, 19

Stanton, P.G., 149

$\mathbf{T}$

Tilmann, Christopher, 1

W

Walker, Cheryl L., 277

Wijayaratne, Ashini, 295

Wilson, Melinda E., 235

Wise, Phyllis M., 235

Wolgemuth, Debra J., 75

Wong, Margaret, 19

Y

Yu-Lee, Li-yuan, 435

Zhao, Liping, 19 


\section{SUBJECT INDEX}

A

Acrosome, 37

Activin, 195

Adjuvant therapy, 317

Adrenal glands, 19

Aging, 235, 411

Aloxifene, 277

Anabolic steroids, 411

Androgen, 149, 385

receptor, 181

Animal models, 37, 129, 277

AR gene, 181

Aromatase, 317

inhibitors, 317

Assisted reproduction, 149

Athletes, 411

B

Bone, 385

loss, 385

mineral density, 257

resorption, 385

Brain, 235, 357

Breast

cancer, 317

development, 181

C

Cancer, breast, 317

Catecholaminergic neurons, 357

Cell cycles

meiotic, 75 mitotic, 75

regulation, 75

Cell survival, 435

Cellular pathways, 1

Central nervous system, 235

Chromatin, 129

Contraception, 75, 149

Cytochrome P450, 19

Cytotrophoblasts, 221

D

Development endocrine, 19

sexual, 181

Differentiation, 435

$\mathbf{E}$

Embryogenesis, 221

Endocrine development, 19

Estradiol, 235

Estrogen, 235, 277, 295, 317, 339, 357, 385

Estrogenopathy, 295

F

Female-specific pathways, 1

Feminization, 181

Fertility, 149

Fertilization, 37

Fibroid tumors, 277

Follicle-stimulating hormone, 149, 195, 257 receptor, 129 
Follicular

activity, 257

development, 195

G

Gamete

binding, 37

male, 103

Gametogenesis, 75, 103

Gene

expression of male germ cells, 103 identification, 1

mutations, 181, 221

transcription, 221

Germ cells, 75, 149

male, 103

Gonad, 1, 19

Gonadotropin, 149

-releasing hormone, 235

H

Hippocampus, 357

Hormonal changes, 257

Hormone, 277

regulation, 149

replacement therapy, 235, 385

Human immunodeficiency virus, 411

Hypothalamus, 235, 357

I

Immune system, 435

Infertility, 181

Inflammation, 435

Inhibin, 195

B, 257

Inhibitors

aromatase, 317

phase III, 317
L

Leiomyoma, uterine, 277

Luteinization, 195

M

Male

germ cells, 103

-specific pathways, 1

Mammals, sex determination, 1

Meiosis, 75, 103

Meiotic cell cycles, 75

Memory, 357

Menopause, 235, 257

Menstruation, 257

Mesenchymal cells, 385

Mitosis, 103

Mitotic cell cycles, 75

Models, animal, 37, 129, 277

Modulation, neuroendocrine, 235

Molecular pathways, 1

Mutations, 181, 22

$\mathbf{N}$

Neuroendocrine modulation, 235

Neurons, catecholaminergic, 357

O

Oligosaccharides, 37

Osteoblastogenesis, 385

Osteoblasts, 385

Osteoclastogenesis, 385

Osteoclasts, 385

Osteoporosis, 295

Ovary, 195, 235, 257

steroids, 357

Ovulation, 195 
$\mathbf{P}$

Pathways

cellular, 1

female-specific, 1

molecular, 1

serotonin, 357

signaling, 195

Peptides, 195

Phase III inhibitors, 317

Pituitary, 235, 435

Placenta, 221

Progesterone receptors, 339

Prolactin, 435

Proliferation, 435

Protein synthesis, 411

$\mathbf{R}$

Receptors

androgen, 181

progesterone, 339

Reproduction, 37, 75, 149, 339, 357

aging, 235

assisted, 149

Reproductive organs, 235

$\mathbf{S}$

Selective estrogen receptor modulators, 277 , 295

Serotonin pathways, 357

Sertoli cells, 129

Sex

determination, 1

steroids, 385

Sexual development, 181

Signaling pathways, 195

Skeleton, 385

Sperm, 37
Spermatogenesis, 129, 149

Spermatogonia, 149

Sry gene, 1

Steroid

anabolic, 411

hormones, 195, 277, 357, 385

hydroxylase, 19

ovary, 357

Steroidogenic factor 1,19

Syncytiotrophoblast, 221

Tamoxifen, 277, 317

Testicular feminization, 181

Testis, 129

Testosterone, 149, 411

Therapy

adjuvant, 317

hormone replacement, 235, 385

Transcription, 435

factors, 1, 129, 221

Trophoblasts, 221

Tumors, fibroid, 277

$\mathbf{U}$

Uterus, leiomyoma, 277

$\mathbf{X}$

Xenoestrogens, 277

Y

Y chromosome, 1 
